\title{
3-Silaazetidine: An Unexplored yet Versatile Organosilane Species for Ring Expansion toward Silaazacycles
}

Wanshu Wang, Song Zhou, Linjie Li, Yuanhang He, Xue Dong, Lu Gao, Qiantao Wang, Zhenlei Song*

Key Laboratory of Drug-Targeting and Drug Delivery System of the Education Ministry and Sichuan Province, Sichuan Engineering Laboratory for Plant-Sourced Drug and Sichuan Research Center for Drug Precision Industrial Technology, West China School of Pharmacy, Sichuan University, Chengdu, 610041, People's Republic of China.

\section{Table of Contents}

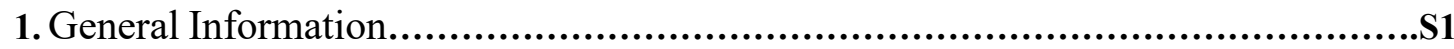

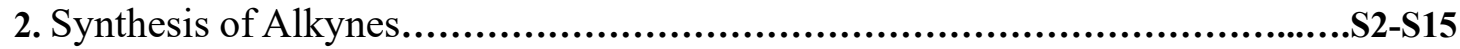

3. Synthesis of 3-silaazetidine Precursor Reagents 2... ...........................S16-S22

4. Optimization of Reaction Conditions..........................................S23-S24

5. Synthesis of 3-silatetrahydropyridines 4....................................S25-S52

6. Data of By-products..............................................................553-S53

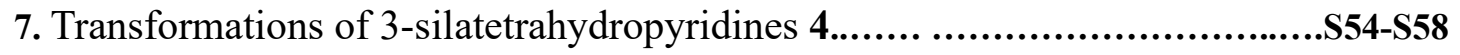

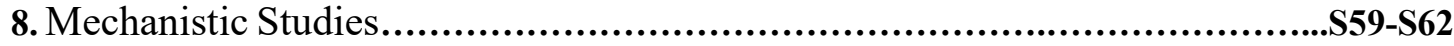

9. X-Ray Crystallographic Data..........................................................563-S75

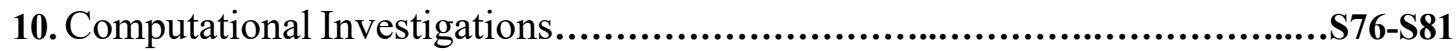

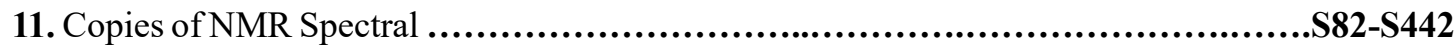




\section{General Information}

$\mathrm{Pd}\left(\mathrm{PPh}_{3}\right)_{2} \mathrm{Cl}_{2}$ and DBU were purchased from TCI, $\mathrm{ClCH}_{2} \mathrm{SiMe}_{2} \mathrm{CH}_{2} \mathrm{Cl}$ was purchased from $\mathrm{WuXi}$ AppTec (WuXi LabNetWork). TsBocNH was purchased from Energy Chemicals. ZnI 2 was purchased from Acros. $\mathrm{Pd}\left(\mathrm{PPh}_{3}\right)_{4}$ was purchased from J\&K. Other reagents were used as purchased from J\&K, Aldrich, Acros, Alfa Aesar, TCI or Energy Chemicals reagent suppliers. Alkynes were purchased for those are commercially available, or prepared according to literatures for those are known compounds, or synthesized as new compounds. $\mathrm{CH}_{3} \mathrm{CN}, \mathrm{DMF}, \mathrm{CH}_{2} \mathrm{Cl}_{2}$ and $\mathrm{Et}_{3} \mathrm{~N}$ were distilled from $\mathrm{CaH}_{2}$. toluene, xylenes, $\mathrm{Et}_{2} \mathrm{O}$ and $\mathrm{THF}$ were distilled from sodium. Inert atmosphere techniques were carried out through Schlenk system and Standard Glovebox (purchased from Vigor Gas Purification Technologies (Suzhou) Co. Ltd.). TLC which was performed on glass-backed silica plates (purchased from Yantai Jiangyou Silica Gel Development Co. Ltd.) and visualized using UV at $254 \mathrm{~nm}, \mathrm{KMnO}_{4}$ stains, $\mathrm{H}_{3} \mathrm{PO}_{4} \cdot 12 \mathrm{MoO}_{3} / \mathrm{EtOH}$ stains. Column chromatography was performed using silica gel (200-300 or 300-400 mesh, purchased from Yantai Jiangyou Silica Gel Development Co. Ltd.). Preparative TLC separations were performed using Kangbino 48-75 $\AA \mathrm{SiO}_{2}$. Melting point were recorded at WRX-4 Melting-point Apparatus (purchased from Shanghai Yice Apparatus \& Equipments Co. Lit.). ${ }^{1} \mathrm{H}$ NMR spectra were recorded at $400 \mathrm{MHz}$ (Varian and Bruker) or 600 $\mathrm{MHz}$ (Agilent) using $\mathrm{CDCl}_{3}$ (except where noted), ${ }^{13} \mathrm{C}$ NMR spectra were recorded at $100 \mathrm{MHz}$ (Bruker) or $150 \mathrm{MHz}$ (Agilent) using $\mathrm{CDCl}_{3}$ (except where noted), ${ }^{19} \mathrm{~F}$ NMR spectra were recorded at $376 \mathrm{MHz}$ (Agilent) using $\mathrm{CDCl}_{3}$ (except where noted), ${ }^{29} \mathrm{Si}$ NMR spectra were recorded at 119 $\mathrm{MHz}$ (Bruker) using toluene- $d_{8}$ as solvent, ${ }^{31} \mathrm{P}$ NMR spectra were recorded at $162 \mathrm{MHz}$ (Bruker) using toluene- $d_{8}$ as solvent. All coupling constants ( $J$ values) were reported in hertz (Hz). Multiplicities are reported as follows: singlet (s), doublet (d), doublet of doublets (dd), triplet (t), quartet (q), and multiplet (m). High-resolution mass spectral analyses performed on Waters Q-TOF in positive mode. Infrared spectra were obtained using PerkinElmer Spectrum Two FTIR Spectrometer. X-ray diffraction experiment was carried out on Xcalibur E and the data obtained was deposited at the Cambridge Crystallographic Data Centre. Enantiomeric excess was determined by HPLC (Agilent Technologies: 1260 Infinity II) analysis on a Chiralpak® AD-H column. Specific optical rotation was measured on PL341 Polarimeter (PerkinElmer). 


\section{Synthesis of Alkynes}

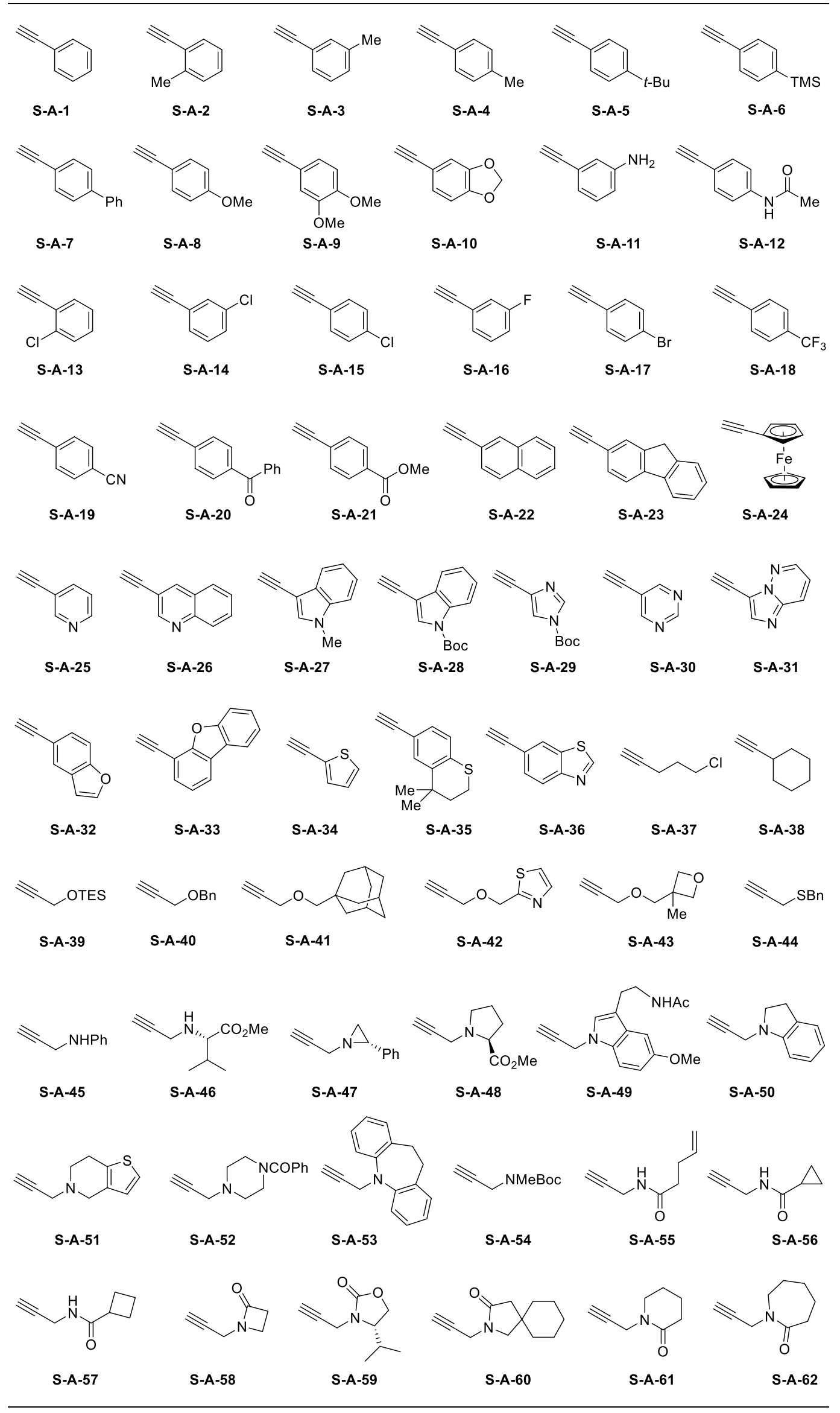




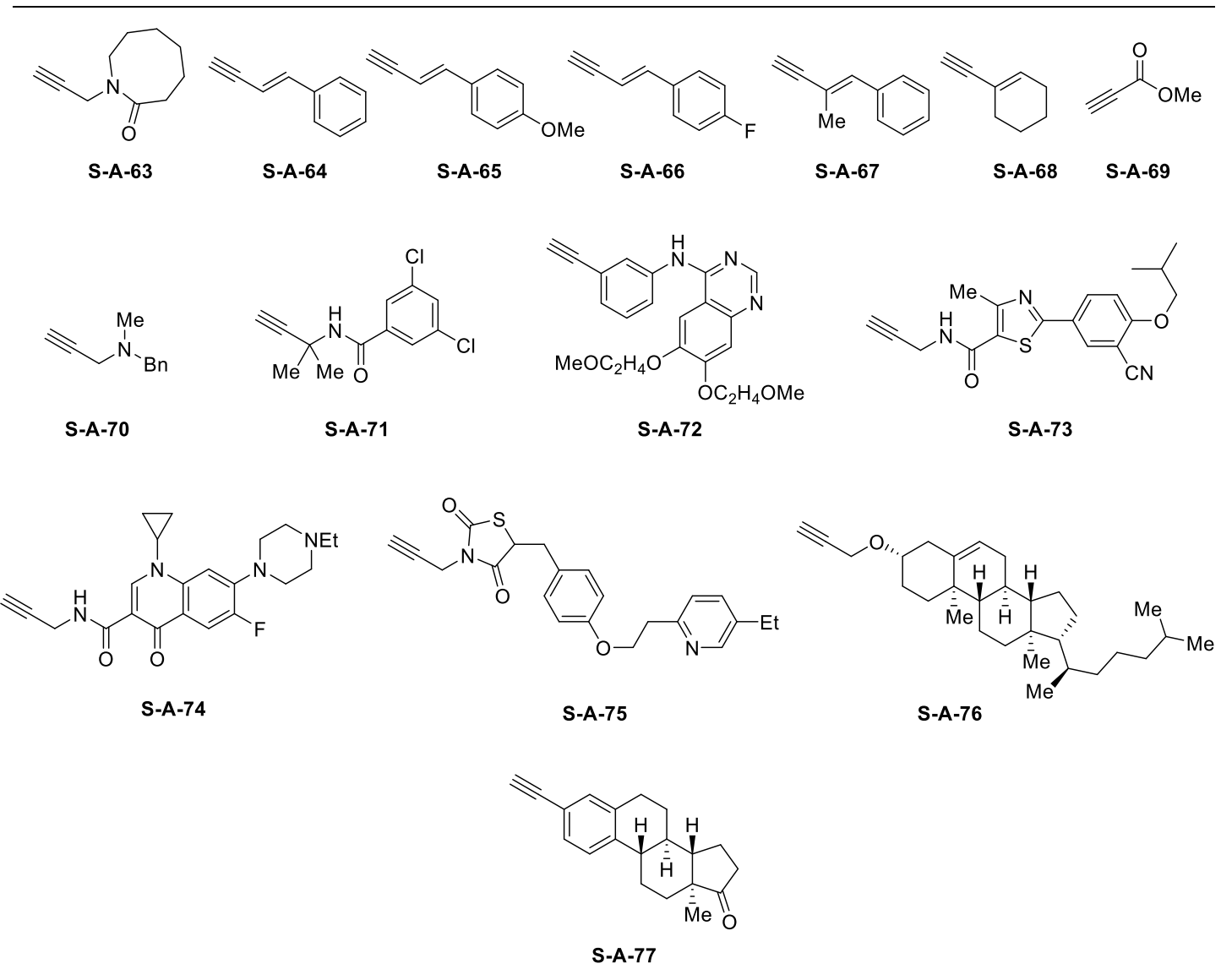

\section{Commercially Available Alkynes:}

S-A-1, S-A-2, S-A-3, S-A-4, S-A-5, S-A-7, S-A-8, S-A-11, S-A-13, S-A-14, S-A-15, S-A-16, S-A17, S-A-18, S-A-19, S-A-21, S-A-22, S-A-24, S-A-25, S-A-34, S-A-35, S-A-37, S-A-38, S-A-68, S-A-69, S-A-71, S-A-72.

\section{Known Alkynes:}

S-A-6, S-A-9, S-A-10, S-A-12, S-A-20, S-A-23, S-A-26, S-A-27, S-A-28, S-A-29, S-A-30, S-A-31, S-A-32, S-A-33, S-A-36, S-A-39, S-A-40, S-A-41, S-A-43, S-A-44, S-A-45, S-A-46, S-A-48, S-A50, S-A-51, S-A-53, S-A-54, S-A-55, S-A-56, S-A-57, S-A-58, S-A-61, S-A-62, S-A-63, S-A-64, S-A-65, S-A-66, S-A-67, S-A-70, S-A-76, S-A-77.

\section{$>$ New Alkynes:}

S-A-42, S-A-47, S-A-49, S-A-52, S-A-59, S-A-60, S-A-73, S-A-74, S-A-75.

\section{General Method A:}

General Method A was used for preparation of known alkynes S-A-43, S-A-44, S-A-53, S-A-61, SA-62, S-A-63 and new alkynes S-A-42, S-A-47, S-A-49, S-A-52, S-A-59, S-A-60, S-A-75.

$$
\underset{(X=N, O, S)}{\mathrm{RXH}}+\mathrm{Br} \sim \frac{\text { base }}{\text { solvent, } 0^{\circ} \mathrm{C}-\mathrm{rt}} \mathrm{RX}
$$

To a solution of RXH (1.0 equiv.) in $\mathrm{MeOH}$ or DMF was added base (1.1 to 2.0 equiv.) at $0{ }^{\circ} \mathrm{C}$ or room temperature. After stirring for $15 \mathrm{~min}$, 3-bromoprop-1-yne was added to the above mixture at $0{ }^{\circ} \mathrm{C}$ or room temperature. The reaction was stirred at room temperature until $\mathrm{RXH}$ was completely consumed (monitored by TLC analysis). The reaction was quenched with water, extracted with EtOAc. The combined organic phases were washed with sat. aq. $\mathrm{NaCl}$, dried over $\mathrm{Na}_{2} \mathrm{SO}_{4}$. and concentrated under reduced pressure. The crude residue was purified by silica gel flash column chromatography to give the desired alkyne. 


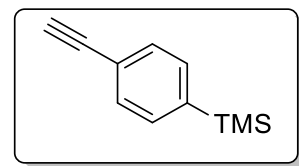

(4-ethynvlphenvl)trimethylsilane (S-A-6)

S-A-6 was prepared according to the literature, and the NMR spectral data are in accordance with the reported data ${ }^{1}$.

$>$ colorless oil, $0.49 \mathrm{~g}, 70 \%$.

$\mathrm{R}_{f}=0.8$ (Petroleum Ether).

$>{ }^{1} \mathrm{H} \mathrm{NMR}\left(400 \mathrm{MHz}, \mathrm{CDCl}_{3}\right) \delta 7.38(\mathrm{~s}, 4 \mathrm{H}), 2.99(\mathrm{~s}, 1 \mathrm{H}), 0.18(\mathrm{~s}, 9 \mathrm{H})$.

$>{ }^{13} \mathrm{C}$ NMR $\left(100 \mathrm{MHz}, \mathrm{CDCl}_{3}\right) \delta 143.0,134.4,132.5,123.6,85.1,78.7,0.0$.

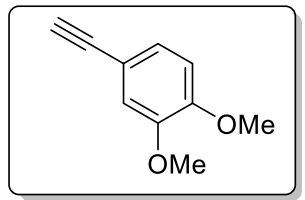

4-ethynyl-1,2-dimethoxybenzene (S-A-9)

S-A-9 was prepared according to the literature, and the NMR spectral data are in accordance with the reported data ${ }^{2}$.

$>\quad$ white solid, $0.27 \mathrm{~g}, 36 \%$.

$>\mathrm{R}_{f}=0.25$ (EtOAc:Petroleum Ether $\left.=1: 25\right)$.

${ }^{1} \mathrm{H}$ NMR $\left(400 \mathrm{MHz}, \mathrm{CDCl}_{3}\right) \delta 7.10(\mathrm{dd}, J=8.0,1.8 \mathrm{~Hz}, 1 \mathrm{H}), 6.99(\mathrm{~d}, J=1.8 \mathrm{~Hz}, 1 \mathrm{H}), 6.80$

(d, $J=8.0 \mathrm{~Hz}, 1 \mathrm{H}), 3.89(\mathrm{~s}, 3 \mathrm{H}), 3.88(\mathrm{~s}, 3 \mathrm{H}), 3.01(\mathrm{~s}, 1 \mathrm{H})$.

$>{ }^{13} \mathrm{C}$ NMR $\left(100 \mathrm{MHz}, \mathrm{CDCl}_{3}\right) \delta 149.9,148.6,125.5,114.7,114.2,110.9,83.8,75.7,55.9$.

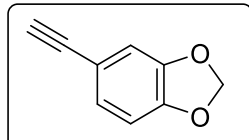

\section{5-ethynylbenzo/d][1,3/dioxole (S-A-10)}

S-A-10 was prepared according to the literature, and the NMR spectral data are in accordance with the reported data ${ }^{3}$.

$>$ yellow solid, $0.52 \mathrm{~g}, 72 \%$.

$>\mathrm{R}_{f}=0.8$ (Petroleum Ether)

$>\quad{ }^{1} \mathrm{H}$ NMR $\left(400 \mathrm{MHz}, \mathrm{CDCl}_{3}\right) \delta 7.02(\mathrm{dd}, J=8.0,1.6 \mathrm{~Hz}, 1 \mathrm{H}), 6.93(\mathrm{~d}, J=1.6 \mathrm{~Hz}, 1 \mathrm{H}), 6.75(\mathrm{~d}$, $J=8.0 \mathrm{~Hz}, 1 \mathrm{H}), 5.96(\mathrm{~s}, 2 \mathrm{H}), 2.97(\mathrm{~s}, 1 \mathrm{H})$.

$>{ }^{13} \mathrm{C} \mathrm{NMR}\left(100 \mathrm{MHz}, \mathrm{CDCl}_{3}\right) \delta 148.3,147.4,126.9,115.3,112.0,108.4,101.4,83.6,75.7$.

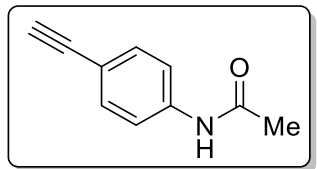

$\mathrm{N}$-(4-ethvnvlphenvl)acetamide (S-A-12)

S-A-12 was prepared according to the literature, and the NMR spectral data are in accordance with the reported data ${ }^{4}$.

$>$ yellow solid, $1.32 \mathrm{~g}, 98 \%$.

$>\mathrm{R}_{f}=0.2(\mathrm{EtOAc} /$ Petroleum Ether $=1: 3)$.

$>{ }^{1} \mathrm{H}$ NMR $\left(400 \mathrm{MHz}, \mathrm{CDCl}_{3}\right) \delta 7.70(\mathrm{br}, \mathrm{s}, 1 \mathrm{H}), 7.50-7.42(\mathrm{~m}, 4 \mathrm{H}), 3.05(\mathrm{~s}, 1 \mathrm{H}), 2.17(\mathrm{~s}, 3 \mathrm{H})$.

$>{ }^{13} \mathrm{C} \mathrm{NMR}\left(100 \mathrm{MHz}, \mathrm{CDCl}_{3}\right) \delta 168.7,138.4,132.9,119.4,117.7,83.4,76.7,24.6$.

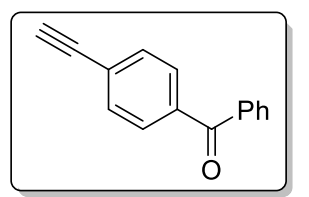

(4-ethynvlphenvl)(phenvl)methanone (S-A-20)

S-A-20 was prepared according to the literature, and the NMR spectral data are in accordance with the reported data ${ }^{5}$.

$>$ pale yellow solid, $0.45 \mathrm{~g}, 61 \%$.

$>\mathrm{R}_{f}=0.4($ EtOAc/Petroleum Ether=1:10).

$>{ }^{1} \mathrm{H} \mathrm{NMR}\left(400 \mathrm{MHz}, \mathrm{CDCl}_{3}\right) \delta 7.80-7.76(\mathrm{~m}, 4 \mathrm{H}), 7.62-7.59(\mathrm{~m}, 3 \mathrm{H}), 7.51-7.47(\mathrm{~m}, 2 \mathrm{H})$, $3.25(\mathrm{~s}, 1 \mathrm{H})$.

$>{ }^{13} \mathrm{C}$ NMR $\left(100 \mathrm{MHz}, \mathrm{CDCl}_{3}\right) \delta 195.9,137.4,137.3,132.7,132.0,130.0,130.0,128.4,126.3$, $82.8,80.1$.

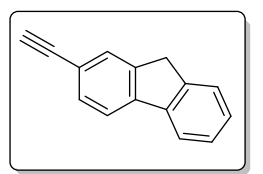

\section{2-ethynyl-9H-fluorene (S-A-23)}

S-A-23 was prepared according to the literature, and the NMR spectral data are in accordance with the reported data ${ }^{6}$.

white solid, $0.58 \mathrm{~g}, 89 \%$.

$\mathrm{R}_{f}=0.5$ (Petroleum Ether).

$>\quad{ }^{1} \mathrm{H}$ NMR $\left(400 \mathrm{MHz}, \mathrm{CDCl}_{3}\right) \delta 7.77(\mathrm{~d}, J=7.5 \mathrm{~Hz}, 1 \mathrm{H}), 7.72(\mathrm{~d}, J=7.9 \mathrm{~Hz}, 1 \mathrm{H}), 7.66(\mathrm{~s}, 1 \mathrm{H})$, $7.57-7.49(\mathrm{~m}, 2 \mathrm{H}), 7.38(\mathrm{td}, J=7.4,1.1 \mathrm{~Hz}, 1 \mathrm{H}), 7.32(\mathrm{td}, J=7.4,1.3 \mathrm{~Hz}, 1 \mathrm{H}), 3.88(\mathrm{~s}, 2 \mathrm{H}), 3.11$ $(\mathrm{s}, 1 \mathrm{H})$.

$>{ }^{13} \mathrm{C} \mathrm{NMR}\left(100 \mathrm{MHz}, \mathrm{CDCl}_{3}\right) \delta 143.6,143.1,142.4,141.0,131.0,128.7,127.3,127.0,125.1$,

1. Wu, J.-S.; Watson, M.-D.; Tchebotareva, N.; Wang, Z.-H.; Müllen, K. J. Org. Chem. 2004, 69, 8194-8204.

2. Feng, L.; Hu, T.; Zhang, S.; Xiong, H.-Y.; Zhang, G.-W. Org. Lett. 2019, 21, 9487-9492.

3. Dong, X.-Y.; Zhang, Y.-F.; Ma, C.-L.; Gu, Q.-S.; Wang, F.-L.; Li, Z.-L.; Jiang, S.-P.; Liu, X.-Y. Nat. Chem. 2019, 11, 1158-1166.

4. Wong, K.-F.; Deng, J.-R.; Wei, X.-Q.; Shao, S.-P.; Xiang, D.-P.; Wong, M.-K. V. Org. Biomol. Chem. 2015, 13, 7408-7411.

5. Zhao, E.-G.; Li, H.-K.; Ling, J.; Wu, H.-Q.; Wang, J.; Zhang, S.; Lam, J. W.-Y.; Sun, J.-Z.; Qin, A. J.; Tang, B. Z. Polym. Chem. 2014, 5, 2301-2308.

6. Görl, C.; Alt. H. G. J. Organomet. Chem. 2007, 692, 4580-4592. 
$120.3,120.1,119.8,84.4,77.0,36.7$.

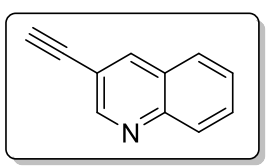

3-ethynylquinoline (S-A-26)

S-A-26 was prepared according to the literature, and the NMR spectral data are in accordance with the reported data ${ }^{7}$.

$>$ white solid, $1.2 \mathrm{~g}, 78 \%$.

$>\mathrm{R}_{f}=0.8$ (EtOAc:Petroleum Ether=1:4).

$>{ }^{1} \mathrm{H} \mathrm{NMR}\left(400 \mathrm{MHz}, \mathrm{CDCl}_{3}\right) \delta 8.95(\mathrm{~d}, J=2.0 \mathrm{~Hz}, 1 \mathrm{H}), 8.28(\mathrm{~d}, J=2.0 \mathrm{~Hz}, 1 \mathrm{H}), 8.10(\mathrm{~d}, J=$ $8.5 \mathrm{~Hz}, 1 \mathrm{H}), 7.79-7.71(\mathrm{~m}, 2 \mathrm{H}), 7.59-7.55(\mathrm{~m}, 1 \mathrm{H}), 3.28(\mathrm{~s}, 1 \mathrm{H})$.

$>{ }^{13} \mathrm{C}$ NMR $\left(100 \mathrm{MHz}, \mathrm{CDCl}_{3}\right) \delta 152.3,147.1,139.4,130.4,129.5,127.6,127.4,127.0,116.3$, 81.0, 80.5.

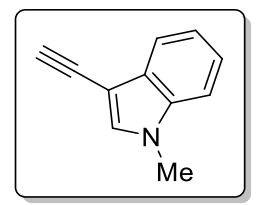

\section{3-ethynyl-1-methyl-1H-indole (S-A-27)}

S-A-27 was prepared according to the literature, and the NMR spectral data are in accordance with the reported data ${ }^{8}$.

$>$ yellow oil, $0.23 \mathrm{~g}, 38 \%$.

$>\mathrm{R}_{f}=0.3$ (EtOAc:Petroleum Ether=1:10).

$>{ }^{1} \mathrm{H}$ NMR $\left(400 \mathrm{MHz}, \mathrm{CDCl}_{3}\right) \delta 7.73(\mathrm{~d}, J=8.0 \mathrm{~Hz}, 1 \mathrm{H}), 7.31-7.17(\mathrm{~m}$, $4 \mathrm{H}), 3.74(\mathrm{~s}, 3 \mathrm{H}), 3.20(\mathrm{~s}, 1 \mathrm{H})$.

$>{ }^{13} \mathrm{CNMR}\left(100 \mathrm{MHz}, \mathrm{CDCl}_{3}\right) \delta 136.1,133.1,129.3,122.7,120.5,120.1,109.6,95.8,78.7,77.7$, 33.1 .

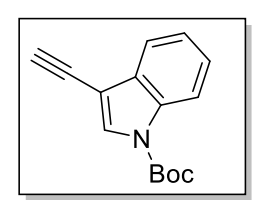

\section{tert-butyl 3-ethynyl-1H-indole-1-carboxylate (S-A-28)}

S-A-28 was prepared according to the literature, and the NMR spectral data are in accordance with the reported data 9 .

$>$ brown oil, $0.23 \mathrm{~g}, 47 \%$.

$>\mathrm{R}_{f}=0.7$ (EtOAc:Petroleum Ether $=1: 10$ ).

$>{ }^{1} \mathrm{H} \mathrm{NMR}\left(400 \mathrm{MHz}, \mathrm{CDCl}_{3}\right) \delta 8.16(\mathrm{~d}, J=8.2 \mathrm{~Hz}, 1 \mathrm{H}), 7.84(\mathrm{~s}, 1 \mathrm{H}), 7.70(\mathrm{~d}, J=7.6 \mathrm{~Hz}, 1 \mathrm{H})$, 7.35 (dtd, $J=24.0,7.3,1.3 \mathrm{~Hz}, 2 \mathrm{H}), 3.27$ (s, $1 \mathrm{H}), 1.68$ (s, 9H).

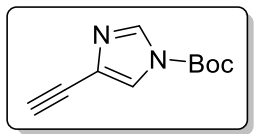

\section{tert-butyl 4-ethynyl-1H-imidazole-1-carboxylate (S-A-29)}

S-A-29 was prepared according to the literature, and the NMR spectral data are in accordance with the reported data ${ }^{10}$.

$>$ white solid, $0.128 \mathrm{~g}, 7 \%$.

$>\mathrm{R}_{f}=0.3$ (EtOAc:Petroleum Ether=1:10).

$>\quad{ }^{1} \mathrm{H} \mathrm{NMR}\left(400 \mathrm{MHz}, \mathrm{CDCl}_{3}\right) \delta 8.00(\mathrm{~d}, J=1.3 \mathrm{~Hz}, 1 \mathrm{H}), 7.55(\mathrm{~d}, J=1.3 \mathrm{~Hz}, 1 \mathrm{H}), 3.10(\mathrm{~s}, 1 \mathrm{H})$, $1.62(\mathrm{~s}, 9 \mathrm{H})$.

$>{ }^{13} \mathrm{C} \mathrm{NMR}\left(100 \mathrm{MHz}, \mathrm{CDCl}_{3}\right) \delta 146.2,136.8,124.8,121.3,86.5,78.7,76.2,27.9$.

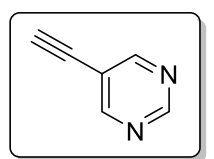

\section{5-ethynylpyrimidine (S-A-30)}

S-A-30 was prepared according to the literature, and the NMR spectral data are in accordance with the reported data ${ }^{11}$.

$>$ white solid, $0.56 \mathrm{~g}, 52 \%$.

$>\mathrm{R}_{f}=0.4$ (EtOAc:Petroleum Ether=1:5).

$>{ }^{1} \mathrm{H} \mathrm{NMR}\left(400 \mathrm{MHz}, \mathrm{CDCl}_{3}\right) \delta 7.92(\mathrm{~s}, 1 \mathrm{H}), 7.57(\mathrm{~s}, 2 \mathrm{H}), 2.15(\mathrm{~s}, 1 \mathrm{H})$.

$>{ }^{13} \mathrm{C} \mathrm{NMR}\left(100 \mathrm{MHz}, \mathrm{CDCl}_{3}\right) \delta 159.4,157.3,118.8,84.5,76.9$.

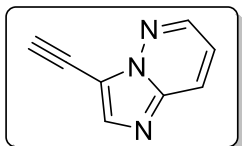

\section{3-ethynylimidazo/1,2-blpyridazine (S-A-31)}

S-A-31 was prepared according to the literature, and the NMR spectral data are in accordance with the reported data ${ }^{12}$.

brown solid, $0.6 \mathrm{~g}, 83 \%$.

$>\mathrm{R}_{f}=0.2$ (EtOAc:Petroleum Ether=1:4).

$>{ }^{1} \mathrm{H}$ NMR $\left(400 \mathrm{MHz}, \mathrm{CDCl}_{3}\right) \delta 8.48(\mathrm{dd}, J=4.4,1.7 \mathrm{~Hz}, 1 \mathrm{H}), 8.04-7.93(\mathrm{~m}, 2 \mathrm{H}), 7.14(\mathrm{dd}, J$

7. Dutta, U.; Lupton, D. W.; Maiti, D. Org. Lett. 2016, 18, 860-863.

8. Sha, F.; Tao, Y.; Tang, C.-Y.; Zhang, F.; Wu, X.-Y. J. Org. Chem., 2015, 80, 8122-8133.

9. Xu, H.-D.; Zhang, R.-W.; Li, X.-X.; Huang, S.-T.; Tang, W.-P.; Hu, W.-H. Org. Lett. 2013, 15, 840-843.

10. Merkul, E.; Klukas, F.; Dorsch, D.; Grädler, U.; Greiner, H. E.; Müller, T. J. J. Org. Biomol. Chem. 2011, 9, 5129-5136.

11. Richardson, C.; Reed, C. A. J. Org. Chem. 2007, 72, 4750-4755.

12. Huang, W.-S.; Metcalf, C. A.; Sundaramoorthi, R.; et al. J. Med. Chem. 2010, 53, 4701-4719. 
$=9.2,4.4 \mathrm{~Hz}, 1 \mathrm{H}), 3.82(\mathrm{~s}, 1 \mathrm{H})$.

$>{ }^{13} \mathrm{C}$ NMR $\left(100 \mathrm{MHz}, \mathrm{CDCl}_{3}\right) \delta 144.0,139.5,139.0,126.0,117.9,112.3,87.3,70.6$.

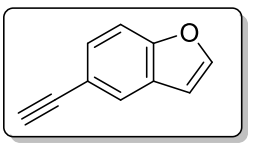

\section{5-ethynylbenzofuran (S-A-32)}

S-A-32 was prepared according to the literature ${ }^{13}$. and the NMR spectral data are in accordance with the reported data ${ }^{14}$.

$>$ yellow oil, $0.29 \mathrm{~g}, 40 \%$.

$>\mathrm{R}_{f}=0.6$ (Petroleum Ether)

$>{ }^{1} \mathrm{H} \mathrm{NMR}\left(400 \mathrm{MHz}, \mathrm{CDCl}_{3}\right) \delta 7.63(\mathrm{~d}, J=1.4 \mathrm{~Hz}, 1 \mathrm{H}), 7.50(\mathrm{~d}, J=2.3 \mathrm{~Hz}, 1 \mathrm{H}), 7.31(\mathrm{~d}, J=$ $1.6 \mathrm{~Hz}, 2 \mathrm{H}), 6.61(\mathrm{~d}, J=2.2 \mathrm{~Hz}, 1 \mathrm{H}), 2.91(\mathrm{~s}, 1 \mathrm{H})$

$>{ }^{13} \mathrm{C}$ NMR $\left(100 \mathrm{MHz}, \mathrm{CDCl}_{3}\right) \delta 154.8,146.0,128.5,127.5,125.5,116.6,111.6,106.5,84.0$, 75.8 .

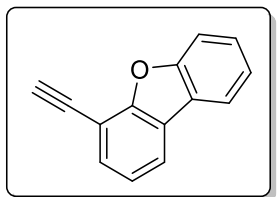

\section{4-ethynyldibenzo/b,dlfuran (S-A-33)}

S-A-33 was prepared according to the literature, and the NMR spectral data are in accordance with the reported data ${ }^{15}$.

yellow solid, $0.15 \mathrm{~g}, 39 \%$.

$\mathrm{R}_{f}=0.8$ (EtOAc:Petroleum Ether=1:10).

$>{ }^{1} \mathrm{H}$ NMR $\left(400 \mathrm{MHz}, \mathrm{CDCl}_{3}\right) \delta 7.95(\mathrm{dt}, J=7.7,1.6 \mathrm{~Hz}, 2 \mathrm{H}), 7.69-7.63$ (m, 1H), 7.60 (dd, $J=7.6,1.3 \mathrm{~Hz}, 1 \mathrm{H}), 7.49$ (ddd, $J=8.4,7.3,1.4 \mathrm{~Hz}, 1 \mathrm{H}), 7.37$ (td, $J=7.5$, $1.0 \mathrm{~Hz}, 1 \mathrm{H}), 7.31(\mathrm{t}, J=7.7 \mathrm{~Hz}, 1 \mathrm{H}), 3.48(\mathrm{~s}, 1 \mathrm{H})$.

$>{ }^{13} \mathrm{C} \mathrm{NMR}\left(100 \mathrm{MHz}, \mathrm{CDCl}_{3}\right) \delta 156.6,156.2,131.0,127.7,124.5,123.8,123.1,122.7,121.5$, $120.8,112.1,106.6,82.2,78.2$.

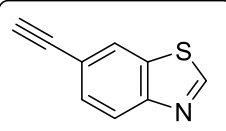

\section{6-ethynvlbenzo[d/thiazole (S-A-36)}

S-A-36 was prepared according to the literature, and the NMR spectral data are in accordance with the reported data ${ }^{14}$. white solid, $0.67 \mathrm{~g}, 94 \%$.

$\mathrm{R}_{f}=0.5$ (EtOAc:Petroleum Ether $\left.=1: 10\right)$.

$>{ }^{1} \mathrm{H} \mathrm{NMR}\left(400 \mathrm{MHz}, \mathrm{CDCl}_{3}\right) \delta 9.04(\mathrm{~s}, 1 \mathrm{H}), 8.10-8.06(\mathrm{~m}, 2 \mathrm{H}), 7.62(\mathrm{dd}, J=8.5,1.6 \mathrm{~Hz}, 1 \mathrm{H})$, $3.17(\mathrm{~s}, 1 \mathrm{H})$.

$>{ }^{13} \mathrm{C} \mathrm{NMR}\left(100 \mathrm{MHz}, \mathrm{CDCl}_{3}\right) \delta 155.3,153.2,133.8,130.1,125.9,123.5,119.6,83.1,78.2$.

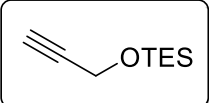

\section{triethyl(prop-2-yn-1-yloxy)silane (S-A-39)}

S-A-39 was prepared according to the literature, and the NMR spectral data are in accordance with the reported data ${ }^{16}$.

$>$ colorless liquid, $6.5 \mathrm{~g}, 90 \%$.

$>\mathrm{R}_{f}=0.4$ (Petroleum Ether).

$>{ }^{1} \mathrm{H} \mathrm{NMR}\left(400 \mathrm{MHz}, \mathrm{CDCl}_{3}\right) \delta 4.31(\mathrm{~d}, J=2.4 \mathrm{~Hz}, 2 \mathrm{H}), 2.39(\mathrm{t}, J=2.4 \mathrm{~Hz}, 1 \mathrm{H}), 0.98(\mathrm{t}, J=$ $7.9 \mathrm{~Hz}, 9 \mathrm{H}), 0.69-0.63(\mathrm{~m}, 6 \mathrm{H})$.

$>{ }^{13} \mathrm{C} \mathrm{NMR}\left(100 \mathrm{MHz}, \mathrm{CDCl}_{3}\right) \delta 82.3,72.8,51.1,6.6,4.4$.

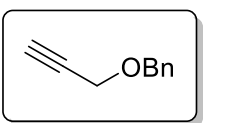

\section{((prop-2-yn-1-yloxy)methyl)benzene (S-A-40)}

S-A-40 was prepared according to the literature, and the NMR spectral data are in accordance with the reported data ${ }^{17}$.

$>$ pale white oil, $2.0 \mathrm{~g}, 53 \%$.

$>\mathrm{R}_{f}=0.3$ (Petroleum Ether).

$>{ }^{1} \mathrm{H}$ NMR $\left(400 \mathrm{MHz}, \mathrm{CDCl}_{3}\right) \delta 7.37-7.34(\mathrm{~m}, 4 \mathrm{H}), 7.33-7.27(\mathrm{~m}, 1 \mathrm{H}), 4.60(\mathrm{~s}, 2 \mathrm{H}), 4.16(\mathrm{~d}$, $J=2.4 \mathrm{~Hz}, 2 \mathrm{H}), 2.45(\mathrm{t}, J=2.4 \mathrm{~Hz}, 1 \mathrm{H})$.

$>{ }^{13} \mathrm{C} \mathrm{NMR}\left(100 \mathrm{MHz}, \mathrm{CDCl}_{3}\right) \delta 137.3,128.5,128.2,128.0,79.7,74.7,71.6,57.1$.

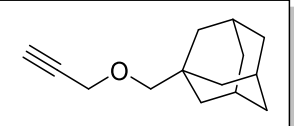

\section{(3r,5r,7r)-1-((prop-2-yn-1-yloxy)methyl)adamantane (S-A-41)}

S-A-41 was prepared according to the literature, and the NMR spectral data

13. Tien, C. H.; Trofimova, A.; Holownia, A.; Kwak, B. S.; Larson, R. T.; Yudin, A. K. Angew. Chem. Int. Ed. 2021, 60, $4342-4349$.

14. Xu, H.; Ma, B.; Fu, Z.-Y.; Li, H.-Y.; Wang, X.; Wang, Z.-Y.; Li, L.-J.; Cheng, T.-J.; Zheng, M.-Y.; Dai, H. X. ACS Catal. 2021, 11, 17581764.

15. Cha, S.-W.; Shin, Y.; Lim, J.-G.; Park, S.-W.; Kim, J.H.; Yoo, J.-H.; Park, Y.-H. US2017/141322, 2017, A1.

16. Abdolreza, A.; Somayeh. J. Chin. Chem. Soc. 2014, 61, 929-934

17. Farran, D.; Slawin, A. M. Z.; Kirsch, P.; O’Hagan, D. J. Org.Chem. 2009, 74, 7168-7171. 
are in accordance with the reported data ${ }^{18}$.

$>$ colorless oil, $1.32 \mathrm{~g}, 72 \%$.

$>\mathrm{R}_{f}=0.5$ (Petroleum Ether).

$>{ }^{1} \mathrm{H} \mathrm{NMR}\left(400 \mathrm{MHz}, \mathrm{CDCl}_{3}\right) \delta 4.11(\mathrm{~d}, J=2.4 \mathrm{~Hz}, 2 \mathrm{H}), 3.08(\mathrm{~s}, 2 \mathrm{H}), 2.39(\mathrm{t}, J=2.4 \mathrm{~Hz}, 1 \mathrm{H})$, $1.97-1.95(\mathrm{~m}, 3 \mathrm{H}), 1.74-1.63(\mathrm{~m}, 6 \mathrm{H}), 1.55(\mathrm{~d}, J=3.0 \mathrm{~Hz}, 6 \mathrm{H})$.

$>{ }^{13} \mathrm{C} \mathrm{NMR}\left(100 \mathrm{MHz}, \mathrm{CDCl}_{3}\right) \delta 81.1,80.4,73.8,58.6,39.6,37.2,33.8,28.2$.

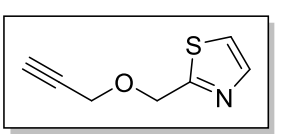

\section{2-((prop-2-yn-1-yloxv)methvl)-4,5-dihvdrothiazole (S-A-42)}

S-A-42 was prepared according to the General Method A. Thiazol-2ylmethanol (4.3 mmol, $0.5 \mathrm{~g}, 1.0$ equiv.), $\mathrm{NaH}$ ( $4.95 \mathrm{mmol}, 0.2 \mathrm{mg}, 60 \%$ dispersion in mineral oil, 1.15 equiv.), 3-bromoprop-1-yne (4.95 mmol, 0.59 g, 1.15 equiv.) in DMF $(5 \mathrm{~mL})$ at room temperature for $0.5 \mathrm{~h}$ to give $\mathbf{S - A - 4 2}$ as brown liquid $(0.62$ g, 94\%).

$>\mathrm{R}_{f}=0.5$ (EtOAc:Petroleum Ether $\left.=1: 3\right)$.

$>{ }^{1} \mathrm{H} \mathrm{NMR}\left(400 \mathrm{MHz}, \mathrm{CDCl}_{3}\right) \delta 7.77(\mathrm{~d}, J=3.2 \mathrm{~Hz}, 1 \mathrm{H}), 7.35(\mathrm{~d}, J=3.2 \mathrm{~Hz}, 1 \mathrm{H}), 4.93(\mathrm{~s}, 2 \mathrm{H})$, $4.31(\mathrm{~d}, J=2.4 \mathrm{~Hz}, 2 \mathrm{H}), 2.51(\mathrm{t}, J=2.4 \mathrm{~Hz}, 1 \mathrm{H})$.

$>{ }^{13} \mathrm{C} \mathrm{NMR}\left(100 \mathrm{MHz}, \mathrm{CDCl}_{3}\right) \delta 167.4,142.7,119.8,78.7,75.5,68.2,58.1$.

$>$ IR (neat) $\mathrm{cm}^{-1} 3290,3116,2856,2116,1503,1351,1189,1145,1079,1022,952,910$.

$>$ HRMS calcd for $\mathrm{C}_{7} \mathrm{H}_{7} \mathrm{NOS}(\mathrm{M}+\mathrm{Na})^{+} 176.0146$ found 176.0137.

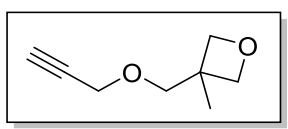

\section{3-methyl-3-((prop-2-yn-1-yloxy)methyl)oxetane (S-A-43)}

S-A-43 was prepared according to the General Method A. (3-Methyloxetan3-yl)methanol (9.7 mmol, $1.0 \mathrm{~g}, 1.0$ equiv.), $\mathrm{NaH}$ (19.5 mmol, $0.75 \mathrm{~g}, 60 \%$ dispersion in mineral oil, 2.0 equiv.), 3-bromoprop-1-yne (11.7 mmol, $1.38 \mathrm{~g}$, 1.2 equiv. $)$ in DMF $(10 \mathrm{~mL})$ at room temperature for $1 \mathrm{~h}$ to give $\mathbf{S - A - 4 3}$ as yellow liquid $(0.78 \mathrm{~g}$, $59 \%)$ and the NMR spectral data are in accordance with the reported data ${ }^{19}$.

$>\mathrm{R}_{f}=0.6($ EtOAc:Petroleum Ether=1:3).

$>{ }^{1} \mathrm{H} \mathrm{NMR}\left(400 \mathrm{MHz}, \mathrm{CDCl}_{3}\right) \delta 4.53(\mathrm{~d}, J=5.8 \mathrm{~Hz}, 2 \mathrm{H}), 4.38(\mathrm{~d}, J=5.8 \mathrm{~Hz}, 2 \mathrm{H}), 4.21(\mathrm{~d}, J=$ $2.3 \mathrm{~Hz}, 2 \mathrm{H}), 3.61(\mathrm{~s}, 2 \mathrm{H}), 2.45(\mathrm{t}, J=2.3 \mathrm{~Hz}, 1 \mathrm{H}), 1.33(\mathrm{~s}, 3 \mathrm{H})$.

$>{ }^{13} \mathrm{C} \mathrm{NMR}\left(100 \mathrm{MHz}, \mathrm{CDCl}_{3}\right) \delta 80.1,79.6,75.1,74.6,58.6,39.6,21.3$.

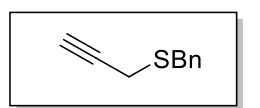

\section{benzyl(prop-2-yn-1-yl)sulfane (S-A-44)}

S-A-44 was prepared according to the General Method A. Phenylmethanethiol ( $8.0 \mathrm{mmol}, 1.0 \mathrm{~g}, 1.0$ equiv.), $\mathrm{NaOH}$ (9.6 mmol, $0.4 \mathrm{~g}, 1.2$ equiv.), 3-bromoprop1-yne ( $8.8 \mathrm{mmol}, 1.07 \mathrm{~g}, 1.1$ equiv.) in $\mathrm{MeOH}(7 \mathrm{~mL})$ at room temperature for $4 \mathrm{~h}$ to give $\mathbf{S}-\mathbf{A}-\mathbf{4 4}$ as yellow liquid $(1.01 \mathrm{~g}, 59 \%)$ and the NMR spectral data are in accordance with the reported data ${ }^{20}$.

$>\mathrm{R}_{f}=0.5$ (Petroleum Ether).

$>{ }^{1} \mathrm{H} \mathrm{NMR}\left(400 \mathrm{MHz}, \mathrm{CDCl}_{3}\right) \delta 7.34-7.29(\mathrm{~m}, 4 \mathrm{H}), 7.27-7.23(\mathrm{~m}, 1 \mathrm{H}), 3.86(\mathrm{~s}, 2 \mathrm{H}), 3.06(\mathrm{~d}$, $J=2.6 \mathrm{~Hz}, 2 \mathrm{H}), 2.28(\mathrm{t}, J=2.6 \mathrm{~Hz}, 1 \mathrm{H})$.

$>{ }^{13} \mathrm{C} \mathrm{NMR}\left(100 \mathrm{MHz}, \mathrm{CDCl}_{3}\right) \delta 137.5,129.1,128.6,127.2,79.9,71.4,35.3,18.4$.

\section{N-(prop-2-yn-1-yl)aniline (S-A-45)}

N NHPh

S-A-45 was prepared according to the literature, and the NMR spectral data are in accordance with the reported data ${ }^{21}$.

$>$ pale yellow oil, $0.93 \mathrm{~g}, 85 \% ; \mathrm{R}_{f}=0.5$ (EtOAc/Petroleum Ether $\left.=1: 20\right)$.

$>{ }^{1} \mathrm{H} \mathrm{NMR}\left(400 \mathrm{MHz}, \mathrm{CDCl}_{3}\right) \delta 7.22-7.18(\mathrm{~m}, 2 \mathrm{H}), 6.77(\mathrm{t}, J=8.0 \mathrm{~Hz}, 1 \mathrm{H}), 6.66(\mathrm{~d}, J=8.0$ $\mathrm{Hz}, 2 \mathrm{H}), 3.89-3.87$ (m, 2H), $3.83(\mathrm{~s}, 1 \mathrm{H}), 2.19$ (d, $J=4.0 \mathrm{~Hz}, 1 \mathrm{H})$.

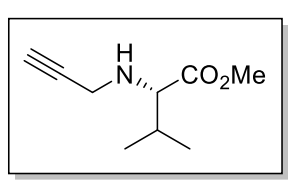

${ }^{13} \mathrm{C}$ NMR $\left(100 \mathrm{MHz}, \mathrm{CDCl}_{3}\right) \delta 146.9,129.3,118.7,113.6,81.2,71.4,33.7$.

\section{methyl prop-2-yn-1-yl-L-valinate (S-A-46)}

S-A-46 was prepared according to the literature, and the NMR spectral data are in accordance with the reported data ${ }^{22}$.

18. Ardes-Guisot, N.; Alonzi, D. S.; Reinkensmeier, G.; Butters, T. D.; Norez, C.; Becq, F.; Shimada, Y.; Nakagawa, S.; Kato, A.; Blériot, Y.; Sollogoub, M.; Vauzeilles, B. Org. Biomol. Chem. 2011, 9, 5373-5388.

19. Schade, G.; Paulus, W.; Schmitt, H. G. Ger. Offen. 1986, 36 pp. CODEN: GWXXBX DE 3510203 A1 19860925 Patent.

20. Kaschula, C. H. Hunter; R.; Stellenboom; N; Caira; M. R.; Winks, S.; Ogunleye, T.; Richards, P.; Cotton, J.; Zilbeyaz, K.; Wang, Y.B.; Siyo, V.; Ngarande, E.; Parker, M. I. Eur. J. Med. Chem. 2012, 50, 236-254.

21. Zhu, D.; Wu, Z.; Luo, B.; Du, Y.; Liu, P.; Chen, Y.; Hu, Y.; Huang, P.; Wen, S. Org. Lett. 2018, 20, 4815-4818.

22. Mindt, T. L.; Struthers, H.; Spingler, B.; Brans, L.; Tourwe, D.; Garcia-Garayoa, E.; Schibli, R. ChemMedChem. 2010, 5, 2026- 
$>$ colorless oil, $0.37 \mathrm{~g}, 18 \%$.

$>\mathrm{R}_{f}=0.3$ (EtOAc:Petroleum Ether=1:10).

$>{ }^{1} \mathrm{H} \mathrm{NMR}\left(400 \mathrm{MHz}, \mathrm{CDCl}_{3}\right) \delta 3.73(\mathrm{~s}, 3 \mathrm{H}), 3.47-3.33(\mathrm{~m}, 2 \mathrm{H}), 3.18(\mathrm{~d}, J=5.6 \mathrm{~Hz}, 1 \mathrm{H}), 2.20$ $(\mathrm{t}, J=2.4 \mathrm{~Hz}, 1 \mathrm{H}), 2.00-1.91(\mathrm{~m}, 1 \mathrm{H}), 1.73(\mathrm{~s}, 1 \mathrm{H}), 0.95(\mathrm{t}, J=6.9 \mathrm{~Hz}, 6 \mathrm{H})$.

$>{ }^{13} \mathrm{C} \mathrm{NMR}\left(100 \mathrm{MHz}, \mathrm{CDCl}_{3}\right) \delta 175.1,81.6,71.5,66.0,51.6,37.6,31.6,19.1,18.4$.

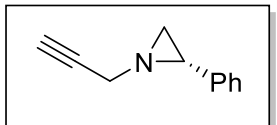

(S)-2-phenyl-1-(prop-2-yn-1-yl)aziridine (S-A-47)

S-A-47 was prepared according to the General Method A. (S)-2phenylaziridine $^{23}$ (1.26 mmol, $0.15 \mathrm{~g}, 1.0$ equiv.), $\mathrm{NaH}$ (1.9 mmol, $76 \mathrm{mg}, 60 \%$ dispersion in mineral oil, 1.5 equiv.), 3-bromoprop-1-yne (1.5 mmol, $0.18 \mathrm{~g}$, 1.2 equiv. $)$ in DMF ( $2 \mathrm{~mL})$ at room temperature for $2 \mathrm{~h}$ to give $\mathbf{S}-\mathbf{A}-\mathbf{4} 7$ as brown liquid $(0.16 \mathrm{~g}$, $82 \%)$.

$>\mathrm{R}_{f}=0.6($ EtOAc:Petroleum Ether=1:5).

$>[\alpha]^{25} \mathrm{D}=+179.4^{\circ}\left(c=15, \mathrm{CHCl}_{3}\right)$.

$>{ }^{1} \mathrm{H} \mathrm{NMR}\left(600 \mathrm{MHz}, \mathrm{CDCl}_{3}\right) \delta 7.31-7.23(\mathrm{~m}, 5 \mathrm{H}), 3.57-3.45(\mathrm{~m}, 2 \mathrm{H}), 2.64$ (ddd, $J=50.1$, $16.7,2.5 \mathrm{~Hz}, 1 \mathrm{H}), 2.27(\mathrm{t}, J=2.5 \mathrm{~Hz}, 1 \mathrm{H}), 1.99(\mathrm{~d}, J=6.7 \mathrm{~Hz}, 1 \mathrm{H}), 1.91(\mathrm{~d}, J=3.5 \mathrm{~Hz}, 1 \mathrm{H})$.

$>{ }^{13} \mathrm{C}$ NMR $\left(150 \mathrm{MHz}, \mathrm{CDCl}_{3}\right) \delta 139.6,128.3,127.1,126.3,78.6,73.1,46.8,39.2,35.8$.

$>$ IR (neat) $\mathrm{cm}^{-1} 3292,3031,2983,2919,1605,1451,1339,1260,1145,1033,924$.

$>$ HRMS calcd for $\mathrm{C}_{11} \mathrm{H}_{11} \mathrm{~N}(\mathrm{M}+\mathrm{H})^{+} 158.0970$, found 158.0969 .

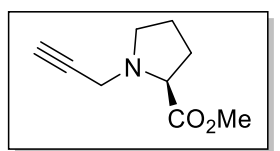

\section{methyl prop-2-yn-1-yl-L-prolinate (S-A-48)}

S-A-48 was prepared according to the literature, and the NMR spectral data are in accordance with the reported data ${ }^{22}$.

pale yellow oil, $1.09 \mathrm{~g}, 54 \%$.

$>\mathrm{R}_{f}=0.6$ (EtOAc:Petroleum Ether=1:3).

$>{ }^{1} \mathrm{H} \mathrm{NMR}\left(600 \mathrm{MHz}, \mathrm{CDCl}_{3}\right) \delta 3.74(\mathrm{~s}, 3 \mathrm{H}), 3.65-3.57(\mathrm{~m}, 2 \mathrm{H}), 3.45(\mathrm{dd}, J=9.2,6.8 \mathrm{~Hz}, 1 \mathrm{H})$, $3.08-3.05(\mathrm{~m}, 1 \mathrm{H}), 2.74(\mathrm{q}, J=8.7 \mathrm{~Hz}, 1 \mathrm{H}), 2.21(\mathrm{t}, J=2.5 \mathrm{~Hz}, 1 \mathrm{H}), 2.19-2.13(\mathrm{~m}, 1 \mathrm{H})$, $2.03-1.97(\mathrm{~m}, 1 \mathrm{H}), 1.96-1.88(\mathrm{~m}, 1 \mathrm{H}), 1.85-1.79(\mathrm{~m}, 1 \mathrm{H})$.

$>{ }^{13} \mathrm{CNMR}\left(100 \mathrm{MHz}, \mathrm{CDCl}_{3}\right) \delta 174.1,78.3,73.2,62.5,52.2,52.0,41.2,29.6,23.3$.

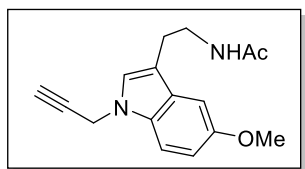

N-(2-(5-methoxy-1-(prop-2-yn-1-yl)-1H-indol-3-yl)ethyl)acetamide (S-A49)

S-A-49 was prepared according to the General Method A. $N$-(2-(5-methoxy$1 \mathrm{H}$ - indol-3-yl)- ethyl)acetamide (4.3 mmol, $1.0 \mathrm{~g}, 1.0$ equiv.), $\mathrm{NaOH}(5.2$ mmol, $0.21 \mathrm{~g}, 1.2$ equiv.), 3-bromoprop -1-yne ( $8.8 \mathrm{mmol}, 1.07 \mathrm{~g}, 2.0$ equiv.) in $\operatorname{DMF}(10 \mathrm{~mL})$ at $0{ }^{\circ} \mathrm{C}$ for $2.5 \mathrm{~h}$ to give $\mathbf{S}-\mathbf{A}-49$ as white solid $(0.98 \mathrm{~g}, 86 \%)$.

$>\mathrm{R}_{f}=0.3$ (EtOAc:Petroleum Ether=4:1).

$>{ }^{1} \mathrm{H} \mathrm{NMR}\left(400 \mathrm{MHz}, \mathrm{CDCl}_{3}\right) \delta 7.27(\mathrm{~d}, J=8.8 \mathrm{~Hz}, 1 \mathrm{H}), 7.03(\mathrm{~d}, J=2.5 \mathrm{~Hz}, 1 \mathrm{H}), 6.99(\mathrm{~s}, 1 \mathrm{H})$, $6.91(\mathrm{dd}, J=8.8,2.5 \mathrm{~Hz}, 1 \mathrm{H}), 5.72(\mathrm{br} \mathrm{s}, 1 \mathrm{H}), 4.78(\mathrm{~d}, J=2.5 \mathrm{~Hz}, 2 \mathrm{H}), 3.85(\mathrm{~s}, 3 \mathrm{H}), 3.55(\mathrm{q}, J$ $=6.6 \mathrm{~Hz}, 2 \mathrm{H}), 2.95-2.86(\mathrm{~m}, 2 \mathrm{H}), 2.39(\mathrm{t}, J=2.5 \mathrm{~Hz}, 1 \mathrm{H}), 1.92(\mathrm{~s}, 3 \mathrm{H})$.

$>{ }^{13} \mathrm{C} \mathrm{NMR}\left(100 \mathrm{MHz}, \mathrm{CDCl}_{3}\right) \delta 170.2,154.2,131.6,128.7,125.8,112.3,112.2,110.3,101.0$, $77.9,73.5,56.0,39.8,35.9,25.2,23.4$.

$>$ IR (neat) $\mathrm{cm}^{-1} 3283,2927,1647,1550,1485,1175,1037$.

$>$ HRMS calcd for $\mathrm{C}_{16} \mathrm{H}_{18} \mathrm{~N}_{2} \mathrm{O}_{2}(\mathrm{M}+\mathrm{Na})^{+}$293.1266, found 293.1267.

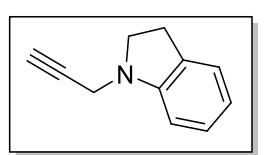

\section{1-(prop-2-yn-1-yl)indoline (S-A-50)}

S-A-50 was prepared according to the literature, and the NMR spectral data are in accordance with the reported data ${ }^{24}$. yellow solid, $2.06 \mathrm{~g}, 65 \%$.

$>\mathrm{R}_{f}=0.7$ (EtOAc:Petroleum Ether $\left.=1: 15\right)$.

$>{ }^{1} \mathrm{H} \mathrm{NMR}\left(400 \mathrm{MHz}, \mathrm{CDCl}_{3}\right) \delta 7.12-7.08(\mathrm{~m}, 2 \mathrm{H}), 6.73(\mathrm{td}, J=7.4,1.0 \mathrm{~Hz}, 1 \mathrm{H}), 6.59(\mathrm{~d}, J=$ $7.9 \mathrm{~Hz}, 1 \mathrm{H}), 3.92(\mathrm{~d}, J=2.4 \mathrm{~Hz}, 2 \mathrm{H}), 3.41(\mathrm{t}, J=8.1 \mathrm{~Hz}, 2 \mathrm{H}), 2.97(\mathrm{t}, J=8.1 \mathrm{~Hz}, 2 \mathrm{H}), 2.14(\mathrm{t}$, $J=2.4 \mathrm{~Hz}, 1 \mathrm{H})$.

$>{ }^{13} \mathrm{C} \mathrm{NMR}\left(100 \mathrm{MHz}, \mathrm{CDCl}_{3}\right) \delta 150.9,130.8,127.2,124.6,119.0,108.3,78.7,72.2,52.9,38.1$, 28.6 . 
$>\mathrm{R}_{f}=0.6$ (EtOAc:Petroleum Ether=1:5).

$>\quad{ }^{1} \mathrm{H} \mathrm{NMR}\left(400 \mathrm{MHz}, \mathrm{CDCl}_{3}\right) \delta 7.08(\mathrm{~d}, J=5.2 \mathrm{~Hz}, 1 \mathrm{H}), 6.73(\mathrm{~d}, J=5.2 \mathrm{~Hz}, 1 \mathrm{H}), 3.69(\mathrm{~s}, 2 \mathrm{H})$, $3.52(\mathrm{~d}, J=2.4 \mathrm{~Hz}, 2 \mathrm{H}), 2.95-2.87(\mathrm{~m}, 4 \mathrm{H}), 2.27(\mathrm{t}, J=2.4 \mathrm{~Hz}, 1 \mathrm{H})$.

$>{ }^{13} \mathrm{C} \mathrm{NMR}\left(100 \mathrm{MHz}, \mathrm{CDCl}_{3}\right) \delta 133.6,132.9,125.2,122.8,78.8,73.3,51.5,49.7,46.4,25.6$.

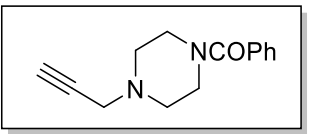

\section{phenvl(4-(prop-2-vn-1-vl)piperazin-1-vl)methanone (S-A-52)}

S-A-52 was prepared according to the General Method A. Phenyl(piperazin1-yl)methanone (2.6 mmol, $0.5 \mathrm{~g}, 1.0$ equiv.), $\mathrm{K}_{2} \mathrm{CO}_{3}$ (3.2 mmol, $0.43 \mathrm{~g}, 1.2$ equiv.), 3-bromoprop-1-yne (3.2 mmol, $0.37 \mathrm{~g}, 1.2$ equiv.) in DMF ( $5 \mathrm{~mL})$ at $60{ }^{\circ} \mathrm{C}$ for $8 \mathrm{~h}$ to give $\mathbf{S - A - 5 2}$ as pale brown solid $(0.17 \mathrm{~g}, 28 \%)$.

$>\mathrm{R}_{f}=0.3\left(\mathrm{CH}_{2} \mathrm{Cl}_{2}: \mathrm{MeOH}=10: 1\right)$.

$>\quad{ }^{1} \mathrm{H} \mathrm{NMR}\left(400 \mathrm{MHz}, \mathrm{CDCl}_{3}\right) \delta 7.41(\mathrm{~s}, 5 \mathrm{H}), 3.83(\mathrm{br} \mathrm{s}, 2 \mathrm{H}), 3.47(\mathrm{br} \mathrm{s}, 2 \mathrm{H}), 3.35(\mathrm{~d}, J=2.5 \mathrm{~Hz}$, $2 \mathrm{H}), 2.65$ (br s, 2H), 2.52 (br s, 2H) 2.29 (t, $J=2.5 \mathrm{~Hz}, 1 \mathrm{H})$.

$>{ }^{13} \mathrm{C} \mathrm{NMR}\left(100 \mathrm{MHz}, \mathrm{CDCl}_{3}\right) \delta 170.3,135.7,129.7,128.5,127.1,78.1,73.7,52.0,51.5,47.6$, $46.8,42.0$.

$>$ IR (neat) $\mathrm{cm}^{-1} 3229,3003,2912,2811,1622,1577,1426,1368,1297,1135,1092,1001,924$.

$>$ HRMS calcd for $\mathrm{C}_{14} \mathrm{H}_{16} \mathrm{~N}_{2} \mathrm{O}(\mathrm{M}+\mathrm{Na})^{+} 251.1160$, found 251.1152 .

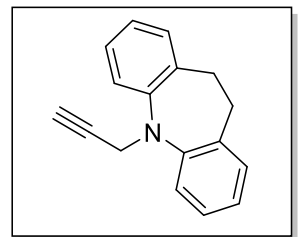

\section{5-(prop-2-vn-1-vl)-10,11-dihvdro-5H-dibenzo/b,flazepine (S-A-53)}

S-A-53 was prepared according to the General Method A. 10,11-Dihydro-5Hdibenzo[b,f]azepine (10 mmol, $2 \mathrm{~g}), \mathrm{NaH}(15 \mathrm{mmol}, 0.6 \mathrm{~g}, 60 \%$ dispersion in mineral oil), 3-bromoprop-1-yne (15 mmol, $1.83 \mathrm{~g})$ in DMF $(10 \mathrm{~mL})$ at room temperature for $3 \mathrm{~h}$ to give $\mathbf{S - A - 5 3}$ as yellow solid (1.01 g, 15\%). The NMR spectral data are in accordance with the reported data ${ }^{26}$.

$>\mathrm{R}_{f}=0.8$ (EtOAc:Petroleum Ether $\left.=1: 10\right)$.

$>{ }^{1} \mathrm{H} \mathrm{NMR}\left(400 \mathrm{MHz}, \mathrm{CDCl}_{3}\right) \delta 7.40(\mathrm{dd}, J=8.2,1.2 \mathrm{~Hz}, 2 \mathrm{H}), 7.16(\mathrm{ddd}, J=8.3,7.3,1.8 \mathrm{~Hz}$, 2H), 7.09 (dd, $J=7.6,1.7 \mathrm{~Hz}, 2 \mathrm{H}), 6.94(\mathrm{td}, J=7.4,1.2 \mathrm{~Hz}, 2 \mathrm{H}), 4.45$ (d, $J=2.4 \mathrm{~Hz}, 2 \mathrm{H})$, $3.14(\mathrm{~s}, 4 \mathrm{H}), 2.34(\mathrm{t}, J=2.4 \mathrm{~Hz}, 1 \mathrm{H})$.

$>{ }^{13} \mathrm{C} \mathrm{NMR}\left(100 \mathrm{MHz}, \mathrm{CDCl}_{3}\right) \delta 147.4,133.8,129.8,126.5,122.7,120.0,81.6,72.9,42.8,32.9$.

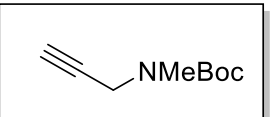

\section{tert-butyl methyl(prop-2-yn-1-yl)carbamate (S-A-54)}

S-A-54 was prepared according to the literature, and the NMR spectral data are in accordance with the reported data ${ }^{27}$.

$>$ colorless oil, $0.55 \mathrm{~g}, 50 \%$.

$\mathrm{R}_{f}=0.6$ (EtOAc:Petroleum Ether=1:5).

$>{ }^{1} \mathrm{H} \mathrm{NMR}\left(400 \mathrm{MHz}, \mathrm{CDCl}_{3}\right) \delta 4.04(\mathrm{br} \mathrm{s}, 2 \mathrm{H}), 2.92(\mathrm{~s}, 3 \mathrm{H}), 2.21(\mathrm{t}, J=2.4 \mathrm{~Hz}, 1 \mathrm{H}), 1.47(\mathrm{~s}$, 9H).

$>{ }^{13} \mathrm{C} \mathrm{NMR}\left(100 \mathrm{MHz}, \mathrm{CDCl}_{3}\right) \delta 155.2,80.1,79.2,71.5,37.6,33.4,28.4$.

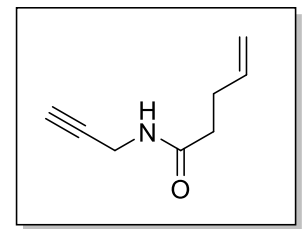

\section{N-(prop-2-yn-1-yl)pent-4-enamide (S-A-55)}

S-A-55 was prepared according to the literature, and the NMR spectral data are in accordance with the reported data ${ }^{28}$.

white solid, $0.65 \mathrm{~g}, 94 \%$.

$\mathrm{R}_{f}=0.7$ (EtOAc:Petroleum Ether $=1: 1$ ).

${ }^{1} \mathrm{H}$ NMR $\left(400 \mathrm{MHz}, \mathrm{CDCl}_{3}\right) \delta 5.98(\mathrm{~s}, 1 \mathrm{H}), 5.83(\mathrm{~m}, 1 \mathrm{H}), 5.11-5.00$ $(\mathrm{m}, 2 \mathrm{H}), 4.05(\mathrm{dd}, J=5.2,2.6 \mathrm{~Hz}, 2 \mathrm{H}), 2.43-2.37(\mathrm{~m}, 2 \mathrm{H}), 2.33-2.09(\mathrm{~m}, 2 \mathrm{H}), 2.23(\mathrm{t}, J=$ $2.6 \mathrm{~Hz}, 1 \mathrm{H})$.

$>{ }^{13} \mathrm{C} \mathrm{NMR}\left(100 \mathrm{MHz}, \mathrm{CDCl}_{3}\right) \delta 172.0,136.8,115.7,79.6,71.5,35.5,29.4,29.1$.

\footnotetext{
25. Toutov, A. A.; Betz, K. N.; Schuman, D. P.; Liu, W.-B.; Fedorov, A.; Stoltz, B. M.; Grubbs, R. H. J. Am. Chem. Soc. 2017, 139, 1668-1674.

26. Gianluigi, B.; Giorgio, M.; Gaetano, Z. J. Chem. Res. Miniprint, 1993, 6, 1263-1278.

27. Ghorai, A.; Samanth Reddy, K.; Achari, B.; Chattopadhyay, P. Org. Lett. 2014, 16, 3196-3199.

28. Poh, J.-S.; Makai, S.; Keutz, T. V.; Tran, D. N.; Battilocchio, C.; Pasau, P.; Ley, S. V. Angew. Chem. Int. Ed. 2017, 56, 1864-1868.
} 


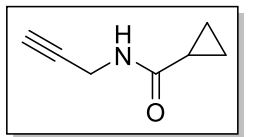

\section{N-(prop-2-vn-1-vl)cyclopropanecarboxamide (S-A-56)}

S-A-56 was prepared according to the literature, and the NMR spectral data are in accordance with the reported data ${ }^{29}$.

white solid, $0.29 \mathrm{~g}, 79 \%$.

$>\mathrm{R}_{f}=0.3$ (EtOAc:Petroleum Ether=1:2).

$>{ }^{1} \mathrm{H} \mathrm{NMR}\left(400 \mathrm{MHz}, \mathrm{CDCl}_{3}\right) \delta 6.23(\mathrm{br} \mathrm{s}, 1 \mathrm{H}), 4.07(\mathrm{dd}, J=5.3,2.6 \mathrm{~Hz}, 2 \mathrm{H}), 2.24$ (t, $J=2.6$ $\mathrm{Hz}, 1 \mathrm{H}), 1.42(\mathrm{tt}, J=7.9,4.5 \mathrm{~Hz}, 1 \mathrm{H}), 1.00-0.97(\mathrm{~m}, 2 \mathrm{H}), 0.79-0.74(\mathrm{~m}, 2 \mathrm{H})$.

$>{ }^{13} \mathrm{C} \mathrm{NMR}\left(100 \mathrm{MHz}, \mathrm{CDCl}_{3}\right) \delta 173.5,79.8,71.4,29.3,14.5,7.4$.

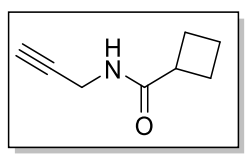

\section{N-(prop-2-vn-1-vl)cyclobutanecarboxamide (S-A-57)}

S-A-57 was prepared according to the literature, and the NMR spectral data are in accordance with the reported data ${ }^{29}$. white solid, $0.34 \mathrm{~g}, 86 \%$.

$>\mathrm{R}_{f}=0.4$ (EtOAc:Petroleum Ether=1:2).

$>{ }^{1} \mathrm{H} \mathrm{NMR}\left(400 \mathrm{MHz}, \mathrm{CDCl}_{3}\right) \delta 5.73(\mathrm{br} \mathrm{s}, 1 \mathrm{H}), 4.05(\mathrm{dd}, J=5.2,2.5 \mathrm{~Hz}, 2 \mathrm{H}), 3.02(\mathrm{pd}, J=8.6$, $1.0 \mathrm{~Hz}, 1 \mathrm{H}), 2.34-2.23(\mathrm{~m}, 2 \mathrm{H}), 2.23(\mathrm{t}, J=2.5 \mathrm{~Hz}, 1 \mathrm{H}), 2.20-2.12(\mathrm{~m}, 2 \mathrm{H}), 2.02-1.82(\mathrm{~m}$, $2 \mathrm{H})$.

$>{ }^{13} \mathrm{C} \mathrm{NMR}\left(100 \mathrm{MHz}, \mathrm{CDCl}_{3}\right) \delta 174.6,79.8,71.5,39.6,29.1,25.3,18.2$.

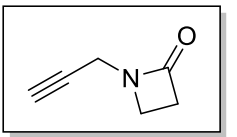

\section{1-(prop-2-yn-1-yl)azetidin-2-one (S-A-58)}

S-A-58 was prepared according to the literature ${ }^{30}$, and the NMR spectral data are in accordance with the reported data ${ }^{31}$.

colorless oil, $0.52 \mathrm{~g}, 33 \%$.

$\mathrm{R}_{f}=0.7$ (EtOAc:Petroleum Ether $=1: 1$ ).

$>{ }^{1} \mathrm{H} \mathrm{NMR}\left(400 \mathrm{MHz}, \mathrm{CDCl}_{3}\right) \delta 4.02(\mathrm{~d}, J=2.5 \mathrm{~Hz}, 2 \mathrm{H}), 3.35(\mathrm{t}, J=4.2 \mathrm{~Hz}, 2 \mathrm{H}), 2.96(\mathrm{t}, J=$ $4.2 \mathrm{~Hz}, 2 \mathrm{H}), 2.34(\mathrm{t}, J=2.5 \mathrm{~Hz}, 1 \mathrm{H})$.

$>{ }^{13} \mathrm{CNMR}\left(100 \mathrm{MHz}, \mathrm{CDCl}_{3}\right) \delta 167.0,76.7,72.5,38.5,36.9,31.2$.

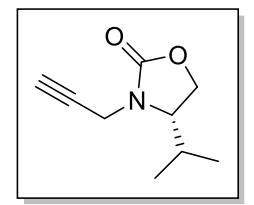

\section{(S)-4-isopropyl-3-(prop-2-yn-1-yl)oxazolidin-2-one (S-A-59)}

S-A-59 was prepared according to the General Method A. (S)-4Isopropyloxazolidin-2-one ( $20 \mathrm{mmol}, 2.58 \mathrm{~g}, 1.0$ equiv.), $\mathrm{NaH}$ ( $22 \mathrm{mmol}, 0.88 \mathrm{~g}$, $60 \%$ dispersion in mineral oil, 1.1 equiv.), 3-bromoprop-1-yne (30 mmol, $3.57 \mathrm{~g}$, 1.5 equiv.) in DMF $(50 \mathrm{~mL})$ at room temperature for $2 \mathrm{~h}$ to give $\mathbf{S - A - 5 9}$ as colorless oil (1.46 g, 44\%).

$>\mathrm{R}_{f}=0.7$ (EtOAc:Petroleum Ether $\left.=1: 1\right)$.

$>[\alpha]^{25}=+20.8^{\circ}\left(c=5, \mathrm{CHCl}_{3}\right)$.

$>\quad{ }^{1} \mathrm{H} \mathrm{NMR}\left(400 \mathrm{MHz}, \mathrm{CDCl}_{3}\right) \delta 4.40(\mathrm{dd}, J=17.9,2.6 \mathrm{~Hz}, 1 \mathrm{H}), 4.28(\mathrm{t}, J=8.9 \mathrm{~Hz}, 1 \mathrm{H}), 4.09$ (dd, $J=8.9,6.4 \mathrm{~Hz}, 1 \mathrm{H}), 3.93(\mathrm{ddd}, J=9.1,6.5,3.8 \mathrm{~Hz}, 1 \mathrm{H}), 3.77$ (dd, $J=17.8,2.5 \mathrm{~Hz}, 1 \mathrm{H})$, 2.30 (t, $J=2.5 \mathrm{~Hz}, 1 \mathrm{H}), 2.14$ (ddp, $J=10.7,7.0,3.8 \mathrm{~Hz}, 1 \mathrm{H}), 0.94$ (d, $J=7.0 \mathrm{~Hz}, 3 \mathrm{H}), 0.90$ (d, $J=7.0 \mathrm{~Hz}, 3 \mathrm{H})$.

$>{ }^{13} \mathrm{C} \mathrm{NMR}\left(100 \mathrm{MHz}, \mathrm{CDCl}_{3}\right) \delta 158.0,73.1,63.2,58.8,32.4,27.5,17.6,14.6$.

$>\mathrm{IR}$ (neat) $\mathrm{cm}^{-1} 3248,2964,1736,1428,1191,1090,928$.

$>$ HRMS calcd for $\mathrm{C}_{9} \mathrm{H}_{13} \mathrm{NO}_{2}(\mathrm{M}+\mathrm{Na})^{+} 190.0844$, found 190.0844 .

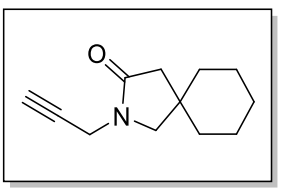

\section{2-(prop-2-yn-1-yl)-2-azaspiro/4.5/decan-3-one (S-A-60)}

S-A-60 was prepared according to the General Method A. 2azaspiro[4.5]decan-1-one (6.5 mmol, $1.0 \mathrm{~g})$, $\mathrm{KOH}(9.8 \mathrm{mmol}, 0.54 \mathrm{~g}), 3-$ bromoprop-1-yne $(7.8 \mathrm{mmol}, 0.93 \mathrm{~g})$ in $\mathrm{DMF}(10 \mathrm{~mL})$ at room temperature for $4.5 \mathrm{~h}$ to give $\mathbf{S - A - 6 0}$ as pale yellow oil $(0.87 \mathrm{~g}, 70 \%)$.

$>\mathrm{R}_{f}=0.2($ EtOAc:Petroleum Ether $=1: 5)$.

$>{ }^{1} \mathrm{H} \mathrm{NMR}\left(400 \mathrm{MHz}, \mathrm{CDCl}_{3}\right) \delta 4.09(\mathrm{~d}, J=2.5 \mathrm{~Hz}, 2 \mathrm{H}), 3.24(\mathrm{~s}, 2 \mathrm{H}), 2.26(\mathrm{~s}, 2 \mathrm{H}), 2.23(\mathrm{t}, J=$ $2.5 \mathrm{~Hz}, 1 \mathrm{H}), 1.50-1.42(\mathrm{~m}, 10 \mathrm{H})$.

$>{ }^{13} \mathrm{C}$ NMR $\left(100 \mathrm{MHz}, \mathrm{CDCl}_{3}\right) \delta 173.6,77.7,72.1,57.7,43.9,36.9,36.2,31.9,25.6,22.8$.

$>$ IR (neat) $\mathrm{cm}^{-1} 3230,2923,2851,2118,1681,1489,1418,1295,1134,851$.

29. Dong, J.-J.; Zhang, S.-L. Adv. Synth. Catal. 2020, 362, 795-800.

30. Nilsson, B. M.; Ringdahl, B.; Hacksell, U. J. Med. Chem. 1990, 33, 580-584.

31. Bahram, R.; Bjorn, R.; Richard, D. Eur. J. Med. Chem. 1981, 16, 379-381. 
D HRMS calcd for $\mathrm{C}_{12} \mathrm{H}_{17} \mathrm{NO}(\mathrm{M}+\mathrm{Na})^{+} 214.1208$, found 214.1223 .

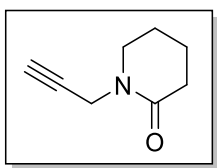

1-(prop-2-yn-1-yl)piperidin-2-one (S-A-61)

S-A-61 was prepared according to the General Method A. Piperidin-2-one (10 mmol, $1.0 \mathrm{~g}, 1.0$ equiv.), $\mathrm{NaOH}$ ( $15 \mathrm{mmol}, 0.59 \mathrm{~g}, 1.5$ equiv.), 3-bromoprop-1-yne $(11 \mathrm{mmol}, 1.44 \mathrm{~g}, 1.1$ equiv.) in DMF $(10 \mathrm{~mL})$ at room temperature for $3.5 \mathrm{~h}$ to give S-A-61 as yellow oil $(0.62 \mathrm{~g}, 45 \%)$. The NMR spectral data are in accordance with the reported data ${ }^{32}$.

$>\mathrm{R}_{f}=0.4$ (EtOAc:Petroleum Ether=3:1).

$>{ }^{1} \mathrm{H}$ NMR $\left(600 \mathrm{MHz}, \mathrm{CDCl}_{3}\right) \delta 4.24(\mathrm{~d}, J=2.5 \mathrm{~Hz}, 2 \mathrm{H}), 3.41(\mathrm{t}, J=6.1 \mathrm{~Hz}, 2 \mathrm{H}), 2.40$ (t, $J=$ $6.6 \mathrm{~Hz}, 2 \mathrm{H}), 2.21(\mathrm{t}, J=2.5 \mathrm{~Hz}, 1 \mathrm{H}), 1.87-1.80(\mathrm{~m}, 4 \mathrm{H})$.

$>{ }^{13} \mathrm{C}$ NMR $\left(100 \mathrm{MHz}, \mathrm{CDCl}_{3}\right) \delta 169.5,78.8,71.7,47.2,35.6,32.4,23.1,21.3$.

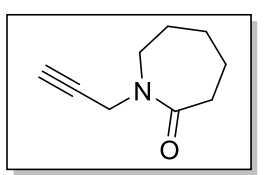

1-(prop-2-yn-1-yl)azocan-2-one (S-A-62)

S-A-62 was prepared according to the General Method A. Azepan-2-one (44 mmol, $5.0 \mathrm{~g}, 1.0$ equiv.), $\mathrm{KOH}$ (52.8 mmol, $2.98 \mathrm{~g}, 1.2$ equiv.), 3-bromoprop1-yne (52.8 mmol, $6.2 \mathrm{~g}, 1.2$ equiv.) in DMF $(50 \mathrm{~mL})$ at room temperature for $3.5 \mathrm{~h}$ to yield give $\mathbf{S}-\mathbf{A}-\mathbf{6 2}$ as yellow solid $(2.8 \mathrm{~g}, 42 \%)$. The NMR spectral data are in accordance with the reported data ${ }^{33}$.

$>\mathrm{R}_{f}=0.3$ (EtOAc:Petroleum Ether=1:2).

$>{ }^{1} \mathrm{H}$ NMR $\left(400 \mathrm{MHz}, \mathrm{CDCl}_{3}\right) \delta 4.23(\mathrm{~d}, J=2.5 \mathrm{~Hz}, 2 \mathrm{H}), 3.49-3.47(\mathrm{~m}, 2 \mathrm{H}), 2.55-2.53(\mathrm{~m}$, $2 \mathrm{H}), 2.20(\mathrm{t}, J=2.5 \mathrm{~Hz}, 1 \mathrm{H}), 1.75-1.66(\mathrm{~m}, 6 \mathrm{H})$.

$>{ }^{13} \mathrm{C} \mathrm{NMR}\left(100 \mathrm{MHz}, \mathrm{CDCl}_{3}\right) \delta 175.4,79.3,71.4,48.7,36.9,36.3,29.9,28.2,23.2$.

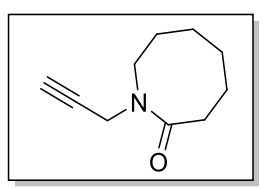

1-(prop-2-pn-1-pl)azocan-2-one (S-A-63)

S-A-63 was prepared according to the General Method A. Azocan-2-one (7.8 mmol, $1.0 \mathrm{~g}, 1.0$ equiv.), $\mathrm{KOH}$ (9.4 mmol, $0.53 \mathrm{~g}, 1.2$ equiv.), 3-bromoprop-1yne $(8.6 \mathrm{mmol}, 1.0 \mathrm{~g}, 1.1$ equiv.) in DMF $(10 \mathrm{~mL})$ at room temperature for overnight to yield product as yellow solid $(0.87 \mathrm{~g}, 69 \%)$. The NMR spectral data are in accordance with the reported data ${ }^{33}$.

$>\mathrm{R}_{f}=0.4$ (EtOAc:Petroleum Ether=1:2).

$>{ }^{1} \mathrm{H} \mathrm{NMR}\left(400 \mathrm{MHz}, \mathrm{CDCl}_{3}\right) \delta 4.21(\mathrm{~d}, J=2.5 \mathrm{~Hz}, 2 \mathrm{H}), 3.64(\mathrm{t}, J=5.92 \mathrm{~Hz}, 2 \mathrm{H}), 2.54-2.51$ $(\mathrm{m}, 2 \mathrm{H}), 2.20(\mathrm{t}, J=2.6 \mathrm{~Hz}, 1 \mathrm{H}), 1.85-1.79(\mathrm{~m}, 2 \mathrm{H}), 1.76-1.69(\mathrm{~m}, 2 \mathrm{H}), 1.59-1.54(\mathrm{~m}$, $2 \mathrm{H}), 1.52-1.46(\mathrm{~m}, 2 \mathrm{H})$.

$>{ }^{13} \mathrm{C} \mathrm{NMR}\left(100 \mathrm{MHz}, \mathrm{CDCl}_{3}\right) \delta 174.7,71.6,46.4,34.1,33.8,28.9,28.8,26.2,24.3$.

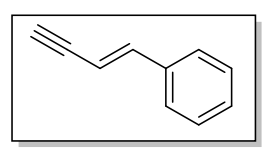

\section{(E)-but-1-en-3-yn-1-ylbenzene (S-A-64)}

S-A-64 was prepared according to the literature, and the NMR spectral data are in accordance with the reported data ${ }^{34}$.

$>$ brown oil, $2.14 \mathrm{~g}, 52 \%$.

$>\mathrm{R}_{f}=0.6$ (Petroleum Ether).

$>{ }^{1} \mathrm{H}$ NMR $\left(400 \mathrm{MHz}, \mathrm{CDCl}_{3}\right) \delta 7.38-7.26(\mathrm{~m}, 5 \mathrm{H}), 7.03(\mathrm{~d}, J=16.4 \mathrm{~Hz}, 1 \mathrm{H}), 6.12(\mathrm{dd}, J=$ $16.4,2.3 \mathrm{~Hz}, 1 \mathrm{H}), 3.04(\mathrm{~d}, J=2.3 \mathrm{~Hz}, 1 \mathrm{H})$.

$>{ }^{13} \mathrm{C} \mathrm{NMR}\left(100 \mathrm{MHz}, \mathrm{CDCl}_{3}\right) \delta 143.2,135.9,129.0,128.8,126.4,107.1,83.0,79.3$.

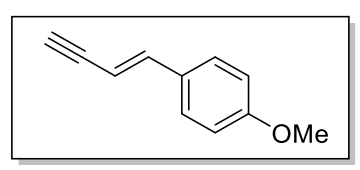

\section{(E)-1-(but-1-en-3-yn-1-vl)-4-methoxpbenzene (S-A-65)}

S-A-65 was prepared according to the literature, and the NMR spectral data are in accordance with the reported data ${ }^{35}$.

yellow solid, $0.3 \mathrm{~g}, 21 \%$.

$>\mathrm{R}_{f}=0.4$ (Petroleum Ether).

$>{ }^{1} \mathrm{H} \mathrm{NMR}\left(400 \mathrm{MHz}, \mathrm{CDCl}_{3}\right) \delta 7.34-7.32(\mathrm{~m}, 2 \mathrm{H}), 6.99(\mathrm{~d}, J=16.3 \mathrm{~Hz}, 1 \mathrm{H}), 6.88-6.84(\mathrm{~m}$, $2 \mathrm{H}), 5.99(\mathrm{dd}, J=16.3,2.4 \mathrm{~Hz}, 1 \mathrm{H}), 3.81(\mathrm{~s}, 3 \mathrm{H}), 3.01(\mathrm{dd}, J=2.4,0.7 \mathrm{~Hz}, 1 \mathrm{H})$.

$>{ }^{13} \mathrm{C} N M R\left(100 \mathrm{MHz}, \mathrm{CDCl}_{3}\right) \delta 160.3,142.7,128.7,127.7,114.2,104.5,83.3,78.4,55.3$.

32. Liu, Y.; Nisi, A. D.; Cerveri, A.; Monari, M.; Bandini, M. Org. Lett. 2017, 19, 5034-5037. 33. Safrygin, A.; Krivosheyeva, E.; Dar'in, D.; Krasavin, M. Synthesis, 2018, 50, 3048-3058. 34. Cai, H.; Nie, J.; Zheng, Y.; Ma, J.-A. J. Org. Chem. 2014, 79, 5484-5493. 35. Mokar, B. D.; Liu, R.-S. Chem. Commun. 2014, 50, 8966-8969. 


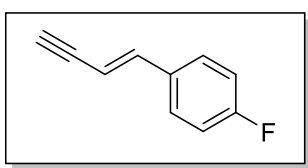

\section{(E)-1-(but-1-en-3-yn-1-yl)-4-fluorobenzene (S-A-66)}

S-A-66 was prepared according to the literature, and the NMR spectral data are in accordance with the reported data ${ }^{36}$.

pale yellow solid, $0.35 \mathrm{~g}, 25 \%$.

$\mathrm{R}_{f}=0.4$ (Petroleum Ether).

$>\quad{ }^{1} \mathrm{H} \mathrm{NMR}\left(400 \mathrm{MHz}, \mathrm{CDCl}_{3}\right) \delta 7.34(\mathrm{dd}, J=8.5,5.4 \mathrm{~Hz}, 2 \mathrm{H}), 7.03-6.96(\mathrm{~m}, 3 \mathrm{H}), 6.03(\mathrm{dd}, J$ $=16.4,2.4 \mathrm{~Hz}, 1 \mathrm{H}), 3.04(\mathrm{~d}, J=2.4 \mathrm{~Hz}, 1 \mathrm{H})$.

$>{ }^{13} \mathrm{C} \mathrm{NMR}\left(100 \mathrm{MHz}, \mathrm{CDCl}_{3}\right) \delta 163.1(\mathrm{~d}, J=247 \mathrm{~Hz}), 141.9,132.1(\mathrm{~d}, J=3.5 \mathrm{~Hz}), 128.0(\mathrm{~d}, J$ $=8.1 \mathrm{~Hz}), 115.8(\mathrm{~d}, J=21.8 \mathrm{~Hz}), 106.8(\mathrm{~d}, J=2.4 \mathrm{~Hz}), 82.7,79.3$.

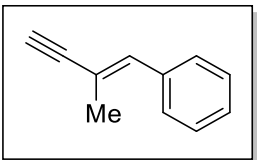

\section{(E)-(2-methylbut-1-en-3-yn-1-yl)benzene (S-A-67)}

S-A-67 was prepared was prepared according to the literature, ${ }^{34}$ and the NMR spectral data are in accordance with the reported data ${ }^{37}$.

$>$ yellow oil, $40 \mathrm{mg}, 5 \%, \mathrm{R}_{f}=0.8$ (Petroleum Ether).

$1 \mathrm{H}), 2.07(\mathrm{~d}, J=1.6 \mathrm{~Hz}, 3 \mathrm{H})$.

${ }^{1} \mathrm{H}$ NMR $\left(400 \mathrm{MHz}, \mathrm{CDCl}_{3}\right) \delta 7.36-7.23(\mathrm{~m}, 5 \mathrm{H}), 6.92(\mathrm{~s}, 1 \mathrm{H}), 2.97(\mathrm{~s}$,

$>{ }^{13} \mathrm{C}$ NMR $\left(100 \mathrm{MHz}, \mathrm{CDCl}_{3}\right) \delta 137.4,136.5,129.0,128.3,127.4,118.9,87.4,75.9,19.0$.

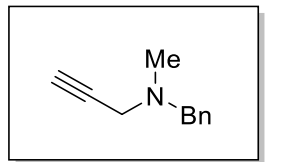

\section{N-benzyl-N-methylprop-2-yn-1-amine (S-A-70)}

S-A-70 was prepared according to the literature, and the NMR spectral data are in accordance with the reported data ${ }^{38}$.

$>$ colorless liquid, $1.43 \mathrm{~g}, 54 \%$.

$>\mathrm{R}_{f}=0.8$ (EtOAc:Petroleum Ether $\left.=1: 5\right)$.

$>\quad{ }^{1} \mathrm{H} \mathrm{NMR}\left(400 \mathrm{MHz}, \mathrm{CDCl}_{3}\right) \delta 7.35-7.30(\mathrm{~m}, 4 \mathrm{H}), 7.28-7.24(\mathrm{~m}, 1 \mathrm{H}), 3.57(\mathrm{~s}, 2 \mathrm{H}), 3.30(\mathrm{~d}$, $J=2.4 \mathrm{~Hz}, 2 \mathrm{H}), 2.34(\mathrm{~s}, 3 \mathrm{H}), 2.27(\mathrm{t}, J=2.4 \mathrm{~Hz}, 1 \mathrm{H})$.

$>{ }^{13} \mathrm{C} \mathrm{NMR}\left(100 \mathrm{MHz}, \mathrm{CDCl}_{3}\right) \delta 138.4,129.2,128.3,127.3,78.6,73.4,60.0,44.9,41.8$.

\section{2-(3-cyano-4-isobutoxyphenyl)-4-methyl-N-(prop-2-yn-1-yl)thiazole-5-carboxamide (S-A-73)}<smiles>Cc1nc(-c2ccc(OCC(C)C)c(C#N)c2)sc1C(=O)O</smiles>

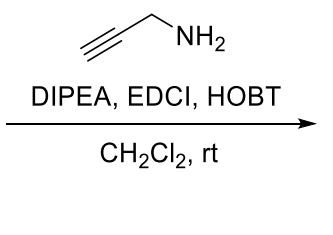

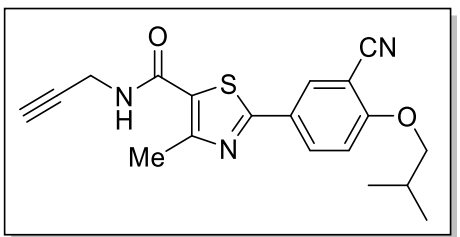

A solution of 2-(3-cyano-4-isobutoxyphenyl) -4-methylthiazole-5-carboxylic acid (1.0 g, 3.2 mmol, 1.0 equiv.), EDCI (0.64 g, $3.8 \mathrm{mmol}, 1.2$ equiv.), DIPEA ( $0.43 \mathrm{~g}, 3.8 \mathrm{mmol}, 1.2$ equiv.) in $\mathrm{CH}_{2} \mathrm{Cl}_{2}(15 \mathrm{~mL})$ was stirred at room temperature for $2 \mathrm{~min}$. Then HOBT $(0.41 \mathrm{~g}, 3.5 \mathrm{mmol}, 1.1$ equiv.) was added to the mixture with stirring for additional $2 \mathrm{~min}$. To the resulting mixture was added prop-2-yn-1-amine $(0.17 \mathrm{~g}, 3.5 \mathrm{mmol}, 1.1$ equiv. $)$. The reaction was stirred at room temperature overnight before quenching with sat. aq. $\mathrm{NaHCO}_{3}(15 \mathrm{~mL})$ and extracting with $\mathrm{CH}_{2} \mathrm{Cl}_{2}$ $(15 \mathrm{~mL} \times 2)$. The combined organic layers were washed with sat. aq. $\mathrm{NaCl}$, dried over $\mathrm{Na}_{2} \mathrm{SO}_{4}$ and concentrated under reduced pressure. The crude residue was purified by silica gel flash column chromatography (eluent: petroleum ether/EtOAc $=3 / 1)$ to afford S-A-73 as white solid $(0.68 \mathrm{~g}$, $73 \%)$.

$>\mathrm{mp}: 180.7-183.2^{\circ} \mathrm{C}$.

$>\mathrm{R}_{f}=0.7$ (EtOAc:Petroleum Ether $\left.=1: 1\right)$.

$>{ }^{1} \mathrm{H} \mathrm{NMR}\left(400 \mathrm{MHz}, \mathrm{CDCl}_{3}\right) \delta 8.14(\mathrm{~d}, J=2.3 \mathrm{~Hz}, 1 \mathrm{H}), 8.06(\mathrm{dd}, J=8.8,2.3 \mathrm{~Hz}, 1 \mathrm{H}), 7.01(\mathrm{~d}$, $J=8.9 \mathrm{~Hz}, 1 \mathrm{H}), 5.97(\mathrm{t}, J=5.4 \mathrm{~Hz}, 1 \mathrm{H}), 4.24(\mathrm{dd}, J=5.2,2.6 \mathrm{~Hz}, 2 \mathrm{H}), 3.90(\mathrm{~d}, J=6.5 \mathrm{~Hz}$, $2 \mathrm{H}), 2.74(\mathrm{~s}, 3 \mathrm{H}), 2.31(\mathrm{t}, J=2.6 \mathrm{~Hz}, 1 \mathrm{H}), 2.25-2.16(\mathrm{~m}, 1 \mathrm{H}), 1.09(\mathrm{~d}, J=6.7 \mathrm{~Hz}, 6 \mathrm{H})$.

$>{ }^{13} \mathrm{C} \mathrm{NMR}\left(100 \mathrm{MHz}, \mathrm{CDCl}_{3}\right) \delta 164.9,162.5,161.2,157.0,132.5,132.0,125.8,124.9,115.4$, $112.7,103.0,79.0,75.7,72.3,29.9,28.2,19.1,17.5$.

$>$ IR (neat) $\mathrm{cm}^{-1} 3410,3294,3006,2231,1634,1603,1431,1170,1133,1012,913$.

$>$ HRMS calcd for $\mathrm{C}_{19} \mathrm{H}_{19} \mathrm{~N}_{3} \mathrm{O}_{2} \mathrm{~S}(\mathrm{M}+\mathrm{Na})^{+} 376.1096$ found 376.1066 .

36. Schabel, T.; Plietker, B. Chem. Eur. J. 2013, 19, 6938-6941.

37. Thummala, Y.; Karunakar, G. V.; Doddi, V. R. Adv. Synth. Catal. 2019, 361, 611-616.

38. Li, H.-J.; Guillot, R.; Gandon, V. J. Org. Chem. 2010, 75, 8435-8449. 
1-cvclopropvl-7-(4-ethvlpiperazin-1-vl)-6-fluoro-4-oxo-N-(prop-2-pn-1-vl)-1,4dihydroquinoline-3-carboxamide (S-A-74)<smiles>CCN1CCCN(c2cc3c(cc2F)c(=O)c(C(=O)O)cn3C2CC2)CC1</smiles>

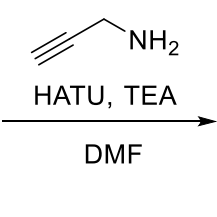<smiles>C#CCNC(=O)c1cn(C2CC2)c2cc(N3CCN(CC)CC3)c(F)cc2c1=O</smiles>

A solution of Enrofloxacin ( $2.8 \mathrm{mmol}, 1.0 \mathrm{~g}, 1.0$ equiv.), prop-2-yn-1-amine (3.0 mmol, 0.19 g, 1.07 equiv.), TEA (14.0 mmol, $1.4 \mathrm{~g}, 5.0$ equiv.) and HATU (3.3 mmol, $1.27 \mathrm{~g}, 1.17$ equiv.) in DMF $(10 \mathrm{~mL})$ was stirred at room temperature for overnight before quenching with water $(10 \mathrm{~mL})$. The mixture was filtered, and filter cake was washed with water and dried by infrared drying lamp. The resulting crude residue was washed with $\mathrm{Et}_{2} \mathrm{O}$ for $10 \mathrm{~min}$ and filtrated to afford $\mathbf{S}-\mathbf{A}-\mathbf{7 4}$ as white solid $(0.6 \mathrm{~g}, 55 \%)$.

$>$ mp: $195.4-198.2^{\circ} \mathrm{C}$.

$>\mathrm{R}_{f}=0.5\left(\mathrm{MeOH}: \mathrm{CH}_{2} \mathrm{Cl}_{2}=1: 10\right)$.

$>{ }^{1} \mathrm{H} \mathrm{NMR}\left(400 \mathrm{MHz}, \mathrm{CDCl}_{3}\right) \delta 10.26(\mathrm{t}, J=5.4 \mathrm{~Hz}, 1 \mathrm{H}), 8.80(\mathrm{~s}, 1 \mathrm{H}), 8.02(\mathrm{~d}, J=13.3 \mathrm{~Hz}$, $1 \mathrm{H}), 7.33(\mathrm{~d}, J=7.1 \mathrm{~Hz}, 1 \mathrm{H}), 4.24(\mathrm{dd}, J=5.4,2.6 \mathrm{~Hz}, 2 \mathrm{H}), 3.50-3.45(\mathrm{~m}, 1 \mathrm{H}), 3.33$ (t, $J=$ $4.9 \mathrm{~Hz}, 4 \mathrm{H}), 2.69(\mathrm{t}, J=4.9 \mathrm{~Hz}, 4 \mathrm{H}), 2.53(\mathrm{q}, J=7.2 \mathrm{~Hz}, 2 \mathrm{H}), 2.22(\mathrm{t}, J=2.6 \mathrm{~Hz}, 1 \mathrm{H}), 1.37-$ $1.30(\mathrm{~m}, 2 \mathrm{H}), 1.18-1.13(\mathrm{~m}, 5 \mathrm{H})$.

$>{ }^{13} \mathrm{C} \mathrm{NMR}\left(100 \mathrm{MHz}, \mathrm{CDCl}_{3}\right) \delta 175.4(\mathrm{~d}, J=2.3 \mathrm{~Hz}), 164.9,153.5(\mathrm{~d}, J=247.9 \mathrm{~Hz}), 146.8$, $145.1(\mathrm{~d}, J=10.6 \mathrm{~Hz}), 138.5,121.7(\mathrm{~d}, J=7.2 \mathrm{~Hz}), 112.5(\mathrm{~d}, J=23.0 \mathrm{~Hz}), 110.8,104.7(\mathrm{~d}, J$ $=3.2 \mathrm{~Hz}), 80.1,70.8,52.5,52.3,50.0(\mathrm{~d}, J=4.7 \mathrm{~Hz}), 34.7,28.8,12.0,8.2$.

$>{ }^{19} \mathrm{~F} \mathrm{NMR}\left(376 \mathrm{MHz}, \mathrm{CDCl}_{3}\right) \delta-122.93$.

$>$ IR (neat) $\mathrm{cm}^{-1} 3221,2816,1662,1626,1534,1478,1343,1149,1003$.

$>$ HRMS calcd for $\mathrm{C}_{22} \mathrm{H}_{25} \mathrm{FN}_{4} \mathrm{O}_{2}(\mathrm{M}+\mathrm{H})^{+} 397.2040$ found 397.2047.

\section{5-(4-(2-(5-ethylpyridin-2-yl)ethoxv)benzyl)-3-(prop-2-yn-1-yl)thiazolidine-2,4-dione (S-A-75)}

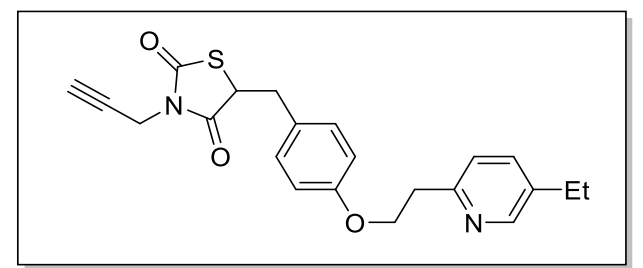

S-A-75 was prepared according to the General Method A. Pioglitazone hydrochloride $(2.5 \mathrm{mmol}$, $1.0 \mathrm{~g}, 1.0$ equiv.), $\mathrm{K}_{2} \mathrm{CO}_{3}(5.0 \mathrm{mmol}, 0.70 \mathrm{~g}, 2.0$ equiv.), 3-bromoprop-1-yne (3.0 mmol, $0.37 \mathrm{~g}, 1.2$ equiv.) in DMF $(10 \mathrm{~mL})$ at room temperature for 2.5 $\mathrm{h}$ to give $\mathbf{S - A}-\mathbf{7 5}$ as white solid $(0.65 \mathrm{~g}, 65 \%)$.

$>\mathrm{mp}: 94.1-94.6^{\circ} \mathrm{C}$.

$>\mathrm{R}_{f}=0.3($ EtOAc:Petroleum Ether $=1: 2)$.

$>{ }^{1} \mathrm{H}$ NMR $\left(400 \mathrm{MHz}, \mathrm{CDCl}_{3}\right) \delta 8.39(\mathrm{~d}, J=2.0 \mathrm{~Hz}, 1 \mathrm{H}), 7.45(\mathrm{dd}, J=7.9,2.3 \mathrm{~Hz}, 1 \mathrm{H}), 7.18$ $(\mathrm{dd}, J=7.9,0.8 \mathrm{~Hz}, 1 \mathrm{H}), 7.12-7.09(\mathrm{~m}, 2 \mathrm{H}), 6.86-6.82(\mathrm{~m}, 2 \mathrm{H}), 4.45(\mathrm{dd}, J=9.3,3.9 \mathrm{~Hz}$, $1 \mathrm{H}), 4.34-4.29(\mathrm{~m}, 4 \mathrm{H}), 3.46(\mathrm{dd}, J=14.2,3.9 \mathrm{~Hz}, 1 \mathrm{H}), 3.22(\mathrm{t}, J=6.7 \mathrm{~Hz}, 2 \mathrm{H}), 3.08(\mathrm{dd}, J$ $=14.2,9.3 \mathrm{~Hz}, 1 \mathrm{H}), 2.63(\mathrm{q}, J=7.6 \mathrm{~Hz}, 2 \mathrm{H}), 2.21(\mathrm{t}, J=2.5 \mathrm{~Hz}, 1 \mathrm{H}), 1.24(\mathrm{t}, J=7.6 \mathrm{~Hz}, 3 \mathrm{H})$.

$>{ }^{13} \mathrm{C} \mathrm{NMR}\left(100 \mathrm{MHz}, \mathrm{CDCl}_{3}\right) \delta 172.7,169.9,158.4,155.6,149.0,137.1,135.9,130.3,127.5$, $123.3,114.9,76.0,72.0,67.3,52.0,37.8,37.6,30.6,25.7,15.4$.

$>\quad \mathrm{IR}$ (neat) $\mathrm{cm}^{-1} 3284,2966,1752,1685,1511,1379,1246,1159,1027,932$.

$>$ HRMS calcd for $\mathrm{C}_{22} \mathrm{H}_{22} \mathrm{~N}_{2} \mathrm{O}_{3} \mathrm{~S}(\mathrm{M}+\mathrm{H})^{+} 395.1429$ found 395.1434 .

(3S,8S,9S,10R,13R,14S,17R)-10,13-dimethyl-17-((R)-6-methylheptan-2-yl)-3-(prop-2-yn-1vloxv)-2,3,4,7, 8,9,10,11,12,13,14,15,16,17-tetradecahvdro-1H-cvclopenta/alphenanthrene (S-A76)

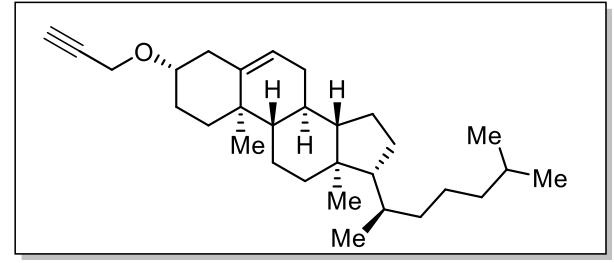

S-A-76 was prepared according to the literature, and the NMR spectral data are in accordance with the reported data $^{39}$.

$38 \% ; \mathrm{R}_{f}=0.3$ (EtOAc:Petroleum Ether $\left.=1: 50\right)$.

${ }^{1} \mathrm{H}$ NMR $\left(600 \mathrm{MHz}, \mathrm{CDCl}_{3}\right) \delta 5.38-5.35(\mathrm{~m}$,

39. Xia, M.; Yao, W.; Meng, X.; Lou, Q.; Li, Z. Tetrahedron Lett. 2017, 58, 2389-2392. 
$1 \mathrm{H}), 4.19(\mathrm{~d}, J=2.4 \mathrm{~Hz}, 2 \mathrm{H}), 3.42-3.35(\mathrm{~m}, 1 \mathrm{H}), 2.41-2.36(\mathrm{~m}, 2 \mathrm{H}), 2.26-2.18(\mathrm{~m}, 1 \mathrm{H})$, $2.04-1.78(\mathrm{~m}, 6 \mathrm{H}), 1.61-1.43(\mathrm{~m}, 8 \mathrm{H}), 1.39-1.27(\mathrm{~m}, 4 \mathrm{H}), 1.18-1.05(\mathrm{~m}, 8 \mathrm{H}), 1.00(\mathrm{~s}$, $3 \mathrm{H}), 0.91(\mathrm{~d}, J=6.5 \mathrm{~Hz}, 3 \mathrm{H}), 0.87(\mathrm{~d}, J=1.8 \mathrm{~Hz}, 3 \mathrm{H}), 0.86(\mathrm{~d}, J=1.8 \mathrm{~Hz}, 3 \mathrm{H}), 0.68(\mathrm{~s}, 3 \mathrm{H})$.

$>{ }^{13} \mathrm{CNMR}\left(100 \mathrm{MHz}, \mathrm{CDCl}_{3}\right) \delta 140.6,121.9,80.5,78.2,73.8,56.8,56.2,55.1,50.2,42.3,39.8$, $39.5,38.7,37.1,36.8,36.2,35.8,32.0,31.9,28.3,28.1,28.0,24.3,23.8,22.8,22.6,21.1,19.4$, $18.7,11.9$.

\section{(8R,9S,13S,14S)-3-ethynvl-13-methvl-6,7,8,9,11,12,13,14,15,16-decahvdro-17H-} cyclopenta/alphenanthren-17-one (S-A-77)

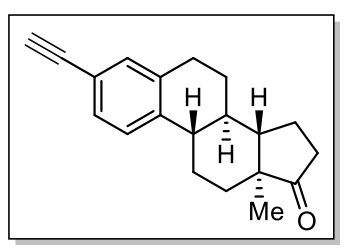

S-A-77 was prepared according to the literature, and the NMR spectral data are in accordance with the reported data ${ }^{40}$.

$>$ yellow solid, $0.35 \mathrm{~g}, 35 \%$.

$>\mathrm{R}_{f}=0.6$ (EtOAc:Petroleum Ether $\left.=1: 5\right)$.

$>{ }^{1} \mathrm{H}$ NMR $\left(400 \mathrm{MHz}, \mathrm{CDCl}_{3}\right) \delta 7.28-7.23(\mathrm{~m}, 3 \mathrm{H}), 3.02(\mathrm{~s}, 1 \mathrm{H}), 2.89$ 1H), $2.32-2.26(\mathrm{~m}, 1 \mathrm{H}), 2.17-1.95(\mathrm{~m}, 4 \mathrm{H}), 1.66-1.41(\mathrm{~m}, 6 \mathrm{H}), 0.91(\mathrm{~s}, 3 \mathrm{H})$.

$>{ }^{13} \mathrm{CNMR}\left(100 \mathrm{MHz}, \mathrm{CDCl}_{3}\right) \delta 220.7,140.8,136.7,132.6,129.4,125.4,119.4,83.8,76.5,50.5$, 47.9, 44.4, 37.9, 35.8, 31.6, 29.1, 26.3, 25.6, 21.6, 13.8 .

40. Chen, M.; Sun, N.; Chen, H.-Y.; Liu, Y.-H. Chem. Commun. 2016, 52, 6324-6327. 


\section{Synthesis of 3-silaazetidine Precursor Reagents 2}

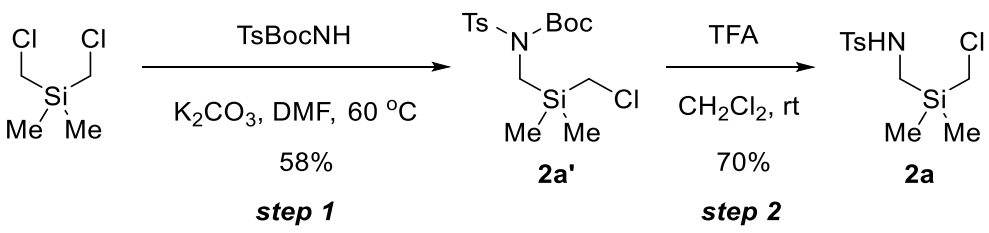

\section{General Method B:}

step 1: To a stirring suspension of TsBocNH ( $48 \mathrm{mmol}, 13.0 \mathrm{~g}, 1.0$ equiv.) and $\mathrm{K}_{2} \mathrm{CO}_{3}(57.6 \mathrm{mmol}$, $7.9 \mathrm{~g}, 1.2$ equiv.) in DMF (200 mL) was added bis(chloromethyl)dimethylsilane (48 mmol, $7.9 \mathrm{~g}$, 1.0 equiv.) at room temperature. The resulting mixture was stirred at $60{ }^{\circ} \mathrm{C}$ for $1.5 \mathrm{~h}$. The reaction was cooled to room temperature and poured into $150 \mathrm{~mL}$ of water. The mixture was extracted with EtOAc $(100 \mathrm{~mL} \times 2)$. The combined organic phases were washed with sat. aq. $\mathrm{NaCl}$, dried over $\mathrm{Na}_{2} \mathrm{SO}_{4}$ and concentrated under reduced pressure. The crude residue was purified by silica gel flash column chromatography (eluent: petroleum ether/EtOAc $=15 / 1$ ) to give the product $\mathbf{2 a}^{\prime}$ as white solid $(11.0 \mathrm{~g}, 58 \%)$.

step 2: To a solution of $\mathbf{2 a}$ ' $\left(28 \mathrm{mmol}, 11.0 \mathrm{~g}, 1.0\right.$ equiv.) in $\mathrm{CH}_{2} \mathrm{Cl}_{2}(150 \mathrm{~mL})$ was added TFA (280 mmol, $32.0 \mathrm{~g}, 10.0$ equiv.) dropwise at room temperature. The reaction was stirred at room temperature for $1 \mathrm{~h}$ before quenching with sat. aq. $\mathrm{NaHCO}_{3}(50 \mathrm{~mL})$ and stirring at room temperature for additional $20 \mathrm{~min}$. The resulting mixture was extracted with $\mathrm{CH}_{2} \mathrm{Cl}_{2}(100 \mathrm{~mL})$. The combined organic phases were washed with sat. aq. $\mathrm{NaCl}$, dried over $\mathrm{Na}_{2} \mathrm{SO}_{4}$ and concentrated under reduced pressure. The crude residue was crystallized $(\mathrm{EA} / \mathrm{PE}=1: 7)$ to give the product $\mathbf{2 a}$ as white solid $(5.8 \mathrm{~g}, 70 \%)$.

\section{N-(((chloromethvl)dimethylsilvl)methyl)-4-methylbenzenesulfonamide (2a)}

$>\quad \mathrm{mp}: 103.7-105.0^{\circ} \mathrm{C}$.

$>\quad \mathrm{R}_{f}=0.4$ (EtOAc:Petroleum Ether $\left.=1: 5\right)$.

$>{ }^{1} \mathrm{H} \mathrm{NMR}\left(400 \mathrm{MHz}, \mathrm{CDCl}_{3}\right) \delta 7.75(\mathrm{~d}, J=8.1 \mathrm{~Hz}, 2 \mathrm{H}), 7.33(\mathrm{~d}, J=8.1 \mathrm{~Hz}, 2 \mathrm{H}), 4.49(\mathrm{t}, J=$ $6.3 \mathrm{~Hz}, 1 \mathrm{H}), 2.82(\mathrm{~s}, 2 \mathrm{H}), 2.45-2.44(\mathrm{~m}, 5 \mathrm{H}), 0.18(\mathrm{~s}, 6 \mathrm{H})$.

$>{ }^{13} \mathrm{C} \mathrm{NMR}\left(100 \mathrm{MHz}, \mathrm{CDCl}_{3}\right) \delta 143.6,135.2,129.7,127.5,30.3,28.7,21.6,-5.6$.

$>$ IR (neat) $\mathrm{cm}^{-1} 3274,2962,1597,1444,1413,1317,1157,1091$.

$>$ HRMS calcd for $\mathrm{C}_{11} \mathrm{H}_{18} \mathrm{ClNO}_{2} \mathrm{SSi}(\mathrm{M}+\mathrm{Na})^{+} 314.0414$ found 314.0404 .

\section{N-(((chloromethyl)dimethylsilyl)methyl)-2-methylpropane-2-sulfonamide (2b)}

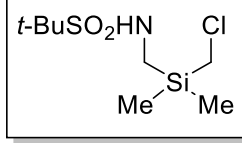

2b was prepared according to the General Method B. $t-\mathrm{BuSO}_{2} \mathrm{BocNH}(5.93 \mathrm{~g}$, 25 mmol, 1.0 equiv.), bis(chloromethyl)dimethylsilane (4.71 g, $30 \mathrm{mmol}, 1.2$ equiv.), $\mathrm{K}_{2} \mathrm{CO}_{3}$ (4.15 g, $30 \mathrm{mmol}, 1.2$ equiv.) in $\operatorname{DMF}(75 \mathrm{~mL})$ at $60{ }^{\circ} \mathrm{C}$ for $3 \mathrm{~h}$, then TFA (49.0 g, $430 \mathrm{mmol}, 20.0$ equiv.) in $\mathrm{CH}_{2} \mathrm{Cl}_{2}(45 \mathrm{~mL})$ at $\mathrm{rt}$ for $1 \mathrm{~h}$ to afford $\mathbf{2 b}$ as white solid (4.01 g, 62\%).

$>\mathrm{mp}: 79.2-81.6^{\circ} \mathrm{C}$.

$\mathrm{R}_{f}=0.4$ (EtOAc:Petroleum Ether $\left.=1: 5\right)$.

$>{ }^{1} \mathrm{H} \mathrm{NMR}\left(400 \mathrm{MHz}, \mathrm{CDCl}_{3}\right) \delta 4.09(\mathrm{t}, J=5.8 \mathrm{~Hz}, 1 \mathrm{H}), 2.90(\mathrm{~s}, 2 \mathrm{H}), 2.82(\mathrm{~d}, J=5.8 \mathrm{~Hz}, 2 \mathrm{H})$, $1.41(\mathrm{~s}, 9 \mathrm{H}), 0.24(\mathrm{~s}, 6 \mathrm{H})$

$>{ }^{13} \mathrm{C} \mathrm{NMR}\left(100 \mathrm{MHz}, \mathrm{CDCl}_{3}\right) \delta 60.4,32.4,28.6,24.5,-5.7$

$>$ IR (neat) $\mathrm{cm}^{-1} 3294,2976,2919,1432,1366,1125,1064$.

$>$ HRMS calcd for $\mathrm{C}_{8} \mathrm{H}_{20} \mathrm{ClNO}_{2} \mathrm{SSi}(\mathrm{M}+\mathrm{Na})^{+} 280.0565$ found 280.0564 .

\section{$\mathrm{N}$-(((chloromethvl)dimethvlsilvl)methvl)methanesulfonamide (2c)}

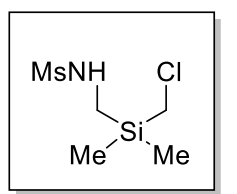

2c was prepared according to the General Method B. MsBocNH (0.97 g, 5.0 mmol, 1.0 equiv.), bis(chloromethyl)dimethylsilane $(0.78 \mathrm{~g}, 5.0 \mathrm{mmol}, 1.0$ equiv.), $\mathrm{K}_{2} \mathrm{CO}_{3}(1.38 \mathrm{~g}, 10 \mathrm{mmol}, 2.0$ equiv. $)$ in $\operatorname{DMF}(10 \mathrm{~mL})$ at $60{ }^{\circ} \mathrm{C}$ for $3 \mathrm{~h}$, then TFA (5.7 g, $50 \mathrm{mmol}, 10.0$ equiv.) in $\mathrm{CH}_{2} \mathrm{Cl}_{2}(40 \mathrm{~mL})$ at $\mathrm{rt}$ for $1 \mathrm{~h}$ afforded $2 \mathbf{c}$ as white solid $(0.51 \mathrm{~g}, 47 \%)$.

$>$ mp: $39.9-43.6{ }^{\circ} \mathrm{C}$.

$>\mathrm{R}_{f}=0.3$ (EtOAc:Petroleum Ether $\left.=1: 3\right)$.

$>{ }^{1} \mathrm{H} \mathrm{NMR}\left(400 \mathrm{MHz}, \mathrm{CDCl}_{3}\right) \delta 4.25(\mathrm{~s}, 1 \mathrm{H}), 2.95(\mathrm{~s}, 3 \mathrm{H}), 2.90(\mathrm{~s}, 2 \mathrm{H}), 2.72(\mathrm{~d}, J=6.2 \mathrm{~Hz}, 2 \mathrm{H})$, 
$0.25(\mathrm{~s}, 6 \mathrm{H})$.

$>{ }^{13} \mathrm{C} \mathrm{NMR}\left(150 \mathrm{MHz}, \mathrm{CDCl}_{3}\right) \delta 38.3,30.8,28.9,-5.3$.

$>$ IR (neat) $\mathrm{cm}^{-1} 3283,2961,1402,1307,1254,1147,1056,974$.

$>$ HRMS calcd for $\mathrm{C}_{5} \mathrm{H}_{14} \mathrm{ClNO}_{2} \mathrm{SSi}(\mathrm{M}+\mathrm{Na})^{+} 238.0101$ found 238.0087 .

N-(((chloromethyl)dimethylsilyl)methyl)-4-(trifluoromethyl)benzamide (2d)

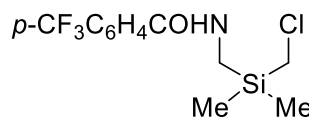

2d was prepared according to the General Method B.

$p-\mathrm{CF}_{3} \mathrm{C}_{6} \mathrm{H}_{4} \mathrm{CONHBoc}^{41}$ (1.0 g, 3.5 mmol, 1.0 equiv.), bis(chloromathyl)dimethylsilane (0.55 g, $3.5 \mathrm{mmol}, 1.0$ equiv.), $\mathrm{K}_{2} \mathrm{CO}_{3}(0.97 \mathrm{~g}, 7$ mmol, 2.0 equiv.) in DMF $(7 \mathrm{~mL})$ at $60{ }^{\circ} \mathrm{C}$ for $3 \mathrm{~h}$, then TFA $(4.0 \mathrm{~g}, 35$ mmol, 10.0 equiv.) in $\mathrm{CH}_{2} \mathrm{Cl}_{2}(30 \mathrm{~mL})$ at $\mathrm{rt}$ for $1 \mathrm{~h}$ to afford $2 \mathbf{d}$ as white

solid $(0.12 \mathrm{~g}, 28 \%)$.

$>$ mp: $53.8-55.6^{\circ} \mathrm{C}$.

$>\mathrm{R}_{f}=0.4$ (EtOAc:Petroleum Ether $\left.=1: 5\right)$.

$>{ }^{1} \mathrm{H}$ NMR $\left(400 \mathrm{MHz}, \mathrm{CDCl}_{3}\right) \delta 7.80(\mathrm{~d}, J=8.0 \mathrm{~Hz}, 2 \mathrm{H}), 7.60(\mathrm{~d}, J=8.0 \mathrm{~Hz}, 2 \mathrm{H}), 6.89(\mathrm{br} \mathrm{s}$, $1 \mathrm{H}), 3.05(\mathrm{dd}, J=5.6,2.0 \mathrm{~Hz}, 2 \mathrm{H}), 2.91(\mathrm{~s}, 2 \mathrm{H}), 0.21(\mathrm{~s}, 6 \mathrm{H})$.

$>{ }^{13} \mathrm{C} \mathrm{NMR}\left(100 \mathrm{MHz}, \mathrm{CDCl}_{3}\right) \delta 166.7,137.9,132.9(\mathrm{q}, J=32 \mathrm{~Hz}), 127.3,125.5(\mathrm{q}, J=4 \mathrm{~Hz})$, $123.6(\mathrm{q}, J=271 \mathrm{~Hz}), 30.0,29.0,-5.1$.

$>{ }^{19} \mathrm{~F} \mathrm{NMR}\left(376 \mathrm{MHz}, \mathrm{CDCl}_{3}\right) \delta-62.95$

$>$ IR (neat) $\mathrm{cm}^{-1} 3275,2962,1634,1549,1508,1324,1259,1167,1127,1068$.

$>$ HRMS calcd for $\mathrm{C}_{12} \mathrm{H}_{15} \mathrm{ClF}_{3} \mathrm{NOSSi}(\mathrm{M}+\mathrm{Na})^{+} 332.0461$ found 332.0445 .

N-(((chloromethvl)diethylsilvl)methvl)-4-methylbenzenesulfonamide (2e)

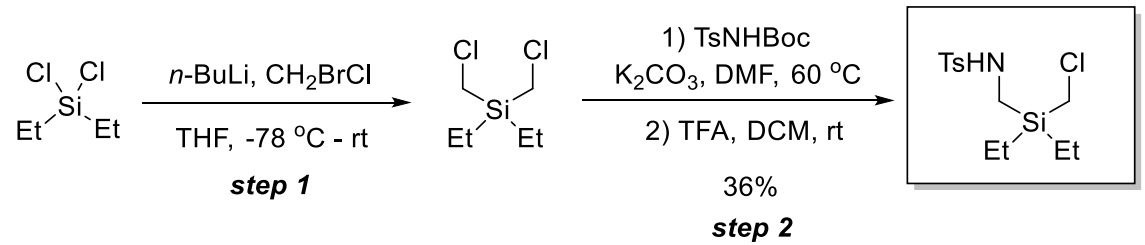

step 1: Bis(chloromethyl)diethylsilane was prepared according to the literature ${ }^{42}$ A solution of dichlorodiethylsilan ( $3.14 \mathrm{~g}, 20.0 \mathrm{mmol}, 1.0$ equiv.), $\mathrm{CH}_{2} \mathrm{BrCl}$ (7.76 g, $60.0 \mathrm{mmol}, 3.0$ equiv.) in dried THF $(120 \mathrm{~mL})$ was cooled to $-78^{\circ} \mathrm{C}$. After $30 \mathrm{~min}, n$-BuLi $(19.2 \mathrm{~mL}, 46 \mathrm{mmol}, 2.4 \mathrm{~mol} / \mathrm{L}$ in hexane, 2.3 equiv.) was added to the solution followed by the cooled wall of the flask during $3 \mathrm{~h}$ and the resulted mixture was stirred at $\mathrm{rt}$ overnight. The reaction was quenched by sat. aq. $\mathrm{NH}_{4} \mathrm{Cl}$ $80 \mathrm{~mL})$ and extracted with $\mathrm{Et}_{2} \mathrm{O}(100 \mathrm{~mL} \times 2)$. The combined organic phases washed with sat. aq. $\mathrm{NaCl}$, dried over $\mathrm{Na}_{2} \mathrm{SO}_{4}$ and concentrated under reduced pressure. The crude product was conducted next step without purification.

step 2: 2e was prepared according to the General Method B. Bis(chloromethyl)diethylsilane (0.37 g, 2.0 mmol, 1.0 equiv., crude product), TsBocNH ( $0.54 \mathrm{~g}, 2.0 \mathrm{mmol}, 1.0$ equiv.), $\mathrm{K}_{2} \mathrm{CO}_{3}(0.33 \mathrm{~g}$, $2.4 \mathrm{mmol}, 1.2$ equiv. $)$ in DMF $(5 \mathrm{~mL})$ at $60^{\circ} \mathrm{C}$ for $4 \mathrm{~h}$, then TFA $(1.14 \mathrm{~g}, 10 \mathrm{mmol}, 20.0$ equiv. $)$ in $\mathrm{CH}_{2} \mathrm{Cl}_{2}$ at $\mathrm{rt}$ for $5 \mathrm{~min}$ afforded $2 \mathrm{e}$ as white solid $(0.13 \mathrm{~g}, 36 \%)$.

$>$ mp: $92.7-95.8^{\circ} \mathrm{C}$.

$>\mathrm{R}_{f}=0.4$ (EtOAc:Petroleum Ether $\left.=1: 7\right)$.

$>{ }^{1} \mathrm{H} \mathrm{NMR}\left(400 \mathrm{MHz}, \mathrm{CDCl}_{3}\right) \delta 7.75(\mathrm{~d}, J=8.0 \mathrm{~Hz}, 2 \mathrm{H}), 7.32(\mathrm{~d}, J=8.0 \mathrm{~Hz}, 2 \mathrm{H}), 4.48(\mathrm{t}, J=$ $6.0 \mathrm{~Hz}, 1 \mathrm{H}), 2.86(\mathrm{~s}, 2 \mathrm{H}), 2.47$ (d, $J=6.0 \mathrm{~Hz}, 2 \mathrm{H}), 2.43$ (s, 3H), 0.95 (t, $J=7.9 \mathrm{~Hz}, 6 \mathrm{H}), 0.70$ (q, $J=7.9 \mathrm{~Hz}, 4 \mathrm{H})$.

$>{ }^{13} \mathrm{C}$ NMR $\left(100 \mathrm{MHz}, \mathrm{CDCl}_{3}\right) \delta 143.5,135.1,129.7,127.5,27.7,26.3,21.5,6.8,1.5$.

$>\mathrm{IR}$ (neat) $\mathrm{cm}^{-1} 3264,3007,2989,1461,1319,1159$.

$>$ HRMS calcd for $\mathrm{C}_{13} \mathrm{H}_{22} \mathrm{ClNO}_{2} \mathrm{SSi}(\mathrm{M}+\mathrm{Na})^{+} 342.0721$ found 342.0722 .

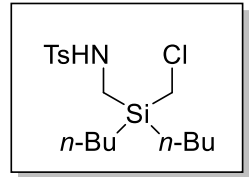

\section{$\mathrm{N}$-((dibutyl(chloromethyl)silyl)methyl)-4-methylbenzenesulfonamide (2f)}

$2 \mathbf{f}$ was prepared according to the similar procedure as that used for $\mathbf{2 e}$.

step 1: Bis(chloromethyl)dibutylsilane was prepared using the same procedure as that used for bis(chloromethyl)diethylsilane ${ }^{42}$. Dibutyldichlorosilane (4.26 g,

41. Dong, G.-Y.; Li, C.-P.; Liu. H. Molecules, 2019, 24, 937-956.

42. Handmann, V. I.; Bertermann, R.; Burschka, C.; Tacke, R. J. Organomet. Chem. 2000, 613, 19-25. 
20.0 mmol, 1.0 equiv.), $\mathrm{CH}_{2} \mathrm{BrCl}$ (7.76 g, $60.0 \mathrm{mmol}, 3.0$ equiv.), $n$-BuLi (19.2 mL, $46 \mathrm{mmol}, 2.4$ $\mathrm{mol} / \mathrm{L}$ in hexane, 2.3 equiv. $)$ in dried THF $(120 \mathrm{~mL})$ at $\mathrm{rt}$ overnight gave bis(chloromethyl)dibutylsilane and conducted next step without purification.

step 2: 2f was prepared according to the General Method B. Bis(chloromethyl)dibutylsilane (0.48 $\mathrm{g}, 2.0 \mathrm{mmol}, 1.0$ equiv., crude product), TsBocNH (0.54 g, $2.0 \mathrm{mmol}, 1.0$ equiv.), $\mathrm{K}_{2} \mathrm{CO}_{3}(0.33 \mathrm{~g}$, 2.4 mmol, 1.2 equiv. $)$ in DMF $(5 \mathrm{~mL})$ at $60^{\circ} \mathrm{C}$ for $4 \mathrm{~h}$, then TFA $(1.14 \mathrm{~g}, 10 \mathrm{mmol}, 20.0$ equiv. $)$ in $\mathrm{CH}_{2} \mathrm{Cl}_{2}$ at $\mathrm{rt}$ for $1 \mathrm{~h}$ afforded $\mathbf{2 f}$ as white solid $(0.08 \mathrm{~g}, 17 \%)$.

$>$ mp: $57.3-59.2^{\circ} \mathrm{C}$.

$>\mathrm{R}_{f}=0.4$ (EtOAc:Petroleum Ether $\left.=1: 10\right)$.

$>{ }^{1} \mathrm{H} \mathrm{NMR}\left(400 \mathrm{MHz}, \mathrm{CDCl}_{3}\right) \delta 7.75(\mathrm{~d}, J=8.0 \mathrm{~Hz}, 2 \mathrm{H}), 7.32(\mathrm{~d}, J=8.0 \mathrm{~Hz}, 2 \mathrm{H}), 4.52(\mathrm{t}, J=$ $6.2 \mathrm{~Hz}, 1 \mathrm{H}), 2.84(\mathrm{~s}, 2 \mathrm{H}), 2.45(\mathrm{~d}, J=6.2 \mathrm{~Hz}, 2 \mathrm{H}), 2.43(\mathrm{~s}, 3 \mathrm{H}), 1.30-1.21(\mathrm{~m}, 8 \mathrm{H}), 0.85(\mathrm{t}, J$ $=7.0 \mathrm{~Hz}, 6 \mathrm{H}), 0.70-0.65(\mathrm{~m}, 4 \mathrm{H})$.

$>{ }^{13} \mathrm{C} \mathrm{NMR}\left(100 \mathrm{MHz}, \mathrm{CDCl}_{3}\right) \delta 143.5,135.2,129.7,127.5,28.2,26.9,26.4,25.3,21.5,13.6$, 9.7 .

$>$ IR (neat) $\mathrm{cm}^{-1} 3278,2957,2923,2871,1990,1598,1456,1321,1157,1093$.

$>$ HRMS calcd for $\mathrm{C}_{17} \mathrm{H}_{30} \mathrm{ClNO}_{2} \mathrm{SSi}(\mathrm{M}+\mathrm{Na})^{+} 398.1347$ found 398.1336 .

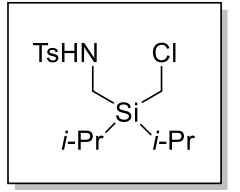

N-(((chloromethyl)diisopropylsilvl)methyl)-4-methylbenzenesulfonamide (2g)

$\mathbf{2 g}$ was prepared according to the similar procedure as that used for $\mathbf{2 e}$.

step 1: Bis(chloromethyl)diisopropylsilane was prepared using the same procedure as that used for bis(chloromethyl)diethylsilane ${ }^{42}$. Dichlorodiisopropylsilane (7.41 g, $40.0 \mathrm{mmol}, 1.0$ equiv.), $\mathrm{CH}_{2} \mathrm{BrCl}(15.5 \mathrm{~g}, 120.0 \mathrm{mmol}, 3.0$ equiv.), $n$ - $\mathrm{BuLi}$ (38.4 mL, $92 \mathrm{mmol}, 2.4 \mathrm{~mol} / \mathrm{L}$ in hexane, 2.3 equiv.) in dried THF $(100 \mathrm{~mL})$ at $\mathrm{rt}$ overnight gave bis(chloromethyl)- diisopropylsilane as colorless oil (4.6 g, 54\%).

step 2: 2g was prepared according to the General Method B. Bis(chloromethyl)diisopropylsilane (1.08 g, 5.1 mmol, 1.0 equiv.), TsBocNH (1.66 g, $6.13 \mathrm{mmol}, 1.2$ equiv.), $\mathrm{K}_{2} \mathrm{CO}_{3}$ (2.12 g, $15.3 \mathrm{mmol}$, 3.0 equiv.) in DMF ( $30 \mathrm{~mL}$ ) at $80{ }^{\circ} \mathrm{C}$ for $12 \mathrm{~h}$, then TFA ( $2.7 \mathrm{~g}, 24 \mathrm{mmol}, 20.0$ equiv.) in $\mathrm{CH}_{2} \mathrm{Cl}_{2}(10$ $\mathrm{mL})$ at $\mathrm{rt}$ for $7 \mathrm{~h}$ afforded $\mathbf{2 g}$ as white solid $(0.42 \mathrm{~g}, 10 \%)$.

$>$ mp: $98.8-101.0^{\circ} \mathrm{C}$.

$>\mathrm{R}_{f}=0.4$ (EtOAc:Petroleum Ether $\left.=1: 5\right)$.

$>{ }^{1} \mathrm{H} \mathrm{NMR}\left(400 \mathrm{MHz}, \mathrm{CDCl}_{3}\right) \delta 7.75(\mathrm{~d}, J=8.0 \mathrm{~Hz}, 2 \mathrm{H}), 7.33(\mathrm{~d}, J=8.0 \mathrm{~Hz}, 2 \mathrm{H}), 4.47(\mathrm{t}, J=$ $6.3 \mathrm{~Hz}, 1 \mathrm{H}), 2.93(\mathrm{~s}, 2 \mathrm{H}), 2.52(\mathrm{~d}, J=6.3 \mathrm{~Hz}, 2 \mathrm{H}), 2.44(\mathrm{~s}, 3 \mathrm{H}), 1.20-1.11(\mathrm{~m}, 2 \mathrm{H}), 1.03(\mathrm{~d}$, $J=6.9 \mathrm{~Hz}, 12 \mathrm{H})$.

$>{ }^{13} \mathrm{C} \mathrm{NMR}\left(100 \mathrm{MHz}, \mathrm{CDCl}_{3}\right) \delta 143.5,135.1,129.7,127.5,26.4,25.2,21.5,17.8,17.8,10.0$.

$>$ IR (neat) $\mathrm{cm}^{-1} 3270,2946,2867,1598,1460,1320,1156,1093,1049$.

$>\quad$ HRMS calcd for $\mathrm{C}_{15} \mathrm{H}_{26} \mathrm{ClNO}_{2} \mathrm{SSi}(\mathrm{M}+\mathrm{Na})^{+} 370.1034$ found 370.1035 .

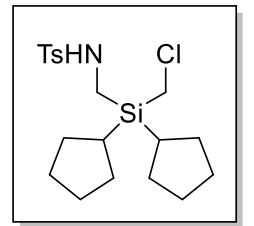

N-(((chloromethvl)dicvclopentylsilvl)methvl)-4-methylbenzenesulfonamide (2h)

2h was prepared according to the similar procedure as that used for $\mathbf{2 e}$.

step 1: Bis(chloromethyl)dicyclopentylsilane was prepared using the same procedure as that used for bis(chloromethyl)diethylsilane ${ }^{42}$. Dichlorodicyclopentylsilane (4.7 g, 20.0 mmol, 1.0 equiv.), $\mathrm{CH}_{2} \mathrm{BrCl}(7.7 \mathrm{~g}, 60.0$ mmol, 3.0 equiv.), $n$-BuLi (19.2 mL, $46 \mathrm{mmol}, 2.4 \mathrm{~mol} / \mathrm{L}$ in hexane, 2.3 equiv.) in dried THF (120 $\mathrm{mL})$ at $\mathrm{rt}$ overnight to give bis(chloromethyl)diisopropylsilane as colorless oil (3.5 g, 66\%).

step 2: $\mathbf{2 h}$ was prepared according to the General Method B. Bis(chloromethyl)diisopropylsilane (1.06 g, 4.0 mmol, 1.0 equiv.), TsBocNH (1.09 g, $4.0 \mathrm{mmol}, 1.0$ equiv.), $\mathrm{K}_{2} \mathrm{CO}_{3}(0.66 \mathrm{~g}, 4.8 \mathrm{mmol}$, 1.2 equiv.) in DMF $(10 \mathrm{~mL})$ at $60^{\circ} \mathrm{C}$ for $4 \mathrm{~h}$, then TFA $(1 \mathrm{~mL})$ in $\mathrm{CH}_{2} \mathrm{Cl}_{2}(5 \mathrm{~mL})$ at $\mathrm{rt}$ for $1 \mathrm{~h}$ to afford $\mathbf{2 h}$ as white solid $(0.12 \mathrm{~g}, 7.5 \%)$.

$>$ mp: $82.8-84.8^{\circ} \mathrm{C}$.

$>\mathrm{R}_{f}=0.5($ EtOAc:Petroleum Ether $=1: 5)$.

$>{ }^{1} \mathrm{H}$ NMR $\left(400 \mathrm{MHz}, \mathrm{CDCl}_{3}\right) \delta 7.74(\mathrm{~d}, J=8.0 \mathrm{~Hz}, 2 \mathrm{H}), 7.32(\mathrm{~d}, J=8.0 \mathrm{~Hz}, 2 \mathrm{H}), 4.42(\mathrm{t}, J=$ $6.3 \mathrm{~Hz}, 1 \mathrm{H}), 2.91(\mathrm{~s}, 2 \mathrm{H}), 2.52(\mathrm{~d}, J=6.3 \mathrm{~Hz}, 2 \mathrm{H}), 2.44(\mathrm{~s}, 3 \mathrm{H}), 1.82-1.75(\mathrm{~m}, 4 \mathrm{H}), 1.64-$ $1.55(\mathrm{~m}, 4 \mathrm{H}), 1.54-1.44(\mathrm{~m}, 4 \mathrm{H}), 1.37-1.26(\mathrm{~m}, 4 \mathrm{H}), 1.14-1.05(\mathrm{~m}, 2 \mathrm{H})$.

$>{ }^{13} \mathrm{C}$ NMR $\left(150 \mathrm{MHz}, \mathrm{CDCl}_{3}\right) \delta 143.4,135.2,129.6,127.5,28.2,27.5,26.6,26.6,26.6,26.2$, 21.7, 21.5 .

$>$ IR (neat) $\mathrm{cm}^{-1} 3281,2947,2863,1598,1448,1321,1157,1093,1042$.

$>$ HRMS calcd for $\mathrm{C}_{19} \mathrm{H}_{30} \mathrm{ClNO}_{2} \mathrm{SSi}(\mathrm{M}+\mathrm{Na})^{+} 422.1347$ found 422.1320 . 


\section{N-(((chloromethvl)divinvlsilvl)methvl)-4-methvlbenzenesulfonamide (2i)}

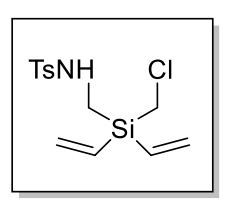

$\mathbf{2 i}$ was prepared according to the similar procedure as that used for $\mathbf{2 e}$.

step 1: Bis(chloromethyl)divinylsilane was prepared using the same procedure as that used for bis(chloromethyl)diethylsilane ${ }^{42}$. Dichlorodivinylsilane (1.48 g, 9.7 mmol, 1.0 equiv.), $\mathrm{CH}_{2} \mathrm{BrCl}$ (3.75 g, $29 \mathrm{mmol}, 3.0$ equiv.), $n$ - $\mathrm{BuLi}(9.2 \mathrm{~mL}, 22.2$ $\mathrm{mmol}, 2.4 \mathrm{~mol} / \mathrm{L}$ in hexane, 2.3 equiv.) in dried THF $(60 \mathrm{~mL})$ at $\mathrm{rt}$ overnight gave bis(chloromethyl)divinylsilane as colorless oil (0.94 g, 54\%).

step 2: $2 \mathbf{i}$ was prepared according to the General Method B. Bis(chloromethyl)divinylsilane ( $0.9 \mathrm{~g}$, $5.0 \mathrm{mmol}, 1.0$ equiv.), TsBocNH (1.35 g, $5.0 \mathrm{mmol}, 1.0$ equiv.), $\mathrm{K}_{2} \mathrm{CO}_{3}(0.82 \mathrm{~g}, 6.0 \mathrm{mmol}, 1.2$ equiv.) in DMF $(10 \mathrm{~mL})$ at $60{ }^{\circ} \mathrm{C}$ for $1.5 \mathrm{~h}$, then TFA $(1.22 \mathrm{~g}, 10.7 \mathrm{mmol}, 5.0$ equiv. $)$ in $\mathrm{CH}_{2} \mathrm{Cl}_{2}(10 \mathrm{~mL})$ at $\mathrm{rt}$ for $0.5 \mathrm{~h}$ afforded $2 \mathrm{i}$ as white solid $(0.6 \mathrm{~g}, 39 \%)$.

$>$ mp: $67.9-69.4^{\circ} \mathrm{C}$.

$>\mathrm{R}_{f}=0.4($ EtOAc:Petroleum Ether $=1: 5)$.

$>{ }^{1} \mathrm{H} \mathrm{NMR}\left(600 \mathrm{MHz}, \mathrm{CDCl}_{3}\right) \delta 7.75(\mathrm{~d}, J=8.0 \mathrm{~Hz}, 2 \mathrm{H}), 7.33(\mathrm{~d}, J=7.9 \mathrm{~Hz}, 2 \mathrm{H}), 6.21(\mathrm{dd}, J=$ $14.8,3.2 \mathrm{~Hz}, 2 \mathrm{H}), 6.06(\mathrm{dd}, J=20.6,14.8 \mathrm{~Hz}, 2 \mathrm{H}), 5.90(\mathrm{dd}, J=20.5,3.3 \mathrm{~Hz}, 2 \mathrm{H}), 4.48(\mathrm{t}, J$ $=6.2 \mathrm{~Hz}, 1 \mathrm{H}), 2.95(\mathrm{~s}, 2 \mathrm{H}), 2.62(\mathrm{~d}, J=6.2 \mathrm{~Hz}, 2 \mathrm{H}), 2.44(\mathrm{~s}, 3 \mathrm{H})$.

$>{ }^{13} \mathrm{C} \mathrm{NMR}\left(150 \mathrm{MHz}, \mathrm{CDCl}_{3}\right) \delta 143.6,138.2,135.1,129.7,129.2,127.5,27.8,26.1,21.5$.

$>$ IR (neat) $\mathrm{cm}^{-1} 3278,3055,2949,1596,1494,1441,1406,1323,1159,1093,1007,964$.

$>$ HRMS calcd for $\mathrm{C}_{13} \mathrm{H}_{18} \mathrm{ClNO}_{2} \mathrm{SSi}(\mathrm{M}+\mathrm{Na})^{+} 338.0414$ found 338.0418 .

\section{N-(((chloromethyl)diphenylsilyl)methyl)-4-methylbenzenesulfonamide $(2 \mathrm{j})$}

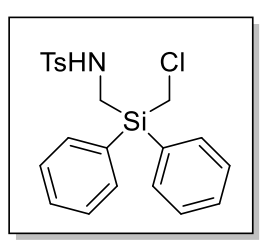

$\mathbf{2} \mathbf{j}$ was prepared according to the similar procedure as that used for $\mathbf{2 e}$.

step 1: Bis(chloromethyl)diphenylsilanewas prepared using the same procedure as that used for bis(chloromethyl)diethylsilane ${ }^{42}$. Dichlorodiphenylsilane (10.3 g, $40 \mathrm{mmol}, 1.0$ equiv.), $\mathrm{CH}_{2} \mathrm{BrCl}$ (15.5 g, 120 mmol, 3.0 equiv.), $n$-BuLi ( $40 \mathrm{~mL}, 92 \mathrm{mmol}, 2.4 \mathrm{~mol} / \mathrm{L}$ in hexane, 2.3 equiv.) colorless oil $(8.7 \mathrm{~g}, 76 \%)$

in dried THF $(250 \mathrm{~mL})$ at $\mathrm{rt}$ overnight gave bis(chloromethyl)divinylsilane as

step 2: $\mathbf{2 j}$ was prepared according to the General Method B. Bis(chloromethyl)diphenylsilanewas (2.81 g, 10.0 mmol, 1.0 equiv.), TsBocNH (2.71 g, 10.0 mmol, 1.0 equiv.), $\mathrm{K}_{2} \mathrm{CO}_{3}$ (2.07 g, 15.0 mmol, 1.5 equiv.) in DMF $(15 \mathrm{~mL})$ at $60{ }^{\circ} \mathrm{C}$ overnight, then TFA ( $4.7 \mathrm{~g}, 41.0 \mathrm{mmol}, 5.0$ equiv.) in $\mathrm{CH}_{2} \mathrm{Cl}_{2}(30 \mathrm{~mL})$ at $\mathrm{rt}$ for $1 \mathrm{~h}$ afforded $\mathbf{2} \mathbf{j}$ as white solid $(1.83 \mathrm{~g}, 58 \%)$.

$>\mathrm{mp}: 141.3-143.5^{\circ} \mathrm{C}$.

$>\mathrm{R}_{f}=0.4($ EtOAc:Petroleum Ether $=1: 5)$.

$>{ }^{1} \mathrm{H} \mathrm{NMR}\left(400 \mathrm{MHz}, \mathrm{CDCl}_{3}\right) \delta 7.71(\mathrm{~d}, J=8.0 \mathrm{~Hz}, 2 \mathrm{H}), 7.50-7.45(\mathrm{~m}, 6 \mathrm{H}), 7.40-7.36(\mathrm{~m}$, $4 \mathrm{H}), 7.31(\mathrm{~d}, J=8.0 \mathrm{~Hz}, 2 \mathrm{H}), 4.29(\mathrm{t}, J=6.2 \mathrm{~Hz}, 1 \mathrm{H}), 3.30(\mathrm{~s}, 2 \mathrm{H}), 3.00(\mathrm{~d}, J=6.2 \mathrm{~Hz}, 2 \mathrm{H})$, $2.44(\mathrm{~s}, 3 \mathrm{H})$.

$>{ }^{13} \mathrm{C}$ NMR $\left(100 \mathrm{MHz}, \mathrm{CDCl}_{3}\right) \delta 143.7,135.0,135.0,130.9,129.9,129.8,128.5,127.6,28.3$, 26.4, 21.6 .

$>$ IR (neat) $\mathrm{cm}^{-1} 3272,3007,1597,1428,1323,1160,1094$.

$>$ HRMS calcd for $\mathrm{C}_{21} \mathrm{H}_{22} \mathrm{ClNO}_{2} \mathrm{SSi}(\mathrm{M}+\mathrm{Na})^{+} 438.0721$ found 438.0701 .

\section{N-(((chloromethyl)bis(3-methoxyphenyl)silyl)methyl)-4-methylbenzenesulfonamide (2k)}
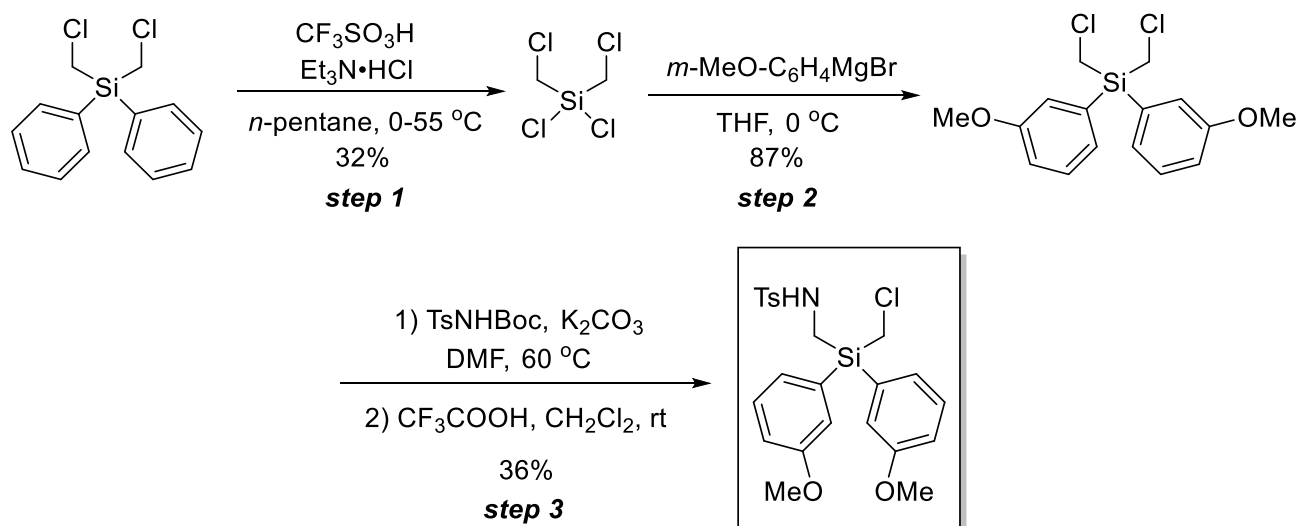
step 1: To a solution of bis(chloromethyl)diphenylsilane (31 mmol, $8.7 \mathrm{~g}, 1.0$ equiv.) in $n$-pentane $(25 \mathrm{~mL})$ was added $\mathrm{CF}_{3} \mathrm{CO}_{3} \mathrm{H}(68 \mathrm{mmol}, 10.2 \mathrm{~g}, 2.2$ equiv.) at room temperature under Ar. The reaction was refluxed at $55^{\circ} \mathrm{C}$ for $1 \mathrm{~h}$ before adding triethylamine hydrochloride $(68 \mathrm{mmol}, 9.3 \mathrm{~g}$, 2.2 equiv.) at $0{ }^{\circ} \mathrm{C}$. The mixture was stirred at room temperature overnight. The upper layer was transferred by a syringe to a dried $50 \mathrm{~mL}$ flask, and the residual was washed with $n$-pentane $(15 \mathrm{~mL})$. The above solution was combined and concentrated under reduced pressure. The residue was distilled to afford dichlorobis(chloromethyl)silane as colorless oil $(2.0 \mathrm{~g}, 32 \%)$.

step 2: To a solution of dichlorobis(chloromethyl)silane ( $8 \mathrm{mmol}, 1.57 \mathrm{~g}, 1.0$ equiv.) in dried THF $(5 \mathrm{~mL})$ was added (3-methoxyphenyl)magnesium bromide $(17.6 \mathrm{mmol}, 17.6 \mathrm{~mL}, 1.0 \mathrm{~mol} / \mathrm{L}$ in THF, 2.2 equiv.) at $0{ }^{\circ} \mathrm{C}$ under Ar. The reaction was stirred at room temperature for $2 \mathrm{~h}$ before quenching with sat. aq. $\mathrm{NH}_{4} \mathrm{Cl}(10 \mathrm{~mL})$ and extracting with EtOAc $(20 \mathrm{~mL} \times 2)$. The combined organic phases were washed with sat. aq. $\mathrm{NaCl}$, dried over $\mathrm{Na}_{2} \mathrm{SO}_{4}$ and concentrated under reduced pressure. The crude residue was purified by silica gel flash column chromatography (eluent: petroleum ether/EtOAc $=50 / 1)$ to give bis(chloromethyl)bis(3-methoxyphenyl)silane as colorless oil ( $2.3 \mathrm{~g}$, $87 \%)$.

step 3: 2k was prepared according to the General Method B. Bis(chloromethyl)bis(3methoxyphenyl)silane ( $2.3 \mathrm{~g}, 7.4 \mathrm{mmol}, 1.0$ equiv.), TsBocNH (1.8 g, $7.4 \mathrm{mmol}, 1.0$ equiv.), $\mathrm{K}_{2} \mathrm{CO}_{3}$ $(1.4 \mathrm{~g}, 10.4 \mathrm{mmol}, 1.4$ equiv. $)$ in DMF $(30 \mathrm{~mL})$ at $60^{\circ} \mathrm{C}$ overnight, then TFA $(1.47 \mathrm{~g}, 12.8 \mathrm{mmol}, 5.0$ equiv.) in $\mathrm{CH}_{2} \mathrm{Cl}_{2}(30 \mathrm{~mL})$ at $\mathrm{rt}$ for $1 \mathrm{~h}$ afforded $2 \mathrm{k}$ as white solid $(1.16 \mathrm{~g}, 36 \%)$.

$>$ mp: $96.2-97.0^{\circ} \mathrm{C}$.

$>\mathrm{R}_{f}=0.3$ (EtOAc:Petroleum Ether $\left.=1: 5\right)$.

$>{ }^{1} \mathrm{H} \mathrm{NMR}\left(400 \mathrm{MHz}, \mathrm{CDCl}_{3}\right) \delta 7.70(\mathrm{~d}, J=8.0 \mathrm{~Hz}, 2 \mathrm{H}), 7.33-7.28(\mathrm{~m}, 4 \mathrm{H}), 7.06-7.04(\mathrm{~m}$, $4 \mathrm{H}), 7.00-6.97(\mathrm{~m}, 2 \mathrm{H}), 4.38(\mathrm{t}, J=6.2 \mathrm{~Hz}, 1 \mathrm{H}), 3.77(\mathrm{~s}, 6 \mathrm{H}), 3.29(\mathrm{~s}, 2 \mathrm{H}), 2.98(\mathrm{~d}, J=6.2$ $\mathrm{Hz}, 2 \mathrm{H}), 2.42(\mathrm{~s}, 3 \mathrm{H})$.

$>{ }^{13} \mathrm{C} \mathrm{NMR}\left(100 \mathrm{MHz}, \mathrm{CDCl}_{3}\right) \delta 159.4,143.6,135.0,131.3,129.8,129.8,127.6,127.1,120.4$, $116.3,55.2,28.3,26.3,21.6$.

$>$ IR (neat) $\mathrm{cm}^{-1} 3276,2935,2834,1570,1403,1281,1158,1039,991$.

$>$ HRMS calcd for $\mathrm{C}_{23} \mathrm{H}_{26} \mathrm{ClNO}_{4} \mathrm{SSi}(\mathrm{M}+\mathrm{Na})^{+} 498.0938$ found 498.0926.

\section{N-(((chloromethvl)bis(4-fluorophenvl)silvl)methvl)-4-methvlbenzenesulfonamide (2I)}

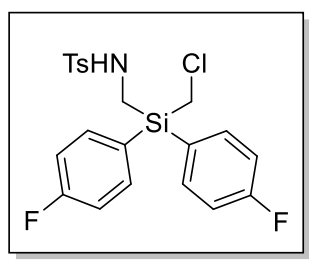

$\mathbf{2 l}$ was prepared according to the similar procedure as that used for $\mathbf{2 k}$.

step 2: Dichlorobis(chloromethyl)silane $(0.4 \mathrm{~g}, 2.0 \mathrm{mmol}$, 1.0 equiv.) obtained from the step $\mathbf{1}$ in the preparation of $\mathbf{2 k}$, (3methoxyphenyl)magnesium bromide $(4.8 \mathrm{mmol}, 4.8 \mathrm{~mL}, 1.0 \mathrm{Mol} / \mathrm{L}$ in THF, 2.4 equiv.) in dried THF $(5 \mathrm{~mL})$ at room temperature for $2 \mathrm{~h}$ gave bis(chloromethyl)bis(4-fluorophenyl)silane as colorless oil ( $0.38 \mathrm{~g}, 60 \%)$.

step 3: 2l was prepared according to the General Method B. Bis(chloromethyl)bis(4-fluorophenyl)silane ( $0.38 \mathrm{~g}, 1.2 \mathrm{mmol}, 1.0$ equiv.), TsBocNH (0.33 g, 1.2 mmol, 1.0 equiv.), $\mathrm{K}_{2} \mathrm{CO}_{3}(0.2 \mathrm{~g}, 1.4 \mathrm{mmol}, 1.2$ equiv. $)$ in DMF $(5 \mathrm{~mL})$ at $60^{\circ} \mathrm{C}$ for $2 \mathrm{~h}$, then TFA $\left(0.4 \mathrm{~g}, 3.5 \mathrm{mmol}, 5.0\right.$ equiv.) in $\mathrm{CH}_{2} \mathrm{Cl}_{2}(5 \mathrm{~mL})$ at room temperature for $2 \mathrm{~h}$ afforded $2 \mathrm{l}$ as white solid $(0.14 \mathrm{~g}, 25 \%)$.

$>\quad \mathrm{mp}: 150.4-154.2^{\circ} \mathrm{C}$.

$>\mathrm{R}_{f}=0.4$ (EtOAc:Petroleum Ether $\left.=1: 5\right)$.

$>{ }^{1} \mathrm{H} \mathrm{NMR}\left(400 \mathrm{MHz}, \mathrm{CDCl}_{3}\right) \delta 7.71(\mathrm{~d}, J=8.0 \mathrm{~Hz}, 2 \mathrm{H}), 7.49-7.46(\mathrm{~m}, 4 \mathrm{H}), 7.32(\mathrm{~d}, J=8.0$ $\mathrm{Hz}, 2 \mathrm{H}), 7.12-7.07(\mathrm{~m}, 4 \mathrm{H}), 4.32(\mathrm{t}, J=6.3 \mathrm{~Hz}, 1 \mathrm{H}), 3.27(\mathrm{~s}, 2 \mathrm{H}), 2.97(\mathrm{~d}, J=6.3 \mathrm{~Hz}, 2 \mathrm{H})$, $2.45(\mathrm{~s}, 3 \mathrm{H})$.

> ${ }^{13} \mathrm{C}$ NMR $\left(100 \mathrm{MHz}, \mathrm{CDCl}_{3}\right) \delta 164.9(\mathrm{~d}, J=250 \mathrm{~Hz}), 143.8,137.1(\mathrm{~d}, J=8 \mathrm{~Hz}), 134.9,129.8$, $127.6,125.3(\mathrm{~d}, J=4 \mathrm{~Hz}), 116.0(\mathrm{~d}, J=20 \mathrm{~Hz}), 28.5,26.3,21.6$.

$>{ }^{19} \mathrm{~F} \mathrm{NMR}\left(376 \mathrm{MHz}, \mathrm{CDCl}_{3}\right) \delta-107.92$.

$>$ IR (neat) $\mathrm{cm}^{-1} 3254,3006,1587,1500,1162,1284,1115$.

$>$ HRMS calcd for $\mathrm{C}_{21} \mathrm{H}_{20} \mathrm{ClF}_{2} \mathrm{NO}_{2} \mathrm{SSi}(\mathrm{M}+\mathrm{Na})^{+} 474.0538$ found 474.0527 .

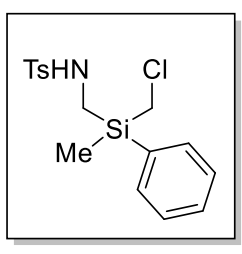

N-(((chloromethvl)(methvl)(phenvl)silvl)methvl)-4-methvlbenzenesulfonami $\underline{\text { de }}(\mathbf{2 m})$

$\mathbf{2 m}$ was prepared according to the similar procedure as that used for $\mathbf{2 e}$.

step 1: Bis(chloromethyl)(methyl)(phenyl)silane was prepared using the same procedure as that used for bis(chloromethyl)diethylsilane ${ }^{42}$. 
Dichloro(methyl)(phenyl)silane (3.9 g, $20 \mathrm{mmol}, 1.0$ equiv.), $\mathrm{CH}_{2} \mathrm{BrCl}$ (7.76 g, $60 \mathrm{mmol}, 3.0$ equiv.), $n$-BuLi (19.2 mL, $46 \mathrm{mmol}, 2.4 \mathrm{~mol} / \mathrm{L}$ in hexane, 2.3 equiv.) in dried THF (120 mL) at room temperature overnight gave bis(chloromethyl)(methyl)(phenyl)silane as colorless oil (3.4 g, 77\%).

step 2: 2m was prepared according to the General Method B. bis(chloromethyl) (methyl)(phenyl)silane (2.7 g, $12.3 \mathrm{mmol}, 1.0$ equiv.), TsBocNH (3.3 g, $12.3 \mathrm{mmol}, 1.0$ equiv.), $\mathrm{K}_{2} \mathrm{CO}_{3}(2.0 \mathrm{~g}, 14.7 \mathrm{mmol}, 1.2$ equiv. $)$ in DMF $(30 \mathrm{~mL})$ at $60^{\circ} \mathrm{C}$ for $4 \mathrm{~h}$, then TFA $(4.7 \mathrm{~g}, 41.0 \mathrm{mmol}$, 5.0 equiv. $)$ in $\mathrm{CH}_{2} \mathrm{Cl}_{2}(30 \mathrm{~mL})$ at $\mathrm{rt}$ for $1 \mathrm{~h}$ afforded $2 \mathrm{~m}$ as white solid $(2.67 \mathrm{~g}, 61 \%)$.

$>$ mp: $122.2-123.0^{\circ} \mathrm{C}$.

$>\mathrm{R}_{f}=0.4$ (EtOAc:Petroleum Ether $\left.=1: 10\right)$.

$>{ }^{1} \mathrm{H} \mathrm{NMR}\left(400 \mathrm{MHz}, \mathrm{CDCl}_{3}\right) \delta 7.71(\mathrm{~d}, J=8.0 \mathrm{~Hz}, 2 \mathrm{H}), 7.47-7.43(\mathrm{~m}, 3 \mathrm{H}), 7.40-7.36(\mathrm{~m}$, $2 \mathrm{H}), 7.31(\mathrm{~d}, J=8.0 \mathrm{~Hz}, 2 \mathrm{H}), 4.23(\mathrm{t}, J=6.3 \mathrm{~Hz}, 1 \mathrm{H}), 3.04(\mathrm{dd}, J=22.2,14.0 \mathrm{~Hz}, 2 \mathrm{H}), 2.70$ (dd, $J=6.3,2.5 \mathrm{~Hz}, 2 \mathrm{H}), 2.44(\mathrm{~s}, 3 \mathrm{H}), 0.47(\mathrm{~s}, 3 \mathrm{H})$.

$>{ }^{13} \mathrm{C}$ NMR $\left(100 \mathrm{MHz}, \mathrm{CDCl}_{3}\right) \delta 143.6,135.1,134.0,131.6,130.8,129.7,128.5,127.5,29.4$, $27.6,21.6,-7.2$.

$>\quad \mathrm{IR}$ (neat) $\mathrm{cm}^{-1} 3267,2926,1595,1446,1426,1321,1159,1093,1017$.

$>$ HRMS calcd for $\mathrm{C}_{16} \mathrm{H}_{20} \mathrm{ClNO}_{2} \mathrm{SSi}(\mathrm{M}+\mathrm{Na})^{+} 376.0570$ found 376.0563 .

N-((1-(chloromethyl)silolan-1-yl)methyl)-4-methylbenzenesulfonamide (2n)

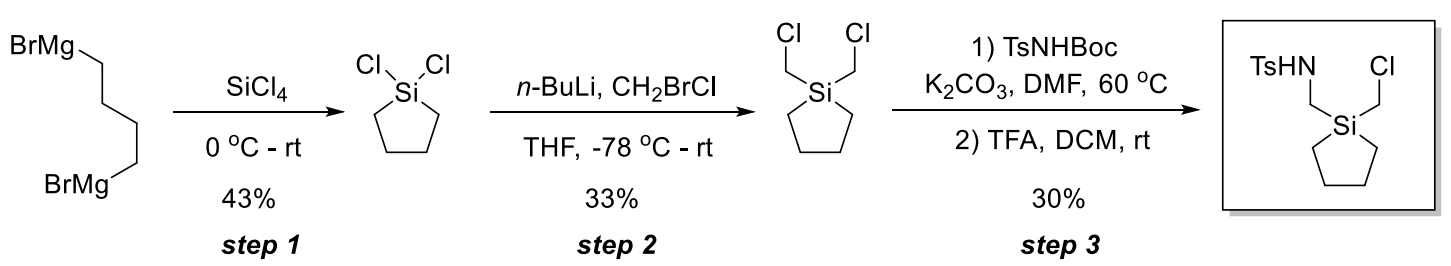

2n was prepared according to the similar procedure as that used for $\mathbf{2 e}$.

step $1^{43}$ : To a stirred suspension of magnesium turnings (5.4 g, $220 \mathrm{mmol}, 2.2$ equiv.) in $\mathrm{Et}_{2} \mathrm{O}$ (60 $\mathrm{mL})$ at room temperature was added a solution of 1,4-dibromobutane (100 mmol, $21.5 \mathrm{~g})$ in $\mathrm{Et}_{2} \mathrm{O}$ $(40 \mathrm{~mL})$ slowly. When the reaction started with gentle heating, the rest of the 1,4-dibromobutane solution was added over $2 \mathrm{~h}$ while keeping the reaction at a gentle reflux. After addition was completed, the mixture was refluxed for an additional $90 \mathrm{~min}$ before cooling to room temperature. The resulting mixture was transferred dropwise over $1 \mathrm{~h}$ by cannula filtration to a second flask containing $\mathrm{SiCl}_{4}\left(12.6 \mathrm{~mL}, 110 \mathrm{mmol}, 1.1\right.$ equiv.) in $\mathrm{Et}_{2} \mathrm{O}(50 \mathrm{~mL})$ at $0{ }^{\circ} \mathrm{C}$. The reaction mixture was stirred at room temperature overnight before concentrating under reduced pressure. The remaining residue was triturated in dry hexanes $(50 \mathrm{~mL} \times 2)$ filtered by cannula. The combined solvent was removed under vacuum and the residue was purified by distillation under reduced pressure to give 1,1-dichlorosilolane as a clear oil $(6.7 \mathrm{~g}, 43 \%)$.

step 2: 1,1-bis(chloromethyl)silolane was prepared using the same procedure as that used for bis(chloromethyl)diethylsilane ${ }^{42}$. 1,1-dichlorosilolane ( $3.1 \mathrm{~g}, 20$ mmol, 1.0 equiv.), $\mathrm{CH}_{2} \mathrm{BrCl}$ (7.76 g, $60 \mathrm{mmol}, 3.0$ equiv.), $n$-BuLi (19.2 mL, $46 \mathrm{mmol}, 2.4 \mathrm{~mol} / \mathrm{L}$ in hexane, 2.3 equiv.) in dried THF $(120 \mathrm{~mL})$ at room temperature overnight gave 1,1-bis(chloromethyl)silolane as colorless oil (1.21 g, 33\%).

step 3: 2n was prepared according to the General Method B. 1,1-bis(chloromethyl)silolane ( $0.37 \mathrm{~g}$, $2.0 \mathrm{mmol}, 1.0$ equiv.), TsBocNH ( $0.54 \mathrm{~g}, 2.0 \mathrm{mmol}, 1.0$ equiv.), $\mathrm{K}_{2} \mathrm{CO}_{3}(0.33 \mathrm{~g}, 2.4 \mathrm{mmol}, 1.2$ equiv.) in DMF $(5 \mathrm{~mL})$ at $60{ }^{\circ} \mathrm{C}$ for $4 \mathrm{~h}$, then TFA $(1.14 \mathrm{~g}, 10.0 \mathrm{mmol}, 20.0$ equiv. $)$ in $\mathrm{CH}_{2} \mathrm{Cl}_{2}(1 \mathrm{~mL})$ at $\mathrm{rt}$ for $5 \mathrm{~min}$ afforded $\mathbf{2 n}$ as white solid $(0.12 \mathrm{~g}, 30 \%)$.

$>\mathrm{mp}: 87.0-89.2^{\circ} \mathrm{C}$.

$>\mathrm{R}_{f}=0.3$ (EtOAc:Petroleum Ether $\left.=1: 8\right)$.

$>{ }^{1} \mathrm{H} \mathrm{NMR}\left(400 \mathrm{MHz}, \mathrm{CDCl}_{3}\right) \delta 7.75(\mathrm{~d}, J=8.0 \mathrm{~Hz}, 2 \mathrm{H}), 7.33(\mathrm{~d}, J=8.0 \mathrm{~Hz}, 2 \mathrm{H}), 4.51(\mathrm{t}, J=$ $5.8 \mathrm{~Hz}, 1 \mathrm{H}), 2.90(\mathrm{~s}, 2 \mathrm{H}), 2.55(\mathrm{~d}, J=6.2 \mathrm{~Hz}, 2 \mathrm{H}), 2.44(\mathrm{~s}, 3 \mathrm{H}), 1.66-1.58(\mathrm{~m}, 4 \mathrm{H}), 0.78-$ $0.67(\mathrm{~m}, 4 \mathrm{H})$.

$>{ }^{13} \mathrm{C} \mathrm{NMR}\left(100 \mathrm{MHz}, \mathrm{CDCl}_{3}\right) \delta 143.6,135.2,129.8,127.5,28.9,27.2,26.8,21.6,8.8$.

$>\quad \mathrm{IR}$ (neat) $\mathrm{cm}^{-1} 3270,2931,1860,1598,1450,1401,1319,1156,1092,1029$. 
$>\quad$ HRMS calcd for $\mathrm{C}_{13} \mathrm{H}_{20} \mathrm{ClNO}_{2} \mathrm{SSi}(\mathrm{M}+\mathrm{Na})^{+} 340.0565$ found 340.0564 .

\section{N-((1-(chloromethyl)silinan-1-yl)methyl)-4-methylbenzenesulfonamide (20)}

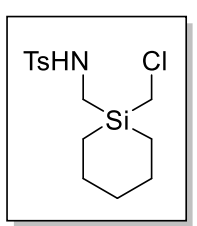

$\mathbf{2 o}$ was prepared according to the similar procedure as that used for $\mathbf{2 n}$.

step 1: 1,5-dibromopentane (23.0 g, $100 \mathrm{mmol}, 1.0$ equiv.), magnesium turnings (4.8 g, 200 mmol, 2.0 equiv.), $\mathrm{SiCl}_{4}\left(17 \mathrm{~g}, 100 \mathrm{mmol}, 1.0\right.$ equiv.) in $\mathrm{Et}_{2} \mathrm{O}$ (300 mL) at room temperature overnight afforded 1,1-dichlorosilinane as a clear oil $(7.33 \mathrm{~g}$, $41 \%)$.

step 2: 1,1-bis(chloromethyl)silinane was prepared using the same procedure as that used for bis(chloromethyl)diethylsilane ${ }^{42}$. 1,1-dichlorosilinane (3.9 g, $20 \mathrm{mmol}, 1.0$ equiv.), $\mathrm{CH}_{2} \mathrm{BrCl}$ (7.76 g, $60 \mathrm{mmol}, 3.0$ equiv.), $n$-BuLi (28.8 mL, $46 \mathrm{mmol}, 1.6 \mathrm{~mol} / \mathrm{L}$ in hexane, 2.3 equiv.) in dried THF $(120 \mathrm{~mL})$ at room temperature overnight gave 1,1-bis(chloromethyl)silinane as colorless oil $(2.56 \mathrm{~g}, 65 \%)$.

step 3: 20 was prepared according to the General Method B. 1,1-bis(chloromethyl)silinane (0.39 g, $2.0 \mathrm{mmol}, 1.0$ equiv.), TsBocNH (0.54 g, $2.0 \mathrm{mmol}, 1.0$ equiv.), $\mathrm{K}_{2} \mathrm{CO}_{3}(0.33 \mathrm{~g}, 2.4 \mathrm{mmol}, 1.2$ equiv. $)$ in DMF $(5 \mathrm{~mL})$ at $60{ }^{\circ} \mathrm{C}$ for $4 \mathrm{~h}$, then TFA $(1.14 \mathrm{~g}, 10.0 \mathrm{mmol}, 20.0$ equiv. $)$ in $\mathrm{CH}_{2} \mathrm{Cl}_{2}(1 \mathrm{~mL})$ at $\mathrm{rt}$ for $5 \mathrm{~min}$ afforded 20 as white solid $(0.08 \mathrm{~g}, 33 \%)$.

$>\quad \mathrm{mp}: 138.2-140.1^{\circ} \mathrm{C}$.

$>\mathrm{R}_{f}=0.4$ (EtOAc:Petroleum Ether $\left.=1: 5\right)$.

$>{ }^{1} \mathrm{H}$ NMR $\left(400 \mathrm{MHz}, \mathrm{CDCl}_{3}\right) \delta 7.75(\mathrm{~d}, J=8.2 \mathrm{~Hz}, 2 \mathrm{H}), 7.32(\mathrm{~d}, J=8.0 \mathrm{z}, 2 \mathrm{H}), 4.67(\mathrm{~s}, 1 \mathrm{H})$, $2.88(\mathrm{~s}, 2 \mathrm{H}), 2.49$ (d, $J=6.2 \mathrm{~Hz}, 2 \mathrm{H}), 2.43$ (br s, 3H), $1.66-1.60(\mathrm{~m}, 4 \mathrm{H}), 1.41-1.37$ (m, 2H), $0.81-0.69(\mathrm{~m}, 4 \mathrm{H})$.

$>{ }^{13} \mathrm{C} \mathrm{NMR}\left(100 \mathrm{MHz}, \mathrm{CDCl}_{3}\right) \delta 143.5,135.1,129.7,127.5,29.2,28.1,26.7,23.9,21.5,8.5$.

$>$ IR (neat) $\mathrm{cm}^{-1} 3273,2918,2852,1598,1445,1401,1320,1156,1093,992,909$.

$>$ HRMS calcd for $\mathrm{C}_{14} \mathrm{H}_{22} \mathrm{ClNO}_{2} \mathrm{SSiNa}(\mathrm{M}+\mathrm{Na})^{+} 354.0721$ found 354.0720.

N-((2-(chloromethyl)-1,2,3,4-tetrahydrobenzo/c/silin-2-yl)methyl)-4-methylbenzenesulfonamide (2p)

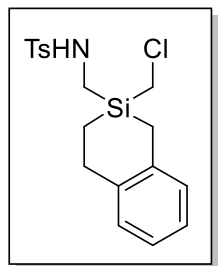

$\mathbf{2 p}$ was prepared according to the similar procedure as that used for $\mathbf{2 n}$.

step 1: 1-bromo-2-(2-bromoethyl)benzene (13.2g, $50 \mathrm{mmol}, 1.0$ equiv.), magnesium turnings $3.6 \mathrm{~g}, 150 \mathrm{mmol}, 3.0$ equiv. $), \mathrm{SiCl}_{4}(9.3 \mathrm{~g}, 55 \mathrm{mmol}, 1.1$ equiv. in THF $(70 \mathrm{~mL})$ at room temperature overnight afforded 1,1-dichloro-2,3-dihydro$1 \mathrm{H}$-benzo[b]silole as a clear oil $(0.55 \mathrm{~g}, 5.4 \%)$.

step 2: 2,2-bis(chloromethyl)-1,2,3,4-tetrahydrobenzo[c]siline was prepared using the same procedure as that used for bis(chloromethyl)diethylsilane ${ }^{42}$. 1,1dichloro-2,3-dihydro-1H-benzo[b] silole (0.55 g, $2.7 \mathrm{mmol}, 1.0$ equiv.), $\mathrm{CH}_{2} \mathrm{BrCl}$ (1.04 g, $8.1 \mathrm{mmol}$, 3.0 equiv.), $n$-BuLi (2.6 mL, $6.3 \mathrm{mmol}, 2.4 \mathrm{~mol} / \mathrm{L}$ in hexane, 2.3 equiv.) in dried THF (10 mL) at room temperature overnight gave 2,2-bis(chloromethyl)-1,2,3,4-tetrahydrobenzo[c]siline as colorless oil $(0.23 \mathrm{~g}, 35 \%)$.

step 3: 2p was prepared according to the General Method B. 2,2-bis(chloromethyl)-1,2,3,4tetrahydrobenzo[c]siline $(0.23 \mathrm{~g}, 0.94 \mathrm{mmol}, 1.0$ equiv.), TsBocNH (0.27 g, $1.0 \mathrm{mmol}, 1.1$ equiv.), $\mathrm{K}_{2} \mathrm{CO}_{3}(0.2 \mathrm{~g}, 1.5 \mathrm{mmol}, 1.6$ equiv. $)$ in DMF $(4 \mathrm{~mL})$ at $60{ }^{\circ} \mathrm{C}$ for $1 \mathrm{~h}$, then TFA $(1.1 \mathrm{~g}, 9.6 \mathrm{mmol}$, 20.0 equiv. $)$ in $\mathrm{CH}_{2} \mathrm{Cl}_{2}(3 \mathrm{~mL})$ at $\mathrm{rt}$ for $15 \mathrm{~min}$ afforded $2 \mathrm{p}$ as white solid $(0.12 \mathrm{~g}, 35 \%)$.

$>\quad \mathrm{mp}: 132.3-134.2^{\circ} \mathrm{C}$.

$>\quad \mathrm{R}_{f}=0.4$ (EtOAc:Petroleum Ether $\left.=1: 5\right)$.

$>{ }^{1} \mathrm{H} \mathrm{NMR}\left(400 \mathrm{MHz}, \mathrm{CDCl}_{3}\right) \delta 7.69(\mathrm{~d}, J=8.1 \mathrm{~Hz}, 2 \mathrm{H}), 7.31(\mathrm{~d}, J=8.0 \mathrm{~Hz}, 2 \mathrm{H}), 7.13-7.04$ $(\mathrm{m}, 4 \mathrm{H}), 4.38(\mathrm{t}, J=6.2 \mathrm{~Hz}, 1 \mathrm{H}), 2.83(\mathrm{~s}, 2 \mathrm{H}), 2.77(\mathrm{t}, J=6.9 \mathrm{~Hz}, 2 \mathrm{H}), 2.48(\mathrm{~d}, J=6.0 \mathrm{~Hz}$, $2 \mathrm{H}), 2.43(\mathrm{~s}, 3 \mathrm{H}), 2.14(\mathrm{q}, J=15.1 \mathrm{~Hz}, 2 \mathrm{H}), 0.96-0.86(\mathrm{~m}, 2 \mathrm{H})$.

$>\quad{ }^{13} \mathrm{C} \mathrm{NMR}\left(100 \mathrm{MHz}, \mathrm{CDCl}_{3}\right) \delta 143.6,140.9,135.1,135.1,129.9,129.7,128.4,127.5,127.2$, $126.0,29.0,28.7,26.8,21.6,15.4,7.1$.

$>\quad$ IR (neat) $\mathrm{cm}^{-1} 3274,2926,1598,1451,1399,1321,1157,1092$.

$>$ HRMS calcd for $\mathrm{C}_{18} \mathrm{H}_{22} \mathrm{ClNO}_{2} \mathrm{SSi}(\mathrm{M}+\mathrm{Na})^{+} 402.0713$ found 402.0727 . 


\section{Optimization of Reaction Conditions}

Table S-1. Screening of Solvent and Base ${ }^{a}$

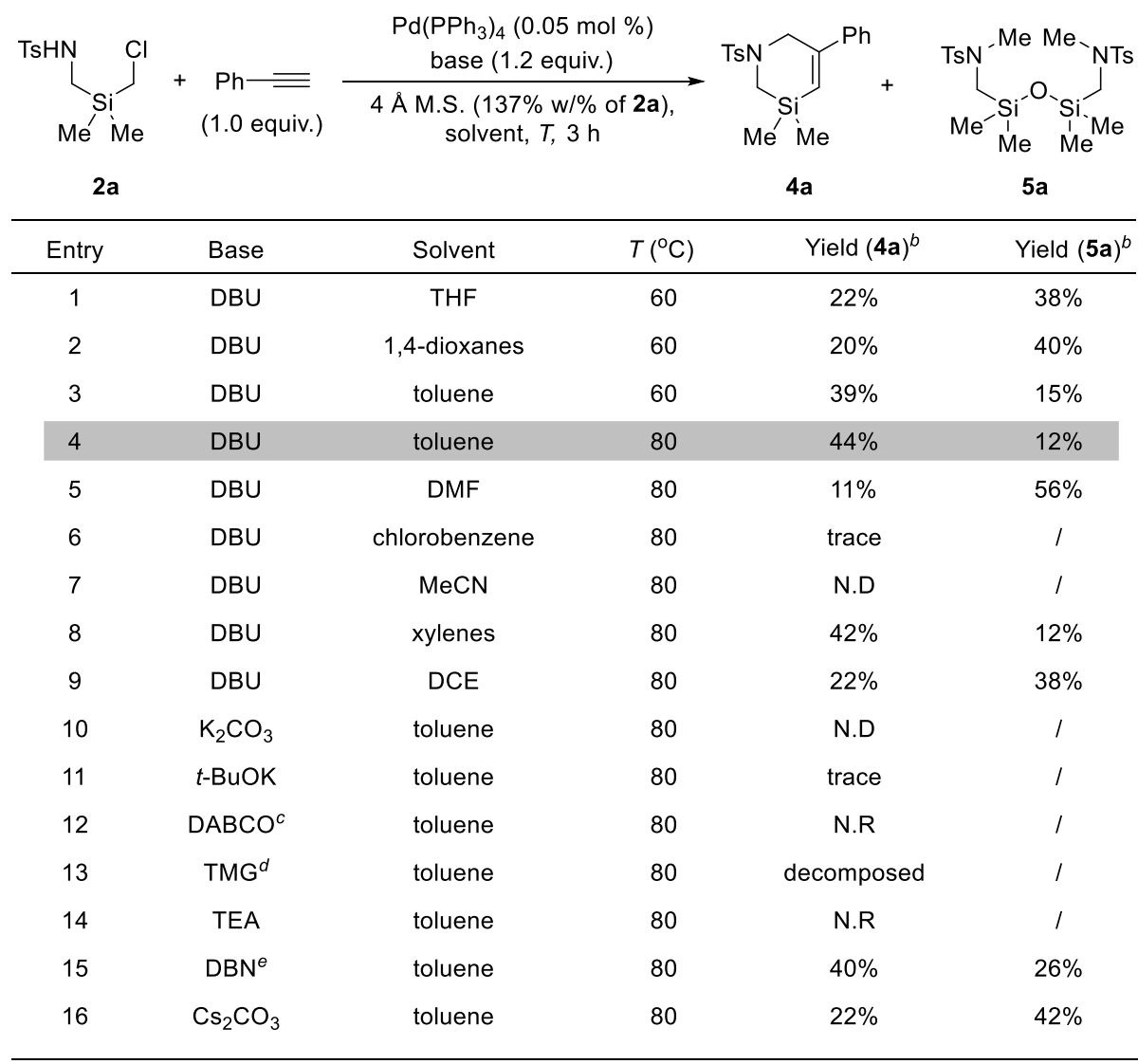

${ }^{a}$ Reaction condition: operated in glovebox, $2 \mathbf{2 a}(0.1 \mathrm{mmol})$, phenylacetylene $(0.1 \mathrm{mmol})$, solvent $(1 \mathrm{~mL}) .{ }^{b}$ Isolated yield. ${ }^{c} \mathrm{DABCO}=$ Triethylenediamine. ${ }^{d} \mathrm{TMG}=$ Tetramethylguanidine. ${ }^{e} \mathrm{DBN}=1,5$-Diazabicyclo[4.3.0]non-5-ene

Table S-2. Screening of Catalysts ${ }^{a}$

\begin{tabular}{|c|c|c|c|c|c|}
\hline $\begin{array}{c}\mathrm{TsHN}_{\mathrm{Si}^{\prime}}^{\mathrm{Cl}} \\
\mathrm{Me}^{\prime} \mathrm{Me} \\
\mathbf{2 a}\end{array}$ & $\begin{array}{r}+\mathrm{Ph} \bar{\equiv} \\
(1.0 \text { equiv. })\end{array}$ & $\begin{array}{c}\text { catalysts }(5 \mathrm{n} \\
\text { M.S. }(137 \% \\
\text { DBU (1.2 } \\
\text { ligand (10 m } \\
\text { solvent, } 80\end{array}$ & $\stackrel{\text { 2a) }}{\longrightarrow}$ & Me & $\begin{array}{l}\text { Me } \mathrm{Me} \text { NTs } \\
\text { 5a }\end{array}$ \\
\hline Entry & Catalyst & Ligand & Solvent & Yield $(\mathbf{4 a})^{b}$ & Yield $(\mathbf{5 a})^{b}$ \\
\hline 1 & $\mathrm{PtCl}_{2}$ & DPPE $^{c}$ & toluene & N.D. & 1 \\
\hline 2 & $\mathrm{Pt}\left(\mathrm{PPh}_{3}\right)_{4}$ & DPPE & toluene & N.D. & I \\
\hline 3 & $\mathrm{Rh}\left(\mathrm{PPh}_{3}\right)_{3} \mathrm{Cl}$ & I & toluene & N.D. & I \\
\hline 4 & $\mathrm{Pd}(\mathrm{acac})_{2}$ & $\mathrm{PPh}_{3}$ & toluene & $17 \%$ & $50 \%$ \\
\hline 5 & $\mathrm{Pd}(\mathrm{MeCN})_{2} \mathrm{Cl}_{2}$ & $\mathrm{PPh}_{3}$ & toluene & $8 \%$ & $68 \%$ \\
\hline 6 & $\mathrm{Pd}\left(\mathrm{PPh}_{3}\right)_{2} \mathrm{Cl}_{2}$ & I & toluene & $43 \%$ & $23 \%$ \\
\hline 7 & $\mathrm{PdCl}_{2}$ & I & toluene & trace & I \\
\hline 8 & $\mathrm{Ni}\left(\mathrm{PPh}_{3}\right)_{2} \mathrm{Cl}_{2}$ & I & toluene & N.D. & I \\
\hline $9^{d}$ & $\mathrm{Rh}\left(\mathrm{PPh}_{3}\right) \mathrm{Cl}$ & I & THF & N.D. & I \\
\hline 10 & $\mathrm{Pd}\left(\mathrm{PPh}_{3}\right)_{4}$ & I & toluene & $44 \%$ & $12 \%$ \\
\hline
\end{tabular}

${ }^{a}$ Reaction condition: operated in glovebox, 2a $(0.1 \mathrm{mmol})$, phenylacetylene $(0.1 \mathrm{mmol})$, solvent $(1 \mathrm{~mL}) .{ }^{b} \mathrm{Isolated}$ yield. ${ }^{c} \mathrm{DPPE}=1,2-\mathrm{Bis}($ diphenylphosphino $)$ ethane. ${ }^{d}$ Phenylacetylene $(0.12 \mathrm{mmol})$. 
Table S-3. Screening of Additives ${ }^{a}$

\begin{tabular}{|c|c|c|c|c|c|c|c|c|}
\hline \multicolumn{2}{|c|}{$\begin{array}{c}\mathrm{TsHN}_{\mathrm{Si}}^{\mathrm{Cl}} \\
\mathbf{2 a} \\
\mathbf{M e}\end{array}$} & $\begin{array}{r}\mathrm{Ph}= \\
(1.2 \text { equ } \\
\mathrm{P}\left(\mathrm{PPh}_{3}\right)_{2} \mathrm{Cl}_{2} \\
\mathrm{DBU}(\mathrm{eq} \\
\text { additives }(0 . \\
\text { solvent, } 12\end{array}$ & $\begin{array}{l}\text { nol \%) } \\
\text { quiv.) } \\
\text { C, } t \text { t }\end{array}$ & a & \multicolumn{3}{|c|}{$\mathrm{TsN}_{\left(\mathrm{Si}_{-}-\mathrm{Me} \mathrm{Me}\right.}^{\mathrm{NTS}}+{ }^{\mathrm{TsHN}}$} & ba \\
\hline Entry & $\mathrm{DBU}(\mathrm{eq})$ & Additive & Solvent & $\mathrm{T}\left({ }^{\circ} \mathrm{C}\right)$ & $t(\min )$ & Yield $(\mathbf{4 a})^{b}$ & Yield $(\mathbf{5 a})^{b}$ & Yield $(\mathbf{6 a})^{b}$ \\
\hline 1 & 1.2 & 1 & toluene $(0.2 \mathrm{M})$ & 120 & 30 & $56 \%$ & trace & $20 \%$ \\
\hline 2 & 2.0 & 1 & xylenes $(0.4 \mathrm{M})$ & 120 & 10 & $60 \%$ & $9 \%$ & $10 \%$ \\
\hline 3 & 2.0 & $\mathrm{MgSO}_{4}{ }^{\mathrm{C}}$ & xylenes $(0.4 \mathrm{M})$ & 120 & 10 & $56 \%$ & trace & $12 \%$ \\
\hline 4 & 2.0 & $\mathrm{Bi}(\mathrm{OTf})_{2}$ & xylenes $(0.4 \mathrm{M})$ & 120 & 10 & $13 \%$ & $13 \%$ & N.D. \\
\hline 5 & 2.0 & $\mathrm{Yb}(\mathrm{OTf})_{3}$ & xylenes $(0.4 \mathrm{M})$ & 120 & 10 & $15 \%$ & $15 \%$ & N.D. \\
\hline 6 & 2.0 & $\mathrm{Sc}(\mathrm{OTf})_{3}$ & xylenes (0.4 M) & 120 & 10 & $10 \%$ & $10 \%$ & N.D. \\
\hline 7 & 2.0 & $\mathrm{Zn}(\mathrm{OTf})_{2}$ & xylenes $(0.4 \mathrm{M})$ & 120 & 10 & $7 \%$ & $7 \%$ & N.D. \\
\hline 8 & 2.0 & $\mathrm{CuF}_{2}$ & xylenes $(0.4 \mathrm{M})$ & 120 & 10 & $42 \%$ & trace & trace \\
\hline 9 & 2.0 & $\mathrm{CeCl}_{3}{ }^{d}$ & xylenes $(0.4 \mathrm{M})$ & 120 & 10 & $56 \%$ & trace & $13 \%$ \\
\hline 10 & 2.0 & $\mathrm{CrCl}_{2}$ & xylenes $(0.4 \mathrm{M})$ & 120 & 10 & $53 \%$ & $10 \%$ & N.D. \\
\hline 11 & 2.0 & $\mathrm{InCl}_{3}$ & xylenes $(0.4 \mathrm{M})$ & 120 & 10 & $35 \%$ & $11 \%$ & N.D. \\
\hline 12 & 2.0 & $\mathrm{CuCl}_{2}$ & xylenes $(0.4 \mathrm{M})$ & 120 & 10 & $43 \%$ & trace & N.D. \\
\hline 13 & 2.0 & $\mathrm{LiCl}$ & xylenes $(0.4 \mathrm{M})$ & 120 & 10 & $26 \%$ & $28 \%$ & N.D. \\
\hline 14 & 2.0 & $\mathrm{FeBr}_{3}$ & xylenes $(0.4 \mathrm{M})$ & 120 & 10 & $53 \%$ & $7 \%$ & N.D. \\
\hline 15 & 2.0 & $\mathrm{ZnBr}_{2}$ & xylenes $(0.4 \mathrm{M})$ & 120 & 10 & $67 \%$ & trace & N.D. \\
\hline 16 & 2.0 & $\mathrm{ZnI}_{2}$ & xylenes (0.4 M) & 120 & 10 & $72 \%$ & trace & N.D. \\
\hline
\end{tabular}

${ }^{a}$ Reaction condition: Operated in glovebox; $2 \mathrm{a}(0.2 \mathrm{mmol}) .{ }^{b}$ Isolated yield. ${ }^{c} 0.5$ equiv. ${ }^{d} 0.1$ equiv. 


\section{Synthesis of 3-silatetrahydropyridines 4}

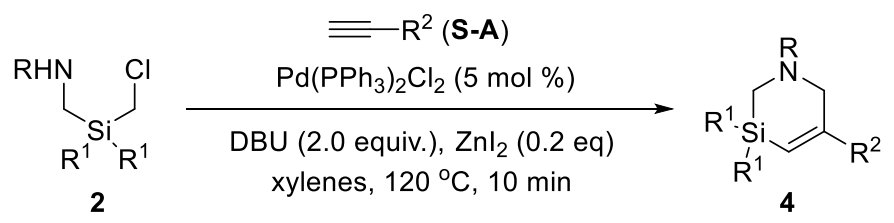

\section{General Method C:}

To a $10 \mathrm{~mL}$ of sealed tube were added 3-silaazetidine precursor $2(0.2 \mathrm{mmol}, 1.0$ equiv.), $\mathrm{Pd}\left(\mathrm{PPh}_{3}\right)_{2} \mathrm{Cl}_{2}(0.01 \mathrm{mmol}, 5 \mathrm{~mol} \%)$ and $\mathrm{ZnI}_{2}(0.04 \mathrm{mmol}, 0.2$ equiv. $)$, dry xylenes $(0.5 \mathrm{~mL})$, alkynes ( $0.24 \mathrm{mmol}, 1.2$ equiv.) and DBU (0.4 mmol, 2.0 equiv.) in the glovebox. The resulting mixture in a sealed tube was removed from glovebox and stirred at $120^{\circ} \mathrm{C}$ for $10 \mathrm{~min}$. The solvent was removed under reduced pressure before the mixture was cooled to room temperature. The residue was dissolved with $\mathrm{CH}_{2} \mathrm{Cl}_{2}(0.2-0.3 \mathrm{~mL})$ and was purified by silica gel flash column chromatography to give 3-silatetrahydropyridines 4 .

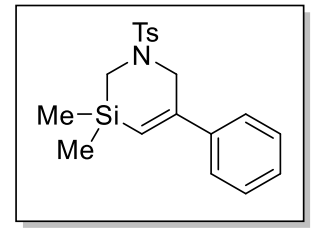

\section{3,3-dimethvl-5-phenvl-1-tosvl-1,2,3,6-tetrahvdro-1,3-azasiline (4a)}

4a was prepared according to General Method C. 2a $(58.4 \mathrm{mg}, 0.20 \mathrm{mmol}$, 1.0 equiv.), $\mathrm{Pd}\left(\mathrm{PPh}_{3}\right)_{2} \mathrm{Cl}_{2}$ (7.2 $\left.\mathrm{mg}, 0.01 \mathrm{mmol}, 5 \mathrm{~mol} \%\right), \mathrm{ZnI}_{2}(12.8 \mathrm{mg}$, 0.04 mmol, 0.2 equiv.), alkyne S-A-1 (25 mg, $0.24 \mathrm{mmol}, 1.2$ equiv.) and DBU (61 mg, $0.4 \mathrm{mmol}, 2.0$ equiv.) in xylenes $(0.5 \mathrm{~mL})$ at $120^{\circ} \mathrm{C}$ for 10 min afforded $4 \mathbf{a}$ as a pale yellow solid (52 $\mathrm{mg}, 72 \%$ ).

scale-up protocol: $2 \mathrm{a}\left(3.6 \mathrm{~g}, 12.4 \mathrm{mmol}, 1.0\right.$ equiv.), $\mathrm{Pd}\left(\mathrm{PPh}_{3}\right)_{2} \mathrm{Cl}_{2}(0.43 \mathrm{~g}$, $0.62 \mathrm{mmol}, 5 \mathrm{~mol} \%), \mathrm{ZnI}_{2}$ (0.79 g, $2.48 \mathrm{mmol}, 0.2$ equiv.), alkyne S-A-1 (1.5 $\mathrm{g}, 14.9 \mathrm{mmol}, 1.2$ equiv.) and DBU (3.7 g, $24.8 \mathrm{mmol}, 2.0$ equiv.) in xylenes $(25 \mathrm{~mL})$ at $120^{\circ} \mathrm{C}$ for 10 min under Ar afforded crude 4a. The crude 4a was purified by silica gel flash column chromatography (eluent: petroleum ether/EtOAc $=20 / 1$ ) and recrystallization (petroleum ether/EtOAc $=60 \mathrm{~mL} / 10$ $\mathrm{mL})$ to give pure $4 \mathrm{a}$ as a pale yellow solid $(3.0 \mathrm{~g}, 68 \%)$.

Growing single crystal of 4a in EtOAc/ $n$-hexane resulted in the formation of colorless single crystals suitable for X-ray analysis.

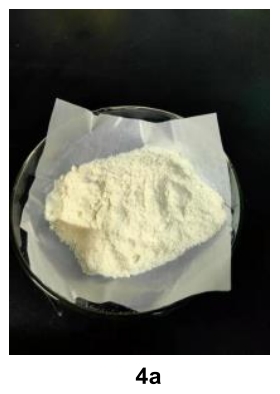

$>\mathrm{mp}: 161.4-164.6^{\circ} \mathrm{C}$.

$>\mathrm{R}_{f}=0.7($ EtOAc:Petroleum Ether $=1: 5)$.

$>{ }^{1} \mathrm{H} \mathrm{NMR}\left(400 \mathrm{MHz}, \mathrm{CDCl}_{3}\right) \delta 7.71(\mathrm{~d}, J=8.0 \mathrm{~Hz}, 2 \mathrm{H}), 7.35-7.29(\mathrm{~m}, 7 \mathrm{H}), 6.02(\mathrm{~s}, 1 \mathrm{H}), 3.93$ $(\mathrm{s}, 2 \mathrm{H}), 2.55(\mathrm{~s}, 2 \mathrm{H}), 2.43(\mathrm{~s}, 3 \mathrm{H}), 0.23(\mathrm{~s}, 6 \mathrm{H})$.

$>{ }^{13} \mathrm{C} \mathrm{NMR}\left(100 \mathrm{MHz}, \mathrm{CDCl}_{3}\right) \delta 152.09,143.5,141.8,132.5,129.6,128.5,128.2,128.0,125.8$, $123.1,50.8,35.3,21.5,-3.0$.

$>{ }^{29} \mathrm{Si} \operatorname{NMR}\left(119 \mathrm{MHz}, \mathrm{CDCl}_{3}\right) \delta-12.82$.

$>$ IR (neat) $\mathrm{cm}^{-1} 2956,159,1492,1441,1336,1160,1089,1031,916$.

$>$ HRMS calcd for $\mathrm{C}_{19} \mathrm{H}_{23} \mathrm{NO}_{2} \mathrm{SSi}(\mathrm{M}+\mathrm{Na})^{+} 380.1116$, found 380.1104 .

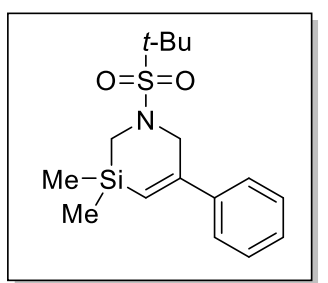

1-(tert-butvlsulfonvl)-3,3-dimethyl-5-phenvl-1,2,3,4-tetrahvdro-1,3azasiline (4b)

4b was prepared according to General Method C. $2 \mathbf{b}(51.6 \mathrm{mg}, 0.20 \mathrm{mmol}$, 1.0 equiv.), $\mathrm{Pd}\left(\mathrm{PPh}_{3}\right)_{2} \mathrm{Cl}_{2}(7.2 \mathrm{mg}, 0.01 \mathrm{mmol}, 5 \mathrm{~mol} \%), \mathrm{ZnI}_{2}(12.8 \mathrm{mg}$, 0.04 mmol, 0.2 equiv.), alkyne S-A-1 $(25 \mathrm{mg}, 0.24 \mathrm{mmol}, 1.2$ equiv. $)$ and $\mathrm{DBU}\left(61 \mathrm{mg}, 0.4 \mathrm{mmol}, 2.0\right.$ equiv.) in xylenes $(0.5 \mathrm{~mL})$ at $120^{\circ} \mathrm{C}$ for 10 min afforded $4 \mathbf{b}$ as a white solid ( $28 \mathrm{mg}, 44 \%$ ).

$>\mathrm{mp}: 108.2-110.3^{\circ} \mathrm{C}$.

$>\mathrm{R}_{f}=0.7$ (EtOAc:Petroleum Ether $\left.=1: 5\right)$.

$>{ }^{1} \mathrm{H}$ NMR $\left(400 \mathrm{MHz}, \mathrm{CDCl}_{3}\right) \delta 7.39-7.26(\mathrm{~m}, 5 \mathrm{H}), 6.06(\mathrm{t}, J=1.7 \mathrm{~Hz}, 1 \mathrm{H}), 4.31(\mathrm{~s}, 2 \mathrm{H}), 3.05$ (s, 2H), $1.43(\mathrm{~s}, 9 \mathrm{H}), 0.24(\mathrm{~s}, 6 \mathrm{H})$.

$>{ }^{13} \mathrm{C} \mathrm{NMR}\left(100 \mathrm{MHz}, \mathrm{CDCl}_{3}\right) \delta 154.6,141.8,128.5,128.2,125.8,123.1,62.4,51.9,38.1,25.2$, -2.8 .

$>\operatorname{IR}$ (neat) $\mathrm{cm}^{-1} 2973,1596,1571,1444,1364,131,1249,1122,934$. 
HRMS calcd for $\mathrm{C}_{16} \mathrm{H}_{25} \mathrm{NO}_{2} \mathrm{SSi}(\mathrm{M}+\mathrm{Na})^{+} 346.1273$, found 346.1275.

\section{3,3-dimethyl-1-(methylsulfonyl)-5-phenyl-1,2,3,6-tetrahydro-1,3-azasiline (4c)}

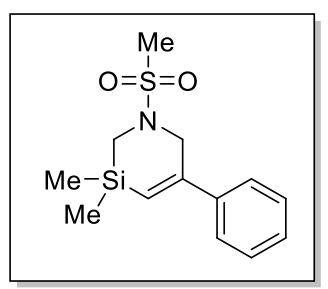

4c was prepared according to General Method C. 2c (44 mg, $0.20 \mathrm{mmol}$, 1.0 equiv.), $\mathrm{Pd}\left(\mathrm{PPh}_{3}\right)_{2} \mathrm{Cl}_{2}$ (7.2 mg, $\left.0.01 \mathrm{mmol}, 5 \mathrm{~mol} \%\right), \mathrm{ZnI}_{2}(12.8 \mathrm{mg}$, 0.04 mmol, 0.2 equiv.), alkyne S-A-1 (25 mg, $0.24 \mathrm{mmol}, 1.2$ equiv.) and DBU (61 mg, $0.4 \mathrm{mmol}, 2.0$ equiv.) in xylenes $(0.5 \mathrm{~mL})$ at $120{ }^{\circ} \mathrm{C}$ for 10 min afforded $4 \mathbf{c}$ as a white solid (25 $\mathrm{mg}, 44 \%)$.

$>\quad \mathrm{mp}: 77.8-79.2^{\circ} \mathrm{C}$

$>\mathrm{R}_{f}=0.3($ EtOAc:Petroleum Ether $=1: 5)$.

$>\quad{ }^{1} \mathrm{H} \mathrm{NMR}\left(400 \mathrm{MHz}, \mathrm{CDCl}_{3}\right) \delta 7.39-7.32(\mathrm{~m}, 5 \mathrm{H}), 6.11(\mathrm{t}, J=1.60$ $\mathrm{Hz}, 1 \mathrm{H}), 4.15$ (d, J=4.0 Hz, 2H), 2.83 (s, 3H), 2.77 (s, 2H), 0.26 (s, 6H).

$>\quad{ }^{13} \mathrm{C} \mathrm{NMR}\left(100 \mathrm{MHz}, \mathrm{CDCl}_{3}\right) \delta 152.8,141.4,128.5,128.3,125.7,123.1,50.5,35.3,32.9,-3.0$.

$>\quad$ IR (neat) $\mathrm{cm}^{-1} 1595,1571,1493,1329,1151,1034,933$.

$>\quad$ HRMS calcd for $\mathrm{C}_{13} \mathrm{H}_{19} \mathrm{NO}_{2} \mathrm{SSi}(\mathrm{M}+\mathrm{Na})^{+}$304.0803, found 304.0798.

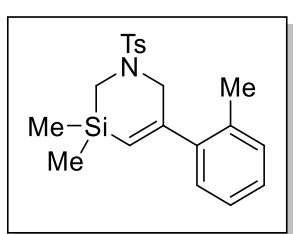

\section{3,3-dimethyl-5-(o-tolyl)-1-tosyl-1,2,3,6-tetrahydro-1,3-azasiline (4e)}

4e was prepared according to General Method C. 2 a $(58.4 \mathrm{mg}, 0.20 \mathrm{mmol}$, 1.0 equiv.), $\mathrm{Pd}\left(\mathrm{PPh}_{3}\right)_{2} \mathrm{Cl}_{2}$ (7.2 mg, $\left.0.01 \mathrm{mmol}, 5 \mathrm{~mol} \%\right), \mathrm{ZnI}_{2}(12.8 \mathrm{mg}, 0.04$ mmol, 0.2 equiv.), alkyne $\mathbf{S}-\mathbf{A}-2$ (27.9 mg, $0.24 \mathrm{mmol}, 1.2$ equiv.) and DBU (61 mg, $0.4 \mathrm{mmol}, 2.0$ equiv.) in xylenes $(0.5 \mathrm{~mL})$ at $120{ }^{\circ} \mathrm{C}$ for $10 \mathrm{~min}$ afforded $4 \mathrm{e}$ as a white solid (44 $\mathrm{mg}, 59 \%)$.

$>\quad$ mp: $104.8-105.4{ }^{\circ} \mathrm{C}$.

$>\mathrm{R}_{f}=0.6($ EtOAc:Petroleum Ether $=1: 5)$.

$>{ }^{1} \mathrm{H}$ NMR $\left(400 \mathrm{MHz}, \mathrm{CDCl}_{3}\right) \delta 7.64(\mathrm{~d}, J=8.0 \mathrm{~Hz}, 2 \mathrm{H}), 7.30(\mathrm{~d}, J=8.0 \mathrm{~Hz}, 2 \mathrm{H}), 7.21-7.11$ $(\mathrm{m}, 3 \mathrm{H}), 7.02(\mathrm{~d}, J=4.0 \mathrm{~Hz}, 1 \mathrm{H}), 5.61(\mathrm{~s}, 1 \mathrm{H}), 3.65(\mathrm{~s}, 2 \mathrm{H}), 2.56(\mathrm{~s}, 2 \mathrm{H}), 2.41(\mathrm{~s}, 3 \mathrm{H}), 2.26(\mathrm{~s}$, $3 \mathrm{H}), 0.24(\mathrm{~s}, 6 \mathrm{H})$.

$>\quad{ }^{13} \mathrm{C}$ NMR $\left(100 \mathrm{MHz}, \mathrm{CDCl}_{3}\right) \delta 155.0,143.4,143.1,134.7,132.5,130.3,129.6,128.0,127.8$, $127.6,125.6,124.5,52.2,35.4,21.5,19.6,-2.9$.

$>\quad$ IR (neat) $\mathrm{cm}^{-1} 2956,2805,1922,1597,1488,1341,1161,1089,1032,926$.

$>$ HRMS calcd for $\mathrm{C}_{20} \mathrm{H}_{25} \mathrm{NO}_{2} \mathrm{SSi}(\mathrm{M}+\mathrm{Na})^{+} 394.1273$, found 394.1269.

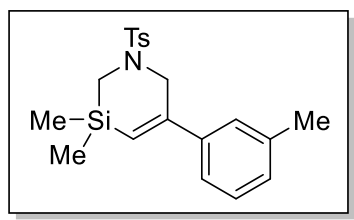

\section{3,3-dimethyl-5-(m-tolyl)-1-tosyl-1,2,3,6-tetrahydro-1,3-azasiline (4f)}

4f was prepared according to General Method C. $2 \mathbf{a}(58.4 \mathrm{mg}, 0.20 \mathrm{mmol}$, 1.0 equiv.), $\mathrm{Pd}\left(\mathrm{PPh}_{3}\right)_{2} \mathrm{Cl}_{2}$ (7.2 mg, $\left.0.01 \mathrm{mmol}, 5 \mathrm{~mol} \%\right), \mathrm{ZnI}_{2}(12.8 \mathrm{mg}$, 0.04 mmol, 0.2 equiv.), alkyne S-A-3 (27.9 mg, 0.24 mmol, 1.2 equiv.) and DBU (61 mg, $0.4 \mathrm{mmol}, 2.0$ equiv.) in xylenes $(0.5 \mathrm{~mL})$ at $120{ }^{\circ} \mathrm{C}$ for $10 \mathrm{~min}$ afforded $\mathbf{4 f}$ as a white solid (45 $\mathrm{mg}, 61 \%)$.

$>$ mp: $112.4-117.0^{\circ} \mathrm{C}$.

$>\mathrm{R}_{f}=0.5($ EtOAc:Petroleum Ether $=1: 10)$.

$>\quad{ }^{1} \mathrm{H} \mathrm{NMR}\left(400 \mathrm{MHz}, \mathrm{CDCl}_{3}\right) \delta 7.71(\mathrm{~d}, J=8.0 \mathrm{~Hz}, 2 \mathrm{H}), 7.33(\mathrm{~d}, J=8.0 \mathrm{~Hz}, 2 \mathrm{H}), 7.23-7.20$ $(\mathrm{m}, 1 \mathrm{H}), 7.15-7.10(\mathrm{~m}, 3 \mathrm{H}), 5.99(\mathrm{t}, J=1.8 \mathrm{~Hz}, 1 \mathrm{H}), 3.92(\mathrm{~d}, J=4.0 \mathrm{~Hz}, 2 \mathrm{H}), 2.54(\mathrm{~s}, 2 \mathrm{H}), \delta$ $2.43(\mathrm{~s}, 3 \mathrm{H}), 2.35(\mathrm{~s}, 3 \mathrm{H}), 0.22(\mathrm{~s}, 6 \mathrm{H})$.

$>{ }^{13} \mathrm{C} \mathrm{NMR}\left(100 \mathrm{MHz}, \mathrm{CDCl}_{3}\right) \delta 153.2,143.5,141.8,138.1,132.5,129.6,129.0,128.4,128.0$, $126.6,123.0,122.8,50.9,35.3,21.6,21.5,-2.9$.

$>\quad \mathrm{IR}$ (neat) $\mathrm{cm}^{-1} 2956,1596,1490,1438,1340,1160,1089,1033,938$.

$>$ HRMS calcd for $\mathrm{C}_{20} \mathrm{H}_{25} \mathrm{NO}_{2} \mathrm{SSi}(\mathrm{M}+\mathrm{Na})^{+} 394.1273$ found 394.1271.

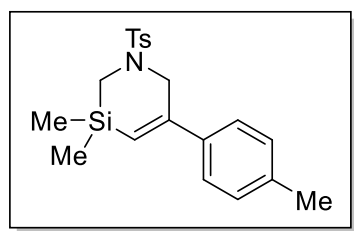

3,3-dimethyl-5-(p-tolyl)-1-tosyl-1,2,3,6-tetrahydro-1,3-azasiline (4g)

4g was prepared according to General Method C. 2a (58.4 mg, 0.20 mmol, 1.0 equiv.), $\mathrm{Pd}\left(\mathrm{PPh}_{3}\right)_{2} \mathrm{Cl}_{2}$ (7.2 $\left.\mathrm{mg}, 0.01 \mathrm{mmol}, 5 \mathrm{~mol} \%\right), \mathrm{ZnI}_{2}$ (12.8 mg, 0.04 mmol, 0.2 equiv.), alkyne S-A-4 (27.9 mg, $0.24 \mathrm{mmol}$, 1.2 equiv.) and $\mathrm{DBU}(61 \mathrm{mg}, 0.4 \mathrm{mmol}, 2.0$ equiv.) in xylenes $(0.5 \mathrm{~mL})$ at $120^{\circ} \mathrm{C}$ for $10 \mathrm{~min}$ afforded $4 \mathrm{~g}$ as a white solid (55 mg, $75 \%$ ).

$>$ mp: $155.8-157.3^{\circ} \mathrm{C}$.

$>\mathrm{R}_{f}=0.5$ (EtOAc:Petroleum Ether $\left.=1: 7\right)$. 
$>\quad{ }^{1} \mathrm{H} \mathrm{NMR}\left(400 \mathrm{MHz}, \mathrm{CDCl}_{3}\right) \delta 7.72(\mathrm{~d}, J=8.2 \mathrm{~Hz}, 2 \mathrm{H}), 7.34(\mathrm{~d}, J=7.9 \mathrm{~Hz}, 2 \mathrm{H}), 7.25(\mathrm{~d}, J=$ $8.1 \mathrm{~Hz}, 2 \mathrm{H}), 7.15(\mathrm{~d}, J=7.9 \mathrm{~Hz}, 2 \mathrm{H}), 6.00(\mathrm{t}, J=1.7 \mathrm{~Hz}, 1 \mathrm{H}), 3.94(\mathrm{~d}, J=1.7 \mathrm{~Hz}, 2 \mathrm{H}), 2.56$ $(\mathrm{s}, 2 \mathrm{H}), 2.44(\mathrm{~s}, 3 \mathrm{H}), 2.35(\mathrm{~s}, 3 \mathrm{H}), 0.24(\mathrm{~s}, 6 \mathrm{H})$.

$>{ }^{13} \mathrm{C} \mathrm{NMR}\left(100 \mathrm{MHz}, \mathrm{CDCl}_{3}\right) \delta 152.8,143.5,138.8138 .2,132.5,129.6,129.1,128.0,125.7$, $122.0,50.8,35.3,21.6,21.1,-2.9$.

$>\quad$ IR (neat) $\mathrm{cm}^{-1} 2957,1596,1511,1340,1162,1089,1034,926$.

$>$ HRMS calcd for $\mathrm{C}_{20} \mathrm{H}_{25} \mathrm{NO}_{2} \mathrm{SSi}(\mathrm{M}+\mathrm{Na})^{+} 394.1273$, found 394.1269.

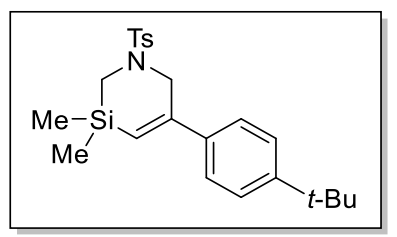

5-(4-(tert-butyl)phenyl)-3,3-dimethyl-1-tosyl-1,2,3,6-tetrahydro-1,3azasiline (4h)

4h was prepared according to General Method C. 2a (58.4 mg, 0.20 mmol, 1.0 equiv.), $\mathrm{Pd}\left(\mathrm{PPh}_{3}\right)_{2} \mathrm{Cl}_{2}$ (7.2 mg, $\left.0.01 \mathrm{mmol}, 5 \mathrm{~mol} \%\right), \mathrm{ZnI}_{2}$ (12.8 mg, $0.04 \mathrm{mmol}, 0.2$ equiv.), alkyne S-A-5 (38.7 mg, $0.24 \mathrm{mmol}$, 1.2 equiv.) and DBU (61 mg, $0.4 \mathrm{mmol}, 2.0$ equiv. $)$ in xylenes $(0.5 \mathrm{~mL})$ at $120{ }^{\circ} \mathrm{C}$ for $10 \mathrm{~min}$ afforded $4 \mathrm{~h}$ as a white solid $(52 \mathrm{mg}, 63 \%)$.

$>$ mp: $103.9-105.6^{\circ} \mathrm{C}$.

$>\mathrm{R}_{f}=0.6$ (EtOAc:Petroleum Ether $\left.=1: 5\right)$.

$>\quad{ }^{1} \mathrm{H} \mathrm{NMR}\left(400 \mathrm{MHz}, \mathrm{CDCl}_{3}\right) \delta 7.71(\mathrm{~d}, J=8.0 \mathrm{~Hz}, 2 \mathrm{H}), 7.37-7.32(\mathrm{~m}, 4 \mathrm{H}), 7.30-7.25(\mathrm{~m}$, $2 \mathrm{H}), 5.99(\mathrm{t}, J=1.8 \mathrm{~Hz}, 1 \mathrm{H}), 3.93(\mathrm{~d}, J=1.7 \mathrm{~Hz}, 2 \mathrm{H}), 2.55(\mathrm{~s}, 2 \mathrm{H}), 2.43(\mathrm{~s}, 3 \mathrm{H}), 1.31(\mathrm{~s}, 9 \mathrm{H})$, $0.22(\mathrm{~s}, 6 \mathrm{H})$.

$>{ }^{13} \mathrm{C} \mathrm{NMR}\left(100 \mathrm{MHz}, \mathrm{CDCl}_{3}\right) \delta 152.7,151.4,143.5,138.8,132.5,129.6,128.0,125.5,125.4$, $122.2,50.8,35.3,34.6,31.3,21.6,-2.9$.

$>\quad \mathrm{IR}$ (neat) $\mathrm{cm}^{-1} 2960,2867,1597,1342,1162,1090,980$.

$>$ HRMS calcd for $\mathrm{C}_{23} \mathrm{H}_{31} \mathrm{NO}_{2} \mathrm{SSi}(\mathrm{M}+\mathrm{H})^{+} 414.1923$ found 414.1917 .

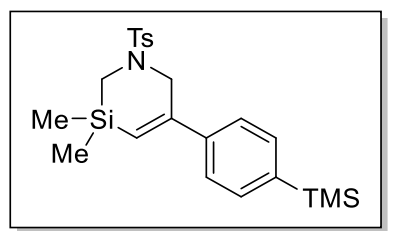

3,3-dimethyl-1-tosvl-5-(4-(trimethylsilvl)phenvl)-1,2,3,6-tetrahvdro1,3-azasiline (4i)

4i was prepared according to General Method C. 2a (58.4 mg, 0.20 mmol, 1.0 equiv.), $\mathrm{Pd}\left(\mathrm{PPh}_{3}\right)_{2} \mathrm{Cl}_{2}$ (7.2 mg, $\left.0.01 \mathrm{mmol}, 5 \mathrm{~mol} \%\right), \mathrm{ZnI}_{2}$ (12.8 mg, 0.04 mmol, 0.2 equiv.), alkyne S-A-6 (42.7 mg, $0.24 \mathrm{mmol}$, 1.2 equiv. ) and DBU (61 mg, $0.4 \mathrm{mmol}, 2.0$ equiv.) in xylenes $(0.5 \mathrm{~mL})$ at $120^{\circ} \mathrm{C}$ for $10 \mathrm{~min}$ afforded $4 \mathbf{i}$ as a white solid $(61.5 \mathrm{mg}, 72 \%)$.

$>$ mp: $100.7-102.1^{\circ} \mathrm{C}$.

$>\mathrm{R}_{f}=0.4$ (EtOAc:Petroleum Ether $\left.=1: 10\right)$.

$>{ }^{1} \mathrm{H} \mathrm{NMR}\left(400 \mathrm{MHz}, \mathrm{CDCl}_{3}\right) \delta 7.70(\mathrm{~d}, J=8.0 \mathrm{~Hz}, 2 \mathrm{H}), 7.49(\mathrm{~d}, J=8.0 \mathrm{~Hz}, 2 \mathrm{H}), 7.34-7.30$ (m, 4H), $6.02(\mathrm{t}, J=1.72 \mathrm{~Hz}, 1 \mathrm{H}), 3.93(\mathrm{~d}, J=1.72 \mathrm{~Hz}, 2 \mathrm{H}), 2.55(\mathrm{~s}, 2 \mathrm{H}), 2.43(\mathrm{~s}, 3 \mathrm{H}), 0.27$ $(\mathrm{s}, 9 \mathrm{H}), 0.23(\mathrm{~s}, 6 \mathrm{H})$.

$>{ }^{13} \mathrm{C} \mathrm{NMR}\left(100 \mathrm{MHz}, \mathrm{CDCl}_{3}\right) \delta 154.1,144.6,143.2,141.9,134.7,133.6,130.8,129.2,126.2$, $124.3,52.0,36.4,22.7,0.0,-1.8$.

$>\quad \mathrm{IR}$ (neat) $\mathrm{cm}^{-1} 2954,1595,1342,1220,1162,1090,1033,927$.

$>$ HRMS calcd for $\mathrm{C}_{22} \mathrm{H}_{31} \mathrm{NO}_{2} \mathrm{SSi}_{2}(\mathrm{M}+\mathrm{Na})^{+} 452.1512$, found 452.1505 .

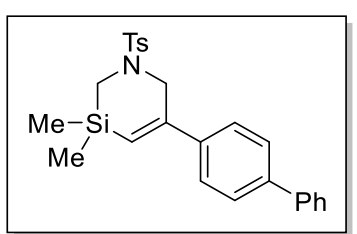

\section{5-([1,1'-biphenyll-4-yl)-3,3-dimethyl-1-tosyl-1,2,3,6-tetrahydro-1,3-}

\section{azasiline (4j)}

4j was prepared according to General Method C. 2a $(58.4 \mathrm{mg}, 0.20 \mathrm{mmol}$, 1.0 equiv.), $\mathrm{Pd}\left(\mathrm{PPh}_{3}\right)_{2} \mathrm{Cl}_{2}$ (7.2 mg, $\left.0.01 \mathrm{mmol}, 5 \mathrm{~mol} \%\right), \mathrm{ZnI}_{2}(12.8 \mathrm{mg}$, $0.04 \mathrm{mmol}, 0.2$ equiv.), alkyne S-A-7 (43.6 mg, $0.24 \mathrm{mmol}, 1.2$ equiv.) and DBU (61 mg, $0.4 \mathrm{mmol}, 2.0$ equiv.) in xylenes $(0.5 \mathrm{~mL})$ at $120{ }^{\circ} \mathrm{C}$

for $10 \mathrm{~min}$ afforded $\mathbf{4 j}$ as a white solid $(43 \mathrm{mg}, 50 \%)$.

$>\mathrm{mp}: 48.3-51.2^{\circ} \mathrm{C}$.

$>\mathrm{R}_{f}=0.7$ (EtOAc:Petroleum Ether $\left.=1: 5\right)$.

$>{ }^{1} \mathrm{H}$ NMR $\left(400 \mathrm{MHz}, \mathrm{CDCl}_{3}\right) \delta 7.73(\mathrm{~d}, J=8.0 \mathrm{~Hz}, 2 \mathrm{H}), 7.61-7.56(\mathrm{~m}, 4 \mathrm{H}), 7.47-7.41(\mathrm{~m}$, $4 \mathrm{H}), 7.38-7.34$ (m, 3H), 6.09 (t, $J=1.6 \mathrm{~Hz}, 1 \mathrm{H}), 3.98(\mathrm{~d}, J=1.6 \mathrm{~Hz}, 2 \mathrm{H}), 2.57$ (s, 2H), 2.44 $(\mathrm{s}, 3 \mathrm{H}), 0.24(\mathrm{~s}, 6 \mathrm{H})$.

$>{ }^{13} \mathrm{C} \mathrm{NMR}\left(100 \mathrm{MHz}, \mathrm{CDCl}_{3}\right) \delta 152.4,143.5,141.1,140.5,140.5,132.5,129.6,128.8,128.1$, $127.5,127.1,127.0,126.2,123.1,50.8,35.3,21.6,-2.9$.

$>$ IR (neat) $\mathrm{cm}^{-1} 2958,1596,1486,1339,1161,1089,928$. 
HRMS calcd for $\mathrm{C}_{25} \mathrm{H}_{27} \mathrm{NO}_{2} \mathrm{SSi}(\mathrm{M}+\mathrm{H})^{+} 434.1610$, found 434.1606.

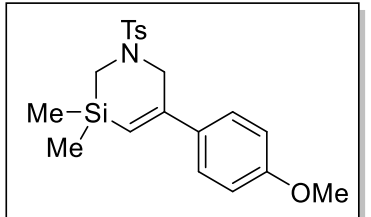

5-(4-methoxyphenyl)-3,3-dimethyl-1-tosyl-1,2,3,6-tetrahydro-1,3azasiline (4k)

4k was prepared according to General Method C. 2a (58.4 mg, 0.20 mmol, 1.0 equiv.), $\mathrm{Pd}\left(\mathrm{PPh}_{3}\right)_{2} \mathrm{Cl}_{2}$ (7.2 $\left.\mathrm{mg}, 0.01 \mathrm{mmol}, 5 \mathrm{~mol} \%\right), \mathrm{ZnI}_{2}$ (12.8 mg, $0.04 \mathrm{mmol}, 0.2$ equiv.), alkyne $\mathbf{S - A - 8}(32.3 \mathrm{mg}, 0.24 \mathrm{mmol}$, 1.2 equiv.) and DBU (61 mg, $0.4 \mathrm{mmol}, 2.0$ equiv.) in xylenes $(0.5 \mathrm{~mL})$ at $120^{\circ} \mathrm{C}$ for $10 \mathrm{~min}$ afforded $4 \mathbf{k}$ as a yellow solid $(56 \mathrm{mg}, 72 \%)$.

$>\mathrm{mp}: 142.1-144.4^{\circ} \mathrm{C}$.

$>\mathrm{R}_{f}=0.3($ EtOAc:Petroleum Ether $=1: 7)$.

$>\quad{ }^{1} \mathrm{H} \mathrm{NMR}\left(400 \mathrm{MHz}, \mathrm{CDCl}_{3}\right) \delta 7.71(\mathrm{~d}, J=8.0 \mathrm{~Hz}, 2 \mathrm{H}), 7.34(\mathrm{~d}, J=8.0 \mathrm{~Hz}, 2 \mathrm{H}), 7.29(\mathrm{~d}, J=$ $8.0 \mathrm{~Hz}, 2 \mathrm{H}), 6.86(\mathrm{~d}, J=8.0 \mathrm{~Hz}, 2 \mathrm{H}), 5.94(\mathrm{~s}, 1 \mathrm{H}), 3.91(\mathrm{~s}, 2 \mathrm{H}), 3.81(\mathrm{~s}, 3 \mathrm{H}), 2.53(\mathrm{~s}, 2 \mathrm{H}), 2.43$ $(\mathrm{s}, 3 \mathrm{H}), 0.22(\mathrm{~s}, 6 \mathrm{H})$.

$>{ }^{13} \mathrm{C} \mathrm{NMR}\left(100 \mathrm{MHz}, \mathrm{CDCl}_{3}\right) \delta 159.7,152.2,143.5,134.1,132.5,129.6,128.0,127.0,121.1$, $113.8,55.3,50.8,35.3,21.5,-2.9$.

$>\quad \mathrm{IR}$ (neat) $\mathrm{cm}^{-1} 2958,1604,1510,1340,1162,1032,924$.

$>$ HRMS calcd for $\mathrm{C}_{20} \mathrm{H}_{25} \mathrm{NO}_{3} \mathrm{SSi}(\mathrm{M}+\mathrm{Na})^{+} 410.1222$ found 410.1213 .

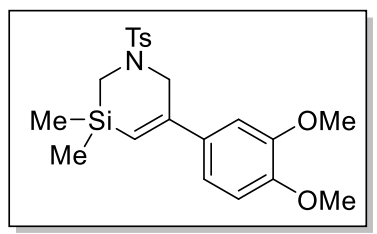

5-(3,4-dimethoxyphenyl)-3,3-dimethyl-1-tosyl-1,2,3,6-tetrahydro-1,3azasiline (4I)

$4 \mathbf{I}$ was prepared according to General Method C. 2a (58.4 mg, 0.20 mmol, 1.0 equiv.), $\mathrm{Pd}\left(\mathrm{PPh}_{3}\right)_{2} \mathrm{Cl}_{2}$ (7.2 $\left.\mathrm{mg}, 0.01 \mathrm{mmol}, 5 \mathrm{~mol} \%\right), \mathrm{ZnI}_{2}$ (12.8 mg, 0.04 mmol, 0.2 equiv.), alkyne S-A-9 $(38.9 \mathrm{mg}, 0.24 \mathrm{mmol}$, 1.2 equiv.) and DBU (61 mg, $0.4 \mathrm{mmol}, 2.0$ equiv.) in xylenes $(0.5 \mathrm{~mL})$ at $120{ }^{\circ} \mathrm{C}$ for $10 \mathrm{~min}$ afforded $4 \mathrm{I}$ as white solid $(68 \mathrm{mg}, 82 \%)$.

$>\mathrm{mp}: 134.7-137.0^{\circ} \mathrm{C}$.

$>\mathrm{R}_{f}=0.4$ (EtOAc:Petroleum Ether $\left.=1: 3\right)$.

$>\quad{ }^{1} \mathrm{H} \mathrm{NMR}\left(600 \mathrm{MHz}, \mathrm{CDCl}_{3}\right) \delta 7.71(\mathrm{~d}, J=7.8 \mathrm{~Hz}, 2 \mathrm{H}), 7.34(\mathrm{~d}, J=7.8, \mathrm{~Hz}, 2 \mathrm{H}), 6.90-6.87$ $(\mathrm{m}, 2 \mathrm{H}), 6.83-6.80(\mathrm{~m}, 1 \mathrm{H}), 5.94(\mathrm{~s}, 1 \mathrm{H}), 3.91(\mathrm{~s}, 2 \mathrm{H}), 3.90(\mathrm{~s}, 3 \mathrm{H}), 3.89(\mathrm{~s}, 3 \mathrm{H}), 2.54(\mathrm{~s}, 2 \mathrm{H})$, $2.43(\mathrm{~s}, 3 \mathrm{H}), 0.23(\mathrm{~s}, 6 \mathrm{H})$.

$>{ }^{13} \mathrm{C} \mathrm{NMR}\left(100 \mathrm{MHz}, \mathrm{CDCl}_{3}\right) \delta 152.6,149.3,148.8,143.5,134.6,132.5,129.6,128.0,121.5$, $118.1,110.8,109.4,56.0,56.0,50.8,35.3,21.5,-2.9$.

$>\quad \mathrm{IR}$ (neat) $\mathrm{cm}^{-1} 2956,2837,1598,1513,1338,1161,1025,941,895$.

$>$ HRMS calcd for $\mathrm{C}_{21} \mathrm{H}_{27} \mathrm{NO}_{4} \mathrm{SSi}(\mathrm{M}+\mathrm{H})^{+} 418.1508$ found 418.1500 .

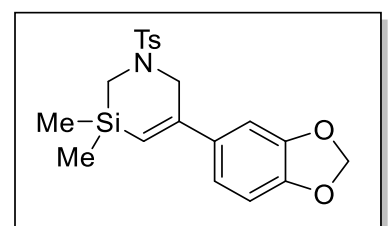

5-(benzo[d][1,3/dioxol-5-vl)-3,3-dimethvl-1-tosvl-1,2,3,6-tetrahvdro1,3-azasiline (4m)

4m was prepared according to General Method C. 2a (58.4 mg, 0.20 mmol, 1.0 equiv.), $\mathrm{Pd}\left(\mathrm{PPh}_{3}\right)_{2} \mathrm{Cl}_{2}$ (7.2 mg, $\left.0.01 \mathrm{mmol}, 5 \mathrm{~mol} \%\right), \mathrm{ZnI}_{2}$ (12.8 $\mathrm{mg}, 0.04 \mathrm{mmol}, 0.2$ equiv.), alkyne S-A-10 (35 mg, $0.24 \mathrm{mmol}$, 1.2 equiv.) and DBU (61 mg, $0.4 \mathrm{mmol}, 2.0$ equiv.) in xylenes $(0.5 \mathrm{~mL})$ at $120^{\circ} \mathrm{C}$ for $10 \mathrm{~min}$ afforded $\mathbf{4 m}$ as white solid (43 $\left.\mathrm{mg}, 51 \%\right)$.

$>$ mp: $148.7-151.4^{\circ} \mathrm{C}$.

$>\mathrm{R}_{f}=0.7$ (EtOAc:Petroleum Ether $\left.=1: 4\right)$.

$>{ }^{1} \mathrm{H}$ NMR $\left(400 \mathrm{MHz}, \mathrm{CDCl}_{3}\right) \delta 7.71(\mathrm{~d}, J=8.3 \mathrm{~Hz}, 2 \mathrm{H}), 7.34(\mathrm{~d}, J=8.0 \mathrm{~Hz}, 2 \mathrm{H}), 6.85-6.79$ $(\mathrm{m}, 2 \mathrm{H}), 6.76(\mathrm{~d}, J=8.0 \mathrm{~Hz}, 1 \mathrm{H}), 5.96(\mathrm{~s}, 2 \mathrm{H}), 5.92(\mathrm{~d}, J=1.7 \mathrm{~Hz}, 1 \mathrm{H}), 3.87(\mathrm{~d}, J=1.7 \mathrm{~Hz}$, $2 \mathrm{H}), 2.53(\mathrm{~s}, 2 \mathrm{H}), 2.43(\mathrm{~s}, 3 \mathrm{H}), 0.21(\mathrm{~s}, 6 \mathrm{H})$.

$>{ }^{13} \mathrm{C} \mathrm{NMR}\left(100 \mathrm{MHz}, \mathrm{CDCl}_{3}\right) \delta 152.2,147.8,147.6,143.5,136.0,132.5,129.6,128.0,121.8$, $119.3,108.1,106.5,101.2,50.9,35.3,21.5,-2.9$.

$>\mathrm{IR}$ (neat) $\mathrm{cm}^{-1} 2958,2896,1588,1503,1486,1339,1238,1161,1090,1036,934$

$>$ HRMS calcd for $\mathrm{C}_{20} \mathrm{H}_{23} \mathrm{NO}_{4} \mathrm{SSi}(\mathrm{M}+\mathrm{H})^{+} 402.1195$, found 402.1185 .

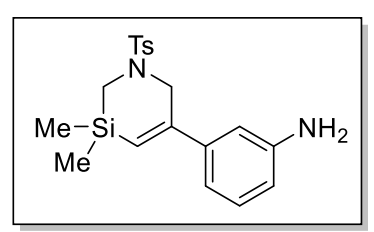

\section{$\underline{\text { 3-(3,3-dimethyl-1-tosyl-1,2,3,6-tetrahydro-1,3-azasilin-5-yl)aniline }}$}

(4n)

4n was prepared according to General Method C. 2a (58.4 mg, 0.20 mmol, 1.0 equiv.), $\mathrm{Pd}\left(\mathrm{PPh}_{3}\right)_{2} \mathrm{Cl}_{2}$ (7.2 $\left.\mathrm{mg}, 0.01 \mathrm{mmol}, 5 \mathrm{~mol} \%\right), \mathrm{ZnI}_{2}$ (12.8 mg, $0.04 \mathrm{mmol}, 0.2$ equiv.), alkyne S-A-11 (28.6 mg, $0.24 \mathrm{mmol}$, 1.2 equiv.) and $\mathrm{DBU}(61 \mathrm{mg}, 0.4 \mathrm{mmol}, 2.0$ equiv. $)$ in xylenes $(0.5 \mathrm{~mL})$ 
at $120^{\circ} \mathrm{C}$ for $10 \mathrm{~min}$ afforded $\mathbf{4 n}$ as white solid $(29 \mathrm{mg}, 38 \%)$.

$>\mathrm{mp}: 150.6-151.8^{\circ} \mathrm{C}$.

$>\mathrm{R}_{f}=0.5$ (EtOAc:Petroleum Ether $\left.=1: 2\right)$.

$>\quad{ }^{1} \mathrm{H} \mathrm{NMR}\left(400 \mathrm{MHz}, \mathrm{CDCl}_{3}\right) \delta 7.70(\mathrm{~d}, J=8.0 \mathrm{~Hz}, 2 \mathrm{H}), 7.33(\mathrm{~d}, J=8.0 \mathrm{~Hz}, 2 \mathrm{H}), 7.11(\mathrm{t}, J=$ $8.0 \mathrm{~Hz}, 1 \mathrm{H}), 6.72-6.70(\mathrm{~m}, 1 \mathrm{H}), 6.66-6.62(\mathrm{~m}, 2 \mathrm{H}), 5.96(\mathrm{t}, J=1.2 \mathrm{~Hz}, 1 \mathrm{H}), 3.88(\mathrm{~d}, J=1.2$ $\mathrm{Hz}, 2 \mathrm{H}), 3.69$ (s, 2H), 2.53 (s, 2H), 2.43 (s, 3H), $0.21(\mathrm{~s}, 6 \mathrm{H})$.

$>{ }^{13} \mathrm{C}$ NMR $\left(100 \mathrm{MHz}, \mathrm{CDCl}_{3}\right) \delta 153.3,146.4,143.5,143.1,132.5,129.6,129.4,128.0,122.5$, $116.3,115.0,112.5,50.9,35.3,21.5,-3.0$.

$>\mathrm{IR}$ (neat) $\mathrm{cm}^{-1} 3469,3377,2956,1620,1597,1491,1334,1158,1088,1033,994,901 ;$ HRMS calcd for $\mathrm{C}_{19} \mathrm{H}_{24} \mathrm{~N}_{2} \mathrm{O}_{2} \mathrm{SSi}(\mathrm{M}+\mathrm{H})^{+} 373.1406$, found 373.1396.

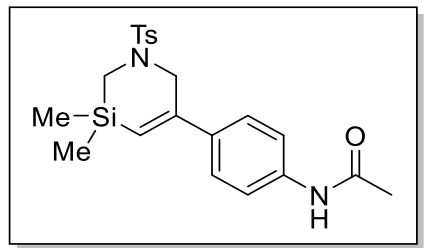

$57 \%)$.

$>\mathrm{mp}: 214.4-216.8^{\circ} \mathrm{C}$.

$>\mathrm{R}_{f}=0.4($ EtOAc:Petroleum Ether $=1: 1)$.

$>\quad{ }^{1} \mathrm{H} \mathrm{NMR}\left(400 \mathrm{MHz}, \mathrm{CDCl}_{3}\right) \delta 7.71(\mathrm{~d}, J=8.2 \mathrm{~Hz}, 2 \mathrm{H}), 7.51-7.48(\mathrm{~m}, 3 \mathrm{H}), 7.35(\mathrm{~d}, J=8.1$ $\mathrm{Hz}, 2 \mathrm{H}), 7.29(\mathrm{~d}, J=8.6 \mathrm{~Hz}, 2 \mathrm{H}), 5.99(\mathrm{~s}, 1 \mathrm{H}), 3.90(\mathrm{~d}, J=1.7 \mathrm{~Hz}, 2 \mathrm{H}), 2.54(\mathrm{~s}, 2 \mathrm{H}), 2.44(\mathrm{~s}$, $3 \mathrm{H}), 2.17(\mathrm{~s}, 3 \mathrm{H}), 0.22(\mathrm{~s}, 6 \mathrm{H})$.

$>{ }^{13} \mathrm{C} \mathrm{NMR}\left(100 \mathrm{MHz}, \mathrm{CDCl}_{3}\right) \delta 168.4,152.0,143.6,138.0,137.3,132.4,129.7,128.0,126.3$, $122.2,119.6,50.7,35.3,24.6,21.5,-2.9$.

$>\quad \mathrm{IR}$ (neat) $\mathrm{cm}^{-1} 3032,2988,1654,1595,1530,1333,1276,1158,1088,929$.

$>$ HRMS calcd for $\mathrm{C}_{21} \mathrm{H}_{26} \mathrm{~N}_{2} \mathrm{O}_{3} \mathrm{SSi}(\mathrm{M}+\mathrm{H})^{+}$415.1512, found 415.1509.

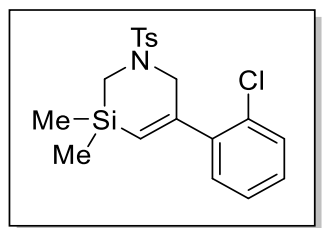

5-(2-chlorophenyl)-3,3-dimethyl-1-tosyl-1,2,3,6-tetrahydro-1,3-azasiline (4p)

4p was prepared according to General Method C. 2a $(58.4 \mathrm{mg}, 0.20 \mathrm{mmol}$, 1.0 equiv.), $\mathrm{Pd}\left(\mathrm{PPh}_{3}\right)_{2} \mathrm{Cl}_{2}$ (7.2 mg, $\left.0.01 \mathrm{mmol}, 5 \mathrm{~mol} \%\right), \mathrm{ZnI}_{2}(12.8 \mathrm{mg}$, $0.04 \mathrm{mmol}, 0.2$ equiv.), alkyne S-A-13 (33.8 $\mathrm{mg}, 0.24 \mathrm{mmol}, 1.2$ equiv.) and DBU (61 mg, $0.4 \mathrm{mmol}, 2.0$ equiv.) in xylenes $(0.5 \mathrm{~mL})$ at $120^{\circ} \mathrm{C}$ for $10 \mathrm{~min}$ afforded $\mathbf{4 p}$ as white solid (51 $\mathrm{mg}, 65 \%)$.

$>$ mp: $111.5-113.1^{\circ} \mathrm{C}$

$>\mathrm{R}_{f}=0.6($ EtOAc:Petroleum Ether $=1: 5)$.

$>{ }^{1} \mathrm{H}$ NMR $\left(400 \mathrm{MHz}, \mathrm{CDCl}_{3}\right) \delta 7.66(\mathrm{~d}, J=8.0 \mathrm{~Hz}, 2 \mathrm{H}), 7.36-7.33(\mathrm{~m}, 1 \mathrm{H}), 7.31(\mathrm{~d}, J=8.0$ $\mathrm{Hz}, 2 \mathrm{H}), 7.23-7.17(\mathrm{~m}, 3 \mathrm{H}), 5.75(\mathrm{t}, J=1.72 \mathrm{~Hz}, 1 \mathrm{H}), 3.78(\mathrm{~d}, J=1.88 \mathrm{~Hz}, 2 \mathrm{H}), 2.57(\mathrm{~s}, 2 \mathrm{H})$, $2.42(\mathrm{~s}, 3 \mathrm{H}), 0.24(\mathrm{~s}, 6 \mathrm{H})$.

$>{ }^{13} \mathrm{C}$ NMR $\left(100 \mathrm{MHz}, \mathrm{CDCl}_{3}\right) \delta 152.5,143.5,141.9,132.5,131.6,129.9,129.6,129.6,128.9$, 128.0, 126.9, 126.5, 51.5, 35.2, 21.6, -3.0.

$>$ IR (neat) $\mathrm{cm}^{-1} 2958,1598,1467,1435,1305,1341,1161,1089,928$.

$>$ HRMS calcd for $\mathrm{C}_{19} \mathrm{H}_{22} \mathrm{ClNO}_{2} \mathrm{SSi}(\mathrm{M}+\mathrm{H})^{+}$392.0907, found 392.0906 .

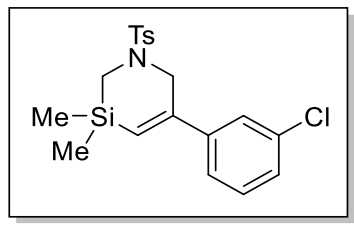

\section{5-(3-chlorophenyl)-3,3-dimethyl-1-tosyl-1,2,3,6-tetrahydro-1,3-} azasiline (4q)

4q was prepared according to General Method C. 2a (58.4 mg, 0.20 mmol, 1.0 equiv.), $\mathrm{Pd}\left(\mathrm{PPh}_{3}\right)_{2} \mathrm{Cl}_{2}$ (7.2 $\mathrm{mg}, 0.01 \mathrm{mmol}, 5 \mathrm{~mol} \%$ ), $\mathrm{ZnI}_{2}$ (12.8 mg, $0.04 \mathrm{mmol}, 0.2$ equiv.), alkyne S-A-14 (33.8 mg, $0.24 \mathrm{mmol}$, 1.2 equiv.) and $\mathrm{DBU}(61 \mathrm{mg}, 0.4 \mathrm{mmol}, 2.0$ equiv.) in xylenes $(0.5 \mathrm{~mL})$ at $120^{\circ} \mathrm{C}$ for $10 \mathrm{~min}$ afforded $\mathbf{4 q}$ as white solid $(45 \mathrm{mg}, 57 \%)$.

$>\mathrm{mp}: 121.1-124.0^{\circ} \mathrm{C}$.

$>\mathrm{R}_{f}=0.6$ (EtOAc:Petroleum Ether $\left.=1: 5\right)$.

$>{ }^{1} \mathrm{H} \mathrm{NMR}\left(400 \mathrm{MHz}, \mathrm{CDCl}_{3}\right) \delta 7.71(\mathrm{~d}, J=8.0 \mathrm{~Hz}, 2 \mathrm{H}), 7.35(\mathrm{~d}, J=8.0 \mathrm{~Hz}, 2 \mathrm{H}), 7.31-7.30$ $(\mathrm{m}, 1 \mathrm{H}), 7.27-7.25(\mathrm{~m}, 2 \mathrm{H}), 7.22-7.19(\mathrm{~m}, 1 \mathrm{H}), 6.04(\mathrm{t}, J=1.76 \mathrm{~Hz}, 1 \mathrm{H}), 3.89(\mathrm{~d}, J=1.64$ $\mathrm{Hz}, 2 \mathrm{H}), 2.56$ (s, 2H), 2.43 (s, 3H), 0.23 (s, 6H). 
$>{ }^{13} \mathrm{C} \mathrm{NMR}\left(100 \mathrm{MHz}, \mathrm{CDCl}_{3}\right) \delta 151.5,143.6,143.5,134.4,132.4,129.7,129.7,128.2,128.0$, 126.0, 124.6, 123.9, 50.6, 35.2, 21.6, -3.0.

$>$ IR (neat) $\mathrm{cm}^{-1} 2959,1592,1562,1438,1341,1161,1089,1034,935$.

$>$ HRMS calcd for $\mathrm{C}_{19} \mathrm{H}_{22} \mathrm{ClNO}_{2} \mathrm{SSi}(\mathrm{M}+\mathrm{Na})^{+} 414.0727$, found 414.0704 .

\section{5-(4-chlorophenyl)-3,3-dimethyl-1-tosyl-1,2,3,6-tetrahydro-1,3-azasiline (4r)}

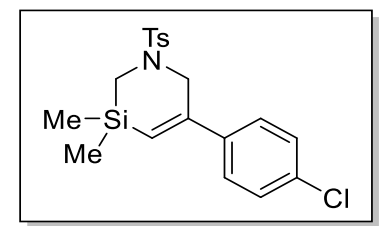

4r was prepared according to General Method C. 2a (58.4 mg, 0.20 mmol, 1.0 equiv.), $\mathrm{Pd}\left(\mathrm{PPh}_{3}\right)_{2} \mathrm{Cl}_{2}$ (7.2 $\left.\mathrm{mg}, 0.01 \mathrm{mmol}, 5 \mathrm{~mol} \%\right), \mathrm{ZnI}_{2}$ (12.8 $\mathrm{mg}, 0.04 \mathrm{mmol}, 0.2$ equiv.), alkyne $\mathbf{S}-\mathbf{A}-\mathbf{1 5}(33.8 \mathrm{mg}, 0.24 \mathrm{mmol}$, 1.2 equiv.) and $\mathrm{DBU}(61 \mathrm{mg}, 0.4 \mathrm{mmol}, 2.0$ equiv. $)$ in xylenes $(0.5 \mathrm{~mL})$ at $120^{\circ} \mathrm{C}$ for $10 \mathrm{~min}$ afforded $4 \mathbf{r}$ as white solid $(57 \mathrm{mg}, 72 \%)$.

$>\quad \mathrm{mp}: 181.1-182.5^{\circ} \mathrm{C}$.

$>\mathrm{R}_{f}=0.7$ (EtOAc:Petroleum Ether $\left.=1: 5\right)$.

$>{ }^{1} \mathrm{H}$ NMR $\left(400 \mathrm{MHz}, \mathrm{CDCl}_{3}\right) \delta 7.71(\mathrm{~d}, J=8.0 \mathrm{~Hz}, 2 \mathrm{H}), 7.35(\mathrm{~d}, J=8.0 \mathrm{~Hz}, 2 \mathrm{H}), 7.31-7.25$ $(\mathrm{m}, 4 \mathrm{H}), 6.02(\mathrm{t}, J=1.76 \mathrm{~Hz}, 1 \mathrm{H}), 3.89(\mathrm{~d}, J=1.64 \mathrm{~Hz}, 2 \mathrm{H}), 2.55(\mathrm{~s}, 2 \mathrm{H}), 2.44(\mathrm{~s}, 3 \mathrm{H}), 0.23$ $(\mathrm{s}, 6 \mathrm{H})$.

$>{ }^{13} \mathrm{C} \mathrm{NMR}\left(100 \mathrm{MHz}, \mathrm{CDCl}_{3}\right) \delta 151.7,143.6,140.1,134.1,132.4,129.7,128.6,128.0,127.1$, $123.8,50.6,35.2,21.5,-3.0$.

$>$ IR (neat) $\mathrm{cm}^{-1} 2958,1598,1577,1488,1438,1339,1158,1089,1033,901$.

$>$ HRMS calcd for $\mathrm{C}_{19} \mathrm{H}_{22} \mathrm{ClNO}_{2} \mathrm{SSi}(\mathrm{M}+\mathrm{Na})^{+} 414.0727$, found 414.0721 .

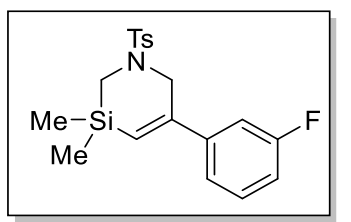

\section{5-(3-fluorophenyl)-3,3-dimethyl-1-tosyl-1,2,3,6-tetrahydro-1,3-} azasiline (4s)

$4 \mathbf{s}$ was prepared according to General Method C. $2 a(58.4 \mathrm{mg}, 0.20 \mathrm{mmol}$, 1.0 equiv.), $\mathrm{Pd}\left(\mathrm{PPh}_{3}\right)_{2} \mathrm{Cl}_{2}$ (7.2 mg, $\left.0.01 \mathrm{mmol}, 5 \mathrm{~mol} \%\right), \mathrm{ZnI}_{2}(12.8 \mathrm{mg}$, 0.04 mmol, 0.2 equiv.), alkyne S-A-16 (29.3 $\mathrm{mg}, 0.24 \mathrm{mmol}, 1.2$ equiv.) and DBU (61 mg, $0.4 \mathrm{mmol}, 2.0$ equiv.) in xylenes $(0.5 \mathrm{~mL})$ at $120^{\circ} \mathrm{C}$ for

$10 \mathrm{~min}$ afforded $\mathbf{4 s}$ as yellow solid (44 $\mathrm{mg}, 58 \%$ ).

$>\mathrm{mp}: 153.3-155.4^{\circ} \mathrm{C}$.

$>\mathrm{R}_{f}=0.6($ EtOAc:Petroleum Ether $=1: 5)$.

$>{ }^{1} \mathrm{H} \mathrm{NMR}\left(400 \mathrm{MHz}, \mathrm{CDCl}_{3}\right) \delta 7.71(\mathrm{~d}, J=8.0 \mathrm{~Hz}, 2 \mathrm{H}), 7.35(\mathrm{~d}, J=8.0 \mathrm{~Hz}, 2 \mathrm{H}), 7.32-7.26$ $(\mathrm{m}, 1 \mathrm{H}), 7.12-7.10(\mathrm{~m}, 1 \mathrm{H}), 7.04-6.96(\mathrm{~m}, 2 \mathrm{H}), 6.06(\mathrm{t}, J=1.80 \mathrm{~Hz}, 1 \mathrm{H}), 3.90(\mathrm{~d}, J=1.80$ $\mathrm{Hz}, 2 \mathrm{H}), 2.56(\mathrm{~s}, 2 \mathrm{H}), 2.44 \mathrm{k}(\mathrm{s}, 3 \mathrm{H}), 0.23$ (s, 6H).

$>{ }^{13} \mathrm{C}$ NMR $\left(150 \mathrm{MHz}, \mathrm{CDCl}_{3}\right) \delta 162.8(\mathrm{~d}, J=245 \mathrm{~Hz}), 151.5,143.8(\mathrm{~d}, J=7 \mathrm{~Hz}), 143.6,132.5$, $129.9(\mathrm{~d}, J=8 \mathrm{~Hz}), 129.6,128.0,124.3,121.3(\mathrm{~d}, J=3 \mathrm{~Hz}), 115.0(\mathrm{~d}, J=21 \mathrm{~Hz}), 112.8(\mathrm{~d}, J$ $=22 \mathrm{~Hz}), 50.6,35.2,21.5,-3.1$.

$>{ }^{19} \mathrm{~F} \mathrm{NMR}(376 \mathrm{MHz}, \mathrm{CDCl} 3) \delta-112.92$.

$>$ IR (neat) $\mathrm{cm}^{-1} 2958,1598,1577,1488,1438,1305,1287,1158,1089,1033,901$.

$>$ HRMS calcd for $\mathrm{C}_{19} \mathrm{H}_{22} \mathrm{FNO}_{2} \mathrm{SSi}(\mathrm{M}+\mathrm{Na})^{+} 398.1022$, found 398.1020.

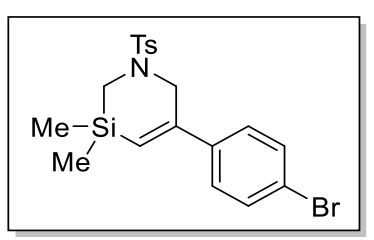

\section{5-(4-bromophenvl)-3,3-dimethvl-1-tosvl-1,2,3,6-tetrahvdro-1,3- azasiline (4t)}

4t was prepared according to General Method C. 2a (58.4 mg, 0.20 mmol, 1.0 equiv.), $\mathrm{Pd}\left(\mathrm{PPh}_{3}\right)_{2} \mathrm{Cl}_{2}$ (7.2 $\left.\mathrm{mg}, 0.01 \mathrm{mmol}, 5 \mathrm{~mol} \%\right), \mathrm{ZnI}_{2}$ (12.8 mg, $0.04 \mathrm{mmol}, 0.2$ equiv.), alkyne S-A-17 (44.8 mg, $0.24 \mathrm{mmol}$, 1.2 equiv.) and $\mathrm{DBU}(61 \mathrm{mg}, 0.4 \mathrm{mmol}, 2.0$ equiv. $)$ in xylenes $(0.5 \mathrm{~mL})$ at $120^{\circ} \mathrm{C}$ for $10 \mathrm{~min}$ afforded $\mathbf{4 t}$ as yellow solid $(11 \mathrm{mg}, 12 \%)$.

$>$ mp: $183.7-185.2^{\circ} \mathrm{C}$.

$>\mathrm{R}_{f}=0.7$ (EtOAc:Petroleum Ether $\left.=1: 5\right)$.

$>{ }^{1} \mathrm{H} \mathrm{NMR}\left(400 \mathrm{MHz}, \mathrm{CDCl}_{3}\right) \delta 7.70(\mathrm{~d}, J=8.0 \mathrm{~Hz}, 2 \mathrm{H}), 7.45(\mathrm{~d}, J=8.0 \mathrm{~Hz}, 2 \mathrm{H}), 7.35(\mathrm{~d}, J=$ $8.0 \mathrm{~Hz}, 2 \mathrm{H}), 7.20(\mathrm{~d}, J=8.0 \mathrm{~Hz}, 2 \mathrm{H}), 6.02(\mathrm{t}, J=1.76 \mathrm{~Hz}, 1 \mathrm{H}), 3.88(\mathrm{~d}, J=1.64 \mathrm{~Hz}, 2 \mathrm{H}), 2.55$ $(\mathrm{s}, 2 \mathrm{H}), 2.44(\mathrm{~s}, 3 \mathrm{H}), 0.23(\mathrm{~s}, 6 \mathrm{H})$.

$>\quad{ }^{13} \mathrm{C} \mathrm{NMR}\left(100 \mathrm{MHz}, \mathrm{CDCl}_{3}\right) \delta 151.7,143.6,140.5,132.4,131.6,129.7,128.0,127.4,123.9$, 122.3, 50.6, 35.2, 21.6, -3.0.

$>$ IR (neat) $\mathrm{cm}^{-1} 2923,1597,1440,1337,1160,1089,1036,927$.

$>$ HRMS calcd for $\mathrm{C}_{19} \mathrm{H}_{22} \mathrm{BrNO}_{2} \mathrm{SSi}(\mathrm{M}+\mathrm{H})^{+} 436.0402$, found 436.0391 . 


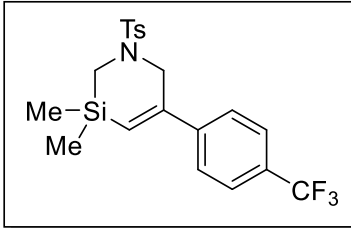

3,3-dimethvl-1-tosyl-5-(4-(trifluoromethvl)phenvl)-1,2,3,6-

tetrahydro-1,3-azasiline (4u)

4u was prepared according to General Method C. 2a (58.4 mg, 0.20 mmol, 1.0 equiv.), $\mathrm{Pd}\left(\mathrm{PPh}_{3}\right)_{2} \mathrm{Cl}_{2}$ (7.2 $\left.\mathrm{mg}, 0.01 \mathrm{mmol}, 5 \mathrm{~mol} \%\right), \mathrm{ZnI}_{2}$ (12.8 mg, $0.04 \mathrm{mmol}, 0.2$ equiv.), alkyne S-A-18 (41.6 mg, $0.24 \mathrm{mmol}$, 1.2 equiv.) and DBU (61 mg, $0.4 \mathrm{mmol}, 2.0$ equiv.) in xylenes $(0.5 \mathrm{~mL})$ at $120^{\circ} \mathrm{C}$ for $10 \mathrm{~min}$ afforded $4 \mathbf{u}$ as yellow solid $(50 \mathrm{mg}, 59 \%)$.

$>\mathrm{mp}: 132.1-133.7^{\circ} \mathrm{C}$.

$>\mathrm{R}_{f}=0.6($ EtOAc:Petroleum Ether $=1: 5)$.

$>{ }^{1} \mathrm{H} \mathrm{NMR}\left(400 \mathrm{MHz}, \mathrm{CDCl}_{3}\right) \delta 7.71(\mathrm{~d}, J=8.0 \mathrm{~Hz}, 2 \mathrm{H}), 7.58(\mathrm{~d}, J=8.0 \mathrm{~Hz}, 2 \mathrm{H}), 7.43(\mathrm{~d}, J=$ $8.0 \mathrm{~Hz}, 2 \mathrm{H}), 7.35(\mathrm{~d}, J=8.0 \mathrm{~Hz}, 2 \mathrm{H}), 6.10(\mathrm{t}, J=1.76 \mathrm{~Hz}, 1 \mathrm{H}), 3.92(\mathrm{~d}, J=1.76 \mathrm{~Hz}, 2 \mathrm{H})$, $2.58(\mathrm{~s}, 2 \mathrm{H}), 2.44(\mathrm{~s}, 3 \mathrm{H}), 0.25(\mathrm{~s}, 6 \mathrm{H})$.

$>{ }^{13} \mathrm{C}$ NMR $\left(100 \mathrm{MHz}, \mathrm{CDCl}_{3}\right) \delta 151.7,145.2,143.7,132.4,130.1(\mathrm{q}, J=32 \mathrm{~Hz}), 129.7,128.0$, 126.1, 125.6, $125.4(\mathrm{q}, J=4 \mathrm{~Hz}), 123.8(\mathrm{q}, J=253 \mathrm{~Hz}), 50.6,35.2,21.6,-3.1$.

$>{ }^{19} \mathrm{~F} \mathrm{NMR}(376 \mathrm{MHz}, \mathrm{CDCl} 3) \delta-62.58$.

$>\mathrm{IR}$ (neat) $\mathrm{cm}^{-1} 2988,1599,1408,1324,1275,1161,1119,1090,1035,928$.

$>$ HRMS calcd for $\mathrm{C}_{20} \mathrm{H}_{22} \mathrm{~F}_{3} \mathrm{NO}_{2} \mathrm{SSi}(\mathrm{M}+\mathrm{H})^{+} 426.1171$, found 426.1162 .

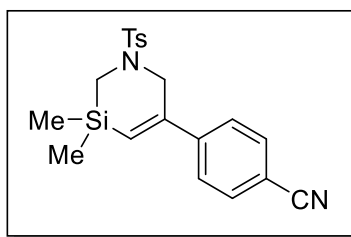

4-(3,3-dimethyl-1-tosyl-1,2,3,6-tetrahydro-1,3-azasilin-5vl)benzonitrile (4v)

4v was prepared according to General Method C. 2a (58.4 mg, 0.20 mmol, 1.0 equiv.), $\mathrm{Pd}\left(\mathrm{PPh}_{3}\right)_{2} \mathrm{Cl}_{2}$ (7.2 $\left.\mathrm{mg}, 0.01 \mathrm{mmol}, 5 \mathrm{~mol} \%\right), \mathrm{ZnI}_{2}$ (12.8 mg, $0.04 \mathrm{mmol}, 0.2$ equiv.), alkyne S-A-19 (31.4 mg, $0.24 \mathrm{mmol}$, 1.2 equiv.) and $\mathrm{DBU}(61 \mathrm{mg}, 0.4 \mathrm{mmol}, 2.0$ equiv. $)$ in xylenes $(0.5 \mathrm{~mL})$ at $120{ }^{\circ} \mathrm{C}$ for $10 \mathrm{~min}$ afforded $\mathbf{4 v}$ as yellow solid (34 $\mathrm{mg}, 39 \%$ ).

$>\mathrm{mp}: 187.3-188.7^{\circ} \mathrm{C}$.

$>\mathrm{R}_{f}=0.4($ EtOAc:Petroleum Ether $=1: 5)$.

$>{ }^{1} \mathrm{H} \mathrm{NMR}\left(400 \mathrm{MHz}, \mathrm{CDCl}_{3}\right) \delta 7.71(\mathrm{~d}, J=8.0 \mathrm{~Hz}, 2 \mathrm{H}), 7.63(\mathrm{~d}, J=8.0 \mathrm{~Hz}, 2 \mathrm{H}), 7.42(\mathrm{~d}, J=$ $8.0 \mathrm{~Hz}, 2 \mathrm{H}), 7.36(\mathrm{~d}, J=8.0 \mathrm{~Hz}, 2 \mathrm{H}), 6.15(\mathrm{t}, J=1.76 \mathrm{~Hz}, 1 \mathrm{H}), 3.91(\mathrm{~d}, J=1.76 \mathrm{~Hz}, 2 \mathrm{H}), 2.58$ (s, 2H), $2.44(\mathrm{~s}, 3 \mathrm{H}), 0.25(\mathrm{~s}, 6 \mathrm{H})$.

$>\quad{ }^{13} \mathrm{C} \mathrm{NMR}\left(100 \mathrm{MHz}, \mathrm{CDCl}_{3}\right) \delta 151.1,145.9,143.7,132.4,132.3,129.7,128.0,126.9,126.4$, $118.6,111.7,50.4,35.2,21.6,-3.1$.

$>$ IR (neat) $\mathrm{cm}^{-1} 2959,2226,1599,1501,1339,1161,1090,1035,928$.

$>$ HRMS calcd for $\mathrm{C}_{20} \mathrm{H}_{22} \mathrm{~N}_{2} \mathrm{O}_{2} \mathrm{SSi}(\mathrm{M}+\mathrm{Na})^{+} 405.1069$, found 405.1062 .

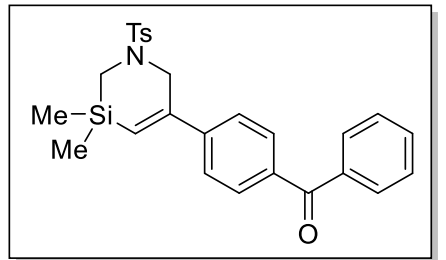

\section{(4-(3,3-dimethyl-1-tosyl-1,2,3,6-tetrahydro-1,3-azasilin-5-} yl)phenyl)(phenyl)methanone $(4 \mathrm{w})$

4w was prepared according to General Method C. $2 \mathbf{a}(58.4 \mathrm{mg}, 0.20$ mmol, 1.0 equiv.), $\mathrm{Pd}\left(\mathrm{PPh}_{3}\right)_{2} \mathrm{Cl}_{2}$ (7.2 $\mathrm{mg}, 0.01 \mathrm{mmol}, 5 \mathrm{~mol} \%$ ), $\mathrm{ZnI}_{2}$ (12.8 mg, $0.04 \mathrm{mmol}, 0.2$ equiv.), alkyne S-A-20 (50.0 mg, 0.24 mmol, 1.2 equiv.) and DBU (61 mg, $0.4 \mathrm{mmol}, 2.0$ equiv.) in

(56 mg, 61\%).

$>\mathrm{mp}: 155.2-156.9^{\circ} \mathrm{C}$.

$>\mathrm{R}_{f}=0.5$ (EtOAc:Petroleum Ether $\left.=1: 2\right)$.

$>{ }^{1} \mathrm{H} \mathrm{NMR}\left(400 \mathrm{MHz}, \mathrm{CDCl}_{3}\right) \delta 7.80-7.77(\mathrm{~m}, 4 \mathrm{H}), 7.73(\mathrm{~d}, J=8.0 \mathrm{~Hz}, 2 \mathrm{H}), 7.62-7.58(\mathrm{~m}$, $1 \mathrm{H}), 7.51-7.47(\mathrm{~m}, 2 \mathrm{H}), 7.44(\mathrm{~d}, J=8.0 \mathrm{~Hz}, 2 \mathrm{H}), 7.36(\mathrm{~d}, J=8.0 \mathrm{~Hz}, 2 \mathrm{H}), 6.17$ (t, $J=1.76$ $\mathrm{Hz}, 1 \mathrm{H}), 3.97(\mathrm{~d}, J=1.76 \mathrm{~Hz}, 2 \mathrm{H}), 2.59(\mathrm{~s}, 2 \mathrm{H}), 2.44(\mathrm{~s}, 3 \mathrm{H}), 0.26$ (s, 6H).

$>{ }^{13} \mathrm{C} \mathrm{NMR}\left(100 \mathrm{MHz}, \mathrm{CDCl}_{3}\right) \delta 196.1,151.9,145.5,143.7,137.5,137.1,132.5,132.4,130.4$, $130.0,129.7,128.4,128.0,125.6,125.5,50.6,35.3,21.6,-3.0$.

$>\quad \mathrm{IR}$ (neat) $\mathrm{cm}^{-1} 2988,1656,1598,1446,1276,1161,1034,925$.

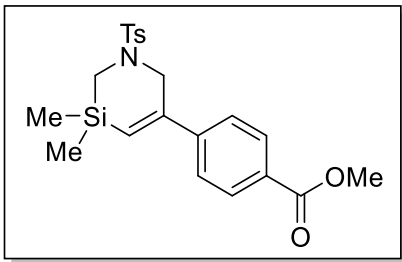

$>$ HRMS calcd for $\mathrm{C}_{26} \mathrm{H}_{27} \mathrm{NO}_{3} \mathrm{SSi}(\mathrm{M}+\mathrm{H})^{+}$462.1559, found 462.1550 .

methyl 4-(3,3-dimethyl-1-tosyl-1,2,3,6-tetrahydro-1,3-azasilin-5yl)benzoate (4x)

4x was prepared according to General Method C. $2 \mathbf{a}(58.4 \mathrm{mg}, 0.20$ mmol, 1.0 equiv.), $\mathrm{Pd}\left(\mathrm{PPh}_{3}\right)_{2} \mathrm{Cl}_{2}$ (7.2 mg, $\left.0.01 \mathrm{mmol}, 5 \mathrm{~mol} \%\right), \mathrm{ZnI}_{2}$ 
(12.8 mg, 0.04 mmol, 0.2 equiv.), alkyne S-A-21 (44.0 mg, 0.24 mmol, 1.2 equiv.) and DBU (61 $\mathrm{mg}, 0.4 \mathrm{mmol}, 2.0$ equiv.) in xylenes $(0.5 \mathrm{~mL})$ at $120^{\circ} \mathrm{C}$ for $10 \mathrm{~min}$ afforded $\mathbf{4 x}$ as yellow solid (51 $\mathrm{mg}, 62 \%)$.

$>$ mp: $140.5-142.8^{\circ} \mathrm{C}$.

$>\mathrm{R}_{f}=0.4$ (EtOAc:Petroleum Ether $\left.=1: 10\right)$.

$>{ }^{1} \mathrm{H} \mathrm{NMR}\left(400 \mathrm{MHz}, \mathrm{CDCl}_{3}\right) \delta 8.00(\mathrm{~d}, J=8.0 \mathrm{~Hz}, 2 \mathrm{H}), 7.72(\mathrm{~d}, J=8.0 \mathrm{~Hz}, 2 \mathrm{H}), 7.39(\mathrm{~d}, J=$ $8.0 \mathrm{~Hz}, 2 \mathrm{H}), 7.35(\mathrm{~d}, J=8.0 \mathrm{~Hz}, 2 \mathrm{H}), 6.14(\mathrm{t}, J=1.80 \mathrm{~Hz}, 1 \mathrm{H}), 3.94(\mathrm{~d}, J=1.84 \mathrm{~Hz}, 2 \mathrm{H}), 3.92$ (s, 3H), $2.57(\mathrm{~s}, 2 \mathrm{H}), 2.44(\mathrm{~s}, 3 \mathrm{H}), 0.24(\mathrm{~s}, 6 \mathrm{H})$.

$>{ }^{13} \mathrm{C} \mathrm{NMR}\left(150 \mathrm{MHz}, \mathrm{CDCl}_{3}\right) \delta 166.6,151.9,145.9,143.6,132.5,129.8,129.7,128.0,125.6$, $125.4,52.2,50.5,35.2,21.5,-3.1$.

$>\mathrm{IR}$ (neat) $\mathrm{cm}^{-1} 2953,1996,1720,1602,1436,1341,1162,1017,929$.

$>$ HRMS calcd for $\mathrm{C}_{21} \mathrm{H}_{25} \mathrm{NO}_{4} \mathrm{SSi}(\mathrm{M}+\mathrm{H})^{+} 416.1352$, found 416.1341 .

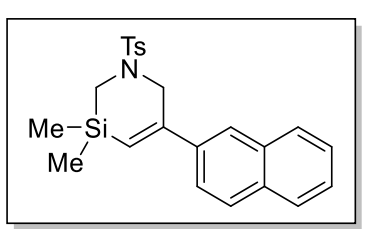

\section{3,3-dimethyl-5-(naphthalen-2-yl)-1-tosyl-1,2,3,6-tetrahydro-1,3- azasiline (4y)}

4y was prepared according to General Method C. 2a (58.4 mg, 0.20 mmol, 1.0 equiv.), $\mathrm{Pd}\left(\mathrm{PPh}_{3}\right)_{2} \mathrm{Cl}_{2}$ (7.2 $\left.\mathrm{mg}, 0.01 \mathrm{mmol}, 5 \mathrm{~mol} \%\right), \mathrm{ZnI}_{2}$ (12.8 mg, 0.04 mmol, 0.2 equiv.), alkyne S-A-22 (36.5 mg, $0.24 \mathrm{mmol}$, 1.2 equiv.) and DBU (61 mg, $0.4 \mathrm{mmol}, 2.0$ equiv.) in xylenes $(0.5 \mathrm{~mL})$ at $120^{\circ} \mathrm{C}$ for $10 \mathrm{~min}$ afforded $4 \mathbf{y}$ as yellow solid $(55 \mathrm{mg}, 68 \%)$.

$>\mathrm{mp}: 155.7-157.1^{\circ} \mathrm{C}$.

$>\mathrm{R}_{f}=0.7$ (EtOAc:Petroleum Ether $\left.=1: 5\right)$.

$>{ }^{1} \mathrm{H} \mathrm{NMR}\left(400 \mathrm{MHz}, \mathrm{CDCl}_{3}\right) \delta 7.85-7.79(\mathrm{~m}, 3 \mathrm{H}), 7.76-7.73(\mathrm{~m}, 3 \mathrm{H}), 7.51-7.45(\mathrm{~m}, 3 \mathrm{H})$, $7.35(\mathrm{~d}, J=8.0 \mathrm{~Hz}, 2 \mathrm{H}), 6.17(\mathrm{t}, J=1.72 \mathrm{~Hz}, 1 \mathrm{H}), 4.06(\mathrm{~d}, J=1.72 \mathrm{~Hz}, 2 \mathrm{H}), 2.59(\mathrm{~s}, 2 \mathrm{H}), 2.43$ $(\mathrm{s}, 3 \mathrm{H}), 0.26(\mathrm{~s}, 6 \mathrm{H})$.

$>{ }^{13} \mathrm{C} \mathrm{NMR}\left(100 \mathrm{MHz}, \mathrm{CDCl}_{3}\right) \delta 152.8,143.5,138.8,133.1,133.1,132.6,129.7,128.3,128.1$, $128.1,127.6,126.4,126.3,124.4,124.2,123.7,50.9,35.3,21.6,-2.9$.

$>$ IR (neat) $\mathrm{cm}^{-1} 2955,2951,2852,1738,1597,1456,1340,1162,1089,928$.

$>$ HRMS calcd for $\mathrm{C}_{23} \mathrm{H}_{25} \mathrm{NO}_{2} \mathrm{SSi}(\mathrm{M}+\mathrm{Na})^{+} 430.1273$, found 430.1271 .

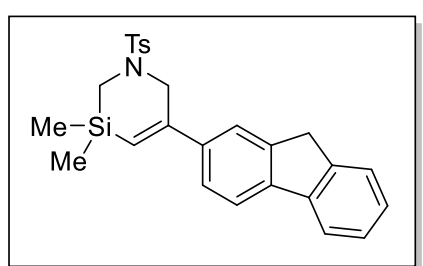

$66 \%)$.

$>$ mp: $60.5-62.1^{\circ} \mathrm{C}$

$>\mathrm{R}_{f}=0.5$ (EtOAc:Petroleum Ether $\left.=1: 5\right)$.

$>{ }^{1} \mathrm{H} \mathrm{NMR}\left(400 \mathrm{MHz}, \mathrm{CDCl}_{3}\right) \delta 7.78(\mathrm{~d}, J=8.0 \mathrm{~Hz}, 1 \mathrm{H}), 7.75-7.71(\mathrm{~m}, 3 \mathrm{H}), 7.55-7.53(\mathrm{~m}$, $2 \mathrm{H}), 7.39-7.29(\mathrm{~m}, 5 \mathrm{H}), 6.08(\mathrm{~s}, 1 \mathrm{H}), 4.00(\mathrm{~s}, 2 \mathrm{H}), 3.89(\mathrm{~s}, 2 \mathrm{H}), 2.57(\mathrm{~s}, 2 \mathrm{H}), 2.43(\mathrm{~s}, 3 \mathrm{H})$, $0.24(\mathrm{~s}, 6 \mathrm{H})$.

$>{ }^{13} \mathrm{C} \mathrm{NMR}\left(100 \mathrm{MHz}, \mathrm{CDCl}_{3}\right) \delta 153.2,143.6,143.5,143.5,141.9,141.2,140.3,132.6,129.6$, $128.1,127.0,126.9,125.1,124.7,122.7,122.5,120.1,119.8,51.0,36.9,35.3,21.6,-2.9$.

$>\quad \mathrm{IR}$ (neat) $\mathrm{cm}^{-1} 3007,1595,1455,1340,1162,1034,938$.

$>$ HRMS calcd for $\mathrm{C}_{26} \mathrm{H}_{27} \mathrm{NO}_{2} \mathrm{SSi}(\mathrm{M}+\mathrm{H})^{+} 446.1610$, found 446.1612 .

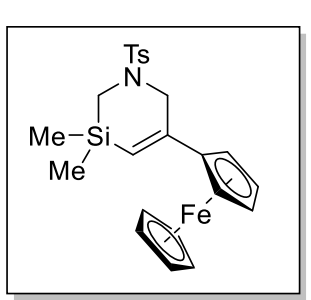

\section{3,3-dimethyl-5-ferrocene-1-tosyl-1,2,3,6-tetrahvdro-1,3-azasiline (4aa)}

4aa was prepared according to General Method C. 2a (58.4 mg, $0.20 \mathrm{mmol}$, 1.0 equiv.), $\mathrm{Pd}\left(\mathrm{PPh}_{3}\right)_{2} \mathrm{Cl}_{2}$ (7.2 mg, $0.01 \mathrm{mmol}, 5 \mathrm{~mol} \%$ ), $\mathrm{ZnI}_{2}(12.8 \mathrm{mg}, 0.04$ mmol, 0.2 equiv.), alkyne S-A-24 (50.4 $\mathrm{mg}, 0.24 \mathrm{mmol}, 1.2$ equiv.) and DBU (61 mg, $0.4 \mathrm{mmol}, 2.0$ equiv.) in xylenes $(0.5 \mathrm{~mL})$ at $120{ }^{\circ} \mathrm{C}$ for 10 min afforded 4 aa as brown solid $(50 \mathrm{mg}, 54 \%)$.

$>\quad \mathrm{mp}: 49.5-50.9^{\circ} \mathrm{C}$.

$>\quad \mathrm{R}_{f}=0.6($ EtOAc:Petroleum Ether $=1: 5)$.

$>\quad{ }^{1} \mathrm{H} \mathrm{NMR}\left(400 \mathrm{MHz}, \mathrm{CDCl}_{3}\right) \delta 7.75(\mathrm{~d}, J=8.0 \mathrm{~Hz}, 2 \mathrm{H}), 7.37(\mathrm{~d}, J=8.0 \mathrm{~Hz}, 2 \mathrm{H}), 5.88(\mathrm{t}, J=$ $1.68 \mathrm{~Hz}, 1 \mathrm{H}), 4.35-4.35(\mathrm{~m}, 2 \mathrm{H}), 4.26-4.25(\mathrm{~m}, 2 \mathrm{H}), 4.12(\mathrm{~s}, 5 \mathrm{H}), 3.88(\mathrm{~d}, \mathrm{~J}=1.68 \mathrm{~Hz}, 2 \mathrm{H})$, $2.54(\mathrm{~s}, 2 \mathrm{H}), 2.45(\mathrm{~s}, 3 \mathrm{H}), 0.18(\mathrm{~s}, 6 \mathrm{H})$. 
$>{ }^{13} \mathrm{C} \mathrm{NMR}\left(100 \mathrm{MHz}, \mathrm{CDCl}_{3}\right) \delta 151.1,143.5,132.7,129.6,128.0,117.1,85.4,69.6,69.2,65.5$, $50.4,35.8,21.6,-2.7$.

$>$ IR (neat) $\mathrm{cm}^{-1}$ 2956, 1591, 1492, 1409, 1339, 1160, 1089, 1034, 935.

$>$ HRMS calcd for Chemical Formula: $\mathrm{C}_{23} \mathrm{H}_{27} \mathrm{FeNO}_{2} \mathrm{SSi}(\mathrm{M}+\mathrm{H})^{+} 466.0959$, found 466.0954.

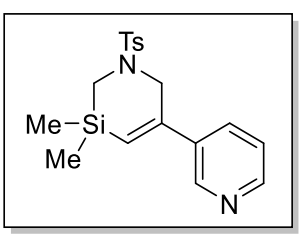

3,3-dimethyl-5-(pyridin-3-yl)-1-tosyl-1,2,3,6-tetrahydro-1,3-azasiline

(4ab)

4ab was prepared according to General Method C. 2a (58.4 mg, $0.20 \mathrm{mmol}$, 1.0 equiv.), $\mathrm{Pd}\left(\mathrm{PPh}_{3}\right)_{2} \mathrm{Cl}_{2}$ (7.2 mg, $\left.0.01 \mathrm{mmol}, 5 \mathrm{~mol} \%\right), \mathrm{ZnI}_{2}(12.8 \mathrm{mg}, 0.04$ mmol, 0.2 equiv.), alkyne alkyne $\mathbf{S}-\mathbf{A}-\mathbf{2 5}$ ( $25.5 \mathrm{mg}, 0.24 \mathrm{mmol}, 1.2$ equiv.) and DBU (61 mg, $0.4 \mathrm{mmol}, 2.0$ equiv.) in xylenes $(0.5 \mathrm{~mL})$ at $120^{\circ} \mathrm{C}$ for $10 \mathrm{~min}$ afforded $4 \mathbf{a b}$ as yellow solid (38 $\mathrm{mg}, 53 \%$ ).

$>$ mp: $112.8-115.3^{\circ} \mathrm{C}$.

$>\mathrm{R}_{f}=0.4($ EtOAc:Petroleum Ether $=1: 1)$.

$>{ }^{1} \mathrm{H} \mathrm{NMR}\left(400 \mathrm{MHz}, \mathrm{CDCl}_{3}\right) \delta 8.58(\mathrm{~d}, J=1.84 \mathrm{~Hz}, 1 \mathrm{H}), 8.53(\mathrm{dd}, J=4.80 \mathrm{~Hz}, J=1.52 \mathrm{~Hz}$, $1 \mathrm{H}), 7.71(\mathrm{~d}, J=8.0 \mathrm{~Hz}, 2 \mathrm{H}), 7.66-7.63(\mathrm{~m}, 1 \mathrm{H}), 7.35(\mathrm{~d}, J=8.0 \mathrm{~Hz}, 2 \mathrm{H}), 7.29-7.26(\mathrm{~m}$, $1 \mathrm{H}), 6.10(\mathrm{t}, J=1.76 \mathrm{~Hz}, 1 \mathrm{H}), 3.92(\mathrm{~d}, J=1.80 \mathrm{~Hz}, 2 \mathrm{H}), 2.58(\mathrm{~s}, 2 \mathrm{H}), 2.44(\mathrm{~s}, 3 \mathrm{H}), 0.25(\mathrm{~s}$, $6 \mathrm{H})$.

$>{ }^{13} \mathrm{C} \mathrm{NMR}\left(100 \mathrm{MHz}, \mathrm{CDCl}_{3}\right) \delta 149.7,149.3,147.1,143.7,137.1,133.0,132.3,129.7,128.0$, 125.6, 123.2, 50.5, 35.2, 21.6, -3.1.

$>\quad \mathrm{IR}$ (neat) $\mathrm{cm}^{-1} 2955,2923,1738,1597,1440,1339,1247,1160,1088,927$.

$>$ HRMS calcd for $\mathrm{C}_{18} \mathrm{H}_{22} \mathrm{~N}_{2} \mathrm{O}_{2} \mathrm{SSi}(\mathrm{M}+\mathrm{Na})^{+} 381.1069$, found 381.1060 .

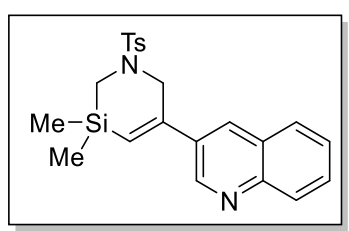

3-(3,3-dimethyl-1-tosyl-1,2,3,6-tetrahydro-1,3-azasilin-5-

vl)quinoline (4ac)

4ac was prepared according to General Method C. 2a (58.4 mg, 0.20 mmol, 1.0 equiv.), $\mathrm{Pd}\left(\mathrm{PPh}_{3}\right)_{2} \mathrm{Cl}_{2}$ (7.2 $\left.\mathrm{mg}, 0.01 \mathrm{mmol}, 5 \mathrm{~mol} \%\right), \mathrm{ZnI}_{2}$ (12.8 mg, $0.04 \mathrm{mmol}, 0.2$ equiv.), alkyne S-A-26 (37.0 mg, $0.24 \mathrm{mmol}$, 1.2 equiv.) and $\mathrm{DBU}(61 \mathrm{mg}, 0.4 \mathrm{mmol}, 2.0$ equiv.) in xylenes $(0.5 \mathrm{~mL})$ at $120^{\circ} \mathrm{C}$ for $10 \mathrm{~min}$ afforded 4 ac as white solid $(58 \mathrm{mg}, 71 \%)$.

$>$ mp: $130.5-132.6^{\circ} \mathrm{C}$.

$>\mathrm{R}_{f}=0.4$ (EtOAc:Petroleum Ether=1:4).

$>{ }^{1} \mathrm{H} \mathrm{NMR}\left(400 \mathrm{MHz}, \mathrm{CDCl}_{3}\right) \delta 8.92(\mathrm{~d}, J=2.28 \mathrm{~Hz}, 1 \mathrm{H}), 8.10-8.05(\mathrm{~m}, 2 \mathrm{H}), 7.84(\mathrm{~d}, J=8.0$ $\mathrm{Hz}, 1 \mathrm{H}), 7.75-7.70(\mathrm{~m}, 3 \mathrm{H}), 7.59-7.55(\mathrm{~m}, 1 \mathrm{H}), 7.36(\mathrm{~d}, J=8.0 \mathrm{~Hz}, 2 \mathrm{H}), 6.26(\mathrm{t}, J=1.80$ $\mathrm{Hz}, 1 \mathrm{H}), 4.05(\mathrm{~d}, J=1.72 \mathrm{~Hz}, 2 \mathrm{H}), 2.62(\mathrm{~s}, 2 \mathrm{H}), 2.44(\mathrm{~s}, 3 \mathrm{H}), 0.29$ (s, 6H).

$>{ }^{13} \mathrm{C} \mathrm{NMR}\left(100 \mathrm{MHz}, \mathrm{CDCl}_{3}\right) \delta 149.7,148.8,147.7,143.7,133.9,132.4,131.8,129.8,129.7$, $129.2,128.0,128.0,127.4,127.2,126.0,50.6,35.2,21.6,-3.0$.

$>\quad$ IR (neat) $\mathrm{cm}^{-1}$ 2957, 2923, 2852, 1598, 1491, 1306, 1338, 1162, 1089, 929.

$>$ HRMS calcd for $\mathrm{C}_{22} \mathrm{H}_{24} \mathrm{~N}_{2} \mathrm{O}_{2} \mathrm{SSi}(\mathrm{M}+\mathrm{H})^{+} 409.1406$, found 409.1396 .

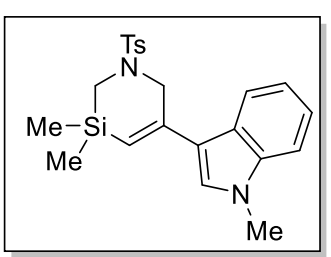

3-(3,3-dimethyl-1-tosyl-1,2,3,6-tetrahydro-1,3-azasilin-5-yl)-1-methyl1H-indole (4ad)

4ad was prepared according to General Method C. 2a (58.4 mg, $0.20 \mathrm{mmol}$, 1.0 equiv.), $\mathrm{Pd}\left(\mathrm{PPh}_{3}\right)_{2} \mathrm{Cl}_{2}$ (7.2 mg, $\left.0.01 \mathrm{mmol}, 5 \mathrm{~mol} \%\right), \mathrm{ZnI}_{2}(12.8 \mathrm{mg}$, $0.04 \mathrm{mmol}, 0.2$ equiv.), alkyne S-A-27 (38.0 mg, $0.24 \mathrm{mmol}, 1.2$ equiv.) and DBU (61 mg, $0.4 \mathrm{mmol}, 2.0$ equiv.) in xylenes $(0.5 \mathrm{~mL})$ at $120^{\circ} \mathrm{C}$ for $10 \mathrm{~min}$ afforded 4 ad as yellow solid (55 $\mathrm{mg}, 67 \%)$.

$>\mathrm{mp}: 138.5-140.2^{\circ} \mathrm{C}$.

$>\mathrm{R}_{f}=0.7$ (EtOAc:Petroleum Ether=1:3).

$>\quad{ }^{1} \mathrm{H}$ NMR $\left(400 \mathrm{MHz}, \mathrm{DMSO}-d_{6}\right) \delta 7.78(\mathrm{~d}, J=8.0 \mathrm{~Hz}, 1 \mathrm{H}), 7.71(\mathrm{~d}, J=7.9 \mathrm{~Hz}, 2 \mathrm{H}), 7.59(\mathrm{~s}$, $1 \mathrm{H}), 7.41(\mathrm{dd}, J=8.3,2.9 \mathrm{~Hz}, 3 \mathrm{H}), 7.15(\mathrm{t}, J=7.6 \mathrm{~Hz}, 1 \mathrm{H}), 7.07(\mathrm{t}, J=7.5 \mathrm{~Hz}, 1 \mathrm{H}), 6.08(\mathrm{~s}$, $1 \mathrm{H}), 3.82(\mathrm{~s}, 2 \mathrm{H}), 3.74(\mathrm{~s}, 3 \mathrm{H}), 2.30(\mathrm{~s}, 2 \mathrm{H}), 2.36(\mathrm{~s}, 3 \mathrm{H}), 0.14(\mathrm{~s}, 6 \mathrm{H})$.

$>{ }^{13} \mathrm{C}$ NMR $\left(100 \mathrm{MHz}, \mathrm{DMSO}-d_{6}\right) \delta 148.9,145.5,139.4,134.2,131.9,130.1,129.9,127.3,123.9$, 122.2, 122.1, 118.6, 116.6, 112.3, 52.6, 37.7, 34.6, 23.1, -0.5.

$>\quad \mathrm{IR}$ (neat) $\mathrm{cm}^{-1} 2954,1592,1469,1371,1335,1161,1089,1033,908$.

$>$ HRMS calcd for $\mathrm{C}_{22} \mathrm{H}_{26} \mathrm{~N}_{2} \mathrm{O}_{2} \mathrm{SSi}(\mathrm{M}+\mathrm{Na})^{+} 433.1382$, found 433.1367 . 


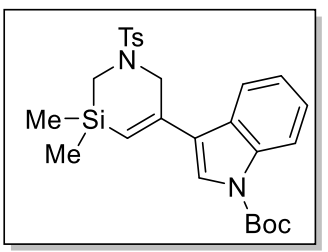

tert-butyl-3-(3,3-dimethyl-1-tosyl-1,2,3,6-tetrahydro-1,3-azasilin-5-yl)1H-indole-1-carboxylate (4ae)

4ae was prepared according to General Method C. 2a (58.4 mg, $0.20 \mathrm{mmol}$, 1.0 equiv.), $\mathrm{Pd}\left(\mathrm{PPh}_{3}\right)_{2} \mathrm{Cl}_{2}$ (7.2 mg, $\left.0.01 \mathrm{mmol}, 5 \mathrm{~mol} \%\right), \mathrm{ZnI}_{2}(12.8 \mathrm{mg}$, 0.04 mmol, 0.2 equiv.), alkyne S-A-28 (57.9 mg, $0.24 \mathrm{mmol}, 1.2$ equiv.) and DBU (61 mg, $0.4 \mathrm{mmol}, 2.0$ equiv.) in xylenes $(0.5 \mathrm{~mL})$ at $120^{\circ} \mathrm{C}$ for $10 \mathrm{~min}$ afforded 4ae as white solid (41 mg, 42\%).

$>\quad \mathrm{mp}: 157.2-159.3^{\circ} \mathrm{C}$

$\mathrm{R}_{f}=0.5$ (EtOAc:Petroleum Ether=1:5).

$>{ }^{1} \mathrm{H} \mathrm{NMR}\left(400 \mathrm{MHz}, \mathrm{CDCl}_{3}\right) \delta 8.15(\mathrm{~d}, J=8.0 \mathrm{~Hz}, 1 \mathrm{H}), 7.73-7.69(\mathrm{~m}, 3 \mathrm{H}), 7.58(\mathrm{~s}, 1 \mathrm{H}), 7.35$ $-7.31(\mathrm{~m}, 3 \mathrm{H}), 7.28-7.24(\mathrm{~m}, 1 \mathrm{H}), 6.17(\mathrm{t}, J=1.76 \mathrm{~Hz}, 1 \mathrm{H}), 3.91(\mathrm{~d}, J=1.80 \mathrm{~Hz}, 2 \mathrm{H}), 2.59$ $(\mathrm{s}, 2 \mathrm{H}), 2.43(\mathrm{~s}, 3 \mathrm{H}), 1.69(\mathrm{~s}, 9 \mathrm{H}), 0.27(\mathrm{~s}, 6 \mathrm{H})$.

$>\quad{ }^{13} \mathrm{C} \mathrm{NMR}\left(100 \mathrm{MHz}, \mathrm{CDCl}_{3}\right) \delta 149.7,145.6,143.5,135.7,132.5,129.6,128.5,128.1,124.8$, $123.4,123.0,122.6,121.9,120.3,115.4,84.3,51.2,35.4,28.2,21.5,-2.8$.

$>\quad$ IR (neat) $\mathrm{cm}^{-1} 2925,1733,1599,1451,1367,1307,1275,1157,1091,911$.

$>\quad$ HRMS calcd for $\mathrm{C}_{26} \mathrm{H}_{32} \mathrm{~N}_{2} \mathrm{O}_{4} \mathrm{SSi}(\mathrm{M}+\mathrm{Na})^{+}$519.1750, found 519.1744.

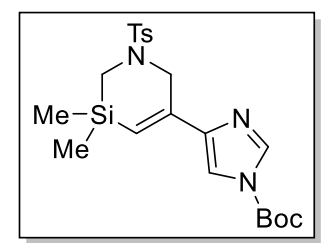

tert-butyl-5-(3,3-dimethyl-1-tosyl-1,2,3,6-tetrahydro-1,3-azasilin-5-yl)1H-imidazole-1-carboxyla te(4af)

4af was prepared according to General Method C. 2a $(58.4 \mathrm{mg}, 0.20 \mathrm{mmol}$, 1.0 equiv.), $\mathrm{Pd}\left(\mathrm{PPh}_{3}\right)_{2} \mathrm{Cl}_{2}$ (7.2 mg, $\left.0.01 \mathrm{mmol}, 5 \mathrm{~mol} \%\right), \mathrm{ZnI}_{2}$ (12.8 mg, 0.04 mmol, 0.2 equiv.), alkyne S-A-29 (46.2 mg, $0.24 \mathrm{mmol}, 1.2$ equiv.) and DBU (61 mg, $0.4 \mathrm{mmol}, 2.0$ equiv.) in xylenes $(0.5 \mathrm{~mL})$ at $120^{\circ} \mathrm{C}$ for $10 \mathrm{~min}$ afforded 4 af as white solid (42 $\mathrm{mg}, 47 \%$ ).

$>\quad \mathrm{mp}: 48.2-49.3^{\circ} \mathrm{C}$

$>\quad \mathrm{R}_{f}=0.4$ (EtOAc:Petroleum Ether=1:4).

$>{ }^{1} \mathrm{H} \mathrm{NMR}\left(400 \mathrm{MHz}, \mathrm{CDCl}_{3}\right) \delta 8.01(\mathrm{~s}, 1 \mathrm{H}), 7.74(\mathrm{~d}, J=8.0 \mathrm{~Hz}, 2 \mathrm{H}), 7.35(\mathrm{~d}, J=8.0 \mathrm{~Hz}, 2 \mathrm{H})$, $7.33(\mathrm{~s}, 1 \mathrm{H}), 6.57(\mathrm{~s}, 1 \mathrm{H}), 3.89(\mathrm{~d}, J=1.72 \mathrm{~Hz}, 2 \mathrm{H}), 2.55(\mathrm{~s}, 2 \mathrm{H}), 2.44(\mathrm{~s}, 3 \mathrm{H}), 1.64(\mathrm{~s}, 9 \mathrm{H})$, $0.21(\mathrm{~s}, 6 \mathrm{H})$.

$>\quad{ }^{13} \mathrm{C} \mathrm{NMR}\left(100 \mathrm{MHz}, \mathrm{CDCl}_{3}\right) \delta 146.9,143.8,143.5,142.2,137.1,132.6,129.7,128.0,120.8$, $112.1,86.2,48.8,35.6,27.9,21.6,-3.0$.

$>\quad$ IR (neat) $\mathrm{cm}^{-1} 2925,1754,1599,1387,1275,1159,1035,1013,914$.

$>$ HRMS calcd for $\mathrm{C}_{21} \mathrm{H}_{29} \mathrm{~N}_{3} \mathrm{O}_{4} \mathrm{SSi}(\mathrm{M}+\mathrm{Na})^{+} 470.1546$, found 470.1542.

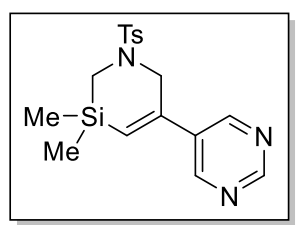

\section{5-(3,3-dimethvl-1-tosvl-1,2,3,6-tetrahvdro-1,3-azasilin-5-} yl)pyrimidine (4ag)

4ag was prepared according to General Method C. 2a (58.4 mg, $0.20 \mathrm{mmol}$, 1.0 equiv.), $\mathrm{Pd}\left(\mathrm{PPh}_{3}\right)_{2} \mathrm{Cl}_{2}$ (7.2 mg, $0.01 \mathrm{mmol}, 5 \mathrm{~mol} \%$ ), $\mathrm{ZnI}_{2}(12.8 \mathrm{mg}, 0.04$ mmol, 0.2 equiv.), alkyne $\mathbf{S - A - 3 0}$ ( $25.0 \mathrm{mg}, 0.24 \mathrm{mmol}, 1.2$ equiv.) and DBU (61 mg, $0.4 \mathrm{mmol}, 2.0$ equiv.) in xylenes $(0.5 \mathrm{~mL})$ at $120{ }^{\circ} \mathrm{C}$ for $10 \mathrm{~min}$ afforded 4 ag as white solid (42 $\mathrm{mg}, 58 \%$ ).

$>\quad \mathrm{mp}: 146.6-148.4^{\circ} \mathrm{C}$.

$>\mathrm{R}_{f}=0.2($ EtOAc:Petroleum Ether $=1: 5)$.

$>{ }^{1} \mathrm{H} \mathrm{NMR}\left(400 \mathrm{MHz}, \mathrm{CDCl}_{3}\right) \delta 9.15(\mathrm{~s}, 1 \mathrm{H}), 8.71(\mathrm{~s}, 2 \mathrm{H}), 7.71(\mathrm{~d}, J=8.0 \mathrm{~Hz}, 2 \mathrm{H}), 7.37(\mathrm{~d}, J=$ $8.0 \mathrm{~Hz}, 2 \mathrm{H}), 6.20(\mathrm{t}, J=1.80 \mathrm{~Hz}, 1 \mathrm{H}), 3.91(\mathrm{~d}, J=1.80 \mathrm{~Hz}, 2 \mathrm{H}), 2.60(\mathrm{~s}, 2 \mathrm{H}), 2.45(\mathrm{~s}, 3 \mathrm{H})$, $0.27(\mathrm{~s}, 6 \mathrm{H})$.

$>\quad{ }^{13} \mathrm{C} \mathrm{NMR}\left(100 \mathrm{MHz}, \mathrm{CDCl}_{3}\right) \delta 158.1,153.7,146.4,143.9,134.6,132.1,129.8,128.0,128.0$, $50.1,35.0,21.6,-3.2$.

$>\quad$ IR (neat) $\mathrm{cm}^{-1} 2923,1597,1549,1413,1340,1161,1089,931$.

$>$ HRMS calcd for $\mathrm{C}_{17} \mathrm{H}_{21} \mathrm{~N}_{3} \mathrm{O}_{2} \mathrm{SSi}(\mathrm{M}+\mathrm{Na})^{+}$382.1021, found 382.1015.

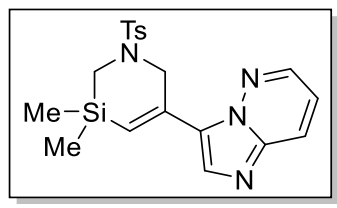

\section{3-(3,3-dimethvl-1-tosvl-1,2,3,6-tetrahvdro-1,3-azasilin-5-} vl)imidazo[1,2-b/pvridazine (4ah)

4ah was prepared according to General Method C. 2a (58.4 mg, 0.20 mmol, 1.0 equiv.), $\mathrm{Pd}\left(\mathrm{PPh}_{3}\right)_{2} \mathrm{Cl}_{2}$ (7.2 mg, $0.01 \mathrm{mmol}, 5 \mathrm{~mol} \%$ ), $\mathrm{ZnI}_{2}(12.8$ $\mathrm{mg}, 0.04 \mathrm{mmol}, 0.2$ equiv.), alkyne S-A-31 (35.2 mg, $0.24 \mathrm{mmol}, 1.2$ equiv.) and $\mathrm{DBU}(61 \mathrm{mg}, 0.4 \mathrm{mmol}, 2.0$ equiv.) in xylenes $(0.5 \mathrm{~mL})$ at

$120{ }^{\circ} \mathrm{C}$ for $10 \mathrm{~min}$ afforded $\mathbf{4 a h}$ as yellow solid (30 mg, 37\%).

$>$ mp: $52.1-53.8^{\circ} \mathrm{C}$. 
$>\mathrm{R}_{f}=0.3$ (EtOAc:Petroleum Ether=1:1).

$>{ }^{1} \mathrm{H} \mathrm{NMR}\left(400 \mathrm{MHz}, \mathrm{CDCl}_{3}\right) \delta 8.41(\mathrm{dd}, J=4.36 \mathrm{~Hz}, J=1.68 \mathrm{~Hz}, 1 \mathrm{H}), 7.98(\mathrm{dd}, J=9.24 \mathrm{~Hz}$, $J=1.76 \mathrm{~Hz}, 1 \mathrm{H}), 7.82(\mathrm{~s}, 1 \mathrm{H}), 7.75(\mathrm{~d}, J=8.0 \mathrm{~Hz}, 2 \mathrm{H}), 7.36(\mathrm{~d}, J=8.0 \mathrm{~Hz}, 2 \mathrm{H}), 7.08(\mathrm{dd}, J=$ $9.16 \mathrm{~Hz}, J=4.40 \mathrm{~Hz}, 1 \mathrm{H}), 7.04(\mathrm{t}, J=1.68 \mathrm{~Hz}, 1 \mathrm{H}), 4.12(\mathrm{~d}, J=1.84 \mathrm{~Hz}, 2 \mathrm{H}), 2.64(\mathrm{~s}, 2 \mathrm{H})$, $2.45(\mathrm{~s}, 3 \mathrm{H}), 0.28(\mathrm{~s}, 6 \mathrm{H})$.

$>\quad{ }^{13} \mathrm{C} \mathrm{NMR}\left(100 \mathrm{MHz}, \mathrm{CDCl}_{3}\right) \delta 143.6,142.8,140.6,139.7,132.8,132.6,129.7,128.0,127.6$, $126.0,123.7,116.7,49.7,35.7,21.6,-2.9$.

$>\quad$ IR (neat) $\mathrm{cm}^{-1} 2957,1590,1528,1336,1160,1088,1032,915$;

$>$ HRMS calcd for $\mathrm{C}_{19} \mathrm{H}_{22} \mathrm{~N}_{4} \mathrm{O}_{2} \mathrm{SSi}(\mathrm{M}+\mathrm{Na})^{+} 421.1130$, found 421.1124 .

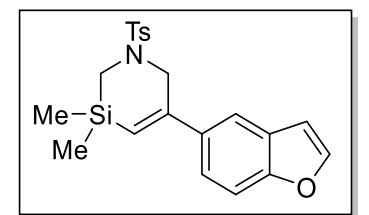

\section{5-(benzofuran-5-pl)-3,3-dimethvl-1-tosvl-1,2,3,6-tetrahvdro-1,3- azasiline (4ai)}

4ai was prepared according to General Method C. 2a (58.4 mg, 0.20 mmol, 1.0 equiv.), $\mathrm{Pd}\left(\mathrm{PPh}_{3}\right)_{2} \mathrm{Cl}_{2}$ (7.2 $\left.\mathrm{mg}, 0.01 \mathrm{mmol}, 5 \mathrm{~mol} \%\right), \mathrm{ZnI}_{2}$ (12.8 mg, $0.04 \mathrm{mmol}, 0.2$ equiv.), alkyne S-A-32 (34.8 mg, $0.24 \mathrm{mmol}$, 1.2 equiv.) and $\mathrm{DBU}(61 \mathrm{mg}, 0.4 \mathrm{mmol}, 2.0$ equiv.) in xylenes $(0.5 \mathrm{~mL})$ at $120^{\circ} \mathrm{C}$ for $10 \mathrm{~min}$ afforded 4 ai as white solid $(53 \mathrm{mg}, 67 \%)$.

$>\mathrm{mp}: 128.1-130.2^{\circ} \mathrm{C}$.

$>\mathrm{R}_{f}=0.6$ (50\% EtOAc:Petroleum Ether=1:5).

$>{ }^{1} \mathrm{H} \mathrm{NMR}\left(400 \mathrm{MHz}, \mathrm{CDCl}_{3}\right) \delta 7.72(\mathrm{~d}, J=8.1 \mathrm{~Hz}, 2 \mathrm{H}), 7.63(\mathrm{~d}, J=2.2 \mathrm{~Hz}, 1 \mathrm{H}), 7.57(\mathrm{~d}, J=$ $1.9 \mathrm{~Hz}, 1 \mathrm{H}), 7.45(\mathrm{~d}, J=8.6 \mathrm{~Hz}, 1 \mathrm{H}), 7.34(\mathrm{~d}, J=8.0 \mathrm{~Hz}, 2 \mathrm{H}), 7.29(\mathrm{dd}, J=8.6,1.9 \mathrm{~Hz}, 1 \mathrm{H})$, $6.77(\mathrm{~d}, J=2.1 \mathrm{~Hz}, 1 \mathrm{H}), 6.00(\mathrm{t}, J=1.8 \mathrm{~Hz}, 1 \mathrm{H}), 3.98(\mathrm{~d}, J=1.7 \mathrm{~Hz}, 2 \mathrm{H}), 2.56(\mathrm{~s}, 2 \mathrm{H}), 2.43$ $(\mathrm{s}, 3 \mathrm{H}), 0.24(\mathrm{~s}, 6 \mathrm{H})$.

$>{ }^{13} \mathrm{C} \mathrm{NMR}\left(100 \mathrm{MHz}, \mathrm{CDCl}_{3}\right) \delta 154.8,153.3,145.7,143.5,137.1,132.5,129.6,128.0,127.5$, $122.8,118.5,111.2,106.8,51.3,35.3,21.5,-2.9$.

$>\quad \mathrm{IR}$ (neat) $\mathrm{cm}^{-1} 2923,1597,1465,1338,1161,1089,1029,941$.

$>$ HRMS calcd for $\mathrm{C}_{21} \mathrm{H}_{23} \mathrm{NO}_{3} \mathrm{SSi}(\mathrm{M}+\mathrm{Na})^{+} 420.1066$, found 420.1056 .

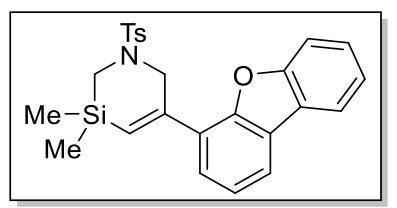

\section{5-(dibenzo[b,d]furan-4-vl)-3,3-dimethvl-1-tosvl-1,2,3,6-tetrahvdro-} 1,3-azasiline(4aj)

4aj was prepared according to General Method C. 2a (58.4 mg, 0.20 mmol, 1.0 equiv.), $\mathrm{Pd}\left(\mathrm{PPh}_{3}\right)_{2} \mathrm{Cl}_{2}$ (7.2 $\left.\mathrm{mg}, 0.01 \mathrm{mmol}, 5 \mathrm{~mol} \%\right), \mathrm{ZnI}_{2}$ (12.8 mg, $0.04 \mathrm{mmol}, 0.2$ equiv.), alkyne S-A-33 (46.2 mg, $0.24 \mathrm{mmol}$, 1.2 equiv.) and $\mathrm{DBU}(61 \mathrm{mg}, 0.4 \mathrm{mmol}, 2.0$ equiv.) in xylenes $(0.5 \mathrm{~mL})$ at $120{ }^{\circ} \mathrm{C}$ for $10 \mathrm{~min}$ afforded 4 aj as white solid $(56 \mathrm{mg}, 63 \%)$.

$>\mathrm{mp}: 180.2-182.9^{\circ} \mathrm{C}$.

$>\mathrm{R}_{f}=0.6($ EtOAc:Petroleum Ether $=1: 5)$.

$>{ }^{1} \mathrm{H} \mathrm{NMR}\left(400 \mathrm{MHz}, \mathrm{CDCl}_{3}\right) \delta 7.94((\mathrm{~d}, J=8.0 \mathrm{~Hz}, 1 \mathrm{H}), 7.87(\mathrm{~d}, J=8.0 \mathrm{~Hz}, 1 \mathrm{H}), 7.74(\mathrm{~d}, J=$ $8.0 \mathrm{~Hz}, 2 \mathrm{H}), 7.54(\mathrm{~d}, J=8.0 \mathrm{~Hz}, 1 \mathrm{H}), 7.49-7.45(\mathrm{~m}, 1 \mathrm{H}), 7.40-7.29(\mathrm{~m}, 5 \mathrm{H}), 6.29(\mathrm{t}, J=$ $1.80 \mathrm{~Hz}, 1 \mathrm{H}), 4.22$ (d, $J=1.76 \mathrm{~Hz}, 2 \mathrm{H}), 2.66(\mathrm{~s}, 2 \mathrm{H}), 2.45$ (s, 3H), 0.29 (s, 6H).

$>{ }^{13} \mathrm{C} \mathrm{NMR}\left(100 \mathrm{MHz}, \mathrm{CDCl}_{3}\right) \delta 155.9,152.5,149.7,143.5,132.5,129.6,128.2,127.4,126.8$, $126.1,125.8,124.8,123.9,123.0,122.9,120.7,120.4,111.7,51.3,35.5,21.6,-2.8$.

$>\mathrm{IR}$ (neat) $\mathrm{cm}^{-1} 2958,1594,1450,1340,1162,1089,1033,934$.

$>$ HRMS calcd for $\mathrm{C}_{25} \mathrm{H}_{25} \mathrm{NO}_{3} \mathrm{SSi}(\mathrm{M}+\mathrm{Na})^{+} 470.1222$, found 470.1219 .

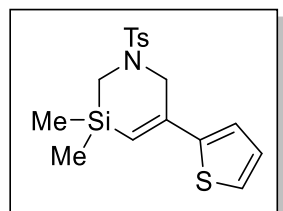

\section{3,3-dimethyl-5-(thiophen-2-yl)-1-tosyl-1,2,3,6-tetrahydro-1,3-} azasiline(4ak)

4ak was prepared according to General Method C. 2a (58.4 mg, $0.20 \mathrm{mmol}$, 1.0 equiv.), $\mathrm{Pd}\left(\mathrm{PPh}_{3}\right)_{2} \mathrm{Cl}_{2}$ (7.2 mg, $0.01 \mathrm{mmol}, 5 \mathrm{~mol} \%$ ), $\mathrm{ZnI}_{2}(12.8 \mathrm{mg}, 0.04$ mmol, 0.2 equiv.), alkyne S-A-34 (26.7 $\mathrm{mg}, 0.24 \mathrm{mmol}, 1.2$ equiv.) and DBU (61 mg, $0.4 \mathrm{mmol}, 2.0$ equiv.) in xylenes $(0.5 \mathrm{~mL})$ at $120{ }^{\circ} \mathrm{C}$ for $10 \mathrm{~min}$ afforded 4 ak as white solid (44 $\mathrm{mg}, 61 \%)$.

$>$ mp: $140.1-142.8^{\circ} \mathrm{C}$.

$>\mathrm{R}_{f}=0.7$ (EtOAc:Petroleum Ether $\left.=1: 7\right)$.

$>{ }^{1} \mathrm{H} \mathrm{NMR}\left(400 \mathrm{MHz}, \mathrm{CDCl}_{3}\right) \delta 7.73(\mathrm{~d}, J=8.0 \mathrm{~Hz}, 2 \mathrm{H}), 7.35(\mathrm{~d}, J=8.0 \mathrm{~Hz}, 2 \mathrm{H}), 7.20(\mathrm{dd}, J=$ $5.1 \mathrm{~Hz}, J=1.2 \mathrm{~Hz}, 1 \mathrm{H}), 7.02(\mathrm{dd}, J=3.7 \mathrm{~Hz}, J=1.2 \mathrm{~Hz}, 1 \mathrm{H}), 6.98(\mathrm{dd}, J=5.1 \mathrm{~Hz}, J=3.7 \mathrm{~Hz}$, $1 \mathrm{H}), 6.12(\mathrm{t}, J=1.72 \mathrm{~Hz}, 1 \mathrm{H}), 3.97(\mathrm{~d}, J=1.72 \mathrm{~Hz}, 2 \mathrm{H}), 2.55(\mathrm{~s}, 2 \mathrm{H}), 2.43(\mathrm{~s}, 3 \mathrm{H}), 0.22(\mathrm{~s}$, $6 \mathrm{H})$. 
$>{ }^{13} \mathrm{C} \mathrm{NMR}\left(100 \mathrm{MHz}, \mathrm{CDCl}_{3}\right) \delta 145.3,145.0,143.6,132.5,129.7,128.0,127.6,125.3,122.9$, 121.0, 50.2, 35.4, 21.6, -3.0.

$>\quad$ IR (neat) $\mathrm{cm}^{-1} 2958,1583,1492,1339,1160,1089,1033,913$.

$>$ HRMS calcd. for $\mathrm{C}_{17} \mathrm{H}_{21} \mathrm{NO}_{2} \mathrm{~S} 2 \mathrm{Si}(\mathrm{M}+\mathrm{Na})^{+} 386.0681$, found 386.0673 .

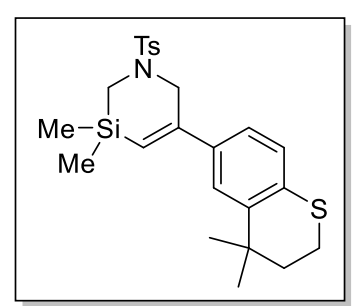

\section{5-(4,4-dimethylthiochroman-6-yl)-3,3-dimethyl-1-tosyl-1,2,3,6- tetrahydro-1,3-azasiline (4al)}

4al was prepared according to General Method C. 2a (58.4 mg, 0.20 mmol, 1.0 equiv.), $\mathrm{Pd}\left(\mathrm{PPh}_{3}\right)_{2} \mathrm{Cl}_{2}$ (7.2 $\left.\mathrm{mg}, 0.01 \mathrm{mmol}, 5 \mathrm{~mol} \%\right), \mathrm{ZnI}_{2}$ (12.8 mg, $0.04 \mathrm{mmol}, 0.2$ equiv.), alkyne S-A-35 (49.5 mg, $0.24 \mathrm{mmol}$, 1.2 equiv.) and $\mathrm{DBU}(61 \mathrm{mg}, 0.4 \mathrm{mmol}, 2.0$ equiv.) in xylenes $(0.5 \mathrm{~mL})$ at $120^{\circ} \mathrm{C}$ for $10 \mathrm{~min}$ afforded $4 \mathrm{al}$ as yellow solid $(60 \mathrm{mg}, 66 \%)$. $>\mathrm{mp}: 60.8-62.2^{\circ} \mathrm{C}$.

$>\mathrm{R}_{f}=0.7$ (EtOAc:Petroleum Ether $\left.=1: 5\right)$.

$>{ }^{1} \mathrm{H} \mathrm{NMR}\left(400 \mathrm{MHz}, \mathrm{CDCl}_{3}\right) \delta 7.70(\mathrm{~d}, J=8.0 \mathrm{~Hz}, 2 \mathrm{H}), 7.34-7.26(\mathrm{~m}, 3 \mathrm{H}), 7.04-6.97(\mathrm{~m}$, $2 \mathrm{H}), 5.93(\mathrm{~s}, 1 \mathrm{H}), 3.89(\mathrm{~s}, 2 \mathrm{H}), 3.04-3.01(\mathrm{~m}, 2 \mathrm{H}), 2.54(\mathrm{~s}, 2 \mathrm{H}), 2.43(\mathrm{~s}, 3 \mathrm{H}), 1.97-1.93(\mathrm{~m}$, $2 \mathrm{H}), 1.33(\mathrm{~s}, 6 \mathrm{H}), 0.22(\mathrm{~s}, 6 \mathrm{H})$.

$>{ }^{13} \mathrm{C} \mathrm{NMR}\left(100 \mathrm{MHz}, \mathrm{CDCl}_{3}\right) \delta 152.8,143.5,142.0,137.6,132.5,132.3,129.6,128.0,126.4$, $123.9,123.3,121.6,50.8,37.5,35.3,33.1,30.1,23.1,21.6,-2.8$.

$>\quad \mathrm{IR}$ (neat) $\mathrm{cm}^{-1} 2961,1596,1474,1341,1162,1090,1034,937$.

$>$ HRMS calcd for $\mathrm{C}_{24} \mathrm{H}_{31} \mathrm{NO}_{2} \mathrm{~S}_{2} \mathrm{Si}(\mathrm{M}+\mathrm{Na})^{+} 480.1463$, found 480.1458 .

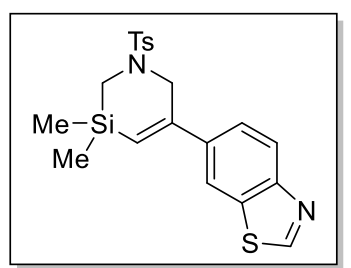

\section{6-(3,3-dimethyl-1-tosyl-1,2,3,6-tetrahydro-1,3-azasilin-5-} yl)benzo/dlthiazole (4am)

4am was prepared according to General Method C. 2a (58.4 mg, 0.20 mmol, 1.0 equiv.), $\mathrm{Pd}\left(\mathrm{PPh}_{3}\right)_{2} \mathrm{Cl}_{2}$ (7.2 $\left.\mathrm{mg}, 0.01 \mathrm{mmol}, 5 \mathrm{~mol} \%\right), \mathrm{ZnI}_{2}(12.8$ $\mathrm{mg}, 0.04 \mathrm{mmol}, 0.2$ equiv.), alkyne $\mathbf{S - A - 3 6}$ (39.0 mg, $0.24 \mathrm{mmol}, 1.2$ equiv.) and DBU (61 mg, $0.4 \mathrm{mmol}, 2.0$ equiv.) in xylenes $(0.5 \mathrm{~mL})$ at $120^{\circ} \mathrm{C}$ for $10 \mathrm{~min}$ afforded $4 \mathrm{am}$ as white solid $(63 \mathrm{mg}, 76 \%)$.

$>$ mp: $130.0-132.7^{\circ} \mathrm{C}$.

$>\mathrm{R}_{f}=0.5$ (EtOAc:Petroleum Ether=1:5).

$>{ }^{1} \mathrm{H} \mathrm{NMR}\left(400 \mathrm{MHz}, \mathrm{CDCl}_{3}\right) \delta 9.00(\mathrm{~s}, 1 \mathrm{H}), 8.08(\mathrm{~d}, J=8.5 \mathrm{~Hz}, 1 \mathrm{H}), 7.93(\mathrm{~d}, J=1.9 \mathrm{~Hz}, 1 \mathrm{H})$, $7.73(\mathrm{~d}, J=8.0 \mathrm{~Hz}, 2 \mathrm{H}), 7.50(\mathrm{dd}, J=8.5,1.9 \mathrm{~Hz}, 1 \mathrm{H}), 7.35(\mathrm{~d}, J=8.0 \mathrm{~Hz}, 2 \mathrm{H}), 6.13(\mathrm{t}, J=$ $1.7 \mathrm{~Hz}, 1 \mathrm{H}), 4.00(\mathrm{~d}, J=1.7 \mathrm{~Hz}, 2 \mathrm{H}), 2.59(\mathrm{~s}, 2 \mathrm{H}), 2.44(\mathrm{~s}, 3 \mathrm{H}), 0.26(\mathrm{~s}, 6 \mathrm{H})$.

$>{ }^{13} \mathrm{C} \mathrm{NMR}\left(100 \mathrm{MHz}, \mathrm{CDCl}_{3}\right) \delta 154.6,153.0,152.2,143.6,139.5,134.2,132.4,129.7,128.0$, $124.7,124.6,123.4,119.0,51.0,35.3,21.6,-3.0$.

$>$ IR (neat) $\mathrm{cm}^{-1} 2924,2852,1684,1438,1339,1161,1089,1032,935$.

$>$ HRMS calcd for $\mathrm{C}_{20} \mathrm{H}_{22} \mathrm{~N}_{2} \mathrm{O}_{2} \mathrm{~S}_{2} \mathrm{Si}(\mathrm{M}+\mathrm{Na})^{+} 437.0790$, found 437.0789.

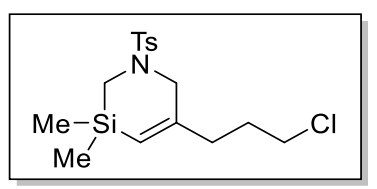

5-(3-chloropropvl)-3,3-dimethvl-1-tosvl-1,2,3,6-tetrahvdro-1,3azasiline(4an)

4an was prepared according to General Method C. 2a (58.4 mg, 0.20 mmol, 1.0 equiv.), $\mathrm{Pd}\left(\mathrm{PPh}_{3}\right)_{2} \mathrm{Cl}_{2}$ (7.2 $\left.\mathrm{mg}, 0.01 \mathrm{mmol}, 5 \mathrm{~mol} \%\right), \mathrm{ZnI}_{2}$ (12.8 mg, $0.04 \mathrm{mmol}, 0.2$ equiv.), alkyne S-A-37 (25.6 mg, $0.24 \mathrm{mmol}$, 1.2 equiv.) and $\mathrm{DBU}(61 \mathrm{mg}, 0.4 \mathrm{mmol}, 2.0$ equiv. $)$ in xylenes $(0.5 \mathrm{~mL})$ at $120^{\circ} \mathrm{C}$ for $10 \mathrm{~min}$ afforded 4an as pale yellow solid (38 mg, 53\%).

$>\mathrm{mp}: 76.8-79.5^{\circ} \mathrm{C}$.

$>\mathrm{R}_{f}=0.7($ EtOAc:Petroleum Ether $=1: 4)$.

$>{ }^{1} \mathrm{H} \mathrm{NMR}\left(600 \mathrm{MHz}, \mathrm{CDCl}_{3}\right) \delta 7.67(\mathrm{~d}, J=7.8 \mathrm{~Hz}, 2 \mathrm{H}), 7.34(\mathrm{~d}, J=7.8 \mathrm{~Hz}, 2 \mathrm{H}), 5.51(\mathrm{~s}, 1 \mathrm{H})$, $3.52(\mathrm{t}, J=6.6 \mathrm{~Hz}, 2 \mathrm{H}), 3.44(\mathrm{~s}, 2 \mathrm{H}), 2.45(\mathrm{~s}, 2 \mathrm{H}), 2.44(\mathrm{~s}, 3 \mathrm{H}), 2.18(\mathrm{t}, J=7.2 \mathrm{~Hz}, 2 \mathrm{H}), 1.94$ $-1.89(\mathrm{~m}, 2 \mathrm{H}), 0.14(\mathrm{~s}, 6 \mathrm{H})$.

$>{ }^{13} \mathrm{C} \mathrm{NMR}\left(100 \mathrm{MHz}, \mathrm{CDCl}_{3}\right) \delta 153.4,143.5,132.3,129.6,128.0,119.6,51.4,44.3,35.4,35.3$, $30.2,21.5,-2.9$.

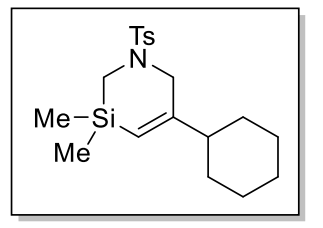

$>\quad \mathrm{IR}$ (neat) $\mathrm{cm}^{-1} 2956,1617,1439,1338,1161,1089,1028,937$.

$>$ HRMS calcd for $\mathrm{C}_{16} \mathrm{H}_{24} \mathrm{ClNO}_{2} \mathrm{SSi}(\mathrm{M}+\mathrm{Na})^{+} 380.0883$, found 380.0877 .

5-cyclohexyl-3,3-dimethyl-1-tosyl-1,2,3,6-tetrahydro-1,3-azasiline (4ao)

4ao was prepared according to General Method C. 2a (58.4 mg, $0.20 \mathrm{mmol}$, 
1.0 equiv.), $\mathrm{Pd}\left(\mathrm{PPh}_{3}\right)_{2} \mathrm{Cl}_{2}$ (7.2 mg, $\left.0.01 \mathrm{mmol}, 5 \mathrm{~mol} \%\right), \mathrm{ZnI}_{2}$ (12.8 mg, $0.04 \mathrm{mmol}, 0.2$ equiv.), alkyne S-A-38 (108.0 mg, $1.0 \mathrm{mmol}, 5.0$ equiv.) and DBU (61 mg, $0.4 \mathrm{mmol}, 2.0$ equiv.) in xylenes $(0.5 \mathrm{~mL})$ at $120^{\circ} \mathrm{C}$ for $10 \mathrm{~min}$ afforded $4 \mathbf{a o}$ as white solid $(27 \mathrm{mg}, 37 \%)$.

$>\mathrm{mp}: 148.5-150.8^{\circ} \mathrm{C}$.

$>\mathrm{R}_{f}=0.6$ (EtOAc:Petroleum Ether $\left.=1: 5\right)$.

$>{ }^{1} \mathrm{H} \mathrm{NMR}\left(400 \mathrm{MHz}, \mathrm{CDCl}_{3}\right) \delta 7.68(\mathrm{~d}, J=8.0 \mathrm{~Hz}, 2 \mathrm{H}), 7.34(\mathrm{~d}, J=8.0 \mathrm{~Hz}, 2 \mathrm{H}), 5.45(\mathrm{~s}, 1 \mathrm{H})$, $3.46(\mathrm{~d}, J=1.76 \mathrm{~Hz}, 2 \mathrm{H}), 2.44(\mathrm{~s}, 3 \mathrm{H}), 2.42(\mathrm{~s}, 2 \mathrm{H}), 1.81-1.66(\mathrm{~m}, 5 \mathrm{H}), 1.30-1.09(\mathrm{~m}, 6 \mathrm{H})$, $0.12(\mathrm{~s}, 6 \mathrm{H})$.

$>{ }^{13} \mathrm{C} \mathrm{NMR}\left(100 \mathrm{MHz}, \mathrm{CDCl}_{3}\right) \delta 160.6,143.3,132.5,129.5,128.0,115.8,50.9,46.6,35.6,32.1$, $26.6,26.2,21.5,-2.8$.

$>\mathrm{IR}$ (neat) $\mathrm{cm}^{-1} 2924,2852,1161,1492,1447,1341,1304,1163,1090,993$.

$>$ HRMS calcd for $\mathrm{C}_{19} \mathrm{H}_{29} \mathrm{NO}_{2} \mathrm{SSi}(\mathrm{M}+\mathrm{H})^{+} 364.1767$, found 364.1759.

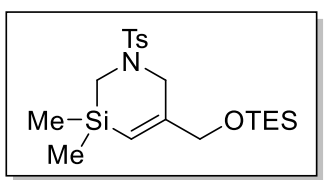

3,3-dimethyl-1-tosyl-5-(((triethylsilyl)oxy)methyl)-1,2,3,6-tetrahydro-1,3azasiline (4ar)

4ar was prepared according to General Method C. 2a (58.4 mg, $0.20 \mathrm{mmol}$, 1.0 equiv.), $\mathrm{Pd}\left(\mathrm{PPh}_{3}\right)_{2} \mathrm{Cl}_{2}$ (7.2 mg, $\left.0.01 \mathrm{mmol}, 5 \mathrm{~mol} \%\right), \mathrm{ZnI}_{2}(12.8 \mathrm{mg}, 0.04$ mmol, 0.2 equiv.), alkyne $\mathbf{S}-\mathbf{A}-\mathbf{3 9}$ ( $40.8 \mathrm{mg}, 0.24 \mathrm{mmol}, 1.2$ equiv.) and DBU

(61 mg, $0.4 \mathrm{mmol}, 2.0$ equiv.) in xylenes $(0.5 \mathrm{~mL})$ at $120^{\circ} \mathrm{C}$ for $10 \mathrm{~min}$ afforded 4 ar as white solid (61 mg, 72\%).

$>$ mp: $79.8-82.3^{\circ} \mathrm{C}$.

$>\mathrm{R}_{f}=0.6$ (EtOAc:Petroleum Ether $\left.=1: 7\right)$.

$>\quad{ }^{1} \mathrm{H} \mathrm{NMR}\left(400 \mathrm{MHz}, \mathrm{CDCl}_{3}\right) \delta 7.67(\mathrm{~d}, J=8.0 \mathrm{~Hz}, 2 \mathrm{H}), 7.31(\mathrm{~d}, J=8.0 \mathrm{~Hz}, 2 \mathrm{H}), 5.78(\mathrm{t}, J=$ $1.6 \mathrm{~Hz}, 1 \mathrm{H}), 4.08(\mathrm{~s}, 2 \mathrm{H}), 3.45(\mathrm{~s}, 2 \mathrm{H}), 2.47(\mathrm{~s}, 2 \mathrm{H}), 2.43(\mathrm{~s}, 3 \mathrm{H}), 0.95(\mathrm{t}, J=8 \mathrm{~Hz}, 9 \mathrm{H}), 0.60$ $(\mathrm{q}, J=8 \mathrm{~Hz}, 6 \mathrm{H}), 0.15(\mathrm{~s}, 6 \mathrm{H})$.

$>{ }^{13} \mathrm{C}$ NMR $\left(100 \mathrm{MHz}, \mathrm{CDCl}_{3}\right) \delta 153.7,143.4,132.2,129.5,128.1,117.4,66.4,48.8,35.8$, $21.5,6.7,4.4,-3.0$.

$>\quad \mathrm{IR}$ (neat) $\mathrm{cm}^{-1} 2955,2876,1626,1598,1440,1413,1342,1163,1090,911$.

$>$ HRMS calcd for $\mathrm{C}_{20} \mathrm{H}_{35} \mathrm{NO}_{3} \mathrm{SSi}_{2}(\mathrm{M}+\mathrm{Na})^{+} 448.1774$, found 448.1768 .

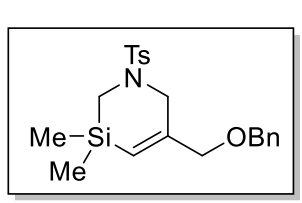

5-((benzyloxy)methyl)-3,3-dimethyl-1-tosyl-1,2,3,6-tetrahydro-1,3azasiline (4as)

4as was prepared according to General Method C. 2a (58.4 mg, $0.20 \mathrm{mmol}$, 1.0 equiv.), $\mathrm{Pd}\left(\mathrm{PPh}_{3}\right)_{2} \mathrm{Cl}_{2}(7.2 \mathrm{mg}, 0.01 \mathrm{mmol}, 5 \mathrm{~mol} \%), \mathrm{ZnI}_{2}(12.8 \mathrm{mg}, 0.04$ mmol, 0.2 equiv.), alkyne $\mathbf{S - A - 4 0}$ (35.0 $\mathrm{mg}, 0.24 \mathrm{mmol}, 1.2$ equiv.) and DBU (61 mg, $0.4 \mathrm{mmol}, 2.0$ equiv.) in xylenes $(0.5 \mathrm{~mL})$ at $120{ }^{\circ} \mathrm{C}$ for $10 \mathrm{~min}$ afforded 4 as as brown oil ( $55 \mathrm{mg}$, $68 \%$ yield).

$>\mathrm{R}_{f}=0.7($ EtOAc:Petroleum Ether $=1: 5)$.

$>{ }^{1} \mathrm{H}$ NMR $\left(400 \mathrm{MHz}, \mathrm{CDCl}_{3}\right) \delta 7.67(\mathrm{~d}, J=8.0 \mathrm{~Hz}, 2 \mathrm{H}), 7.37-7.27(\mathrm{~m}, 7 \mathrm{H}), 5.82(\mathrm{t}, J=1.60$ $\mathrm{Hz}, 1 \mathrm{H}), 4.46(\mathrm{~s}, 2 \mathrm{H}), 3.94(\mathrm{~d}, J=1.28 \mathrm{~Hz}, 2 \mathrm{H}), 3.94(\mathrm{~d}, J=1.60 \mathrm{~Hz}, 2 \mathrm{H}), 2.48(\mathrm{~s}, 2 \mathrm{H}), 2.43$ (s, 3H), $0.17(\mathrm{~s}, 6 \mathrm{H})$.

$>{ }^{13} \mathrm{C} \mathrm{NMR}\left(100 \mathrm{MHz}, \mathrm{CDCl}_{3}\right) \delta 151.4,143.4,137.9,132.4,129.6,128.4,128.1,127.9,127.8$, $121.3,74.3,72.3,49.2,35.6,21.5,-3.1$.

$>\mathrm{IR}$ (neat) $\mathrm{cm}^{-1} 2955,2923,2853,1624,1452,1342,1163,1090$.

$>$ HRMS calcd for $\mathrm{C}_{21} \mathrm{H}_{27} \mathrm{NO}_{3} \mathrm{SSi}(\mathrm{M}+\mathrm{Na})^{+} 424.1379$, found 424.1374 .

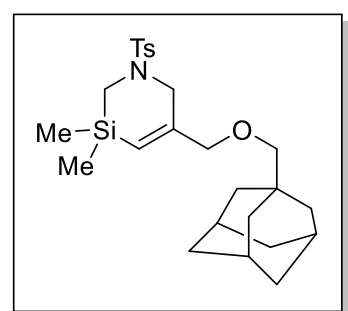

\section{5-((((1S,3s)-adamantan-1-yl)methoxy)methyl)-3,3-dimethyl-1-tosyl-} 1,2,3,6-tetrahydro-1,3-azasiline(4at)

4at was prepared according to General Method C. $2 \mathbf{a}(58.4 \mathrm{mg}, 0.20 \mathrm{mmol}$, 1.0 equiv.), $\mathrm{Pd}\left(\mathrm{PPh}_{3}\right)_{2} \mathrm{Cl}_{2}$ (7.2 mg, $\left.0.01 \mathrm{mmol}, 5 \mathrm{~mol} \%\right), \mathrm{ZnI}_{2}(12.8 \mathrm{mg}$, $0.04 \mathrm{mmol}, 0.2$ equiv.), alkyne $\mathbf{S - A - 4 1}(50.0 \mathrm{mg}, 0.24 \mathrm{mmol}, 1.2$ equiv.) and DBU (61 mg, $0.4 \mathrm{mmol}, 2.0$ equiv.) in xylenes $(0.5 \mathrm{~mL})$ at $120^{\circ} \mathrm{C}$ for $10 \mathrm{~min}$ afforded 4 at as white solid (73 $\mathrm{mg}, 80 \%$ ).

$>\mathrm{mp}: 38.1-39.9^{\circ} \mathrm{C}$.

$>\mathrm{R}_{f}=0.6($ EtOAc:Petroleum Ether $=1: 4)$.

$>\quad{ }^{1} \mathrm{H} \mathrm{NMR}\left(400 \mathrm{MHz}, \mathrm{CDCl}_{3}\right) \delta 7.67(\mathrm{~d}, J=8.0 \mathrm{~Hz}, 2 \mathrm{H}), 7.32(\mathrm{~d}, J=8.0 \mathrm{~Hz}, 2 \mathrm{H}), 5.72(\mathrm{~s}, 1 \mathrm{H})$, $3.87(\mathrm{~s}, 2 \mathrm{H}), 3.47(\mathrm{~s}, 2 \mathrm{H}), 2.91(\mathrm{~s}, 2 \mathrm{H}), 2.47(\mathrm{~s}, 2 \mathrm{H}), 2.43(\mathrm{~s}, 3 \mathrm{H}), 1.97(\mathrm{~s}, 3 \mathrm{H}), 1.75-1.62(\mathrm{~m}$, $6 \mathrm{H}), 1.51(\mathrm{~d}, J=1.56 \mathrm{~Hz}, 6 \mathrm{H}), 0.17(\mathrm{~s}, 6 \mathrm{H})$. 
$>{ }^{13} \mathrm{C} \mathrm{NMR}\left(100 \mathrm{MHz}, \mathrm{CDCl}_{3}\right) \delta 152.0,143.4,132.1,129.5,128.1,120.2,81.3,75.7,49.2,39.7$, 37.2, 35.6, 34.0, 28.3, 21.6, -3.0.

$>\quad$ IR (neat) $\mathrm{cm}^{-1} 2900,2846,1723,1598,1446,1343,1163,1090,1019,936$.

$>$ HRMS calcd for $\mathrm{C}_{25} \mathrm{H}_{37} \mathrm{NO}_{3} \mathrm{SSiNa}(\mathrm{M}+\mathrm{Na})^{+} 482.2161$, found 482.2160 .

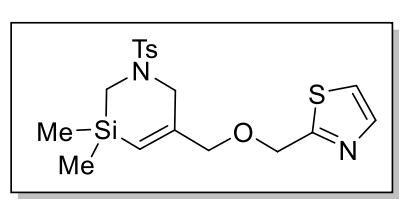

\section{2-(((3,3-dimethyl-1-tosyl-1,2,3,6-tetrahydro-1,3-azasilin-5- yl)methoxy)methyl)thiazole (4au)}

4au was prepared according to General Method C. 2a (58.4 mg, 0.20 mmol, 1.0 equiv.), $\mathrm{Pd}\left(\mathrm{PPh}_{3}\right)_{2} \mathrm{Cl}_{2}$ (7.2 $\left.\mathrm{mg}, 0.01 \mathrm{mmol}, 5 \mathrm{~mol} \%\right), \mathrm{ZnI}_{2}$ (12.8 mg, $0.04 \mathrm{mmol}, 0.2$ equiv.), alkyne S-A-42 (36.8 mg, $0.24 \mathrm{mmol}$, 1.2 equiv.) and DBU (61 mg, $0.4 \mathrm{mmol}, 2.0$ equiv.) in xylenes $(0.5$ $\mathrm{mL})$ at $120^{\circ} \mathrm{C}$ for $10 \mathrm{~min}$ afforded 4 au as yellow oil $(50 \mathrm{mg}, 61 \%)$.

$>\quad \mathrm{R}_{f}=0.4$ (EtOAc:Petroleum Ether $\left.=1: 2\right)$.

$>{ }^{1} \mathrm{H}$ NMR $\left(400 \mathrm{MHz}, \mathrm{CDCl}_{3}\right) \delta 7.75(\mathrm{~d}, J=4.0 \mathrm{~Hz}, 1 \mathrm{H}), 7.68(\mathrm{~d}, J=8.0 \mathrm{~Hz}, 2 \mathrm{H}), 7.35(\mathrm{~d}, J=$ $4.0 \mathrm{~Hz}, 1 \mathrm{H}), 7.33(\mathrm{~d}, J=8.0 \mathrm{~Hz}, 2 \mathrm{H}), 5.84(\mathrm{~s}, 1 \mathrm{H}), 4.77$ (s, 2H), 4.05 (s, 2H), $3.53(\mathrm{~s}, 2 \mathrm{H}), 2.49$ (s, 2H), 2.43 (s, 3H), 0.17 (s, 6H).

$>{ }^{13} \mathrm{C} \mathrm{NMR}\left(100 \mathrm{MHz}, \mathrm{CDCl}_{3}\right) \delta 168.2,150.3,143.5,142.6,132.2,129.6,128.1,122.1,119.7$, $75.1,69.1,49.1,35.5,21.5,-3.1$.

$>$ IR (neat) $\mathrm{cm}^{-1} 2923,2853,1624,1597,1440,1339,1161,1089,942$.

$>$ HRMS calcd for $\mathrm{C}_{18} \mathrm{H}_{24} \mathrm{~N}_{2} \mathrm{O}_{3} \mathrm{~S}_{2} \mathrm{Si}(\mathrm{M}+\mathrm{Na})^{+} 431.0890$, found 431.0885 .

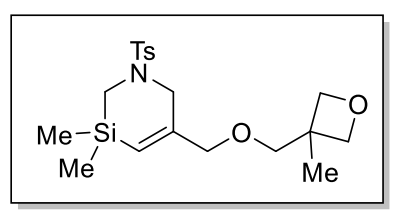

\section{3,3-dimethvl-5-(((3-methvloxetan-3-vl)methoxv)methvl)-1-tosyl-} 1,2,3,6-tetrahydro-1,3-azasiline (4av)

4av was prepared according to General Method C. 2a (58.4 mg, 0.20 mmol, 1.0 equiv.), $\mathrm{Pd}\left(\mathrm{PPh}_{3}\right)_{2} \mathrm{Cl}_{2}$ (7.2 $\left.\mathrm{mg}, 0.01 \mathrm{mmol}, 5 \mathrm{~mol} \%\right), \mathrm{ZnI}_{2}$ (12.8 mg, $0.04 \mathrm{mmol}, 0.2$ equiv.), alkyne S-A-43 (33.6 mg, $0.24 \mathrm{mmol}$, 1.2 equiv.) and DBU (61 mg, $0.4 \mathrm{mmol}, 2.0$ equiv.) in xylenes $(0.5 \mathrm{~mL})$ at $120{ }^{\circ} \mathrm{C}$ for $10 \mathrm{~min}$ afforded 4 av as brown oil $(54 \mathrm{mg}, 68 \%)$.

$>\mathrm{R}_{f}=0.3$ (EtOAc:Petroleum Ether $\left.=1: 5\right)$.

$>{ }^{1} \mathrm{H} \mathrm{NMR}\left(400 \mathrm{MHz}, \mathrm{CDCl}_{3}\right) \delta 7.67(\mathrm{~d}, J=8.0 \mathrm{~Hz}, 2 \mathrm{H}), 7.33(\mathrm{~d}, J=8.0 \mathrm{~Hz}, 2 \mathrm{H}), 5.76(\mathrm{~s}, 1 \mathrm{H})$, $4.51(\mathrm{~d}, J=8.0 \mathrm{~Hz}, 2 \mathrm{H}), 4.37(\mathrm{~d}, J=8.0 \mathrm{~Hz}, 2 \mathrm{H}), 3.96(\mathrm{~s}, 2 \mathrm{H}), 3.49(\mathrm{~s}, 2 \mathrm{H}), 3.43(\mathrm{~s}, 2 \mathrm{H}), 2.49$ (s, 2H), 2.43 (s, 3H), 1.32 (s, 3H), 0.17 (s, 6H).

$>{ }^{13} \mathrm{CNMR}\left(100 \mathrm{MHz}, \mathrm{CDCl}_{3}\right) \delta 151.3,143.5,132.3,129.6,128.0,121.4,80.1,75.7,75.5,49.0$, $39.8,35.6,21.5,21.4,-3.0$.

$>$ IR (neat) $\mathrm{cm}^{-1} 2926,2865,1623,1598,1441,1340,1161,1089,1040,978$.

$>$ HRMS calcd for $\mathrm{C}_{19} \mathrm{H}_{29} \mathrm{NO}_{4} \mathrm{SSi}(\mathrm{M}+\mathrm{Na})^{+} 418.1484$, found 418.1472 .

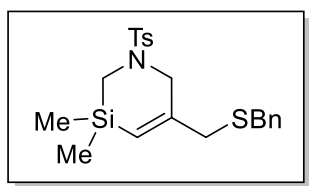

5-((benzylthio)methyl)-3,3-dimethyl-1-tosyl-1,2,3,6-tetrahydro-1,3azasiline (4aw)

4aw was prepared according to General Method C. 2a (58.4 mg, $0.20 \mathrm{mmol}$, 1.0 equiv.), $\mathrm{Pd}\left(\mathrm{PPh}_{3}\right)_{2} \mathrm{Cl}_{2}$ (7.2 mg, $0.01 \mathrm{mmol}, 5 \mathrm{~mol} \%$ ), $\mathrm{ZnI}_{2}(12.8 \mathrm{mg}, 0.04$ mmol, 0.2 equiv.), alkyne $\mathbf{S}-\mathbf{A}-\mathbf{4 4}(38.9 \mathrm{mg}, 0.24 \mathrm{mmol}, 1.2$ equiv.) and $\mathrm{DBU}\left(61 \mathrm{mg}, 0.4 \mathrm{mmol}, 2.0\right.$ equiv.) in xylenes $(0.5 \mathrm{~mL})$ at $120^{\circ} \mathrm{C}$ for $10 \mathrm{~min}$ afforded 4 aw as yellow oil (34 mg, 41\%).

$>\quad \mathrm{R}_{f}=0.7$ (EtOAc:Petroleum Ether $\left.=1: 5\right)$.

$>{ }^{1} \mathrm{H}$ NMR $\left(400 \mathrm{MHz}, \mathrm{CDCl}_{3}\right) \delta 7.68(\mathrm{~d}, J=8.0 \mathrm{~Hz}, 2 \mathrm{H}), 7.34(\mathrm{~d}, J=8.0 \mathrm{~Hz}, 2 \mathrm{H}), 7.33-7.20$ $(\mathrm{m}, 5 \mathrm{H}), 5.82(\mathrm{t}, J=1.48 \mathrm{~Hz}, 1 \mathrm{H}), 3.59(\mathrm{~d}, J=1.64 \mathrm{~Hz}, 2 \mathrm{H}), 3.57(\mathrm{~s}, 2 \mathrm{H}), 3.05(\mathrm{~s}, 2 \mathrm{H}), 2.46$ $(\mathrm{s}, 2 \mathrm{H}), 2.44(\mathrm{~s}, 3 \mathrm{H}), 0.17(\mathrm{~s}, 6 \mathrm{H})$.

$>{ }^{13} \mathrm{C} \mathrm{NMR}\left(100 \mathrm{MHz}, \mathrm{CDCl}_{3}\right) \delta 149.9,143.4,137.8,132.5,129.6,129.0,128.5,128.0,127.0$, 123.6, 50.3, 39.6, 35.4, 35.4, 21.5, -3.0.

$>$ IR (neat) $\mathrm{cm}^{-1} 3028,2955,1600,1493,1414,1339,1161,1089,1031,944$.

$>$ HRMS calcd for $\mathrm{C}_{21} \mathrm{H}_{27} \mathrm{NO}_{2} \mathrm{~S}_{2} \mathrm{Si}(\mathrm{M}+\mathrm{Na})^{+} 440.1150$, found 440.1143 .

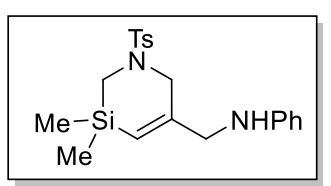

N-((3,3-dimethvl-1-tosvl-1,2,3,6-tetrahvdro-1,3-azasilin-5-vl)methvl)anil ine (4ax)

4ax was prepared according to General Method C. 2a $(58.4 \mathrm{mg}, 0.20 \mathrm{mmol}$, 1.0 equiv.), $\mathrm{Pd}\left(\mathrm{PPh}_{3}\right)_{2} \mathrm{Cl}_{2}$ (7.2 $\left.\mathrm{mg}, 0.01 \mathrm{mmol}, 5 \mathrm{~mol} \%\right), \mathrm{ZnI}_{2}(12.8 \mathrm{mg}$, 0.04 mmol, 0.2 equiv.), alkyne S-A-45 (31.4 mg, $0.24 \mathrm{mmol}, 1.2$ equiv.) and DBU (61 mg, $0.4 \mathrm{mmol}, 2.0$ equiv.) in xylenes $(0.5 \mathrm{~mL})$ at $120^{\circ} \mathrm{C}$ for $10 \mathrm{~min}$ afforded $4 \mathrm{ax}$ as 
white solid (57 mg, 74\%).

$>$ mp: $43.2-45.1^{\circ} \mathrm{C}$.

$>\mathrm{R}_{f}=0.5$ (EtOAc:Petroleum Ether $\left.=1: 5\right)$.

$>{ }^{1} \mathrm{H} \mathrm{NMR}\left(400 \mathrm{MHz}, \mathrm{CDCl}_{3}\right) \delta 7.67(\mathrm{~d}, J=8.0 \mathrm{~Hz}, 2 \mathrm{H}), 7.33(\mathrm{~d}, J=8.0 \mathrm{~Hz}, 2 \mathrm{H}), 7.15(\mathrm{t}, J=$ $8.0 \mathrm{~Hz}, 2 \mathrm{H}), 6.71(\mathrm{t}, J=8.0 \mathrm{~Hz}, 1 \mathrm{H}), 6.55(\mathrm{~d}, J=8.0 \mathrm{~Hz}, 2 \mathrm{H}), 5.80(\mathrm{t}, J=1.76 \mathrm{~Hz}, 1 \mathrm{H}), 3.89$ (s, 1H), $3.70(\mathrm{~s}, 2 \mathrm{H}), 3.51(\mathrm{~d}, J=1.52 \mathrm{~Hz}, 2 \mathrm{H}), 2.48(\mathrm{~s}, 2 \mathrm{H}), 2.44(\mathrm{~s}, 3 \mathrm{H}), 0.14(\mathrm{~s}, 6 \mathrm{H})$.

$>{ }^{13} \mathrm{C}$ NMR $\left(100 \mathrm{MHz}, \mathrm{CDCl}_{3}\right) \delta 151.8,147.9,143.5,132.2,129.6,129.2,128.1,119.3,117.7$, $112.8,50.3,49.8,35.7,21.6,-3.0$.

$>\operatorname{IR}$ (neat) $\mathrm{cm}^{-1} 3414,1602,1508,1440,1335,1162,1090,986$.

$>$ HRMS calcd for $\mathrm{C}_{20} \mathrm{H}_{26} \mathrm{~N}_{2} \mathrm{O}_{2} \mathrm{SSi}(\mathrm{M}+\mathrm{H})^{+} 387.1563$, found 387.1551 .

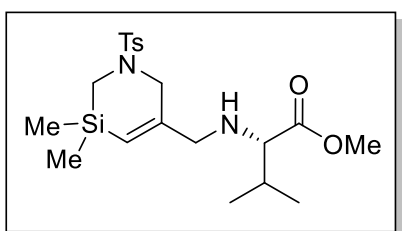

methyl((3,3-dimethyl-1-tosyl-1,2,3,6-tetrahydro-1,3-azasilin-5yl)methyl)-L-valinate(4ay)

4ay was prepared according to General Method C. 2a (58.4 mg, 0.20 mmol, 1.0 equiv.), $\mathrm{Pd}\left(\mathrm{PPh}_{3}\right)_{2} \mathrm{Cl}_{2}$ (7.2 mg, $\left.0.01 \mathrm{mmol}, 5 \mathrm{~mol} \%\right), \mathrm{ZnI}_{2}$ (12.8 mg, $0.04 \mathrm{mmol}, 0.2$ equiv.), alkyne S-A-46 (40.5 mg, 0.24 mmol, 1.2 equiv.) and DBU (61 mg, $0.4 \mathrm{mmol}, 2.0$ equiv.) in xylenes

$(0.5 \mathrm{~mL})$ at $120^{\circ} \mathrm{C}$ for $10 \mathrm{~min}$ afforded 4 ay as yellow oil $(41 \mathrm{mg}, 48 \%)$.

$>\mathrm{R}_{f}=0.5$ (EtOAc:Petroleum Ether $=1: 2$ ).

$>[\alpha]^{25} \mathrm{D}=-24.2^{\circ}\left(c=1.0, \mathrm{CHCl}_{3}\right)$.

$>{ }^{1} \mathrm{H} \mathrm{NMR}\left(400 \mathrm{MHz}, \mathrm{CDCl}_{3}\right) \delta 7.67(\mathrm{~d}, J=8.0 \mathrm{~Hz}, 2 \mathrm{H}), 7.31(\mathrm{~d}, J=8.0 \mathrm{~Hz}, 2 \mathrm{H}), 5.69(\mathrm{t}, J=$ $1.64 \mathrm{~Hz}, 1 \mathrm{H}), 3.70(\mathrm{~s}, 3 \mathrm{H}), 3.54-3.43(\mathrm{~m}, 2 \mathrm{H}), 3.26-3.22(\mathrm{~m}, 1 \mathrm{H}), 2.95-2.89(\mathrm{~m}, 2 \mathrm{H}), 2.49$ $-2.39(\mathrm{~m}, 5 \mathrm{H}), 1.92-1.83(\mathrm{~m}, 1 \mathrm{H}), 1.58(\mathrm{~s}, 1 \mathrm{H}), 0.92(\mathrm{~m}, 3 \mathrm{H}), 0.91(\mathrm{~s}, 3 \mathrm{H}), 0.14(\mathrm{~s}, 3 \mathrm{H}), 0.13$ $(\mathrm{s}, 3 \mathrm{H})$.

$>{ }^{13} \mathrm{CNMR}\left(100 \mathrm{MHz}, \mathrm{CDCl}_{3}\right) \delta 175.7,153.1,143.4,132.2,129.5,128.1,119.9,66.4,55.1,51.5$, $50.2,35.6,31.7,21.6,19.5,18.5,-2.9,-3.0$.

$>$ IR (neat) $\mathrm{cm}^{-1} 2958,1730,1621,1598,1436,1340,1197,1161,1090,996,939$.

$>$ HRMS calcd for $\mathrm{C}_{20} \mathrm{H}_{32} \mathrm{~N}_{2} \mathrm{O}_{4} \mathrm{SSi}(\mathrm{M}+\mathrm{Na})^{+} 447.1750$, found 447.1747 .
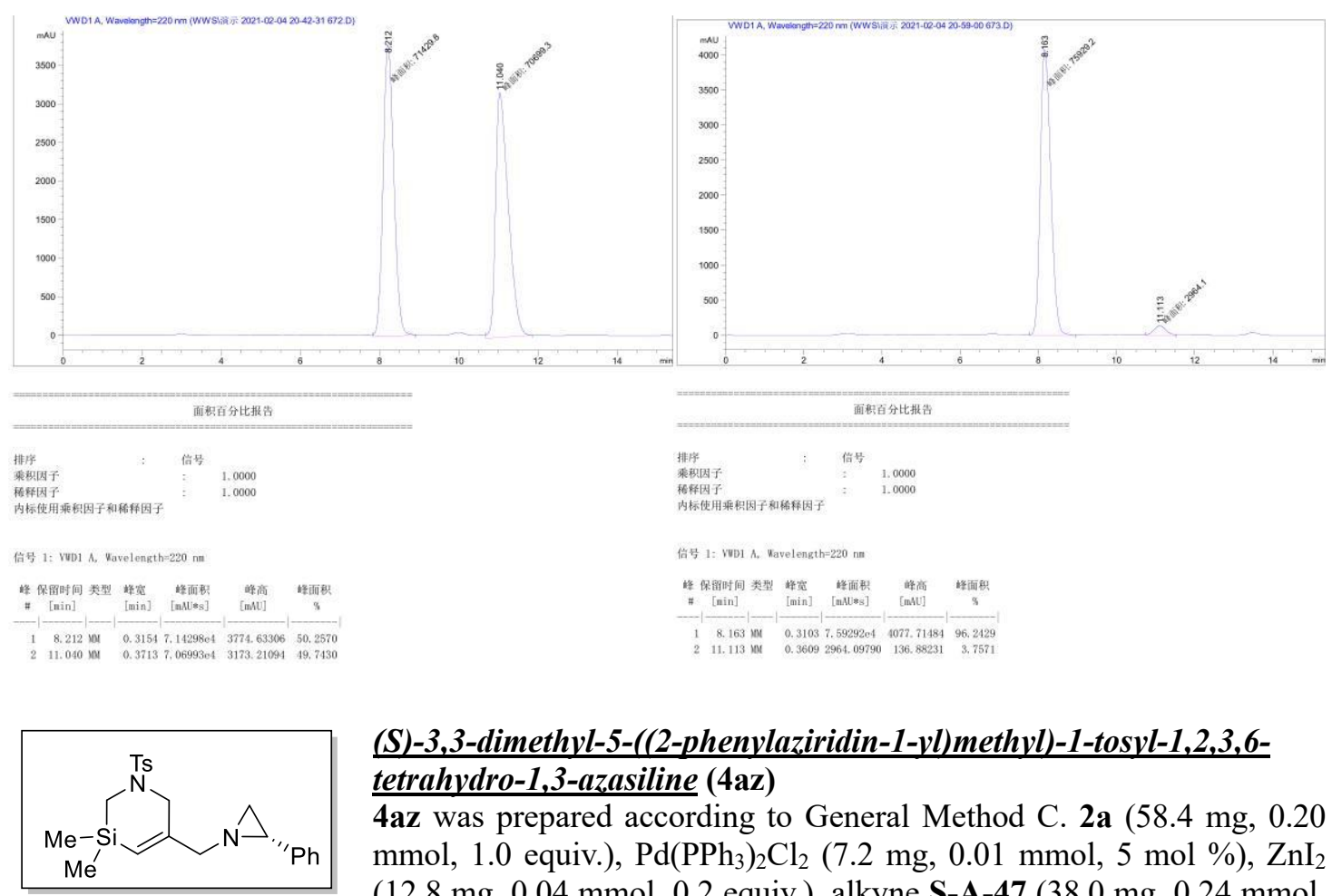

(S)-3,3-dimethyl-5-((2-phenvlaziridin-1-vl)methvl)-1-tosvl-1,2,3,6tetrahydro-1,3-azasiline (4az)

4az was prepared according to General Method C. 2a (58.4 mg, 0.20 mmol, 1.0 equiv.), $\mathrm{Pd}\left(\mathrm{PPh}_{3}\right)_{2} \mathrm{Cl}_{2}$ (7.2 $\left.\mathrm{mg}, 0.01 \mathrm{mmol}, 5 \mathrm{~mol} \%\right), \mathrm{ZnI}_{2}$ (12.8 mg, $0.04 \mathrm{mmol}, 0.2$ equiv.), alkyne S-A-47 (38.0 mg, $0.24 \mathrm{mmol}$, 1.2 equiv.) and $\mathrm{DBU}\left(61 \mathrm{mg}, 0.4 \mathrm{mmol}, 2.0\right.$ equiv. ) in xylenes $(0.5 \mathrm{~mL})$ at $120^{\circ} \mathrm{C}$ for $10 \mathrm{~min}$ afforded 4az as yellow oil (62 $\mathrm{mg}, 75 \%)$.

$>\mathrm{R}_{f}=0.5($ EtOAc:Petroleum Ether $=1: 5)$.

$>[\alpha]^{25} \mathrm{D}=+33.4^{\circ}\left(c=0.75, \mathrm{CHCl}_{3}\right)$.

$>{ }^{1} \mathrm{H}$ NMR $\left(400 \mathrm{MHz}, \mathrm{CDCl}_{3}\right) \delta 7.59(\mathrm{~d}, J=8.0 \mathrm{~Hz}, 2 \mathrm{H}), 7.32-7.21(\mathrm{~m}, 7 \mathrm{H}), 5.75(\mathrm{~s}, 1 \mathrm{H}), 3.62$ 
$-3.47(\mathrm{~m}, 2 \mathrm{H}), 3.16(\mathrm{~d}, J=12.0 \mathrm{~Hz}, 1 \mathrm{H}), 2.90(\mathrm{~d}, J=12.0 \mathrm{~Hz}, 1 \mathrm{H}), 2.49-2.38(\mathrm{~m}, 5 \mathrm{H}), 2.36$ $-2.33(\mathrm{~m}, 1 \mathrm{H}), 1.94(\mathrm{~d}, J=4.0 \mathrm{~Hz}, 1 \mathrm{H}), 1.71(\mathrm{~d}, J=4.0 \mathrm{~Hz}, 1 \mathrm{H}), 0.13(\mathrm{~s}, 3 \mathrm{H}), 0.12(\mathrm{~s}, 3 \mathrm{H})$.

$>{ }^{13} \mathrm{C} \mathrm{NMR}\left(100 \mathrm{MHz}, \mathrm{CDCl}_{3}\right) \delta 152.1,143.2,139.8,132.2,129.5,128.3,128.0,127.0,126.1$, $120.2,67.6,50.2,41.7,37.8,35.5,21.5,-3.0$.

$>\quad \mathrm{IR}$ (neat) $\mathrm{cm}^{-1} 2955,2922,2852,1737,1599,1442,1339,1161,1089,1030$.

$>$ HRMS calcd forC $\mathrm{C}_{22} \mathrm{H}_{28} \mathrm{~N}_{2} \mathrm{O}_{2} \mathrm{SSi}(\mathrm{M}+\mathrm{Na})^{+} 435.1538$, found 435.1546.

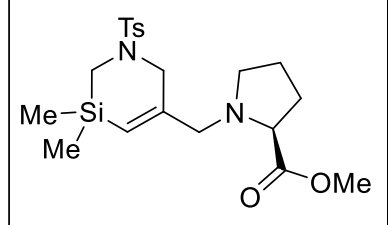

$66 \%)$.

\section{methyl((3,3-dimethyl-1-tosyl-1,2,3,6-tetrahydro-1,3-azasilin-5- vl)methyl)-L-prolinate(4ba)}

4ba was prepared according to General Method C. 2a (58.4 mg, 0.20 mmol, 1.0 equiv.), $\mathrm{Pd}\left(\mathrm{PPh}_{3}\right)_{2} \mathrm{Cl}_{2}$ (7.2 $\left.\mathrm{mg}, 0.01 \mathrm{mmol}, 5 \mathrm{~mol} \%\right), \mathrm{ZnI}_{2}$ (12.8 $\mathrm{mg}, 0.04 \mathrm{mmol}, 0.2$ equiv.), alkyne S-A-48 (40.0 mg, 0.24 mmol, 1.2 equiv.) and DBU (61 mg, $0.4 \mathrm{mmol}, 2.0$ equiv.) in xylenes $(0.5 \mathrm{~mL})$ at $120{ }^{\circ} \mathrm{C}$ for $10 \mathrm{~min}$ afforded $4 \mathrm{ba}$ as yellow oil $(55 \mathrm{mg}$,

$>\mathrm{R}_{f}=0.3$ (EtOAc:Petroleum Ether=1:5).

$>[\alpha]^{25} \mathrm{D}=-23.4^{\circ}\left(c=0.35, \mathrm{CHCl}_{3}\right)$.

$>{ }^{1} \mathrm{H} \mathrm{NMR}\left(400 \mathrm{MHz}, \mathrm{CDCl}_{3}\right) \delta 7.69(\mathrm{~d}, J=8.0 \mathrm{~Hz}, 2 \mathrm{H}), 7.33(\mathrm{~d}, J=8.0 \mathrm{~Hz}, 2 \mathrm{H}), 5.69(\mathrm{~s}, 1 \mathrm{H})$, $3.69(\mathrm{~s}, 3 \mathrm{H}), 3.57(\mathrm{q}, J=16 \mathrm{~Hz}, 2 \mathrm{H}), 3.29-3.20(\mathrm{~m}, 2 \mathrm{H}), 3.03-2.97(\mathrm{~m}, 2 \mathrm{H}), 2.50-2.45(\mathrm{~m}$, $2 \mathrm{H}), 2.43(\mathrm{~s}, 3 \mathrm{H}), 2.35(\mathrm{q}, J=8.0 \mathrm{~Hz}, 1 \mathrm{H}), 2.15-2.05(\mathrm{~m}, 1 \mathrm{H}), 1.95-1.82(\mathrm{~m}, 2 \mathrm{H}), 1.82-$ $1.74(\mathrm{~m}, 1 \mathrm{H}), 0.14(\mathrm{~s}, 6 \mathrm{H})$.

$>{ }^{13} \mathrm{CNMR}\left(100 \mathrm{MHz}, \mathrm{CDCl}_{3}\right) \delta 174.6,152.8,143.3,132.6,129.5,128.1,121.5,65.3,62.3,53.3$, 51.7, 50.3, 35.6, 29.4, 23.3, 21.5, -3.0, -3.0.

$>$ IR (neat) $\mathrm{cm}^{-1} 2954,1733,1437,1342,1163,1090,1036$.

$>$ HRMS calcd for $\mathrm{C}_{20} \mathrm{H}_{30} \mathrm{~N}_{2} \mathrm{O}_{4} \mathrm{SSi}(\mathrm{M}+\mathrm{Na})^{+} 445.1593$, found 445.1595 .

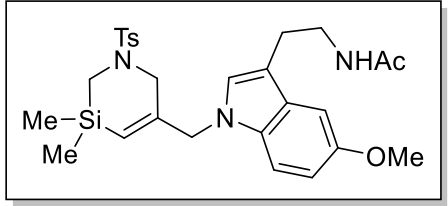

N-(2-(1-((3,3-dimethyl-1-tosyl-1,2,3,6-tetrahydro-1,3-azasilin-5yl)methyl)-5-methoxy-1H-indol-3-yl)ethyl)acetamide (4bb)

4bb was prepared according to General Method C. 2a $(58.4 \mathrm{mg}$, $0.2 \mathrm{mmol}, 2.0$ equiv.), $\mathrm{Pd}\left(\mathrm{PPh}_{3}\right)_{2} \mathrm{Cl}_{2}(7.2 \mathrm{mg}, 0.01 \mathrm{mmol}, 10$ mol \%), $\mathrm{ZnI}_{2}$ (6.4 mg, $0.02 \mathrm{mmol}, 0.2$ equiv.), alkyne S-A-49 (27 $\mathrm{mg}, 0.1 \mathrm{mmol}, 1.0$ equiv.) and DBU (31 mg, $0.2 \mathrm{mmol}, 2.0$ equiv.) in xylenes $(0.5 \mathrm{~mL})$ at $120^{\circ} \mathrm{C}$ for $10 \mathrm{~min}$ afforded $\mathbf{4 b b}$ as yellow oil $(34 \mathrm{mg}, 66 \%)$.

$>\mathrm{R}_{f}=0.4\left(\mathrm{CH}_{2} \mathrm{Cl}_{2}: \mathrm{MeOH}=10: 1\right)$.

$>{ }^{1} \mathrm{H} \mathrm{NMR}\left(400 \mathrm{MHz}, \mathrm{CDCl}_{3}\right) \delta 7.42(\mathrm{~d}, J=7.9 \mathrm{~Hz}, 2 \mathrm{H}), 7.25(\mathrm{~d}, J=7.9 \mathrm{~Hz}, 2 \mathrm{H}), 7.06(\mathrm{dd}, J=$ $14.2,5.7 \mathrm{~Hz}, 2 \mathrm{H}), 6.87(\mathrm{dd}, J=8.8,2.4 \mathrm{~Hz}, 1 \mathrm{H}), 6.84(\mathrm{~s}, 1 \mathrm{H}), 5.99(\mathrm{t}, J=5.9 \mathrm{~Hz}, 1 \mathrm{H}), 5.50(\mathrm{~s}$, $1 \mathrm{H}), 4.60(\mathrm{~s}, 2 \mathrm{H}), 3.88(\mathrm{~s}, 3 \mathrm{H}), 3.56(\mathrm{q}, J=6.3 \mathrm{~Hz}, 2 \mathrm{H}), 3.19(\mathrm{~s}, 2 \mathrm{H}), 2.94(\mathrm{t}, J=6.5 \mathrm{~Hz}, 2 \mathrm{H})$, $2.44(\mathrm{~s}, 2 \mathrm{H}), 2.41(\mathrm{~s}, 3 \mathrm{H}), 1.93$ (s, 3H), 0.11 (s, 6H).

$>{ }^{13} \mathrm{C}$ NMR $\left(100 \mathrm{MHz}, \mathrm{CDCl}_{3}\right) \delta 170.5,154.0,150.3,143.7,132.2,131.9,129.6,128.4,127.8$, $126.4,121.4,112.8,112.4,110.4,100.7,55.9,52.6,48.9,39.9,35.5,25.1,23.3,21.5,-3.1$.

$>$ IR (neat) $\mathrm{cm}^{-1} 3290,2926,1656,1542,1486,1336,1161,1089,1036,974$.

$>$ HRMS calcd for $\mathrm{C}_{27} \mathrm{H}_{35} \mathrm{~N}_{3} \mathrm{O}_{4} \mathrm{SSi}(\mathrm{M}+\mathrm{H})^{+}$526.2196, found 526.2198.

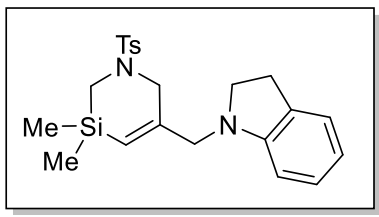

\section{1-((3,3-dimethvl-1-tosvl-1,2,3,6-tetrahvdro-1,3-azasilin-5-}

\section{vl)methvl)indoline (4bc)}

4bc was prepared according to General Method C. 2a (58.4 mg, 0.20 mmol, 1.0 equiv.), $\mathrm{Pd}\left(\mathrm{PPh}_{3}\right)_{2} \mathrm{Cl}_{2}$ (7.2 mg, $\left.0.01 \mathrm{mmol}, 5 \mathrm{~mol} \%\right), \mathrm{ZnI}_{2}$ (12.8 $\mathrm{mg}, 0.04 \mathrm{mmol}, 0.2$ equiv.), alkyne S-A-50 (37.2 mg, $0.24 \mathrm{mmol}$, 1.2 equiv.) and DBU (61 mg, $0.4 \mathrm{mmol}, 2.0$ equiv.) in xylenes $(0.5 \mathrm{~mL})$

at $120{ }^{\circ} \mathrm{C}$ for $10 \mathrm{~min}$ afforded $\mathbf{4 b c}$ as yellow solid (48 $\mathrm{mg}, 56 \%$ ).

$>\mathrm{mp}: 53.5-54.5^{\circ} \mathrm{C}$.

$>\mathrm{R}_{f}=0.5$ (EtOAc:Petroleum Ether=1:5).

$>\quad{ }^{1} \mathrm{H} \mathrm{NMR}\left(400 \mathrm{MHz}, \mathrm{CDCl}_{3}\right) \delta 7.65(\mathrm{~d}, J=8.0 \mathrm{~Hz}, 2 \mathrm{H}), 7.31(\mathrm{~d}, J=8.0 \mathrm{~Hz}, 2 \mathrm{H}), 7.09-7.02$ $(\mathrm{m}, 2 \mathrm{H}), 6.67(\mathrm{t}, J=7.28 \mathrm{~Hz}, 1 \mathrm{H}), 6.39(\mathrm{~d}, J=8.0 \mathrm{~Hz}, 1 \mathrm{H}), 5.80(\mathrm{~s}, 1 \mathrm{H}), 3.60(\mathrm{~s}, 2 \mathrm{H}), 3.52(\mathrm{~s}$, $2 \mathrm{H}), 3.26(\mathrm{t}, J=8.0 \mathrm{~Hz}, 2 \mathrm{H}), 2.96(\mathrm{t}, J=8.0 \mathrm{~Hz}, 2 \mathrm{H}), 2.50(\mathrm{~s}, 2 \mathrm{H}), 2.43(\mathrm{~s}, 3 \mathrm{H}), 0.16(\mathrm{~s}, 6 \mathrm{H})$;

$>{ }^{13} \mathrm{C} \mathrm{NMR}\left(100 \mathrm{MHz}, \mathrm{CDCl}_{3}\right) \delta 152.3,151.3,143.4,132.4,129.9,129.6,128.1,127.3,124.5$, $120.9,117.8,106.9,57.0,53.7,49.8,35.7,28.6,21.6,-3.0$. 
$>$ IR (neat) $\mathrm{cm}^{-1}$ 2956, 2922, 2816, 1605, 1488, 1339, 1161, 1089, 906.

$>$ HRMS calcd for $\mathrm{C}_{22} \mathrm{H}_{28} \mathrm{~N}_{2} \mathrm{O}_{2} \mathrm{SSi}(\mathrm{M}+\mathrm{Na})^{+} 435.1538$, found 435.1534.

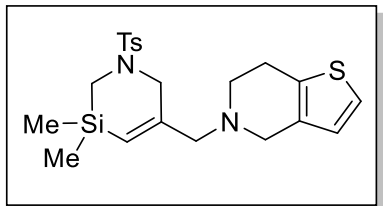

5-((3,3-dimethyl-1-tosyl-1,2,3,6-tetrahydro-1,3-azasilin-5yl)methyl)-4,5,6,7-tetrahydrothieno [3,2-c/pyridine(4bd)

4bd was prepared according to General Method C. 2a (58.4 mg, 0.20 mmol, 1.0 equiv.), $\mathrm{Pd}\left(\mathrm{PPh}_{3}\right)_{2} \mathrm{Cl}_{2}$ (7.2 $\left.\mathrm{mg}, 0.01 \mathrm{mmol}, 5 \mathrm{~mol} \%\right), \mathrm{ZnI}_{2}$ (12.8 mg, $0.04 \mathrm{mmol}, 0.2$ equiv.), alkyne S-A-51 (43.4 mg, $0.24 \mathrm{mmol}$, 1.2 equiv.) and DBU (61 mg, $0.4 \mathrm{mmol}, 2.0$ equiv.) in xylenes $(0.5 \mathrm{~mL})$ at $120{ }^{\circ} \mathrm{C}$ for $10 \mathrm{~min}$ afforded $\mathbf{4 b d}$ as white solid $(65 \mathrm{mg}, 75 \%)$.

$>\mathrm{mp}: 125.9-130.8^{\circ} \mathrm{C}$.

$>\mathrm{R}_{f}=0.5$ (EtOAc:Petroleum Ether $\left.=1: 5\right)$.

$>{ }^{1} \mathrm{H} \mathrm{NMR}\left(400 \mathrm{MHz}, \mathrm{CDCl}_{3}\right) \delta 7.68(\mathrm{~d}, J=8.0 \mathrm{~Hz}, 2 \mathrm{H}), 7.31(\mathrm{~d}, J=8.0 \mathrm{~Hz}, 2 \mathrm{H}), 7.07(\mathrm{~d}, J=$ $4.0 \mathrm{~Hz}, 1 \mathrm{H}), 6.71(\mathrm{~d}, J=4.0 \mathrm{~Hz}, 1 \mathrm{H}), 5.77(\mathrm{~s}, 1 \mathrm{H}), 3.56(\mathrm{~s}, 2 \mathrm{H}), 3.48(\mathrm{~s}, 2 \mathrm{H}), 3.12(\mathrm{~s}, 2 \mathrm{H}), 2.85$ $-2.83(\mathrm{~m}, 2 \mathrm{H}), 2.69-2.66(\mathrm{~m}, 2 \mathrm{H}), 2.50(\mathrm{~s}, 2 \mathrm{H}), 2.42(\mathrm{~s}, 3 \mathrm{H}), 0.17(\mathrm{~s}, 6 \mathrm{H})$.

$>{ }^{13} \mathrm{C} \mathrm{NMR}\left(100 \mathrm{MHz}, \mathrm{CDCl}_{3}\right) \delta 152.3,143.3,133.8,133.6,132.6,129.5,128.0,125.2,122.6$, $122.1,65.6,53.2,50.2,50.1,35.7,25.4,21.5,-2.9$.

$>$ IR (neat) $\mathrm{cm}^{-1} 2956,2804,1619,1596,1436,1338,1160,1089,1035,903$.

$>$ HRMS calcd for $\mathrm{C}_{21} \mathrm{H}_{28} \mathrm{~N}_{2} \mathrm{O}_{2} \mathrm{~S}_{2} \mathrm{Si}(\mathrm{M}+\mathrm{H})^{+} 433.1440$, found 433.1439 .

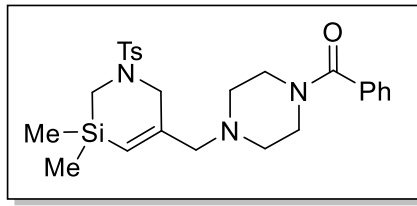

(4-((3,3-dimethyl-1-tosyl-1,2,3,6-tetrahydro-1,3-azasilin-5vl)methvl)piperazin-1-vl)(phenvl)methanone (4be)

4be was prepared according to General Method C. 2a $(58.4 \mathrm{mg}$, $0.20 \mathrm{mmol}, 1.0$ equiv.), $\mathrm{Pd}\left(\mathrm{PPh}_{3}\right)_{2} \mathrm{Cl}_{2}(7.2 \mathrm{mg}, 0.01 \mathrm{mmol}, 5 \mathrm{~mol} \%)$, $\mathrm{ZnI}_{2}$ (12.8 mg, $0.04 \mathrm{mmol}, 0.2$ equiv.), alkyne S-A-52 (54.8 mg, $0.24 \mathrm{mmol}, 1.2$ equiv.) and DBU (61 mg, $0.4 \mathrm{mmol}, 2.0$ equiv.) in xylenes $(0.5 \mathrm{~mL})$ at $120^{\circ} \mathrm{C}$ for $10 \mathrm{~min}$ afforded 4 be as yellow oil $(44 \mathrm{mg}, 45 \%)$.

$>\mathrm{R}_{f}=0.5$ (EtOAc:Petroleum Ether $\left.=1: 1\right)$.

$>\quad{ }^{1} \mathrm{H} \mathrm{NMR}\left(400 \mathrm{MHz}, \mathrm{CDCl}_{3}\right) \delta 7.69(\mathrm{~d}, J=8.2 \mathrm{~Hz}, 2 \mathrm{H}), 7.40(\mathrm{~d}, J=2.7 \mathrm{~Hz}, 5 \mathrm{H}), 7.34(\mathrm{~d}, J=$ $8.0 \mathrm{~Hz}, 2 \mathrm{H}), 5.69(\mathrm{~s}, 1 \mathrm{H}), 3.77(\mathrm{~s}, 2 \mathrm{H}), 3.57-3.50(\mathrm{~m}, 2 \mathrm{H}), 3.41(\mathrm{~s}, 2 \mathrm{H}), 2.94(\mathrm{~s}, 2 \mathrm{H}), 2.48(\mathrm{~s}$, $2 \mathrm{H}), 2.44(\mathrm{~s}, 5 \mathrm{H}), 2.27(\mathrm{~s}, 2 \mathrm{H}), 0.14(\mathrm{~s}, 6 \mathrm{H})$.

$>{ }^{13} \mathrm{C} \mathrm{NMR}\left(100 \mathrm{MHz}, \mathrm{CDCl}_{3}\right) \delta 170.3,151.6,143.4,135.9,132.6,129.6,129.5,128.5,128.0$, 127.0, 123.1, 66.3, 52.9, 49.9, 47.8, 35.7, 21.6, -3.0.

$>\quad$ IR (neat) $\mathrm{cm}^{-1} 2923,2855,2807,1643,1433,1339,1305,1161,1090,1035,996,942$.

$>$ HRMS calcd for $\mathrm{C}_{25} \mathrm{H}_{33} \mathrm{~N}_{3} \mathrm{O}_{3} \mathrm{SSi}(\mathrm{M}+\mathrm{H})^{+}$484.2090, found 484.2090.

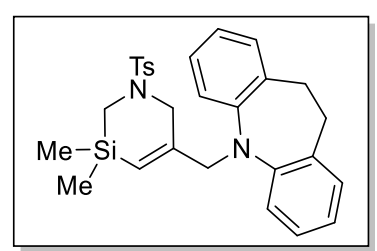

\section{5-((3,3-dimethvl-1-tosyl-1,2,3,6-tetrahvdro-1,3-azasilin-5-vl)methvl)- 10,11-dihvdro-5H-dibenzo/b,flazepine (4bf)}

4bf was prepared according to General Method C. 2a (58.4 mg, 0.20 mmol, 1.0 equiv.), $\mathrm{Pd}\left(\mathrm{PPh}_{3}\right)_{2} \mathrm{Cl}_{2}$ (7.2 $\left.\mathrm{mg}, 0.01 \mathrm{mmol}, 5 \mathrm{~mol} \%\right), \mathrm{ZnI}_{2}$ (12.8 mg, $0.04 \mathrm{mmol}, 0.2$ equiv.), alkyne S-A-53 (58.4 mg, $0.24 \mathrm{mmol}$, 1.2 equiv.) and DBU (61 mg, $0.4 \mathrm{mmol}, 2.0$ equiv.) in xylenes $(0.5 \mathrm{~mL})$ at $120^{\circ} \mathrm{C}$ for $10 \mathrm{~min}$ afforded $\mathbf{4 b f}$ as white solid $(66 \mathrm{mg}, 68 \%$ ).

$>$ mp: $166.6-168.5^{\circ} \mathrm{C}$.

$>\mathrm{R}_{f}=0.8$ (EtOAc:Petroleum Ether $\left.=1: 4\right)$.

$>{ }^{1} \mathrm{H} \mathrm{NMR}\left(400 \mathrm{MHz}, \mathrm{CDCl}_{3}\right) \delta 7.60(\mathrm{~d}, J=8.3 \mathrm{~Hz}, 2 \mathrm{H}), 7.29(\mathrm{~d}, J=8.0 \mathrm{~Hz}, 2 \mathrm{H}), 7.04(\mathrm{ddd}, J$ $=7.4,6.0,1.9 \mathrm{~Hz}, 4 \mathrm{H}), 6.95(\mathrm{dd}, J=8.4,1.2 \mathrm{~Hz}, 2 \mathrm{H}), 6.88(\mathrm{td}, J=7.3,1.3 \mathrm{~Hz}, 2 \mathrm{H}), 5.80(\mathrm{t}, J$ $=1.6 \mathrm{~Hz}, 1 \mathrm{H}), 4.37(\mathrm{~s}, 2 \mathrm{H}), 3.42(\mathrm{~s}, 2 \mathrm{H}), 3.14(\mathrm{~s}, 4 \mathrm{H}), 2.42(\mathrm{~s}, 3 \mathrm{H}), 2.29(\mathrm{~s}, 2 \mathrm{H}), 0.04(\mathrm{~s}, 6 \mathrm{H})$.

$>{ }^{13} \mathrm{C} \mathrm{NMR}\left(100 \mathrm{MHz}, \mathrm{CDCl}_{3}\right) \delta 150.8,147.6,143.4,133.9,132.4,129.9,129.5,128.0,126.2$, $124.0,122.8,120.1,59.8,50.2,35.5,32.5,21.6,-3.2$.

$>$ IR (neat) $\mathrm{cm}^{-1} 2920,2843,1596,1486,1455,1393,1161,1037,936$.

$>$ HRMS calcd for $\mathrm{C}_{28} \mathrm{H}_{32} \mathrm{~N}_{2} \mathrm{O}_{2} \mathrm{SSi}(\mathrm{M}+\mathrm{Na})^{+}$511.1851, found 511.1844.

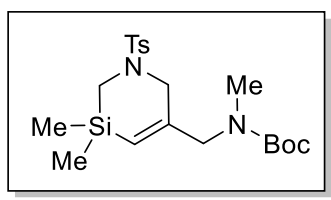

tert-butyl((3,3-dimethyl-1-tosyl-1,2,3,6-tetrahydro-1,3-azasilin-5vl)methvl)(methvl) carbamate(4bg)

4bg was prepared according to General Method C. 2a (58.4 mg, $0.20 \mathrm{mmol}$, 1.0 equiv.), $\mathrm{Pd}\left(\mathrm{PPh}_{3}\right)_{2} \mathrm{Cl}_{2}$ (7.2 mg, $\left.0.01 \mathrm{mmol}, 5 \mathrm{~mol} \%\right), \mathrm{ZnI}_{2}(12.8 \mathrm{mg}$, $0.04 \mathrm{mmol}, 0.2$ equiv.), alkyne S-A-54 (40.6 mg, $0.24 \mathrm{mmol}, 1.2$ equiv.) 
and DBU (61 mg, $0.4 \mathrm{mmol}, 2.0$ equiv.) in xylenes $(0.5 \mathrm{~mL})$ at $120{ }^{\circ} \mathrm{C}$ for $10 \mathrm{~min}$ afforded $4 \mathrm{bg}$ as white solid (57 mg, 67\%).

$>\mathrm{mp}: 83.2-85.1^{\circ} \mathrm{C}$.

$>\mathrm{R}_{f}=0.6$ (EtOAc:Petroleum Ether=1:4).

$>\quad{ }^{1} \mathrm{H} \mathrm{NMR}\left(400 \mathrm{MHz}, \mathrm{CDCl}_{3}\right) \delta 7.66(\mathrm{~d}, J=8.0 \mathrm{~Hz}, 2 \mathrm{H}), 7.33(\mathrm{~d}, J=8.0 \mathrm{~Hz}, 2 \mathrm{H}), 5.82(\mathrm{t}, J=1$. $60 \mathrm{~Hz}, 1 \mathrm{H}), 3.77(\mathrm{~d}, J=12 \mathrm{~Hz}, 2 \mathrm{H}), 3.40(\mathrm{~s}, 2 \mathrm{H}), 2.79$ (s, 3H), 2.47 (s, 2H), $2.43(\mathrm{~s}, 3 \mathrm{H}), 1.48$ $-1.45(\mathrm{~m}, 9 \mathrm{H}), 0.16(\mathrm{~s}, 6 \mathrm{H})$.

$>{ }^{13} \mathrm{C}$ NMR $\left(100 \mathrm{MHz}, \mathrm{CDCl}_{3}\right) \delta 150.9(155.5), 150.6(150.2), 143.5,132.2,129.6,128.0$, 120.2(119.5), 79.8, 55.4(54.6), 49.4(49.3), 35.5, 34.1, 28.4, 21.5, -3.0.

$>$ IR (neat) $\mathrm{cm}^{-1} 2972,1693,1622,1451,1392,1342,1163,1090,974$.

$>$ HRMS calcd for $\mathrm{C}_{20} \mathrm{H}_{32} \mathrm{~N}_{2} \mathrm{O}_{4} \mathrm{SSi}(\mathrm{M}+\mathrm{Na})^{+} 447.1750$, found 447.1725.

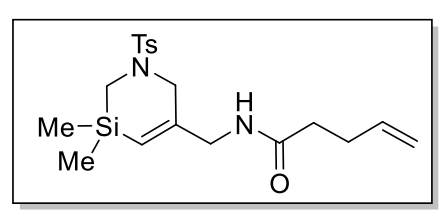

N-((3,3-dimethyl-1-tosyl-1,2,3,6-tetrahydro-1,3-azasilin-5yl)methyl)pent-4-enamide( $4 \mathrm{bh})$

4bh was prepared according to General Method C. 2a (58.4 mg, 0.20 mmol, 1.0 equiv.), $\mathrm{Pd}\left(\mathrm{PPh}_{3}\right)_{2} \mathrm{Cl}_{2}(7.2 \mathrm{mg}, 0.01 \mathrm{mmol}, 5$ mol \%), $\mathrm{ZnI}_{2}(12.8 \mathrm{mg}, 0.04 \mathrm{mmol}, 0.2$ equiv.), alkyne $\mathbf{S}-\mathbf{A}-\mathbf{5 5}$ (33.6 mg, $0.24 \mathrm{mmol}, 1.2$ equiv.) and DBU (61 mg, $0.4 \mathrm{mmol}, 2.0$ equiv.) in xylenes $(0.5 \mathrm{~mL})$ at $120^{\circ} \mathrm{C}$ for $10 \mathrm{~min}$ afforded $\mathbf{4 b h}$ as white solid (46 mg, 59\%).

$>$ mp: $123.1-124.8^{\circ} \mathrm{C}$.

$>\mathrm{R}_{f}=0.2($ EtOAc:Petroleum Ether $=1: 3)$.

$>{ }^{1} \mathrm{H} \mathrm{NMR}\left(400 \mathrm{MHz}, \mathrm{CDCl}_{3}\right) \delta 7.65(\mathrm{~d}, J=8.0 \mathrm{~Hz}, 2 \mathrm{H}), 7.34(\mathrm{~d}, J=8.0 \mathrm{~Hz}, 2 \mathrm{H}), 5.87-5.78$ $(\mathrm{m}, 2 \mathrm{H}), 5.62(\mathrm{t}, J=1.60 \mathrm{~Hz}, 1 \mathrm{H}), 5.10-5.00(\mathrm{~m}, 2 \mathrm{H}), 3.80(\mathrm{~d}, J=5.92 \mathrm{~Hz}, 2 \mathrm{H}), 3.45(\mathrm{~s}, 2 \mathrm{H})$, $2.46-2.39(\mathrm{~m}, 7 \mathrm{H}), 2.35-2.31(\mathrm{~m}, 2 \mathrm{H}), 0.15(\mathrm{~s}, 6 \mathrm{H})$.

$>{ }^{13} \mathrm{C} \mathrm{NMR}\left(100 \mathrm{MHz}, \mathrm{CDCl}_{3}\right) \delta 172.4,151.1,143.6,137.0,132.0,129.6,128.0,119.7,115.8$, 49.6, 45.0, 35.8, 35.5, 29.6, 21.5, -3.1.

$>$ IR (neat) $\mathrm{cm}^{-1} 3293,2958,1649,1543,1439,1339,1259,1161,1089,1020,910$.

$>$ HRMS calcd for $\mathrm{C}_{19} \mathrm{H}_{28} \mathrm{~N}_{2} \mathrm{O}_{3} \mathrm{SSi}(\mathrm{M}+\mathrm{Na})^{+} 415.1488$, found 415.1480 .

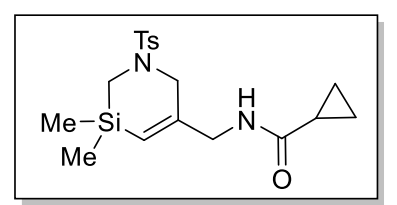

N-((3,3-dimethyl-1-tosyl-1,2,3,6-tetrahydro-1,3-azasilin-5yl)methyl)cyclopropanecarboxamide (4bi)

4bi was prepared according to General Method C. 2a (58.4 mg, 0.20 mmol, 1.0 equiv.), $\mathrm{Pd}\left(\mathrm{PPh}_{3}\right)_{2} \mathrm{Cl}_{2}$ (7.2 $\left.\mathrm{mg}, 0.01 \mathrm{mmol}, 5 \mathrm{~mol} \%\right), \mathrm{ZnI}_{2}$ (12.8 mg, 0.04 mmol, 0.2 equiv.), alkyne S-A-56 (30.0 mg, $0.24 \mathrm{mmol}$, 1.2 equiv.) and DBU (61 mg, $0.4 \mathrm{mmol}, 2.0$ equiv.) in xylenes $(0.5 \mathrm{~mL})$ at $120^{\circ} \mathrm{C}$ for $10 \mathrm{~min}$ afforded $4 \mathrm{bi}$ as yellow solid $(50 \mathrm{mg}, 66 \%)$.

$>$ mp: $139.5-143.0^{\circ} \mathrm{C}$.

$>\mathrm{R}_{f}=0.3$ (EtOAc:Petroleum Ether $\left.=1: 3\right)$.

$>{ }^{1} \mathrm{H} \mathrm{NMR}\left(400 \mathrm{MHz}, \mathrm{CDCl}_{3}\right) \delta 7.66(\mathrm{~d}, J=8.0 \mathrm{~Hz}, 2 \mathrm{H}), 7.34(\mathrm{~d}, J=8.0 \mathrm{~Hz}, 2 \mathrm{H}), 5.95-5.87$ $(\mathrm{m}, 1 \mathrm{H}), 5.65(\mathrm{~s}, 1 \mathrm{H}), 3.82(\mathrm{~d}, J=4.0 \mathrm{~Hz}, 2 \mathrm{H}), 3.45(\mathrm{~s}, 2 \mathrm{H}), 2.46(\mathrm{~s}, 2 \mathrm{H}), 2.43(\mathrm{~s}, 3 \mathrm{H}), 1.44-$ $1.38(\mathrm{~m}, 1 \mathrm{H}), 1.00-0.96(\mathrm{~m}, 2 \mathrm{H}), 0.79-0.74(\mathrm{~m}, 2 \mathrm{H}), 0.16(\mathrm{~s}, 6 \mathrm{H})$.

$>{ }^{13} \mathrm{CNMR}\left(150 \mathrm{MHz}, \mathrm{CDCl}_{3}\right) \delta 173.6,151.4,143.5,132.1,129.6,128.0,119.6,49.6,45.3,35.4$, $21.5,14.7,7.3,-3.1$.

$>$ IR (neat) $\mathrm{cm}^{-1} 3291,2958,1647,1597,1534,1399,1337,1160,1089,1031,939$.

$>$ HRMS calcd for $\mathrm{C}_{18} \mathrm{H}_{26} \mathrm{~N}_{2} \mathrm{O}_{3} \mathrm{SSi}(\mathrm{M}+\mathrm{Na})^{+}$401.1331, found 401.1333 .

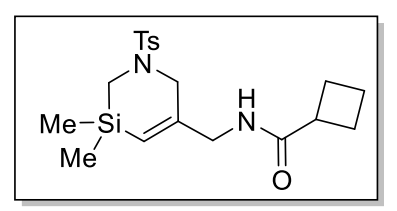

\section{N-((3,3-dimethyl-1-tosyl-1,2,3,6-tetrahydro-1,3-azasilin-5- yl)methyl)cyclobutanecarboxamide (4bj)}

4bj was prepared according to General Method C. 2a (58.4 mg, 0.20 mmol, 1.0 equiv.), $\mathrm{Pd}\left(\mathrm{PPh}_{3}\right)_{2} \mathrm{Cl}_{2}$ (7.2 $\left.\mathrm{mg}, 0.01 \mathrm{mmol}, 5 \mathrm{~mol} \%\right), \mathrm{ZnI}_{2}$ (12.8 mg, $0.04 \mathrm{mmol}, 0.2$ equiv.), alkyne S-A-57 (33.0 mg, $0.24 \mathrm{mmol}$, 1.2 equiv.) and DBU (61 mg, $0.4 \mathrm{mmol}, 2.0$ equiv.) in xylenes $(0.5 \mathrm{~mL})$

at $120^{\circ} \mathrm{C}$ for $10 \mathrm{~min}$ afforded $\mathbf{4 b j}$ as yellow solid $(57 \mathrm{mg}, 73 \%)$.

$>$ mp: $120.2-121.6^{\circ} \mathrm{C}$.

$>\mathrm{R}_{f}=0.3($ EtOAc:Petroleum Ether $=1: 3)$;

$>{ }^{1} \mathrm{H}$ NMR $\left(400 \mathrm{MHz}, \mathrm{CDCl}_{3}\right) \delta 7.65(\mathrm{~d}, J=8.2 \mathrm{~Hz}, 2 \mathrm{H}), 7.34(\mathrm{~d}, J=8.0 \mathrm{~Hz}, 2 \mathrm{H}), 5.64-5.57$ $(\mathrm{m}, 2 \mathrm{H}), 3.80(\mathrm{~d}, J=5.9 \mathrm{~Hz}, 2 \mathrm{H}), 3.45(\mathrm{~s}, 2 \mathrm{H}), 3.10-3.01(\mathrm{~m}, 1 \mathrm{H}), 2.46(\mathrm{~s}, 2 \mathrm{H}), 2.44(\mathrm{~s}, 3 \mathrm{H})$, $2.35-2.25(\mathrm{~m}, 2 \mathrm{H}), 2.23-2.14(\mathrm{~m}, 2 \mathrm{H}), 2.04-1.85(\mathrm{~m}, 2 \mathrm{H}), 0.15(\mathrm{~s}, 6 \mathrm{H})$.

$>{ }^{13} \mathrm{CNMR}\left(150 \mathrm{MHz}, \mathrm{CDCl}_{3}\right) \delta 175.0,151.5,143.5,132.1,129.6,128.0,119.6,49.5,44.9,39.8$, 
$35.5,25.4,21.5,18.2,-3.1$.

$>$ IR (neat) $\mathrm{cm}^{-1} 3292,2946,1648,1531,1338,1160,1089,993$.

$>$ HRMS calcd for $\mathrm{C}_{19} \mathrm{H}_{28} \mathrm{~N}_{2} \mathrm{O}_{3} \mathrm{SSi}(\mathrm{M}+\mathrm{Na})^{+} 415.1488$, found 415.1497 .

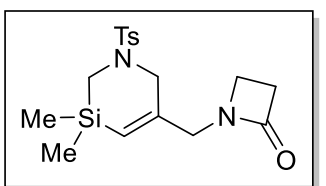

1-((3,3-dimethyl-1-tosyl-1,2,3,6-tetrahydro-1,3-azasilin-5-yl)methyl) azetidin-2-one (4bk)

4bk was prepared according to General Method C. $2 \mathbf{a}(58.4 \mathrm{mg}, 0.2 \mathrm{mmol}$, 1.0 equiv.), $\mathrm{Pd}\left(\mathrm{PPh}_{3}\right)_{2} \mathrm{Cl}_{2}$ (7.2 mg, $\left.0.01 \mathrm{mmol}, 5 \mathrm{~mol} \%\right), \mathrm{ZnI}_{2}(12.8 \mathrm{mg}$, 0.04 mmol, 0.2 equiv.), alkyne S-A-58 (44 mg, $0.4 \mathrm{mmol}, 2.0$ equiv.) and DBU (61 mg, $0.4 \mathrm{mmol}, 2.0$ equiv.) in xylenes $(0.5 \mathrm{~mL})$ at $120^{\circ} \mathrm{C}$ for $10 \mathrm{~min}$ afforded $4 \mathbf{b k}$ as white solid (37 mg, 50\%).

$>\quad \mathrm{mp}: 91.9-93.9^{\circ} \mathrm{C}$.

$>\mathrm{R}_{f}=0.4$ (EtOAc:Petroleum Ether $\left.=1: 1\right)$.

$>\quad{ }^{1} \mathrm{H} \mathrm{NMR}\left(400 \mathrm{MHz}, \mathrm{CDCl}_{3}\right) \delta 7.67(\mathrm{~d}, J=8.0 \mathrm{~Hz}, 2 \mathrm{H}), 7.34(\mathrm{~d}, J=8.0 \mathrm{~Hz}, 2 \mathrm{H}), 5.66(\mathrm{t}, J=$ $1.56 \mathrm{~Hz}, 1 \mathrm{H}), 3.75(\mathrm{~s}, 2 \mathrm{H}), 3.41(\mathrm{~s}, 2 \mathrm{H}), 3.22(\mathrm{t}, J=4.08 \mathrm{~Hz}, 2 \mathrm{H}), 3.01(\mathrm{t}, J=4.08 \mathrm{~Hz}, 2 \mathrm{H})$, $2.49(\mathrm{~s}, 2 \mathrm{H}), 2.44(\mathrm{~s}, 3 \mathrm{H}), 0.17(\mathrm{~s}, 6 \mathrm{H})$.

$>{ }^{13} \mathrm{C} \mathrm{NMR}\left(100 \mathrm{MHz}, \mathrm{CDCl}_{3}\right) \delta 168.1,148.7,143.6,132.2,129.7,128.0,122.8,49.3,48.6,39.3$, $37.0,35.5,21.5,-3.0$.

$>\quad$ IR (neat) $\mathrm{cm}^{-1} 2958,1746,1623,1400,1339,1162,1089,932$.

$>$ HRMS calcd for $\mathrm{C}_{17} \mathrm{H}_{24} \mathrm{~N}_{2} \mathrm{O}_{3} \mathrm{SSi}(\mathrm{M}+\mathrm{Na})^{+}$387.1175, found 387.1166.

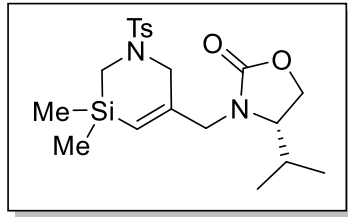

(S)-3-((3,3-dimethvl-1-tosvl-1,2,3,6-tetrahvdro-1,3-azasilin-5yl)methyl)-4-isopropyloxazolidin-2-one (4bl)

4bl was prepared according to General Method C. $2 \mathbf{a}(58.4 \mathrm{mg}, 0.20$ mmol, 1.0 equiv.), $\mathrm{Pd}\left(\mathrm{PPh}_{3}\right)_{2} \mathrm{Cl}_{2}(7.2 \mathrm{mg}, 0.01 \mathrm{mmol}, 5 \mathrm{~mol} \%), \mathrm{ZnI}_{2}$ (12.8 mg, 0.04 mmol, 0.2 equiv.), alkyne S-A-59 (40.0 mg, 0.24 mmol, 1.2 equiv.) and DBU (61 mg, 0.4 mmol, 2.0 equiv.) in xylenes $(0.5 \mathrm{~mL})$ at $120{ }^{\circ} \mathrm{C}$ for $10 \mathrm{~min}$ afforded $4 \mathrm{bl}$ as yellow oil $(51 \mathrm{mg}, 60 \%)$.

$>\mathrm{R}_{f}=0.4$ (EtOAc:Petroleum Ether $\left.=1: 2\right)$.

$>[\alpha]^{25} \mathrm{D}=+2.5^{\circ}\left(c=0.4, \mathrm{CHCl}_{3}\right)$.

$>{ }^{1} \mathrm{H}$ NMR $\left(400 \mathrm{MHz}, \mathrm{CDCl}_{3}\right) \delta 7.66(\mathrm{~d}, J=8.0 \mathrm{~Hz}, 2 \mathrm{H}), 7.34(\mathrm{~d}, J=8.0 \mathrm{~Hz}, 2 \mathrm{H}), 5.69(\mathrm{~s}, 1 \mathrm{H})$, $4.32(\mathrm{t}, J=8.0 \mathrm{~Hz}, 1 \mathrm{H}), 4.17-4.11(\mathrm{~m}, 2 \mathrm{H}), 3.69-3.65(\mathrm{~m}, 1 \mathrm{H}), 3.58(\mathrm{~d}, J=16 \mathrm{~Hz}, 1 \mathrm{H})$, $3.47(\mathrm{~d}, J=16 \mathrm{~Hz}, 1 \mathrm{H}), 3.26(\mathrm{~d}, J=20 \mathrm{~Hz}, 1 \mathrm{H}), 2.69(\mathrm{~d}, J=12 \mathrm{~Hz}, 1 \mathrm{H}), 2.44(\mathrm{~s}, 3 \mathrm{H}), 2.33(\mathrm{~d}$, $J=16 \mathrm{~Hz}, 1 \mathrm{H}), 2.07-1.99(\mathrm{~m}, 1 \mathrm{H}), 0.87(\mathrm{t}, J=8.0 \mathrm{~Hz}, 6 \mathrm{H}), 0.20(\mathrm{~s}, 3 \mathrm{H}), 0.16(\mathrm{~s}, 3 \mathrm{H})$.

$>\quad{ }^{13} \mathrm{C} \mathrm{NMR}\left(100 \mathrm{MHz}, \mathrm{CDCl}_{3}\right) \delta 158.6,149.0,143.6,132.2,129.7,127.9,123.3,63.0,58.8,48.9$, 48.6, 35.6, 27.3, 21.5, 17.7, 14.3, -2.9, -3.2.

$>\quad$ IR (neat) $\mathrm{cm}^{-1} 2961,2925,1774,1597,1432,1339,1161,1089,1045,974$.

$>\quad \mathrm{HRMS}$ calcd for $\mathrm{C}_{20} \mathrm{H}_{30} \mathrm{~N}_{2} \mathrm{O}_{4} \mathrm{SSi}(\mathrm{M}+\mathrm{Na})^{+} 445.1593$, found 445.1593 .

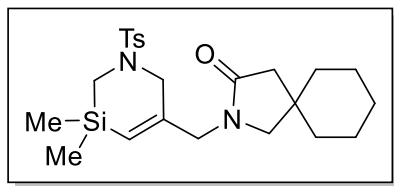

2-((3,3-dimethyl-1-tosyl-1,2,3,6-tetrahydro-1,3-azasilin-5yl)methyl)-2-azaspiro[4.5/decan-3-one (4bm)

4bm was prepared according to General Method C. 2a $(58.4 \mathrm{mg}, 0.2$ mmol, 1.0 equiv.), $\mathrm{Pd}\left(\mathrm{PPh}_{3}\right)_{2} \mathrm{Cl}_{2}$ (7.2 mg, $\left.0.01 \mathrm{mmol}, 5 \mathrm{~mol} \%\right), \mathrm{ZnI}_{2}$ (12.8 mg, $0.04 \mathrm{mmol}, 0.2$ equiv.), alkyne S-A-60 (76.0 mg, $0.4 \mathrm{mmol}$, 2.0 equiv.) and DBU (61 mg, $0.4 \mathrm{mmol}, 2.0$ equiv.) in xylenes $(0.5$ $\mathrm{mL})$ at $120^{\circ} \mathrm{C}$ for $10 \mathrm{~min}$ afforded $\mathbf{4 b m}$ as yellow oil $(52 \mathrm{mg}, 58 \%)$.

$>\mathrm{R}_{f}=0.2($ EtOAc:Petroleum Ether $=1: 3)$.

$>\quad{ }^{1} \mathrm{H} \mathrm{NMR}\left(400 \mathrm{MHz}, \mathrm{CDCl}_{3}\right) \delta 7.65(\mathrm{~d}, J=8.0 \mathrm{~Hz}, 2 \mathrm{H}), 7.33(\mathrm{~d}, J=8.0 \mathrm{~Hz}, 2 \mathrm{H}), 5.60(\mathrm{~s}, 1 \mathrm{H})$, $3.80(\mathrm{~s}, 2 \mathrm{H}), 3.37(\mathrm{~s}, 2 \mathrm{H}), 3.04(\mathrm{~s}, 2 \mathrm{H}), 2.47(\mathrm{~s}, 2 \mathrm{H}), 2.43(\mathrm{~s}, 3 \mathrm{H}), 2.32(\mathrm{~s}, 2 \mathrm{H}), 1.53-1.40$ (m, 10H) 0.17 (s, 6H).

$>{ }^{13} \mathrm{C} \mathrm{NMR}\left(100 \mathrm{MHz}, \mathrm{CDCl}_{3}\right) \delta 174.5,149.4,143.5,132.1,129.6,128.0,122.2,58.3,49.2,49.0$, $43.9,37.0,36.1,35.5,25.6,22.8,21.5,-3.0$.

$>\operatorname{IR}$ (neat) $\mathrm{cm}^{-1} 2924,2852,1686,1623,1490,1440,1341,1247,1220$,

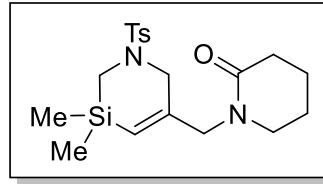
$1168,1032,950$.

$>$ HRMS calcd for $\mathrm{C}_{23} \mathrm{H}_{34} \mathrm{~N}_{2} \mathrm{O}_{3} \mathrm{SSi}(\mathrm{M}+\mathrm{Na})^{+} 469.1957$, found 469.1947 .

1-((3,3-dimethyl-1-tosyl-1,2,3,6-tetrahydro-1,3-azasilin-5yl)methyl)piperidin-2-one (4bn) 
4bn was prepared according to General Method C. 2a (58.4 mg, $0.20 \mathrm{mmol}$, 1.0 equiv.), $\mathrm{Pd}\left(\mathrm{PPh}_{3}\right)_{2} \mathrm{Cl}_{2}$ (7.2 mg, $\left.0.01 \mathrm{mmol}, 5 \mathrm{~mol} \%\right), \mathrm{ZnI}_{2}(12.8 \mathrm{mg}, 0.04 \mathrm{mmol}, 0.2$ equiv.), alkyne S-A-61 (33.0 mg, $0.24 \mathrm{mmol}, 1.2$ equiv.) and DBU (61 mg, $0.4 \mathrm{mmol}, 2.0$ equiv.) in xylenes $(0.5 \mathrm{~mL})$ at $120^{\circ} \mathrm{C}$ for $10 \mathrm{~min}$ afforded $\mathbf{4 b n}$ as yellow solid $(47 \mathrm{mg}, 60 \%)$.

$>$ mp: $104.4-107.2^{\circ} \mathrm{C}$.

$>\mathrm{R}_{f}=0.3$ (EtOAc:Petroleum Ether $\left.=1: 1\right)$.

$>\quad{ }^{1} \mathrm{H} \mathrm{NMR}\left(400 \mathrm{MHz}, \mathrm{CDCl}_{3}\right) \delta 7.75(\mathrm{~d}, J=8.0 \mathrm{~Hz}, 2 \mathrm{H}), 7.33(\mathrm{~d}, J=8.0 \mathrm{~Hz}, 2 \mathrm{H}), 5.55(\mathrm{~s}, 1 \mathrm{H})$, $3.97(\mathrm{~s}, 2 \mathrm{H}), 3.39(\mathrm{~s}, 2 \mathrm{H}), 3.18(\mathrm{~s}, 2 \mathrm{H}), 2.47-2.43(\mathrm{~m}, 7 \mathrm{H}), 1.86-1.81(\mathrm{~m}, 4 \mathrm{H}), 0.16(\mathrm{~s}, 6 \mathrm{H})$.

$>{ }^{13} \mathrm{CNMR}\left(150 \mathrm{MHz}, \mathrm{CDCl}_{3}\right) \delta 170.0,150.0,143.4,132.3,129.6,127.9,121.0,52.5,49.0,47.4$, 35.6, 32.3, 23.1, 21.5, 21.3, -3.0.

$>\quad \mathrm{IR}$ (neat) $\mathrm{cm}^{-1} 2950,2866,1637,1493,1338,1160,1089,1033,940$.

$>$ HRMS calcd for $\mathrm{C}_{19} \mathrm{H}_{28} \mathrm{~N}_{2} \mathrm{O}_{3} \mathrm{SSi}(\mathrm{M}+\mathrm{Na})^{+} 415.1488$, found 415.1482 .

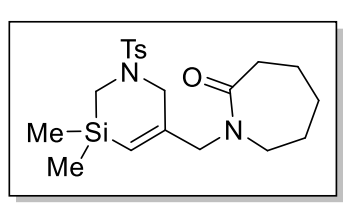

1-((3,3-dimethvl-1-tosvl-1,2,3,6-tetrahvdro-1,3-azasilin-5-vl)methvl) azepan-2-one (4bo)

4bo was prepared according to General Method C. 2a (58.4 mg, 0.20 mmol, 1.0 equiv.), $\mathrm{Pd}\left(\mathrm{PPh}_{3}\right)_{2} \mathrm{Cl}_{2}$ (7.2 mg, $\left.0.01 \mathrm{mmol}, 5 \mathrm{~mol} \%\right), \mathrm{ZnI}_{2}(12.8$ $\mathrm{mg}, 0.04 \mathrm{mmol}, 0.2$ equiv.), alkyne $\mathbf{S}-\mathbf{A}-62(37.0 \mathrm{mg}, 0.24 \mathrm{mmol}, 1.2$ equiv.) and DBU (61 mg, $0.4 \mathrm{mmol}, 2.0$ equiv.) in xylenes $(0.5 \mathrm{~mL})$ at

$120{ }^{\circ} \mathrm{C}$ for $10 \mathrm{~min}$ afforded $4 \mathrm{bo}$ as yellow solid (46 mg, $56 \%$ ).

$>\mathrm{mp}: 54.6-57.0^{\circ} \mathrm{C}$,

$>\mathrm{R}_{f}=0.2$ (EtOAc:Petroleum Ether=1:2).

$>{ }^{1} \mathrm{H} \mathrm{NMR}\left(400 \mathrm{MHz}, \mathrm{CDCl}_{3}\right) \delta 7.65(\mathrm{~d}, J=8.2 \mathrm{~Hz}, 2 \mathrm{H}), 7.33(\mathrm{~d}, J=8.0 \mathrm{~Hz}, 2 \mathrm{H}), 5.59(\mathrm{~s}, 1 \mathrm{H})$, $3.95(\mathrm{~s}, 2 \mathrm{H}), 3.40$ (s, 2H), $3.30-3.28(\mathrm{~m}, 2 \mathrm{H}), 2.60-2.57$ (m, 2H), 2.47 (s, 2H), $2.43(\mathrm{~s}, 3 \mathrm{H})$, $1.74(\mathrm{dt}, J=6.2,2.8 \mathrm{~Hz}, 4 \mathrm{H}), 1.61(\mathrm{t}, J=5.3 \mathrm{~Hz}, 2 \mathrm{H}), 0.16(\mathrm{~s}, 6 \mathrm{H})$.

$>{ }^{13} \mathrm{CNMR}\left(100 \mathrm{MHz}, \mathrm{CDCl}_{3}\right) \delta 176.2,150.9,143.5,132.2,129.6,128.0,122.1,54.0,49.1,48.9$, 37.1, 35.6, 30.0, 28.0, 23.3, 21.5, -3.0.

$>\quad \mathrm{IR}$ (neat) $\mathrm{cm}^{-1} 2928,1641,1480,1443,1340,1162,1089,970$.

$>$ HRMS calcd for $\mathrm{C}_{20} \mathrm{H}_{30} \mathrm{~N}_{2} \mathrm{O}_{3} \mathrm{SSi}(\mathrm{M}+\mathrm{Na})^{+} 429.1644$, found 429.1633 .

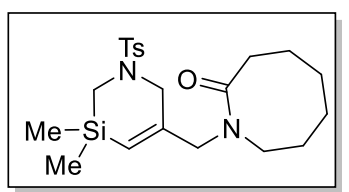

\section{1-((3,3-dimethyl-1-tosyl-1,2,3,6-tetrahydro-1,3-azasilin-5-yl)methyl)} azocan-2-one (4bp)

4bp was prepared according to General Method C. 2a (58.4 mg, 0.20 mmol, 1.0 equiv.), $\mathrm{Pd}\left(\mathrm{PPh}_{3}\right)_{2} \mathrm{Cl}_{2}$ (7.2 mg, $\left.0.01 \mathrm{mmol}, 5 \mathrm{~mol} \%\right), \mathrm{ZnI}_{2}(12.8$ $\mathrm{mg}, 0.04 \mathrm{mmol}, 0.2$ equiv.), alkyne S-A-63 ( $40.0 \mathrm{mg}, 0.24 \mathrm{mmol}, 1.2$ equiv.) and $\mathrm{DBU}\left(61 \mathrm{mg}, 0.4 \mathrm{mmol}, 2.0\right.$ equiv.) in xylenes $(0.5 \mathrm{~mL})$ at $120^{\circ} \mathrm{C}$ for $10 \mathrm{~min}$ afforded 4bp as yellow oil $(59 \mathrm{mg}, 70 \%)$.

$>\mathrm{R}_{f}=0.3$ (EtOAc:Petroleum Ether=1:2).

$>{ }^{1} \mathrm{H} \mathrm{NMR}\left(400 \mathrm{MHz}, \mathrm{CDCl}_{3}\right) \delta 7.66(\mathrm{~d}, J=8.0 \mathrm{~Hz}, 2 \mathrm{H}), 7.33(\mathrm{~d}, J=8.0 \mathrm{~Hz}, 2 \mathrm{H}), 5.57(\mathrm{~s}, 1 \mathrm{H})$, $3.94(\mathrm{~s}, 2 \mathrm{H}), 3.44-3.41(\mathrm{~m}, 4 \mathrm{H}), 2.58-2.55(\mathrm{~m}, 2 \mathrm{H}), 2.46(\mathrm{~s}, 2 \mathrm{H}), 2.43(\mathrm{~s}, 3 \mathrm{H}), 1.83-1.79$ (m, 2H), $1.63-1.47(\mathrm{~m}, 6 \mathrm{H}), 0.16(\mathrm{~s}, 6 \mathrm{H})$.

$>{ }^{13} \mathrm{CNMR}\left(100 \mathrm{MHz}, \mathrm{CDCl}_{3}\right) \delta 175.3,150.2,143.5,132.1,129.6,128.0,120.9,50.0,49.5,46.0$, $35.5,33.7,28.8,28.7,26.2,24.4,21.5,-3.0$.

$>$ IR (neat) $\mathrm{cm}^{-1} 2924,2855,1637,1464,1340,1163,1090,991$

$>$ HRMS calcd for $\mathrm{C}_{21} \mathrm{H}_{32} \mathrm{~N}_{2} \mathrm{O}_{3} \mathrm{SSi}(\mathrm{M}+\mathrm{H})^{+}$421.1981, found 421.1967 .

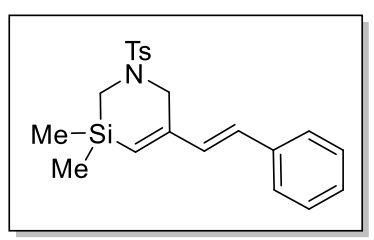

\section{(E)-3,3-dimethyl-5-styryl-1-tosyl-1,2,3,6-tetrahydro-1,3-azasiline (4bq)}

4bq was prepared according to General Method C. 2a (58.4 mg, 0.20 mmol, 1.0 equiv.), $\mathrm{Pd}\left(\mathrm{PPh}_{3}\right)_{2} \mathrm{Cl}_{2}$ (7.2 $\left.\mathrm{mg}, 0.01 \mathrm{mmol}, 5 \mathrm{~mol} \%\right), \mathrm{ZnI}_{2}$ (12.8 mg, $0.04 \mathrm{mmol}, 0.2$ equiv.), alkyne S-A-64 (31.4 mg, $0.24 \mathrm{mmol}$, 1.2 equiv.) and $\mathrm{DBU}(61 \mathrm{mg}, 0.4 \mathrm{mmol}, 2.0$ equiv. $)$ in xylenes $(0.5 \mathrm{~mL})$ at $120^{\circ} \mathrm{C}$ for $10 \mathrm{~min}$ afforded $\mathbf{4 b q}$ as yellow solid (44 $\left.\mathrm{mg}, 57 \%\right)$.

$>$ mp: $128.4-130.8^{\circ} \mathrm{C}$.

$>\mathrm{R}_{f}=0.5$ (EtOAc:Petroleum Ether=1:5).

$>{ }^{1} \mathrm{H} \mathrm{NMR}\left(400 \mathrm{MHz}, \mathrm{CDCl}_{3}\right) \delta 7.75(\mathrm{~d}, J=8.0 \mathrm{~Hz}, 2 \mathrm{H}), 7.43-7.31(\mathrm{~m}, 6 \mathrm{H}), 7.27-7.24(\mathrm{~m}$, $1 \mathrm{H}), 6.69(\mathrm{~d}, J=20.0 \mathrm{~Hz}, 1 \mathrm{H}), 6.52(\mathrm{~d}, J=20.0 \mathrm{~Hz}, 1 \mathrm{H}), 5.89(\mathrm{~s}, 1 \mathrm{H}), 3.87(\mathrm{~s}, 2 \mathrm{H}), 2.53$ (s, $2 \mathrm{H}), 2.45$ (s, 3H), 0.20 (s, 6H). 
$>{ }^{13} \mathrm{C} \mathrm{NMR}\left(100 \mathrm{MHz}, \mathrm{CDCl}_{3}\right) \delta 149.6,143.5,136.7,132.6,131.4,129.7,128.7,128.0,128.0$, $128.0,127.0,126.7,48.5,35.6,21.6,-3.1$.

$>\quad \mathrm{IR}$ (neat) $\mathrm{cm}^{-1} 3029,2958,1597,1566,1493,1340,1162,1089,1031,962,946$.

$>$ HRMS calcd for $\mathrm{C}_{21} \mathrm{H}_{25} \mathrm{NO}_{2} \mathrm{SSi}(\mathrm{M}+\mathrm{Na})^{+} 406.1273$, found 406.1273.

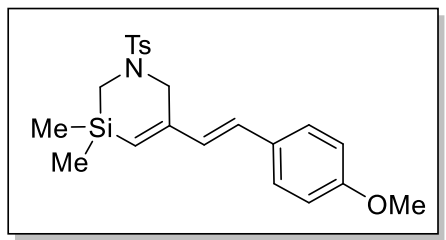

yellow solid (56 mg, 68\%).

$>$ mp: $155.4-157.1^{\circ} \mathrm{C}$.

$>\mathrm{R}_{f}=0.6($ EtOAc:Petroleum Ether $=1: 5)$.

$>{ }^{1} \mathrm{H}$ NMR $\left(400 \mathrm{MHz}, \mathrm{CDCl}_{3}\right) \delta 7.75(\mathrm{~d}, J=8.2 \mathrm{~Hz}, 2 \mathrm{H}), 7.36(\mathrm{dd}, J=8.3,5.5 \mathrm{~Hz}, 4 \mathrm{H}), 6.87(\mathrm{~d}$, $J=8.7 \mathrm{~Hz}, 2 \mathrm{H}), 6.59-6.45(\mathrm{~m}, 2 \mathrm{H}), 5.83(\mathrm{~s}, 1 \mathrm{H}), 3.85(\mathrm{~s}, 2 \mathrm{H}), 3.81(\mathrm{~s}, 3 \mathrm{H}), 2.52(\mathrm{~s}, 2 \mathrm{H}), 2.44$ $(\mathrm{s}, 3 \mathrm{H}), 0.19(\mathrm{~s}, 6 \mathrm{H})$

$>{ }^{13} \mathrm{C} \mathrm{NMR}\left(100 \mathrm{MHz}, \mathrm{CDCl}_{3}\right) \delta 159.6,149.8,143.5,132.6,129.7,129.4,129.4,128.0,127.9$, 127.5, 125.6, 114.2, 55.3, 48.5, 35.7, 21.6, -3.0.

$>\quad \mathrm{IR}$ (neat) $\mathrm{cm}^{-1} 2957,1603,1566,1510,1339,1162,1089,1031,963$.

$>$ HRMS calcd for $\mathrm{C}_{22} \mathrm{H}_{27} \mathrm{NO}_{3} \mathrm{SSi}(\mathrm{M}+\mathrm{Na})^{+} 436.1375$, found436.1379.

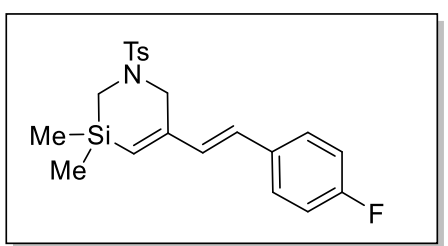

\section{(E)-5-(4-fluorostyryl)-3,3-dimethyl-1-tosyl-1,2,3,6-tetrahydro- 1,3-azasiline (4bs)}

4bs was prepared according to General Method C. 2a (58.4 mg, $0.20 \mathrm{mmol}, 1.0$ equiv.), $\mathrm{Pd}\left(\mathrm{PPh}_{3}\right)_{2} \mathrm{Cl}_{2}$ (7.2 $\mathrm{mg}, 0.01 \mathrm{mmol}, 5$ mol \%), $\mathrm{ZnI}_{2}$ (12.8 mg, $0.04 \mathrm{mmol}, 0.2$ equiv.), alkyne $\mathbf{S - A - 6 6}$ (35.4 mg, $0.24 \mathrm{mmol}, 1.2$ equiv.) and DBU (61 mg, $0.4 \mathrm{mmol}, 2.0$ equiv.) in xylenes $(0.5 \mathrm{~mL})$ at $120^{\circ} \mathrm{C}$ for $10 \mathrm{~min}$ afforded $4 \mathbf{b s}$ as yellow solid ( $49 \mathrm{mg}, 61 \%$ ).

$>\mathrm{mp:} 143.0-145.8^{\circ} \mathrm{C}$.

$>\mathrm{R}_{f}=0.7$ (EtOAc:Petroleum Ether $\left.=1: 5\right)$.

$>{ }^{1} \mathrm{H}$ NMR $\left(400 \mathrm{MHz}, \mathrm{CDCl}_{3}\right) \delta 7.75(\mathrm{~d}, J=8.0 \mathrm{~Hz}, 2 \mathrm{H}), 7.40-7.36(\mathrm{~m}, 4 \mathrm{H}), 7.02(\mathrm{t}, J=8.7$ $\mathrm{Hz}, 2 \mathrm{H}), 6.54(\mathrm{dd}, J=50.2,16.5 \mathrm{~Hz}, 2 \mathrm{H}), 5.88(\mathrm{~s}, 1 \mathrm{H}), 3.85(\mathrm{~s}, 2 \mathrm{H}), 2.53(\mathrm{~s}, 2 \mathrm{H}), 2.45(\mathrm{~s}, 3 \mathrm{H})$, $0.20(\mathrm{~s}, 6 \mathrm{H})$.

$>{ }^{13} \mathrm{C}$ NMR $\left(100 \mathrm{MHz}, \mathrm{CDCl}_{3}\right) \delta 162.6(\mathrm{~d}, J=246 \mathrm{~Hz}), 149.4,143.6,132.9(\mathrm{~d}, J=4 \mathrm{~Hz}), 132.6$, $131.2(\mathrm{~d}, J=2 \mathrm{~Hz}), 129.7,128.2(\mathrm{~d}, J=8 \mathrm{~Hz}), 128.0,127.1,126.7,115.7(\mathrm{~d}, J=21 \mathrm{~Hz}), 48.4$, $35.6,21.6,-3.1$.

$>{ }^{19} \mathrm{~F}$ NMR $\left(376 \mathrm{MHz}, \mathrm{CDCl}_{3}\right) \delta-113.56$.

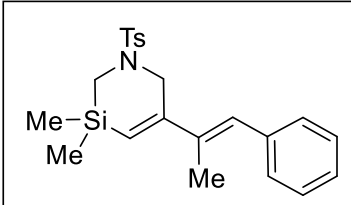

IR (neat) $\mathrm{cm}^{-1} 2960,1598,1507,1338,1160,1089,1031,946$.

$>\quad$ HRMS calcd for $\mathrm{C}_{21} \mathrm{H}_{24} \mathrm{FNO}_{2} \mathrm{SSi}(\mathrm{M}+\mathrm{Na})^{+} 424.1179$, found 424.1175 .

\section{(E)-3,3-dimethyl-5-(1-phenylprop-1-en-2-yl)-1-tosyl-1,2,3,6-tetrahy- dro-1,3-azasiline (4bt)}

4bt was prepared according to General Method C. 2a (58.4 mg, 0.20 mmol, 1.0 equiv.), $\mathrm{Pd}\left(\mathrm{PPh}_{3}\right)_{2} \mathrm{Cl}_{2}$ (7.2 mg, $\left.0.01 \mathrm{mmol}, 5 \mathrm{~mol} \%\right), \mathrm{ZnI}_{2}(12.8 \mathrm{mg}, 0.04 \mathrm{mmol}, 0.2$ equiv.), alkyne S-A-67 (34.0 mg, $0.24 \mathrm{mmol}, 1.2$ equiv.) and DBU (61 mg, $0.4 \mathrm{mmol}, 2.0$ equiv.) in xylenes $(0.5 \mathrm{~mL})$ at $120^{\circ} \mathrm{C}$ for $10 \mathrm{~min}$ afforded $\mathbf{4 b t}$ as yellow solid $(54 \mathrm{mg}, 68 \%)$.

$>$ mp: $95.2-97.3^{\circ} \mathrm{C}$.

$>\mathrm{R}_{f}=0.7$ (EtOAc:Petroleum Ether=1:5).

$>{ }^{1} \mathrm{H} \mathrm{NMR}\left(400 \mathrm{MHz}, \mathrm{CDCl}_{3}\right) \delta 7.73(\mathrm{~d}, J=8.0 \mathrm{~Hz}, 2 \mathrm{H}), 7.37-7.33(\mathrm{~m}, 4 \mathrm{H}), 7.26-7.23(\mathrm{~m}$, $3 \mathrm{H}), 6.54(\mathrm{~s}, 1 \mathrm{H}), 5.95(\mathrm{~s}, 1 \mathrm{H}), 3.89(\mathrm{~s}, 2 \mathrm{H}), 2.53(\mathrm{~s}, 2 \mathrm{H}), 2.44(\mathrm{~s}, 3 \mathrm{H}), 1.98(\mathrm{~s}, 3 \mathrm{H}), 0.21(\mathrm{~s}$, $6 \mathrm{H})$.

$>{ }^{13} \mathrm{C} \mathrm{NMR}\left(150 \mathrm{MHz}, \mathrm{CDCl}_{3}\right) \delta 153.5,143.4,137.8,137.4,132.8,129.6,129.2,128.1,128.0$, $126.7,126.5,121.7,49.7,35.5,21.5,15.9,-3.0$.

$>\mathrm{IR}$ (neat) $\mathrm{cm}^{-1} 2988,1597,1568,1340,1162,1089,1030,926$. 
$>\quad$ HRMS calcd for $\mathrm{C}_{22} \mathrm{H}_{27} \mathrm{NO}_{2} \mathrm{SSi}(\mathrm{M}+\mathrm{Na})^{+} 420.1429$, found 420.1427 .

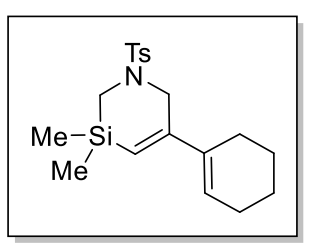

5-(cyclohex-1-en-1-yl)-3,3-dimethyl-1-tosyl-1,2,3,6-tetrahydro-1,3azasiline (4bu)

4bu was prepared according to General Method C. 2a (58.4 mg, $0.20 \mathrm{mmol}$, 1.0 equiv.), $\mathrm{Pd}\left(\mathrm{PPh}_{3}\right)_{2} \mathrm{Cl}_{2}(7.2 \mathrm{mg}, 0.01 \mathrm{mmol}, 5 \mathrm{~mol} \%), \mathrm{ZnI}_{2}(12.8 \mathrm{mg}, 0.04$ mmol, 0.2 equiv.), alkyne $\mathbf{S - A - 6 8}(26.0 \mathrm{mg}, 0.24 \mathrm{mmol}, 1.2$ equiv.) and $\mathrm{DBU}$ (61 mg, $0.4 \mathrm{mmol}, 2.0$ equiv.) in xylenes $(0.5 \mathrm{~mL})$ at $120^{\circ} \mathrm{C}$ for $10 \mathrm{~min}$ afforded $\mathbf{4 b u}$ as white solid ( $45 \mathrm{mg}, 63 \%$ ).

$>\mathrm{mp}: 128.8-130.2^{\circ} \mathrm{C}$

$>\mathrm{R}_{f}=0.6$ (EtOAc:Petroleum Ether $\left.=1: 10\right)$.

$>\quad{ }^{1} \mathrm{H} \mathrm{NMR}\left(400 \mathrm{MHz}, \mathrm{CDCl}_{3}\right) \delta 7.70(\mathrm{~d}, J=8.0 \mathrm{~Hz}, 2 \mathrm{H}), 7.34(\mathrm{~d}, J=8.0 \mathrm{~Hz}, 2 \mathrm{H}), 5.85-5.82$ $(\mathrm{m}, 1 \mathrm{H}), 5.70(\mathrm{~s}, 1 \mathrm{H}), 3.75(\mathrm{~d}, J=1.44 \mathrm{~Hz}, 2 \mathrm{H}), 2.47(\mathrm{~s}, 2 \mathrm{H}), 2.43(\mathrm{~s}, 3 \mathrm{H}), 2.17-2.11(\mathrm{~m}, 4 \mathrm{H})$, $1.68-1.63(\mathrm{~m}, 2 \mathrm{H}), 1.58-1.53(\mathrm{~m}, 2 \mathrm{H}), 0.16(\mathrm{~s}, 6 \mathrm{H})$.

$>{ }^{13} \mathrm{C} \mathrm{NMR}\left(100 \mathrm{MHz}, \mathrm{CDCl}_{3}\right) \delta 152.3,143.3,136.4,132.8,129.6,128.0,124.7,117.7,49.4$, $35.7,26.0,25.8,22.9,22.0,21.5,-2.9$.

$>\mathrm{IR}$ (neat) $\mathrm{cm}^{-1} 2926,2857,1571,1445,1339,1161,1089,1031,953$.

$>$ HRMS calcd for $\mathrm{C}_{19} \mathrm{H}_{27} \mathrm{NO}_{2} \mathrm{SSi}(\mathrm{M}+\mathrm{Na})^{+} 384.1429$, found 384.1427 .

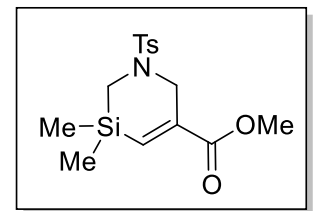

methyl 3,3-dimethyl-1-tosyl-1,2,3,6-tetrahydro-1,3-azasiline-5-carboxylate (4bw)

In glovebox, 2a ( $0.2 \mathrm{mmol}, 1.0$ equiv.) and DBU (34 $\mathrm{mg}, 0.22 \mathrm{mmol}$, 1.1equiv.) were dissolved in xylenes $(0.5 \mathrm{~mL})$ in sealed tube-I, and alkyne S-A-69 (40 mg, $0.48 \mathrm{mmol}, 2.4$ equiv.), $\mathrm{PdCp}\left(\eta^{3}-\mathrm{C}_{3} \mathrm{H}_{5}\right)(2 \mathrm{mg}, 0.01 \mathrm{mmol}$, $5 \mathrm{~mol} \%$ ), $\mathrm{PPh}_{3}(5.3 \mathrm{mg}, 0.02 \mathrm{mmol}, 10 \mathrm{~mol} \%$ ) were dissolved in xylenes $(0.25 \mathrm{~mL})$ in sealed tube-II. Two tubes were sealed and removed from glovebox. The mixture in sealed tube-I was stirred at $120^{\circ} \mathrm{C}$ for $10 \mathrm{~min}$, and the mixture in sealed tube-II was stirred at $60{ }^{\circ} \mathrm{C}$ for $10 \mathrm{~min}$. The solution in in sealed tube-II was added into the sealed tube-I. The resulting mixture was stirred at $120^{\circ} \mathrm{C}$ for $5 \mathrm{~min}$. The mixture in the sealed tube-I was cooled to room temperature and concentrated under reduced pressure. The residue was purified by silica gel flash column chromatography (eluent: petroleum ether/EtOAc $=15 / 1$ ) to afford the product $\mathbf{4 b w}$ as white solid (22 mg, 32\%).

$>\mathrm{mp}: 108.3-110.6^{\circ} \mathrm{C}$.

$>\mathrm{R}_{f}=0.4$ (EtOAc:Petroleum Ether=1:5).

$>{ }^{1} \mathrm{H} \mathrm{NMR}\left(400 \mathrm{MHz}, \mathrm{CDCl}_{3}\right) \delta 7.71(\mathrm{~d}, J=8.0 \mathrm{~Hz}, 2 \mathrm{H}), 7.35(\mathrm{~d}, J=8.0 \mathrm{~Hz}, 2 \mathrm{H}), 7.09(\mathrm{t}, J=$ $2.0 \mathrm{~Hz}, 1 \mathrm{H}), 3.83(\mathrm{~d}, J=2.0 \mathrm{~Hz}, 2 \mathrm{H}), 3.76(\mathrm{~s}, 3 \mathrm{H}), 2.51(\mathrm{~s}, 2 \mathrm{H}), 2.44(\mathrm{~s}, 3 \mathrm{H}), 0.23(\mathrm{~s}, 6 \mathrm{H})$.

$>{ }^{13} \mathrm{CNMR}\left(150 \mathrm{MHz}, \mathrm{CDCl}_{3}\right) \delta 165.1,143.6,143.4,137.2,132.5,129.6,128.0,52.2,48.1,35.0$, 21.5, -3.8.

$>$ IR (neat) $\mathrm{cm}^{-1} 2955,1711,1599,1437,1342,1276,1216,1162,1090,1014,923$.

$>$ HRMS calcd for $\mathrm{C}_{15} \mathrm{H}_{21} \mathrm{NO}_{4} \mathrm{SSi}(\mathrm{M}+\mathrm{Na})^{+} 362.0858$, found 362.0857 .

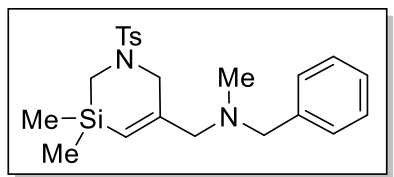

N-benzyl-1-(3,3-dimethyl-1-tosyl-1,2,3,6-tetrahydro-1,3-azasilin-5yl)-N-methylmethanamine (4bx)

4bx was prepared according to General Method C. 2a (58.4 mg, 0.20 mmol, 1.0 equiv.), $\mathrm{Pd}\left(\mathrm{PPh}_{3}\right)_{2} \mathrm{Cl}_{2}$ (7.2 $\left.\mathrm{mg}, 0.01 \mathrm{mmol}, 5 \mathrm{~mol} \%\right), \mathrm{ZnI}_{2}$ (12.8 mg, $0.04 \mathrm{mmol}, 0.2$ equiv.), alkyne S-A-70 (38.2 mg, $0.24 \mathrm{mmol}$, 1.2 equiv.) and $\mathrm{DBU}(61 \mathrm{mg}, 0.4 \mathrm{mmol}, 2.0$ equiv. $)$ in xylenes $(0.5 \mathrm{~mL})$ at $120^{\circ} \mathrm{C}$ for $10 \mathrm{~min}$ afforded 4bx as yellow oil ( $50 \mathrm{mg}, 60 \%)$.

$>\mathrm{R}_{f}=0.5$ (EtOAc:Petroleum Ether=1:5).

$>{ }^{1} \mathrm{H} \mathrm{NMR}\left(400 \mathrm{MHz}, \mathrm{CDCl}_{3}\right) \delta 7.70(\mathrm{~d}, J=8.0 \mathrm{~Hz}, 2 \mathrm{H}), 7.35-7.25(\mathrm{~m}, 7 \mathrm{H}), 5.71(\mathrm{~s}, 1 \mathrm{H}), 3.56$ $(\mathrm{s}, 2 \mathrm{H}), 3.40(\mathrm{~s}, 2 \mathrm{H}), 2.93(\mathrm{~d}, J=1.28 \mathrm{~Hz}, 2 \mathrm{H}), 2.45(\mathrm{~s}, 2 \mathrm{H}), 2.44(\mathrm{~s}, 3 \mathrm{H}), 2.11(\mathrm{~s}, 3 \mathrm{H}), 0.14(\mathrm{~s}$, $6 \mathrm{H})$.

$>{ }^{13} \mathrm{C} \mathrm{NMR}\left(150 \mathrm{MHz}, \mathrm{CDCl}_{3}\right) \delta 153.1,143.3,139.2,132.4,129.5,128.8,128.2,128.1,127.0$, $121.8,65.9,61.7,50.3,42.3,35.6,21.5,-3.0$.

$>\mathrm{IR}$ (neat) $\mathrm{cm}^{-1} 2953,2791,1620,1493,1450,1342,1260,1163,1090,1028,9881$.

$>$ HRMS calcd for $\mathrm{C}_{22} \mathrm{H}_{30} \mathrm{~N}_{2} \mathrm{O}_{2} \mathrm{SSi}(\mathrm{M}+\mathrm{H})^{+} 415.1876$, found 415.1866. 


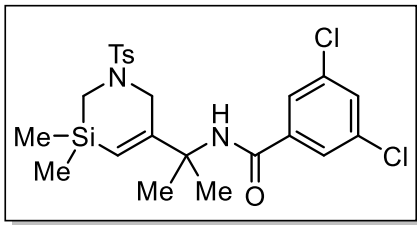

(60 mg, 61\%).

mp: $131.9-134.4^{\circ} \mathrm{C}$.

$\mathrm{R}_{f}=0.5($ EtOAc:Petroleum Ether $=1: 5)$

$>{ }^{1} \mathrm{H} \mathrm{NMR}\left(400 \mathrm{MHz}, \mathrm{CDCl}_{3}\right) \delta 7.56(\mathrm{~d}, J=8.0 \mathrm{~Hz}, 2 \mathrm{H}), 7.54(\mathrm{~d}, J=1.88 \mathrm{~Hz}, 2 \mathrm{H}), 7.40(\mathrm{t}, J=$ $1.84 \mathrm{~Hz}, 1 \mathrm{H}), 7.21(\mathrm{~d}, J=8.0 \mathrm{~Hz}, 2 \mathrm{H}), 6.35(\mathrm{~s}, 1 \mathrm{H}), 5.73(\mathrm{~s}, 1 \mathrm{H}), 3.53(\mathrm{~s}, 2 \mathrm{H}), 2.45(\mathrm{~s}, 2 \mathrm{H})$, $2.39(\mathrm{~s}, 3 \mathrm{H}), 1.56(\mathrm{~s}, 6 \mathrm{H}), 0.17(\mathrm{~s}, 6 \mathrm{H})$.

$>\quad{ }^{13} \mathrm{C} \mathrm{NMR}\left(100 \mathrm{MHz}, \mathrm{CDCl}_{3}\right) \delta 164.5,157.9,143.7,138.1,135.4,131.8,131.1,129.5,127.8$, $125.6,117.7,58.4,48.4,35.6,27.3,21.5,-2.7$.

$>\quad \mathrm{IR}$ (neat) $\mathrm{cm}^{-1} 3346,2987,1648,1528,1160,1090,1035,930$.

$>\quad$ HRMS calcd for $\mathrm{C}_{23} \mathrm{H}_{28} \mathrm{Cl}_{2} \mathrm{~N}_{2} \mathrm{O}_{3} \mathrm{SSi}(\mathrm{M}+\mathrm{Na})^{+} 533.0865$, found 533.0859.

N-(3-(3,3-dimethyl-1-tosyl-1,2,3,6-tetrahydro-1,3-azasilin-5-yl)phenyl)-6,7-bis(2-methoxyethox y)quinazolin-4-amine (4bz)

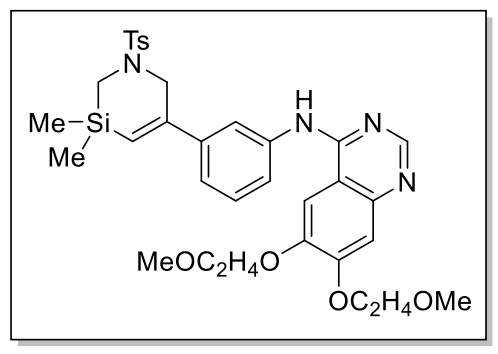

4bz was prepared according to General Method C. 2a (58.4 mg, $0.2 \mathrm{mmol}, 2.0$ equiv.), $\mathrm{Pd}\left(\mathrm{PPh}_{3}\right)_{2} \mathrm{Cl}_{2}$ (7.2 mg, $0.01 \mathrm{mmol}, 10$ mol \%), $\mathrm{ZnI}_{2}$ (6.4 mg, $0.02 \mathrm{mmol}, 0.2$ equiv.), alkyne $\mathbf{S}-\mathbf{A}-\mathbf{7 2}$ (40.0 mg, $0.1 \mathrm{mmol}, 1.0$ equiv.) and DBU (31.8 $\mathrm{mg}, 0.2 \mathrm{mmol}$, 2.0 equiv. $)$ in xylenes $(0.5 \mathrm{~mL})$ at $120{ }^{\circ} \mathrm{C}$ for $10 \mathrm{~min}$ afforded $\mathbf{4 b z}$ as yellow solid (47.6 mg, $73 \%)$.

$>$ mp: $78.9-81.8^{\circ} \mathrm{C}$.

$>\mathrm{R}_{f}=0.7\left(\mathrm{MeOH}: \mathrm{CH}_{2} \mathrm{Cl}_{2}=1: 10\right)$.

${ }^{1} \mathrm{H}$ NMR $\left(400 \mathrm{MHz}, \mathrm{DMSO}-d_{6}\right) \delta 9.46(\mathrm{~s}, 1 \mathrm{H}), 8.42(\mathrm{~s}, 1 \mathrm{H})$ $7.83(\mathrm{~d}, J=7.7 \mathrm{~Hz}, 2 \mathrm{H}), 7.75-7.65(\mathrm{~m}, 3 \mathrm{H}), 7.42(\mathrm{~d}, J=7.9 \mathrm{~Hz}, 2 \mathrm{H}), 7.36(\mathrm{~d}, J=8.0 \mathrm{~Hz}, 1 \mathrm{H})$, $7.18(\mathrm{~s}, 1 \mathrm{H}), 7.09(\mathrm{~d}, J=7.9 \mathrm{~Hz}, 1 \mathrm{H}), 6.13(\mathrm{~s}, 1 \mathrm{H}), 4.25(\mathrm{~s}, 4 \mathrm{H}), 3.87(\mathrm{~s}, 2 \mathrm{H}), 3.72(\mathrm{~d}, J=15.0$ $\mathrm{Hz}, 4 \mathrm{H}), 3.32$ (s, 6H), 2.47 (s, 2H), 2.36 (s, 3H), 0.15 (s, 6H).

$>\quad{ }^{13} \mathrm{C} \mathrm{NMR}\left(150 \mathrm{MHz}, \mathrm{DMSO}-d_{6}\right) \delta 156.8,154.1,153.3,152.5,148.5,147.4,144.0,141.6,140.1$, $132.6,130.3,129.1,128.2,123.4,122.6,120.6,119.4,109.4,108.7,103.8,70.6,70.5,68.9$, $68.5,58.8,58.8,50.8,35.6,21.5,-2.6$.

$>\quad \mathrm{IR}$ (neat) $\mathrm{cm}^{-1} 2926,1620,1575,1505,1430,1391,1157,1090,1033,924$.

$>$ HRMS calcd for $\mathrm{C}_{33} \mathrm{H}_{40} \mathrm{~N}_{4} \mathrm{O}_{6} \mathrm{SSi}(\mathrm{M}+\mathrm{Na})^{+}$671.2336, found 671.2338.

\section{2-(3-cyano-4-isobutoxyphenyl)-N-((3,3-dimethyl-1-tosyl-1,2,3,6-tetrahydro-1,3-azasilin-5-} yl)methyl)-4-methylthiazole-5-carboxamide (4ca)

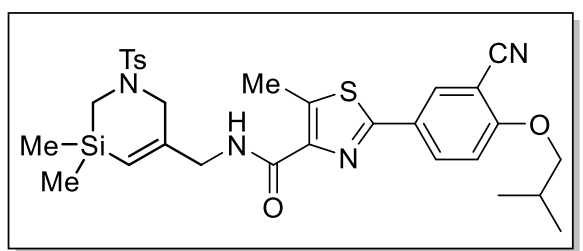

4ca was prepared according to General Method C. 2a (58.4 mg, $0.2 \mathrm{mmol}, 2.0$ equiv.), $\mathrm{Pd}\left(\mathrm{PPh}_{3}\right)_{2} \mathrm{Cl}_{2}(7.2 \mathrm{mg}$, $0.01 \mathrm{mmol}, 10 \mathrm{~mol} \%), \mathrm{ZnI}_{2}(6.4 \mathrm{mg}, 0.02 \mathrm{mmol}, 0.2$ equiv.), alkyne S-A-73 (34.0 mg, $0.1 \mathrm{mmol}, 1.0$ equiv.) and DBU (31.8 mg, $0.2 \mathrm{mmol}, 2.0$ equiv.) in xylenes $(0.5$ $\mathrm{mL})$ at $120{ }^{\circ} \mathrm{C}$ for $10 \mathrm{~min}$ afforded $4 \mathbf{c a}$ as yellow solid (35 mg, 57\%).

$>\quad m p: 115.3-117.6^{\circ} \mathrm{C}$.

$>\mathrm{R}_{f}=0.5$ (EtOAc:Petroleum Ether $\left.=1: 1\right)$.

$>{ }^{1} \mathrm{H}$ NMR $\left(400 \mathrm{MHz}, \mathrm{CDCl}_{3}\right) \delta 8.15(\mathrm{~d}, J=2.3 \mathrm{~Hz}, 1 \mathrm{H}), 8.07(\mathrm{dd}, J=8.8,2.3 \mathrm{~Hz}, 1 \mathrm{H}), 7.67(\mathrm{~d}$, $J=8.0 \mathrm{~Hz}, 2 \mathrm{H}), 7.34(\mathrm{~d}, J=7.9 \mathrm{~Hz}, 2 \mathrm{H}), 7.02(\mathrm{~d}, J=8.9 \mathrm{~Hz}, 1 \mathrm{H}), 5.99(\mathrm{t}, J=6.0 \mathrm{~Hz}, 1 \mathrm{H})$, $5.70(\mathrm{~s}, 1 \mathrm{H}), 4.00(\mathrm{~d}, J=6.1 \mathrm{~Hz}, 2 \mathrm{H}), 3.90(\mathrm{~d}, J=6.5 \mathrm{~Hz}, 2 \mathrm{H}), 3.53(\mathrm{~s}, 2 \mathrm{H}), 2.74(\mathrm{~s}, 3 \mathrm{H}), 2.50$ $(\mathrm{s}, 2 \mathrm{H}), 2.44(\mathrm{~s}, 3 \mathrm{H}), 2.21(\mathrm{dt}, J=13.3,6.7 \mathrm{~Hz}, 1 \mathrm{H}), 1.10(\mathrm{~s}, 3 \mathrm{H}), 1.08(\mathrm{~s}, 3 \mathrm{H}), 0.17(\mathrm{~s}, 6 \mathrm{H})$.

$>{ }^{13} \mathrm{C}$ NMR $\left(100 \mathrm{MHz}, \mathrm{CDCl}_{3}\right) \delta 164.7,162.4,161.6,156.7,150.6,143.7,132.5,132.1,132.0$, $129.7,128.0,125.9,125.2,120.2,115.4,112.7,103.0,75.7,49.6,45.6,35.5,28.2,21.6,19.1$, $17.5,-3.0$

$>\quad$ IR (neat) $\mathrm{cm}^{-1} 2925,2228,1641,1605,1504,1439,1335,1162,1090,1012$.

$>$ HRMS calcd for $\mathrm{C}_{30} \mathrm{H}_{36} \mathrm{~N}_{4} \mathrm{O}_{4} \mathrm{~S}_{2} \mathrm{Si}(\mathrm{M}+\mathrm{Na})^{+}$631.1845, found 631.1834. 
1-cvclopropvl-N-((3,3-dimethvl-1-tosvl-1,2,3,6-tetrahvdro-1,3-azasilin-5-yl)methvl)-7-(4ethylpiperazin-1-yl)-6-fluoro-4-oxo-1,4-dihydroquinoline-3-carboxamide (4cb)<smiles>CCN1CCN(c2cc3c(cc2F)c(=O)c(C(=O)NCC2=CS(C)(C)C[N]C2)cn3C2CC2)CC1</smiles>

$>$ mp: $76.1-79.4^{\circ} \mathrm{C}$

$\mathrm{R}_{f}=0.5\left(\mathrm{CH}_{2} \mathrm{Cl}_{2}: \mathrm{MeOH}=10: 1\right)$.

$>{ }^{1} \mathrm{H}$ NMR $\left(400 \mathrm{MHz}, \mathrm{CDCl}_{3}\right) \delta 10.09(\mathrm{t}, J=6.1 \mathrm{~Hz}, 1 \mathrm{H}), 8.67(\mathrm{~s}, 1 \mathrm{H}), 7.90(\mathrm{~d}, J=13.3 \mathrm{~Hz}$, $1 \mathrm{H}), 7.54(\mathrm{~d}, J=7.9 \mathrm{~Hz}, 2 \mathrm{H}), 7.20(\mathrm{t}, J=7.2 \mathrm{~Hz}, 3 \mathrm{H}), 5.59(\mathrm{~s}, 1 \mathrm{H}), 3.88(\mathrm{~d}, J=6.0 \mathrm{~Hz}, 2 \mathrm{H})$, $3.40(\mathrm{~s}, 2 \mathrm{H}), 3.34(\mathrm{p}, J=3.2 \mathrm{~Hz}, 1 \mathrm{H}), 3.21(\mathrm{t}, J=4.8 \mathrm{~Hz}, 4 \mathrm{H}), 2.57(\mathrm{t}, J=4.8 \mathrm{~Hz}, 4 \mathrm{H}), 2.40$ $(\mathrm{q}, J=7.2 \mathrm{~Hz}, 2 \mathrm{H}), 2.33(\mathrm{~s}, 2 \mathrm{H}), 2.30(\mathrm{~s}, 3 \mathrm{H}), 1.20(\mathrm{~d}, J=6.8 \mathrm{~Hz}, 2 \mathrm{H}), 1.06-1.01(\mathrm{~m}, 5 \mathrm{H})$, $0.00(\mathrm{~s}, 6 \mathrm{H})$.

$>{ }^{13} \mathrm{C}$ NMR $\left(100 \mathrm{MHz}, \mathrm{CDCl}_{3}\right) \delta 175.6(\mathrm{~d}, J=2 \mathrm{~Hz}), 165.1,153.5(\mathrm{~d}, J=248 \mathrm{~Hz}), 151.1,146.9$, $145.0(\mathrm{~d}, J=11 \mathrm{~Hz}), 143.4,138.5,132.2,129.6,128.1,121.7(\mathrm{~d}, J=8 \mathrm{~Hz}), 117.9,112.6(\mathrm{~d}, J$ $=23 \mathrm{~Hz}), 111.0,104.7(\mathrm{~d}, J=3 \mathrm{~Hz}), 52.5,52.3,49.9,49.9,44.4,35.5,34.7,21.5,11.9,8.2,-$ 3.0.

$>{ }^{19} \mathrm{~F} \mathrm{NMR}(376 \mathrm{MHz}, \mathrm{CDCl} 3) \delta-122.97$.

$>$ IR (neat) $\mathrm{cm}^{-1}$ 2926, 2815, 1663, 1478, 1336, 1161, 1025,950.

$>$ HRMS calcd for $\mathrm{C}_{33} \mathrm{H}_{42} \mathrm{FN}_{5} \mathrm{O}_{4} \mathrm{SSi}(\mathrm{M}+\mathrm{H})^{+} 652.2789$, found 652.2785 .

3-((3,3-dimethyl-1-tosyl-1,2,3,6-tetrahydro-1,3-azasilin-5-yl)methyl)-5-(4-(2-(5-ethylpyridin-2vl)ethoxv)benzyl)thiazolidine-2,4-dione (4cc)

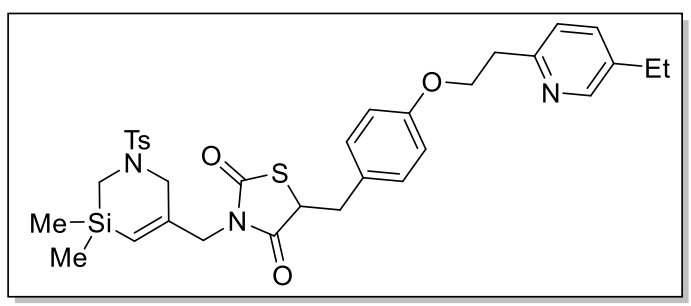

4cc was prepared according to General Method $\mathrm{C}$. 2a (58.4 mg, $0.2 \mathrm{mmol}, 2.0$ equiv.), $\mathrm{Pd}\left(\mathrm{PPh}_{3}\right)_{2} \mathrm{Cl}_{2}$ (7.2 $\mathrm{mg}, 0.01 \mathrm{mmol}, 10 \mathrm{~mol} \%), \mathrm{ZnI}_{2}(6.4 \mathrm{mg}$, $0.02 \mathrm{mmol}, 0.2$ equiv.), alkyne S-A-75 (40.0 mg, $0.1 \mathrm{mmol}, 1.0$ equiv.) and DBU (31.8 $\mathrm{mg}, 0.2$ mmol, 2.0 equiv. $)$ in xylenes $(0.5 \mathrm{~mL})$ at $120^{\circ} \mathrm{C}$ for $20 \mathrm{~min}$ afforded $\mathbf{4 c c}$ as white solid $(42 \mathrm{mg}$, $70 \%)$.

$>\mathrm{mp}: 63.5-64.4^{\circ} \mathrm{C}$.

$>\mathrm{R}_{f}=0.4$ (EtOAc:Petroleum Ether=1:1).

$>{ }^{1} \mathrm{H} \mathrm{NMR}\left(400 \mathrm{MHz}, \mathrm{CDCl}_{3}\right) \delta 8.39(\mathrm{~d}, J=2.28 \mathrm{~Hz}, 1 \mathrm{H}), 7.65(\mathrm{~d}, J=8.0 \mathrm{~Hz}, 2 \mathrm{H}), 7.45(\mathrm{dd}, J$ $=7.88 \mathrm{~Hz}, J=2.04 \mathrm{~Hz}, 1 \mathrm{H}), 7.33(\mathrm{~d}, J=8.0 \mathrm{~Hz}, 2 \mathrm{H}), 7.18(\mathrm{~d}, J=8.0 \mathrm{~Hz}, 1 \mathrm{H}), 7.11(\mathrm{~d}, J=$ $8.52 \mathrm{~Hz}, 2 \mathrm{H}), 6.82(\mathrm{~d}, J=8.52 \mathrm{~Hz}, 2 \mathrm{H}), 5.30(\mathrm{t}, J=1.72 \mathrm{~Hz}, 1 \mathrm{H}), 4.48(\mathrm{dd}, J=9.12 \mathrm{~Hz}, J=$ $3.88 \mathrm{~Hz}, 1 \mathrm{H}), 4.31(\mathrm{t}, J=6.68 \mathrm{~Hz}, 2 \mathrm{H}), 4.09-3.97(\mathrm{~m}, 2 \mathrm{H}), 3.51-3.38(\mathrm{~m}, 3 \mathrm{H}), 3.21(\mathrm{t}, J=$ $6.64 \mathrm{~Hz}, 2 \mathrm{H}), 3.11-3.05(\mathrm{~m}, 1 \mathrm{H}), 2.63(\mathrm{q}, J=7.64 \mathrm{~Hz}, 2 \mathrm{H}), 2.50-2.35(\mathrm{~m}, 5 \mathrm{H}), 1.26-1.23$ (m, 3H), $0.13(\mathrm{~s}, 3 \mathrm{H}), 0.09(\mathrm{~s}, 3 \mathrm{H})$.

$>{ }^{13} \mathrm{C} \mathrm{NMR}\left(100 \mathrm{MHz}, \mathrm{CDCl}_{3}\right) \delta 173.4,170.6,158.3,155.6,149.1,146.5,143.6,137.1,135.8$, $132.0,130.5,129.7,128.0,127.5,123.3,120.8,114.8,67.3,51.9,49.7,46.3,37.7,37.6,35.2$, $25.7,21.6,15.4,-3.1,-3.3$.

$>\mathrm{IR}$ (neat) $\mathrm{cm}^{-1} 2961,2925,1751,1683,1511,1338,1163,1089,1029,986$.

$>$ HRMS calcd for $\mathrm{C}_{33} \mathrm{H}_{39} \mathrm{~N}_{3} \mathrm{O}_{5} \mathrm{~S}_{2} \mathrm{Si}(\mathrm{M}+\mathrm{Na})^{+} 672.1998$, found 672.2013 .

\section{5-((((3S,8S,9S,10R,13R,14S)-10,13-dimethyl-17-(6-methylheptan-2-yl)-}

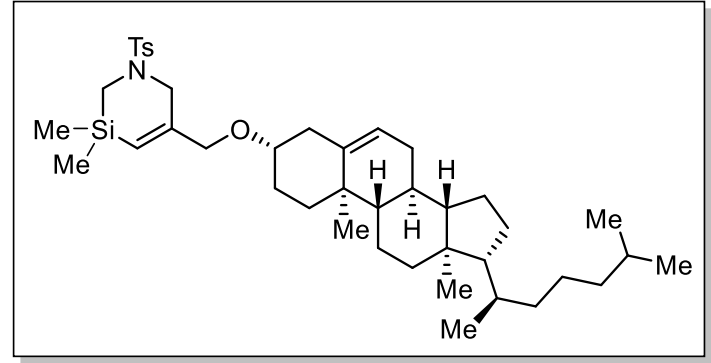

$\underline{2,3,4,7,8,9,10,11,12,13,14,15,16,17-}$ tetradecahydro-1H-cyclopenta/a/phenanthren3-yl)oxy)methyl)-3,3-dimethyl-1-tosyl-1,2,3,6tetrahydro-1,3-azasiline (4cd)

4cd was prepared according to General Method C. $2 \mathrm{a}$ (58.4 mg, $0.2 \mathrm{mmol}, 2.0$ equiv.), $\mathrm{Pd}\left(\mathrm{PPh}_{3}\right)_{2} \mathrm{Cl}_{2}$ (3.5 mg, $0.005 \mathrm{mmol}, 5 \mathrm{~mol} \%$ ), $\mathrm{ZnI}_{2}(6.4 \mathrm{mg}, 0.02 \mathrm{mmol}, 0.2$ equiv.), alkyne SA-76 (43.4 mg, $0.1 \mathrm{mmol}, 1.0$ equiv.) and DBU 
(31.8 mg, $0.2 \mathrm{mmol}, 2.0$ equiv.) in xylenes $(0.5 \mathrm{~mL})$ at $120{ }^{\circ} \mathrm{C}$ for $10 \mathrm{~min}$ afforded $\mathbf{4 c d}$ as white solid (44 mg, 64\%).

$>$ mp: $52.4-54.3^{\circ} \mathrm{C}$.

$>[\alpha]^{25} \mathrm{D}=-12.6^{\circ}\left(c=0.35, \mathrm{CHCl}_{3}\right)$.

$>\mathrm{R}_{f}=0.6($ EtOAc:Petroleum Ether $=1: 5)$.

$>{ }^{1} \mathrm{H} \mathrm{NMR}\left(400 \mathrm{MHz}, \mathrm{CDCl}_{3}\right) \delta 7.68(\mathrm{~d}, J=8.0 \mathrm{~Hz}, 2 \mathrm{H}), 7.33(\mathrm{~d}, J=8.0 \mathrm{~Hz}, 2 \mathrm{H}), 5.78(\mathrm{~s}, 1 \mathrm{H})$, $5.34-5.32(\mathrm{~m}, 1 \mathrm{H}), 3.93(\mathrm{~s}, 2 \mathrm{H}), 3.55-3.45(\mathrm{~m}, 2 \mathrm{H}), 3.18-3.10(\mathrm{~m}, 1 \mathrm{H}), 2.56-2.47(\mathrm{~m}$, $2 \mathrm{H}), 2.43(\mathrm{~s}, 3 \mathrm{H}), 2.36-2.31(\mathrm{~m}, 1 \mathrm{H}), 2.23-2.17(\mathrm{~m}, 1 \mathrm{H}), 2.04-1.94(\mathrm{~m}, 2 \mathrm{H}), 1.90-1.78$ (m, 3H), $1.61-1.41(\mathrm{~m}, 7 \mathrm{H}), 1.38-1.26(\mathrm{~m}, 5 \mathrm{H}), 1.19-1.08(\mathrm{~m}, 5 \mathrm{H}), 1.03-1.00(\mathrm{~m}, 6 \mathrm{H})$, $0.91(\mathrm{~d}, J=4.0 \mathrm{~Hz}, 4 \mathrm{H}), 0.87-0.86(\mathrm{~m}, 6 \mathrm{H}), 0.68(\mathrm{~s}, 3 \mathrm{H}), 0.16(\mathrm{~s}, 3 \mathrm{H}), 0.15(\mathrm{~s}, 3 \mathrm{H})$.

$>{ }^{13} \mathrm{C}$ NMR $\left(100 \mathrm{MHz}, \mathrm{CDCl}_{3}\right) \delta 152.1,143.4,140.8,132.3,129.5,128.1,121.8,120.1,79.1$, $72.2,56.8,56.2,50.2,49.1,42.3,39.8,39.5,39.1,37.2,36.9,36.2,35.8,35.6,32.0,31.9,28.4$, $28.3,28.0,24.3,23.8,22.8,22.6,21.5,21.1,19.4,18.7,11.9,-3.0,-3.0$.

$>$ IR (neat) $\mathrm{cm}^{-1} 2933,2866,1625,1598,1463,1440,1343,1164,1124,936,907$.

$>$ HRMS calcd for $\mathrm{C}_{41} \mathrm{H}_{65} \mathrm{NO}_{3} \mathrm{SSi}(\mathrm{M}+\mathrm{Na})^{+} 702.4352$, found 702.4350 .

(8R,9S,13S,14S)-3-(3,3-dimethyl-1-tosyl-1,2,3,6-tetrahydro-1,3-azasilin-5-yl)-13-methyl6,7,8,9,11,12,13,14,15,16-decahydro-17H-cyclopenta/alphenanthren-17-one (4ce)

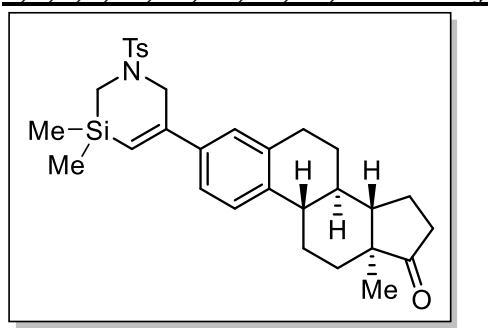

4ce was prepared according to General Method C. 2a (58.4 mg, 0.20 mmol, 1.0 equiv.), $\mathrm{Pd}\left(\mathrm{PPh}_{3}\right)_{2} \mathrm{Cl}_{2}(7.2 \mathrm{mg}, 0.01 \mathrm{mmol}, 5$ mol \%), $\mathrm{ZnI}_{2}(12.8 \mathrm{mg}, 0.04 \mathrm{mmol}, 0.2$ equiv.), alkyne S-A-77 (66.8 mg, $0.24 \mathrm{mmol}, 1.2$ equiv.) and DBU (61 mg, $0.4 \mathrm{mmol}, 2.0$ equiv.) in xylenes $(0.5 \mathrm{~mL})$ at $120^{\circ} \mathrm{C}$ for 10 min afforded 4 ce as yellow solid (61 mg, $57 \%)$.

mp: $206.8-208.1^{\circ} \mathrm{C}$.

$>\quad[\alpha]^{25} \mathrm{D}=+74.3^{\circ}\left(c=0.35, \mathrm{CHCl}_{3}\right)$.

$>\mathrm{R}_{f}=0.4$ (EtOAc:Petroleum Ether $\left.=1: 5\right)$.

$>{ }^{1} \mathrm{H}$ NMR $\left(400 \mathrm{MHz}, \mathrm{CDCl}_{3}\right) \delta 7.70(\mathrm{~d}, J=8.0 \mathrm{~Hz}, 2 \mathrm{H}), 7.33(\mathrm{~d}, J=8.0 \mathrm{~Hz}, 2 \mathrm{H}), 7.27-7.25$ $(\mathrm{m}, 2 \mathrm{H}), 7.13-7.08(\mathrm{~m}, 2 \mathrm{H}), 5.96(\mathrm{t}, J=1.8 \mathrm{~Hz}, 1 \mathrm{H}), 3.92(\mathrm{~d}, J=1.8 \mathrm{~Hz}, 2 \mathrm{H}), 2.92(\mathrm{dd}, J=$ 9.0, $4.2 \mathrm{~Hz}, 2 \mathrm{H}), 2.55-2.48(\mathrm{~m}, 3 \mathrm{H}), 2.45-2.27(\mathrm{~m}, 4 \mathrm{H}), 2.20-1.95(\mathrm{~m}, 4 \mathrm{H}), 1.69-1.40(\mathrm{~m}$, $6 \mathrm{H}), 0.91(\mathrm{~s}, 3 \mathrm{H}), 0.22(\mathrm{~s}, 6 \mathrm{H})$.

$>{ }^{13} \mathrm{C} \mathrm{NMR}\left(100 \mathrm{MHz}, \mathrm{CDCl}_{3}\right) \delta 220.7,152.9,143.4,140.0,139.4,136.6,132.6,129.6,128.0$, $126.4,125.5,123.4,122.4,50.8,50.5,48.0,44.4,38.1,35.8,35.3,31.6,29.5,26.5,25.7,21.6$, 21.5, 13.9, -2.9.

$>\quad \mathrm{IR}$ (neat) $\mathrm{cm}^{-1} 2927,1737,1596,1405,1341,1162,1088,1035,936$.

$>$ HRMS calcd for $\mathrm{C}_{31} \mathrm{H}_{39} \mathrm{NO}_{3} \mathrm{SSi}(\mathrm{M}+\mathrm{Na})^{+}$556.2312, found 556.2316.

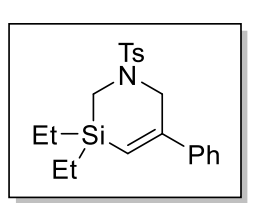

\section{3,3-diethyl-5-phenyl-1-tosyl-1,2,3,6-tetrahydro-1,3-azasiline (4cf)}

4cf was prepared according to General Method C. 2e (64 mg, $0.2 \mathrm{mmol}, 1.0$ equiv.), $\mathrm{Pd}\left(\mathrm{PPh}_{3}\right)_{2} \mathrm{Cl}_{2}$ (7.2 $\mathrm{mg}, 0.01 \mathrm{mmol}, 5 \mathrm{~mol} \%$ ), $\mathrm{ZnI}_{2}(12.8 \mathrm{mg}, 0.04 \mathrm{mmol}$, 0.2 equiv.), alkyne S-A-1 (24.5 mg, $0.24 \mathrm{mmol}, 1.2$ equiv.) and DBU (61 mg, $0.4 \mathrm{mmol}, 2.0$ equiv. $)$ in xylenes $(0.5 \mathrm{~mL})$ at $120^{\circ} \mathrm{C}$ for $10 \mathrm{~min}$ afforded $4 \mathbf{c f}$ as white solid (47 mg, 61\%).

$>\quad m p: 92.7-95.8^{\circ} \mathrm{C}$.

$>\mathrm{R}_{f}=0.7$ (EtOAc: Petroleum Ether $=1: 7$ ).

$>{ }^{1} \mathrm{H} \mathrm{NMR}\left(400 \mathrm{MHz}, \mathrm{CDCl}_{3}\right) \delta 7.71(\mathrm{~d}, J=8.0 \mathrm{~Hz}, 2 \mathrm{H}), 7.38-7.27(\mathrm{~m}, 7 \mathrm{H}), 6.03(\mathrm{t}, J=1.72$ $\mathrm{Hz}, 1 \mathrm{H}), 3.93(\mathrm{~d}, J=1.72 \mathrm{~Hz}, 2 \mathrm{H}), 2.57(\mathrm{~s}, 2 \mathrm{H}), 2.43(\mathrm{~s}, 3 \mathrm{H}), 1.01(\mathrm{t}, J=8.0 \mathrm{~Hz}, 6 \mathrm{H}), 0.72(\mathrm{q}$, $J=8.0 \mathrm{~Hz}, 4 \mathrm{H})$.

$>{ }^{13} \mathrm{C} \mathrm{NMR}\left(100 \mathrm{MHz}, \mathrm{CDCl}_{3}\right) \delta 154.3,143.5,141.9,132.4,129.6,128.5,128.2,128.0,125.8$, $120.9,50.9,32.3,21.6,7.4,4.1$.

$>$ IR (neat) $\mathrm{cm}^{-1} 2953,2873,1596,1492,1340,1160,1089,1120,929$.

$>$ HRMS calcd for $\mathrm{C}_{21} \mathrm{H}_{27} \mathrm{NO}_{2} \mathrm{SSi}(\mathrm{M}+\mathrm{Na})^{+} 408.1429$, found 408.1424 .

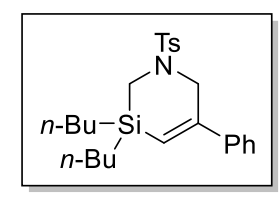

3,3-dibutvl-5-phenvl-1-tosvl-1,2,3,6-tetrahvdro-1,3-azasiline (4cg)

4eg was prepared according to General Method C. $2 \mathrm{f}(76 \mathrm{mg}, 0.2 \mathrm{mmol}, 1.0$ equiv.), $\mathrm{Pd}\left(\mathrm{PPh}_{3}\right)_{2} \mathrm{Cl}_{2}$ (7.2 mg, $0.01 \mathrm{mmol}, 5 \mathrm{~mol} \%$ ), $\mathrm{ZnI}_{2}(12.8 \mathrm{mg}, 0.04 \mathrm{mmol}$, 0.2 equiv.), alkyne S-A-1 (24.5 mg, $0.24 \mathrm{mmol}, 1.2$ equiv.) and $\mathrm{DBU}(61 \mathrm{mg}$, 0.4 mmol, 2.0 equiv. $)$ in xylenes $(0.5 \mathrm{~mL})$ at $120^{\circ} \mathrm{C}$ for $10 \mathrm{~min}$ afforded $4 \mathrm{cg}$ as 
yellow oil (53 mg, 60\%).

$>\mathrm{R}_{f}=0.6($ EtOAc:Petroleum Ether $=1: 10)$.

$>{ }^{1} \mathrm{H}$ NMR $\left(400 \mathrm{MHz}, \mathrm{CDCl}_{3}\right) \delta 7.71(\mathrm{~d}, J=8.0 \mathrm{~Hz}, 2 \mathrm{H}), 7.40-7.26(\mathrm{~m}, 7 \mathrm{H}), 6.03(\mathrm{t}, J=1.88$ $\mathrm{Hz}, 1 \mathrm{H}), 3.92(\mathrm{~d}, J=1.68 \mathrm{~Hz}, 2 \mathrm{H}), 2.56(\mathrm{~s}, 2 \mathrm{H}), 2.43(\mathrm{~s}, 3 \mathrm{H}), 1.39-1.26(\mathrm{~m}, 8 \mathrm{H}), 3.92(\mathrm{t}, J=$ $1.80 \mathrm{~Hz}, 6 \mathrm{H}), 0.72(\mathrm{q}, J=8.0 \mathrm{~Hz}, 4 \mathrm{H})$.

$>{ }^{13} \mathrm{C} \mathrm{NMR}\left(100 \mathrm{MHz}, \mathrm{CDCl}_{3}\right) \delta 153.7,143.5,142.0,132.5,129.6,128.5,128.2,128.1,125.8$, $121.7,50.8,33.1,26.5,25.9,21.6,13.8,12.3$.

$>$ IR (neat) $\mathrm{cm}^{-1} 255,2921,2869,1597,1443,1342,1162,1088,1029,930$.

$>$ HRMS calcd for $\mathrm{C}_{25} \mathrm{H}_{35} \mathrm{NO}_{2} \mathrm{SSi}(\mathrm{M}+\mathrm{Na})^{+} 464.2055$, found 464.2040.

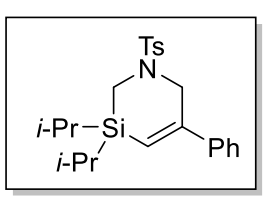

\section{3,3-diisopropyl-5-phenyl-1-tosyl-1,2,3,6-tetrahydro-1,3-azasiline (4ch)}

4ch was prepared according to General Method C. $2 \mathrm{~g}$ (34.8 $\mathrm{mg}, 0.2 \mathrm{mmol}, 1.0$ equiv.), $\mathrm{Pd}\left(\mathrm{PPh}_{3}\right)_{2} \mathrm{Cl}_{2}(3.5 \mathrm{mg}, 0.005 \mathrm{mmol}, 5 \mathrm{~mol} \%), \mathrm{ZnI}_{2}(6.4 \mathrm{mg}, 0.02 \mathrm{mmol}$, 0.2 equiv.), alkyne S-A-1 (12.3 mg, $0.12 \mathrm{mmol}, 1.2$ equiv.) and DBU (30.8 mg, $0.2 \mathrm{mmol}, 2.0$ equiv. $)$ in xylenes $(0.25 \mathrm{~mL})$ at $120^{\circ} \mathrm{C}$ for $10 \mathrm{~min}$ afforded $4 \mathrm{ch}$ as white solid (21 mg, 51\%).

$>\mathrm{mp}: 98.8-101.0^{\circ} \mathrm{C}$.

$>\mathrm{R}_{f}=0.6$ (EtOAc:Petroleum Ether $\left.=1: 5\right)$.

$>{ }^{1} \mathrm{H}$ NMR $\left(400 \mathrm{MHz}, \mathrm{CDCl}_{3}\right) \delta 7.72(\mathrm{~d}, J=8.0 \mathrm{~Hz}, 2 \mathrm{H}), 7.35-7.29(\mathrm{~m}, 7 \mathrm{H}), 6.03(\mathrm{t}, J=1.72$ $\mathrm{Hz}, 1 \mathrm{H}), 3.90(\mathrm{~d}, J=1.68 \mathrm{~Hz}, 2 \mathrm{H}), 2.56(\mathrm{~s}, 2 \mathrm{H}), 2.44(\mathrm{~s}, 3 \mathrm{H}), 1.14-1.03(\mathrm{~m}, 14 \mathrm{H})$.

$>{ }^{13} \mathrm{C}$ NMR $\left(100 \mathrm{MHz}, \mathrm{CDCl}_{3}\right) \delta 155.1,143.4,142.1,132.4,129.6,128.5,128.2,128.0,125.9$, $119.4,50.9,30.2,21.5,18.2,18.0,11.2$.

$>$ IR (neat) $\mathrm{cm}^{-1} 2926,2862,1597,1461,1340,1161,1089,1031,929$.

$>$ HRMS calcd for $\mathrm{C}_{23} \mathrm{H}_{31} \mathrm{NO}_{2} \mathrm{SSi}(\mathrm{M}+\mathrm{Na})^{+} 436.1737$, found 436.1731 .

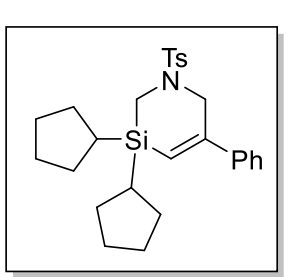

\section{3,3-dicvclopentyl-5-phenvl-1-tosyl-1,2,3,6-tetrahvdro-1,3-azasiline (4ci)}

4ci was prepared according to General Method C. $2 \mathbf{h}(80.0 \mathrm{mg}, 0.2 \mathrm{mmol}, 1.0$ equiv.), $\mathrm{Pd}\left(\mathrm{PPh}_{3}\right)_{2} \mathrm{Cl}_{2}$ (7.2 mg, $0.01 \mathrm{mmol}, 5 \mathrm{~mol} \%$ ), $\mathrm{ZnI}_{2}(12.8 \mathrm{mg}, 0.04$ mmol, 0.2 equiv.), alkyne S-A-1 (24.5 $\mathrm{mg}, 0.24 \mathrm{mmol}, 1.2$ equiv.) and $\mathrm{DBU}$ (61 mg, $0.4 \mathrm{mmol}, 2.0$ equiv.) in xylenes $(0.5 \mathrm{~mL})$ at $120{ }^{\circ} \mathrm{C}$ for $10 \mathrm{~min}$ afforded 4ci as pale yellow oil ( $47 \mathrm{mg}, 50 \%$ ).

$>\mathrm{R}_{f}=0.7$ (EtOAc:Petroleum Ether $=1: 5$ ).

$>{ }^{1} \mathrm{H} \mathrm{NMR}\left(400 \mathrm{MHz}, \mathrm{CDCl}_{3}\right) \delta 7.71(\mathrm{~d}, J=4.0 \mathrm{~Hz}, 2 \mathrm{H}), 7.35-7.29(\mathrm{~m}, 7 \mathrm{H}), 6.03(\mathrm{t}, J=1.60$ $\mathrm{Hz}, 1 \mathrm{H}), 3.91(\mathrm{~d}, J=4.0 \mathrm{~Hz}, 2 \mathrm{H}), 2.58(\mathrm{~s}, 2 \mathrm{H}), 2.43(\mathrm{~s}, 3 \mathrm{H}), 1.87-1.77(\mathrm{~m}, 4 \mathrm{H}), 1.63-1.57$ $(\mathrm{m}, 4 \mathrm{H}), 1.54-1.45(\mathrm{~m}, 4 \mathrm{H}), 1.44-1.32(\mathrm{~m}, 4 \mathrm{H}), 1.16-1.06(\mathrm{~m}, 2 \mathrm{H})$.

$>{ }^{13} \mathrm{C} \mathrm{NMR}\left(100 \mathrm{MHz}, \mathrm{CDCl}_{3}\right) \delta 154.6,143.4,142.2,132.4,129.6,128.4,128.2,128.0,125.9$, $120.0,50.9,31.7,28.5,26.9,26.8,23.2,21.6$.

$>$ IR (neat) $\mathrm{cm}^{-1}$ 2944, 2860, 1596, 1444, 1342, 1161, 1089, 929.

$>$ HRMS calcd for $\mathrm{C}_{27} \mathrm{H}_{35} \mathrm{NO}_{2} \mathrm{SSi}(\mathrm{M}+\mathrm{Na})^{+} 488.2055$, found 488.2053 .

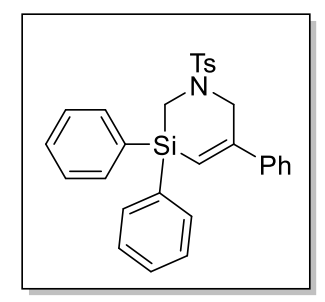

\section{3,3,5-triphenyl-1-tosyl-1,2,3,6-tetrahydro-1,3-azasiline (4ck)}

4ck was prepared according to General Method C. $\mathbf{2 j}(83.2 \mathrm{mg}, 0.2 \mathrm{mmol}$, 1.0 equiv.), $\mathrm{Pd}\left(\mathrm{PPh}_{3}\right)_{2} \mathrm{Cl}_{2}$ (7.2 mg, $0.01 \mathrm{mmol}, 5 \mathrm{~mol} \%$ ), $\mathrm{ZnI}_{2}(12.8 \mathrm{mg}, 0.04$ mmol, 0.2 equiv.), alkyne S-A-1 ( $24.5 \mathrm{mg}, 0.24 \mathrm{mmol}, 1.2$ equiv.) and DBU (61 mg, $0.4 \mathrm{mmol}, 2.0$ equiv.) in xylenes $(0.5 \mathrm{~mL})$ at $120{ }^{\circ} \mathrm{C}$ for $10 \mathrm{~min}$ afforded 4ck as white solid (62.5 mg, 65\%). mp: $141.3-143.5^{\circ} \mathrm{C}$.

$\mathrm{R}_{f}=0.5$ (EtOAc:Petroleum Ether $\left.=1: 7\right)$.

$>{ }^{1} \mathrm{H} \mathrm{NMR}\left(400 \mathrm{MHz}, \mathrm{CDCl}_{3}\right) \delta 7.71(\mathrm{~d}, J=8.0 \mathrm{~Hz}, 2 \mathrm{H}), 7.63-7.61(\mathrm{~m}, 4 \mathrm{H}), 7.46-7.30(\mathrm{~m}$, $13 \mathrm{H}), 6.39(\mathrm{t}, J=1.72 \mathrm{~Hz}, 1 \mathrm{H}), 4.10(\mathrm{~d}, J=1.72 \mathrm{~Hz}, 2 \mathrm{H}), 3.05(\mathrm{~s}, 2 \mathrm{H}), 2.41(\mathrm{~s}, 3 \mathrm{H})$.

$>{ }^{13} \mathrm{C} \mathrm{NMR}\left(100 \mathrm{MHz}, \mathrm{CDCl}_{3}\right) \delta 155.6,143.7,141.6,135.2,133.1,132.2,130.2,129.7,128.6$,

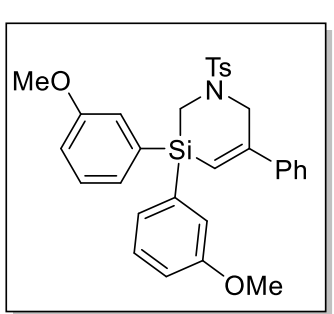
$128.6,128.2,128.1,125.9,119.3,50.9,34.2,21.5$.

$>$ IR (neat) $\mathrm{cm}^{-1} 3049,2922,2851,1595,1569,1428,1341,1161,1088$, 1219, 998.

$>$ HRMS calcd for $\mathrm{C}_{29} \mathrm{H}_{27} \mathrm{NO}_{2} \mathrm{SSi}(\mathrm{M}+\mathrm{Na})^{+} 504.1429$, found 504.1426

3,3-bis(3-methoxyphenyl)-5-phenyl-1-tosyl-1,2,3,6-tetrahydro-1,3azasiline (4cl)

4cl was prepared according to General Method C. $2 \mathbf{k}(95.2 \mathrm{mg}, 0.2 \mathrm{mmol}$, 
1.0 equiv.), $\mathrm{Pd}\left(\mathrm{PPh}_{3}\right)_{2} \mathrm{Cl}_{2}$ (7.2 mg, $\left.0.01 \mathrm{mmol}, 5 \mathrm{~mol} \%\right), \mathrm{ZnI}_{2}(12.8 \mathrm{mg}, 0.04 \mathrm{mmol}, 0.2$ equiv.), alkyne S-A-1 (24.5 mg, $0.24 \mathrm{mmol}, 1.2$ equiv.) and DBU (61 mg, $0.4 \mathrm{mmol}, 2.0$ equiv.) in xylenes $(0.5 \mathrm{~mL})$ at $120^{\circ} \mathrm{C}$ for $10 \mathrm{~min}$ afforded $\mathbf{4 c l}$ as yellow solid $(83 \mathrm{mg}, 77 \%)$.

$>$ mp: $58.4-60.4^{\circ} \mathrm{C}$.

$>\mathrm{R}_{f}=0.4($ EtOAc:Petroleum Ether $=1: 5)$.

$>{ }^{1} \mathrm{H}$ NMR $\left(400 \mathrm{MHz}, \mathrm{CDCl}_{3}\right) \delta 7.70(\mathrm{~d}, J=8.0 \mathrm{~Hz}, 2 \mathrm{H}), 7.43-7.39(\mathrm{~m}, 2 \mathrm{H}), 7.38-7.29(\mathrm{~m}$, $7 \mathrm{H}), 7.22-7.15(\mathrm{~m}, 4 \mathrm{H}), 6.96(\mathrm{ddd}, J=8.2,2.7,1.0 \mathrm{~Hz}, 2 \mathrm{H}), 6.37(\mathrm{t}, J=1.7 \mathrm{~Hz}, 1 \mathrm{H}), 4.09(\mathrm{~s}$, $2 \mathrm{H}), 3.80(\mathrm{~s}, 6 \mathrm{H}), 3.04(\mathrm{~s}, 2 \mathrm{H}), 2.41(\mathrm{~s}, 3 \mathrm{H})$.

$>{ }^{13} \mathrm{C} \mathrm{NMR}\left(100 \mathrm{MHz}, \mathrm{CDCl}_{3}\right) \delta 159.2,155.7,143.7,141.6,134.5,132.2,129.7,129.4,128.6$, 128.6, 128.1, 127.4, 126.0, 120.2, 119.1, 115.8, 55.3, 51.0, 34.3, 21.6.

$>$ IR (neat) $\mathrm{cm}^{-1} 2932,2834,1588,1569,1405,1340,1229,1160,1088,929$.

$>$ HRMS calcd for $\mathrm{C}_{31} \mathrm{H}_{31} \mathrm{NO}_{4} \mathrm{SSi}(\mathrm{M}+\mathrm{Na})^{+}$564.1641, found 564.1627.

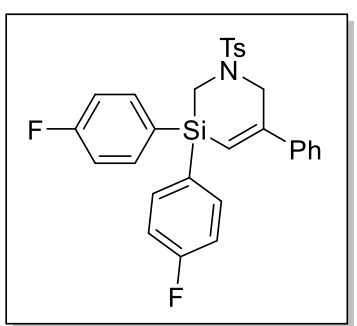

\section{3,3-bis(4-fluorophenyl)-5-phenyl-1-tosyl-1,2,3,6-tetrahydro-1,3-} azasiline (4cm)

4cm was prepared according to General Method C. $2 \mathbf{l}(90.2 \mathrm{mg}, 0.2$ mmol, 1.0 equiv.), $\mathrm{Pd}\left(\mathrm{PPh}_{3}\right)_{2} \mathrm{Cl}_{2}$ (7.2 $\left.\mathrm{mg}, 0.01 \mathrm{mmol}, 5 \mathrm{~mol} \%\right), \mathrm{ZnI}_{2}$ (12.8 $\mathrm{mg}, 0.04 \mathrm{mmol}, 0.2$ equiv.), alkyne S-A-1 (24.5 mg, $0.24 \mathrm{mmol}$, 1.2 equiv.) and $\mathrm{DBU}(61 \mathrm{mg}, 0.4 \mathrm{mmol}, 2.0$ equiv.) in xylenes $(0.5 \mathrm{~mL})$ at $120^{\circ} \mathrm{C}$ for $10 \mathrm{~min}$ afforded $4 \mathbf{c m}$ as yellow solid $(60 \mathrm{mg}, 58 \%)$.

$>\mathrm{mp}: 130.0-131.3^{\circ} \mathrm{C}$.

$>\mathrm{R}_{f}=0.6($ EtOAc:Petroleum Ether $=1: 5)$.

$>{ }^{1} \mathrm{H} \mathrm{NMR}\left(400 \mathrm{MHz}, \mathrm{CDCl}_{3}\right) \delta 7.70(\mathrm{~d}, J=8.0 \mathrm{~Hz}, 2 \mathrm{H}), 7.60-7.56(\mathrm{~m}, 4 \mathrm{H}), 7.43-7.31(\mathrm{~m}$, $7 \mathrm{H}), 7.09(\mathrm{~d}, J=8.0 \mathrm{~Hz}, 4 \mathrm{H}), 6.03(\mathrm{t}, J=2.0 \mathrm{~Hz}, 1 \mathrm{H}), 4.11(\mathrm{~s}, 2 \mathrm{H}), 3.01(\mathrm{~s}, 2 \mathrm{H}), 2.42(\mathrm{~s}, 3 \mathrm{H})$.

$>{ }^{13} \mathrm{C} \mathrm{NMR}\left(100 \mathrm{MHz}, \mathrm{CDCl}_{3}\right) \delta 164.4(\mathrm{~d}, J=248 \mathrm{~Hz}), 156.1,143.9,141.4,137.2(\mathrm{~d}, J=8 \mathrm{~Hz})$, $132.1,129.8,128.8,128.6,128.4(\mathrm{~d}, J=3 \mathrm{~Hz}), 128.1,125.9,118.7,115.6(\mathrm{~d}, J=20 \mathrm{~Hz}), 51.0$, 34.3, 21.6.

$>{ }^{19} \mathrm{~F}$ NMR $(376 \mathrm{MHz}, \mathrm{CDCl} 3) \delta-107.92$.

$>\mathrm{IR}$ (neat) $\mathrm{cm}^{-1} 3026,1586,1497,1341,1160,1089,1029,929$.

$>$ HRMS calcd for $\mathrm{C}_{29} \mathrm{H}_{25} \mathrm{~F}_{2} \mathrm{NO}_{2} \mathrm{SSi}(\mathrm{M}+\mathrm{Na})^{+} 540.1241$, found 540.1236.

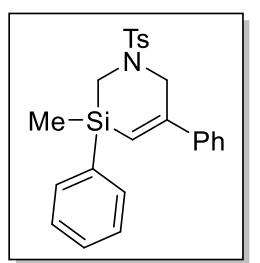

\section{3-methyl-3,5-diphenyl-1-tosyl-1,2,3,6-tetrahydro-1,3-azasiline (4cn)}

4cn was prepared according to General Method C. $2 \mathbf{m ~ ( 7 0 ~ m g , ~} 0.2 \mathrm{mmol}, 1.0$ equiv.), $\mathrm{Pd}\left(\mathrm{PPh}_{3}\right)_{2} \mathrm{Cl}_{2}$ (7.2 $\mathrm{mg}, 0.01 \mathrm{mmol}, 5 \mathrm{~mol} \%$ ), $\mathrm{ZnI}_{2}(12.8 \mathrm{mg}, 0.04 \mathrm{mmol}$, 0.2 equiv.), alkyne S-A-1 (24.5 mg, $0.24 \mathrm{mmol}, 1.2$ equiv.) and DBU (61 mg, $0.4 \mathrm{mmol}, 2.0$ equiv. $)$ in xylenes $(0.5 \mathrm{~mL})$ at $120^{\circ} \mathrm{C}$ for $10 \mathrm{~min}$ afforded $4 \mathrm{cn}$ as white solid (65 mg, 77\%).

$>$ mp: $129.6-131.2^{\circ} \mathrm{C}$.

$>\mathrm{R}_{f}=0.7$ (EtOAc:Petroleum Ether $\left.=1: 5\right)$.

$>{ }^{1} \mathrm{H}$ NMR $\left(400 \mathrm{MHz}, \mathrm{CDCl}_{3}\right) \delta 7.70(\mathrm{~d}, J=8.0 \mathrm{~Hz}, 2 \mathrm{H}), 7.58-7.56(\mathrm{~m}, 2 \mathrm{H}), 7.40-7.30(\mathrm{~m}$, $10 \mathrm{H}), 6.16(\mathrm{t}, J=2.0 \mathrm{~Hz}, 1 \mathrm{H}), 4.22(\mathrm{~d}, J=16.0 \mathrm{~Hz}, 1 \mathrm{H}), 3.86(\mathrm{~d}, J=16.0 \mathrm{~Hz}, 1 \mathrm{H}), 2.92(\mathrm{~d}, J$ $=12.0 \mathrm{~Hz}, 1 \mathrm{H}), 2.57(\mathrm{~d}, J=16.0 \mathrm{~Hz}, 1 \mathrm{H}), 2.42(\mathrm{~s}, 3 \mathrm{H}), 0.56(\mathrm{~s}, 3 \mathrm{H})$.

$>{ }^{13} \mathrm{C} \mathrm{NMR}\left(100 \mathrm{MHz}, \mathrm{CDCl}_{3}\right) \delta 154.6,143.6,141.6,135.0,134.3,132.3,130.0,129.7,128.5$, $128.5,128.1,128.1,125.9,121.1,50.9,35.1,21.6,-4.8$.

$>\quad \mathrm{IR}$ (neat) $\mathrm{cm}^{-1} 3051,2922,2852,1596,1340,1160,1088,1030,930$.

$>$ HRMS calcd for $\mathrm{C}_{24} \mathrm{H}_{25} \mathrm{NO}_{2} \mathrm{SSi}(\mathrm{M}+\mathrm{Na})^{+} 442.1273$, found 442.1263 .

\section{9-phenyl-7-tosyl-7-aza-5-silaspiro[4.5/dec-9-ene (4co)}

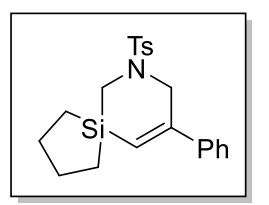

4co was prepared according to General Method C. 2 n $(63.8 \mathrm{mg}, 0.2 \mathrm{mmol}, 1.0$ equiv.), $\mathrm{Pd}\left(\mathrm{PPh}_{3}\right)_{2} \mathrm{Cl}_{2}$ (7.2 mg, $\left.0.01 \mathrm{mmol}, 5 \mathrm{~mol} \%\right), \mathrm{ZnI}_{2}(12.8 \mathrm{mg}, 0.04 \mathrm{mmol}$, 0.2 equiv.), alkyne S-A-1 (24.5 mg, $0.24 \mathrm{mmol}, 1.2$ equiv.) and DBU (61 mg, $0.4 \mathrm{mmol}, 2.0$ equiv.) in xylenes $(0.5 \mathrm{~mL})$ at $120^{\circ} \mathrm{C}$ for 10 min afforded $4 \mathrm{co}$ as white solid (34 mg, 45\%).

$>$ mp: $126.1-128.8^{\circ} \mathrm{C}$.

$>\mathrm{R}_{f}=0.4($ EtOAc:Petroleum Ether $=1: 8)$.

$>{ }^{1} \mathrm{H} \mathrm{NMR}\left(400 \mathrm{MHz}, \mathrm{CDCl}_{3}\right) \delta 7.71(\mathrm{~d}, J=8.0 \mathrm{~Hz}, 2 \mathrm{H}), 7.35-7.31(\mathrm{~m}, 7 \mathrm{H}), 6.03(\mathrm{t}, J=2.0$ $\mathrm{Hz}, 1 \mathrm{H}), 3.96(\mathrm{~d}, J=2.0 \mathrm{~Hz}, 2 \mathrm{H}), 2.64(\mathrm{~s}, 2 \mathrm{H}), 2.43(\mathrm{~s}, 3 \mathrm{H}), 1.68-1.64(\mathrm{~m}, 4 \mathrm{H}), 0.76(\mathrm{t}, J=$ $4.0 \mathrm{~Hz}, 4 \mathrm{H})$. 
$>{ }^{13} \mathrm{C} \mathrm{NMR}\left(100 \mathrm{MHz}, \mathrm{CDCl}_{3}\right) \delta 154.5,143.6,141.7,132.4,129.6,128.5,128.3,128.1,125.8$, 121.3, 50.7, 34.0, 27.0, 21.6, 10.9.

$>\quad \mathrm{IR}$ (neat) $\mathrm{cm}^{-1} 2924,2852,1738,1595,1443,1340,1161,1088,1020$.

$>$ HRMS calcd for $\mathrm{C}_{21} \mathrm{H}_{25} \mathrm{NO}_{2} \mathrm{SSi}(\mathrm{M}+\mathrm{Na})^{+} 406.1273$, found 406.1275 .

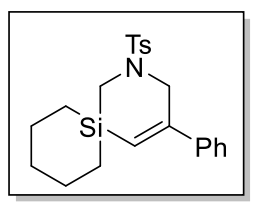

4-phenyl-2-tosyl-2-aza-6-silaspiro/5.5/undec-4-ene (4cp)

4ep was prepared according to General Method C. 20 (66.4 mg, $0.2 \mathrm{mmol}, 1.0$ equiv.), $\mathrm{Pd}\left(\mathrm{PPh}_{3}\right)_{2} \mathrm{Cl}_{2}$ (7.2 mg, $0.01 \mathrm{mmol}, 5 \mathrm{~mol} \%$ ), $\mathrm{ZnI}_{2}(12.8 \mathrm{mg}, 0.04 \mathrm{mmol}$, 0.2 equiv.), alkyne S-A-1 (24.5 mg, $0.24 \mathrm{mmol}, 1.2$ equiv.) and DBU (61 mg, $0.4 \mathrm{mmol}, 2.0$ equiv.) in xylenes $(0.5 \mathrm{~mL})$ at $120^{\circ} \mathrm{C}$ for $10 \mathrm{~min}$ afforded $4 \mathbf{c p}$ as white solid (50 mg, 63\%).

$>$ mp: $138.2-140.1^{\circ} \mathrm{C}$.

$>\mathrm{R}_{f}=0.7$ (EtOAc:Petroleum Ether $\left.=1: 5\right)$.

$>\quad{ }^{1} \mathrm{H} \mathrm{NMR}\left(400 \mathrm{MHz}, \mathrm{CDCl}_{3}\right) \delta 7.72(\mathrm{~d}, J=8.0 \mathrm{~Hz}, 2 \mathrm{H}), 7.35-7.29(\mathrm{~m}, 7 \mathrm{H}), 6.07(\mathrm{t}, J=1.72$ $\mathrm{Hz}, 1 \mathrm{H}), 3.95(\mathrm{~d}, J=1.72 \mathrm{~Hz}, 2 \mathrm{H}), 2.63(\mathrm{~s}, 2 \mathrm{H}), 2.43(\mathrm{~s}, 3 \mathrm{H}), 1.90-1.82(\mathrm{~m}, 2 \mathrm{H}), 1.67-1.53$ $(\mathrm{m}, 3 \mathrm{H}), 1.41-1.31(\mathrm{~m}, 1 \mathrm{H}), 0.86-0.72(\mathrm{~m}, 4 \mathrm{H})$.

$>{ }^{13} \mathrm{C} \mathrm{NMR}\left(100 \mathrm{MHz}, \mathrm{CDCl}_{3}\right) \delta 154.2,143.5,141.8,132.6,129.6,128.5,128.3,128.0,125.8$, $121.5,51.0,33.0,29.6,24.5,21.6,11.4$.

$>$ IR (neat) $\mathrm{cm}^{-1}$ 2916, 2649, 1596, 1443, 1341, 1161, 1089, 1031, 929, 909.

$>$ HRMS calcd for $\mathrm{C}_{22} \mathrm{H}_{27} \mathrm{NO}_{2} \mathrm{SSi}(\mathrm{M}+\mathrm{Na})^{+} 420.1429$, found 420.1429 .

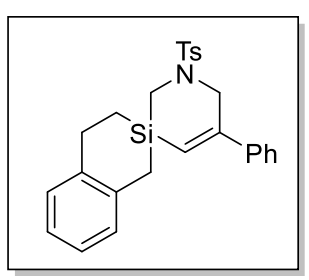

5'-phenyl-1'-tosyl-1',3,4,6'-tetrahydro-1H,2'H-spiro/benzo/c/siline-2,3'1,3]azasiline](4cq)

4cq was prepared according to General Method C. 2p (36.6 mg, $0.096 \mathrm{mmol}$, 1.0 equiv.), $\mathrm{Pd}\left(\mathrm{PPh}_{3}\right)_{2} \mathrm{Cl}_{2}$ (3.5 mg, $\left.0.005 \mathrm{mmol}, 5.2 \mathrm{~mol} \%\right), \mathrm{ZnI}_{2}(6.4 \mathrm{mg}$, $0.02 \mathrm{mmol}, 0.21$ equiv.), alkyne S-A-1 (12.4 mg, $0.12 \mathrm{mmol}, 1.25$ equiv.) and DBU (30.8 mg, $0.2 \mathrm{mmol}, 2.08$ equiv.) in xylenes $(0.5 \mathrm{~mL})$ at $120^{\circ} \mathrm{C}$ for $10 \mathrm{~min}$ afforded $\mathbf{4 c q}$ as yellow solid $(24 \mathrm{mg}, 56 \%)$.

$>$ mp: $56.5-57.9^{\circ} \mathrm{C}$.

$>\mathrm{R}_{f}=0.7$ (EtOAc:Petroleum Ether $\left.=1: 5\right)$.

$>{ }^{1} \mathrm{H} \mathrm{NMR}\left(400 \mathrm{MHz}, \mathrm{CDCl}_{3}\right) \delta 7.68(\mathrm{~d}, J=8.0 \mathrm{~Hz}, 2 \mathrm{H}), 7.33-7.30(\mathrm{~m}, 7 \mathrm{H}), 7.18-7.10(\mathrm{~m}$, $4 \mathrm{H}), 5.99(\mathrm{t}, J=2.0 \mathrm{~Hz}, 1 \mathrm{H}), 4.10(\mathrm{~d}, J=16.0 \mathrm{~Hz}, 1 \mathrm{H}), 3.86(\mathrm{~d}, J=16.0 \mathrm{~Hz}, 1 \mathrm{H}), 2.83(\mathrm{t}, J=$ $8.0 \mathrm{~Hz}, 2 \mathrm{H}), 2.63(\mathrm{~d}, J=8.0 \mathrm{~Hz}, 1 \mathrm{H}), 2.44-2.40(\mathrm{~m}, 4 \mathrm{H}), 2.23-2.13(\mathrm{~m}, 2 \mathrm{H}), 1.05-0.91$ $(\mathrm{m}, 2 \mathrm{H})$.

$>{ }^{13} \mathrm{C} \mathrm{NMR}\left(100 \mathrm{MHz}, \mathrm{CDCl}_{3}\right) \delta 155.0,143.6,141.4,141.3,136.3,132.4,130.1,129.7,128.5$, $128.5,128.2,128.0,126.8,125.8,125.5,120.5,50.9,33.6,29.3,21.6,17.7,8.9$.

$>$ IR (neat) $\mathrm{cm}^{-1} 2923,2985,1596,1341,1162,1088,1031,921$.

$>$ HRMS calcd for $\mathrm{C}_{26} \mathrm{H}_{27} \mathrm{NO}_{2} \mathrm{SSi}(\mathrm{M}+\mathrm{Na})^{+} 468.1429$, found 468.1406 . 


\section{Data of By-products}

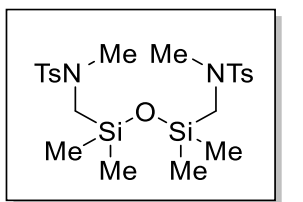

N,N'-((1,1,3,3-tetramethyldisiloxane-1,3-diyl)bis(methylene))bis(N,4-

dimethylbenzenesulfonam-ide) (5a)

$>$ white solid, mp: $71.5-73.2^{\circ} \mathrm{C}$.

$>\mathrm{R}_{f}=0.4$ (EtOAc:Petroleum Ether $\left.=1: 5\right)$.

$>{ }^{1} \mathrm{H}$ NMR $\left(400 \mathrm{MHz}, \mathrm{CDCl}_{3}\right) \delta 7.64(\mathrm{~d}, J=8.0 \mathrm{~Hz}, 4 \mathrm{H}), 7.32(\mathrm{~d}, J=8.0$

$\mathrm{Hz}, 4 \mathrm{H}), 2.66(\mathrm{~s}, 6 \mathrm{H}), 2.43(\mathrm{~s}, 6 \mathrm{H}), 2.37(\mathrm{~s}, 4 \mathrm{H}), 0.20(\mathrm{~s}, 12 \mathrm{H})$.

$>{ }^{13} \mathrm{C} \mathrm{NMR}\left(100 \mathrm{MHz}, \mathrm{CDCl}_{3}\right) \delta 143.2,132.1,129.4,127.9,41.4,37.8,21.4,0.0$.

$>\quad \mathrm{IR}$ (neat) $\mathrm{cm}^{-1} 2958,1597,1455,1338,1158,1052,963$.

$>$ HRMS calcd for $\mathrm{C}_{22} \mathrm{H}_{36} \mathrm{~N}_{2} \mathrm{O}_{5} \mathrm{~S}_{2} \mathrm{Si}_{2}(\mathrm{M}+\mathrm{Na})^{+}$551.1502, found 551.1501.

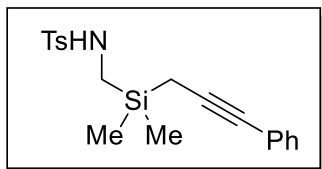

N-((dimethyl(3-phenylprop-2-yn-1-yl)silyl)methyl)-4-methylbenzenesul fonamide (6a)

white solid; mp: $131.5-133.6^{\circ} \mathrm{C}$.

$>\mathrm{R}_{f}=0.5$ (EtOAc:Petroleum Ether $\left.=1: 5\right)$.

$>{ }^{1} \mathrm{H} \mathrm{NMR}\left(400 \mathrm{MHz}, \mathrm{CDCl}_{3}\right) \delta 7.68(\mathrm{~d}, J=8.0 \mathrm{~Hz}, 2 \mathrm{H}), 7.31-7.26$ $(\mathrm{m}, 5 \mathrm{H}), 7.23(\mathrm{~d}, J=8.0 \mathrm{~Hz}, 2 \mathrm{H}), 4.57(\mathrm{t}, J=6.2 \mathrm{~Hz}, 1 \mathrm{H}), 2.48(\mathrm{~d}, J=6.2 \mathrm{~Hz}, 2 \mathrm{H}), 2.39(\mathrm{~s}$, $3 \mathrm{H}), 1.77$ (s, 2H), 0.21 (s, 6H).

$>{ }^{13} \mathrm{C} \mathrm{NMR}\left(100 \mathrm{MHz}, \mathrm{CDCl}_{3}\right) \delta 143.4,135.3,131.5,129.7,128.3,127.6,127.5,123.8,86.5$, $80.7,31.3,21.5,5.5,-4.6$.

$>$ IR (neat) $\mathrm{cm}^{-1} 3285,2924,1597,1489,1304,1155,1065,905$.

$>$ HRMS calcd for $\mathrm{C}_{19} \mathrm{H}_{23} \mathrm{NO}_{2} \mathrm{SSi}(\mathrm{M}+\mathrm{Na})^{+} 380.1116$, found 380.1116 .

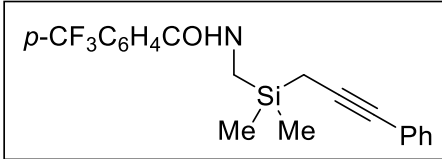

\section{N-((dimethyl(3-phenylprop-2-yn-1-yl)silyl)methyl)-4-}

(trifluoromethyl)benzamide(6d)

$>$ colorless oil.

$>\mathrm{R}_{f}=0.4($ EtOAc:Petroleum Ether $=1: 5)$.

$>{ }^{1} \mathrm{HNMR}\left(400 \mathrm{MHz}, \mathrm{CDCl}_{3}\right) \delta 7.68(\mathrm{~d}, J=8.0 \mathrm{~Hz}, 2 \mathrm{H}), 7.36$ $(\mathrm{d}, J=8.0 \mathrm{~Hz}, 2 \mathrm{H}), 7.28-7.23(\mathrm{~m}, 5 \mathrm{H}), 6.69(\mathrm{~s}, 1 \mathrm{H}) 3.17(\mathrm{~d}, J=5.6 \mathrm{~Hz}, 2 \mathrm{H}), 1.91(\mathrm{~s}, 2 \mathrm{H})$, $0.30(\mathrm{~s}, 6 \mathrm{H})$.

$>{ }^{13} \mathrm{C}$ NMR $\left(100 \mathrm{MHz}, \mathrm{CDCl}_{3}\right) \delta 166.6,137.8,132.7(\mathrm{q}, J=32 \mathrm{~Hz}), 131.4,128.4,127.8,127.2$, $125.4(\mathrm{q}, J=4 \mathrm{~Hz}), 123.7,123.7(\mathrm{q}, J=271 \mathrm{~Hz}), 87.9,80.7,29.7,6.4,-4.3,-4.3$.

$>{ }^{19} \mathrm{~F} \mathrm{NMR}\left(376 \mathrm{MHz}, \mathrm{CDCl}_{3}\right) \delta-63.03$

$>$ IR (neat) $\mathrm{cm}^{-1} 3294,2922,1636,1550,1408,1326,1276,1129,1168,1017$.

$>$ HRMS calcd for $\mathrm{C}_{20} \mathrm{H}_{20} \mathrm{~F}_{3} \mathrm{NOSiSSi}(\mathrm{M}+\mathrm{Na})^{+} 398.1164$, found 398.1148 . 


\section{Transformations of 3-silatetrahydropyridines 4}

\section{ethyl(6R,7S,8aS)-1,1-dimethyl-6-phenyl-3-tosyl-1,2,3,4,6,7,8,8a-}

octahvdrobenzo[d][1,3/azasiline-7-carboxvlate (7)
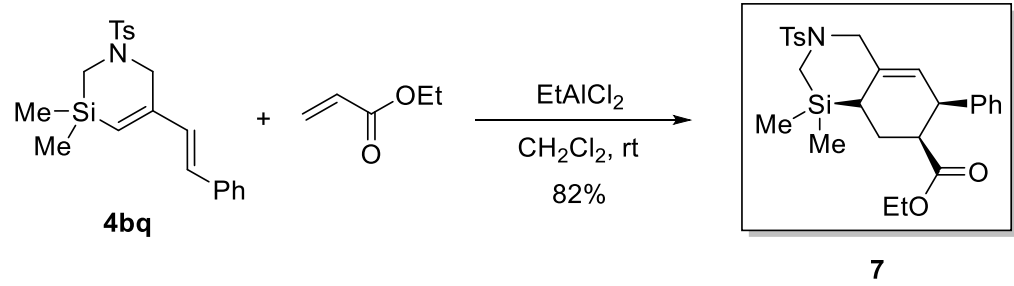

To a solution of $4 \mathbf{b q}(38.4 \mathrm{mg}, 0.1 \mathrm{mmol}, 1.0$ equiv.) and ethyl acrylate $(24 \mathrm{mg}, 0.2 \mathrm{mmol}, 2.0$ equiv.) in $\mathrm{CH}_{2} \mathrm{Cl}_{2}(1 \mathrm{~mL})$ was added $\mathrm{EtAlCl}_{2}\left(0.05 \mathrm{mmol}, 28 \mu \mathrm{L}, 1.8 \mathrm{M}\right.$ in toluene) at $0{ }^{\circ} \mathrm{C}$ under Ar. The reaction was stirred at room temperature for $6 \mathrm{~h}$ before quenching with water $(3 \mathrm{~mL})$ and extracting with $\mathrm{CH}_{2} \mathrm{Cl}_{2}(5 \mathrm{~mL} \times 3)$. The combined organic phases were washed with sat. aq. $\mathrm{NaCl}$, dried over $\mathrm{Na}_{2} \mathrm{SO}_{4}$. and concentrated under reduced pressure. The residue was purified by $\mathrm{Al}_{2} \mathrm{O}_{3}$ flash column chromatography (eluent: petroleum ether/EtOAc $=8 / 1$ ) to afford 7 as a white solid (39.7 mg, 82\%).

$>\mathrm{mp}: 52.3-55.0^{\circ} \mathrm{C}$.

$>\mathrm{R}_{f}=0.3($ EtOAc:Petroleum Ether $=1: 5)$.

$>{ }^{1} \mathrm{H} \mathrm{NMR}\left(400 \mathrm{MHz}, \mathrm{CDCl}_{3}\right) 7.68(\mathrm{~d}, J=8.0 \mathrm{~Hz}, 2 \mathrm{H}), 7.34(\mathrm{~d}, J=8.0 \mathrm{~Hz}, 2 \mathrm{H}), 7.28-7.22(\mathrm{~m}$, $3 \mathrm{H}), 7.14-7.11(\mathrm{~m}, 2 \mathrm{H}), 5.75(\mathrm{~d}, J=3.8 \mathrm{~Hz}, 1 \mathrm{H}), 4.24(\mathrm{dd}, J=12.8,2.5 \mathrm{~Hz}, 1 \mathrm{H}), 4.02-3.93$ $(\mathrm{m}, 2 \mathrm{H}), 3.92-3.88(\mathrm{~m}, 1 \mathrm{H}), 3.22(\mathrm{dd}, J=13.4,2.4 \mathrm{~Hz}, 1 \mathrm{H}), 2.77-2.69(\mathrm{~m}, 2 \mathrm{H}), 2.44(\mathrm{~s}, 3 \mathrm{H})$, $1.86-1.78(\mathrm{~m}, 2 \mathrm{H}), 1.75-1.65(\mathrm{~m}, 1 \mathrm{H}), 1.56-1.52(\mathrm{~m}, 1 \mathrm{H}), 1.15(\mathrm{t}, J=7.1 \mathrm{~Hz}, 3 \mathrm{H}), 0.32$ $(\mathrm{s}, 3 \mathrm{H}), 0.11(\mathrm{~s}, 3 \mathrm{H})$.

$>{ }^{13} \mathrm{C} \operatorname{NMR}\left(100 \mathrm{MHz}, \mathrm{CDCl}_{3}\right) \delta 173.7,143.4,139.4,135.3,132.4,129.6,129.6,128.1,127.9$, $127.1,125.2,60.2,55.8,45.2,42.7,37.6,26.6,21.6,19.5,14.1,-4.7,-6.5$.

$>$ IR (neat) $\mathrm{cm}^{-1} 3985,1729,1372,1339,1158,1090,1052,985$.

$>$ HRMS calcd for $\mathrm{C}_{26} \mathrm{H}_{33} \mathrm{NO}_{4} \mathrm{SSi}(\mathrm{M}+\mathrm{Na})^{+}$506.1792, found 506.1792.

(6R,6aS,9aR,9bS)-1,1-dimethyl-6,8-diphenyl-3-tosyl-1,2,3,4,6,6a,9a,9b-octahydro-7H[1,3]azasilino[4,5-e]isoindole-7,9(8H)-dione (8)

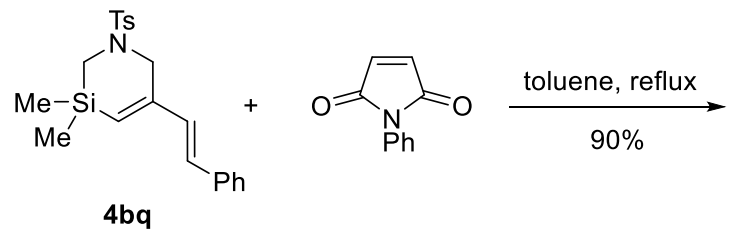

$4 \mathbf{b q}$

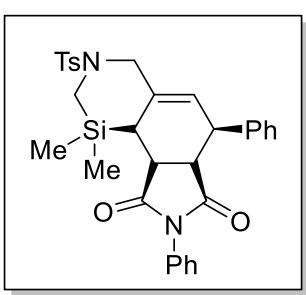

A solution of $\mathbf{4 b q}$ (76.8 $\mathrm{mg}, 0.2 \mathrm{mmol}, 1.0$ equiv.), 1-phenylpyrrolidine-2,5-dione (41.6 mg, $0.24 \mathrm{mmol}, 1.2$ equiv.) in toluene $(3 \mathrm{~mL})$ was refluxed at $110^{\circ} \mathrm{C}$ for $24 \mathrm{~h}$. Then the mixture was cooled to room temperature and concentrated under reduced pressure. The residue was purified by $\mathrm{Al}_{2} \mathrm{O}_{3}$ flash column chromatography (eluent: petroleum ether/EtOAc $=8 / 1$ ) to afford 8 as yellow solid (100 mg, 90\%).

$>\quad$ mp: the compound decomposed during heating.

$>\mathrm{R}_{f}=0.4$ (EtOAc:Petroleum Ether $\left.=1: 2\right)$.

$>{ }^{1} \mathrm{H}$ NMR $\left(400 \mathrm{MHz}, \mathrm{CDCl}_{3}\right) \delta 7.69(\mathrm{~d}, J=8.0 \mathrm{~Hz}, 2 \mathrm{H}), 7.34(\mathrm{~d}, J=7.9 \mathrm{~Hz}, 2 \mathrm{H}), 7.29-7.14$ $(\mathrm{m}, 9 \mathrm{H}), 6.32(\mathrm{dd}, J=6.7,3.0 \mathrm{~Hz}, 2 \mathrm{H}), 5.90(\mathrm{dd}, J=6.4,2.2 \mathrm{~Hz}, 1 \mathrm{H}), 4.30(\mathrm{dd}, J=12.8,2.3$ $\mathrm{Hz}, 1 \mathrm{H}), 4.04$ (t, $J=7.1 \mathrm{~Hz}, 1 \mathrm{H}), 3.52(\mathrm{dd}, J=10.9,8.3 \mathrm{~Hz}, 1 \mathrm{H}), 3.28(\mathrm{t}, J=8.0 \mathrm{~Hz}, 1 \mathrm{H}), 3.14$ $(\mathrm{dd}, J=13.2,2.3 \mathrm{~Hz}, 1 \mathrm{H}), 2.92(\mathrm{~d}, J=12.6 \mathrm{~Hz}, 1 \mathrm{H}), 2.45(\mathrm{~s}, 3 \mathrm{H}), 2.08(\mathrm{~d}, J=10.8 \mathrm{~Hz}, 1 \mathrm{H})$, $1.87(\mathrm{~d}, J=13.2 \mathrm{~Hz}, 1 \mathrm{H}), 0.57(\mathrm{~s}, 3 \mathrm{H}), 0.36(\mathrm{~s}, 3 \mathrm{H})$.

$>{ }^{13} \mathrm{C} \mathrm{NMR}\left(100 \mathrm{MHz}, \mathrm{CDCl}_{3}\right) \delta 177.6,176.4,143.5,137.3,134.9,132.2,131.0,129.8,129.6$, $128.8,128.5,128.5,128.1,127.8,126.2,122.3,56.5,43.3,40.4,40.1,40.0,26.3,21.6,-2.4$, 2.7 .

$>\quad \mathrm{IR}$ (neat) $\mathrm{cm}^{-1} 2923,2852,1711,1383,1159,1089,1024,961$. 
$>$ HRMS calcd for $\mathrm{C}_{31} \mathrm{H}_{32} \mathrm{~N}_{2} \mathrm{O}_{4} \mathrm{SSi}(\mathrm{M}+\mathrm{Na})^{+} 579.1744$, found 579.1743 .

(1R,6S)-2,2-dimethyl-6-phenyl-4-tosyl-7-oxa-4-aza-2-silabicyclo[4.1.0/heptane (9)

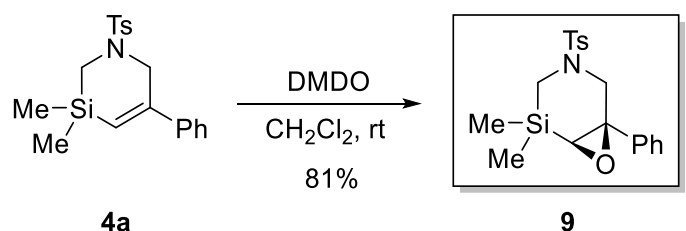

A solution of $4 \mathbf{a}$ ( $357 \mathrm{mg}, 1.0 \mathrm{mmol}, 1.0$ equiv.) in $\mathrm{CH}_{2} \mathrm{Cl}_{2}(20 \mathrm{~mL})$ was added fresh DMDO $(15 \mathrm{~mL}, 1.5 \mathrm{mmol}, 0.1 \mathrm{Mol} / \mathrm{L}, 1.5$ equiv.) and stirred at room temperature for $6 \mathrm{~h}$. Then the reaction was quenched with sat. aq. $\mathrm{Na}_{2} \mathrm{~S}_{2} \mathrm{O}_{3}(10 \mathrm{~mL})$ and extracted with $\mathrm{CH}_{2} \mathrm{Cl}_{2}(10 \mathrm{~mL} \times 3)$. The combined organic phases were washed with sat. aq. $\mathrm{NaCl}$, dried over $\mathrm{Na}_{2} \mathrm{SO}_{4}$. and concentrated under reduced pressure. The residue was purified by silica gel flash column chromatography (eluent: petroleum ether/EtOAc $=3 / 1)$ to afford 9 as white solid $(302 \mathrm{mg}, 81 \%)$.

$>\mathrm{mp}: 105.8-109.0^{\circ} \mathrm{C}$.

$>\mathrm{R}_{f}=0.3($ EtOAc:Petroleum Ether $=1: 2)$.

$>{ }^{1} \mathrm{H} \mathrm{NMR}\left(600 \mathrm{MHz}, \mathrm{CDCl}_{3}\right) \delta 7.65(\mathrm{~d}, J=8.2 \mathrm{~Hz}, 2 \mathrm{H}), 7.42-7.39(\mathrm{~m}, 2 \mathrm{H}), 7.37-7.35(\mathrm{~m}$, $2 \mathrm{H}), 7.34-7.28(\mathrm{~m}, 3 \mathrm{H}), 3.66-3.55(\mathrm{~m}, 2 \mathrm{H}), 2.65(\mathrm{~d}, J=12.0 \mathrm{~Hz}, 1 \mathrm{H}), 2.43(\mathrm{~s}, 3 \mathrm{H}), 2.33(\mathrm{~s}$, $1 \mathrm{H}), 2.27(\mathrm{~d}, J=14.0 \mathrm{~Hz}, 1 \mathrm{H}), 0.33(\mathrm{~s}, 3 \mathrm{H}), 0.27(\mathrm{~s}, 3 \mathrm{H})$.

$>{ }^{13} \mathrm{C} \mathrm{NMR}\left(100 \mathrm{MHz}, \mathrm{CDCl}_{3}\right) \delta 143.6,140.7,133.0,129.7,128.6,128.0,127.9,125.5,61.7$, $55.0,51.2,34.7,21.6,-4.8,-5.3$.

$>$ IR (neat) $\mathrm{cm}^{-1} 3060,2956,1733,1598,1446,1340,1160,1089,1058,962$.

$>$ HRMS calcd for $\mathrm{C}_{19} \mathrm{H}_{23} \mathrm{NO}_{3} \mathrm{SSi}(\mathrm{M}+\mathrm{Na})^{+} 396.1060$, found 396.1069.

(4R,5R)-4-azido-3,3-dimethyl-5-phenvl-1-tosvl-1,3-azasilinan-5-ol (10)

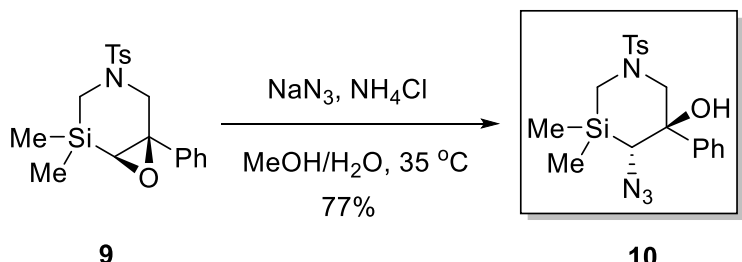

A solution of 9 (37.3 mg, $0.1 \mathrm{mmol}, 1.0$ equiv.), $\mathrm{NaN}_{3}\left(26 \mathrm{mg}, 0.4 \mathrm{mmol}, 4.0\right.$ equiv.) and $\mathrm{NH}_{4} \mathrm{Cl}$ (12 mg, 0.22 mmol, 2.2 equiv.) in $\mathrm{MeOH}(0.8 \mathrm{~mL})$ and $\mathrm{H}_{2} \mathrm{O}(0.1 \mathrm{~mL})$ was stirred at $35^{\circ} \mathrm{C}$ for $1 \mathrm{~h}$. The reaction was extracted with $\mathrm{CH}_{2} \mathrm{Cl}_{2}(5 \mathrm{~mL} \times 3)$ before adding $\mathrm{H}_{2} \mathrm{O}(5 \mathrm{~mL})$. The combined organic phases were washed with sat. aq. $\mathrm{NaCl}$, dried over $\mathrm{Na}_{2} \mathrm{SO}_{4}$. and concentrated under reduced pressure. The residue was purified by silica gel flash column chromatography (eluent:petroleum ether/EtOAc $=20 / 1$ ) to afford product 10 as white solid ( $32 \mathrm{mg}, 77 \%$ ). Growing single crystal of $\mathbf{1 0}$ in EtOAc/ $n$-hexane resulted in the formation of colorless single crystals suitable for X-ray analysis.

$>$ mp: $129.5-131.5^{\circ} \mathrm{C}$.

$>\mathrm{R}_{f}=0.6($ EtOAc:Petroleum Ether $=1: 5)$.

$>{ }^{1} \mathrm{H} \mathrm{NMR}\left(400 \mathrm{MHz}, \mathrm{CDCl}_{3}\right) \delta 7.66(\mathrm{~d}, J=8.0 \mathrm{~Hz}, 2 \mathrm{H}), 7.46-7.44(\mathrm{~m}, 2 \mathrm{H}), 7.40-7.30(\mathrm{~m}$, $5 \mathrm{H}), 3.70(\mathrm{dt}, J=12.9,1.9 \mathrm{~Hz}, 1 \mathrm{H}), 3.31(\mathrm{dd}, J=13.7,2.2 \mathrm{~Hz}, 1 \mathrm{H}), 3.10(\mathrm{~d}, J=12.9 \mathrm{~Hz}, 1 \mathrm{H})$, $3.02(\mathrm{~d}, J=15.9 \mathrm{~Hz}, 2 \mathrm{H}), 2.47$ (s, 3H), $1.98(\mathrm{~d}, J=13.7 \mathrm{~Hz}, 1 \mathrm{H}), 0.51(\mathrm{~s}, 3 \mathrm{H}), 0.18(\mathrm{~s}, 3 \mathrm{H})$.

$>{ }^{13} \mathrm{CNMR}\left(100 \mathrm{MHz}, \mathrm{CDCl}_{3}\right) \delta 146.8,146.4,135.5,132.4,130.9,130.4,127.4,76.5,61.2,54.7$, $37.5,24.1,0.0,-2.2$.

$>$ IR (neat) $\mathrm{cm}^{-1} 3485,2957,2921,2095,157,1447,139,1161,1089,991,948$.

$>$ HRMS calcd for $\mathrm{C}_{19} \mathrm{H}_{24} \mathrm{~N}_{4} \mathrm{O}_{3} \mathrm{SSi}(\mathrm{M}+\mathrm{Na})^{+} 439.1236$, found 439.1230 .

3-methvl-3,5-diphenvl-1-tosyl-1,3-azasilinane (11)

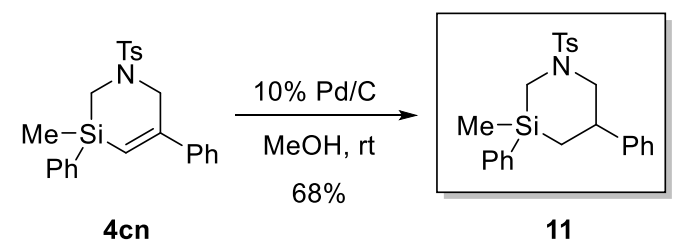


A suspension of $4 \mathbf{c n}(300 \mathrm{mg}, 4 \mathrm{mmol}, 1.0$ equiv.) and 10\% $\mathrm{Pd} / \mathrm{C}(60 \mathrm{mg}, 20 \% \mathrm{w} / \mathrm{t})$ in $\mathrm{MeOH}(10$ $\mathrm{mL}$ ) was stirred at room temperature under $1 \mathrm{~atm}$ of $\mathrm{H}_{2}$ overnight. The mixture was filtrated and the filter cake washed with $\mathrm{CH}_{2} \mathrm{Cl}_{2}(10 \mathrm{~mL})$. The combined solutions were concentrated under reduced pressure to give the product 11 as white solid $(206 \mathrm{mg}, 68 \%)$.

$>\mathrm{R}_{f}=0.5($ EtOAc:Petroleum Ether $=1: 5)$.

$>{ }^{1} \mathrm{H} \mathrm{NMR}\left(400 \mathrm{MHz}, \mathrm{CDCl}_{3}\right) \delta 7.75(\mathrm{dd}, J=6.5,3.0 \mathrm{~Hz}, 2 \mathrm{H}), 7.64-7.59(\mathrm{~m}, 4 \mathrm{H}), 7.50-7.47$ $(\mathrm{m}, 2 \mathrm{H}), 7.45-7.17(\mathrm{~m}, 20 \mathrm{H}), 4.01-3.92(\mathrm{~m}, 2 \mathrm{H}), 3.71(\mathrm{dt}, J=13.8,1.7 \mathrm{~Hz}, 1 \mathrm{H}), 3.45(\mathrm{dt}, J$ $=13.4,1.8 \mathrm{~Hz}, 1 \mathrm{H}), 3.27$ (ddt, $J=14.2,11.3,3.4 \mathrm{~Hz}, 1 \mathrm{H}), 3.11(\mathrm{ddd}, J=13.7,8.5,3.1 \mathrm{~Hz}, 1 \mathrm{H})$, $2.43(\mathrm{~s}, 3 \mathrm{H}), 2.40(\mathrm{~s}, 4 \mathrm{H}), 2.16(\mathrm{q}, J=11.2 \mathrm{~Hz}, 3 \mathrm{H}), 1.93(\mathrm{~s}, 1 \mathrm{H}), 1.89(\mathrm{~s}, 1 \mathrm{H}), 1.35-1.13(\mathrm{~m}$, $4 \mathrm{H}), 0.99(\mathrm{t}, J=13.9 \mathrm{~Hz}, 1 \mathrm{H}), 0.61(\mathrm{~s}, 3 \mathrm{H}), 0.31(\mathrm{~s}, 3 \mathrm{H})$.

$>{ }^{13} \mathrm{C} \mathrm{NMR}\left(100 \mathrm{MHz}, \mathrm{CDCl}_{3}\right) \delta 145.3,145.2,143.3,143.3,134.8,134.7,134.2,133.7,132.7$, $132.4,130.1,129.8,129.5,128.7,128.6,128.2,128.1,128.0,127.8,126.8,126.7,56.0,55.8$, $41.7,41.2,36.6,35.5,21.5,21.5,18.9,18.1,-4.0,-6.3$.

$>\quad \mathrm{IR}$ (neat) $\mathrm{cm}^{-1} 2967,2889,1601,1493,1349,1307,1119,1021,964,849$.

$>$ HRMS calcd for $\mathrm{C}_{16} \mathrm{H}_{27} \mathrm{NO}_{2} \mathrm{SSi}(\mathrm{M}+\mathrm{Na})^{+} 444.1429$, found 444.1422 .

3-methyl-5-phenyl-1-tosyl-1,3-azasilinane (12)<smiles>[Y5]C1CC(c2ccccc2)C[Si]([Y6])(c2ccccc2)C1</smiles>

11

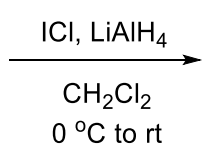

$69 \%$

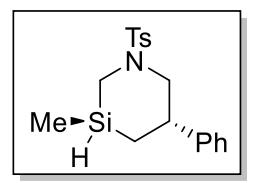

$12 a$

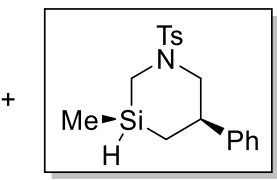

$12 b$

To a solution of 11 (106 mg, $0.25 \mathrm{mmol}, 1.0$ equiv.) in $\mathrm{CH}_{2} \mathrm{Cl}_{2}(2.5 \mathrm{~mL})$ was added $\mathrm{ICl}$ ( $62 \mathrm{mg}$, 0.38 mmol, 1.5 equiv., dissolved in $2.5 \mathrm{~mL} \mathrm{CH}_{2} \mathrm{Cl}_{2}$ ) dropwise at room temperature under Ar. After stirring for $1 \mathrm{~h}$, the mixture was cooled to $0{ }^{\circ} \mathrm{C}$ and $\mathrm{LiAlH}_{4}(386 \mu \mathrm{L}, 0.38 \mathrm{mmol}, 1.5$ equiv., $1 \mathrm{~mol} / \mathrm{L}$ in THF) was added dropwise. The reaction was stirred at $0{ }^{\circ} \mathrm{C}$ for $1 \mathrm{~h}$ before quenching with cooled $\mathrm{H}_{2} \mathrm{O}(10 \mathrm{~mL})$ and extracting with $\mathrm{CH}_{2} \mathrm{Cl}_{2}(10 \mathrm{~mL} \times 3)$. The combined organic phases were washed with sat. aq. $\mathrm{NaCl}$, dried over $\mathrm{Na}_{2} \mathrm{SO}_{4}$. and concentrated under reduced pressure. The residue was purified by silica gel flash column chromatography (eluent: petroleum ether/EtOAc $=20 / 1$ ) to afford $\mathbf{1 2}$ as white solid. (12a: $28 \mathrm{mg}$ and $\mathbf{1 2 b}$ : $32 \mathrm{mg}, 69 \%$ ).

12a:

$>28 \mathrm{mg}, \mathrm{mp}: 155.8-158.1{ }^{\circ} \mathrm{C}$;

$>\mathrm{R}_{f}=0.6($ EtOAc:Petroleum Ether $=1: 5)$.

$>{ }^{1} \mathrm{H} \mathrm{NMR}\left(400 \mathrm{MHz}, \mathrm{CDCl}_{3}\right) \delta 7.60(\mathrm{~d}, J=8.0 \mathrm{~Hz}, 2 \mathrm{H}), 7.34-7.20(\mathrm{~m}, 7 \mathrm{H}), 4.07-4.04(\mathrm{~m}$, $1 \mathrm{H}), 3.89(\mathrm{dq}, J=12.3,2.3,1.7 \mathrm{~Hz}, 1 \mathrm{H}), 3.31(\mathrm{dt}, J=13.7,1.7 \mathrm{~Hz}, 1 \mathrm{H}), 3.14(\mathrm{tt}, J=11.7,5.9$ $\mathrm{Hz}, 1 \mathrm{H}), 2.42(\mathrm{~s}, 3 \mathrm{H}), 2.10(\mathrm{dd}, J=12.3,10.9 \mathrm{~Hz}, 1 \mathrm{H}), 1.96(\mathrm{~d}, J=13.6 \mathrm{~Hz}, 1 \mathrm{H}), 1.10-0.98$ $(\mathrm{m}, 2 \mathrm{H}), 0.40(\mathrm{~d}, J=3.6 \mathrm{~Hz}, 3 \mathrm{H})$.

$>{ }^{13} \mathrm{C} \mathrm{NMR}\left(100 \mathrm{MHz}, \mathrm{CDCl}_{3}\right) \delta 145.2,143.4,132.6,129.6,128.7,127.9,126.8,126.8,55.8$, 41.2, 34.7, 21.5, 15.9, -7.4.

$>\quad \mathrm{IR}$ (neat) $\mathrm{cm}^{-1} 2922,2137,1597,1399,1162,1089,1007,958$.

$>$ HRMS calcd for $\mathrm{C}_{18} \mathrm{H}_{23} \mathrm{NO}_{2} \mathrm{SSi}(\mathrm{M}+\mathrm{Na})^{+} 368.1116$, found 368.1115.

12b:

> $\quad 32 \mathrm{mg}, \mathrm{mp}: 164.5-167.1^{\circ} \mathrm{C}$.

$>\mathrm{R}_{f}=0.5$ (EtOAc:Petroleum Ether $\left.=1: 5\right)$.

$>{ }^{1} \mathrm{H} \mathrm{NMR}\left(400 \mathrm{MHz}, \mathrm{CDCl}_{3}\right) \delta 7.61(\mathrm{~d}, J=8.0 \mathrm{~Hz}, 2 \mathrm{H}), 7.33-7.29(\mathrm{~m}, 4 \mathrm{H}), 7.25-7.18(\mathrm{~m}$, $3 \mathrm{H}), 4.16-4.11(\mathrm{~m}, 1 \mathrm{H}), 3.90(\mathrm{dq}, J=12.1,2.2 \mathrm{~Hz}, 1 \mathrm{H}), 3.50(\mathrm{dq}, J=13.8,2.0 \mathrm{~Hz}, 1 \mathrm{H}), 3.16$ (ddt, $J=13.8,10.9,3.0 \mathrm{~Hz}, 1 \mathrm{H}), 2.42(\mathrm{~s}, 3 \mathrm{H}), 2.09(\mathrm{dd}, J=12.2,10.9 \mathrm{~Hz}, 1 \mathrm{H}), 1.72$ (dd, $J=$ 13.6, $7.2 \mathrm{~Hz}, 1 \mathrm{H}), 1.27-1.22(\mathrm{~m}, 1 \mathrm{H}), 0.84-0.76(\mathrm{~m}, 1 \mathrm{H}), 0.17(\mathrm{~d}, J=3.5 \mathrm{~Hz}, 3 \mathrm{H})$.

$>{ }^{13} \mathrm{C} \mathrm{NMR}\left(100 \mathrm{MHz}, \mathrm{CDCl}_{3}\right) \delta 145.3,143.3,132.6,129.6,128.7,127.9,126.8,126.8,55.5$, $42.4,34.5,21.5,17.2,-6.6$.

$>\mathrm{IR}$ (neat) $\mathrm{cm}^{-1} 2920,2851,2135,1598,1492,1337,1160,1087,1006,963$.

$>$ HRMS calcd for $\mathrm{C}_{18} \mathrm{H}_{23} \mathrm{NO}_{2} \mathrm{SSi}(\mathrm{M}+\mathrm{Na})^{+} 368.1116$, found 368.1113.

\section{3,3-dimethyl-5-phenvl-1,3-azasilinane hydrogen chloride (13)}



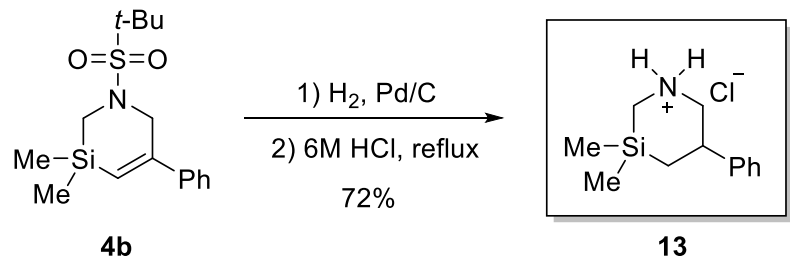

A suspension of $4 \mathbf{b}$ (1.30 g, 4 mmol, 1.0 equiv.) and $10 \% \mathrm{Pd} / \mathrm{C}(20 \% \mathrm{w} / \mathrm{t}, 260 \mathrm{mg})$ in $\mathrm{MeOH}(60$ $\mathrm{mL}$ ) was charged with $\mathrm{H}_{2}$ by balloon and stirred at room temperature for overnight. The mixture was filtrated and the filter cake washed with $\mathrm{CH}_{2} \mathrm{Cl}_{2}(20 \mathrm{~mL})$. The combine solutions were concentrated under reduced pressure to give hydrogenated product as white solid (1.16 g, 90\%). Then a solution of the above hydrogenated product (195 mg, $0.6 \mathrm{mmol}, 1.0$ equiv.) in aq. $\mathrm{HCl}$ (12 $\mathrm{mL} 6 \mathrm{~mol} / \mathrm{L}$ ) were heated at $115^{\circ} \mathrm{C}$ for $3 \mathrm{~h}$. The mixture was concentrated under high vacuum to give the product $\mathbf{1 3}$ as white solid (104.1 mg, 72\%). Growing single crystal of $\mathbf{1 3}$ in $\mathrm{MeOH}$ resulted in the formation of colorless single crystals suitable for X-ray analysis.

$>\mathrm{mp}: 148.5-150.3^{\circ} \mathrm{C}$.

$>{ }^{1} \mathrm{H}$ NMR $\left(400 \mathrm{MHz}, \mathrm{D}_{2} \mathrm{O}\right) \delta 7.47-47.43(\mathrm{~m}, 2 \mathrm{H}), 7.38-7.34(\mathrm{~m}, 3 \mathrm{H}), 3.36(\mathrm{~d}, J=12.7 \mathrm{~Hz}$, $1 \mathrm{H}), 3.25(\mathrm{tt}, J=12.1,3.9 \mathrm{~Hz}, 1 \mathrm{H}), 3.02(\mathrm{t}, J=12.4 \mathrm{~Hz}, 1 \mathrm{H}), 2.83(\mathrm{~d}, J=14.8 \mathrm{~Hz}, 1 \mathrm{H}), 2.57$ $(\mathrm{d}, J=14.9 \mathrm{~Hz}, 1 \mathrm{H}), 1.29-1.18(\mathrm{~m}, 2 \mathrm{H}), 0.37(\mathrm{~s}, 3 \mathrm{H}), 0.25(\mathrm{~s}, 3 \mathrm{H})$.

$>{ }^{13} \mathrm{C}$ NMR $\left(100 \mathrm{MHz}, \mathrm{D}_{2} \mathrm{O}\right) \delta 144.6,129.1,127.3,126.6,51.5,38.0,33.8,18.5,-4.8,-5.5$.

$>$ IR (neat) $\mathrm{cm}^{-1} 3406,3025,2948,2744,2668,2526,1596,1454,1491,1173,1039,949$.

$>$ HRMS calcd for $\mathrm{C}_{12} \mathrm{H}_{19} \mathrm{NSi}(\mathrm{M}+\mathrm{H})^{+} 206.1360$, found 206.1368 .

\section{1-cinnamyl-3,3-dimethyl-5-phenyl-1,3-azasilinane (14)}

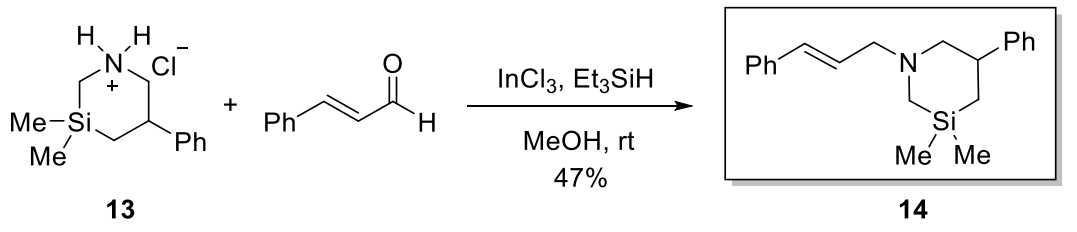

A solution of 13 (48.4 mg, $0.2 \mathrm{mmol}, 1.0$ equiv.) and cinnamaldehyde ( $29.1 \mathrm{mg}, 0.22 \mathrm{mmol}, 1.1$ equiv.) in $\mathrm{MeOH}(1 \mathrm{~mL})$ was stirred at room temperature for $1 \mathrm{~h}$. To the resulting mixture was added $\mathrm{InCl}_{3}$ (13.3 mg, $0.06 \mathrm{mmol}, 0.3$ equiv.) and $\mathrm{Et}_{3} \mathrm{SiH}$ ( $70.0 \mathrm{mg}, 0.6 \mathrm{mmol}, 3.0$ equiv.) The reaction was stirred at room temperature overnight before quenching with sat. aq. $\mathrm{K}_{2} \mathrm{CO}_{3}(5.0 \mathrm{~mL})$ and extracting with EtOAc $(5 \mathrm{~mL} \times 3)$. The combined organic phases washed with sat. aq. $\mathrm{NaCl}$, dried over $\mathrm{Na}_{2} \mathrm{SO}_{4}$ and concentrated under reduced pressure. The residue was purified by silica gel flash column chromatography (eluent: $\left.\mathrm{CH}_{2} \mathrm{Cl}_{2} / \mathrm{MeOH}=50 / 1\right)$ to afford 14 as yellow oil (30 $\mathrm{mg}, 47 \%$ ).

$>\mathrm{R}_{f}=0.4\left(\mathrm{MeOH}: \mathrm{CH}_{2} \mathrm{Cl}_{2}=1: 20\right)$.

$>{ }^{1} \mathrm{H}$ NMR $\left(600 \mathrm{MHz}, \mathrm{CDCl}_{3}\right) \delta 7.37(\mathrm{~d}, J=7.6 \mathrm{~Hz}, 2 \mathrm{H}), 7.29$ (q, $\left.J=7.8,7.2 \mathrm{~Hz}, 4 \mathrm{H}\right), 7.31-$ $7.16(\mathrm{~m}, 4 \mathrm{H}), 6.48(\mathrm{~d}, J=15.8 \mathrm{~Hz}, 1 \mathrm{H}), 6.31-6.25(\mathrm{~m}, 1 \mathrm{H}), 3.33(\mathrm{dd}, J=13.6,6.0 \mathrm{~Hz}, 1 \mathrm{H})$, $3.06(\mathrm{dd}, J=13.6,6.8 \mathrm{~Hz}, 2 \mathrm{H}), 2.91(\mathrm{~d}, J=11.9 \mathrm{~Hz}, 1 \mathrm{H}), 2.38(\mathrm{~d}, J=13.8 \mathrm{~Hz}, 1 \mathrm{H}), 2.09(\mathrm{t}, J$ $=11.4 \mathrm{~Hz}, 1 \mathrm{H}), 1.49(\mathrm{~d}, J=13.8 \mathrm{~Hz}, 1 \mathrm{H}), 0.99(\mathrm{~d}, J=14.0 \mathrm{~Hz}, 1 \mathrm{H}), 0.81(\mathrm{t}, J=13.8 \mathrm{~Hz}, 1 \mathrm{H})$, $0.25(\mathrm{~s}, 3 \mathrm{H}), 0.05(\mathrm{~d}, J=1.8 \mathrm{~Hz}, 3 \mathrm{H})$.

$>{ }^{13} \mathrm{C} \mathrm{NMR}\left(150 \mathrm{MHz}, \mathrm{CDCl}_{3}\right) \delta 147.7,137.1,132.6,128.5,128.4,127.4,127.3,126.7,126.3$, $126.0,65.8,64.9,44.8,42.2,20.0,-3.1,-4.0$.

$>$ IR (neat) $\mathrm{cm}^{-1} 3026,2919,2785,1728,1600,1493,1450,1089,1027,967$.

$>$ HRMS calcd for $\mathrm{C}_{21} \mathrm{H}_{27} \mathrm{NSi}(\mathrm{M}+\mathrm{H})^{+} 322.1986$, found 322.1980 .

\section{(3,3-dimethvl-5-phenvl-1,3-azasilinan-1-yl)(2-hvdroxvphenvl)methanone (15)}

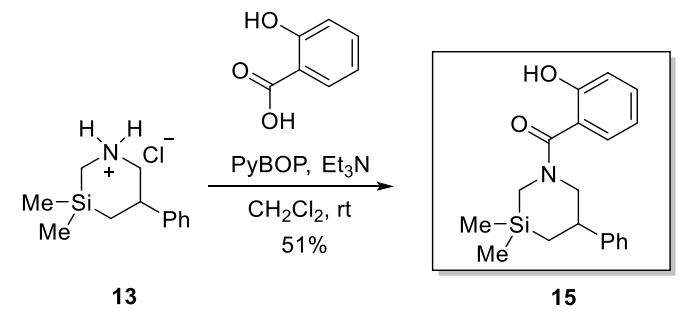


To a solution of 13 ( $83 \mathrm{mg}, 0.35 \mathrm{mmol}, 1.0$ equiv.) and salicylic acid (120 mg, $0.87 \mathrm{mmol}, 2.5$ equiv.) in $\mathrm{CH}_{2} \mathrm{Cl}_{2}(5 \mathrm{~mL})$ were added $\mathrm{PyBOP}\left(312 \mathrm{mg}, 0.6 \mathrm{mmol}, 1.7\right.$ equiv.) and $\mathrm{Et}_{3} \mathrm{~N}$ (121 mg, 1.2 mmol, 3.4 equiv.) at room temperature. The reaction was stirred overnight under Ar before diluting with by $\mathrm{CH}_{2} \mathrm{Cl}_{2}(5 \mathrm{~mL})$ and washing with aq. $\mathrm{NaOH}(10 \mathrm{~mL}, 1 \mathrm{M})$, aq. $\mathrm{HCl}(10 \mathrm{~mL}, 1 \mathrm{M})$, aq. $\mathrm{NaHCO}_{3}(10 \mathrm{~mL}, 1 \mathrm{M})$ and sat. aq. $\mathrm{NaCl}(10 \mathrm{~mL})$. The organic phases were dried over $\mathrm{Na}_{2} \mathrm{SO}_{4}$ and concentrated under reduced pressure. The residue was purified by silica gel flash column chromatography (eluent: petroleum ether/EtOAc $=5 / 1$ ) to afford 15 as a yellow oil ( $58 \mathrm{mg}, 51 \%$ ).

$>\mathrm{R}_{f}=0.8($ EtOAc:Petroleum Ether $=1: 1)$.

$>{ }^{1} \mathrm{H} \mathrm{NMR}\left(400 \mathrm{MHz}, \mathrm{CDCl}_{3}\right) 9.51(\mathrm{~s}, 1 \mathrm{H}), 7.36-7.27(\mathrm{~m}, 4 \mathrm{H}), 7.25-7.20(\mathrm{~m}, 3 \mathrm{H}), 6.98(\mathrm{~d}, J$ $=8.3 \mathrm{~Hz}, 1 \mathrm{H}), 6.84(\mathrm{t}, J=7.3 \mathrm{~Hz}, 1 \mathrm{H}), 4.32(\mathrm{~d}, J=13.6 \mathrm{~Hz}, 1 \mathrm{H}), 4.06(\mathrm{~d}, J=15.2 \mathrm{~Hz}, 1 \mathrm{H})$, $3.16(\mathrm{tt}, J=12.0,3.3 \mathrm{~Hz}, 1 \mathrm{H}), 2.84(\mathrm{t}, J=12.5 \mathrm{~Hz}, 1 \mathrm{H}), 2.55(\mathrm{~d}, J=14.8 \mathrm{~Hz}, 1 \mathrm{H}), 1.21-1.08$ $(\mathrm{m}, 2 \mathrm{H}), 0.19(\mathrm{~d}, J=7.9 \mathrm{~Hz}, 6 \mathrm{H})$.

$>13 \mathrm{C} \mathrm{NMR}\left(100 \mathrm{MHz}, \mathrm{CDCl}_{3}\right) \delta 171.0,158.5,145.0,132.2,128.7,127.9,126.8,126.6,118.5$, $118.1,118.0,56.7,41.7,38.4,20.3,-3.5,-4.8$.

$>\quad$ IR (neat) $\mathrm{cm}-1$ 3422, 2924, 2854, 1662, 1602, 1449, 1388, 1097.

$>$ HRMS calcd for $\mathrm{C}_{19} \mathrm{H}_{23} \mathrm{NO}_{2} \mathrm{Si}(\mathrm{M}+\mathrm{H})^{+} 348.1390$, found 348.1397 . 


\section{Mechanistic Studies}

Preparation of 3a: To a solution of $2 \mathbf{a}(58.4 \mathrm{mg}, 0.2 \mathrm{mmol}, 1.0$ equiv. $)$ in toluene- $d_{8}(0.5 \mathrm{~mL}$, dried by sodium) was added DBU $(30.5 \mathrm{mg}, 0.2 \mathrm{mmol}, 1.0$ equiv.) in glovebox. The resulting mixture in a sealed tube was then removed from glovebox and stirred at $120^{\circ} \mathrm{C}$ for $10 \mathrm{~min}$. Then the flask was cooled by liquid nitrogen for $3 \mathrm{~min}$ and warmed to $\mathrm{rt}$ slowly (repeat 3 times). The supernatant containing crude 3a was drawn off by syringe into an NMR tube for NMR experiment studies.

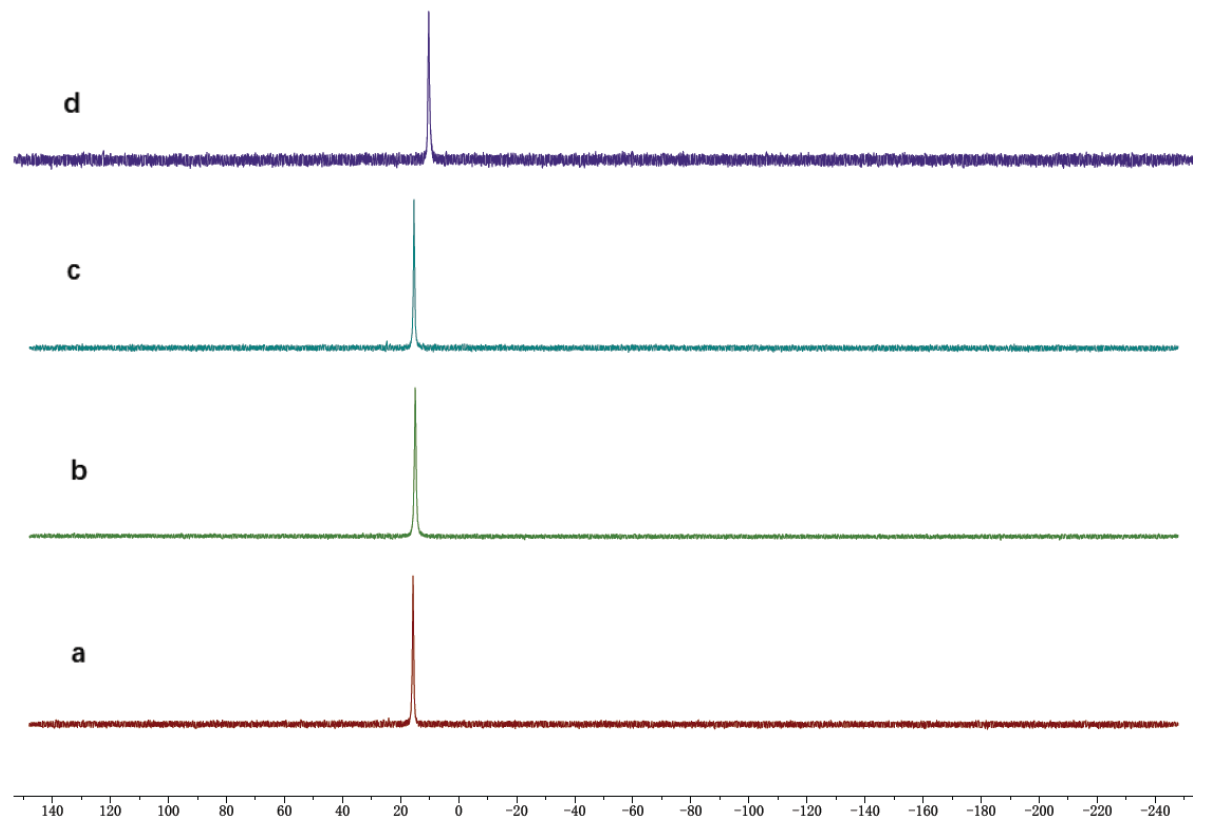

Fig. S-1 ${ }^{31} \mathrm{P}$ NMR spectra in toluene- $d_{8}$. a) $\mathrm{Pd}\left(\mathrm{PPh}_{3}\right)_{4}$ at $25{ }^{\circ} \mathrm{C}$; b) $3 \mathrm{a} / \mathrm{Pd}\left(\mathrm{PPh}_{3}\right)_{4}(20: 1)$ at $25{ }^{\circ} \mathrm{C}$; c) $3 a / P d\left(\mathrm{PPh}_{3}\right)_{4}(1: 1)$ at $\left.25^{\circ} \mathrm{C} ; \mathrm{d}\right) 3 \mathrm{a} / \mathrm{Pd}\left(\mathrm{PPh}_{3}\right)_{4}(1: 1)$ heating at $90{ }^{\circ} \mathrm{C}$ for $10 \mathrm{~min}$, then monitored at 25 ${ }^{\circ} \mathrm{C}$.

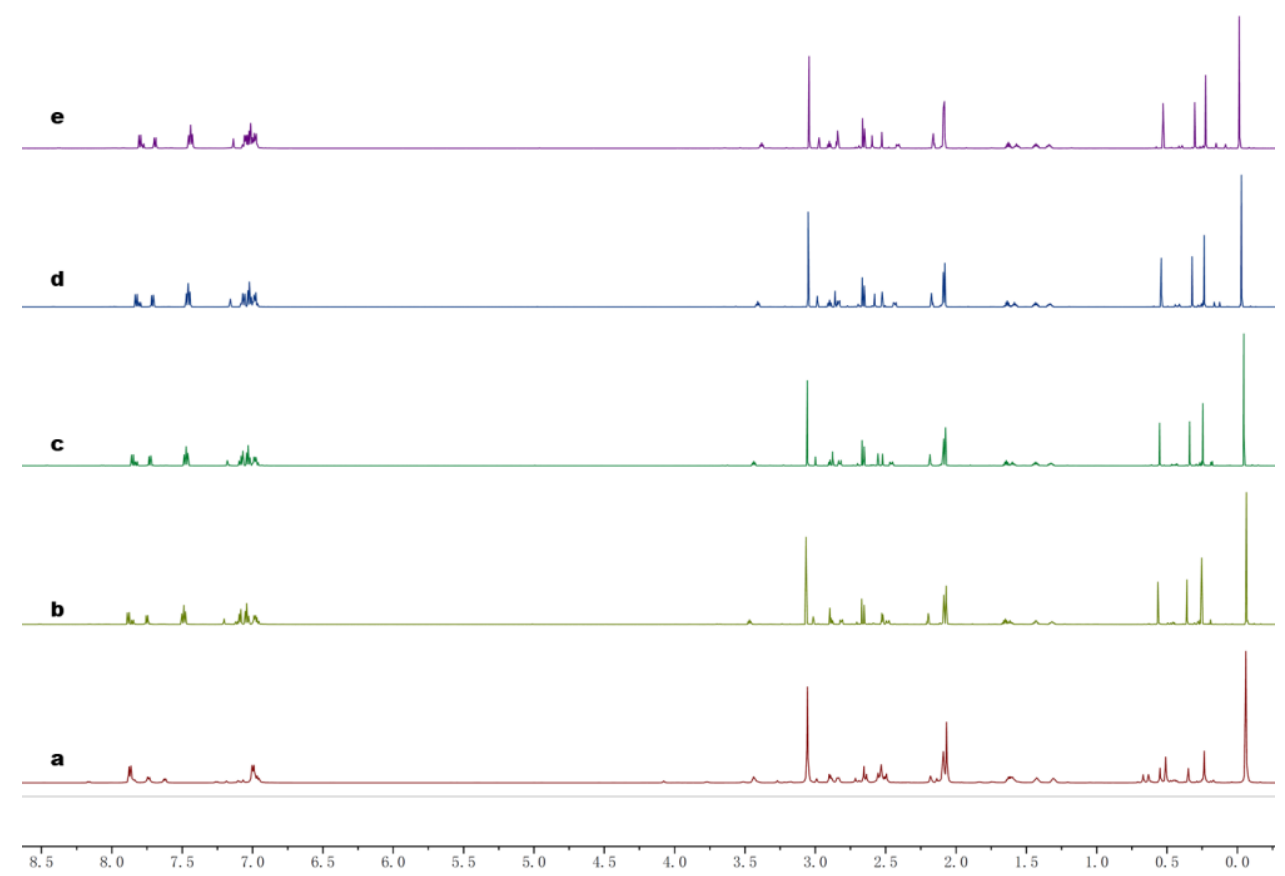

Fig. S-2 ${ }^{1} \mathrm{H}$ NMR spectra of in toluene- $d_{8}$. a) $3 a$ at $25{ }^{\circ} \mathrm{C}$; b) $3 a / P d\left(P P h_{3}\right)_{4}(1: 1)$ at $25{ }^{\circ} \mathrm{C} ;$ c)

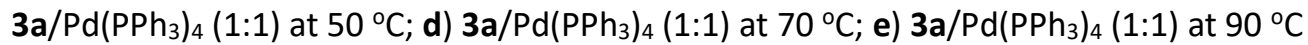




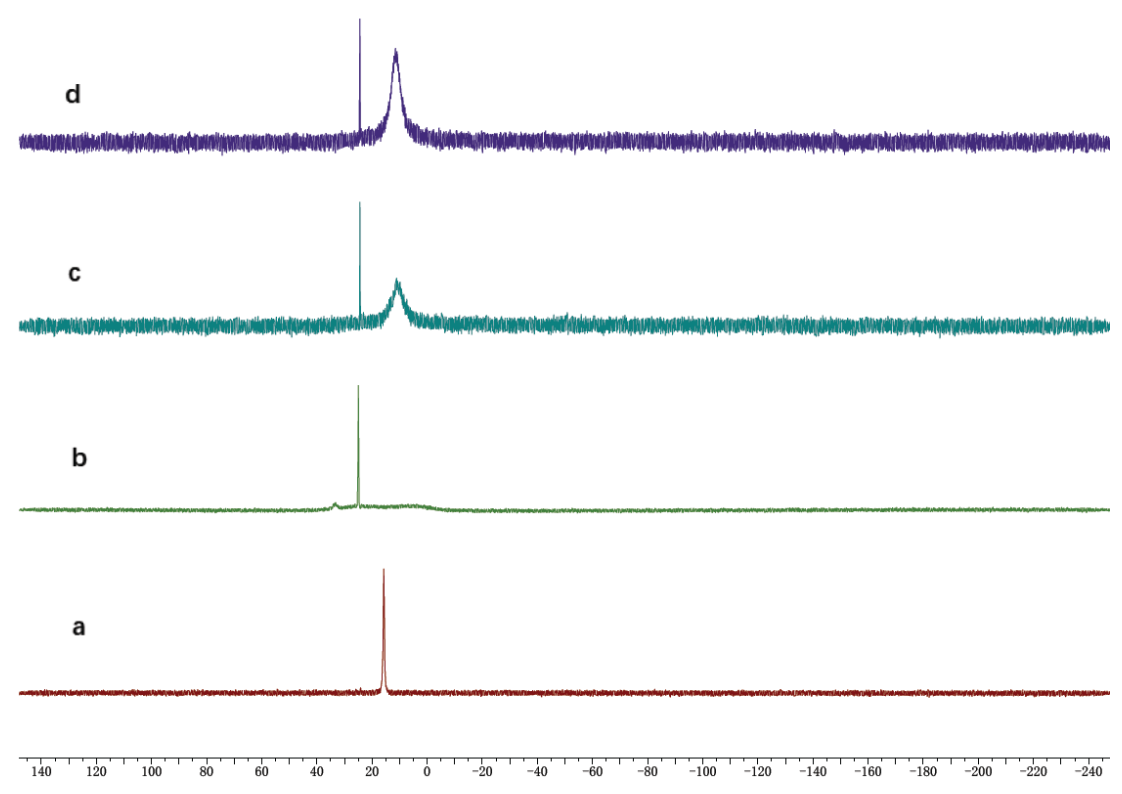

Fig. S-3 ${ }^{31} \mathrm{P}$ NMR spectra in toluene- $d_{8}$. a) $\mathrm{Pd}\left(\mathrm{PPh}_{3}\right)_{4}$ at $25^{\circ} \mathrm{C}$; b) $\mathrm{CF}_{3}-p-\mathrm{C}_{6} \mathrm{H}_{4} \mathrm{CCH} / \mathrm{Pd}\left(\mathrm{PPh}_{3}\right)_{4}(24: 1)$ at $25^{\circ} \mathrm{C}$; c) $\mathrm{CF}_{3}-p-\mathrm{C}_{6} \mathrm{H}_{4} \mathrm{CCH} / \mathrm{Pd}\left(\mathrm{PPh}_{3}\right)_{4}(1: 1)$ at $25^{\circ} \mathrm{C}$; d) $\mathrm{CF}_{3}-p-\mathrm{C}_{6} \mathrm{H}_{4} \mathrm{CCH} / \mathrm{Pd}\left(\mathrm{PPh}_{3}\right)_{4}(1: 1)$ heating at $90{ }^{\circ} \mathrm{C}$ for $10 \mathrm{~min}$, then monitored at $25^{\circ} \mathrm{C}$.

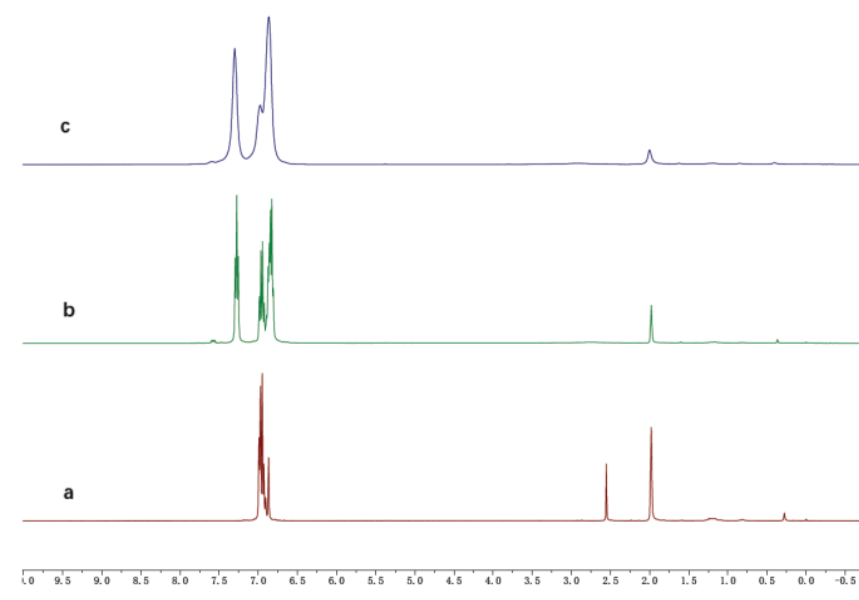

$p-\mathrm{CF}_{3}-\mathrm{C}_{6} \mathrm{H}_{4}=\mathrm{H}$

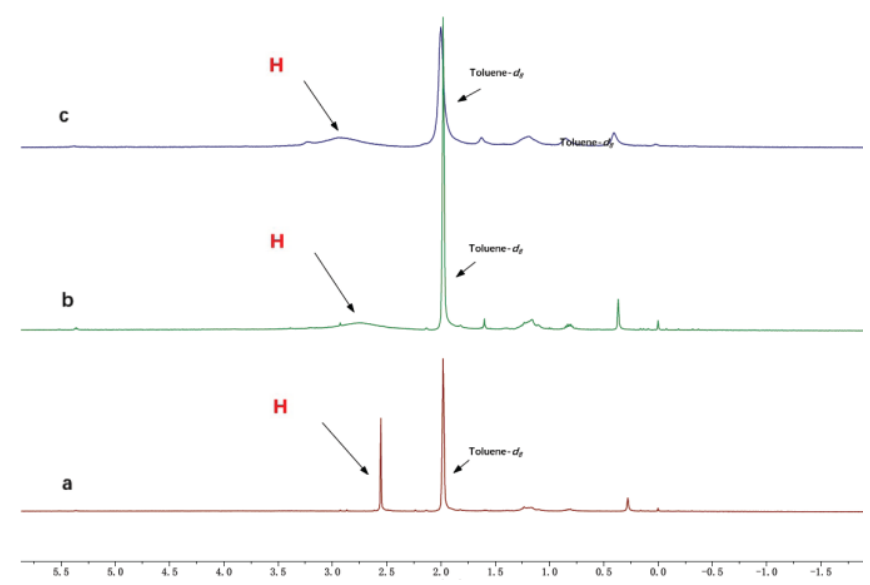

Fig. S-4 ${ }^{1} \mathrm{H}$ NMR spectra in toluene- $d_{8}$. a) $\mathrm{CF}_{3}-p-\mathrm{C}_{6} \mathrm{H}_{4} \mathrm{CCH}$ at $25^{\circ} \mathrm{C}$; b) $\mathrm{CF}_{3}-p-\mathrm{C}_{6} \mathrm{H}_{4} \mathrm{CCH} / \mathrm{Pd}\left(\mathrm{PPh}_{3}\right)_{4}(1: 1)$ at $\left.25^{\circ} \mathrm{C} ; \mathrm{c}\right) \mathrm{CF}_{3}-p-\mathrm{C}_{6} \mathrm{H}_{4} \mathrm{CCH} / \mathrm{Pd}\left(\mathrm{PPh}_{3}\right)_{4}(1: 1)$ heating at $90^{\circ} \mathrm{C}$ for $10 \mathrm{~min}$, then monitored at $25^{\circ} \mathrm{C}$. 


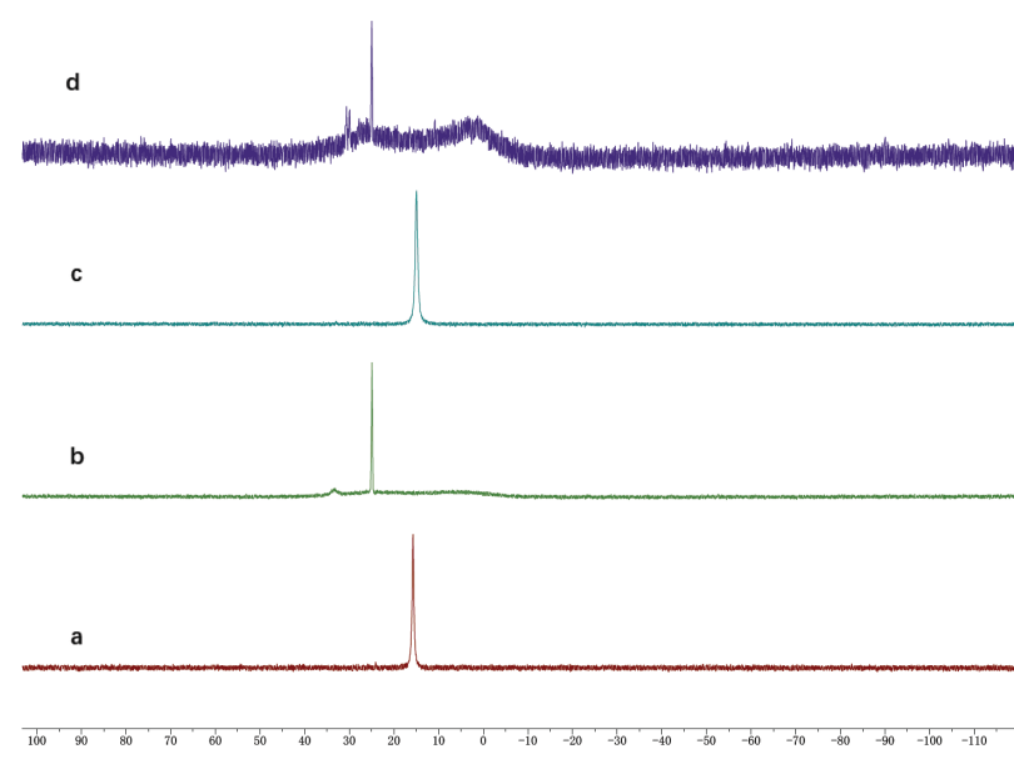

Fig. S-5 ${ }^{31} \mathrm{P}$ NMR spectra in toluene- $d_{8}$. a) $\mathrm{Pd}\left(\mathrm{PPh}_{3}\right)_{4}$ at $25^{\circ} \mathrm{C}$ b) $\mathrm{CF}_{3}-p-\mathrm{C}_{6} \mathrm{H}_{4} \mathrm{CCH} / \mathrm{Pd}\left(\mathrm{PPh}_{3}\right)_{4}(24: 1)$ at $\left.25^{\circ} \mathrm{C} ; \mathrm{c}\right) 3 \mathrm{a} / \mathrm{Pd}\left(\mathrm{PPh}_{3}\right)_{4}(20: 1)$ at $\left.25^{\circ} \mathrm{C} ; \mathrm{d}\right) \mathrm{CF}_{3}-p-\mathrm{C}_{6} \mathrm{H}_{4} \mathrm{CCH} / 3 \mathrm{a} / \mathrm{Pd}\left(\mathrm{PPh}_{3}\right)_{4}(24: 20: 1) 25^{\circ} \mathrm{C}$.

a)

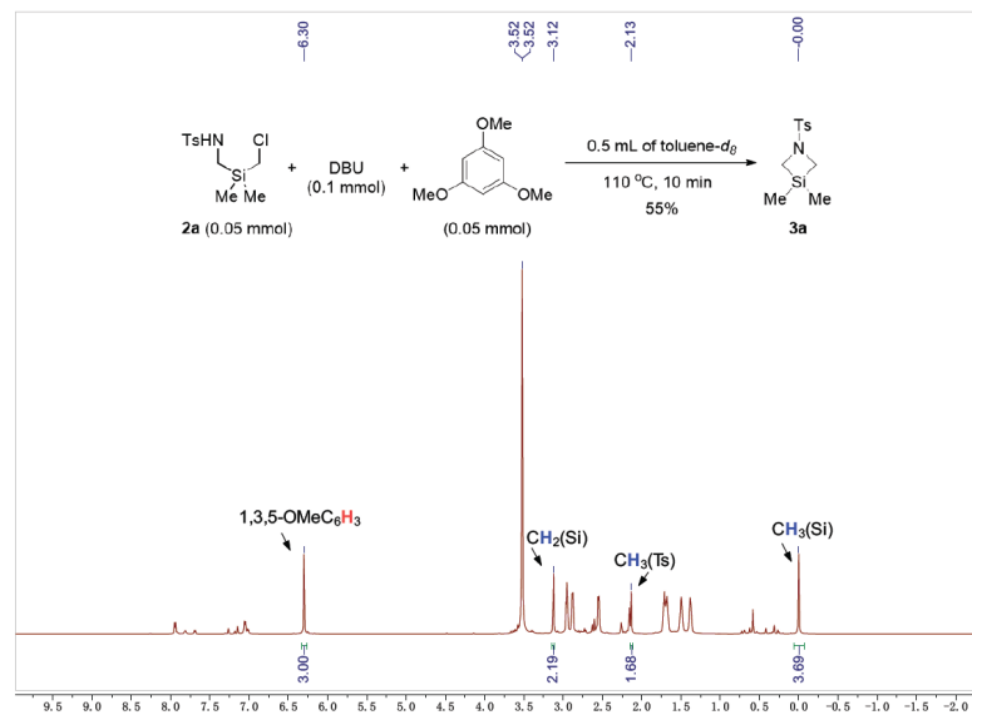

b)

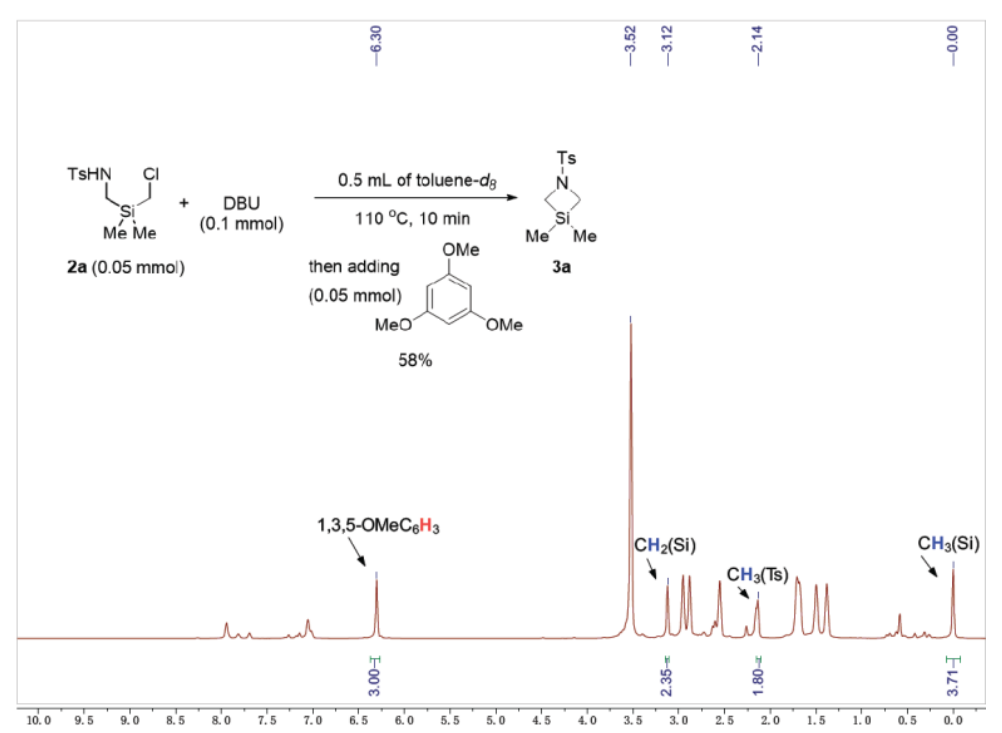




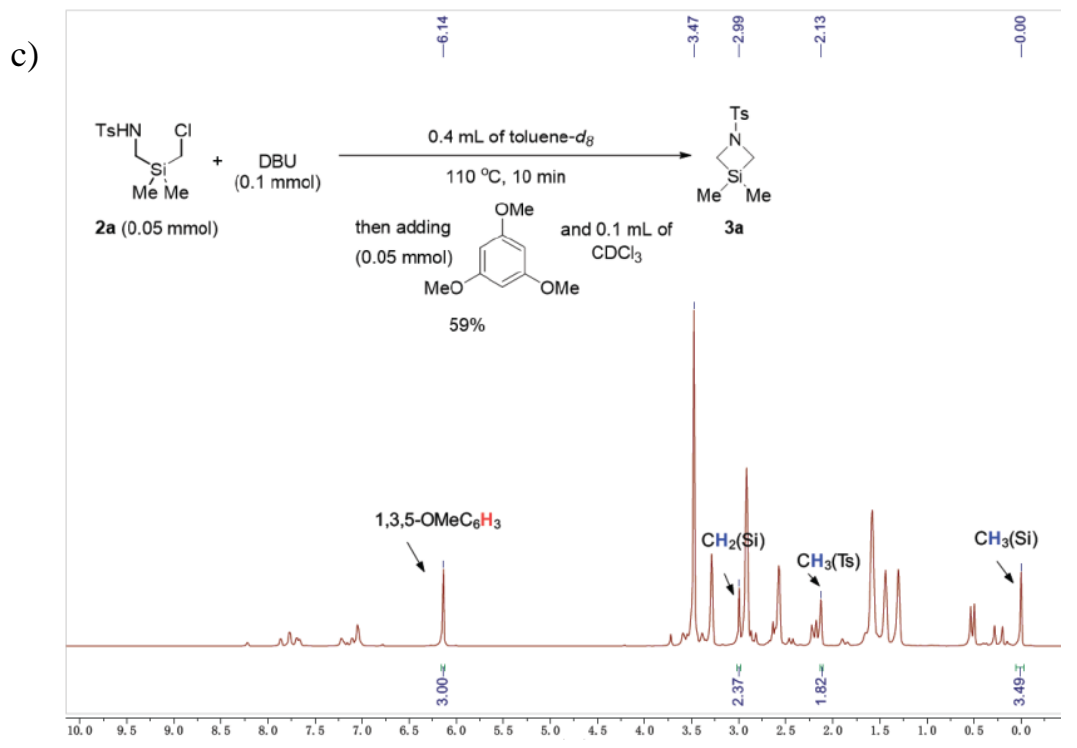

Fig. S-6 In situ ${ }^{1} \mathrm{H}$ NMR methods to monitor the yield of 3a using $1,3,5-\mathrm{OMeC}_{6} \mathrm{H}_{3}$ as internal reference. a) heating the mixture of $\mathbf{2 a}(0.05 \mathrm{mmol}), \mathrm{DBU}(0.1 \mathrm{mmol})$ and $1,3,5-\mathrm{OMeC}_{6} \mathrm{H}_{3}(0.05$ $\mathrm{mmol}$ ) in $0.5 \mathrm{~mL}$ of toluene- $d_{8}$ at $110^{\circ} \mathrm{C}$ for $10 \mathrm{~min}$ gave rise to a $55 \%$ NMR yield of $3 \mathbf{a}$; $\mathbf{b}$ ) heating the mixture of $2 \mathrm{a}(0.05 \mathrm{mmol})$ and $\mathrm{DBU}(0.1 \mathrm{mmol})$ in $0.5 \mathrm{~mL}$ of toluene- $d_{8}$ at $110^{\circ} \mathrm{C}$ for $10 \mathrm{~min}$ followed by the addition of $1,3,5-\mathrm{OMeC}_{6} \mathrm{H}_{3}(0.05 \mathrm{mmol})$ gave rise to a $58 \% \mathrm{NMR}$ yield of $3 \mathrm{a}$; c) heating the mixture of $2 \mathrm{a}(0.05 \mathrm{mmol})$ and DBU $(0.1 \mathrm{mmol})$ in $0.4 \mathrm{~mL}$ of toluene- $d_{8}$ at $110^{\circ} \mathrm{C}$ for 10 min followed by the addition of $1,3,5-\mathrm{OMeC}_{6} \mathrm{H}_{3}(0.05 \mathrm{mmol})$, and $0.1 \mathrm{~mL}$ of $\mathrm{CDCl}_{3}$ to dissolve the insoluble stuff. This mixed solvent system gave the NMR spectrum is more complex than that without $\mathrm{CDCl}_{3}$, giving a $59 \%$ NMR yield of $3 a$. The in situ ${ }^{1} \mathrm{H}$ NMR yield of 3 a monitored by the above experiments appeared being not consistent with the observed high yields (up to $84 \%$ ) of 3silatetrahydropyridines 4 . A reasonable explanation of this inconsistence is that in the reaction to form 3-silatetrahydropyridines $\mathbf{4}$, once $\mathbf{3} \mathbf{a}$ was generated from $\mathbf{2 a}$, it can be quickly trapped by alkyne via ring expansion, thus minimizing the undesired consumption as much as possible. 


\section{X-Ray Crystallographic Data}

\section{Compound 4a}

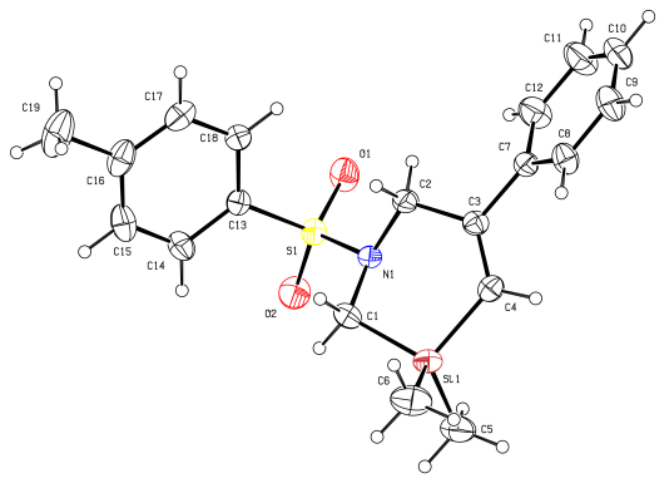

Table 1 Crystal data and structure refinement for $4 a$.

Identification code

4a

Empirical formula

$\mathrm{C}_{19} \mathrm{H}_{23} \mathrm{NO}_{2} \mathrm{SSi}$

Formula weight

357.53

Temperature/K

293.15

Crystal system

orthorhombic

Space group

Pca $2_{1}$

$\mathrm{a} / \AA ̊ \AA$

$12.4636(9)$

$\mathrm{b} / \AA$

$8.4119(6)$

$\mathrm{c} / \AA$

$18.4767(15)$

$\alpha /^{\circ}$

90

$\beta /{ }^{\circ}$

90

$\gamma /{ }^{\circ}$

90

Volume $/ \AA^{3}$

1937.1(3)

Z

4

$\rho_{\text {calc }} / \mathrm{cm}^{3} \quad 1.226$

$\mu / \mathrm{mm}^{-1} \quad 0.239$

$\mathrm{F}(000) \quad 760.0$

Crystal size $/ \mathrm{mm}^{3}$

$0.35 \times 0.3 \times 0.25$

Radiation

$\operatorname{MoK} \alpha(\lambda=0.71073)$

$2 \Theta$ range for data collection/ ${ }^{\circ}$

6.246 to 52.72

Index ranges

$-15 \leq \mathrm{h} \leq 8,-10 \leq \mathrm{k} \leq 10,-23 \leq 1 \leq 21$

Reflections collected

5320

Independent reflections

$3214\left[\mathrm{R}_{\text {int }}=0.0199, \mathrm{R}_{\text {sigma }}=0.0378\right]$

Data/restraints/parameters

$3214 / 1 / 220$

Goodness-of-fit on $\mathrm{F}^{2}$

1.072

Final $\mathrm{R}$ indexes $[\mathrm{I}>=2 \sigma(\mathrm{I})]$

$\mathrm{R}_{1}=0.0391, \mathrm{wR}_{2}=0.0858$

Final $\mathrm{R}$ indexes [all data]

$\mathrm{R}_{1}=0.0552, \mathrm{wR}_{2}=0.0960$

Largest diff. peak/hole / e $\AA^{-3}$

$0.16 /-0.23$

Flack parameter

$-0.02(7)$

Table 2 Fractional Atomic Coordinates $\left(\times 10^{4}\right)$ and Equivalent Isotropic Displacement Parameters $\left(\AA^{2} \times 10^{3}\right)$ for $4 \mathrm{a} . \mathrm{U}_{\text {eq }}$ is defined as $1 / 3$ of of the trace of the orthogonalised U IJ tensor. 


$\begin{array}{ccccc}\text { Atom } & \boldsymbol{x} & \boldsymbol{y} & \boldsymbol{z} & \mathbf{U}(\mathbf{e q}) \\ \mathrm{S} 1 & 2560.7(8) & 192.8(10) & 3562.7(6) & 40.9(2) \\ \text { Si1 } & 2984.2(10) & 70.4(13) & 1294.5(7) & 44.5(3) \\ \text { O1 } & 2795(3) & 1565(3) & 3986.2(16) & 60.0(8) \\ \text { O2 } & 1470(2) & -258(4) & 3455.8(19) & 60.6(8) \\ \text { N1 } & 3076(2) & 501(3) & 2757.5(16) & 36.4(7) \\ \text { C1 } & 2936(4) & -801(5) & 2229(2) & 53.3(11) \\ \text { C2 } & 4143(3) & 1235(5) & 2741(2) & 47.2(10) \\ \text { C3 } & 4362(3) & 2135(4) & 2048(2) & 39.5(8) \\ \text { C4 } & 3910(3) & 1768(4) & 1418(2) & 44.5(9) \\ \text { C5 } & 1636(4) & 751(6) & 999(3) & 65.2(13) \\ \text { C6 } & 3535(4) & -1385(6) & 644(3) & 75.4(15) \\ \text { C7 } & 5166(3) & 3449(5) & 2124(2) & 40.2(8) \\ \text { C8 } & 5974(3) & 3658(5) & 1619(2) & 51.1(10) \\ \text { C9 } & 6736(4) & 4840(5) & 1697(3) & 66.1(13) \\ \text { C10 } & 6692(4) & 5849(6) & 2275(3) & 71.9(14) \\ \text { C11 } & 5894(5) & 5679(6) & 2773(3) & 77.7(16) \\ \text { C12 } & 5149(4) & 4493(6) & 2703(3) & 64.6(13) \\ \text { C13 } & 3233(3) & -1457(4) & 3941.2(19) & 37.1(8) \\ \text { C14 } & 2758(3) & -2946(5) & 3918(2) & 51.3(11) \\ \text { C15 } & 3298(4) & -4228(5) & 4202(3) & 61.6(13) \\ \text { C16 } & 4303(4) & -4077(6) & 4497(2) & 61.4(13) \\ \text { C17 } & 4775(4) & -2576(6) & 4497(2) & 58.9(11) \\ \text { C18 } & 4237(3) & -1271(5) & 4226.4(19) & 44.8(9) \\ \text { C19 } & 4901(6) & -5498(6) & 4797(4) & 97(2) \\ & & & & \end{array}$

Table 3 Anisotropic Displacement Parameters $\left(\AA^{2} \times 10^{3}\right)$ for $4 \mathrm{a}$. The Anisotropic displacement factor exponent takes the form: $-2 \pi^{2}\left[h^{2} a^{* 2} U_{11}+2 h k a * b * U_{12}+\ldots\right]$.

$\begin{array}{ccccccc}\text { Atom } & \mathbf{U}_{\mathbf{1 1}} & \mathbf{U}_{\mathbf{2 2}} & \mathbf{U}_{\mathbf{3 3}} & \mathbf{U}_{\mathbf{2 3}} & \mathbf{U}_{\mathbf{1 3}} & \mathbf{U}_{\mathbf{1 2}} \\ \mathrm{S} 1 & 35.5(5) & 42.2(4) & 45.0(5) & 2.7(5) & 3.6(5) & 2.5(4) \\ \mathrm{S} 11 & 43.4(6) & 50.7(6) & 39.4(6) & -7.9(5) & -3.6(6) & -1.5(5) \\ \mathrm{O} 1 & 86(2) & 41.5(15) & 53.0(15) & -7.7(14) & 7.6(16) & 8.1(16) \\ \mathrm{O} 2 & 30.7(14) & 76.7(19) & 74(2) & 12.9(18) & 6.0(17) & 2.2(14) \\ \mathrm{N} 1 & 33.5(17) & 37.9(15) & 37.9(16) & 1.8(14) & -4.5(14) & -3.1(14) \\ \mathrm{C} 1 & 63(3) & 41(2) & 55(3) & -7.4(19) & -9(2) & -11(2) \\ \mathrm{C} 2 & 38(2) & 59(2) & 45(2) & 10(2) & -7.3(19) & -15.2(19) \\ \mathrm{C} 3 & 31.5(18) & 45(2) & 42.1(19) & 0.1(17) & 0.9(18) & -0.7(17) \\ \mathrm{C} 4 & 48(2) & 48(2) & 37(2) & 1.5(18) & -0.4(18) & 1(2) \\ \mathrm{C} 5 & 55(3) & 64(3) & 76(3) & -13(3) & -18(3) & -1(2) \\ \mathrm{C} 6 & 70(3) & 90(4) & 67(3) & -32(3) & 2(3) & -1(3) \\ \mathrm{C} 7 & 37.6(19) & 42.9(19) & 40.2(19) & 2.4(17) & -0.9(18) & -3.7(18) \\ \mathrm{C} 8 & 53(3) & 45(2) & 55(2) & 0(2) & 13(2) & -4(2) \\ \mathrm{C} 9 & 55(3) & 63(3) & 80(3) & 9(3) & 17(3) & -18(2) \\ \mathrm{C} 10 & 81(4) & 57(3) & 77(3) & 5(3) & -3(3) & -31(3) \\ \mathrm{C} 11 & 97(4) & 66(3) & 71(3) & -15(3) & 9(3) & -32(3) \\ \mathrm{C} 12 & 68(3) & 71(3) & 55(3) & -10(3) & 14(3) & -19(3) \\ \mathrm{C} 13 & 38(2) & 39.1(19) & 34.2(17) & 0.4(17) & 5.4(17) & 2.3(18) \\ \mathrm{C} 14 & 46(2) & 47(2) & 62(2) & 2(2) & 4(2) & -9(2) \\ \text { C15 } & 74(3) & 38(2) & 73(3) & 4(2) & 20(3) & 2(2) \\ \text { C16 } & 82(4) & 56(3) & 47(2) & 7(2) & 12(3) & 25(3) \\ \text { C17 } & 56(3) & 73(3) & 48(2) & 8(2) & -6(2) & 9(3) \\ & & & & & & \end{array}$




$\begin{array}{llllccc}\mathrm{C} 18 & 48(2) & 45(2) & 41(2) & 0.2(18) & -2.3(19) & -2.5(19) \\ \mathrm{C} 19 & 130(6) & 71(3) & 90(5) & 21(4) & 10(4) & 51(4)\end{array}$

Table 4 Bond Lengths for 4 a.

\begin{tabular}{|c|c|c|c|c|c|}
\hline Atom & Atom & Length/Å & Atom & Atom & Length/Å \\
\hline S1 & $\mathrm{O} 1$ & $1.425(3)$ & $\mathrm{C} 7$ & $\mathrm{C} 8$ & $1.385(5)$ \\
\hline S1 & $\mathrm{O} 2$ & $1.426(3)$ & $\mathrm{C} 7$ & $\mathrm{C} 12$ & $1.384(6)$ \\
\hline S1 & N1 & $1.641(3)$ & $\mathrm{C} 8$ & C9 & $1.382(6)$ \\
\hline S1 & $\mathrm{C} 13$ & $1.765(4)$ & C9 & $\mathrm{C} 10$ & $1.366(7)$ \\
\hline Si1 & $\mathrm{C} 1$ & $1.877(4)$ & $\mathrm{C} 10$ & $\mathrm{C} 11$ & $1.363(7)$ \\
\hline Si1 & $\mathrm{C} 4$ & $1.850(4)$ & $\mathrm{C} 11$ & $\mathrm{C} 12$ & $1.369(6)$ \\
\hline Si1 & $\mathrm{C} 5$ & $1.858(5)$ & $\mathrm{C} 13$ & $\mathrm{C} 14$ & $1.387(5)$ \\
\hline Sil & C6 & $1.848(5)$ & $\mathrm{C} 13$ & $\mathrm{C} 18$ & $1.367(5)$ \\
\hline N1 & $\mathrm{C} 1$ & $1.478(5)$ & $\mathrm{C} 14$ & $\mathrm{C} 15$ & $1.376(6)$ \\
\hline N1 & $\mathrm{C} 2$ & $1.467(4)$ & $\mathrm{C} 15$ & $\mathrm{C} 16$ & $1.371(7)$ \\
\hline $\mathrm{C} 2$ & $\mathrm{C} 3$ & $1.512(5)$ & $\mathrm{C} 16$ & $\mathrm{C} 17$ & $1.394(6)$ \\
\hline $\mathrm{C} 3$ & $\mathrm{C} 4$ & $1.331(5)$ & $\mathrm{C} 16$ & C19 & $1.513(7)$ \\
\hline C3 & $\mathrm{C} 7$ & $1.498(5)$ & $\mathrm{C} 17$ & $\mathrm{C} 18$ & $1.380(5)$ \\
\hline
\end{tabular}

Table 5 Bond Angles for $4 a$.

$\begin{array}{cccccccc}\text { Atom } & \text { Atom } & \text { Atom } & \text { Angle }^{\circ} & \text { Atom } & \text { Atom } & \text { Atom } & \text { Angle }{ }^{\circ} \\ \text { O1 } & \text { S1 } & \text { O2 } & 119.2(2) & \text { C3 } & \text { C4 } & \text { Si1 } & 123.4(3) \\ \text { O1 } & \text { S1 } & \text { N1 } & 106.83(17) & \text { C8 } & \text { C7 } & \text { C3 } & 121.1(3) \\ \text { O1 } & \text { S1 } & \text { C13 } & 108.76(19) & \text { C12 } & \text { C7 } & \text { C3 } & 122.0(3) \\ \text { O2 } & \text { S1 } & \text { N1 } & 106.83(19) & \text { C12 } & \text { C7 } & \text { C8 } & 116.8(4) \\ \text { O2 } & \text { S1 } & \text { C13 } & 107.37(18) & \text { C9 } & \text { C8 } & \text { C7 } & 121.4(4) \\ \text { N1 } & \text { S1 } & \text { C13 } & 107.32(16) & \text { C10 } & \text { C9 } & \text { C8 } & 120.1(5) \\ \text { C4 } & \text { Si1 } & \text { C1 } & 102.03(18) & \text { C11 } & \text { C10 } & \text { C9 } & 119.5(5) \\ \text { C4 } & \text { Si1 } & \text { C5 } & 111.3(2) & \text { C10 } & \text { C11 } & \text { C12 } & 120.4(5) \\ \text { C5 } & \text { Si1 } & \text { C1 } & 111.2(2) & \text { C11 } & \text { C12 } & \text { C7 } & 121.8(4) \\ \text { C6 } & \text { Si1 } & \text { C1 } & 110.6(2) & \text { C14 } & \text { C13 } & \text { S1 } & 119.7(3) \\ \text { C6 } & \text { Si1 } & \text { C4 } & 111.1(2) & \text { C18 } & \text { C13 } & \text { S1 } & 119.9(3) \\ \text { C6 } & \text { Si1 } & \text { C5 } & 110.4(2) & \text { C18 } & \text { C13 } & \text { C14 } & 120.4(4) \\ \text { C1 } & \text { N1 } & \text { S1 } & 115.8(2) & \text { C15 } & \text { C14 } & \text { C13 } & 119.1(4) \\ \text { C2 } & \text { N1 } & \text { S1 } & 116.1(2) & \text { C16 } & \text { C15 } & \text { C14 } & 121.8(4) \\ \text { C2 } & \text { N1 } & \text { C1 } & 113.9(3) & \text { C15 } & \text { C16 } & \text { C17 } & 118.0(4) \\ \text { N1 } & \text { C1 } & \text { Si1 } & 108.3(2) & \text { C15 } & \text { C16 } & \text { C19 } & 121.5(5) \\ \text { N1 } & \text { C2 } & \text { C3 } & 113.1(3) & \text { C17 } & \text { C16 } & \text { C19 } & 120.5(5) \\ \text { C4 } & \text { C3 } & \text { C2 } & 123.3(3) & \text { C18 } & \text { C17 } & \text { C16 } & 121.0(4) \\ \text { C4 } & \text { C3 } & \text { C7 } & 122.4(3) & \text { C13 } & \text { C18 } & \text { C17 } & 119.6(4) \\ \text { C7 } & \text { C3 } & \text { C2 } & 114.3(3) & & & & \end{array}$


Table 6 Torsion Angles for 4a.

\begin{tabular}{|c|c|c|c|c|c|c|c|c|c|}
\hline $\mathbf{A}$ & B & $\mathbf{C}$ & D & Angle $/^{\circ}$ & $\mathbf{A}$ & B & $\mathbf{C}$ & D & Angle ${ }^{\circ}$ \\
\hline $\mathrm{S} 1$ & $\mathrm{~N} 1$ & $\mathrm{C} 1$ & Si1 & $154.5(2)$ & $\mathrm{C} 4$ & Si1 & $\mathrm{C} 1$ & $\mathrm{~N} 1$ & $31.4(3)$ \\
\hline $\mathrm{S} 1$ & $\mathrm{~N} 1$ & $\mathrm{C} 2$ & $\mathrm{C} 3$ & $-154.4(3)$ & $\mathrm{C} 4$ & $\mathrm{C} 3$ & $\mathrm{C} 7$ & $\mathrm{C} 8$ & $-42.8(6)$ \\
\hline S1 & $\mathrm{C} 13$ & $\mathrm{C} 14$ & $\mathrm{C} 15$ & $178.8(3)$ & $\mathrm{C} 4$ & $\mathrm{C} 3$ & $\mathrm{C} 7$ & $\mathrm{C} 12$ & 138.2(4) \\
\hline S1 & $\mathrm{C} 13$ & $\mathrm{C} 18$ & $\mathrm{C} 17$ & $-177.6(3)$ & $\mathrm{C} 5$ & Si1 & $\mathrm{C} 1$ & $\mathrm{~N} 1$ & $-87.3(3)$ \\
\hline $\mathrm{O} 1$ & $\mathrm{~S} 1$ & $\mathrm{~N} 1$ & $\mathrm{C} 1$ & 179.2(3) & $\mathrm{C} 5$ & Si1 & $\mathrm{C} 4$ & $\mathrm{C} 3$ & 118.3(4) \\
\hline $\mathrm{O} 1$ & $\mathrm{~S} 1$ & $\mathrm{~N} 1$ & $\mathrm{C} 2$ & $41.6(3)$ & C6 & Si1 & $\mathrm{C} 1$ & $\mathrm{~N} 1$ & $149.6(3)$ \\
\hline $\mathrm{O} 1$ & $\mathrm{~S} 1$ & $\mathrm{C} 13$ & $\mathrm{C} 14$ & $145.4(3)$ & C6 & Si1 & $\mathrm{C} 4$ & $\mathrm{C} 3$ & $-118.2(4)$ \\
\hline $\mathrm{O} 1$ & $\mathrm{~S} 1$ & $\mathrm{C} 13$ & C18 & $-37.4(3)$ & $\mathrm{C} 7$ & $\mathrm{C} 3$ & $\mathrm{C} 4$ & Si 1 & $175.8(3)$ \\
\hline $\mathrm{O} 2$ & $\mathrm{~S} 1$ & $\mathrm{~N} 1$ & $\mathrm{C} 1$ & $-52.2(3)$ & $\mathrm{C} 7$ & $\mathrm{C} 8$ & C9 & $\mathrm{C} 10$ & $-1.1(7)$ \\
\hline $\mathrm{O} 2$ & $\mathrm{~S} 1$ & $\mathrm{~N} 1$ & $\mathrm{C} 2$ & $170.2(3)$ & $\mathrm{C} 8$ & $\mathrm{C} 7$ & $\mathrm{C} 12$ & $\mathrm{C} 11$ & $0.1(7)$ \\
\hline $\mathrm{O} 2$ & $\mathrm{~S} 1$ & $\mathrm{C} 13$ & $\mathrm{C} 14$ & $15.2(4)$ & $\mathrm{C} 8$ & $\mathrm{C} 9$ & $\mathrm{C} 10$ & $\mathrm{C} 11$ & $0.1(8)$ \\
\hline $\mathrm{O} 2$ & $\mathrm{~S} 1$ & $\mathrm{C} 13$ & $\mathrm{C} 18$ & $-167.6(3)$ & $\mathrm{C} 9$ & $\mathrm{C} 10$ & $\mathrm{C} 11$ & $\mathrm{C} 12$ & $0.9(9)$ \\
\hline N1 & $\mathrm{S} 1$ & $\mathrm{C} 13$ & $\mathrm{C} 14$ & $-99.3(3)$ & $\mathrm{C} 10$ & $\mathrm{C} 11$ & $\mathrm{C} 12$ & $\mathrm{C} 7$ & $-1.1(9)$ \\
\hline N1 & $\mathrm{S} 1$ & $\mathrm{C} 13$ & $\mathrm{C} 18$ & 77.8(3) & $\mathrm{C} 12$ & $\mathrm{C} 7$ & $\mathrm{C} 8$ & C9 & $1.0(6)$ \\
\hline N1 & $\mathrm{C} 2$ & $\mathrm{C} 3$ & $\mathrm{C} 4$ & $-28.1(5)$ & $\mathrm{C} 13$ & $\mathrm{~S} 1$ & $\mathrm{~N} 1$ & $\mathrm{C} 1$ & $62.7(3)$ \\
\hline N1 & $\mathrm{C} 2$ & $\mathrm{C} 3$ & $\mathrm{C} 7$ & $154.0(3)$ & $\mathrm{C} 13$ & $\mathrm{~S} 1$ & $\mathrm{~N} 1$ & $\mathrm{C} 2$ & $-75.0(3)$ \\
\hline $\mathrm{C} 1$ & Si1 & $\mathrm{C} 4$ & $\mathrm{C} 3$ & $-0.4(4)$ & $\mathrm{C} 13$ & $\mathrm{C} 14$ & $\mathrm{C} 15$ & $\mathrm{C} 16$ & $-1.2(6)$ \\
\hline $\mathrm{C} 1$ & $\mathrm{~N} 1$ & $\mathrm{C} 2$ & $\mathrm{C} 3$ & $67.2(4)$ & $\mathrm{C} 14$ & $\mathrm{C} 13$ & $\mathrm{C} 18$ & $\mathrm{C} 17$ & $-0.5(6)$ \\
\hline $\mathrm{C} 2$ & $\mathrm{~N} 1$ & $\mathrm{C} 1$ & Si1 & $-66.9(4)$ & $\mathrm{C} 14$ & $\mathrm{C} 15$ & $\mathrm{C} 16$ & $\mathrm{C} 17$ & $-0.5(7)$ \\
\hline $\mathrm{C} 2$ & $\mathrm{C} 3$ & $\mathrm{C} 4$ & $\mathrm{Si} 1$ & $-1.9(5)$ & $\mathrm{C} 14$ & $\mathrm{C} 15$ & $\mathrm{C} 16$ & C19 & $-178.9(4)$ \\
\hline $\mathrm{C} 2$ & $\mathrm{C} 3$ & $\mathrm{C} 7$ & $\mathrm{C} 8$ & 135.2(4) & $\mathrm{C} 15$ & C16 & $\mathrm{C} 17$ & $\mathrm{C} 18$ & $1.7(7)$ \\
\hline $\mathrm{C} 2$ & $\mathrm{C} 3$ & $\mathrm{C} 7$ & $\mathrm{C} 12$ & $-43.9(5)$ & $\mathrm{C} 16$ & $\mathrm{C} 17$ & $\mathrm{C} 18$ & $\mathrm{C} 13$ & $-1.2(6)$ \\
\hline C3 & $\mathrm{C} 7$ & $\mathrm{C} 8$ & $\mathrm{C} 9$ & $-178.1(4)$ & $\mathrm{C} 18$ & $\mathrm{C} 13$ & $\mathrm{C} 14$ & $\mathrm{C} 15$ & $1.6(6)$ \\
\hline $\mathrm{C} 3$ & $\mathrm{C} 7$ & $\mathrm{C} 12$ & $\mathrm{C} 11$ & $179.2(5)$ & C19 & $\mathrm{C} 16$ & $\mathrm{C} 17$ & $\mathrm{C} 18$ & $-179.9(4)$ \\
\hline
\end{tabular}

Table 7 Hydrogen Atom Coordinates $\left(\AA \times 10^{4}\right)$ and Isotropic Displacement Parameters $\left(\AA^{2} \times 10^{3}\right)$ for $4 \mathrm{a}$.

$\begin{array}{ccccc}\text { Atom } & \boldsymbol{x} & \boldsymbol{y} & \boldsymbol{z} & \mathbf{U}(\mathbf{e q}) \\ \text { H1A } & 2252 & -1325 & 2307 & 64 \\ \text { H1B } & 3502 & -1581 & 2287 & 64 \\ \text { H2A } & 4681 & 412 & 2799 & 57 \\ \text { H2B } & 4208 & 1959 & 3147 & 57 \\ \text { H4 } & 4068 & 2397 & 1018 & 53 \\ \text { H5A } & 1334 & 1434 & 1362 & 98 \\ \text { H5B } & 1177 & -154 & 930 & 98 \\ \text { H5C } & 1699 & 1323 & 551 & 98 \\ \text { H6A } & 3618 & -889 & 179 & 113 \\ \text { H6B } & 3053 & -2271 & 602 & 113 \\ \text { H6C } & 4220 & -1752 & 812 & 113 \\ \text { H8 } & 6005 & 2989 & 1218 & 61 \\ \text { H9 } & 7280 & 4947 & 1355 & 79\end{array}$




$\begin{array}{ccccc}\text { H10 } & 7202 & 6647 & 2328 & 86 \\ \text { H11 } & 5854 & 6374 & 3164 & 93 \\ \text { H12 } & 4618 & 4386 & 3053 & 78 \\ \text { H14 } & 2082 & -3077 & 3713 & 62 \\ \text { H15 } & 2974 & -5224 & 4195 & 74 \\ \text { H17 } & 5464 & -2451 & 4683 & 71 \\ \text { H18 } & 4556 & -272 & 4238 & 54 \\ \text { H19A } & 5395 & -5150 & 5163 & 146 \\ \text { H19B } & 5290 & -6011 & 4414 & 146 \\ \text { H19C } & 4399 & -6233 & 5004 & 146\end{array}$

\section{Compound 10}

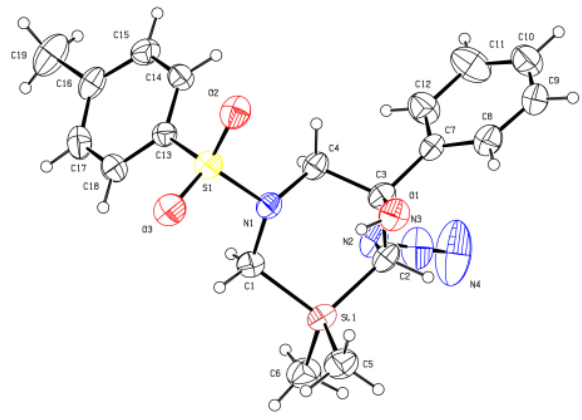

Table 1 Crystal data and structure refinement for 10 .

Identification code

10

Empirical formula

$\mathrm{C}_{19} \mathrm{H}_{24} \mathrm{~N}_{4} \mathrm{O}_{3} \mathrm{SSi}$

Formula weight

416.57

Temperature/K

293.15

Crystal system

tetragonal

Space group

$\mathrm{P}_{3}$

$\mathrm{a} / \AA ̊$

$11.8765(4)$

$\mathrm{b} / \AA$

$11.8765(4)$

c/Å

15.0989(7)

$\alpha /^{\circ}$

90

$\beta /{ }^{\circ}$

90

$\gamma /{ }^{\circ}$

90

Volume $/ \AA^{3}$

2129.72(17)

Z

4

$\rho_{\text {calc }} \mathrm{g} / \mathrm{cm}^{3}$

1.299

$\mu / \mathrm{mm}^{-1}$

0.235

$\mathrm{F}(000)$

880.0

Crystal size $/ \mathrm{mm}^{3}$

$0.0 \times 0.0 \times 0.0$

Radiation

$\operatorname{MoK} \alpha(\lambda=0.71073)$

$2 \Theta$ range for data collection/ ${ }^{\circ}$

6.394 to 52.722

Index ranges

$-14 \leq \mathrm{h} \leq 13,-14 \leq \mathrm{k} \leq 10,-11 \leq 1 \leq 18$

Reflections collected

5215

Independent reflections

$3227\left[R_{\text {int }}=0.0218, R_{\text {sigma }}=0.0457\right]$

Data/restraints/parameters

$3227 / 1 / 258$ 
Goodness-of-fit on $\mathrm{F}^{2}$

Final $\mathrm{R}$ indexes $[\mathrm{I}>=2 \sigma(\mathrm{I})]$

Final $\mathrm{R}$ indexes [all data]

Largest diff. peak/hole / e $\AA^{-3}$

Flack parameter
1.052

$$
\begin{aligned}
& \mathrm{R}_{1}=0.0425, \mathrm{wR}_{2}=0.0707 \\
& \mathrm{R}_{1}=0.0575, \mathrm{wR}_{2}=0.0763 \\
& 0.17 /-0.23 \\
& -0.15(6)
\end{aligned}
$$

\begin{tabular}{|c|c|c|c|c|}
\hline Atom & $x$ & $y$ & $z$ & $\mathbf{U}(\mathbf{e q})$ \\
\hline $\mathrm{S} 1$ & $8153.7(12)$ & $4929.8(14)$ & $7142.0(10)$ & $54.6(3)$ \\
\hline Si1 & $9514.4(13)$ & $3937.6(14)$ & $4605.4(10)$ & $57.5(4)$ \\
\hline $\mathrm{O} 1$ & $10759(3)$ & $5907(4)$ & $5599(3)$ & $66.6(11)$ \\
\hline $\mathrm{O} 2$ & $8580(3)$ & $5866(4)$ & $7640(2)$ & $70.3(12)$ \\
\hline $\mathrm{O} 3$ & $8303(4)$ & $3816(4)$ & $7471(2)$ & $70.9(12)$ \\
\hline N1 & $8778(3)$ & $4973(4)$ & $6156(3)$ & $50.0(11)$ \\
\hline $\mathrm{N} 2$ & $8689(4)$ & $5795(4)$ & $3770(3)$ & $62.6(14)$ \\
\hline N3 & $8839(5)$ & $6194(6)$ & $3043(4)$ & $89.0(19)$ \\
\hline N4 & $8872(7)$ & $6567(9)$ & $2365(5)$ & $167(4)$ \\
\hline $\mathrm{C} 1$ & $8504(5)$ & $4021(5)$ & $5571(4)$ & $58.0(16)$ \\
\hline $\mathrm{C} 2$ & $9707(5)$ & $5478(5)$ & $4297(4)$ & $56.2(15)$ \\
\hline $\mathrm{C} 3$ & $9774(4)$ & $6248(5)$ & $5129(4)$ & $52.6(14)$ \\
\hline $\mathrm{C} 4$ & $8741(4)$ & $6085(5)$ & $5729(4)$ & $52.6(14)$ \\
\hline C5 & $10837(5)$ & $3231(5)$ & $4942(4)$ & $77(2)$ \\
\hline $\mathrm{C} 6$ & $8844(6)$ & $3127(6)$ & $3685(4)$ & $80(2)$ \\
\hline $\mathrm{C} 7$ & $9900(5)$ & $7485(5)$ & $4889(4)$ & $55.0(16)$ \\
\hline $\mathrm{C} 8$ & $10940(6)$ & $8015(7)$ & $4906(5)$ & $84(2)$ \\
\hline C9 & $11084(7)$ & $9108(7)$ & $4650(7)$ & $102(3)$ \\
\hline $\mathrm{C} 10$ & 10181(10) & $9745(7)$ & $4407(6)$ & $102(3)$ \\
\hline C11 & $9120(8)$ & $9259(7)$ & $4405(6)$ & $99(3)$ \\
\hline $\mathrm{C} 12$ & $8994(6)$ & $8128(6)$ & $4649(5)$ & 79.0(19) \\
\hline C13 & $6704(4)$ & $5152(5)$ & $6956(3)$ & $45.3(14)$ \\
\hline C14 & $6277(5)$ & $6222(5)$ & $6919(4)$ & $56.5(15)$ \\
\hline C15 & $5148(6)$ & $6400(5)$ & $6811(4)$ & $64.4(17)$ \\
\hline C16 & $4406(5)$ & $5505(7)$ & $6741(4)$ & $62.4(18)$ \\
\hline C17 & $4868(6)$ & $4439(6)$ & $6721(4)$ & $66.5(19)$ \\
\hline C18 & $6005(6)$ & $4247(5)$ & $6847(4)$ & $59.3(17)$ \\
\hline C19 & $3150(6)$ & $5682(8)$ & $6658(5)$ & $102(3)$ \\
\hline
\end{tabular}

Table 2 Fractional Atomic Coordinates $\left(\times 10^{4}\right)$ and Equivalent Isotropic Displacement Parameters $\left(\AA^{2} \times 10^{3}\right)$ for 10 . $U_{e q}$ is defined as $1 / 3$ of of the trace of the orthogonalised UIJ tensor.

Table 3 Anisotropic Displacement Parameters $\left(\AA^{2} \times 10^{3}\right)$ for 10 . The Anisotropic displacement factor exponent takes the form: $-2 \pi^{2}\left[h^{2} a * 2 U_{11}+2 h k a * b * U_{12}+\ldots\right]$.

$\begin{array}{ccccccc}\text { Atom } & \mathbf{U}_{\mathbf{1 1}} & \mathbf{U}_{\mathbf{2 2}} & \mathbf{U}_{\mathbf{3 3}} & \mathbf{U}_{\mathbf{2 3}} & \mathbf{U}_{\mathbf{1 3}} & \mathbf{U}_{\mathbf{1 2}} \\ \mathrm{S} 1 & 55.6(9) & 66.6(10) & 41.4(6) & 4.1(8) & -5.7(8) & 4.6(7) \\ \text { Si1 } & 53.2(11) & 67.2(12) & 52.0(8) & -7.6(10) & 2.5(9) & 7.9(8) \\ \text { O1 } & 41(2) & 83(4) & 76(3) & 4(2) & -12(2) & 7.1(19) \\ \text { O2 } & 70(3) & 94(3) & 48(2) & -5(2) & -5(2) & -8(2) \\ \text { O3 } & 79(3) & 76(3) & 58(2) & 20(2) & -3(2) & 20(2) \\ \text { N1 } & 50(3) & 57(3) & 43(2) & 1(2) & 2(2) & 3(2) \\ \text { N2 } & 48(3) & 90(4) & 50(3) & 6(3) & -2(2) & 4(2) \\ \text { N3 } & 79(4) & 121(6) & 67(3) & 19(4) & -19(3) & -17(4)\end{array}$




$\begin{array}{lcccccc}\text { N4 } & 123(7) & 281(11) & 97(6) & 104(7) & -28(5) & -34(6) \\ \text { C1 } & 62(4) & 56(4) & 56(3) & -5(3) & 4(3) & 1(3) \\ \text { C2 } & 40(3) & 78(4) & 51(3) & 1(3) & 3(3) & 9(3) \\ \text { C3 } & 40(3) & 62(4) & 56(3) & -1(3) & -2(3) & 9(3) \\ \text { C4 } & 43(3) & 66(4) & 48(3) & 4(3) & 1(3) & 6(3) \\ \text { C5 } & 65(4) & 78(5) & 89(5) & 1(4) & -1(4) & 21(4) \\ \text { C6 } & 86(5) & 88(5) & 66(4) & -19(4) & 0(4) & -1(4) \\ \text { C7 } & 52(4) & 62(4) & 51(4) & -2(3) & 13(3) & 2(3) \\ \text { C8 } & 64(5) & 85(6) & 104(5) & -12(5) & 24(4) & -6(4) \\ \text { C9 } & 87(6) & 79(6) & 139(8) & -15(6) & 55(6) & -18(5) \\ \text { C10 } & 151(9) & 63(6) & 93(6) & -3(4) & 36(7) & -17(6) \\ \text { C11 } & 118(7) & 70(6) & 110(6) & 10(5) & -35(6) & 5(5) \\ \text { C12 } & 82(5) & 64(4) & 91(5) & 7(5) & -5(5) & 0(3) \\ \text { C13 } & 50(3) & 46(3) & 40(3) & 2(3) & 1(2) & 3(3) \\ \text { C14 } & 66(4) & 47(4) & 56(4) & 1(3) & 5(3) & -4(3) \\ \text { C15 } & 60(4) & 60(4) & 73(4) & 1(3) & 10(4) & 15(3) \\ \text { C16 } & 54(4) & 94(6) & 39(3) & 8(3) & 4(3) & 5(4) \\ \text { C17 } & 67(5) & 78(5) & 55(3) & 2(3) & 3(4) & -26(4) \\ \text { C18 } & 66(4) & 50(4) & 61(4) & 9(3) & 4(3) & 0(3) \\ \text { C19 } & 51(5) & 168(9) & 88(5) & 1(5) & 1(4) & 12(5) \\ & & & & & & \end{array}$

Table 4 Bond Lengths for 10 .

\begin{tabular}{|c|c|c|c|c|c|}
\hline Atom & Atom & Length/Å & Atom & Atom & Length/Å \\
\hline S1 & $\mathrm{O} 2$ & $1.435(5)$ & $\mathrm{C} 3$ & $\mathrm{C} 4$ & $1.537(7)$ \\
\hline S1 & $\mathrm{O} 3$ & $1.424(4)$ & $\mathrm{C} 3$ & $\mathrm{C} 7$ & $1.520(7)$ \\
\hline S1 & N1 & $1.663(4)$ & $\mathrm{C} 7$ & $\mathrm{C} 8$ & $1.386(8)$ \\
\hline S1 & $\mathrm{C} 13$ & $1.764(6)$ & $\mathrm{C} 7$ & $\mathrm{C} 12$ & $1.368(9)$ \\
\hline Sil & $\mathrm{C} 1$ & $1.890(5)$ & $\mathrm{C} 8$ & C9 & $1.366(10)$ \\
\hline Sil & $\mathrm{C} 2$ & $1.902(6)$ & C9 & $\mathrm{C} 10$ & $1.362(11)$ \\
\hline Sil & $\mathrm{C} 5$ & $1.853(6)$ & $\mathrm{C} 10$ & $\mathrm{C} 11$ & $1.387(11)$ \\
\hline Si1 & C6 & $1.869(6)$ & C11 & $\mathrm{C} 12$ & $1.401(10)$ \\
\hline $\mathrm{O} 1$ & $\mathrm{C} 3$ & $1.427(6)$ & $\mathrm{C} 13$ & $\mathrm{C} 14$ & $1.370(7)$ \\
\hline $\mathrm{N} 1$ & $\mathrm{C} 1$ & $1.471(7)$ & $\mathrm{C} 13$ & C18 & $1.368(8)$ \\
\hline $\mathrm{N} 1$ & $\mathrm{C} 4$ & $1.470(7)$ & C14 & $\mathrm{C} 15$ & $1.366(8)$ \\
\hline $\mathrm{N} 2$ & N3 & $1.210(7)$ & $\mathrm{C} 15$ & $\mathrm{C} 16$ & $1.386(8)$ \\
\hline $\mathrm{N} 2$ & $\mathrm{C} 2$ & $1.494(7)$ & $\mathrm{C} 16$ & $\mathrm{C} 17$ & $1.380(9)$ \\
\hline N3 & N4 & $1.115(7)$ & $\mathrm{C} 16$ & C19 & $1.511(8)$ \\
\hline $\mathrm{C} 2$ & $\mathrm{C} 3$ & $1.557(8)$ & $\mathrm{C} 17$ & $\mathrm{C} 18$ & $1.382(8)$ \\
\hline
\end{tabular}

Table 5 Bond Angles for 10.

$\begin{array}{cccccccc}\text { Atom } & \text { Atom } & \text { Atom } & \text { Angle }^{\circ} & \text { Atom } & \text { Atom } & \text { Atom } & \text { Angle }{ }^{\circ} \\ \text { O2 } & \text { S1 } & \text { N1 } & 106.7(2) & \text { O1 } & \text { C3 } & \text { C7 } & 108.1(5) \\ \text { O2 } & \text { S1 } & \text { C13 } & 108.2(3) & \text { C4 } & \text { C3 } & \text { C2 } & 111.2(5) \\ \text { O3 } & \text { S1 } & \text { O2 } & 119.5(3) & \text { C7 } & \text { C3 } & \text { C2 } & 112.3(5) \\ \text { O3 } & \text { S1 } & \text { N1 } & 106.6(2) & \text { C7 } & \text { C3 } & \text { C4 } & 110.0(4)\end{array}$




$\begin{array}{llllllll}\text { O3 } & \text { S1 } & \text { C13 } & 108.4(3) & \text { N1 } & \text { C4 } & \text { C3 } & 110.4(4) \\ \text { N1 } & \text { S1 } & \text { C13 } & 106.7(2) & \text { C8 } & \text { C7 } & \text { C3 } & 121.5(6) \\ \text { C1 } & \text { Si1 } & \text { C2 } & 102.4(2) & \text { C12 } & \text { C7 } & \text { C3 } & 121.6(6) \\ \text { C5 } & \text { Si1 } & \text { C1 } & 110.5(3) & \text { C12 } & \text { C7 } & \text { C8 } & 116.9(6) \\ \text { C5 } & \text { Si1 } & \text { C2 } & 113.7(3) & \text { C9 } & \text { C8 } & \text { C7 } & 122.5(8) \\ \text { C5 } & \text { Si1 } & \text { C6 } & 109.4(3) & \text { C10 } & \text { C9 } & \text { C8 } & 120.4(8) \\ \text { C6 } & \text { Si1 } & \text { C1 } & 109.3(3) & \text { C9 } & \text { C10 } & \text { C11 } & 119.0(8) \\ \text { C6 } & \text { Si1 } & \text { C2 } & 111.4(3) & \text { C10 } & \text { C11 } & \text { C12 } & 119.7(8) \\ \text { C1 } & \text { N1 } & \text { S1 } & 114.6(4) & \text { C7 } & \text { C12 } & \text { C11 } & 121.4(7) \\ \text { C4 } & \text { N1 } & \text { S1 } & 114.0(3) & \text { C14 } & \text { C13 } & \text { S1 } & 120.5(4) \\ \text { C4 } & \text { N1 } & \text { C1 } & 114.8(5) & \text { C18 } & \text { C13 } & \text { S1 } & 119.6(5) \\ \text { N3 } & \text { N2 } & \text { C2 } & 117.5(5) & \text { C18 } & \text { C13 } & \text { C14 } & 119.9(5) \\ \text { N4 } & \text { N3 } & \text { N2 } & 173.5(8) & \text { C15 } & \text { C14 } & \text { C13 } & 120.8(5) \\ \text { N1 } & \text { C1 } & \text { Si1 } & 111.3(4) & \text { C14 } & \text { C15 } & \text { C16 } & 121.0(6) \\ \text { N2 } & \text { C2 } & \text { Si1 } & 106.0(4) & \text { C15 } & \text { C16 } & \text { C19 } & 121.8(7) \\ \text { N2 } & \text { C2 } & \text { C3 } & 108.8(4) & \text { C17 } & \text { C16 } & \text { C15 } & 116.9(6) \\ \text { C3 } & \text { C2 } & \text { Si1 } & 111.9(4) & \text { C17 } & \text { C16 } & \text { C19 } & 121.3(7) \\ \text { O1 } & \text { C3 } & \text { C2 } & 106.1(4) & \text { C16 } & \text { C17 } & \text { C18 } & 122.5(6) \\ \text { O1 } & \text { C3 } & \text { C4 } & 108.9(5) & \text { C13 } & \text { C18 } & \text { C17 } & 118.7(6)\end{array}$

Table 6 Torsion Angles for 10.

$\begin{array}{lccccccccc}\text { A } & \text { B } & \text { C } & \text { D } & \text { Angle } /^{\circ} & \text { A } & \text { B } & \text { C } & \text { D } & \text { Angle }{ }^{\circ} \\ \text { S1 } & \text { N1 } & \text { C1 } & \text { Si1 } & -165.4(3) & \text { C2 } & \text { C3 } & \text { C4 } & \text { N1 } & 70.0(6) \\ \text { S1 } & \text { N1 } & \text { C4 } & \text { C3 } & 150.5(4) & \text { C2 } & \text { C3 } & \text { C7 } & \text { C8 } & -99.4(6) \\ \text { S1 } & \text { C13 } & \text { C14 } & \text { C15 } & -177.4(4) & \text { C2 } & \text { C3 } & \text { C7 } & \text { C12 } & 80.9(7) \\ \text { S1 } & \text { C13 } & \text { C18 } & \text { C17 } & 178.7(4) & \text { C3 } & \text { C7 } & \text { C8 } & \text { C9 } & 176.5(7) \\ \text { Si1 } & \text { C2 } & \text { C3 } & \text { O1 } & 63.3(5) & \text { C3 } & \text { C7 } & \text { C12 } & \text { C11 } & -178.2(7) \\ \text { Si1 } & \text { C2 } & \text { C3 } & \text { C4 } & -54.9(5) & \text { C4 } & \text { N1 } & \text { C1 } & \text { Si1 } & 59.8(5) \\ \text { Si1 } & \text { C2 } & \text { C3 } & \text { C7 } & -178.7(4) & \text { C4 } & \text { C3 } & \text { C7 } & \text { C8 } & 136.2(6) \\ \text { O1 } & \text { C3 } & \text { C4 } & \text { N1 } & -46.5(6) & \text { C4 } & \text { C3 } & \text { C7 } & \text { C12 } & -43.5(8) \\ \text { O1 } & \text { C3 } & \text { C7 } & \text { C8 } & 17.3(8) & \text { C5 } & \text { Si1 } & \text { C1 } & \text { N1 } & 81.4(5) \\ \text { O1 } & \text { C3 } & \text { C7 } & \text { C12 } & -162.4(6) & \text { C6 } & \text { Si1 } & \text { C1 } & \text { N1 } & -158.2(4) \\ \text { O2 } & \text { S1 } & \text { N1 } & \text { C1 } & 175.7(4) & \text { C7 } & \text { C3 } & \text { C4 } & \text { N1 } & -164.9(5) \\ \text { O2 } & \text { S1 } & \text { N1 } & \text { C4 } & -49.0(4) & \text { C7 } & \text { C8 } & \text { C9 } & \text { C10 } & 3.6(13) \\ \text { O2 } & \text { S1 } & \text { C13 } & \text { C14 } & 27.7(5) & \text { C8 } & \text { C7 } & \text { C12 } & \text { C11 } & 2.1(10) \\ \text { O2 } & \text { S1 } & \text { C13 } & \text { C18 } & -152.4(5) & \text { C8 } & \text { C9 } & \text { C10 } & \text { C11 } & -1.6(14) \\ \text { O3 } & \text { S1 } & \text { N1 } & \text { C1 } & 46.9(4) & \text { C9 } & \text { C10 } & \text { C11 } & \text { C12 } & 0.0(13) \\ \text { O3 } & \text { S1 } & \text { N1 } & \text { C4 } & -177.9(4) & \text { C10 } & \text { C11 } & \text { C12 } & \text { C7 } & -0.3(12) \\ \text { O3 } & \text { S1 } & \text { C13 } & \text { C14 } & 158.7(4) & \text { C12 } & \text { C7 } & \text { C8 } & \text { C9 } & -3.8(11) \\ \text { O3 } & \text { S1 } & \text { C13 } & \text { C18 } & -21.4(5) & \text { C13 } & \text { S1 } & \text { N1 } & \text { C1 } & -68.8(4) \\ \text { N1 } & \text { S1 } & \text { C13 } & \text { C14 } & -86.8(5) & \text { C13 } & \text { S1 } & \text { N1 } & \text { C4 } & 66.4(4)\end{array}$




$\begin{array}{llllllllll}\text { N1 } & \text { S1 } & \text { C13 } & \text { C18 } & 93.1(5) & \text { C13 } & \text { C14 } & \text { C15 } & \text { C16 } & 0.4(10) \\ \text { N2 } & \text { C2 } & \text { C3 } & \text { O1 } & -179.8(4) & \text { C14 } & \text { C13 } & \text { C18 } & \text { C17 } & -1.5(9) \\ \text { N2 } & \text { C2 } & \text { C3 } & \text { C4 } & 61.9(6) & \text { C14 } & \text { C15 } & \text { C16 } & \text { C17 } & -4.6(9) \\ \text { N2 } & \text { C2 } & \text { C3 } & \text { C7 } & -61.9(6) & \text { C14 } & \text { C15 } & \text { C16 } & \text { C19 } & 177.5(6) \\ \text { N3 } & \text { N2 } & \text { C2 } & \text { Si1 } & -125.1(6) & \text { C15 } & \text { C16 } & \text { C17 } & \text { C18 } & 6.0(9) \\ \text { N3 } & \text { N2 } & \text { C2 } & \text { C3 } & 114.4(6) & \text { C16 } & \text { C17 } & \text { C18 } & \text { C13 } & -3.0(9) \\ \text { C1 } & \text { N1 } & \text { C4 } & \text { C3 } & -74.4(6) & \text { C18 } & \text { C13 } & \text { C14 } & \text { C15 } & 2.8(9) \\ \text { C2 } & \text { Si1 } & \text { C1 } & \text { N1 } & -40.0(5) & \text { C19 } & \text { C16 } & \text { C17 } & \text { C18 } & -176.1(6)\end{array}$

Table 7 Hydrogen Atom Coordinates $\left(\AA \times 10^{4}\right)$ and Isotropic Displacement Parameters $\left(\AA^{2} \times 10^{3}\right)$ for 10 .

$\begin{array}{ccccc}\text { Atom } & \boldsymbol{x} & \boldsymbol{y} & \boldsymbol{z} & \mathbf{U}(\mathbf{e q )} \\ \text { H1 } & 10617 & 5346 & 5895 & 100 \\ \text { H1A } & 7743 & 4107 & 5349 & 70 \\ \text { H1B } & 8539 & 3325 & 5906 & 70 \\ \text { H2 } & 10386 & 5570 & 3935 & 67 \\ \text { H4A } & 8729 & 6668 & 6179 & 63 \\ \text { H4B } & 8059 & 6150 & 5380 & 63 \\ \text { H5A } & 11172 & 3634 & 5426 & 116 \\ \text { H5B } & 11350 & 3218 & 4451 & 116 \\ \text { H5C } & 10676 & 2473 & 5124 & 116 \\ \text { H6A } & 8826 & 2342 & 3836 & 120 \\ \text { H6B } & 9273 & 3228 & 3151 & 120 \\ \text { H6C } & 8090 & 3394 & 3594 & 120 \\ \text { H8 } & 11564 & 7610 & 5100 & 101 \\ \text { H9 } & 11802 & 9419 & 4640 & 122 \\ \text { H10 } & 10276 & 10495 & 4246 & 123 \\ \text { H11 } & 8494 & 9681 & 4241 & 119 \\ \text { H12 } & 8280 & 7807 & 4649 & 95 \\ \text { H14 } & 6760 & 6835 & 6968 & 68 \\ \text { H15 } & 4875 & 7133 & 6783 & 77 \\ \text { H17 } & 4398 & 3827 & 6619 & 80 \\ \text { H18 } & 6288 & 3517 & 6858 & 71 \\ \text { H19A } & 2806 & 5010 & 6431 & 154 \\ \text { H19B } & 3006 & 6296 & 6261 & 154 \\ \text { H19C } & 2840 & 5852 & 7230 & 154\end{array}$

\section{Compound 13}

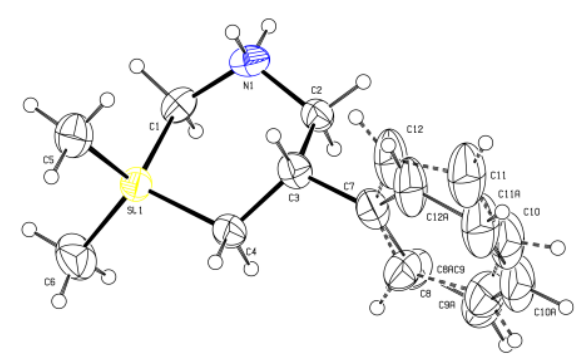


Table 1 Crystal data and structure refinement for 13.

Identification code

Empirical formula

Formula weight

Temperature/K

Crystal system

Space group

$\mathrm{a} / \AA$

$\mathrm{b} / \AA$

c/Å

$\alpha /{ }^{\circ}$

$\beta /{ }^{\circ}$

$\gamma /{ }^{\circ}$

Volume $/ \AA^{3}$

$\mathrm{Z}$

$\rho_{\text {calc }} \mathrm{g} / \mathrm{cm}^{3}$

$\mu / \mathrm{mm}^{-1}$

$\mathrm{F}(000)$

Crystal size $/ \mathrm{mm}^{3}$

Radiation

$2 \Theta$ range for data collection/ ${ }^{\circ}$

Index ranges

Reflections collected

Independent reflections

Data/restraints/parameters

Goodness-of-fit on $\mathrm{F}^{2}$

Final $\mathrm{R}$ indexes $[\mathrm{I}>=2 \sigma(\mathrm{I})]$

Final $\mathrm{R}$ indexes [all data]

Largest diff. peak/hole / e $\AA^{-3}$
13

$\mathrm{C}_{12} \mathrm{H}_{20} \mathrm{CINSi}$

241.83

293.15

monoclinic

$\mathrm{P} 2{ }_{1} / \mathrm{c}$

$8.0155(6)$

$7.1976(4)$

25.5836(18)

90

93.107(7)

90

1473.80(17)

4

1.090

0.314

520.0

$0.35 \times 0.3 \times 0.25$

$\operatorname{MoK} \alpha(\lambda=0.71073)$

5.858 to 52.742

$-10 \leq \mathrm{h} \leq 7,-8 \leq \mathrm{k} \leq 8,-31 \leq 1 \leq 30$

6929

$3001\left[R_{\text {int }}=0.0225, R_{\text {sigma }}=0.0329\right]$

$3001 / 2 / 154$

1.200

$\mathrm{R}_{1}=0.0804, \mathrm{wR}_{2}=0.1639$

$\mathrm{R}_{1}=0.1035, \mathrm{wR}_{2}=0.1726$

$0.34 /-0.35$

Table 2 Fractional Atomic Coordinates $\left(\times 10^{4}\right)$ and Equivalent Isotropic Displacement Parameters $\left(\AA^{2} \times 10^{3}\right)$ for 13 . $U_{e q}$ is defined as $1 / 3$ of of the trace of the orthogonalised $U_{I J}$ tensor.

$\begin{array}{ccccc}\text { Atom } & \boldsymbol{x} & \boldsymbol{y} & \boldsymbol{z} & \mathbf{U}(\mathbf{e q}) \\ & & & & \\ \text { C11 } & 3880(2) & 12912.0(18) & 3045.3(6) & 86.9(5) \\ \text { Si1 } & 1043.0(15) & 7728.3(17) & 3407.1(5) & 52.2(3) \\ \text { N1 } & 3933(5) & 8681(5) & 2886.5(13) & 57.3(9) \\ \text { C1 } & 2293(5) & 7702(6) & 2806.7(16) & 55.9(11) \\ \text { C2 } & 5017(5) & 7957(6) & 3337.7(15) & 52.6(10) \\ \text { C3 } & 4295(5) & 8301(7) & 3871.2(16) & 56.4(11) \\ \text { C4 } & 2678(5) & 7251(7) & 3931.5(16) & 60.2(12) \\ \text { C5 } & 55(7) & 10042(8) & 3488(2) & 85.4(17) \\ \text { C6 } & -531(7) & 5851(8) & 3344(2) & 93.2(18) \\ \text { C7 } & 5655(6) & 7854(9) & 4288.5(18) & 68.6(14) \\ \text { C8 } & 5940(60) & 6560(70) & 4537(16) & 86(3) \\ \text { C9 } & 7480(70) & 6470(110) & 4940(20) & 120(6) \\ \text { C10 } & 8190(90) & 8080(150) & 4990(30) & 132(8) \\ \text { C11 } & 7720(70) & 9710(100) & 4780(18) & 122(9)\end{array}$




$\begin{array}{ccccc}\text { C12 } & 6280(60) & 9620(40) & 4408(18) & 98(4) \\ \text { C8A } & 6161(16) & 5941(18) & 4372(6) & 86(3) \\ \text { C9A } & 7354(17) & 5520(30) & 4745(6) & 120(6) \\ \text { C10A } & 8180(20) & 6870(30) & 5043(7) & 132(8) \\ \text { C11A } & 7740(20) & 8700(30) & 4968(7) & 122(9) \\ \text { C12A } & 6531(16) & 9171(19) & 4580(5) & 98(4)\end{array}$

Table 3 Anisotropic Displacement Parameters $\left(\check{A}^{2} \times 10^{3}\right)$ for 13. The Anisotropic displacement factor exponent takes the form: $-2 \pi^{2}\left[h^{2} a^{* 2} U_{11}+2 h k a * b * U_{12}+\ldots\right]$.

$\begin{array}{lcccccc}\text { Atom } & \mathbf{U}_{\mathbf{1 1}} & \mathbf{U}_{\mathbf{2 2}} & \mathbf{U}_{\mathbf{3 3}} & \mathbf{U}_{\mathbf{2 3}} & \mathbf{U}_{\mathbf{1 3}} & \mathbf{U}_{\mathbf{1 2}} \\ \text { C11 } & 131.4(12) & 47.4(7) & 87.8(9) & -1.0(6) & 59.6(9) & -1.3(7) \\ \text { Si1 } & 48.2(6) & 49.7(7) & 58.3(7) & 0.6(6) & -0.4(5) & 1.3(6) \\ \text { N1 } & 78(3) & 46(2) & 49(2) & 2.5(16) & 17.0(18) & 8.4(19) \\ \text { C1 } & 67(3) & 51(2) & 49(2) & -2(2) & -6(2) & 8(2) \\ \text { C2 } & 48(2) & 59(3) & 51(2) & 2(2) & 7.2(18) & -2(2) \\ \text { C3 } & 54(2) & 67(3) & 49(2) & -7(2) & 5.6(19) & -2(2) \\ \text { C4 } & 52(2) & 77(3) & 53(2) & 9(2) & 10.3(19) & 1(2) \\ \text { C5 } & 72(3) & 81(4) & 104(4) & 2(3) & 15(3) & 20(3) \\ \text { C6 } & 71(4) & 84(4) & 123(5) & -8(4) & -3(3) & -22(3) \\ \text { C7 } & 49(2) & 108(4) & 49(3) & -2(3) & 3(2) & -7(3) \\ \text { C8 } & 74(5) & 108(9) & 74(8) & 23(6) & -6(5) & 11(6) \\ \text { C9 } & 85(6) & 183(17) & 89(10) & 46(9) & -25(7) & -6(9) \\ \text { C10 } & 63(5) & 250(30) & 80(7) & 22(12) & -14(5) & 17(14) \\ \text { C11 } & 75(7) & 200(30) & 85(11) & -44(13) & -21(9) & 17(13) \\ \text { C12 } & 56(6) & 152(8) & 85(10) & -43(6) & -21(6) & 8(6) \\ \text { C8A } & 74(5) & 108(9) & 74(8) & 23(6) & -6(5) & 11(6) \\ \text { C9A } & 85(6) & 183(17) & 89(10) & 46(9) & -25(7) & -6(9) \\ \text { C10A } & 63(5) & 250(30) & 80(7) & 22(12) & -14(5) & 17(14) \\ \text { C11A } & 75(7) & 200(30) & 85(11) & -44(13) & -21(9) & 17(13) \\ \text { C12A } & 56(6) & 152(8) & 85(10) & -43(6) & -21(6) & 8(6)\end{array}$

Table 4 Bond Lengths for 13.

\begin{tabular}{|c|c|c|c|c|c|}
\hline Atom & Atom & Length/Å & Atom & Atom & Length/Å \\
\hline Si1 & $\mathrm{C} 1$ & $1.879(4)$ & C7 & C8A & $1.448(13)$ \\
\hline Si1 & $\mathrm{C} 4$ & $1.856(4)$ & C7 & $\mathrm{C} 12 \mathrm{~A}$ & $1.375(11)$ \\
\hline Si1 & $\mathrm{C} 5$ & $1.861(5)$ & $\mathrm{C} 8$ & C9 & $1.56(7)$ \\
\hline Si1 & C6 & $1.849(5)$ & C9 & $\mathrm{C} 10$ & $1.30(12)$ \\
\hline N1 & $\mathrm{C} 1$ & $1.496(5)$ & $\mathrm{C} 10$ & C11 & $1.33(12)$ \\
\hline N1 & $\mathrm{C} 2$ & $1.500(5)$ & $\mathrm{C} 11$ & $\mathrm{C} 12$ & $1.46(6)$ \\
\hline $\mathrm{C} 2$ & $\mathrm{C} 3$ & $1.531(5)$ & C8A & C9A & $1.348(17)$ \\
\hline C3 & $\mathrm{C} 4$ & $1.515(6)$ & C9A & $\mathrm{C} 10 \mathrm{~A}$ & $1.38(2)$ \\
\hline C3 & C7 & $1.518(6)$ & C10A & C11A & $1.38(2)$ \\
\hline $\mathrm{C} 7$ & $\mathrm{C} 8$ & $1.15(5)$ & C11A & $\mathrm{C} 12 \mathrm{~A}$ & $1.391(14)$ \\
\hline
\end{tabular}

Table 5 Bond Angles for 13.

\begin{tabular}{cccccccc}
\multicolumn{2}{c}{ Atom Atom Atom } & Angle $^{\circ}$ & Atom & Atom & Atom & Angle $^{\circ}$ \\
C4 & Si 1 & C1 & $101.67(19)$ & C12 & C7 & C3 & $101.2(18)$ \\
C4 & Si 1 & C5 & $111.9(2)$ & C8A & C7 & C3 & $119.3(7)$ \\
C5 & Si1 & C1 & $110.4(2)$ & C12A & C7 & C3 & $124.2(8)$ \\
C6 & Si1 & C1 & $108.2(2)$ & C12A & C7 & C8A & $116.4(8)$
\end{tabular}




$\begin{array}{llllcccc}\text { C6 } & \text { Si1 } & \text { C4 } & 112.3(2) & \text { C7 } & \text { C8 } & \text { C9 } & 122(5) \\ \text { C6 } & \text { Si1 } & \text { C5 } & 111.8(3) & \text { C10 } & \text { C9 } & \text { C8 } & 111(6) \\ \text { C1 } & \text { N1 } & \text { C2 } & 114.2(3) & \text { C9 } & \text { C10 } & \text { C11 } & 129(7) \\ \text { N1 } & \text { C1 } & \text { Si1 } & 112.8(3) & \text { C10 } & \text { C11 } & \text { C12 } & 115(6) \\ \text { N1 } & \text { C2 } & \text { C3 } & 113.5(3) & \text { C7 } & \text { C12 } & \text { C11 } & 116(3) \\ \text { C4 } & \text { C3 } & \text { C2 } & 112.1(4) & \text { C9A } & \text { C8A } & \text { C7 } & 120.0(11) \\ \text { C4 } & \text { C3 } & \text { C7 } & 114.1(4) & \text { C8A } & \text { C9A } & \text { C10A } & 122.3(14) \\ \text { C7 } & \text { C3 } & \text { C2 } & 107.5(3) & \text { C11A } & \text { C10A } & \text { C9A } & 118.9(14) \\ \text { C3 } & \text { C4 } & \text { Si1 } & 114.1(3) & \text { C10A } & \text { C11A } & \text { C12A } & 119.9(14) \\ \text { C8 } & \text { C7 } & \text { C3 } & 133(3) & \text { C7 } & \text { C12A } & \text { C11A } & 122.3(12) \\ \text { C8 } & \text { C7 } & \text { C12 } & 124(3) & & & & \end{array}$

Table 6 Torsion Angles for 13.

\begin{tabular}{|c|c|c|c|c|c|c|c|c|}
\hline A B & C & D & Angle $/^{\circ}$ & $\mathbf{A}$ & B & C & D & Angle $/^{\circ}$ \\
\hline $\mathrm{N} 1 \mathrm{C} 2$ & $\mathrm{C} 3$ & $\mathrm{C} 4$ & $64.8(5)$ & $\mathrm{C} 4$ & $\mathrm{C} 3$ & $\mathrm{C} 7$ & $\mathrm{C} 12 \mathrm{~A}$ & $-128.8(9)$ \\
\hline $\mathrm{N} 1 \mathrm{C} 2$ & $\mathrm{C} 3$ & $\mathrm{C} 7$ & $-169.0(4)$ & $\mathrm{C} 5$ & Sil & $\mathrm{C} 1$ & $\mathrm{~N} 1$ & $76.9(3)$ \\
\hline C1 Sil & $\mathrm{C} 4$ & $\mathrm{C} 3$ & $42.6(4)$ & $\mathrm{C} 5$ & Si1 & $\mathrm{C} 4$ & $\mathrm{C} 3$ & $-75.3(4)$ \\
\hline $\mathrm{C} 1 \mathrm{~N} 1$ & $\mathrm{C} 2$ & C3 & $-66.2(5)$ & C6 & Si1 & $\mathrm{C} 1$ & N1 & $-160.4(3)$ \\
\hline $\mathrm{C} 2 \mathrm{~N} 1$ & $\mathrm{C} 1$ & Si1 & $55.8(4)$ & C6 & Si1 & $\mathrm{C} 4$ & $\mathrm{C} 3$ & $158.1(4)$ \\
\hline $\mathrm{C} 2 \mathrm{C} 3$ & $\mathrm{C} 4$ & Si1 & $-55.2(5)$ & C7 & $\mathrm{C} 3$ & $\mathrm{C} 4$ & Si1 & $-177.8(3)$ \\
\hline $\mathrm{C} 2 \mathrm{C} 3$ & C7 & $\mathrm{C} 8$ & $-97(3)$ & C7 & C8 & C9 & C10 & $8(7)$ \\
\hline $\mathrm{C} 2 \mathrm{C} 3$ & $\mathrm{C} 7$ & $\mathrm{C} 12$ & $98(2)$ & C7 & $\mathrm{C} 8 \mathrm{~A}$ & C9A & C10A & $-3(2)$ \\
\hline $\mathrm{C} 2 \mathrm{C} 3$ & $\mathrm{C} 7$ & $\mathrm{C} 8 \mathrm{~A}$ & $-69.9(8)$ & $\mathrm{C} 8$ & $\mathrm{C} 7$ & $\mathrm{C} 12$ & $\mathrm{C} 11$ & $18(6)$ \\
\hline $\mathrm{C} 2 \mathrm{C} 3$ & $\mathrm{C} 7$ & $\mathrm{C} 12 \mathrm{~A}$ & $106.2(9)$ & $\mathrm{C} 8$ & C9 & $\mathrm{C} 10$ & $\mathrm{C} 11$ & $5(11)$ \\
\hline C3 C7 & $\mathrm{C} 8$ & C9 & $179(3)$ & C9 & $\mathrm{C} 10$ & $\mathrm{C} 11$ & $\mathrm{C} 12$ & $-6(11)$ \\
\hline C3 C7 & $\mathrm{C} 12$ & C11 & $-176(4)$ & C10 & C11 & $\mathrm{C} 12$ & C7 & $-4(7)$ \\
\hline C3 C7 & C8A & C9A & $-179.3(9)$ & C12 & $\mathrm{C} 7$ & C8 & C9 & $-20(5)$ \\
\hline C3 C7 & $\mathrm{C} 12 \mathrm{~A}$ & $\mathrm{C} 11 \mathrm{~A}$ & $178.4(13)$ & $\mathrm{C} 8 \mathrm{~A}$ & $\mathrm{C} 7$ & $\mathrm{C} 12 \mathrm{~A}$ & $\mathrm{C} 11 \mathrm{~A}$ & $-5.4(19)$ \\
\hline C4 Si1 & $\mathrm{C} 1$ & N1 & $-42.0(3)$ & $\mathrm{C} 8 \mathrm{~A}$ & C9A & $\mathrm{C} 10 \mathrm{~A}$ & $\mathrm{C} 11 \mathrm{~A}$ & 2(3) \\
\hline C4 C3 & C7 & C8 & $28(3)$ & C9A & C10A & C11A & $\mathrm{C} 12 \mathrm{~A}$ & $-3(3)$ \\
\hline $\mathrm{C} 4 \mathrm{C} 3$ & $\mathrm{C} 7$ & $\mathrm{C} 12$ & $-137(2)$ & $\mathrm{C} 10 \mathrm{~A}$ & $\mathrm{C} 11 \mathrm{~A}$ & $\mathrm{C} 12 \mathrm{~A}$ & $\mathrm{C} 7$ & $5(3)$ \\
\hline $\mathrm{C} 4 \mathrm{C} 3$ & $\mathrm{C} 7$ & C8A & $55.1(8)$ & $\mathrm{C} 12 \mathrm{~A}$ & C7 & $\mathrm{C} 8 \mathrm{~A}$ & C9A & $4.3(16)$ \\
\hline
\end{tabular}

Table 7 Hydrogen Atom Coordinates $\left(\AA \times 10^{4}\right)$ and Isotropic Displacement Parameters $\left(\AA^{2} \times 10^{3}\right)$ for 13 .

$\begin{array}{ccccc}\text { Atom } & \boldsymbol{x} & \boldsymbol{y} & \boldsymbol{z} & \mathbf{U}(\mathbf{e q}) \\ \text { H1A } & 3742 & 9883 & 2938 & 69 \\ \text { H1B } & 4487 & 8577 & 2596 & 69 \\ \text { H1C } & 2492 & 6424 & 2707 & 67 \\ \text { H1D } & 1648 & 8291 & 2521 & 67 \\ \text { H2A } & 6105 & 8545 & 3333 & 63 \\ \text { H2B } & 5174 & 6632 & 3292 & 63 \\ \text { H3 } & 4047 & 9630 & 3896 & 68 \\ \text { H4A } & 2244 & 7573 & 4266 & 72 \\ \text { H4B } & 2916 & 5930 & 3936 & 72 \\ \text { H5A } & -655 & 10321 & 3184 & 128 \\ \text { H5B } & 908 & 10974 & 3531 & 128 \\ \text { H5C } & -599 & 10025 & 3792 & 128 \\ \text { H6A } & 14 & 4699 & 3271 & 140 \\ \text { H6B } & -1330 & 6138 & 3062 & 140 \\ \text { H6C } & -1092 & 5743 & 3664 & 140\end{array}$




$\begin{array}{ccccc}\text { H8 } & 5241 & 5534 & 4500 & 103 \\ \text { H9 } & 7832 & 5401 & 5115 & 144 \\ \text { H10 } & 9170 & 8101 & 5197 & 158 \\ \text { H11 } & 8266 & 10813 & 4866 & 146 \\ \text { H12 } & 5797 & 10690 & 4258 & 118 \\ \text { H8A } & 5662 & 5002 & 4169 & 103 \\ \text { H9A } & 7629 & 4280 & 4804 & 144 \\ \text { H10A } & 9029 & 6547 & 5290 & 158 \\ \text { H11A } & 8248 & 9622 & 5176 & 146 \\ \text { H12A } & 6306 & 10421 & 4516 & 118\end{array}$

Table 8 Atomic Occupancy for 13.

$\begin{array}{cccccc}\text { Atom } & \text { Occupancy } & \text { Atom } & \text { Occupancy } & \text { Atom } & \text { Occupancy } \\ \text { C8 } & 0.25(2) & \text { H8 } & 0.25(2) & \text { C9 } & 0.25(2) \\ \text { H9 } & 0.25(2) & \text { C10 } & 0.25(2) & \text { H10 } & 0.25(2) \\ \text { C11 } & 0.25(2) & \text { H11 } & 0.25(2) & \text { C12 } & 0.25(2) \\ \text { H12 } & 0.25(2) & \text { C8A } & 0.75(2) & \text { H8A } & 0.75(2) \\ \text { C9A } & 0.75(2) & \text { H9A } & 0.75(2) & \text { C10A } & 0.75(2) \\ \text { H10A } & 0.75(2) & \text { C11A } & 0.75(2) & \text { H11A } & 0.75(2) \\ \text { C12A } & 0.75(2) & \text { H12A } & 0.75(2) & & \end{array}$




\section{Computational Investigations}

Computational Details: All structures were optimized at the B3LYP/6-311G+(d,p) level of theory along with PCM method to model the solvent effect (toluene, dielectric constant of 2.38).

Energy and frequency calculations were done using the same method. All calculations were done using QChem5.1.

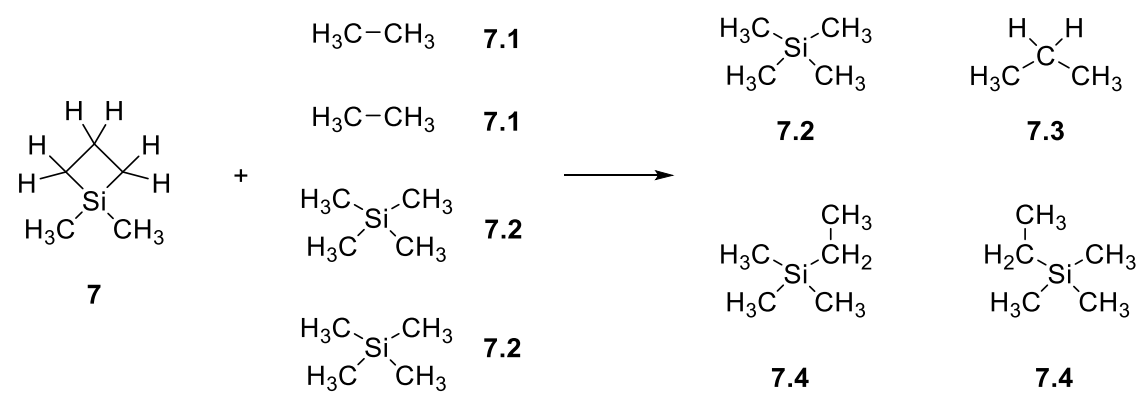

Scheme 1. The ring strain energy of 7 was calculation as: - (free energy of the four molecules at the right - free energy of the five molecules at the left). The detailed energy components and XYZ structures of each molecules are given below.

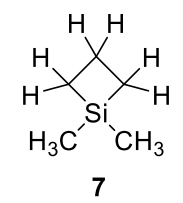

\begin{tabular}{|c|c|c|c|c|}
\hline \multicolumn{2}{|c|}{ Energy component } & \multicolumn{3}{|c|}{ Value (kcal/mol) } \\
\hline \multicolumn{2}{|c|}{ Electronic Energy (PCM) } & & -305815.42 & \\
\hline \multicolumn{2}{|c|}{ Zero Point Energy Correction } & & 97.33 & \\
\hline \multicolumn{2}{|c|}{ Enthalpy } & & 103.42 & \\
\hline \multicolumn{2}{|c|}{$-\mathrm{T} \times \mathrm{dS}(298.15 \mathrm{~K})$} & & -26.50 & \\
\hline \multicolumn{2}{|c|}{ Free Energy } & & -305641.18 & \\
\hline \multicolumn{2}{|c|}{ Dipole Moment (Debye) } & & 0.59 & \\
\hline \multicolumn{5}{|c|}{ XYZ coordinate } \\
\hline 1 & $\mathrm{C}$ & -0.9029096514 & -0.5201051679 & -1.5107603280 \\
\hline 2 & $\mathrm{C}$ & -1.0707814837 & 1.0183722753 & -1.7552940540 \\
\hline 3 & $\mathrm{Si}$ & -0.0306169508 & -0.0731980809 & 0.1256286623 \\
\hline 4 & $\mathrm{C}$ & -0.9020768735 & 1.5684064275 & -0.2984051272 \\
\hline 5 & $\mathrm{H}$ & -2.0102005741 & 1.3007250180 & -2.2403090970 \\
\hline 6 & $\mathrm{H}$ & -0.2560826324 & 1.3844569430 & -2.3865548055 \\
\hline 7 & $\mathrm{C}$ & 1.8502330519 & 0.0017742988 & -0.0128842414 \\
\hline 8 & $\mathrm{C}$ & -0.5240378989 & -0.9831789033 & 1.7020322513 \\
\hline 9 & $\mathrm{H}$ & -1.8714808195 & -0.9995482217 & -1.3369338018 \\
\hline 10 & $\mathrm{H}$ & -0.3846790282 & -1.0689628270 & -2.2995171330 \\
\hline 11 & $\mathrm{H}$ & -1.8703007250 & 1.6521780092 & 0.2055814919 \\
\hline 12 & $\mathrm{H}$ & -0.3866460756 & 2.5268271733 & -0.2121243928 \\
\hline 13 & $\mathrm{H}$ & 2.2854405458 & 0.5430126138 & 0.8336109497 \\
\hline 14 & $\mathrm{H}$ & 2.2807317998 & -1.0048076937 & -0.0214715354 \\
\hline 15 & $\mathrm{H}$ & 2.1586135330 & 0.5081931416 & -0.9321398404 \\
\hline 16 & $\mathrm{H}$ & -1.6113666682 & -1.0508533609 & 1.7982339230 \\
\hline 17 & $\mathrm{H}$ & -0.1379513599 & -0.4663320020 & 2.5869549418 \\
\hline 18 & $\mathrm{H}$ & -0.1198881893 & -2.0009596433 & 1.7083521365 \\
\hline
\end{tabular}


$\mathrm{H}_{3} \mathrm{C}-\mathrm{CH}_{3}$

7.1

\begin{tabular}{|c|c|c|c|c|}
\hline \multicolumn{2}{|c|}{ Energy component } & \multicolumn{2}{|c|}{ Value (kcal/mol) } & \\
\hline \multicolumn{2}{|c|}{ Electronic Energy (PCM) } & \multicolumn{2}{|c|}{-50110.95} & \\
\hline \multicolumn{2}{|c|}{ Zero Point Energy Correction } & \multicolumn{2}{|r|}{46.49} & \\
\hline \multicolumn{2}{|c|}{ Enthalpy } & \multicolumn{2}{|r|}{49.28} & \\
\hline \multicolumn{2}{|c|}{$-\mathrm{T} \times \mathrm{dS}(298.15 \mathrm{~K})$} & \multicolumn{3}{|c|}{-17.29} \\
\hline \multicolumn{2}{|c|}{ Free Energy } & \multicolumn{2}{|c|}{-50032.46} & \\
\hline \multicolumn{5}{|c|}{$\mathrm{XYZ}$ coordinate } \\
\hline 1 & $\mathrm{C}$ & 1.5306688714 & -0.0000108672 & 0.0000000000 \\
\hline 2 & $\mathrm{C}$ & -0.0006688714 & 0.0000108672 & 0.0000000000 \\
\hline 3 & $\mathrm{H}$ & 1.9282718002 & -0.5095974100 & -0.8825276112 \\
\hline 4 & $\mathrm{H}$ & 1.9282718002 & -0.5095974100 & 0.8825276112 \\
\hline 5 & $\mathrm{H}$ & 1.9281163146 & 1.0190620141 & 0.0000000000 \\
\hline 6 & $\mathrm{H}$ & -0.3982718003 & 0.5095974100 & 0.8825276112 \\
\hline 7 & $\mathrm{H}$ & -0.3981163146 & -1.0190620141 & 0.0000000000 \\
\hline 8 & $\mathrm{H}$ & -0.3982718003 & 0.5095974100 & -0.8825276112 \\
\hline
\end{tabular}

$\mathrm{H}_{3} \mathrm{C}_{-} \mathrm{Si}^{-} \mathrm{CH}_{3}$

$\mathrm{H}_{3} \mathrm{C}^{\prime} \mathrm{CH}_{3}$

7.2

\begin{tabular}{lr} 
Energy component & Value (kcal/mol) \\
\hline Electronic Energy (PCM) & -281923.19 \\
Zero Point Energy Correction & 91.75 \\
Enthalpy & 98.24 \\
$-\mathrm{T} \times \mathrm{dS}(298.15 \mathrm{~K})$ & -26.97 \\
Free Energy & -281760.16
\end{tabular}

\begin{tabular}{llrrr}
\hline XYZ coordinate & & & & \\
1 & $\mathrm{C}$ & 1.5585487912 & -0.8572580286 & 2.5183317087 \\
2 & $\mathrm{Si}$ & 1.1892051017 & 0.4959669522 & 1.2505914556 \\
3 & $\mathrm{C}$ & -0.5952754308 & 1.0847139019 & 1.4621410476 \\
4 & $\mathrm{C}$ & 1.4208488085 & -0.1946498675 & -0.4941566879 \\
5 & $\mathrm{C}$ & 2.3695170102 & 1.9488779805 & 1.5148989085 \\
6 & $\mathrm{H}$ & 1.5873169440 & -1.2193139561 & 2.4226315602 \\
7 & $\mathrm{H}$ & 1.4292971228 & -0.4874585954 & 3.5406461550 \\
8 & $\mathrm{H}$ & -8902752002 & -1.7144446952 & 2.3867396962 \\
9 & $\mathrm{H}$ & -0.8414693753 & 1.8686309646 & 0.7387375591 \\
10 & $\mathrm{H}$ & -0.7608855274 & 1.4913410278 & 2.4649285802 \\
11 & $\mathrm{H}$ & -1.3022426125 & 0.2618107488 & 1.3153724488 \\
12 & $\mathrm{H}$ & 2.4461474999 & -0.5462398491 & -0.6474880835 \\
13 & $\mathrm{H}$ & 1.2129224389 & 0.5675720965 & -1.2521255502 \\
14 & $\mathrm{H}$ & 0.7472927906 & -1.0382040552 & -0.6763183896 \\
15 & $\mathrm{H}$ & 2.1808186598 & 2.7470763679 & 0.7898653286 \\
16 & $\mathrm{H}$ & 3.4124742763 & 1.6358650856 & 1.4023064754 \\
17 & $\mathrm{H}$ & 2.2552083016 & 2.3747139216 & 2.5168977872 \\
\hline
\end{tabular}

$\mathrm{H}_{3} \mathrm{C}^{-} \mathrm{C}^{\prime}-\mathrm{CH}_{3}$

7.3

\begin{tabular}{lr}
\hline Energy component & Value (kcal/mol) \\
\hline Electronic Energy (PCM) & -74787.51 \\
Zero Point Energy Correction & 64.34 \\
Enthalpy & 67.81 \\
\hline
\end{tabular}




\begin{tabular}{llrrr}
\hline $\begin{array}{l}\text {-T } \times \text { dS }(298.15 K) \\
\text { Free Energy }\end{array}$ & \multicolumn{3}{c}{-74674.97} \\
\hline XYZ coordinate \\
1 & $\mathrm{C}$ & -0.0156691336 & 0.0112529075 & 0.0219658596 \\
2 & $\mathrm{C}$ & 1.5155888194 & -0.0071462449 & -0.0193888554 \\
3 & $\mathrm{C}$ & 2.0684212762 & -0.7241329417 & -1.2548166848 \\
4 & $\mathrm{H}$ & 1.8975347365 & -0.4944421677 & 0.8850369536 \\
5 & $\mathrm{H}$ & 1.8932570029 & 1.0214233663 & 0.0055391860 \\
6 & $\mathrm{H}$ & -0.3872833734 & 0.5224725025 & 0.9148040095 \\
7 & $\mathrm{H}$ & -0.4192356322 & -1.0065950299 & 0.0268993137 \\
8 & $\mathrm{H}$ & -0.4273686848 & 0.5257220885 & -0.8526390118 \\
9 & $\mathrm{H}$ & 1.7402103397 & -0.2315520379 & -2.1760106283 \\
10 & $\mathrm{H}$ & 1.7213160791 & -1.7620665161 & -1.2936868862 \\
11 & $\mathrm{H}$ & 3.1622285702 & -0.7379359267 & -1.2557032560 \\
\hline
\end{tabular}

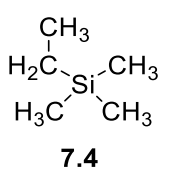

\begin{tabular}{lcrrr}
\hline Energy component & \multicolumn{3}{c}{ Value (kcal/mol) } \\
\hline Electronic Energy (PCM) & \multicolumn{3}{c}{-306596.41} \\
Zero Point Energy Correction & \multicolumn{3}{c}{109.75} \\
Enthalpy & \multicolumn{3}{c}{117.12} & -29.58 \\
-T $\times$ dS $(298.15 K)$ & \multicolumn{3}{c}{-306399.12} & \\
Free Energy & & & \\
\hline XYZ coordinate & & & \\
1 & $\mathrm{C}$ & 1.4362815901 & 1.4689151117 & 1.1572593596 \\
2 & $\mathrm{Si}$ & 0.8194136896 & 0.7367691799 & -0.4741142892 \\
3 & $\mathrm{C}$ & 1.4431441366 & 1.7942895743 & -1.9123357927 \\
4 & $\mathrm{C}$ & -1.0780883013 & 0.7210167037 & -0.5139458683 \\
5 & $\mathrm{C}$ & 1.4888134434 & -1.0234182166 & -0.6550717643 \\
6 & $\mathrm{C}$ & -1.7726223251 & -0.0681960905 & 0.6107662057 \\
7 & $\mathrm{H}$ & 1.1156755789 & 0.8684976402 & 2.0142423891 \\
8 & $\mathrm{H}$ & 2.5302961580 & 1.5101830993 & 1.1731808088 \\
9 & $\mathrm{H}$ & 1.0633204616 & 2.4875195884 & 1.3052942774 \\
10 & $\mathrm{H}$ & 1.1080503457 & 1.3919372281 & -2.8737904273 \\
11 & $\mathrm{H}$ & 2.5371159199 & 1.8321547221 & -1.9310577665 \\
12 & $\mathrm{H}$ & 1.0760836673 & 2.8230631379 & -1.8359960143 \\
13 & $\mathrm{H}$ & -1.3867863434 & 0.3225012974 & -1.4888817024 \\
14 & $\mathrm{H}$ & -1.4226213120 & 1.7632925145 & -0.4995333796 \\
15 & $\mathrm{H}$ & 1.1701175406 & -1.4753171355 & -1.6002498083 \\
16 & $\mathrm{H}$ & 1.1435077348 & -1.6704137622 & 0.1574113564 \\
17 & $\mathrm{H}$ & 2.5836390593 & -1.0281603685 & -0.6385852298 \\
$\mathrm{~s} 18$ & $\mathrm{H}$ & -2.8624029902 & -0.0323865868 & 0.5133916111 \\
19 & $\mathrm{H}$ & -1.4802362888 & -1.1226421337 & 0.6023759312 \\
20 & $\mathrm{H}$ & -1.5217017650 & 0.3323944963 & 1.5976401034 \\
\hline
\end{tabular}




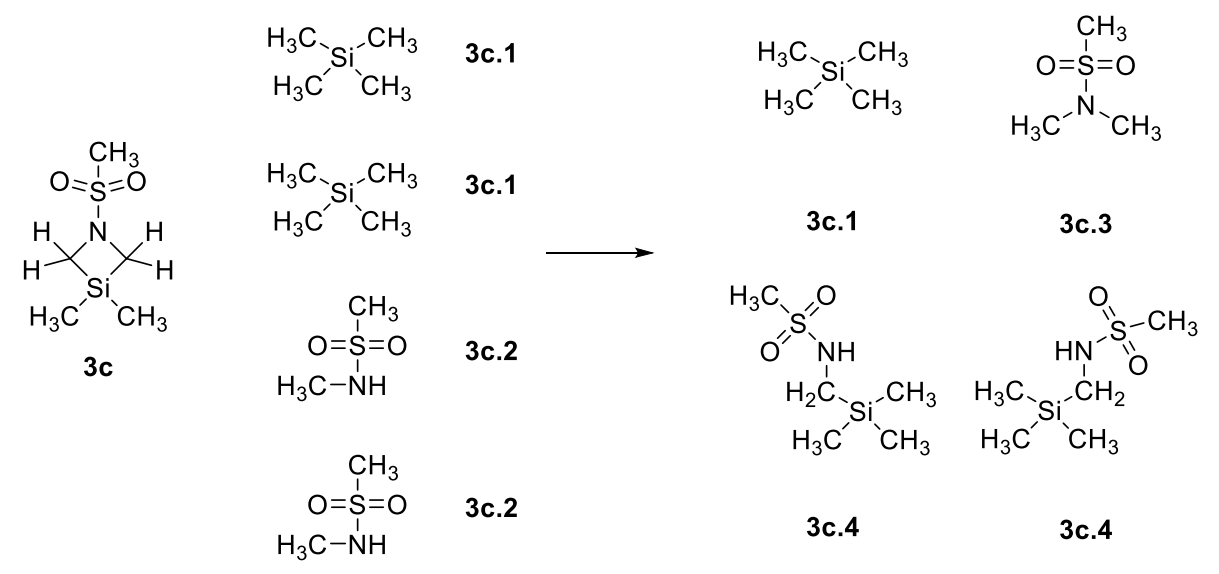

Scheme 2. The ring strain energy of $\mathbf{3 c}$ was calculation as: - (free energy of the four molecules at the right - free energy of the five molecules at the left). The detailed energy components and XYZ structures of each molecules are given below. As 3c.1 is same as $\mathbf{7 . 2}$ above, its energy and coordinate are not listed below.

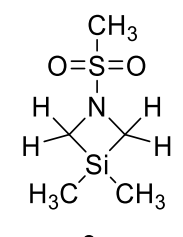

$3 c$

\begin{tabular}{|c|c|c|c|c|}
\hline \multicolumn{2}{|c|}{ Energy component } & \multicolumn{2}{|c|}{ Value (kcal/mol) } & \\
\hline \multicolumn{2}{|c|}{ Electronic Energy (PCM) } & \multicolumn{2}{|c|}{-684841.91} & \\
\hline \multicolumn{2}{|c|}{ Zero Point Energy Correction } & \multicolumn{2}{|c|}{113.93} & \\
\hline \multicolumn{2}{|c|}{ Enthalpy } & \multicolumn{2}{|r|}{122.98} & \\
\hline \multicolumn{2}{|c|}{$-\mathrm{T} \times \mathrm{dS}(298.15 \mathrm{~K})$} & \multicolumn{2}{|r|}{-34.34} & \\
\hline \multicolumn{2}{|c|}{ Free Energy } & \multicolumn{2}{|c|}{-684639.34} & \\
\hline \multicolumn{2}{|c|}{ Dipole Moment (Debye) } & \multirow{2}{*}{\multicolumn{2}{|c|}{6.43}} & \\
\hline $\mathrm{XY}$ & & & & \\
\hline 1 & $\mathrm{C}$ & 1.7118587043 & 1.9307185756 & 0.6161268186 \\
\hline 2 & $\mathrm{~S}$ & 1.9439084279 & 0.2156446036 & 0.1313884677 \\
\hline 3 & $\mathrm{~N}$ & 0.4536079646 & -0.1706230762 & -0.5632977544 \\
\hline 4 & $\mathrm{O}$ & 3.0315143558 & 0.1792063983 & -0.8612114079 \\
\hline 5 & $\mathrm{O}$ & 2.0721014102 & -0.6137387034 & 1.3362856990 \\
\hline 6 & $\mathrm{C}$ & 0.1569745302 & 0.2869407717 & -1.9600319464 \\
\hline 7 & $\mathrm{C}$ & 0.0100008599 & -1.6055425849 & -0.5301115194 \\
\hline 8 & $\mathrm{Si}$ & -1.0312893318 & -1.2118673243 & -2.0895256112 \\
\hline 9 & $\mathrm{C}$ & -0.8216019627 & -2.3484956702 & -3.5659862808 \\
\hline 10 & $\mathrm{C}$ & -2.8332058272 & -0.8108263116 & -1.7538465820 \\
\hline 11 & $\mathrm{H}$ & -0.4660267221 & -1.8640619851 & 0.4153416070 \\
\hline 12 & $\mathrm{H}$ & 0.8271662816 & -2.3082511851 & -0.7268862919 \\
\hline 13 & $\mathrm{H}$ & -0.2312182157 & 1.3066897879 & -1.9860608456 \\
\hline 14 & $\mathrm{H}$ & 1.0319772466 & 0.2095556247 & -2.6147968668 \\
\hline 15 & $\mathrm{H}$ & 1.4748531363 & 2.5150454254 & -0.2717465647 \\
\hline 16 & $\mathrm{H}$ & 2.6589317739 & 2.2579945915 & 1.0449235081 \\
\hline 17 & $\mathrm{H}$ & 0.9106944890 & 1.9732575378 & 1.3510547711 \\
\hline 18 & $\mathrm{H}$ & 0.2334267922 & -2.5624859498 & -3.7550054679 \\
\hline 19 & $\mathrm{H}$ & -1.2467157839 & -1.8954009827 & -4.4671435539 \\
\hline 20 & $\mathrm{H}$ & -1.3374003668 & -3.2992026793 & -3.3973052103 \\
\hline 21 & $\mathrm{H}$ & -2.9325331301 & -0.1450248984 & -0.8924512556 \\
\hline 22 & $\mathrm{H}$ & -3.2903047208 & -0.3217398585 & -2.6196447054 \\
\hline 23 & $\mathrm{H}$ & -3.4017199115 & -1.7227921071 & -1.5470690073 \\
\hline
\end{tabular}




$$
\begin{array}{r}
\begin{array}{r}
\mathrm{CH}_{1} \\
\mathrm{O}=\underset{\mathrm{S}}{\mathrm{H}}=\mathrm{O}
\end{array} \\
\mathrm{H}_{3} \mathrm{C}-\mathrm{N} \mathrm{H} \\
3 \mathrm{c} .2
\end{array}
$$

\begin{tabular}{|c|c|c|c|c|}
\hline \multicolumn{2}{|c|}{ Energy component } & \multicolumn{2}{|c|}{ Value (kcal/mol) } & \\
\hline \multicolumn{2}{|c|}{ Electronic Energy (PCM) } & \multicolumn{2}{|c|}{-429138.44} & \\
\hline \multicolumn{2}{|c|}{ Zero Point Energy Correction } & \multicolumn{2}{|r|}{64.02} & \\
\hline \multicolumn{2}{|c|}{ Enthalpy } & \multicolumn{2}{|r|}{69.31} & \\
\hline \multicolumn{2}{|c|}{$-\mathrm{T} \times \mathrm{dS}(298.15 \mathrm{~K})$} & \multicolumn{2}{|r|}{-25.18} & \\
\hline \multicolumn{2}{|c|}{ Free Energy } & \multicolumn{2}{|c|}{-429030.29} & \\
\hline \multicolumn{5}{|c|}{ XYZ coordinate } \\
\hline 1 & $\mathrm{C}$ & 1.4206344379 & -0.7481815259 & -2.2132050026 \\
\hline 2 & $\mathrm{~S}$ & 1.6419112459 & -0.5081990751 & -0.4483811527 \\
\hline 3 & $\mathrm{O}$ & 2.1563826142 & -1.7468920258 & 0.1435853411 \\
\hline 4 & $\mathrm{~N}$ & 0.0456810643 & -0.3388656304 & 0.0819589717 \\
\hline 5 & $\mathrm{O}$ & 2.3933324300 & 0.7405981884 & -0.2577355795 \\
\hline 6 & $\mathrm{C}$ & -0.2646315211 & -0.8419128768 & 1.4350496257 \\
\hline 7 & $\mathrm{H}$ & 0.9097358707 & 0.1237343308 & -2.6195230887 \\
\hline 8 & $\mathrm{H}$ & 0.8422672187 & -1.6568128497 & -2.3658998957 \\
\hline 9 & $\mathrm{H}$ & 2.4205090354 & -0.8396283599 & -2.6371997567 \\
\hline 10 & $\mathrm{H}$ & -0.2324971180 & 0.6321207882 & -0.0371813482 \\
\hline 11 & $\mathrm{H}$ & -1.3365194860 & -0.7082318733 & 1.5851150667 \\
\hline 12 & $\mathrm{H}$ & 0.2779984592 & -0.3166735597 & 2.2266159133 \\
\hline 13 & $\mathrm{H}$ & -0.0338042512 & -1.9040555307 & 1.4838009054 \\
\hline
\end{tabular}

\begin{tabular}{|c|c|c|c|c|}
\hline \multicolumn{2}{|c|}{ Energy component } & \multicolumn{2}{|c|}{ Value (kcal/mol) } & \\
\hline \multicolumn{2}{|c|}{ Electronic Energy (PCM) } & \multicolumn{2}{|c|}{-453809.27} & \\
\hline \multicolumn{2}{|c|}{ Zero Point Energy Correction } & \multicolumn{2}{|r|}{81.28} & \\
\hline \multicolumn{2}{|c|}{ Enthalpy } & \multicolumn{2}{|r|}{87.47} & \\
\hline \multicolumn{2}{|c|}{$-\mathrm{T} \times \mathrm{dS}(298.15 \mathrm{~K})$} & \multicolumn{3}{|c|}{-27.07} \\
\hline \multicolumn{2}{|c|}{ Free Energy } & \multicolumn{2}{|c|}{-453667.58} & \\
\hline \multicolumn{5}{|c|}{ XYZ coordinate } \\
\hline 1 & $\mathrm{C}$ & 3.1893760237 & -0.8759019268 & 1.4051536337 \\
\hline 2 & $\mathrm{~S}$ & 1.9555878905 & 0.3659063409 & 0.9608720337 \\
\hline 3 & $\mathrm{O}$ & 1.7524948018 & 1.2190293308 & 2.1341026197 \\
\hline 4 & $\mathrm{~N}$ & 0.5001253850 & -0.4221723957 & 0.6793965543 \\
\hline 5 & $\mathrm{O}$ & 2.3670873674 & 0.9584448431 & -0.3139313921 \\
\hline 6 & $\mathrm{C}$ & -0.1763187820 & -1.0029279782 & 1.8474662407 \\
\hline 7 & $\mathrm{C}$ & 0.4070897176 & -1.2318820070 & -0.5434468758 \\
\hline 8 & $\mathrm{H}$ & 3.2999458101 & -1.5737270118 & 0.5761091435 \\
\hline 9 & $\mathrm{H}$ & 2.8672488212 & -1.3801408678 & 2.3153517105 \\
\hline 10 & $\mathrm{H}$ & 4.1206275498 & -0.3364176655 & 1.5768666525 \\
\hline 11 & $\mathrm{H}$ & -1.2340256336 & -1.1116470216 & 1.6001865480 \\
\hline 12 & $\mathrm{H}$ & -0.0885300974 & -0.3298236518 & 2.6977277742 \\
\hline 13 & $\mathrm{H}$ & 0.2244368457 & -1.9904979905 & 2.1127393244 \\
\hline 14 & $\mathrm{H}$ & -0.6517425526 & -1.3596823825 & -0.7761677016 \\
\hline 15 & $\mathrm{H}$ & 0.8609626005 & -2.2243748318 & -0.4219377121 \\
\hline 16 & $\mathrm{H}$ & 0.8816342523 & -0.7081847839 & -1.3704885536 \\
\hline
\end{tabular}

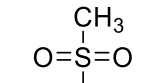

$$
\begin{aligned}
& \mathrm{H}_{3} \mathrm{C}^{-\stackrel{1}{N}}-\mathrm{CH}_{3} \\
& 3 c .3
\end{aligned}
$$




$$
\begin{aligned}
& \mathrm{H}_{3} \mathrm{C} \text {, } \mathrm{O} \\
& \text { O" } \mathrm{NH} \\
& \mathrm{H}_{2} \mathrm{C} \cdot \mathrm{Si}-\mathrm{CH}_{3} \\
& \mathrm{H}_{3} \mathrm{C}^{\prime} \mathrm{CH}_{3} \\
& \text { 3c. } 4
\end{aligned}
$$

\begin{tabular}{|c|c|c|c|c|}
\hline \multicolumn{2}{|c|}{ Energy component } & \multicolumn{2}{|c|}{ Value (kcal/mol) } & \\
\hline \multicolumn{2}{|c|}{ Electronic Energy (PCM) } & \multicolumn{2}{|c|}{-685625.19} & \\
\hline \multicolumn{2}{|c|}{ Zero Point Energy Correction } & \multicolumn{2}{|r|}{127.11} & \\
\hline \multicolumn{2}{|c|}{ Enthalpy } & & 137.16 & \\
\hline \multicolumn{2}{|c|}{$-\mathrm{T} \times \mathrm{dS}(298.15 \mathrm{~K})$} & \multicolumn{3}{|c|}{-37.11} \\
\hline \multicolumn{2}{|c|}{ Free Energy } & \multicolumn{3}{|c|}{-685398.03} \\
\hline \multicolumn{5}{|c|}{ XYZ coordinate } \\
\hline 1 & $\mathrm{C}$ & 2.5615381515 & -0.8723026571 & 3.7159950840 \\
\hline 2 & $\mathrm{~S}$ & 1.9145599270 & -1.2509500079 & 2.0842824527 \\
\hline 3 & $\mathrm{O}$ & 2.6799356883 & -2.3890148209 & 1.5508896738 \\
\hline 4 & $\mathrm{~N}$ & 2.2641266919 & 0.1791828225 & 1.2392649659 \\
\hline 5 & $\mathrm{O}$ & 0.4538078983 & -1.3558429960 & 2.1570462111 \\
\hline 6 & $\mathrm{C}$ & 1.3682976264 & 0.5190093997 & 0.1086909464 \\
\hline 7 & $\mathrm{Si}$ & 1.8120494683 & 2.2093014639 & -0.6673882419 \\
\hline 8 & $\mathrm{C}$ & 1.5854576485 & 3.5691358891 & 0.6177805026 \\
\hline 9 & $\mathrm{C}$ & 0.6383581825 & 2.4582916480 & -2.1216538107 \\
\hline 10 & $\mathrm{C}$ & 3.6007661077 & 2.1649465536 & -1.2712151713 \\
\hline 11 & $\mathrm{H}$ & 2.4067465356 & -1.7644719433 & 4.3227903846 \\
\hline 12 & $\mathrm{H}$ & 3.6244678321 & -0.6523375694 & 3.6240267473 \\
\hline 13 & $\mathrm{H}$ & 2.0107975461 & -0.0231625759 & 4.1145736370 \\
\hline 14 & $\mathrm{H}$ & 3.2405948391 & 0.1370144607 & 0.9556354038 \\
\hline 15 & $\mathrm{H}$ & 0.3494627576 & 0.5800989203 & 0.4978941780 \\
\hline 16 & $\mathrm{H}$ & 1.3717503565 & -0.2577481015 & -0.6676514880 \\
\hline 17 & $\mathrm{H}$ & 0.5557189256 & 3.5940920966 & 0.9873965179 \\
\hline 18 & $\mathrm{H}$ & 1.8108754205 & 4.5511071658 & 0.1898291236 \\
\hline 19 & $\mathrm{H}$ & 2.2457728315 & 3.4183654090 & 1.4766185594 \\
\hline 20 & $\mathrm{H}$ & -0.4037097796 & 2.4911298458 & -1.7894303237 \\
\hline 21 & $\mathrm{H}$ & 0.8551590703 & 3.4003575031 & -2.6348449347 \\
\hline 22 & $\mathrm{H}$ & 0.7346605325 & 1.6493286377 & -2.8519690828 \\
\hline 23 & $\mathrm{H}$ & 4.3085920104 & 2.0011940574 & -0.4522616093 \\
\hline 24 & $\mathrm{H}$ & 3.8682188738 & 3.1142901702 & -1.7463852350 \\
\hline 25 & $\mathrm{H}$ & 3.7499948575 & 1.3709846288 & -2.0099144906 \\
\hline
\end{tabular}




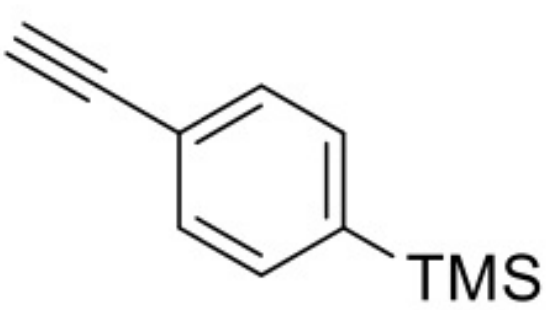

\section{S-A-6}




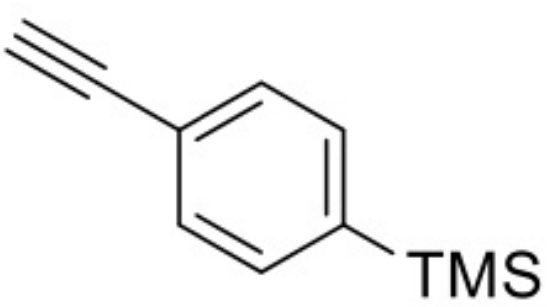

\section{S-A-6}

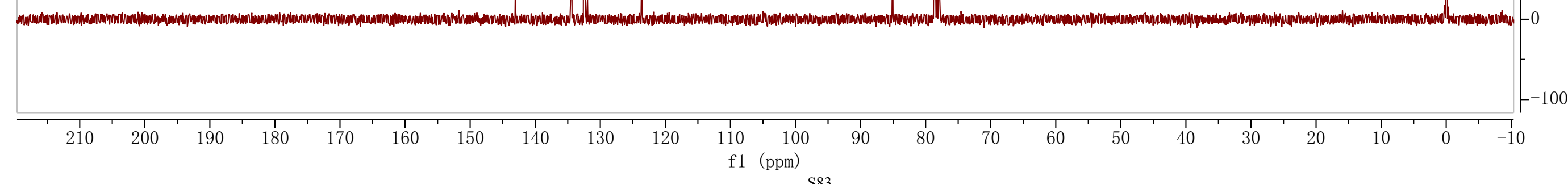




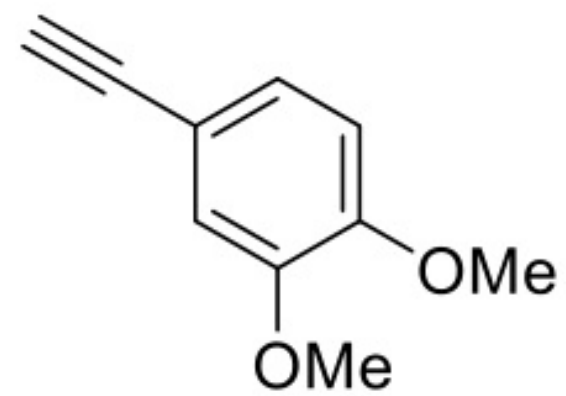

S-A-9

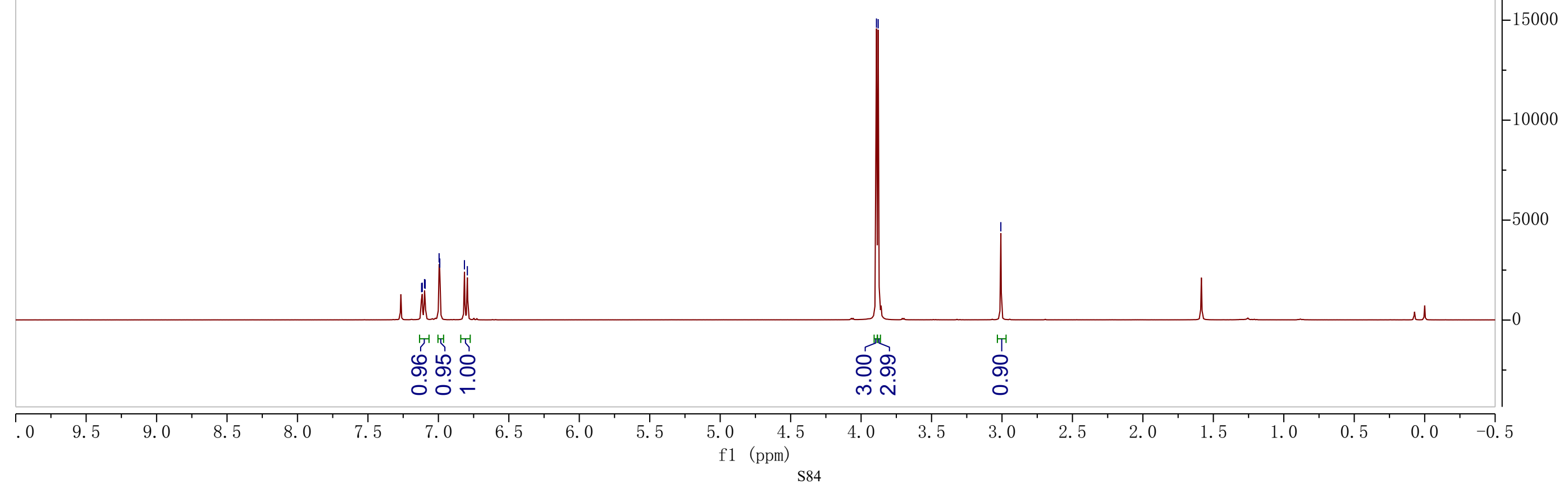




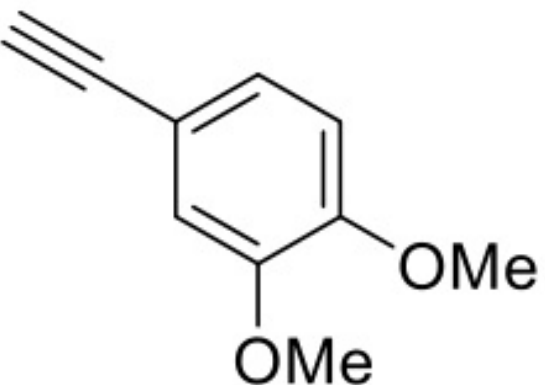

S-A-9 


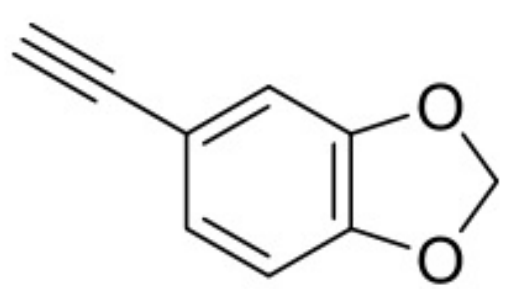

S-A-10

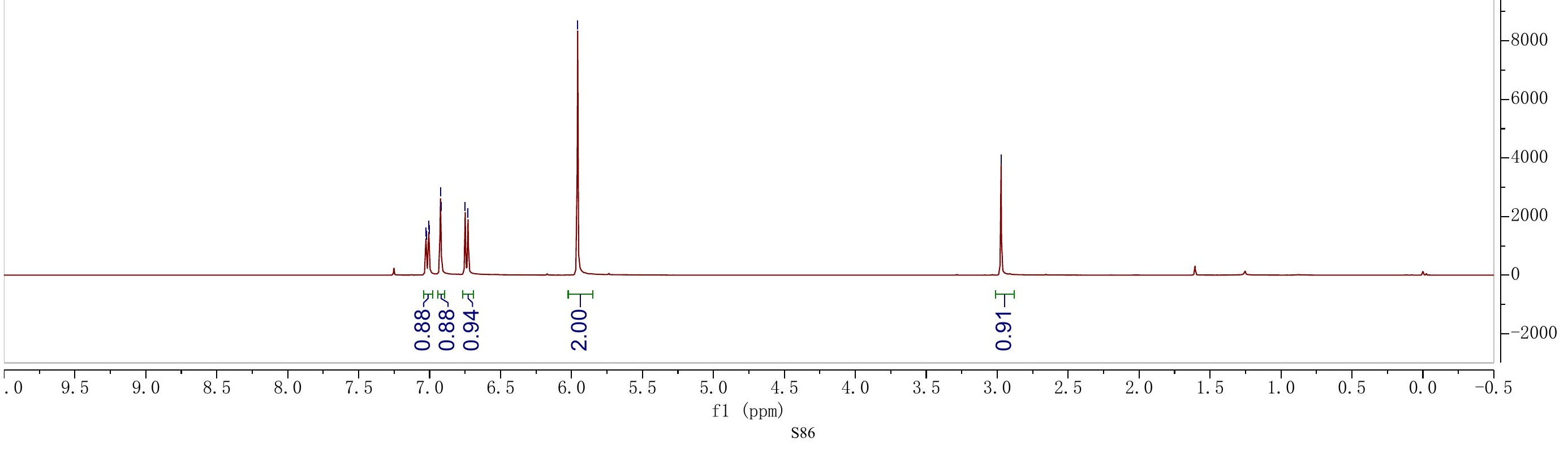




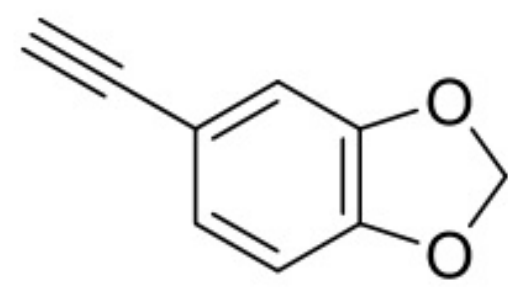

\section{S-A-10}

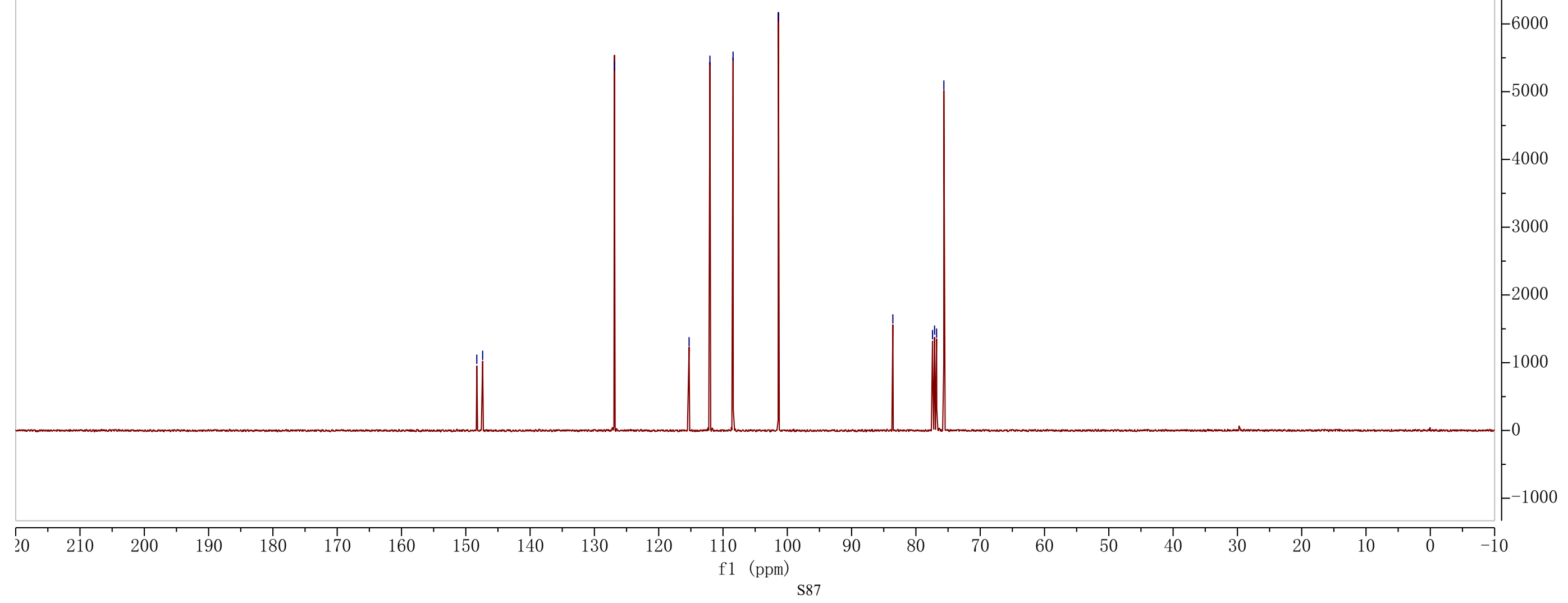




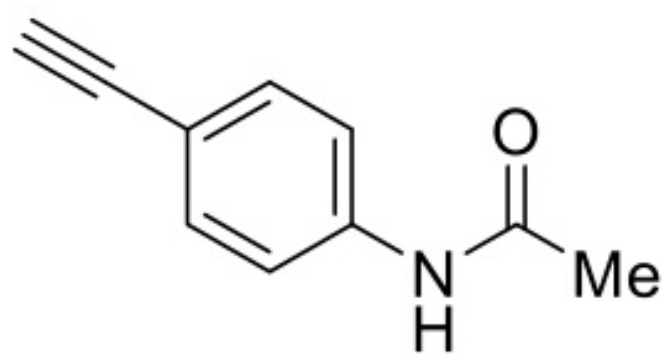

S-A-12

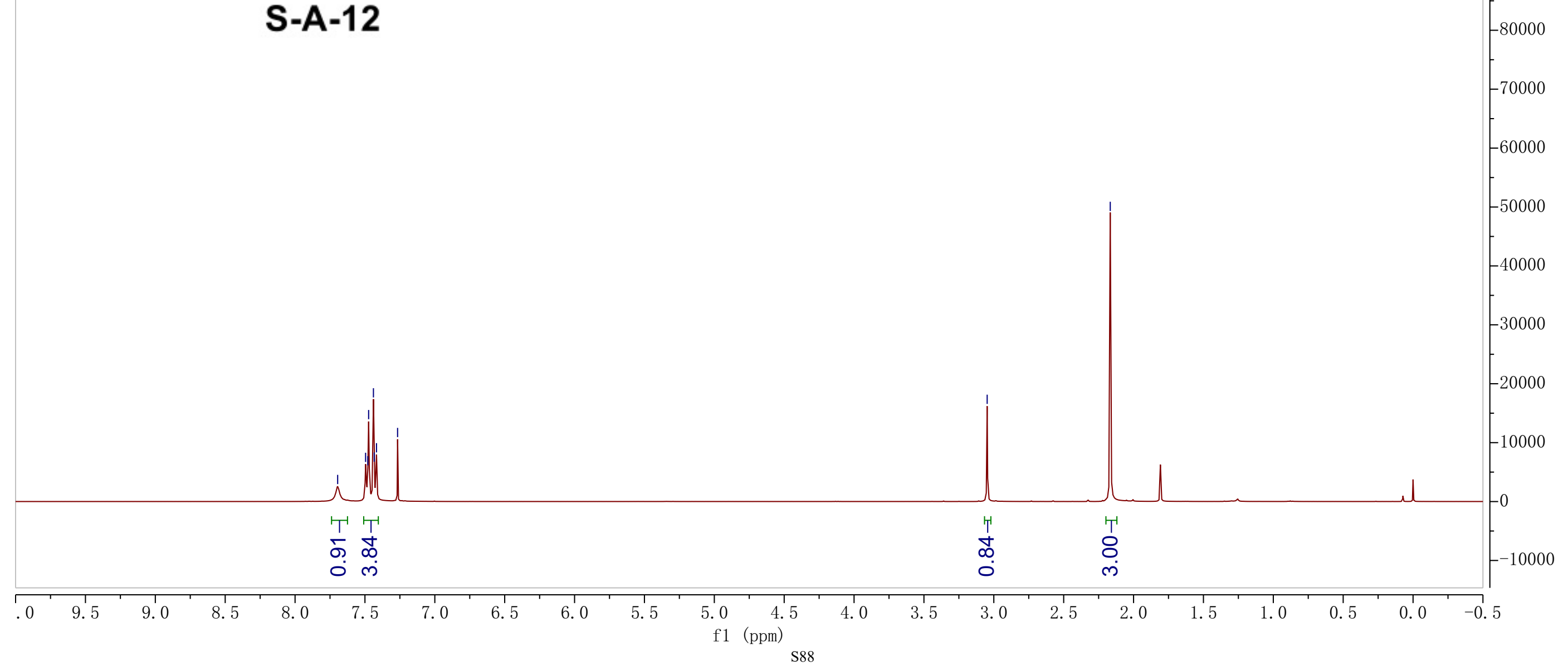




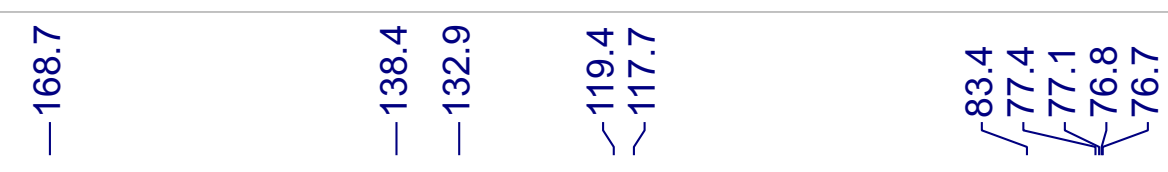

i

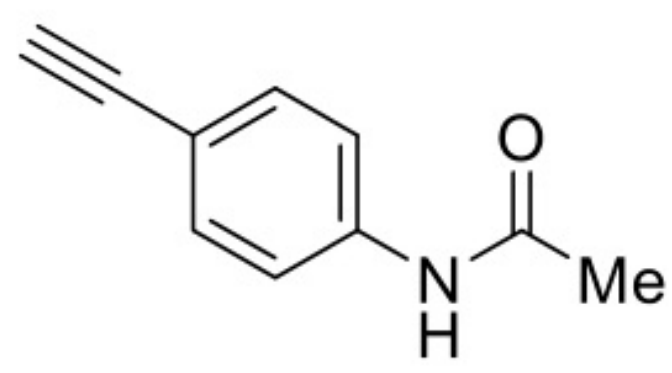

S-A-12

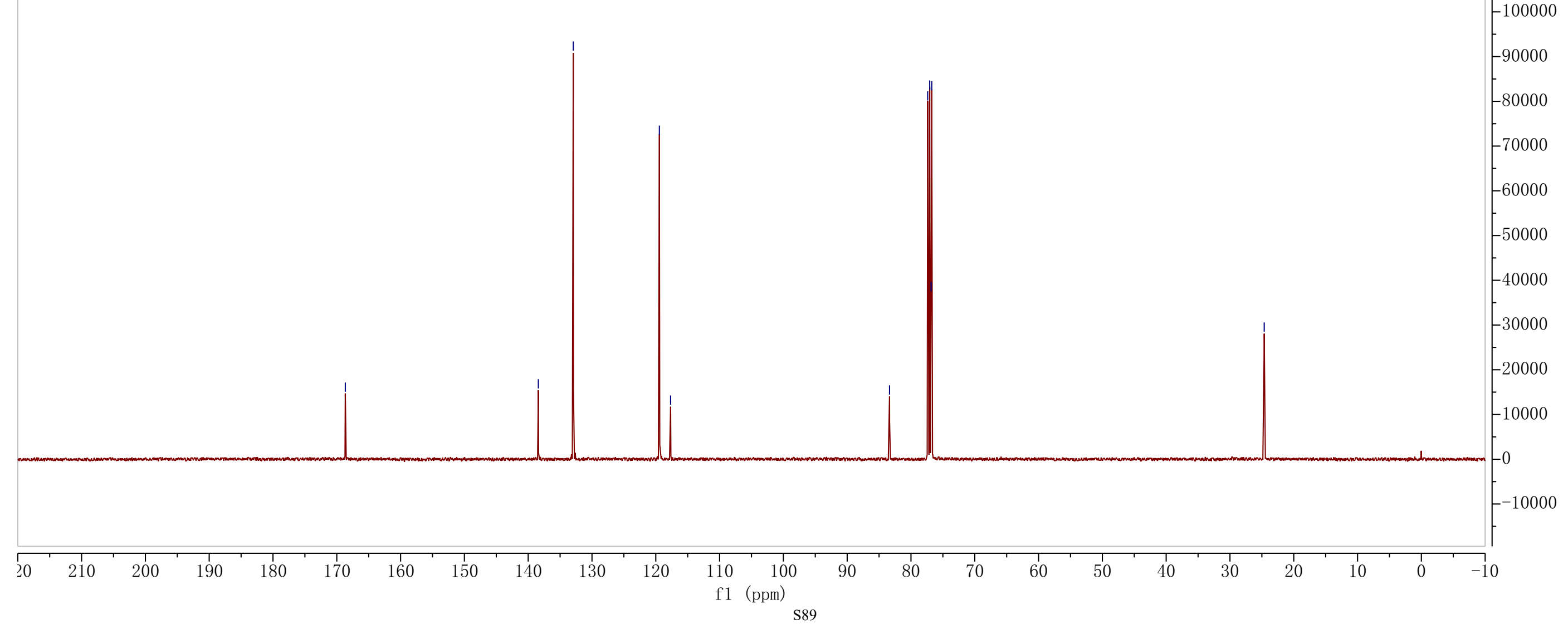


<smiles>C#Cc1ccc(C(=O)c2ccccc2)cc1</smiles>

S-A-20

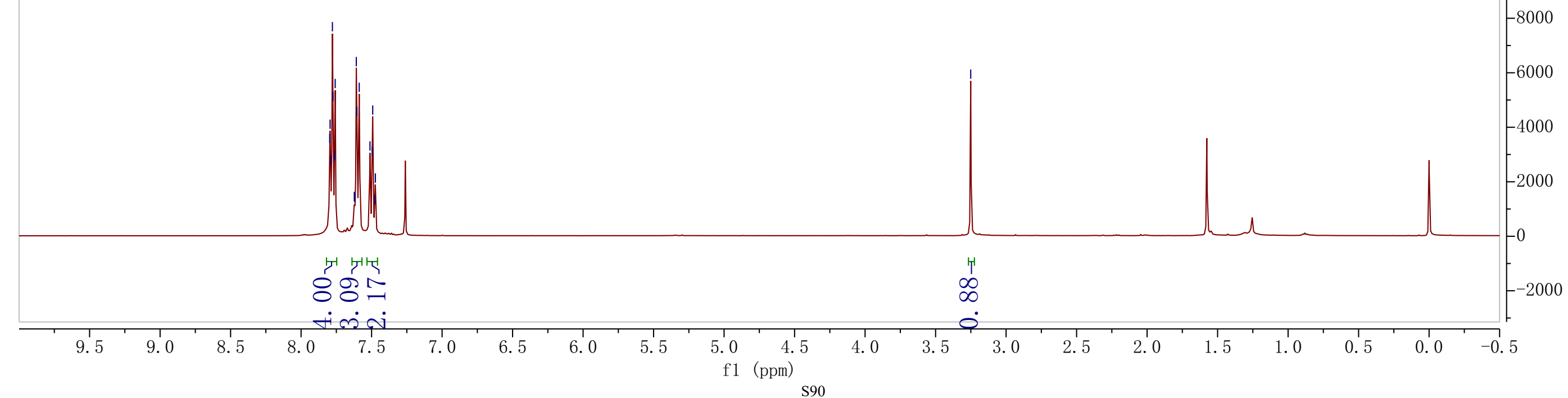




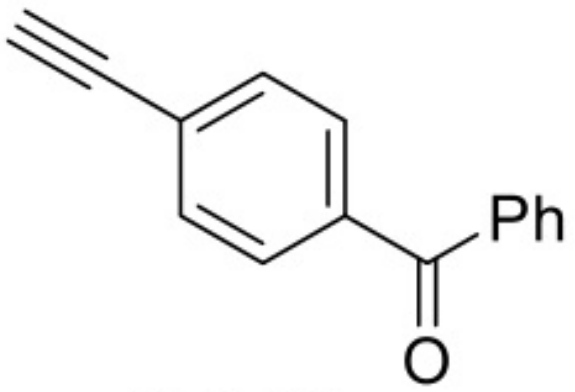

S-A-20

\section{h}

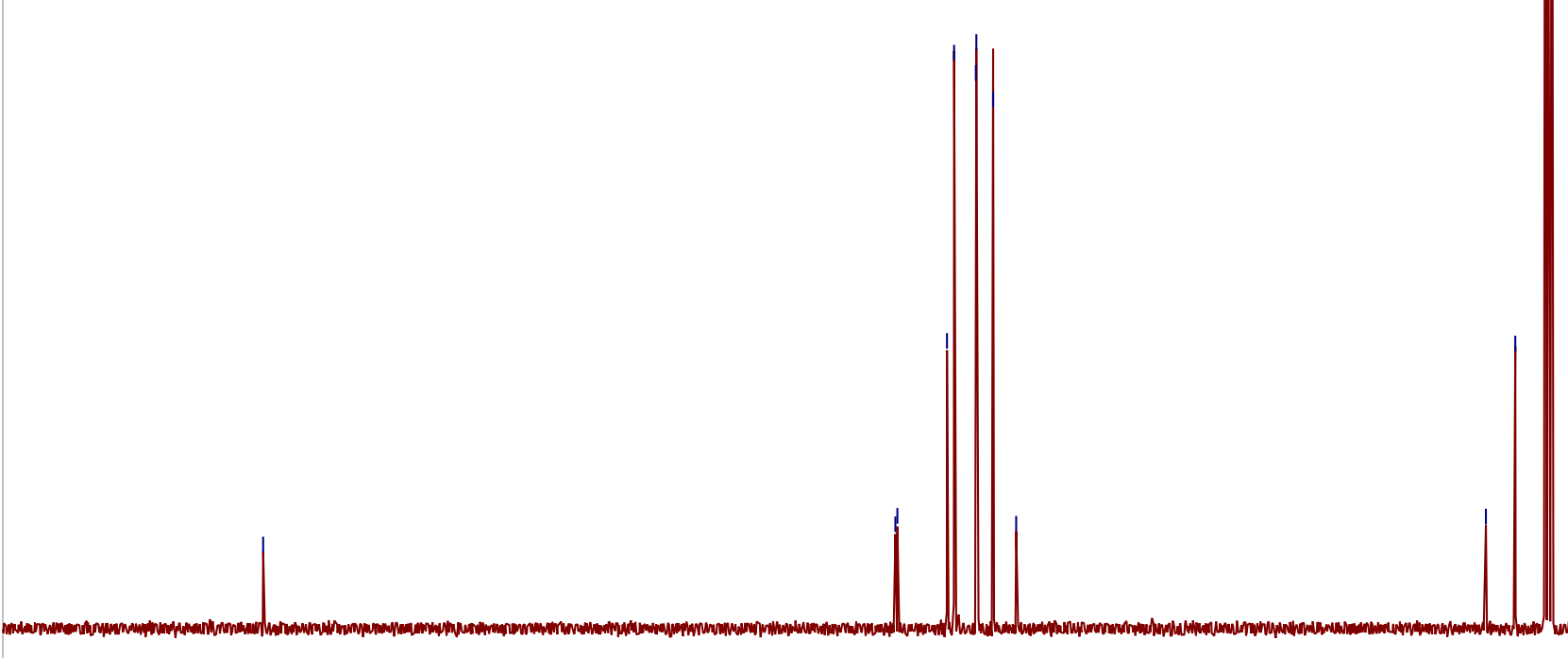




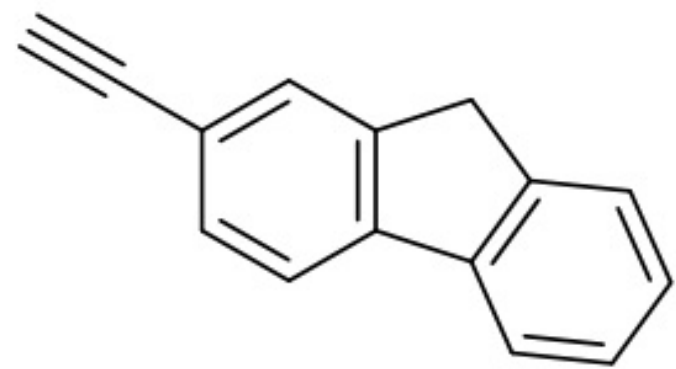

S-A-23
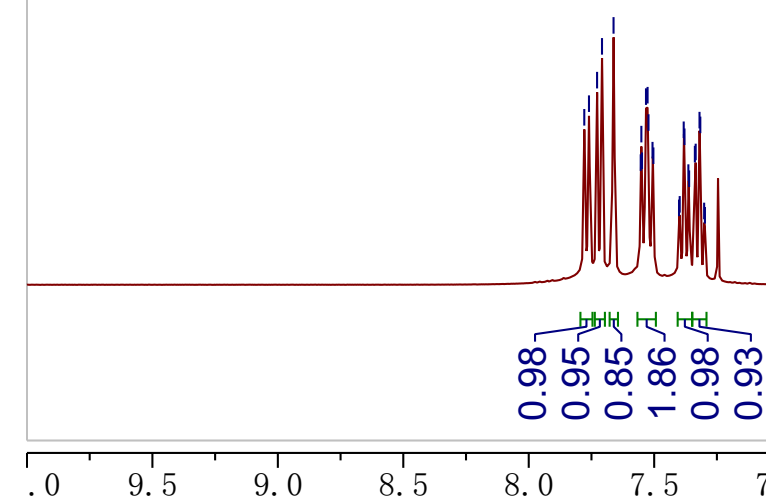


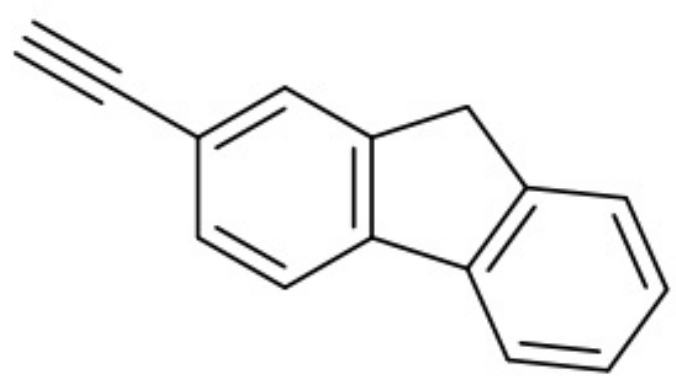

S-A-23

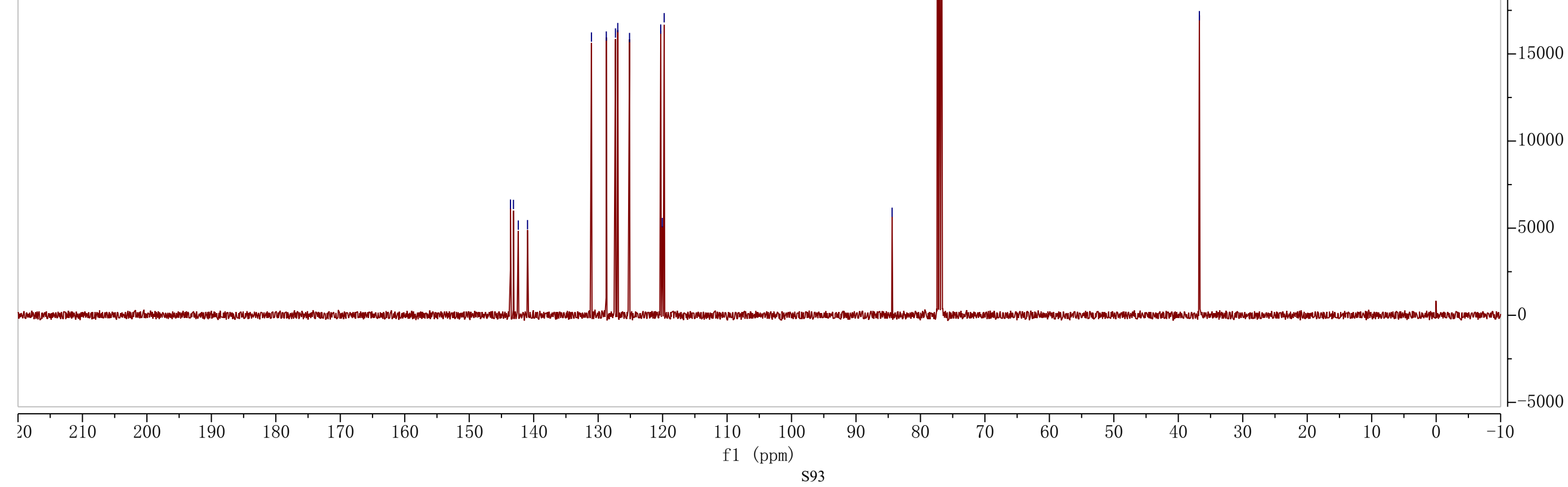


<smiles>C#Cc1cnc2ccccc2c1</smiles>

S-A-26
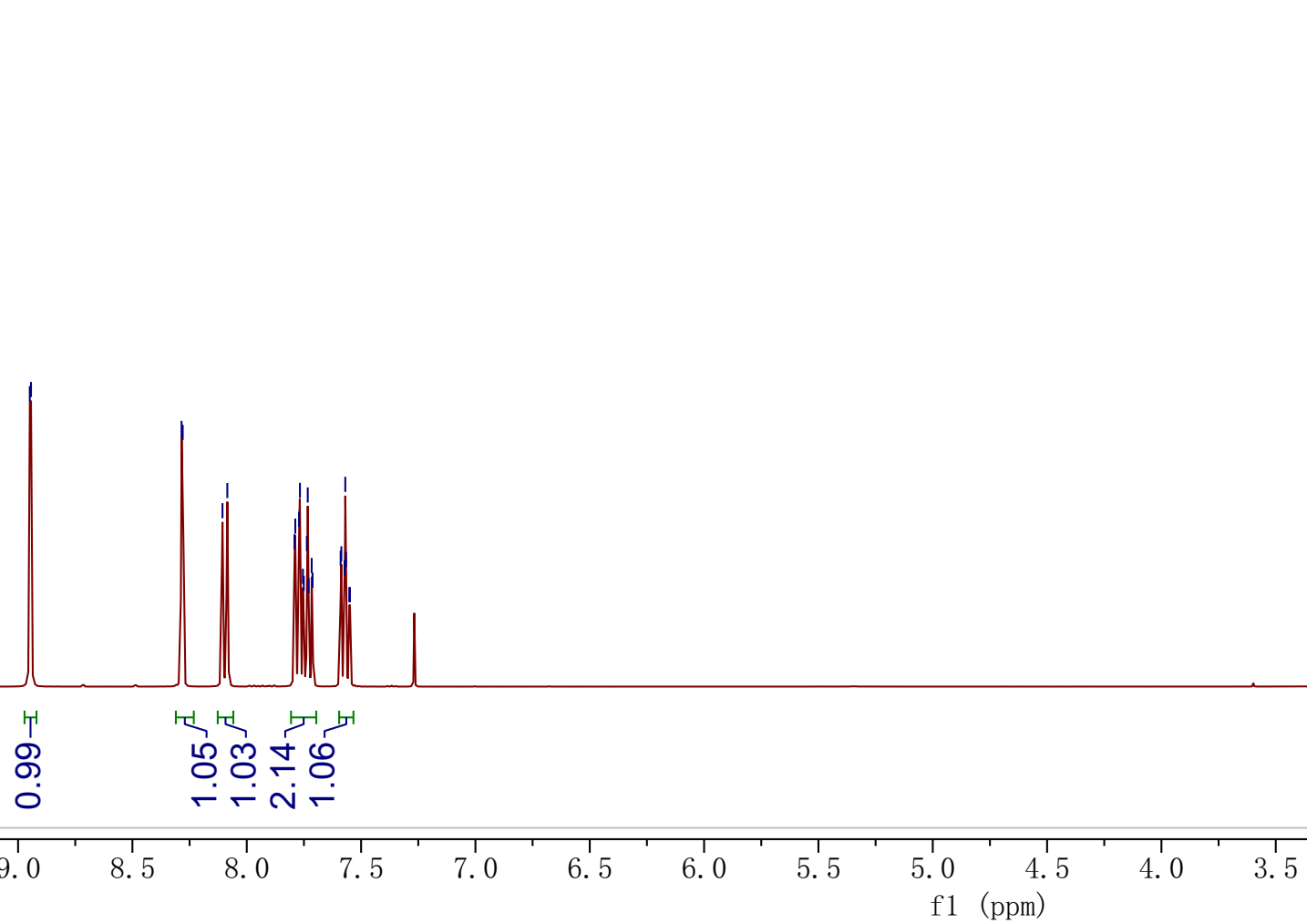

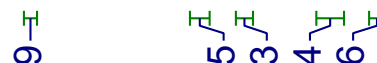

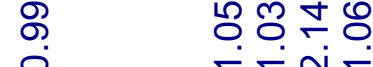

$\stackrel{+}{\stackrel{1}{\circ}}$ 


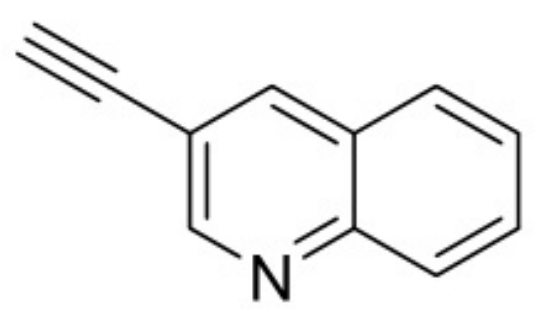

\section{S-A-26}

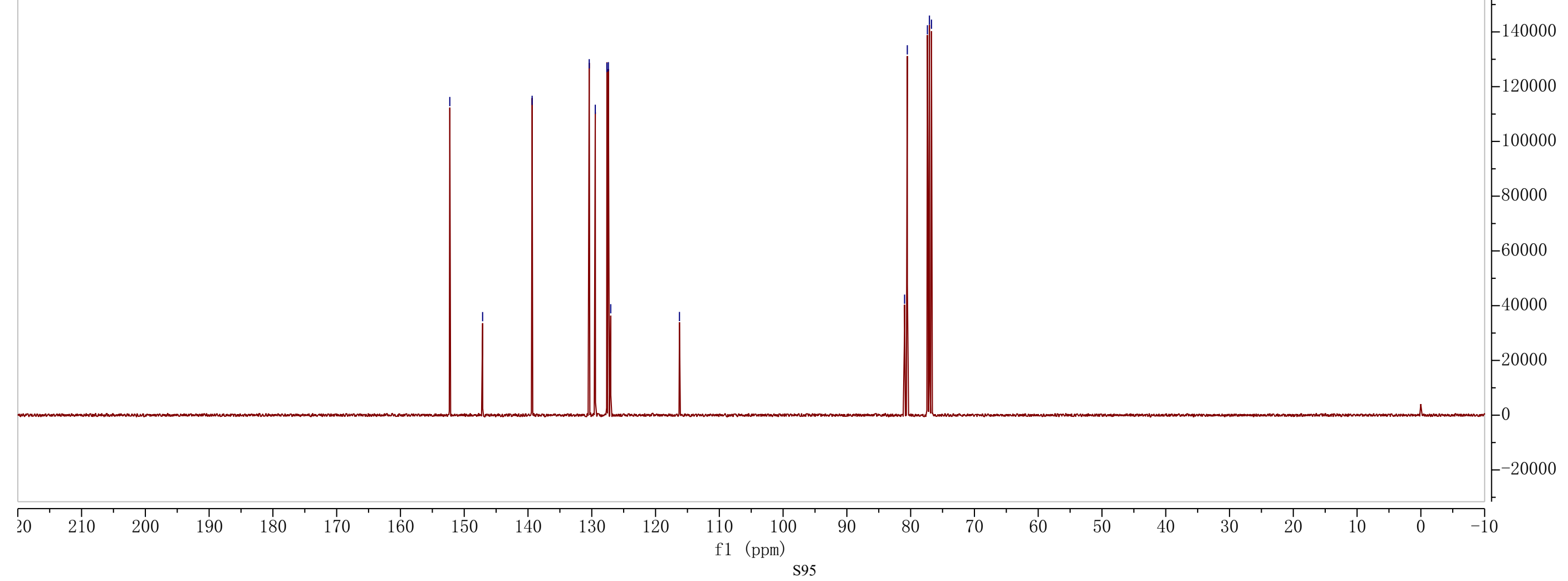


) r

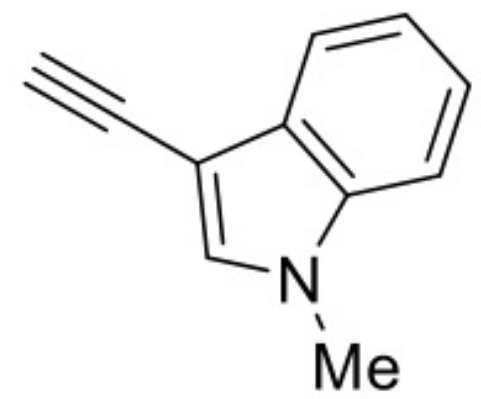

S-A-27

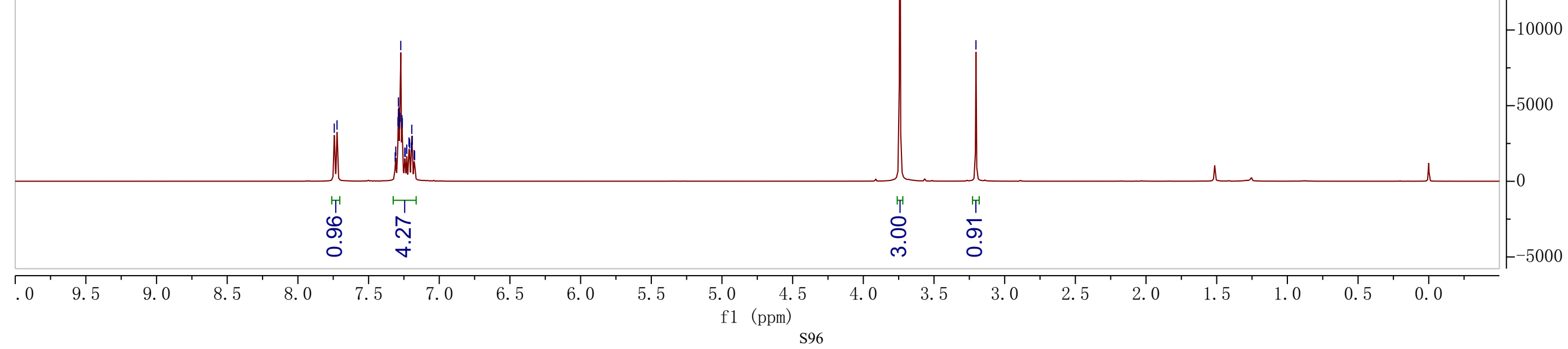




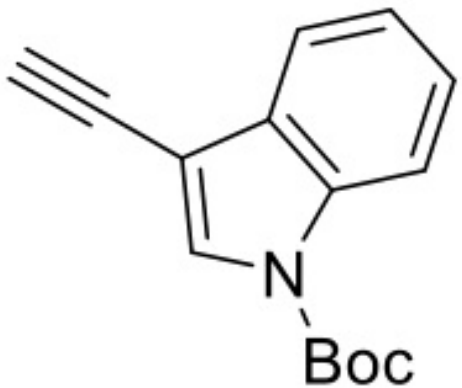

S-A-28

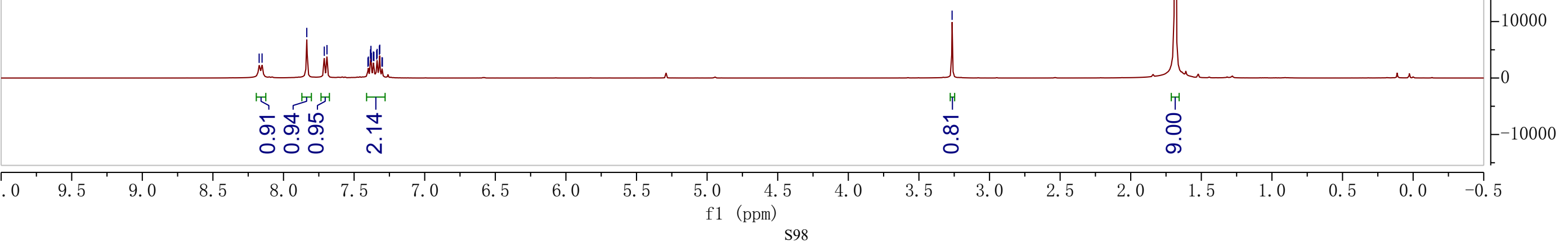




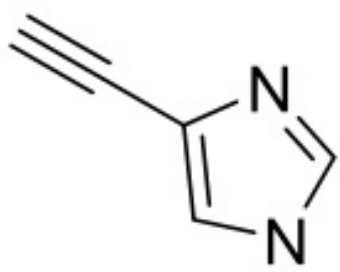

N

Boc

S-A-29

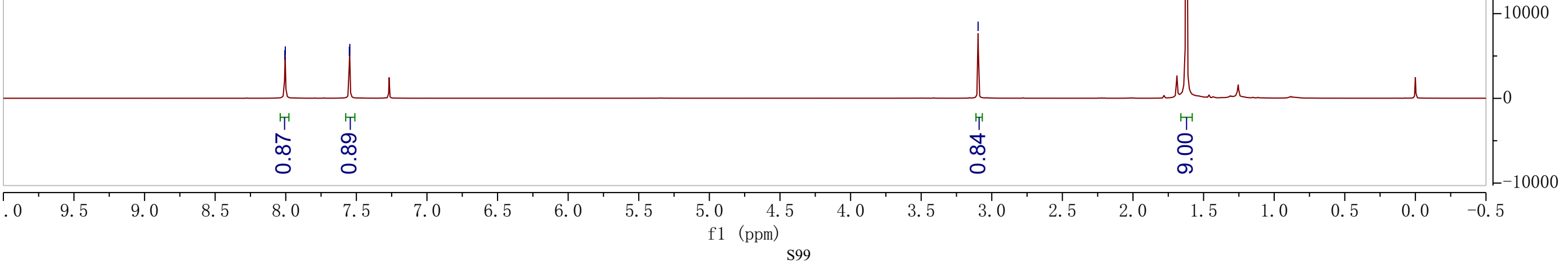




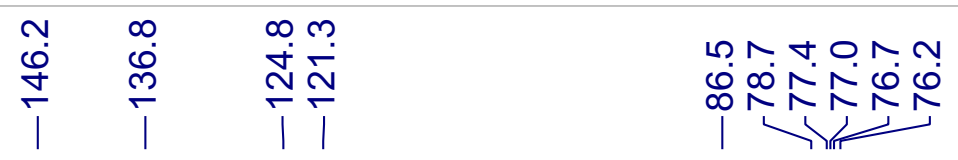


<smiles>C#Cc1cncnc1</smiles>

S-A-30

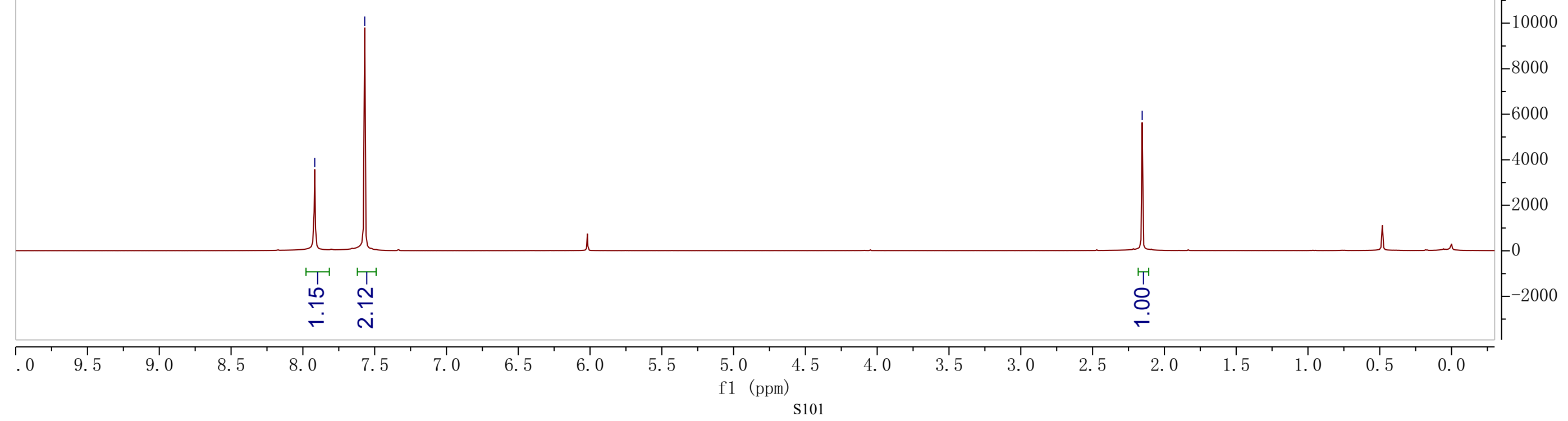


<smiles>C#Cc1cncnc1</smiles>

S-A-30

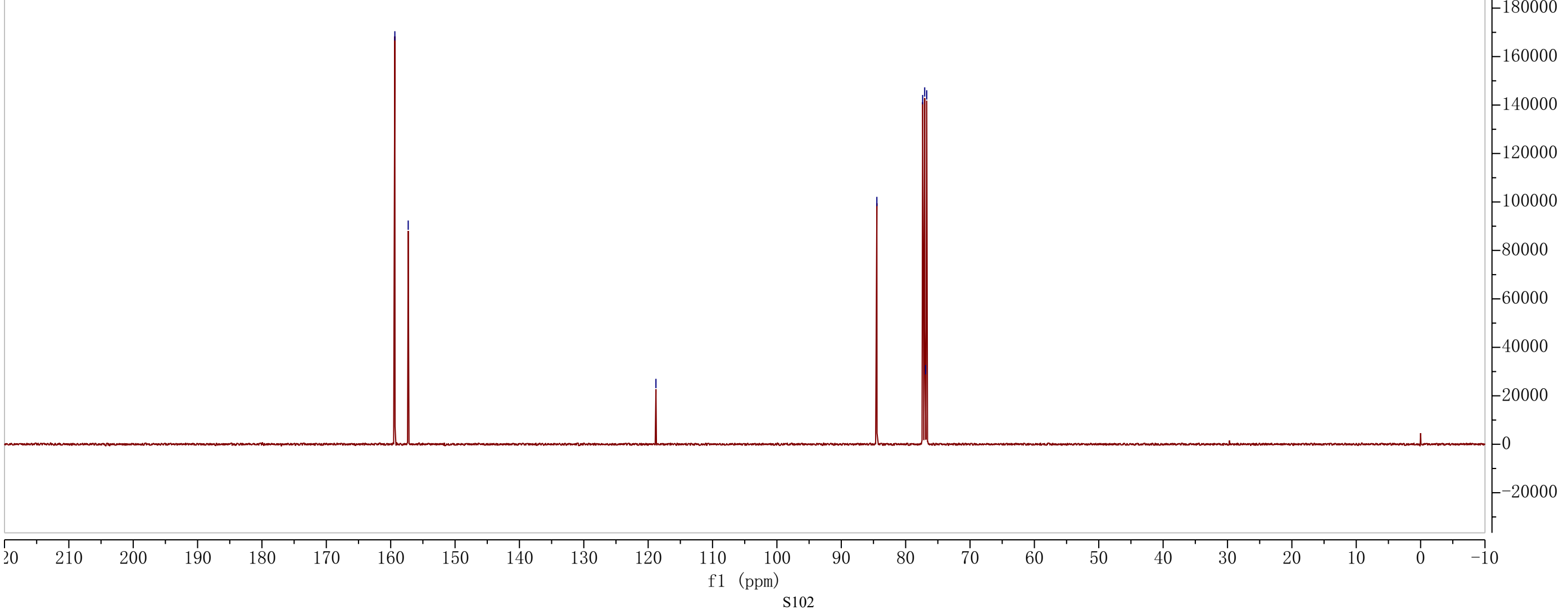




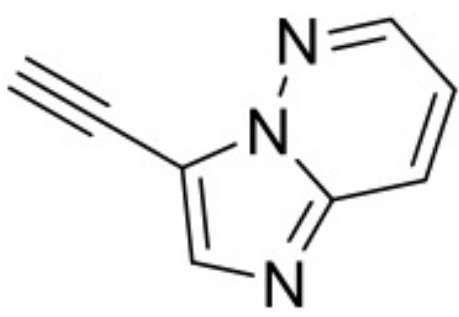

S-A-31

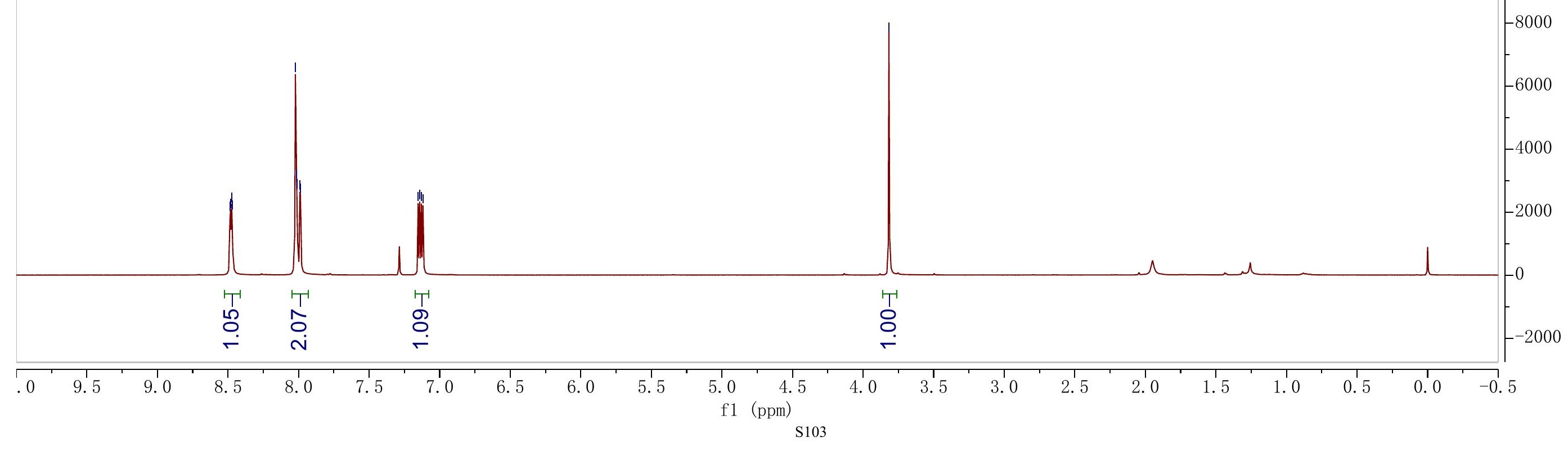




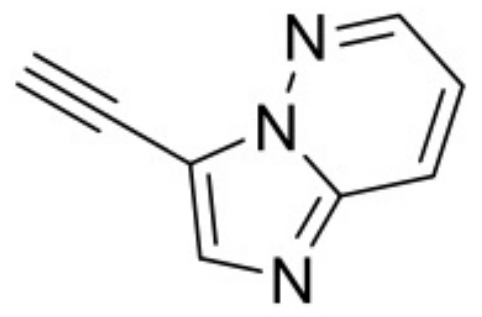

\section{S-A-31}




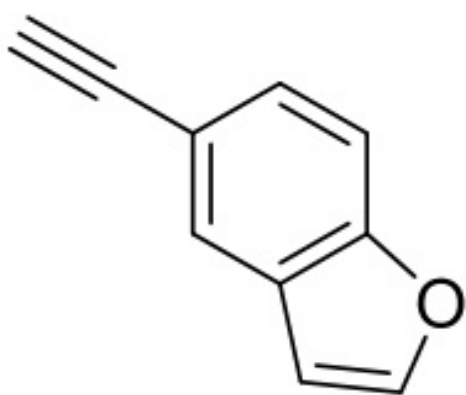

\section{S-A-32}

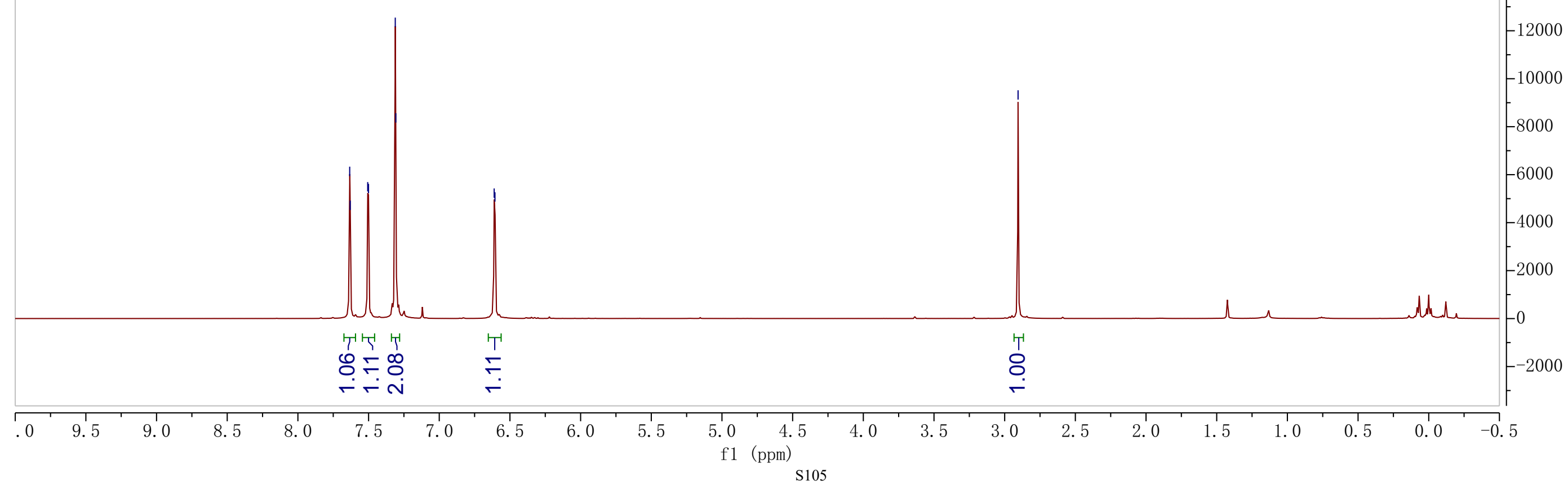




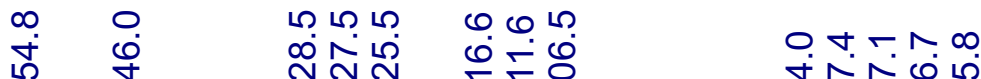

$$
\begin{aligned}
& \text { ᄂ }
\end{aligned}
$$

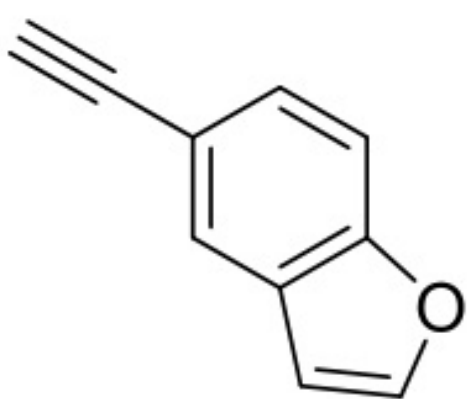

S-A-32 


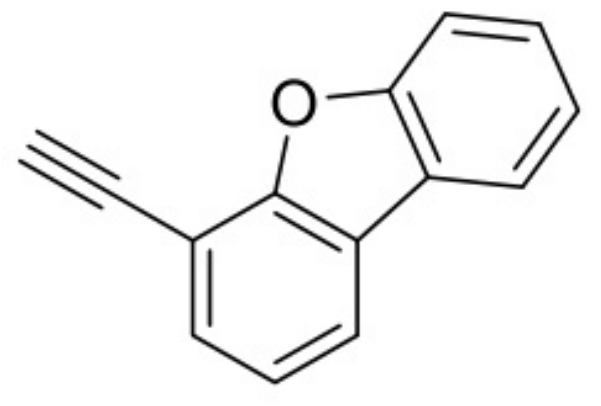

\section{S-A-33}

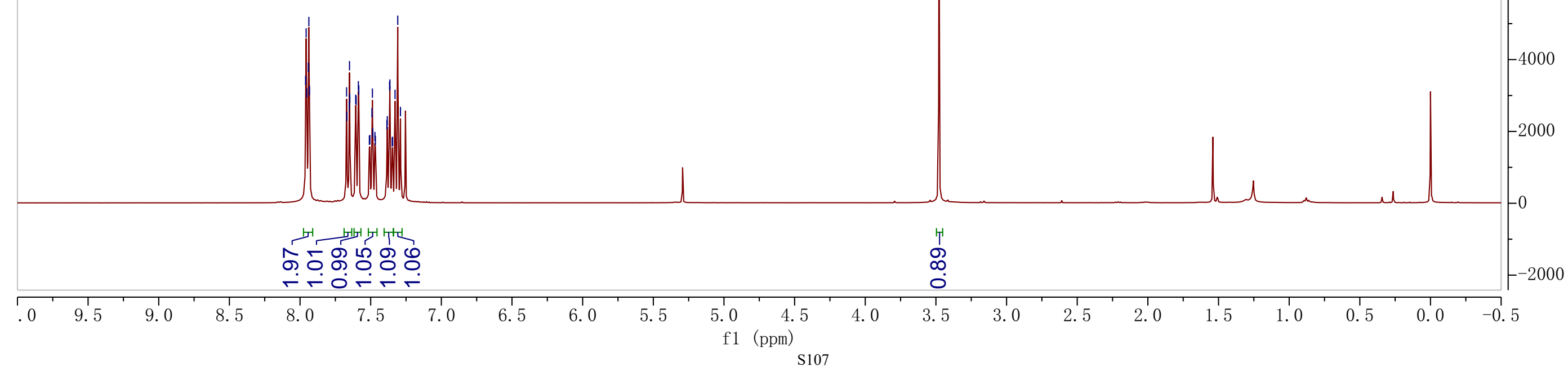




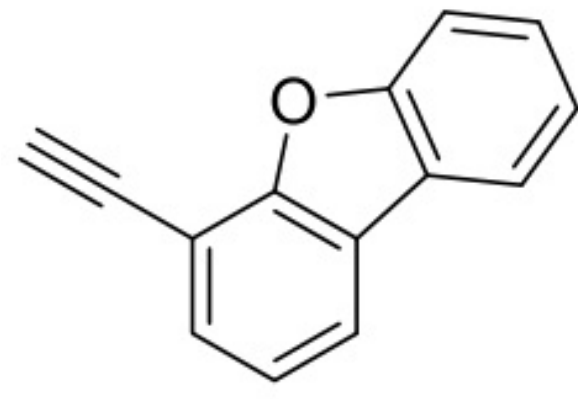

\section{S-A-33}

$210 \quad 200 \quad 190$

180

170

$160 \quad 150$

130 


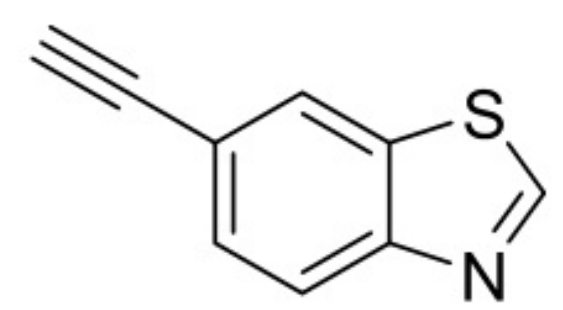

\section{S-A-36}

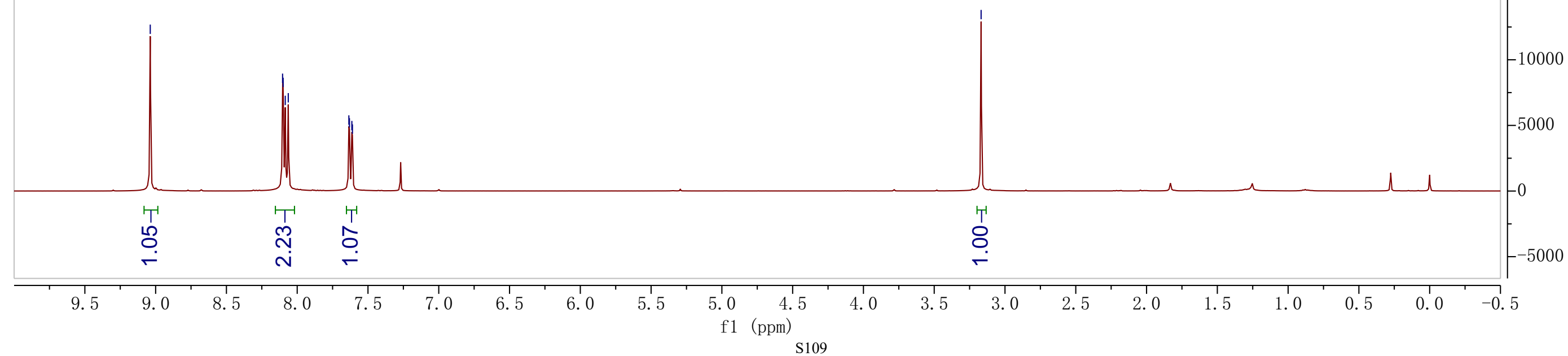




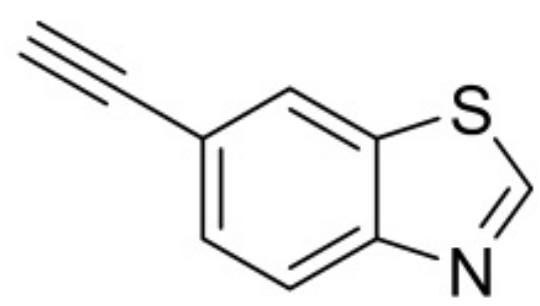

S-A-36

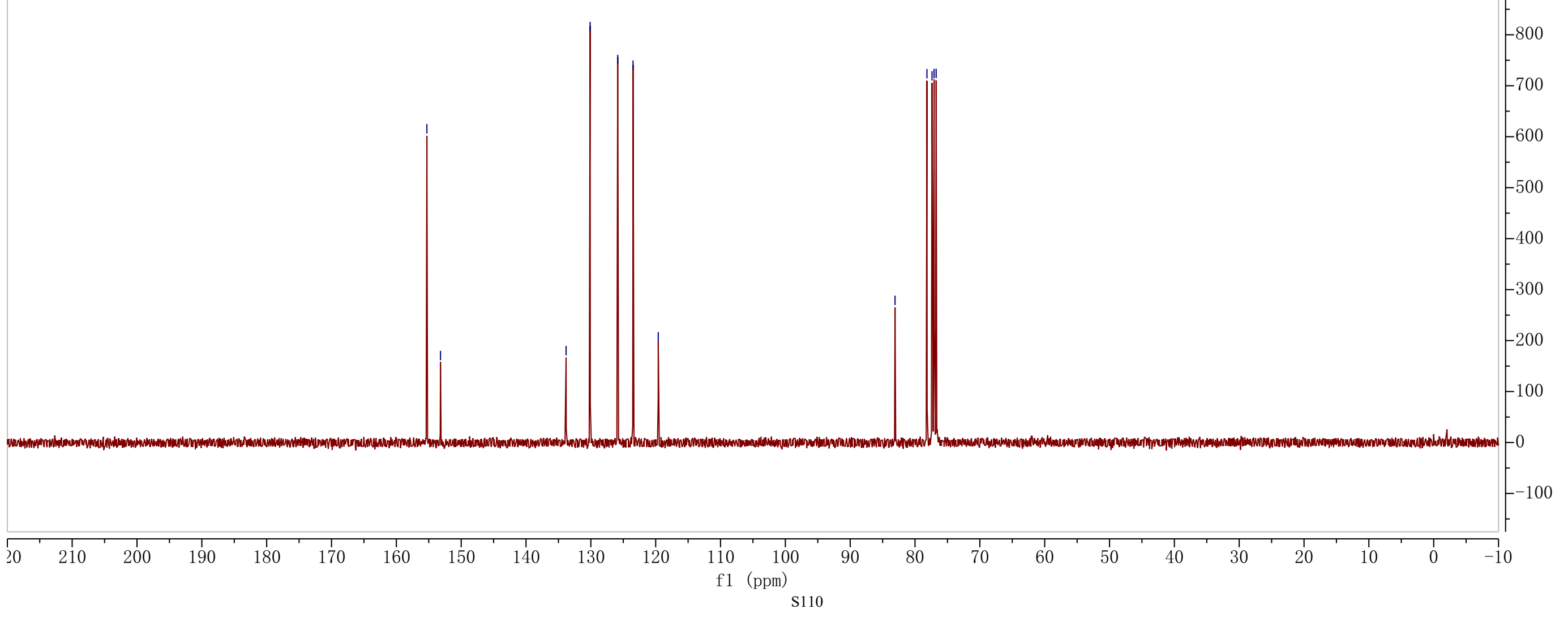




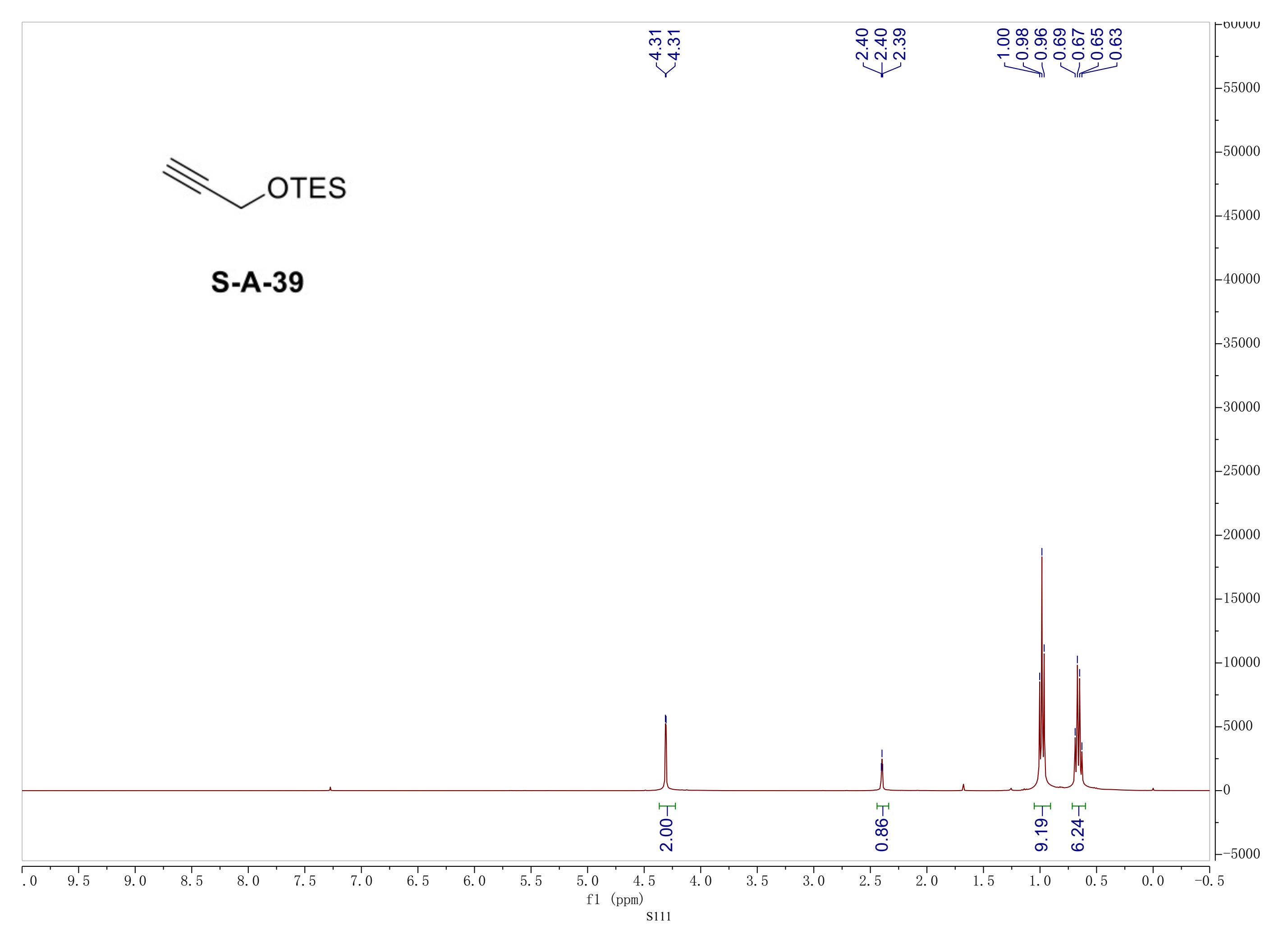


Notes

S-A-39

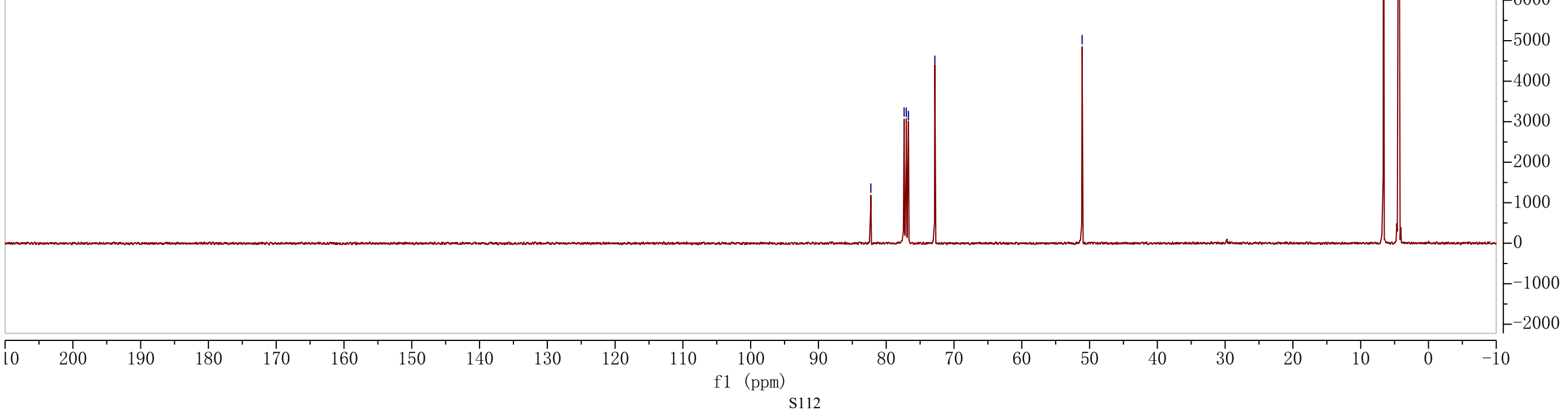




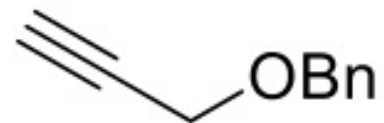

S-A-40 


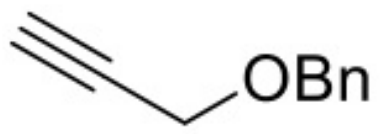

\section{S-A-40}

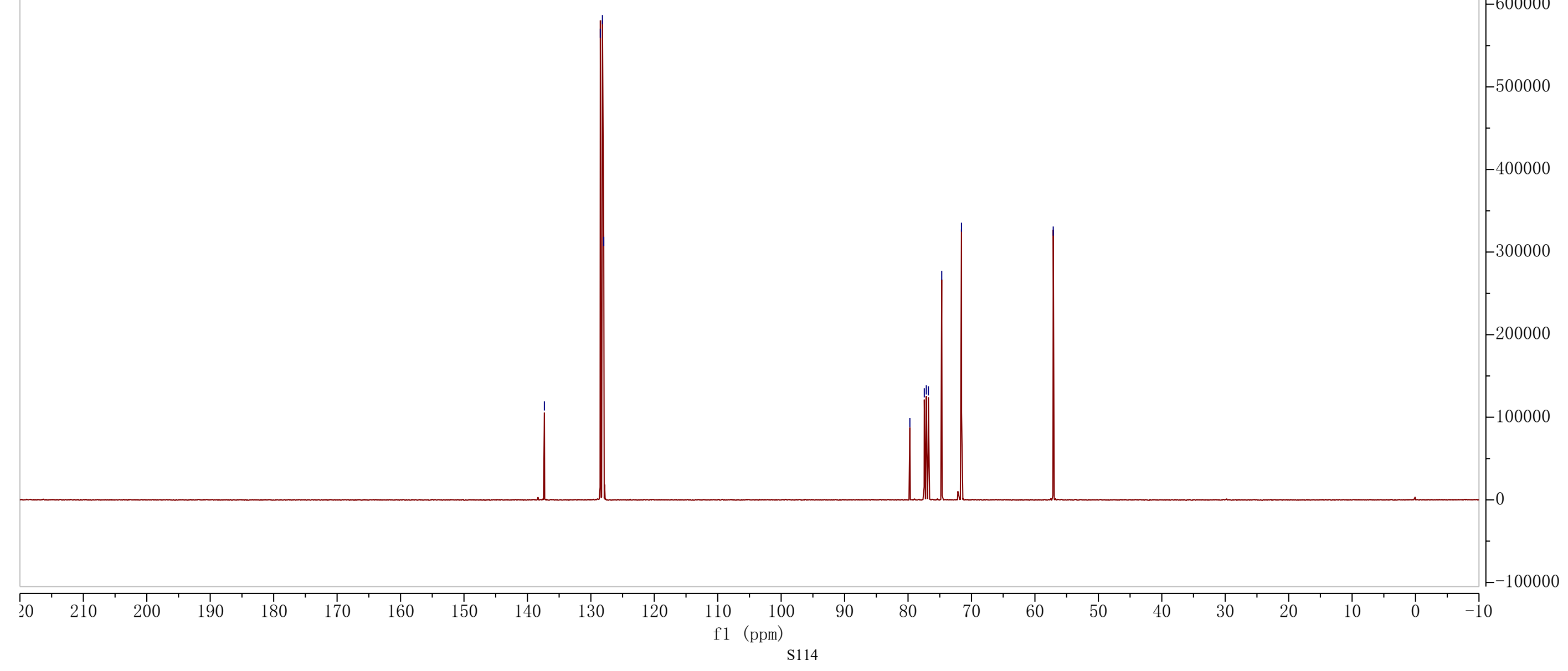




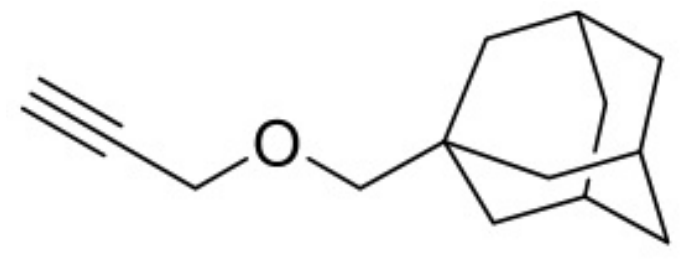

S-A-41

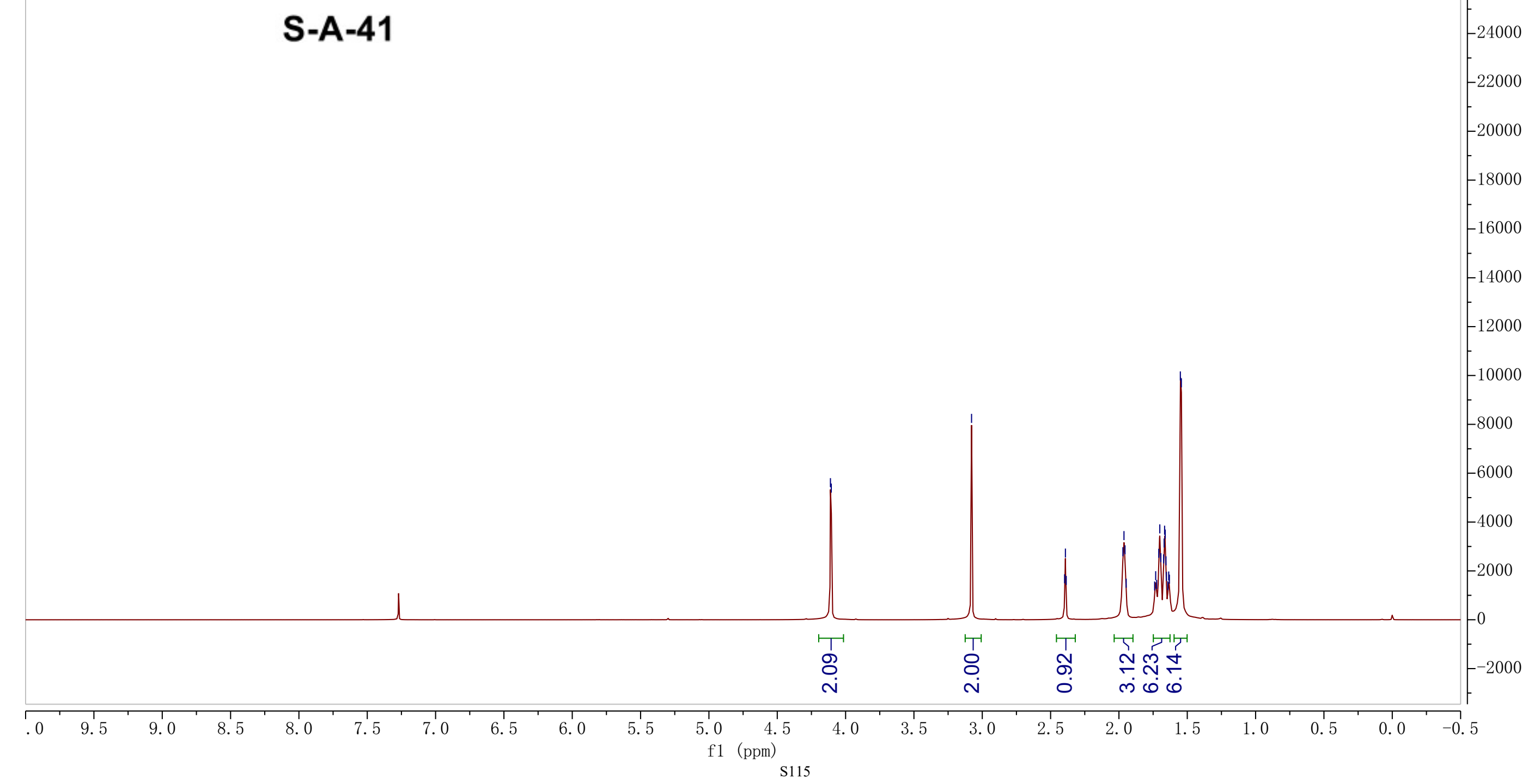




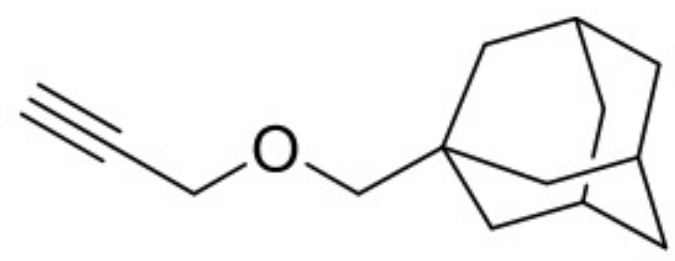

\section{S-A-41}

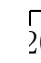




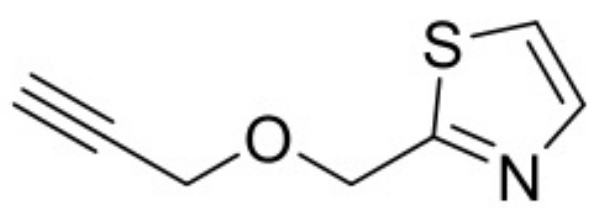

\section{S-A-42}

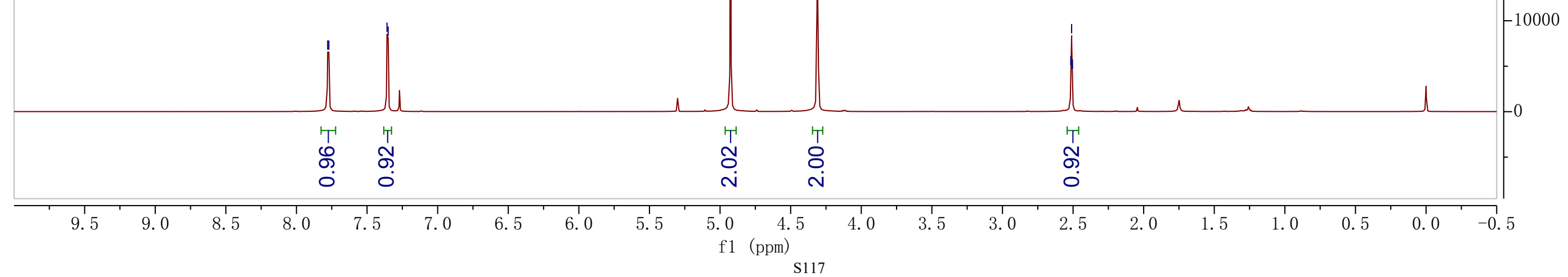


<smiles>C#CCOCc1nccs1</smiles>

S-A-42

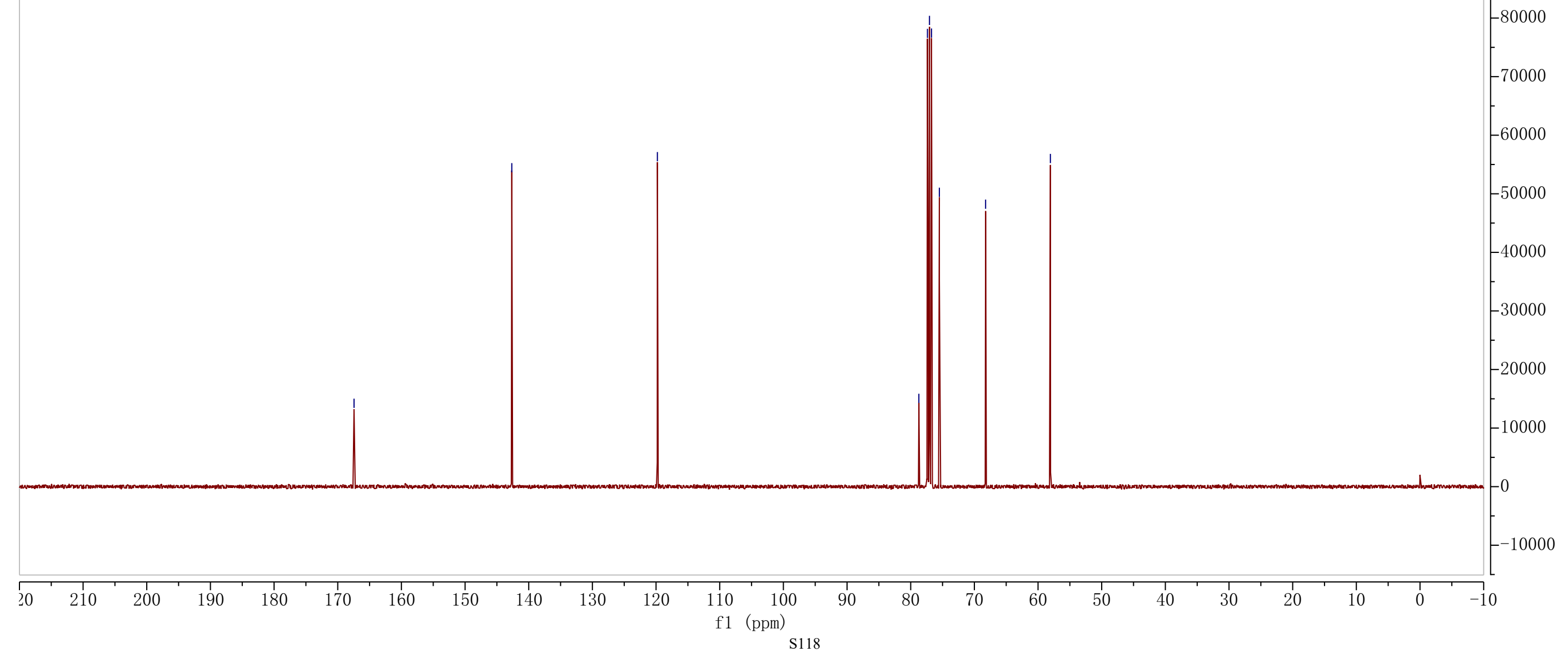




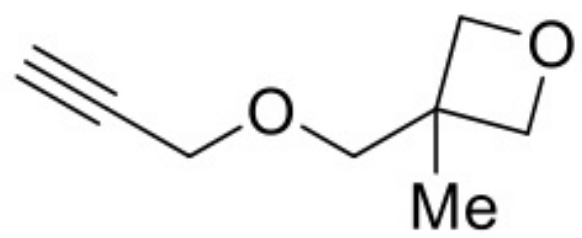

S-A-43

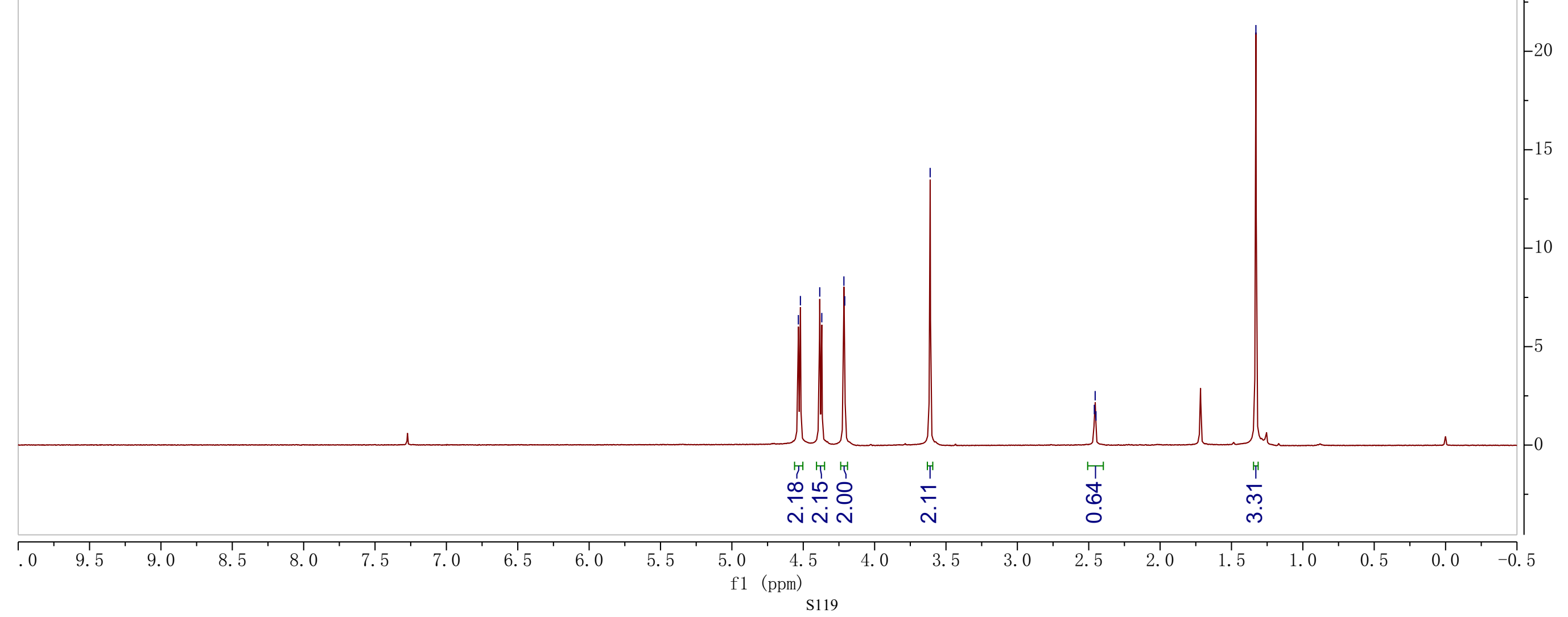




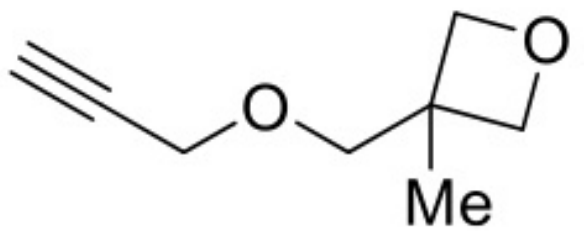

S-A-43 


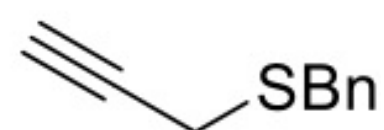

\section{S-A-44}

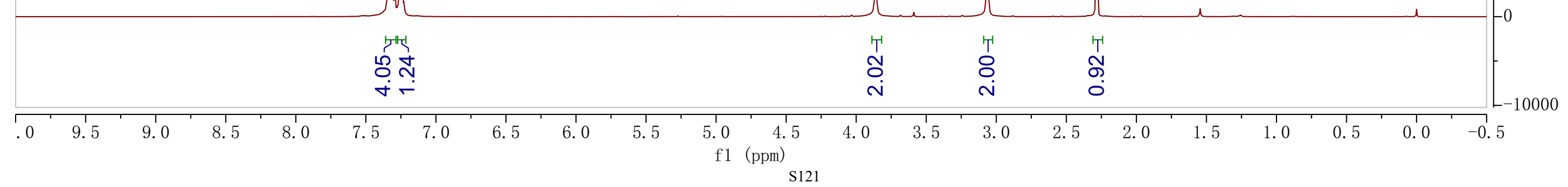




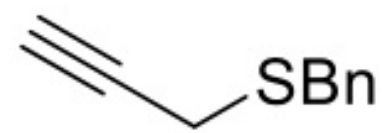

$\mathrm{SBn}$

\section{S-A-44}




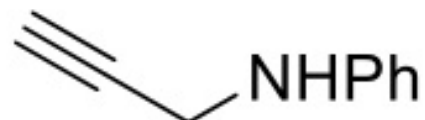

S-A-45

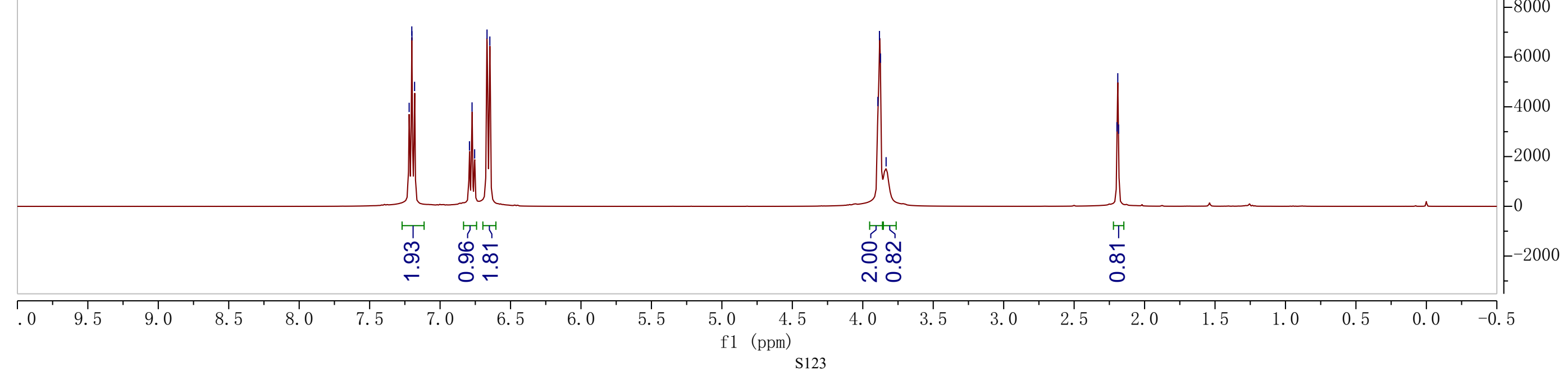


$\mathrm{NHPh}$

S-A-45

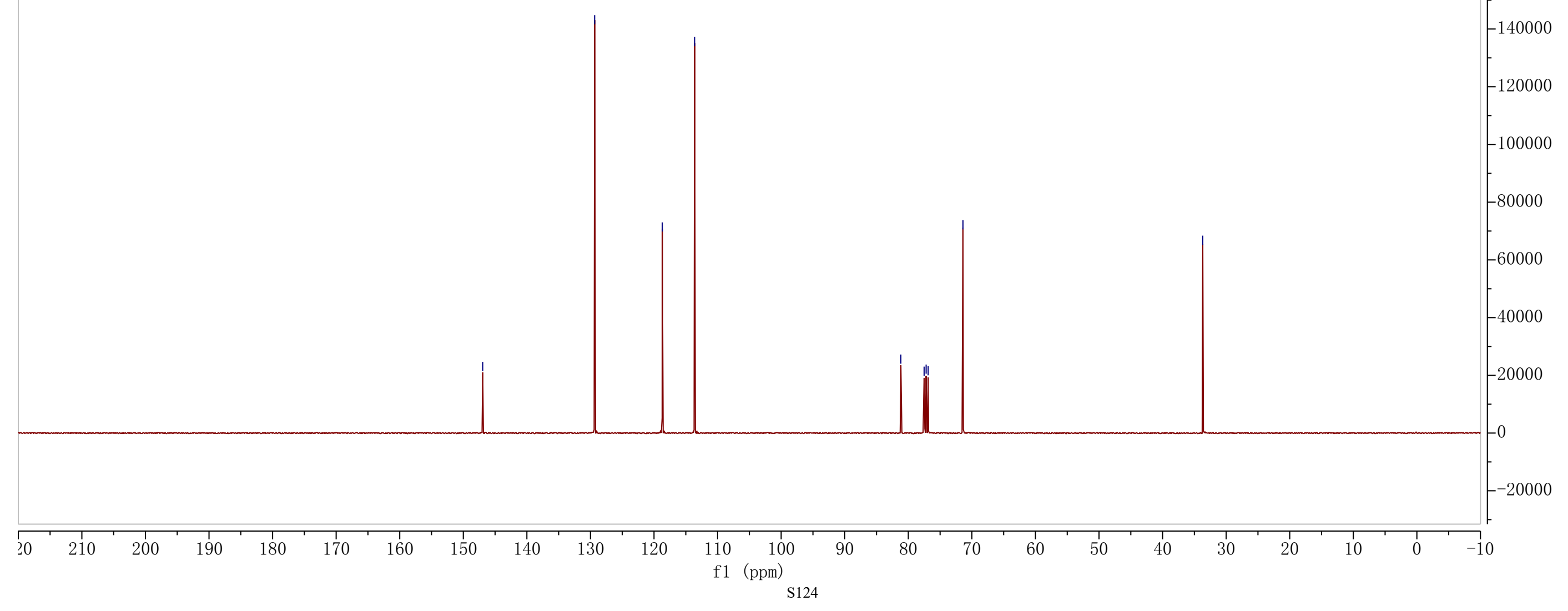




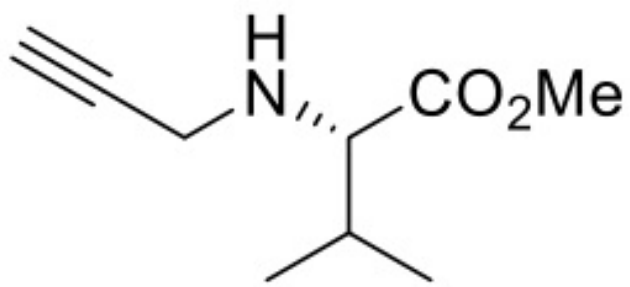

S-A-46

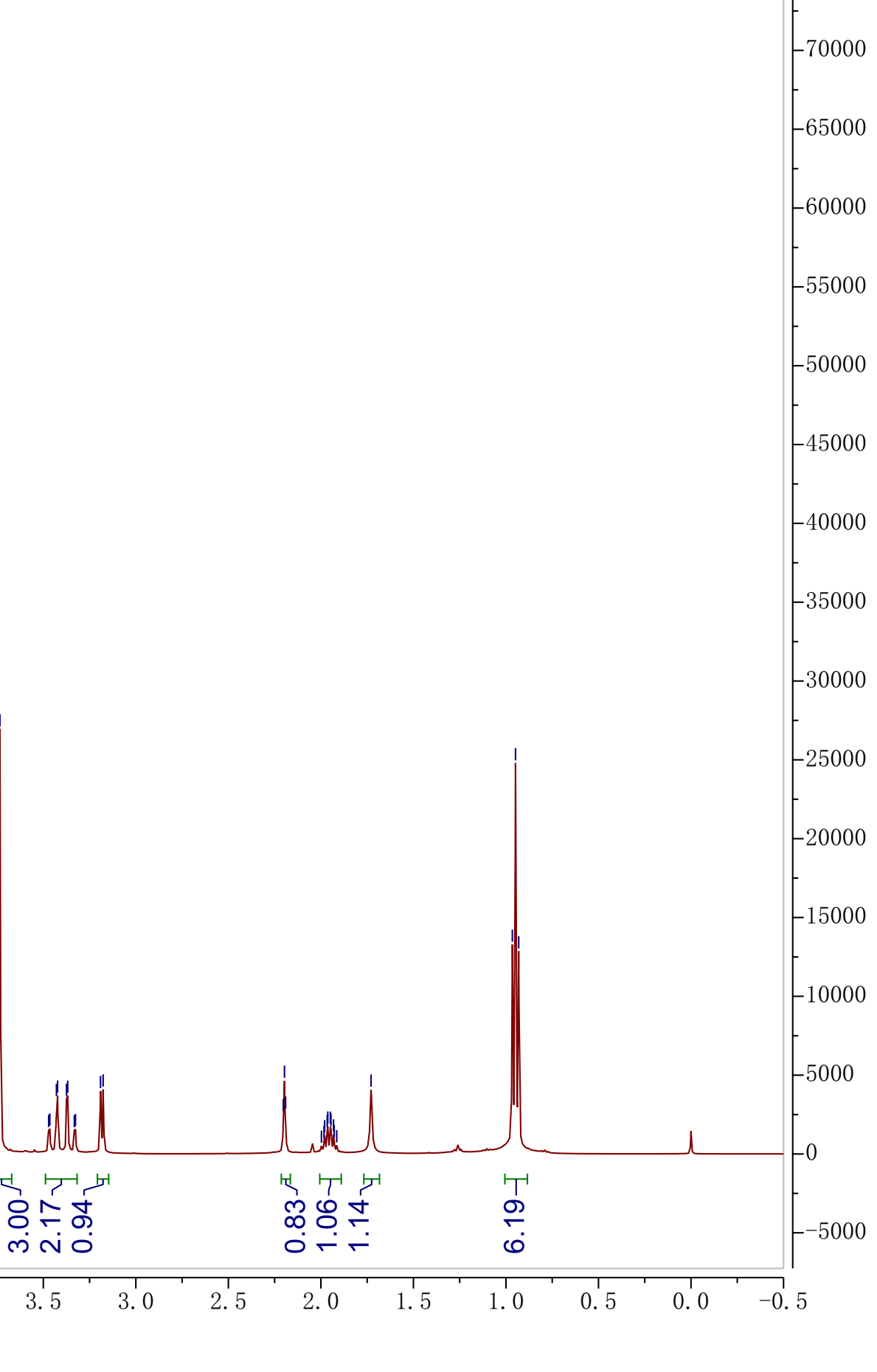




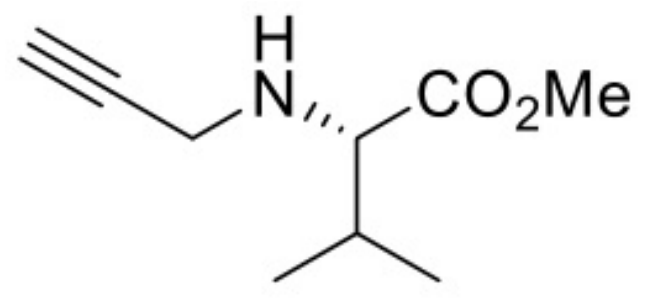

S-A-46

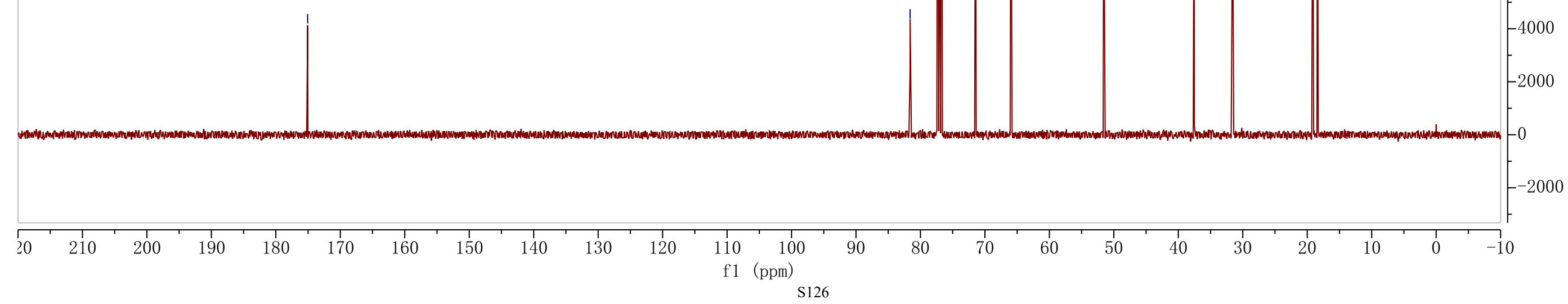




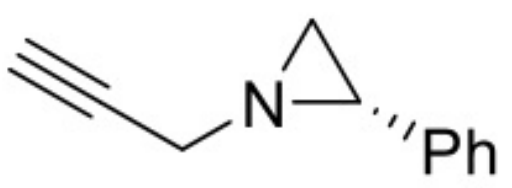

\section{S-A-47}

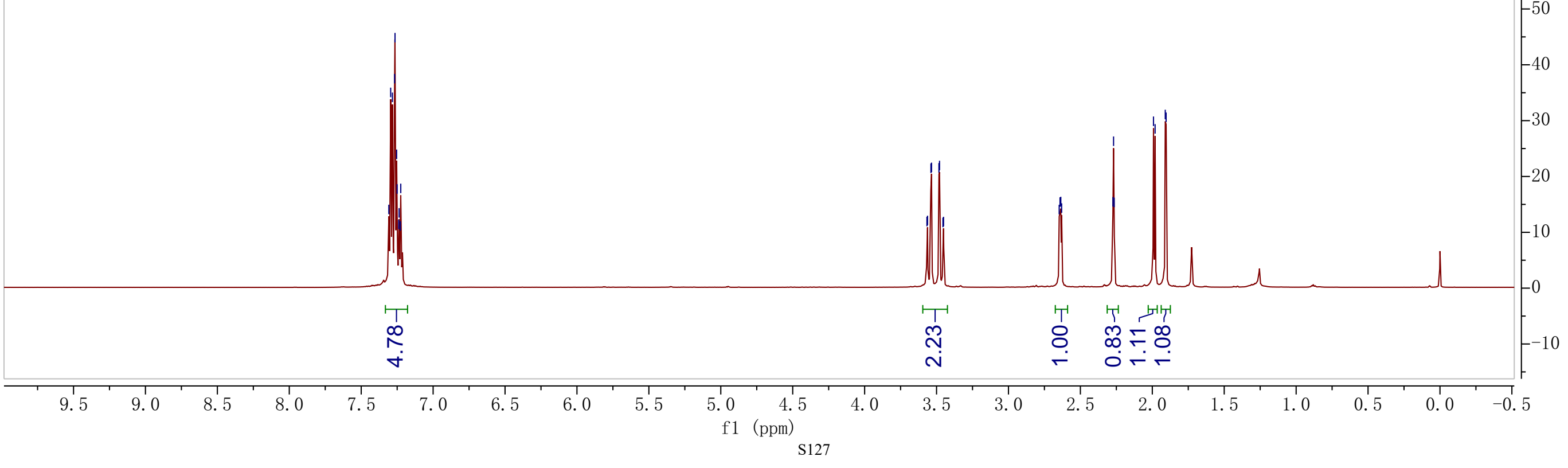




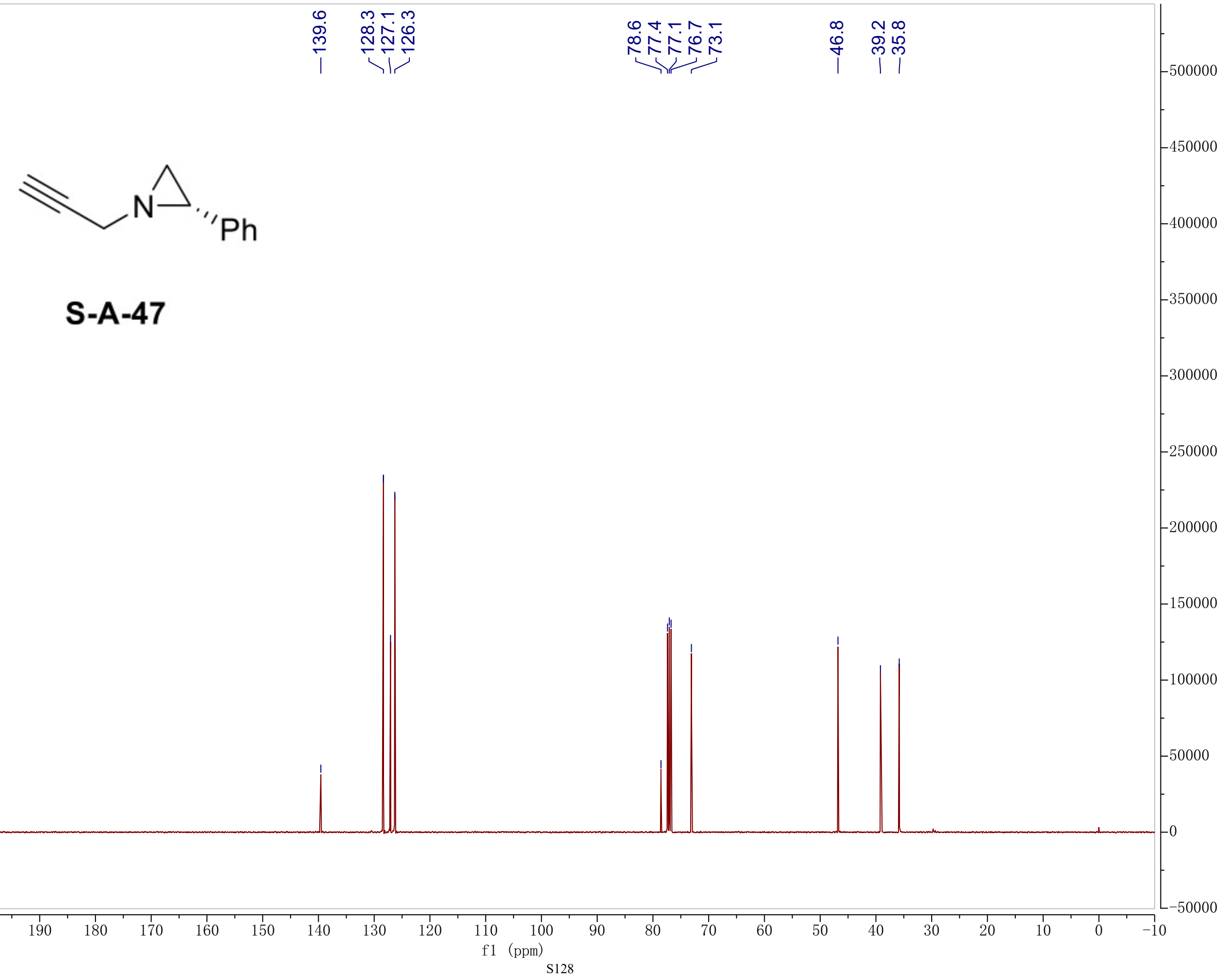




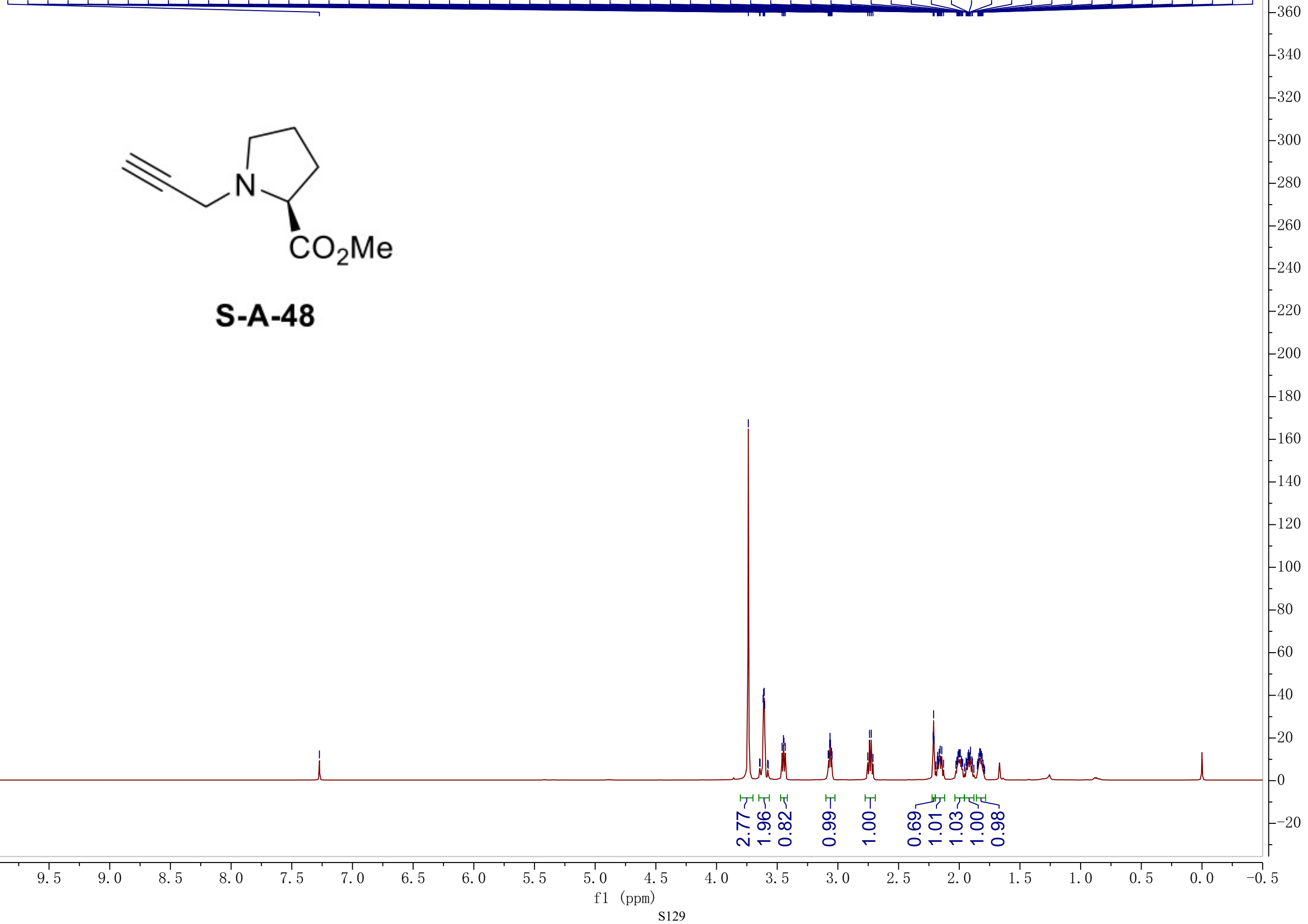




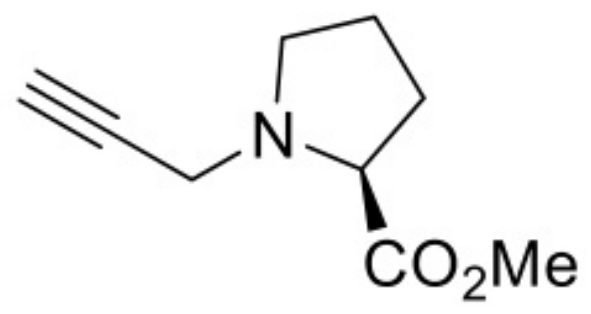

S-A-48

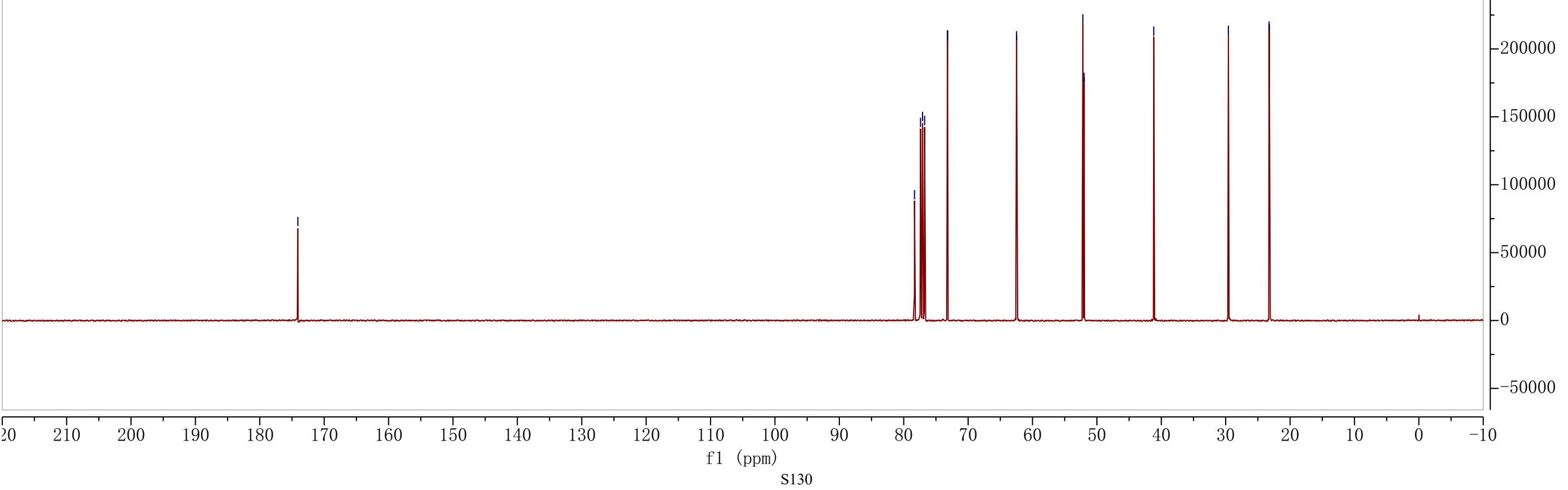




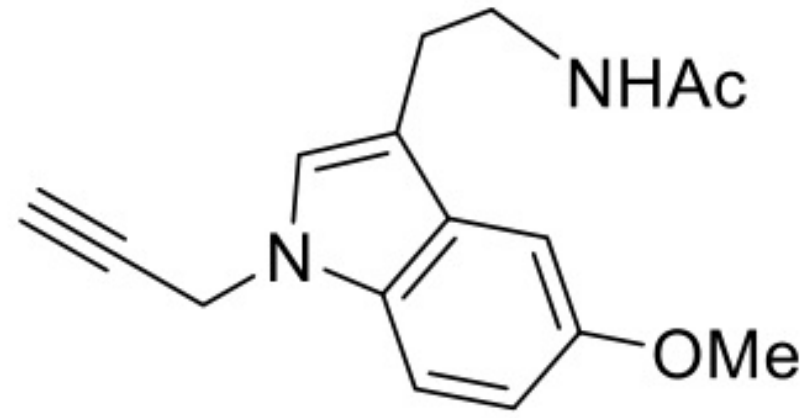

\section{S-A-49}

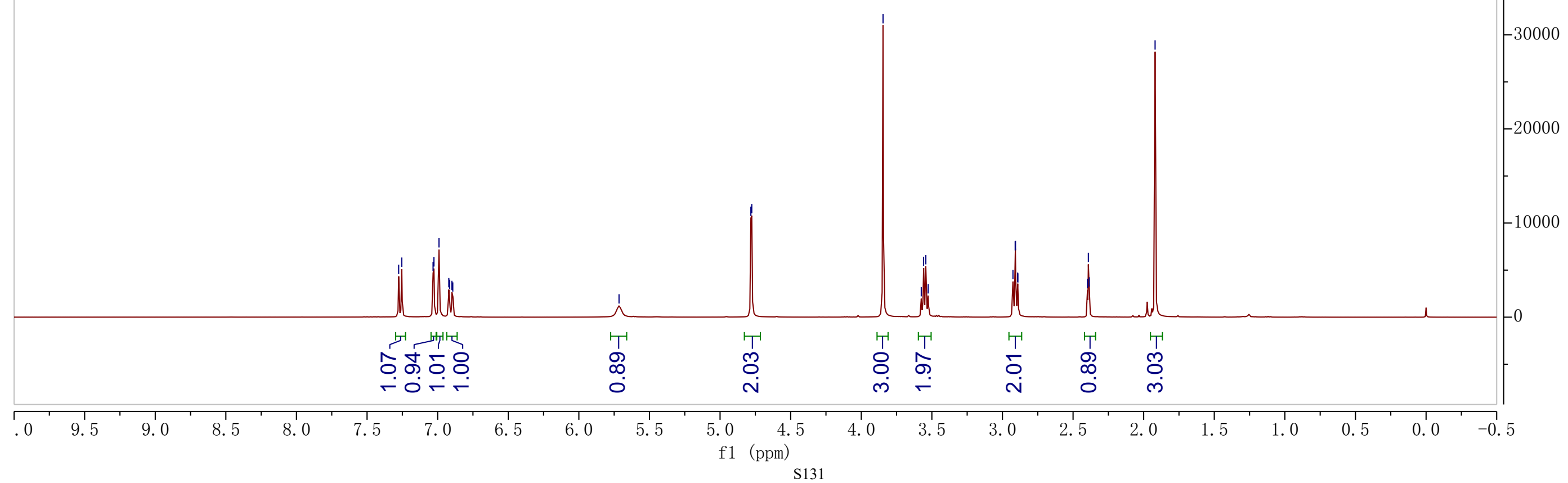




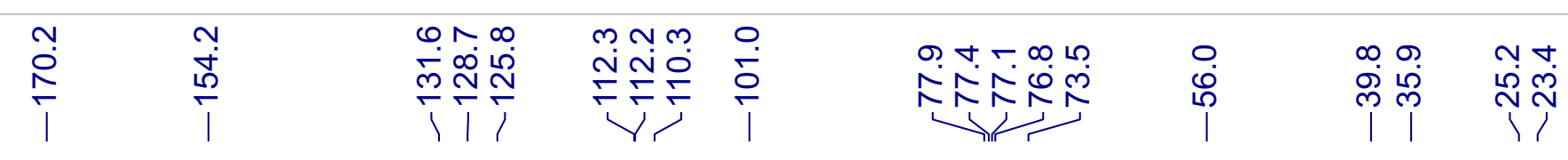

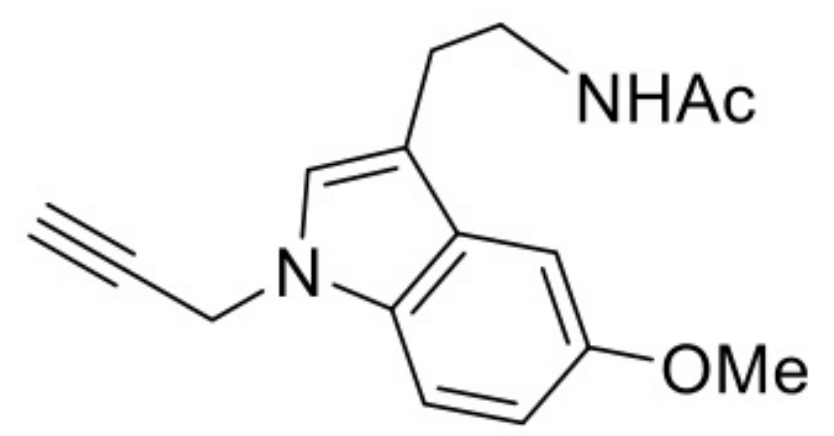

S-A-49 


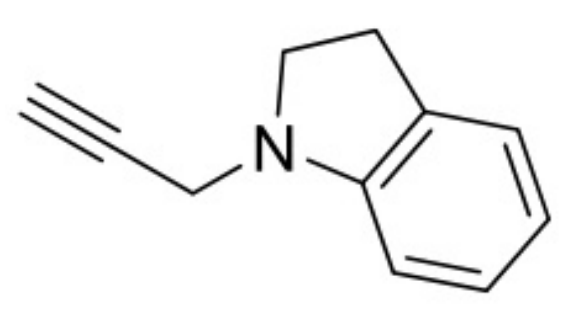

S-A-50

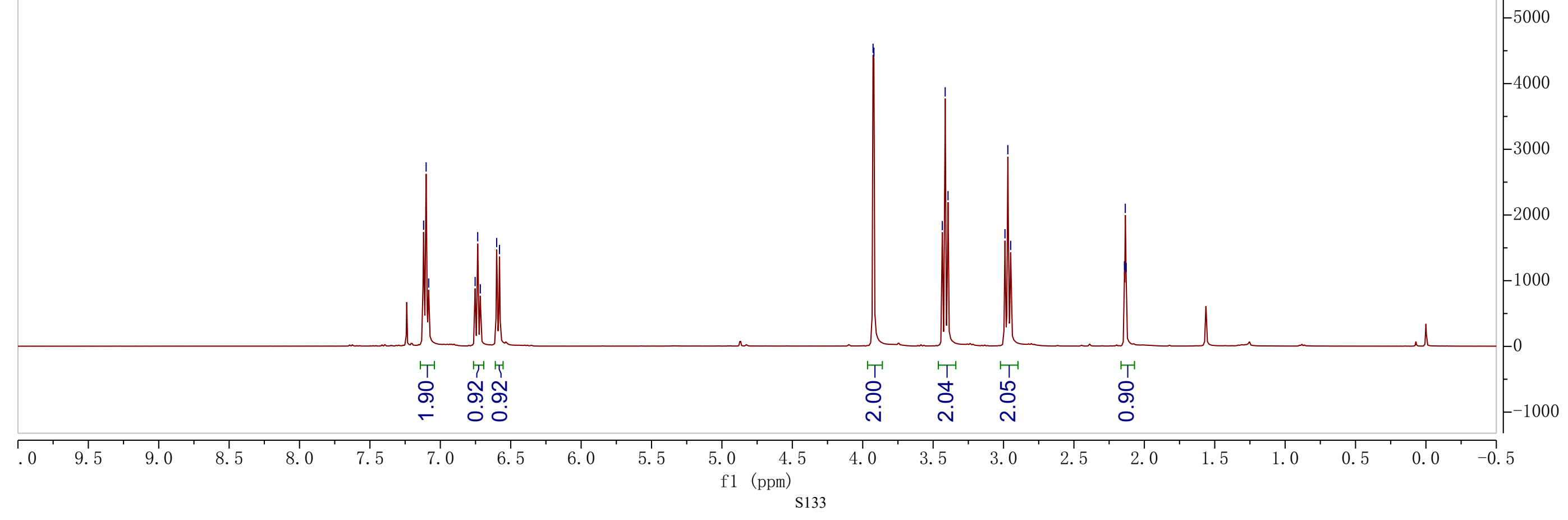




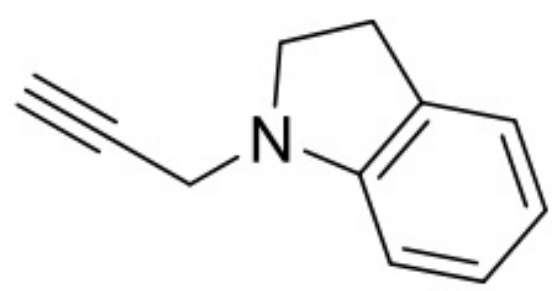

S-A-50 


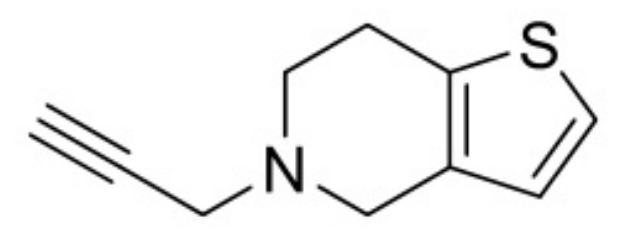

S-A-51

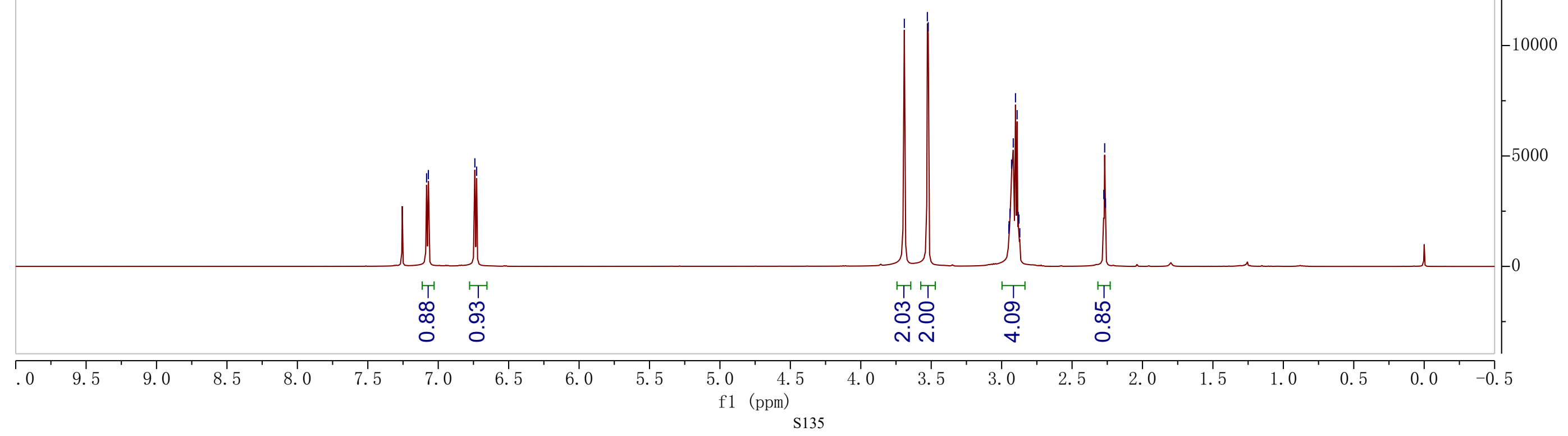


<smiles>C#CCN1CCc2sccc2C1</smiles>

S-A-51

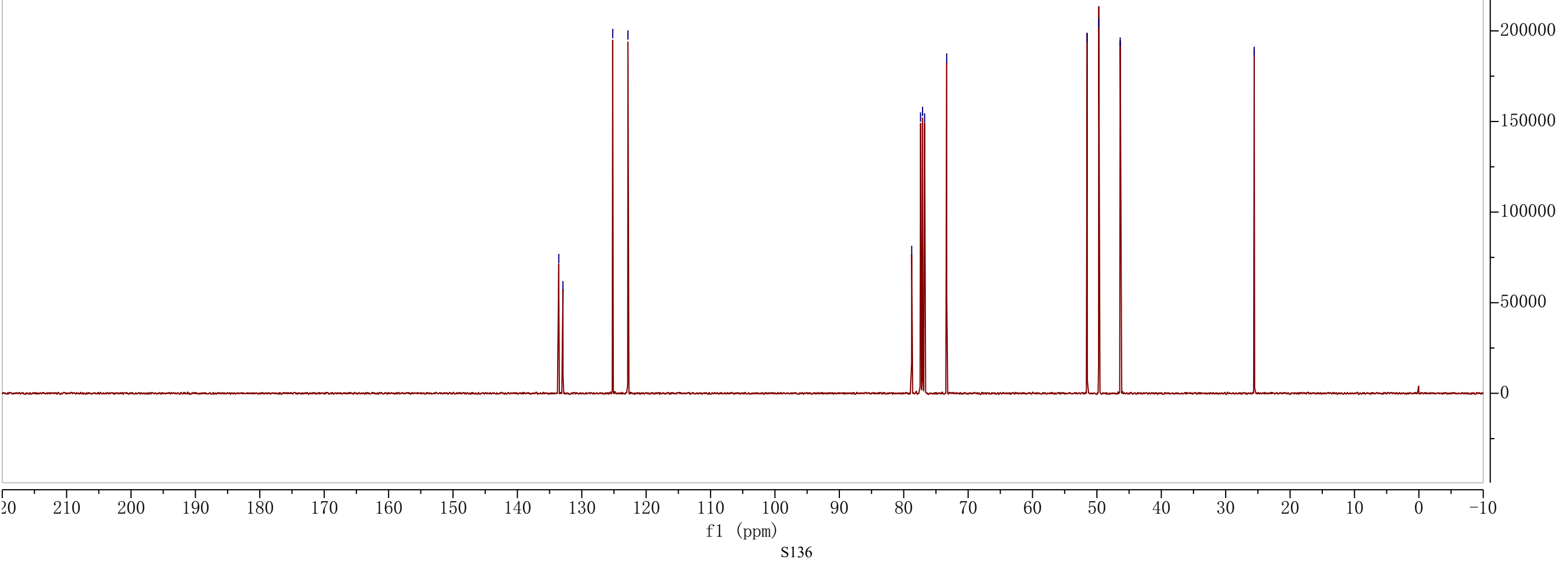




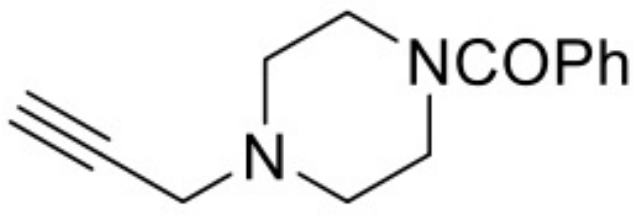

S-A-52

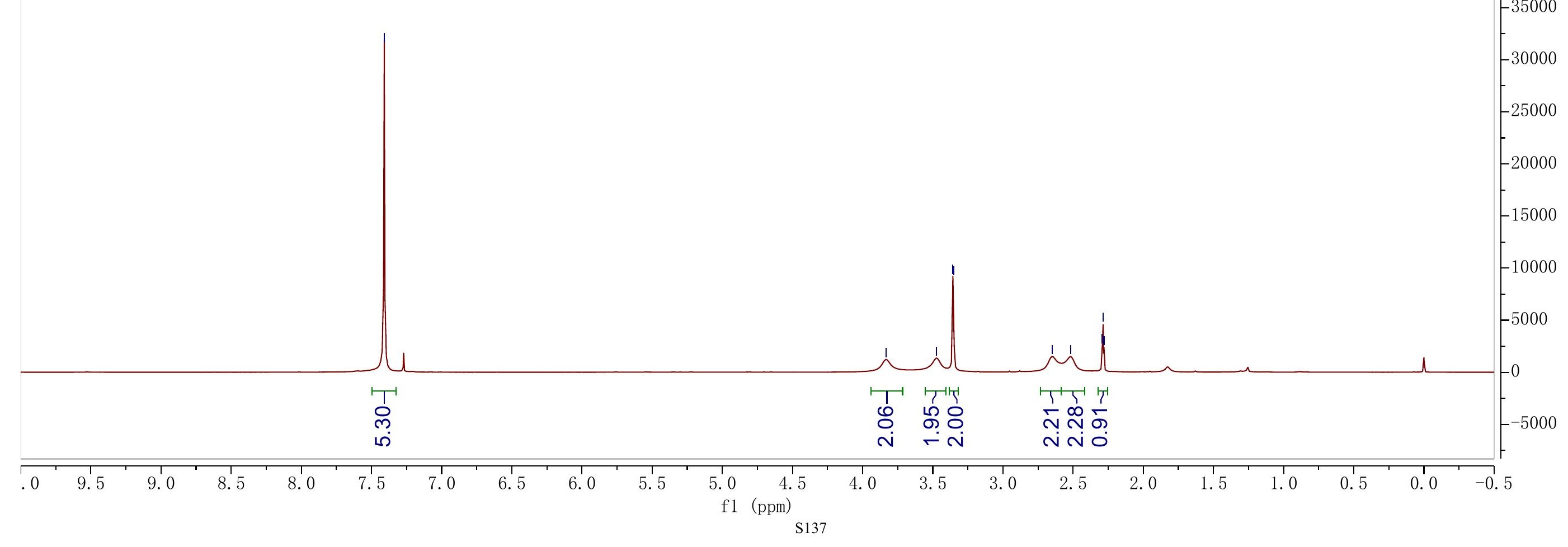


S-A-52 


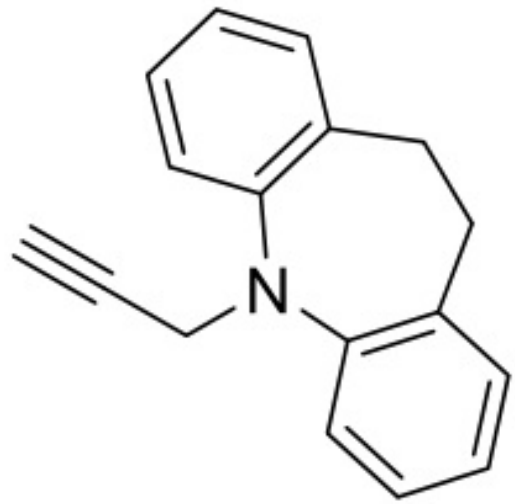

S-A-53

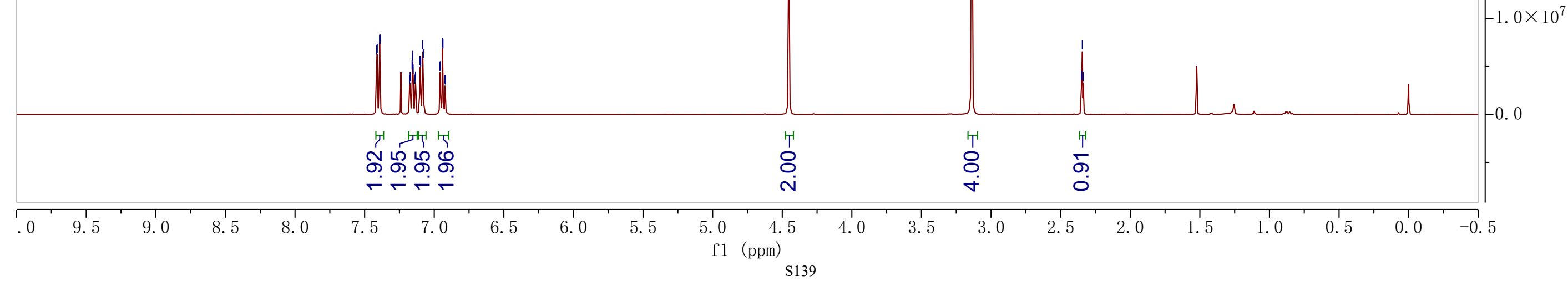



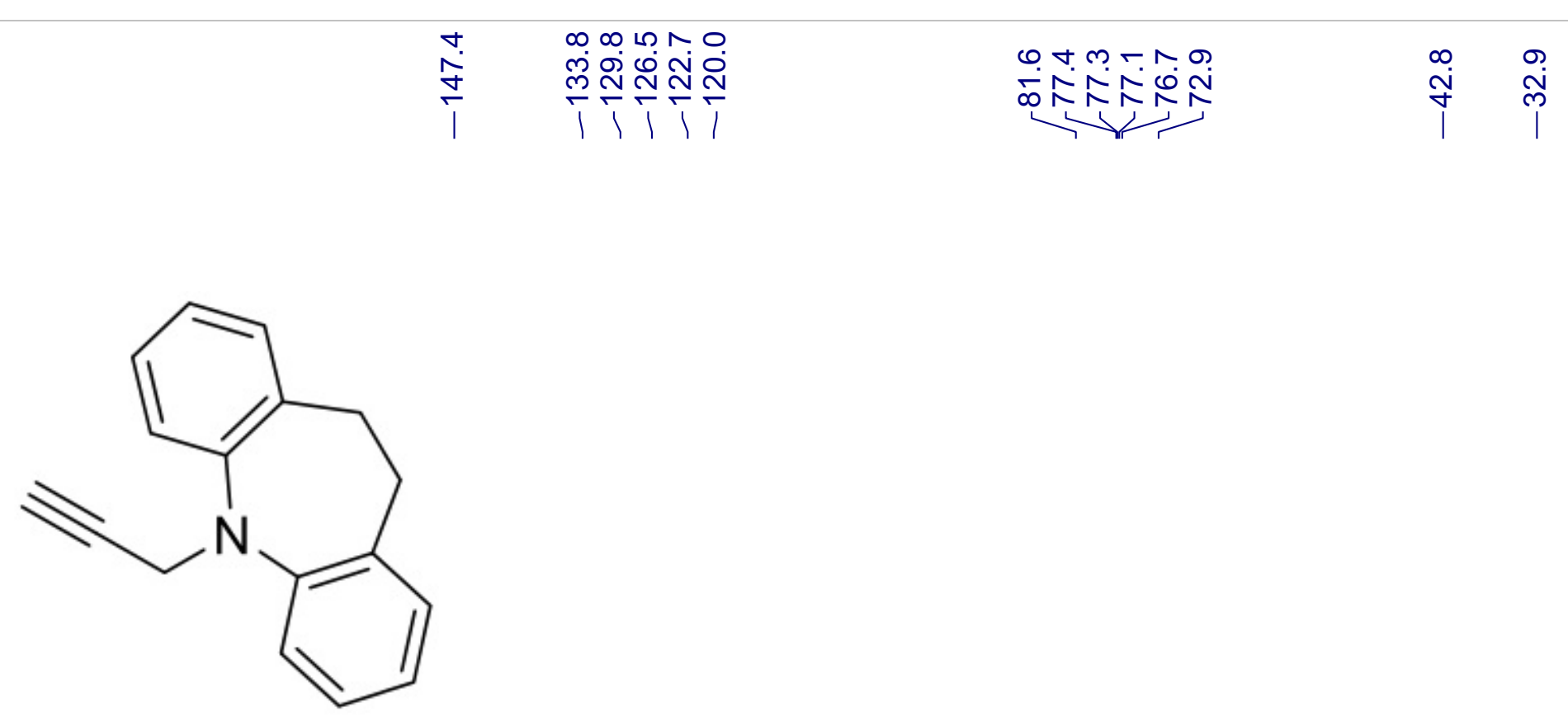

S-A-53

।

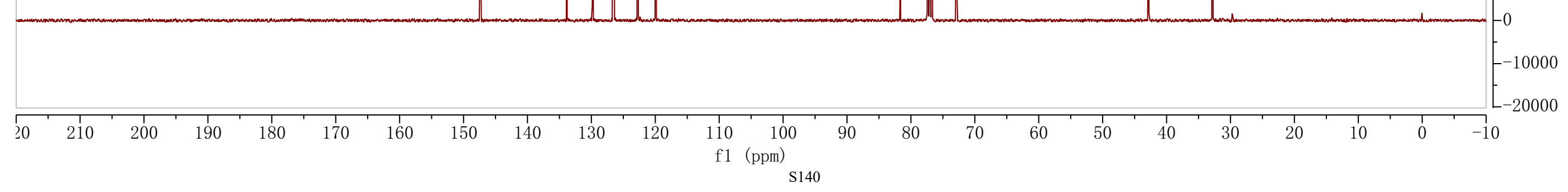


NMeBoc

S-A-54 


\section{S-A-54}

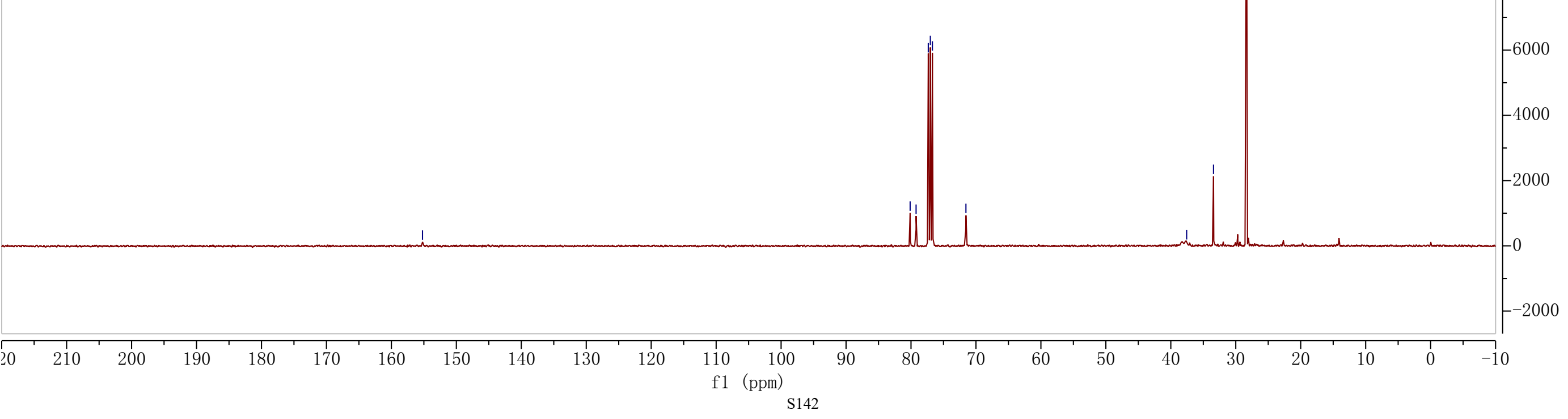




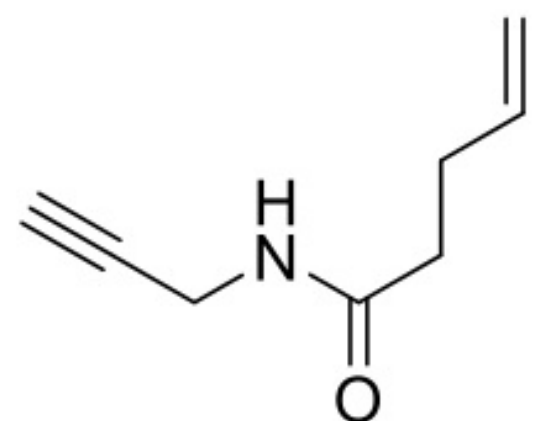

S-A-55

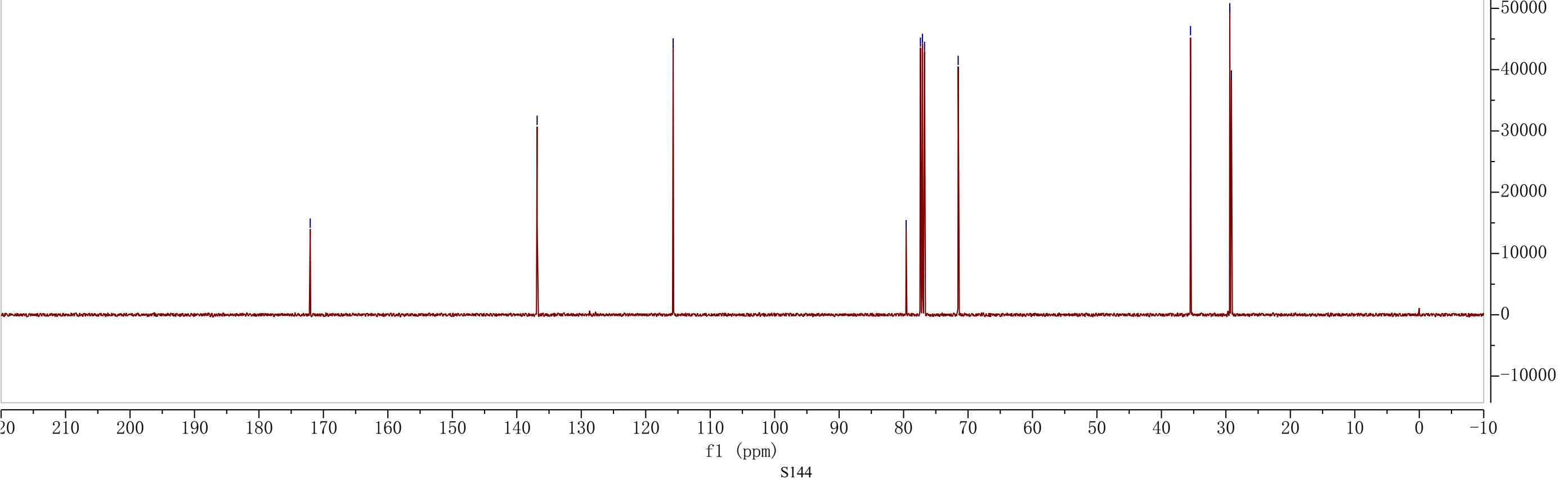




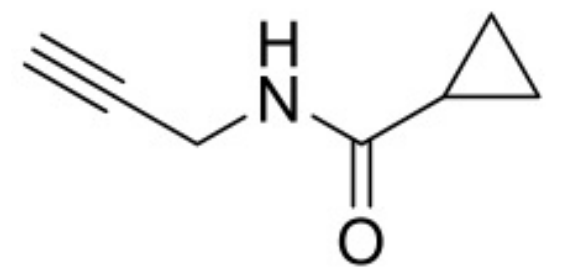

S-A-56

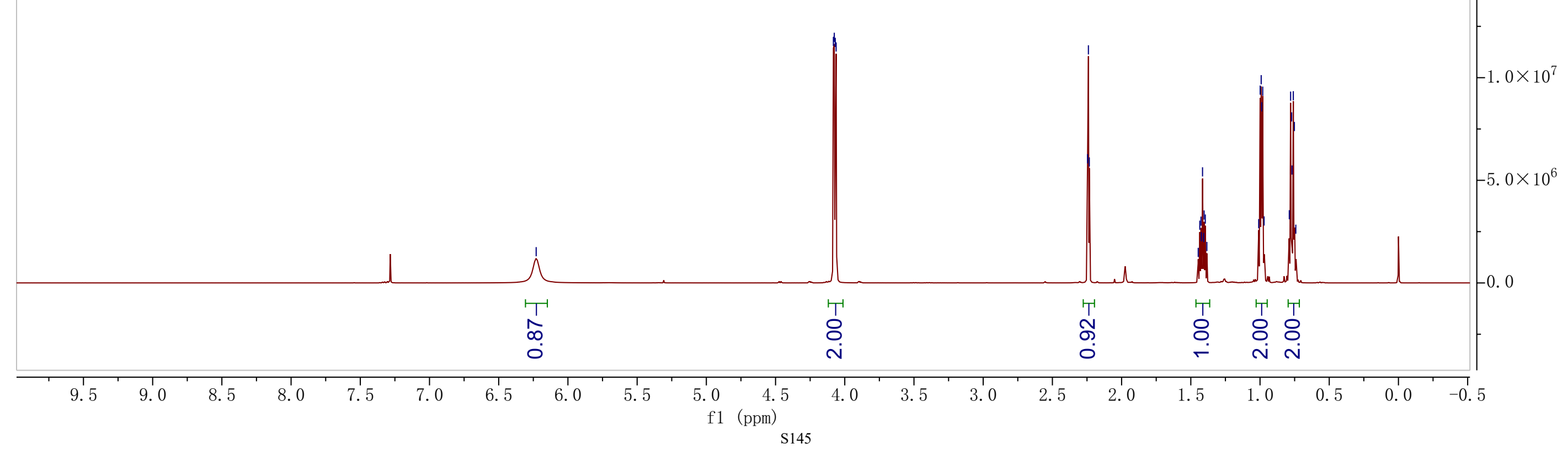




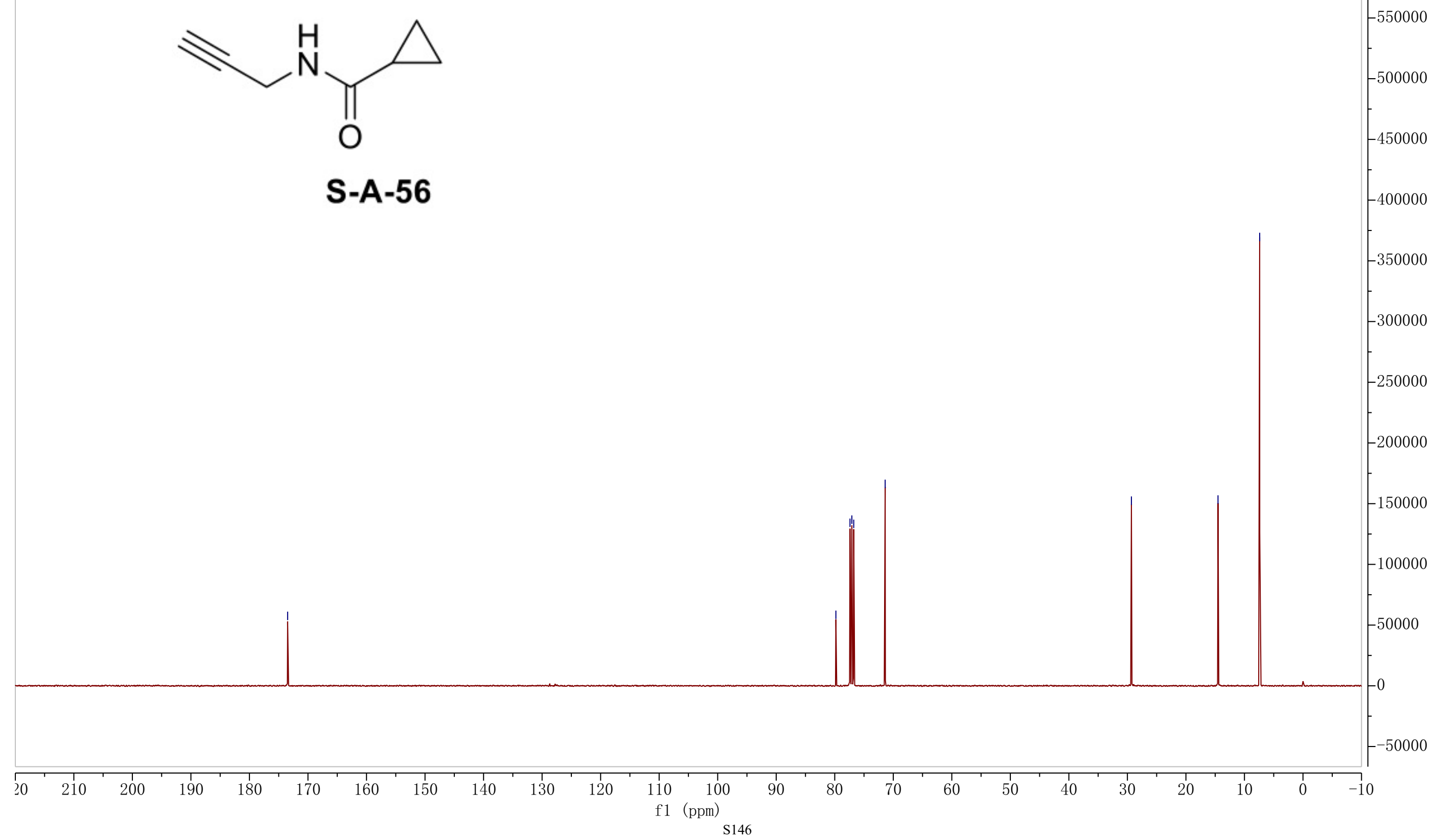

S-A-56 


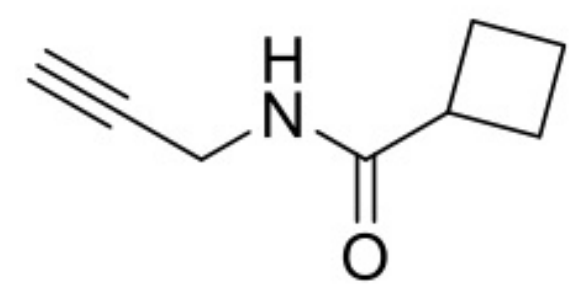

S-A-57

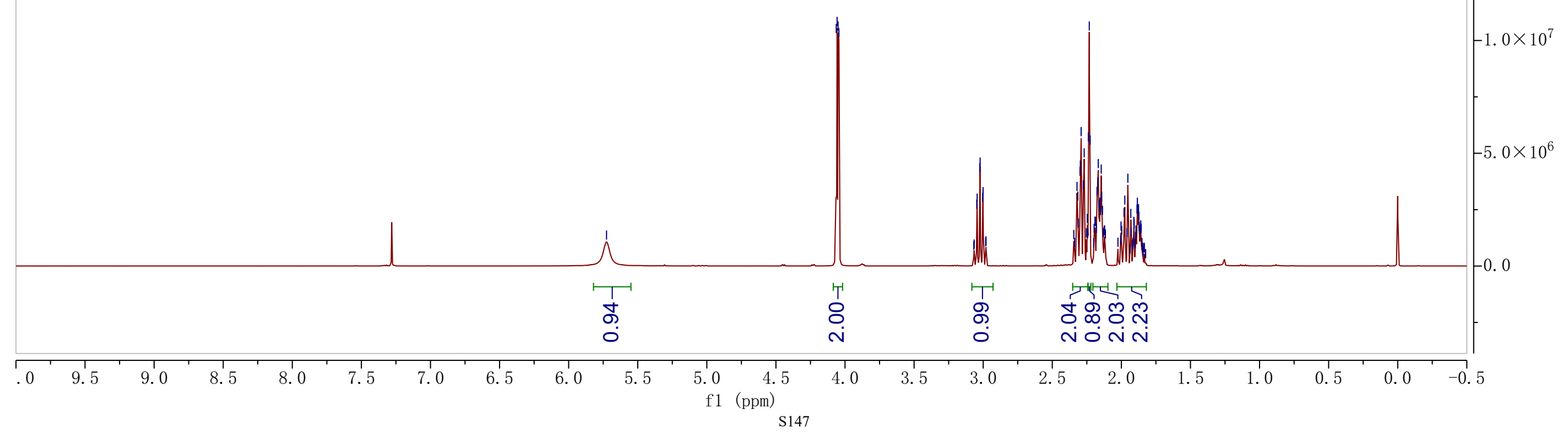




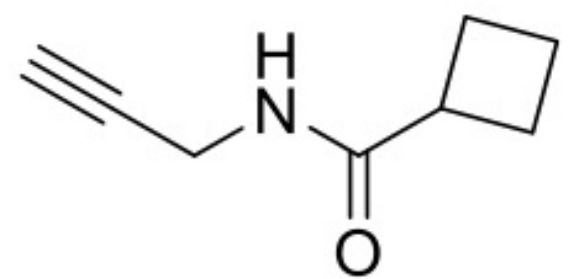

\section{S-A-57}

$210 \quad 200 \quad 190$ 


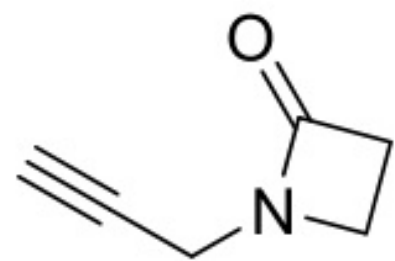

S-A-58

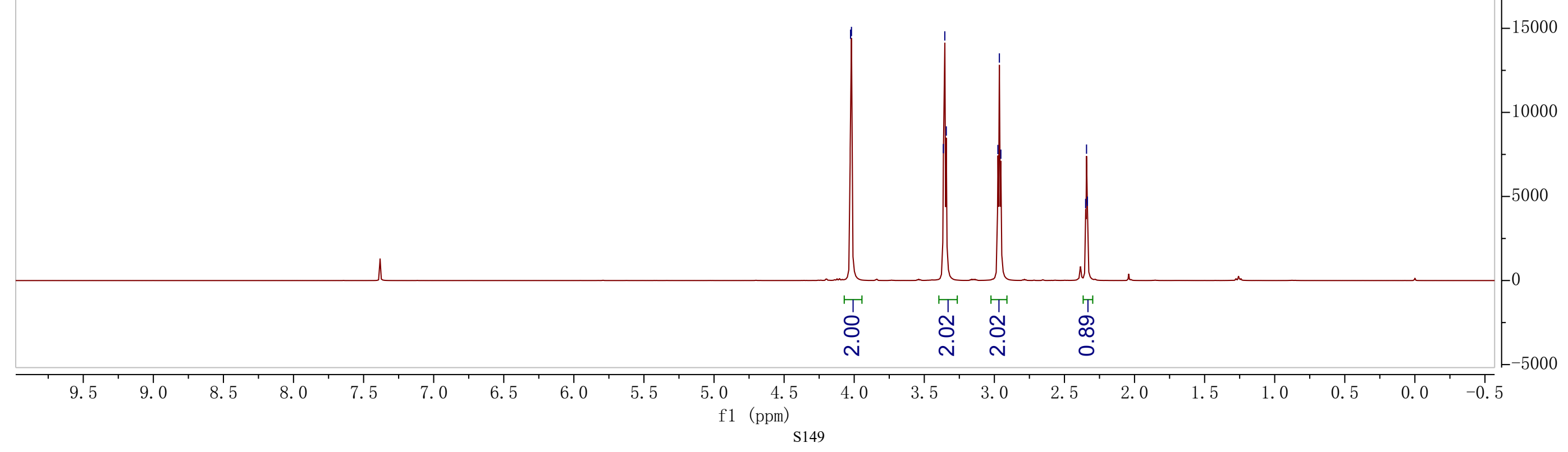




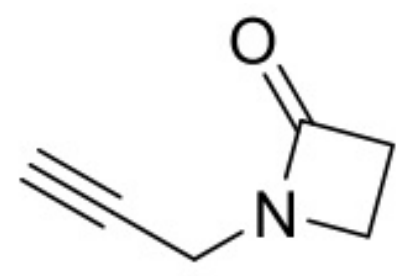

S-A-58 


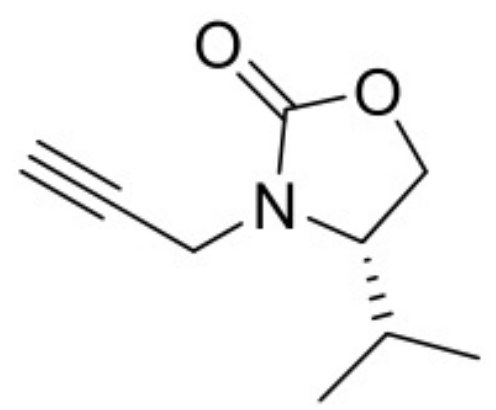

S-A-59

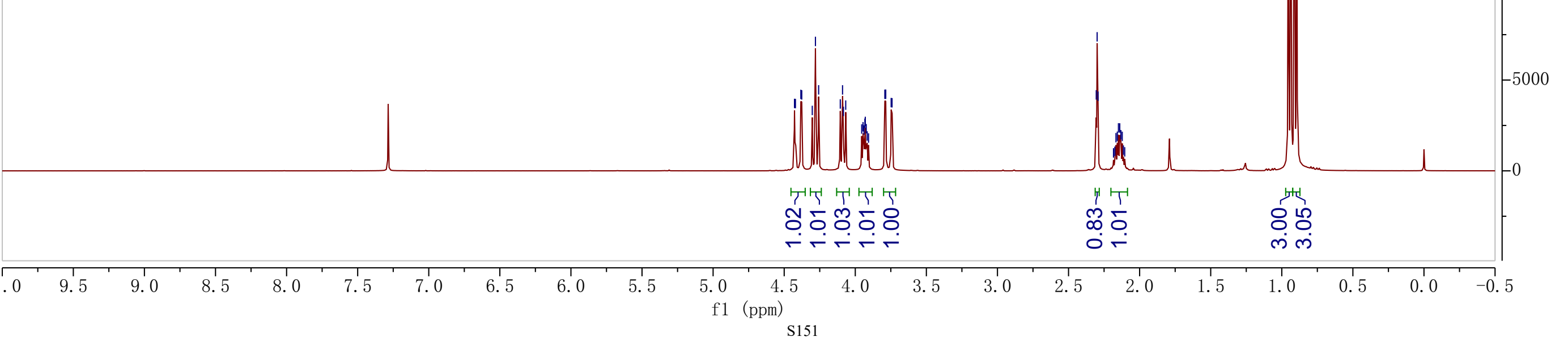




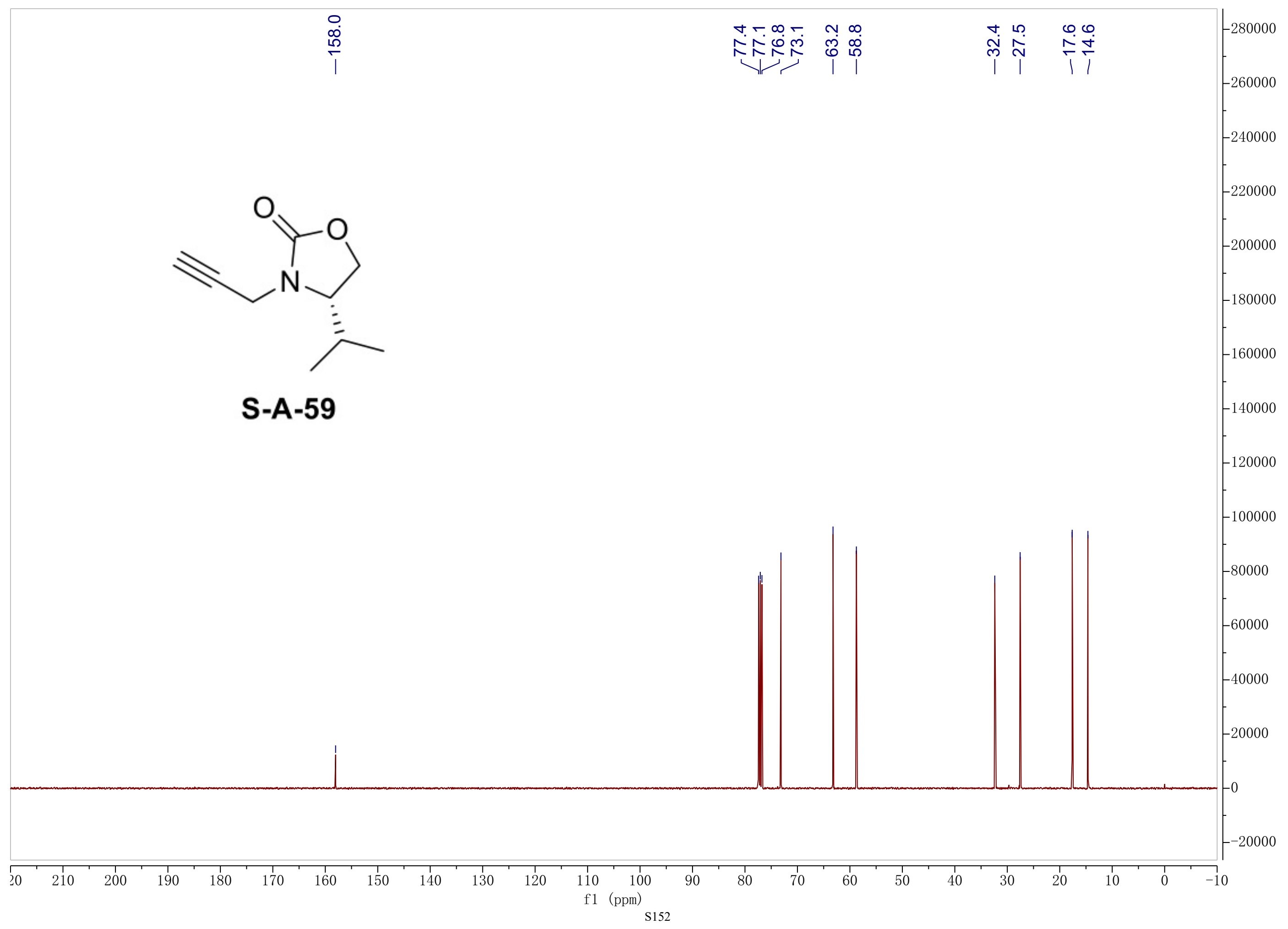




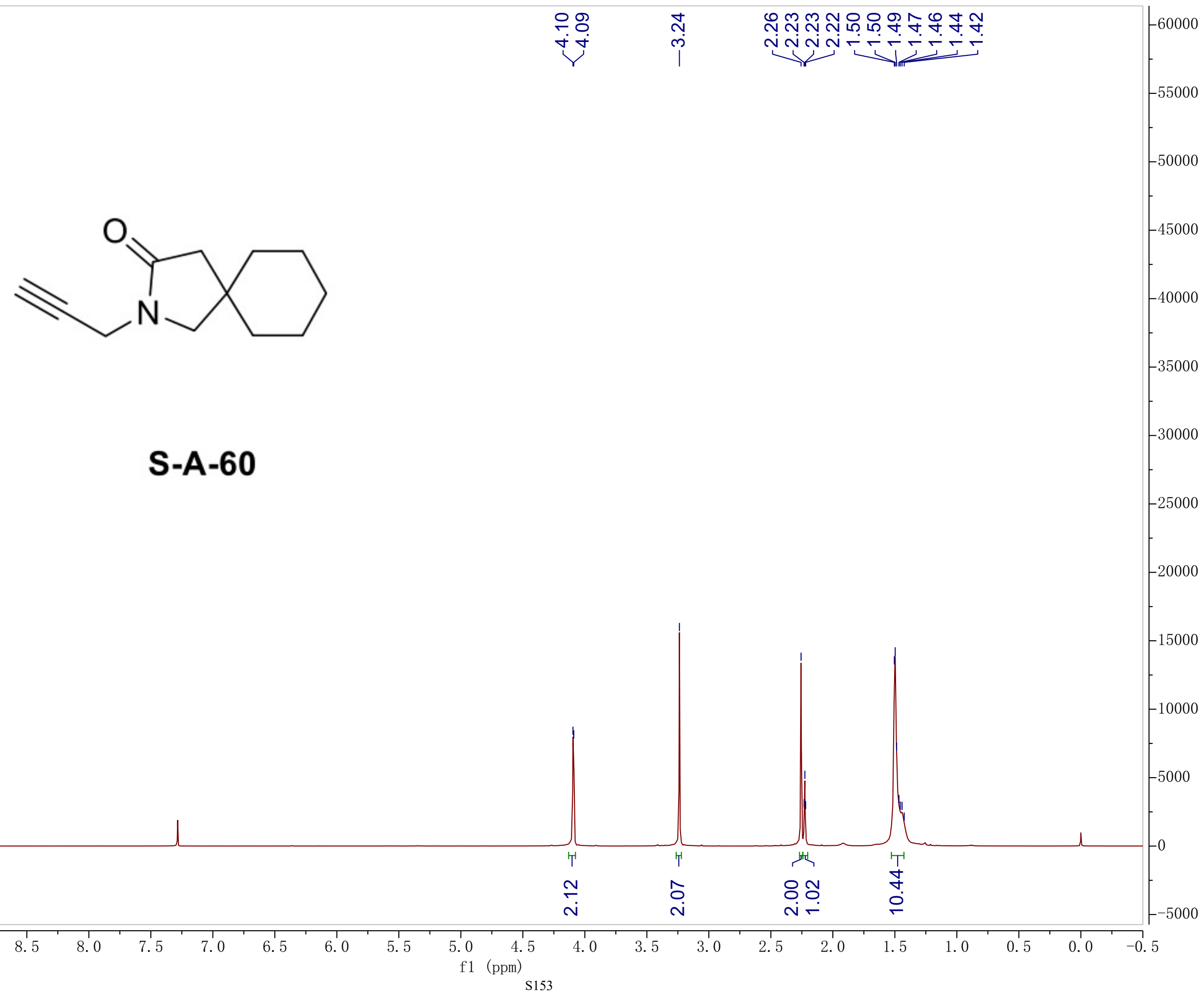




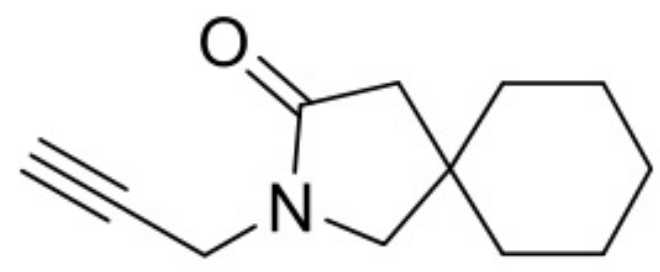

\section{S-A-60}

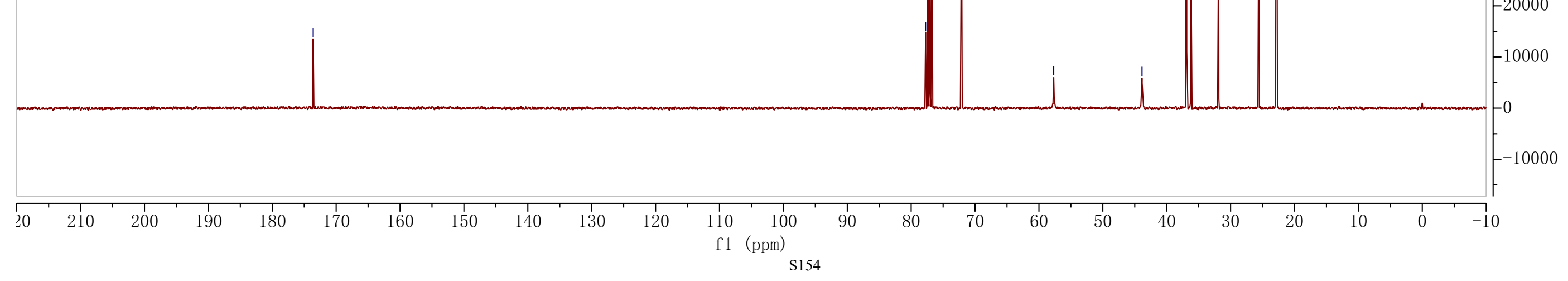




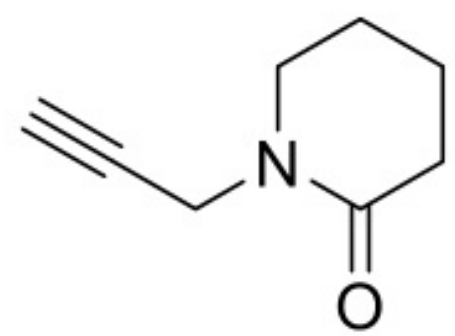

S-A-61
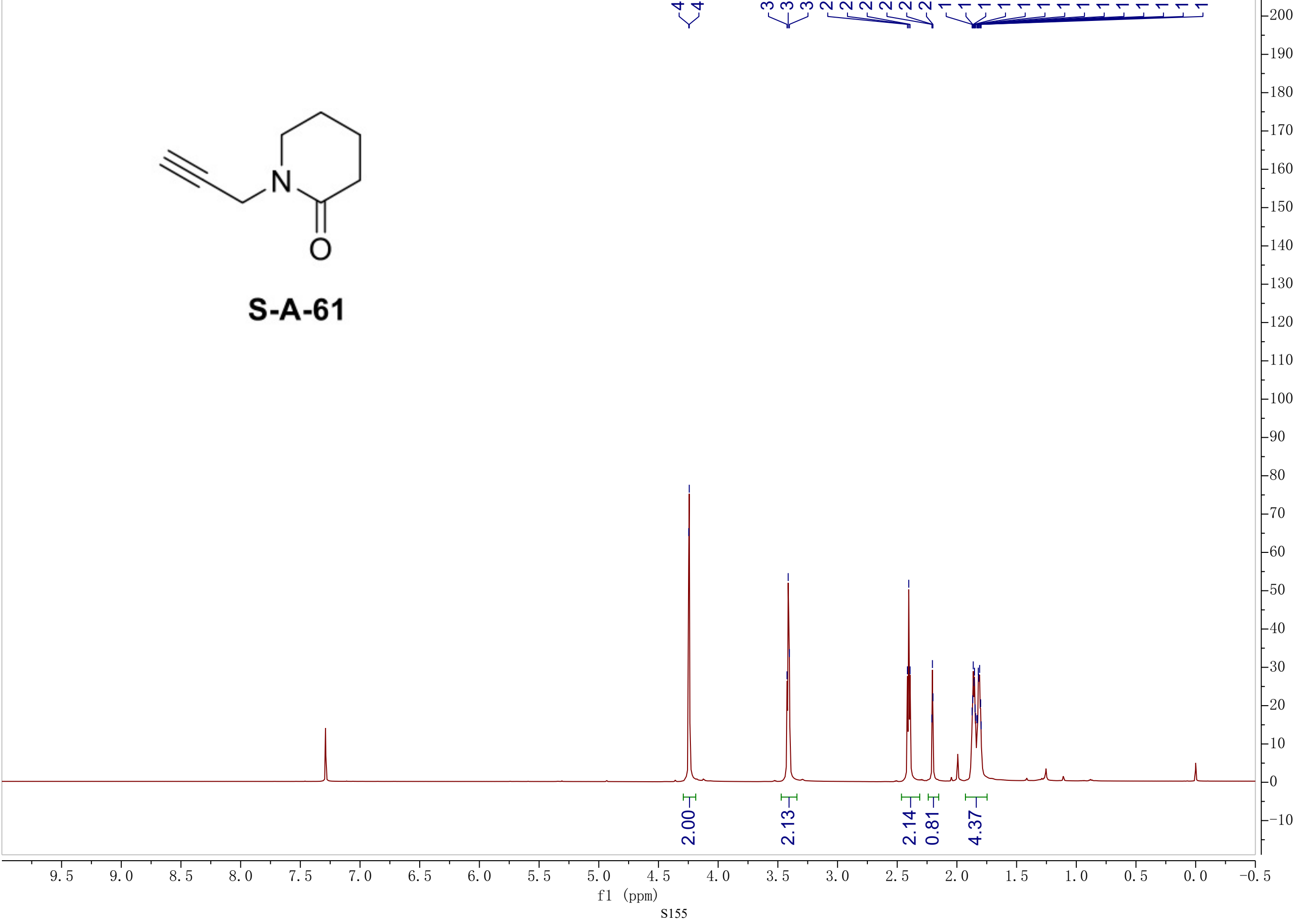


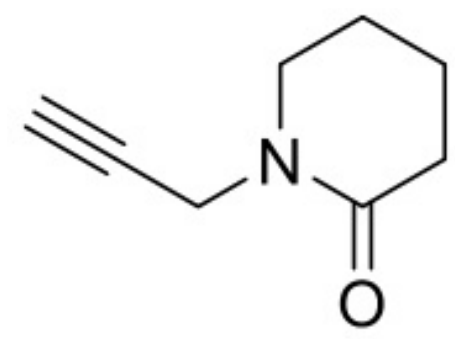

S-A-61

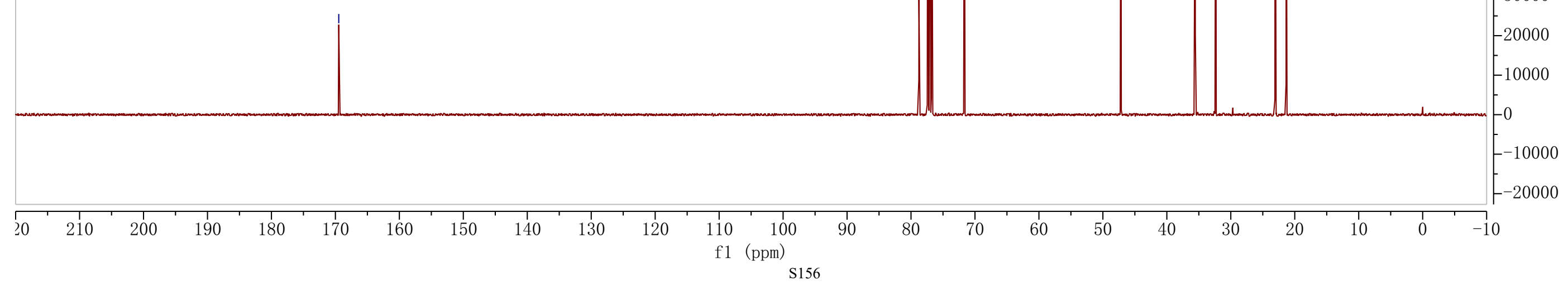




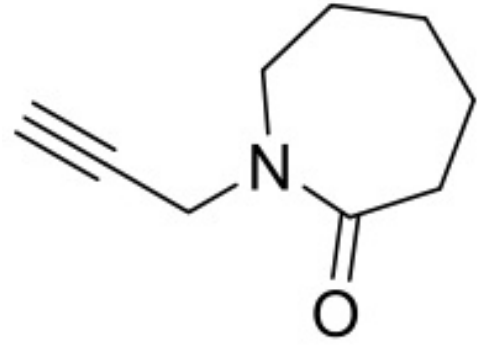

S-A-62

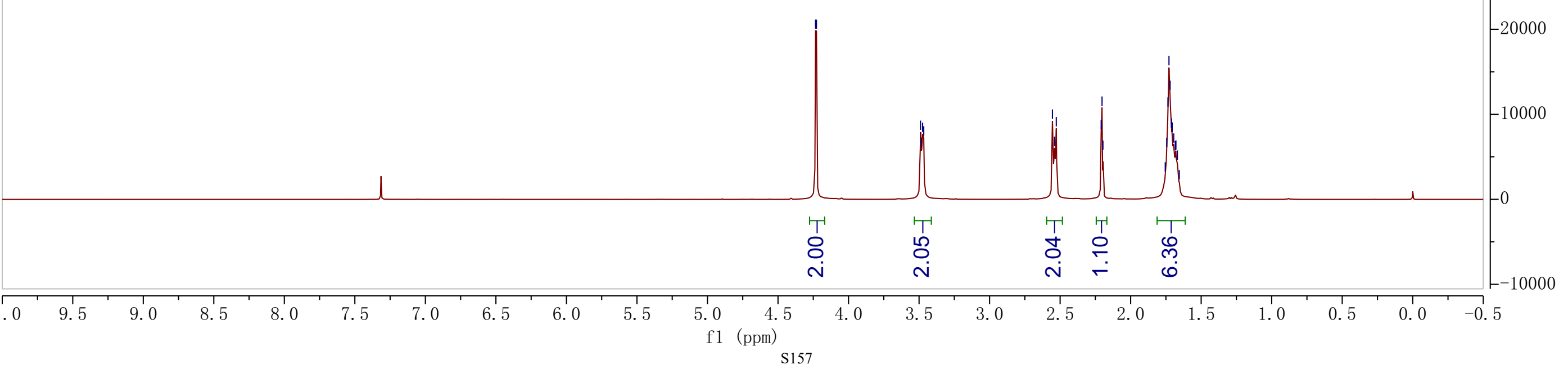




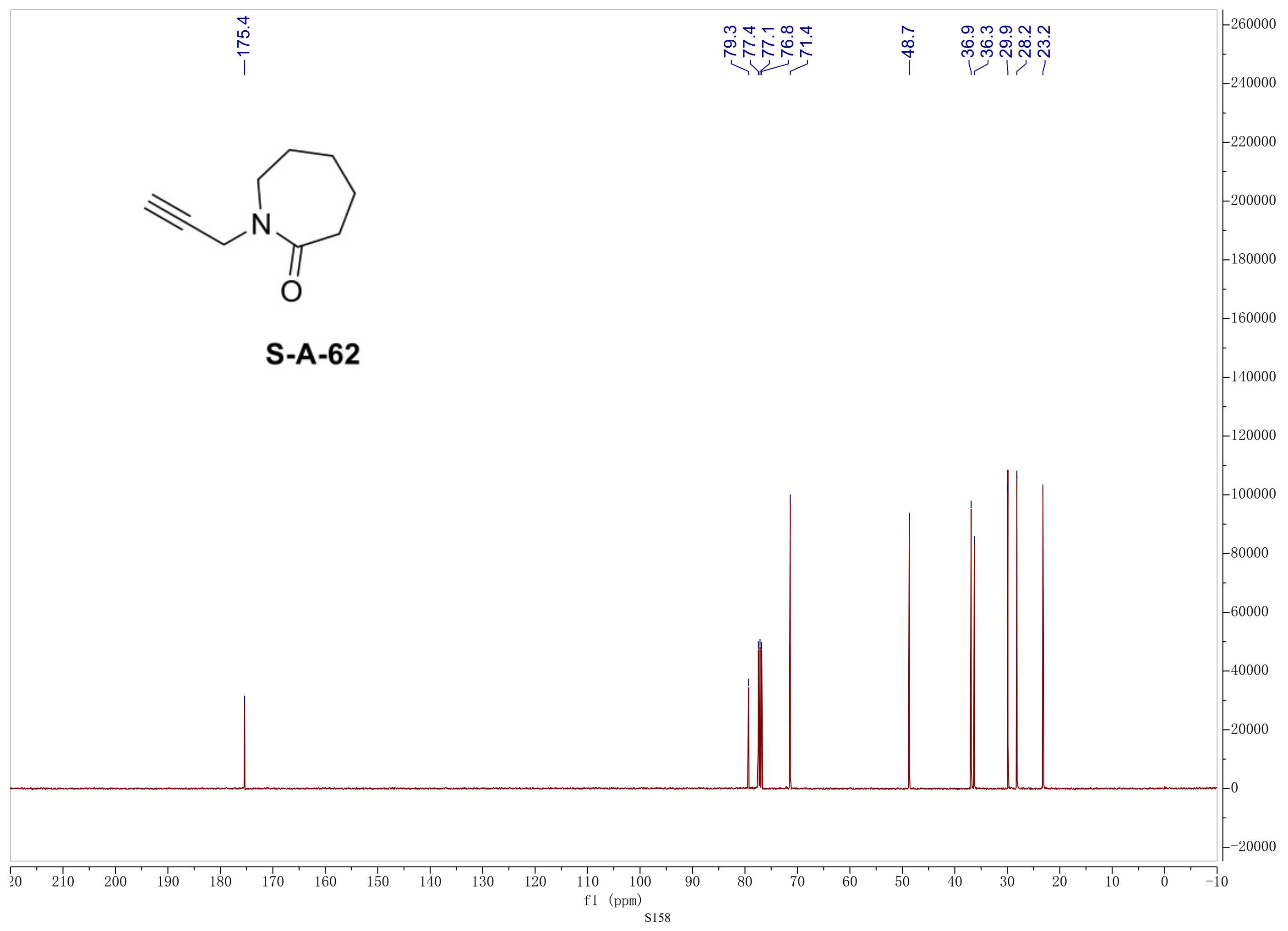




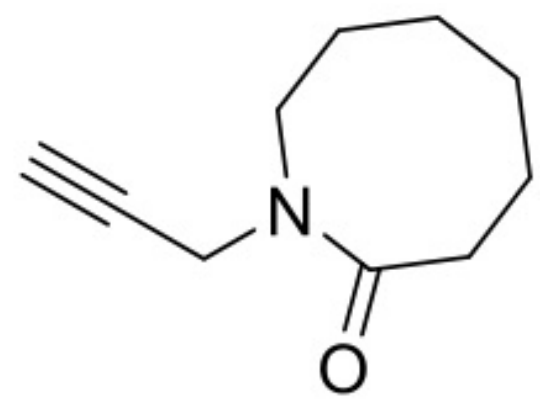

S-A-63

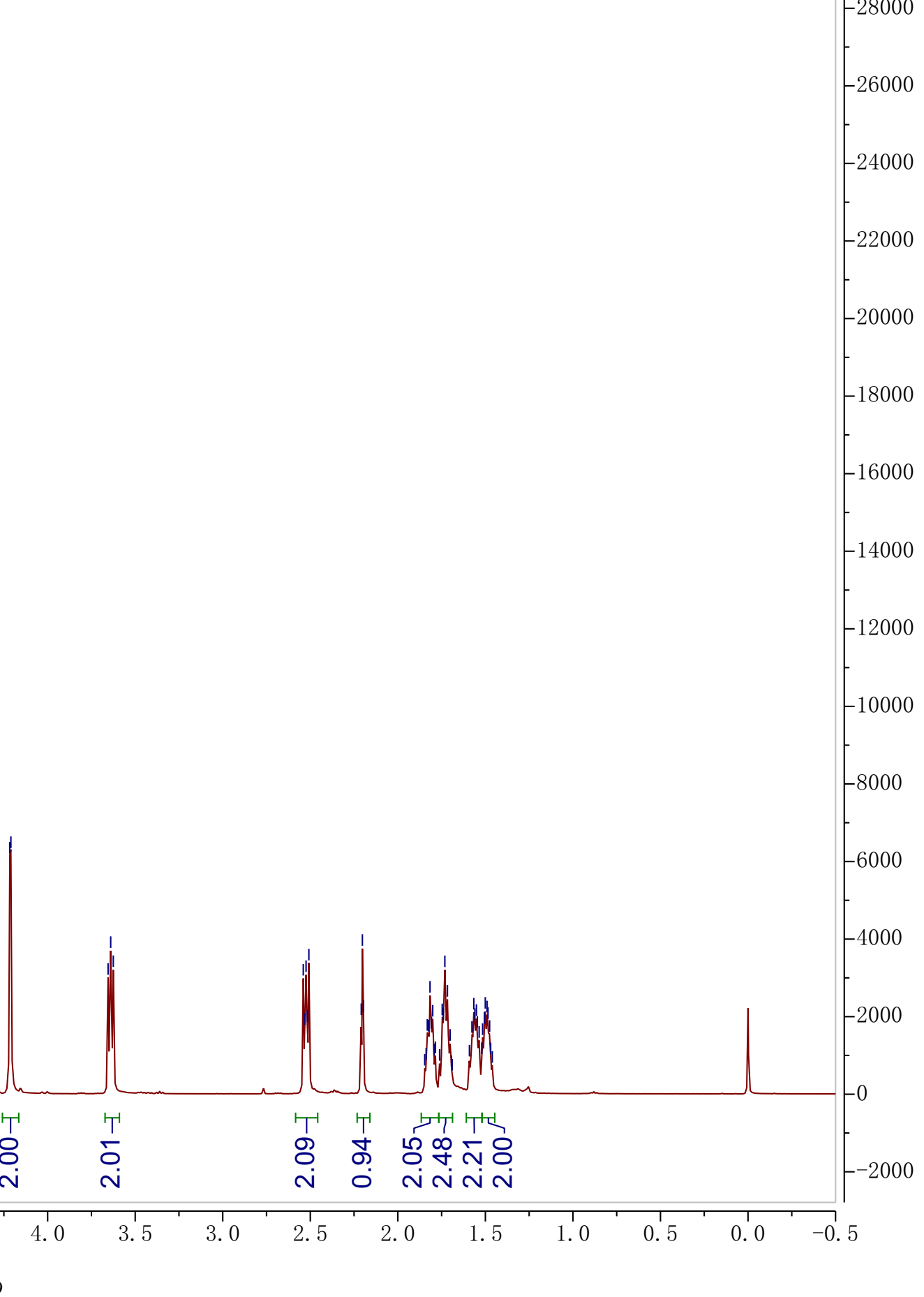




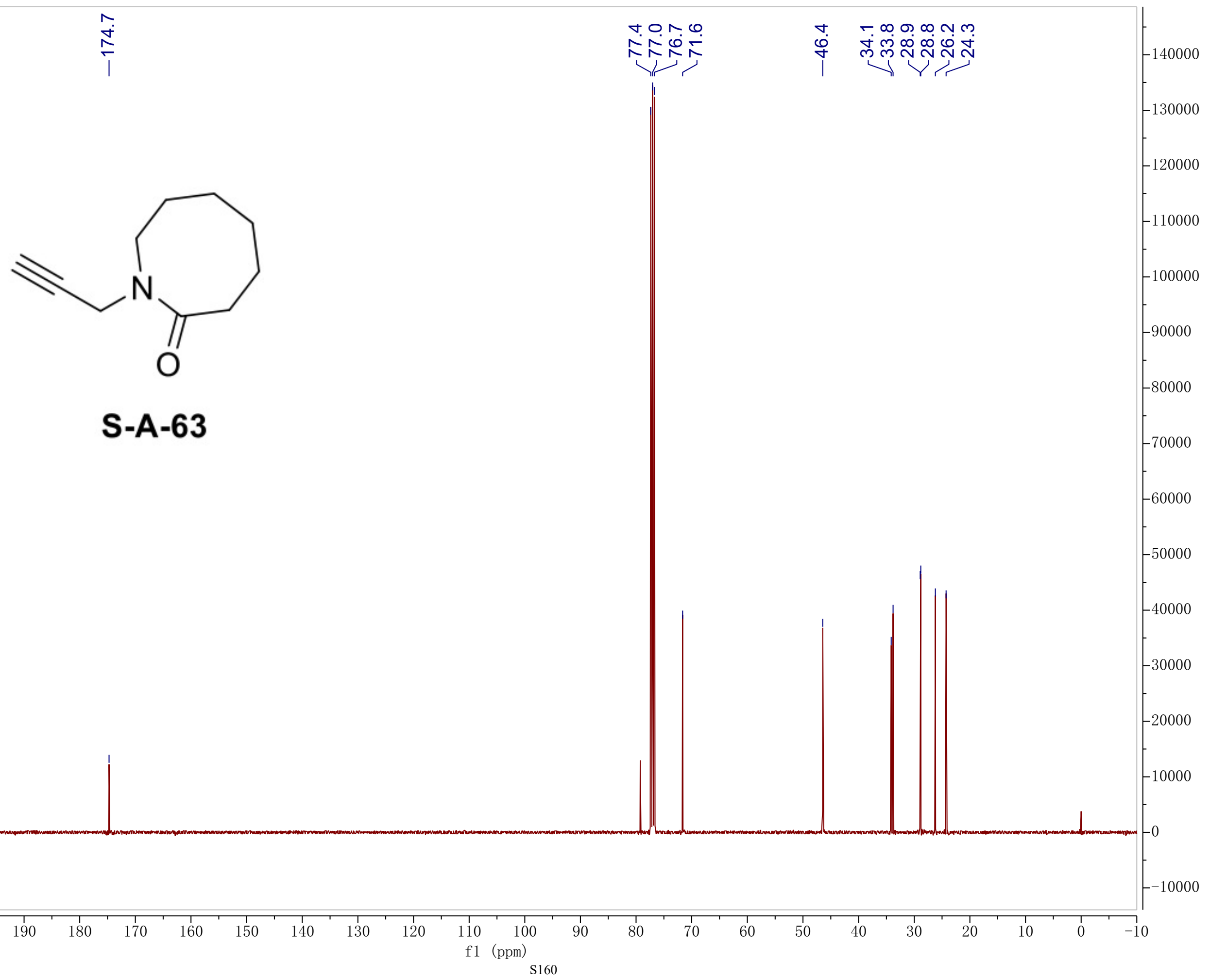




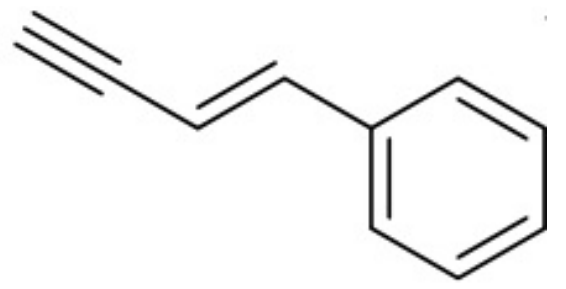

\section{S-A-64}

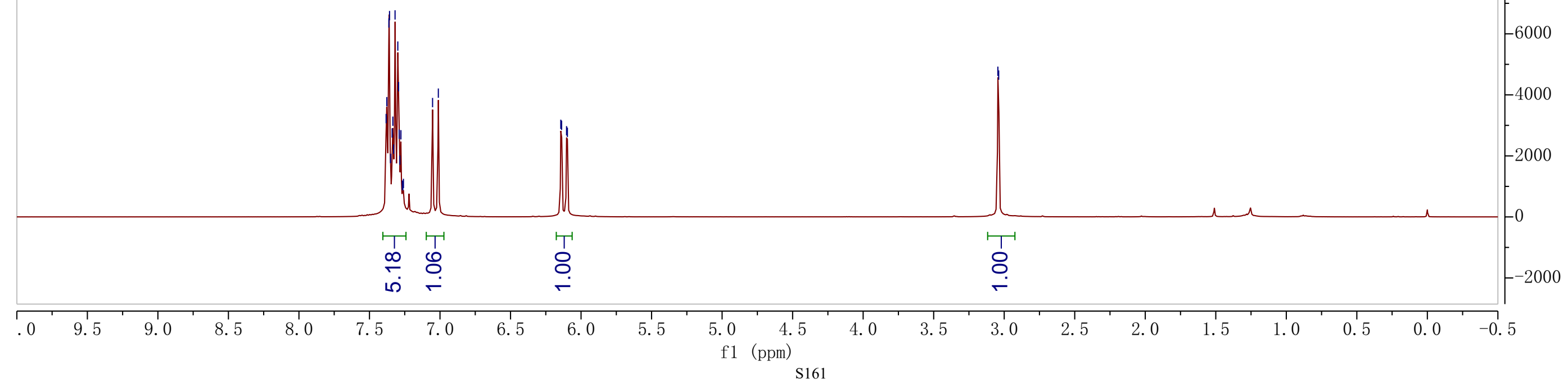




i

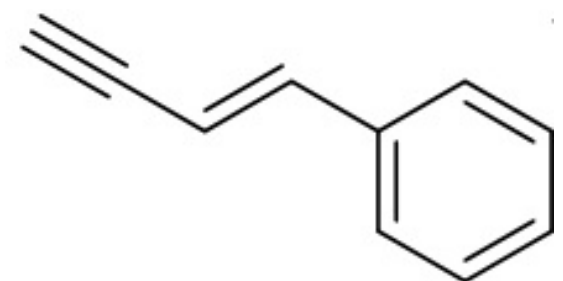

S-A-64 


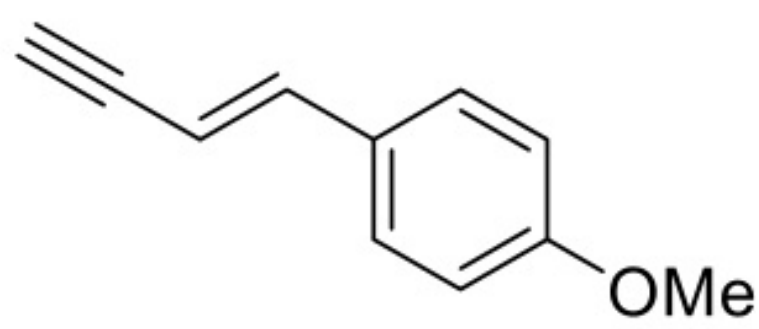

\section{S-A-65}




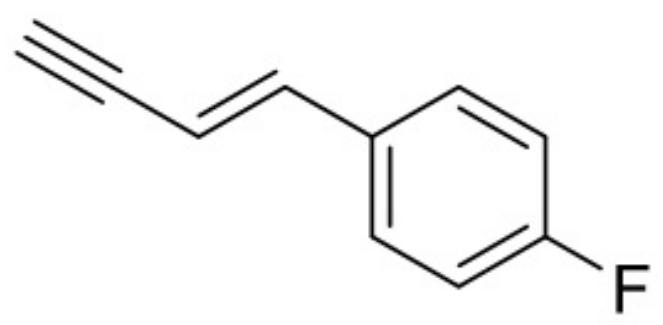

S-A-66

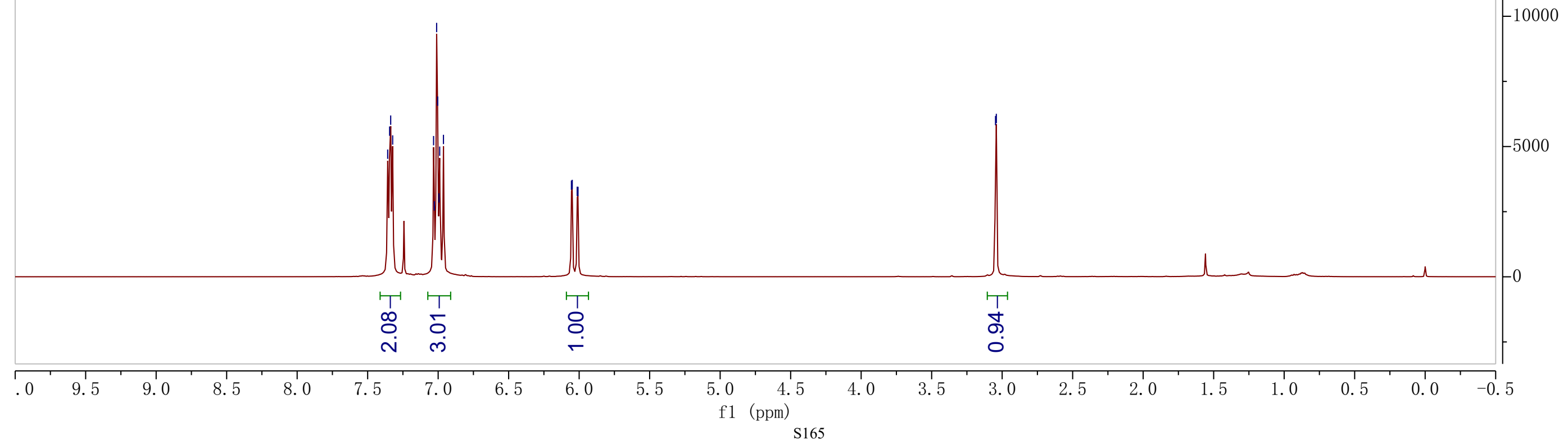




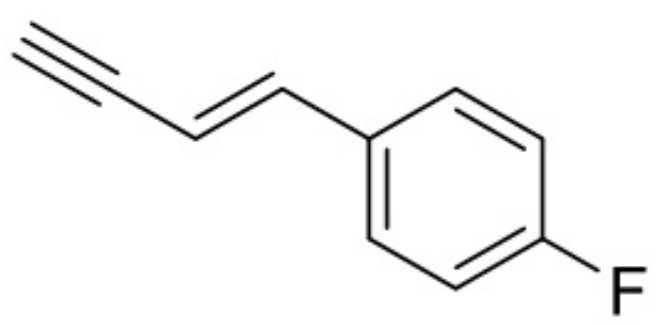

S-A-66

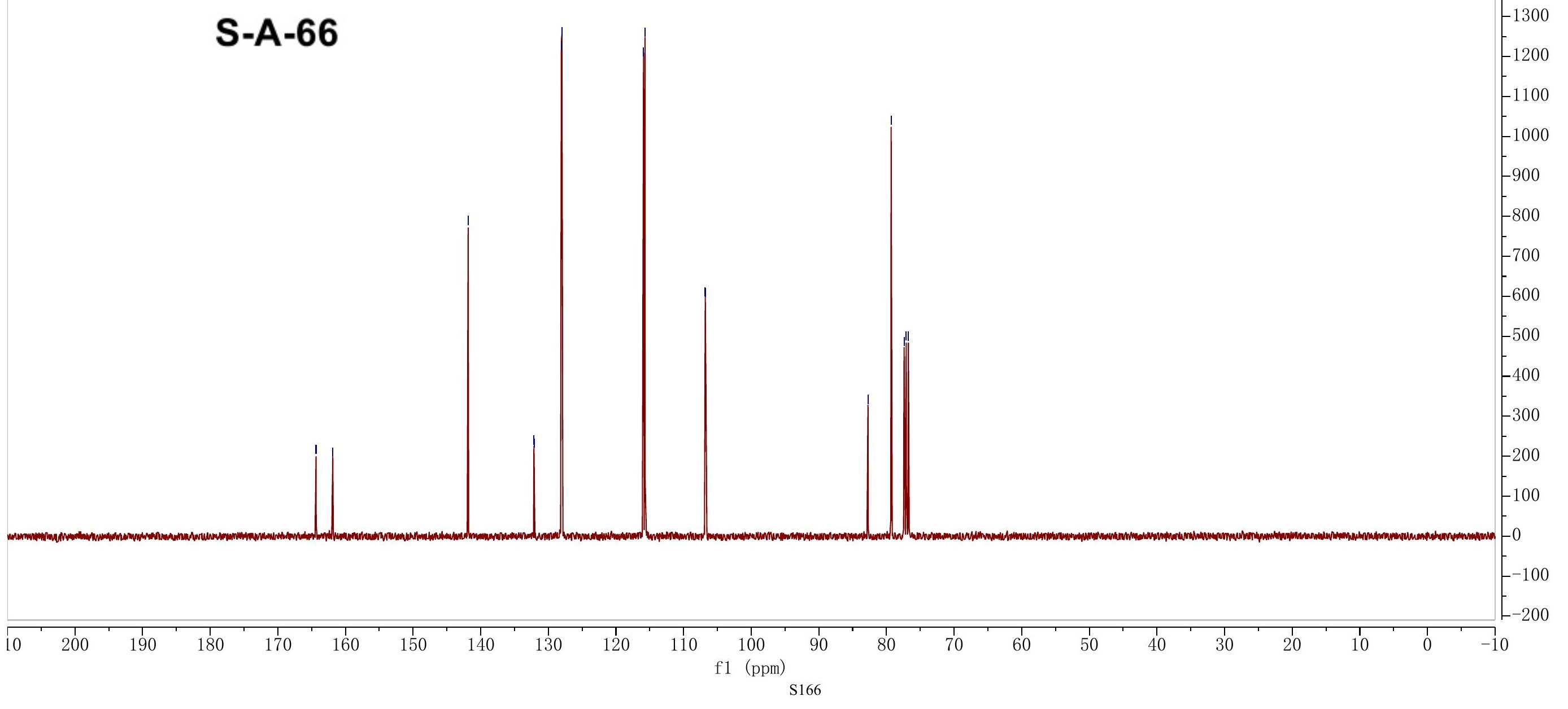

\section{F}




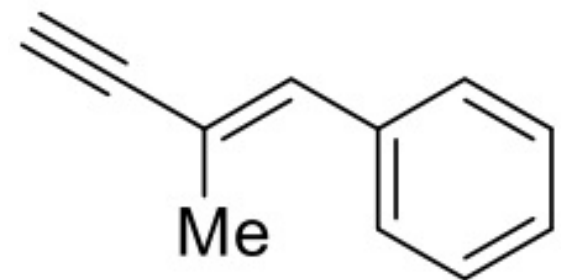

S-A-67

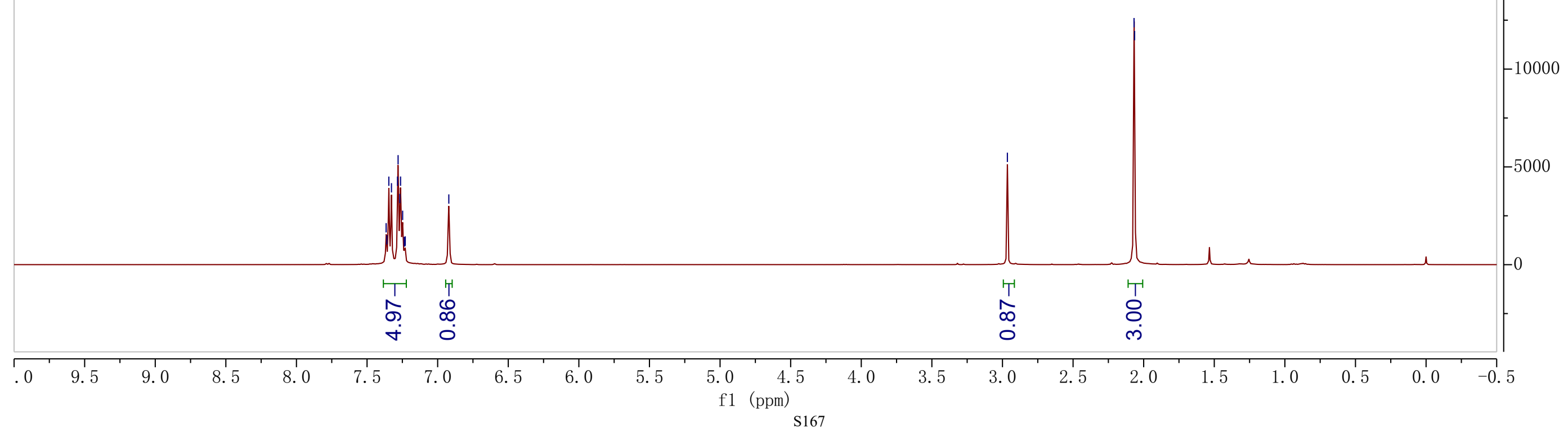




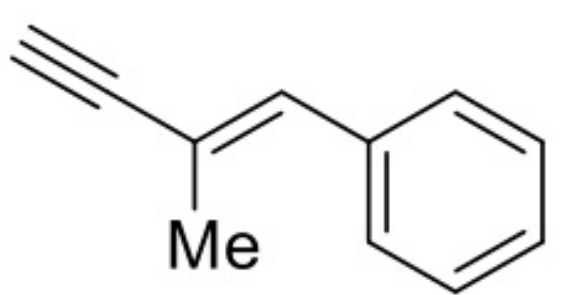

\section{S-A-67}

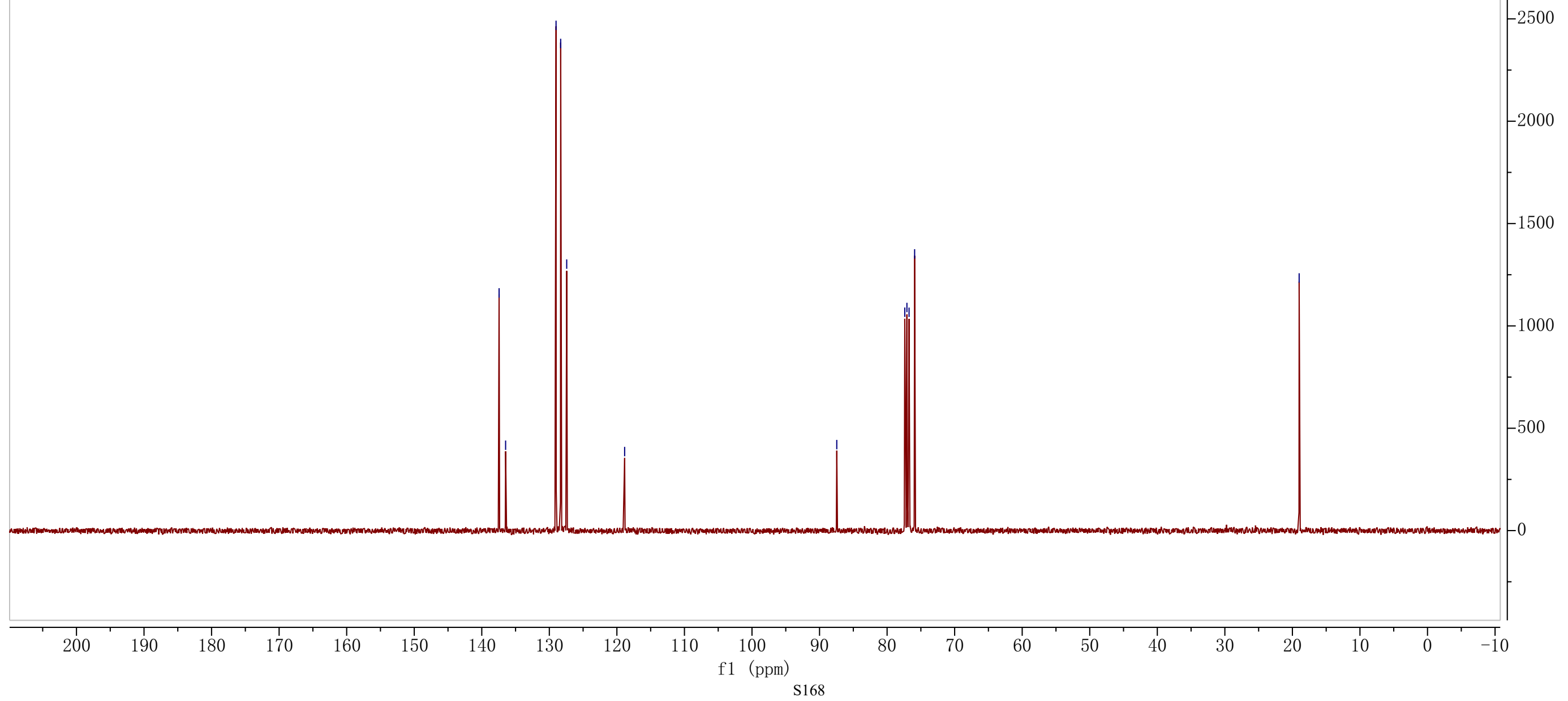




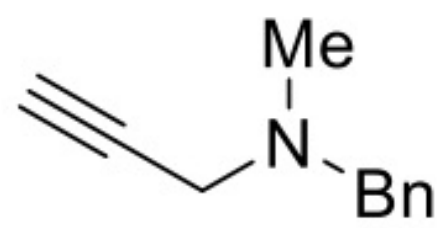

S-A-70

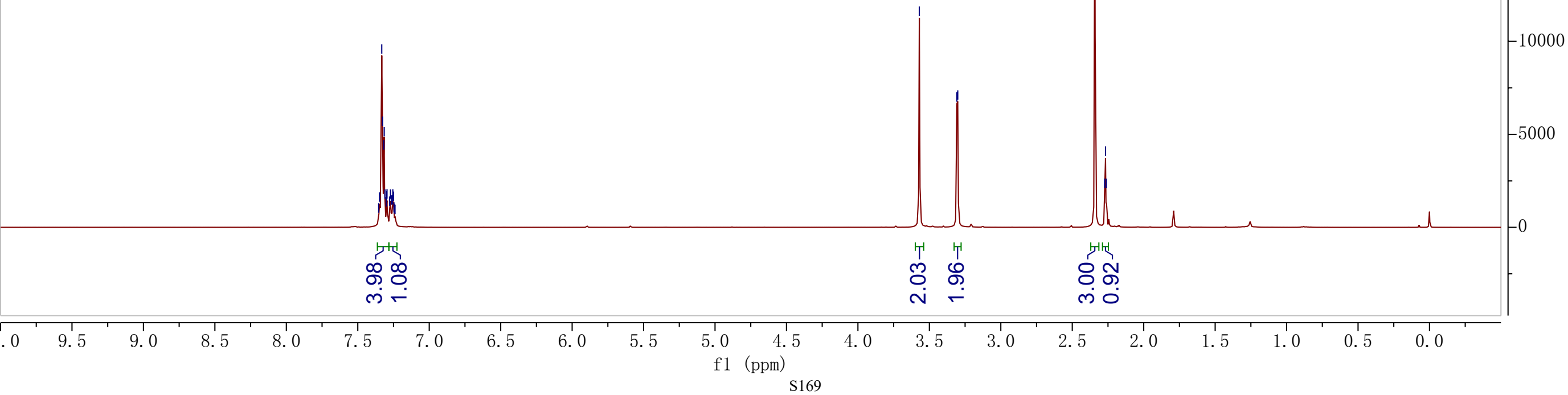




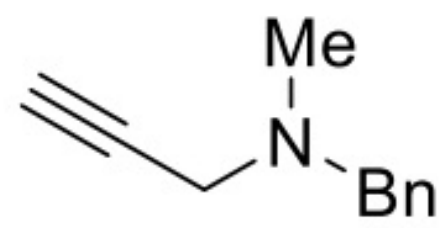

S-A-70 


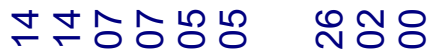

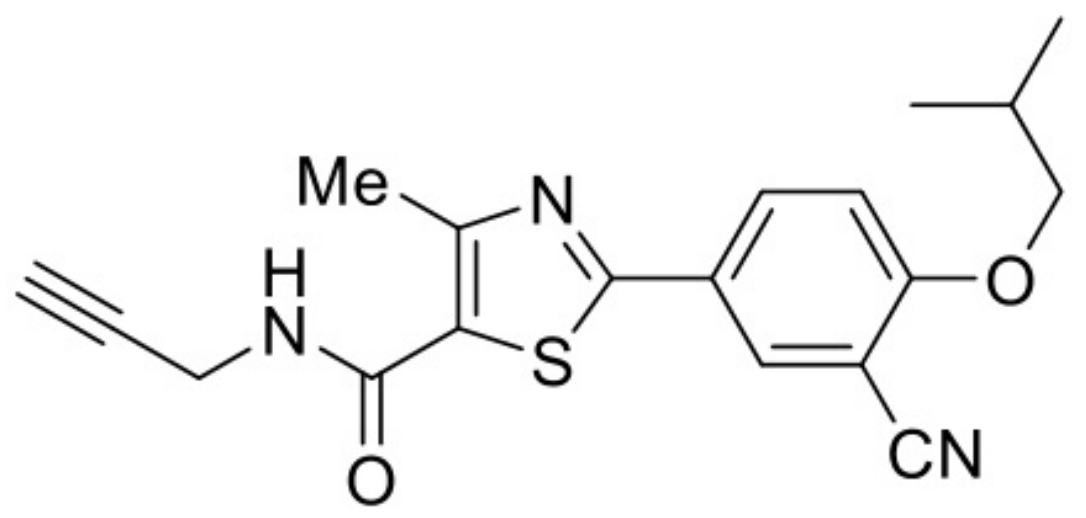

S-A-73

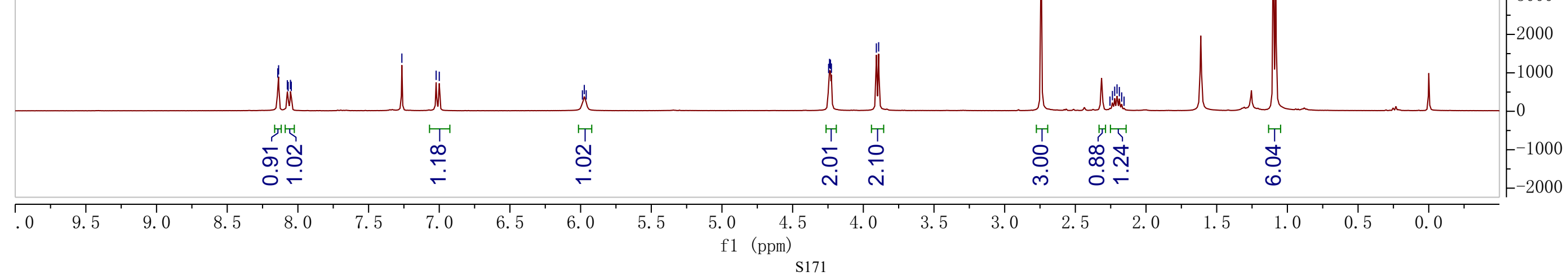




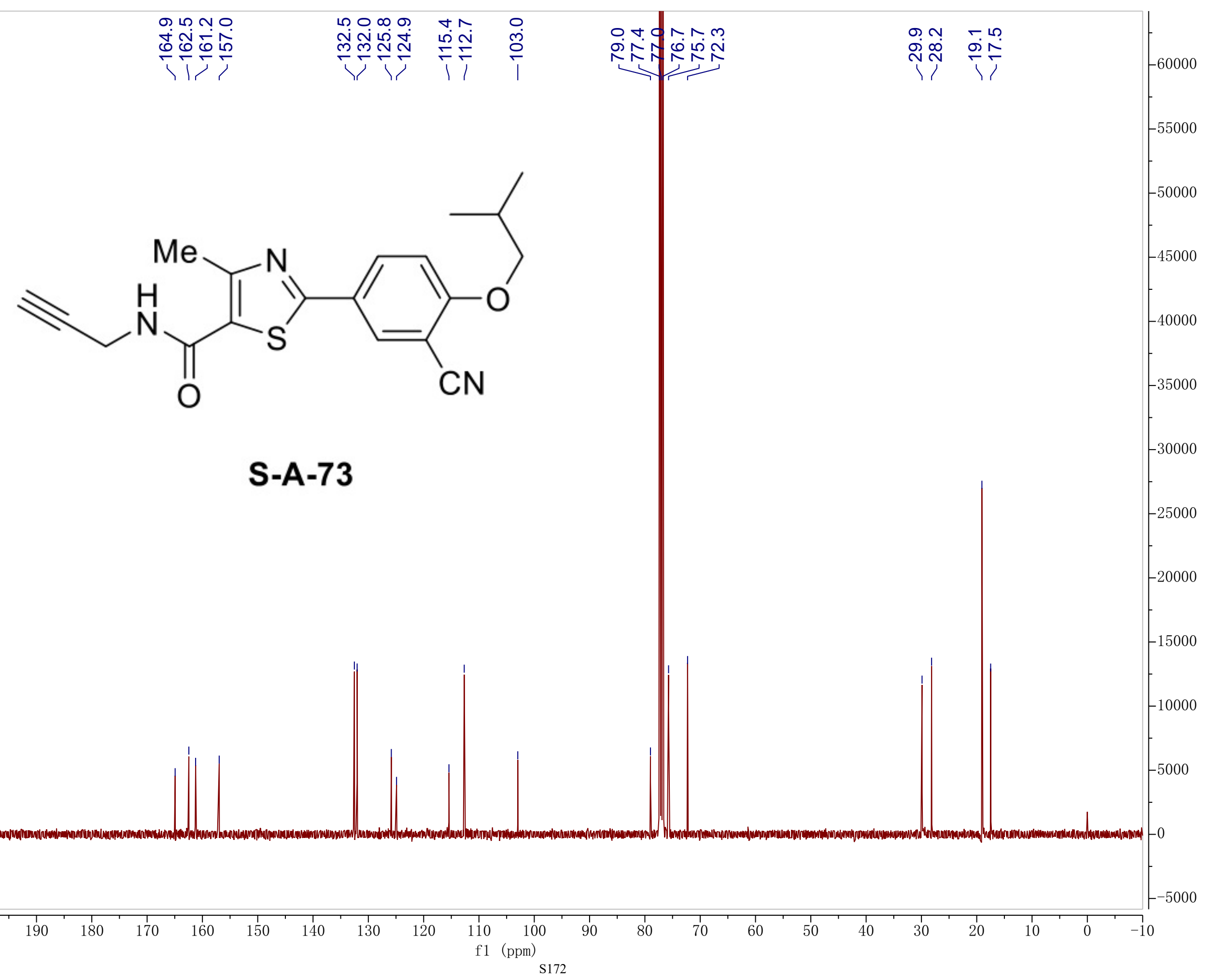


<smiles>C#CCNC(=O)c1cn(C2CC2)c2cc(N3CCN(CC)CC3)c(F)cc2c1=O</smiles>

S-A-74 
<smiles>C#CCNC(=O)c1cn(C2CC2)c2cc(N3CCN(CC)CC3)c(F)cc2c1=O</smiles>
S-A-74

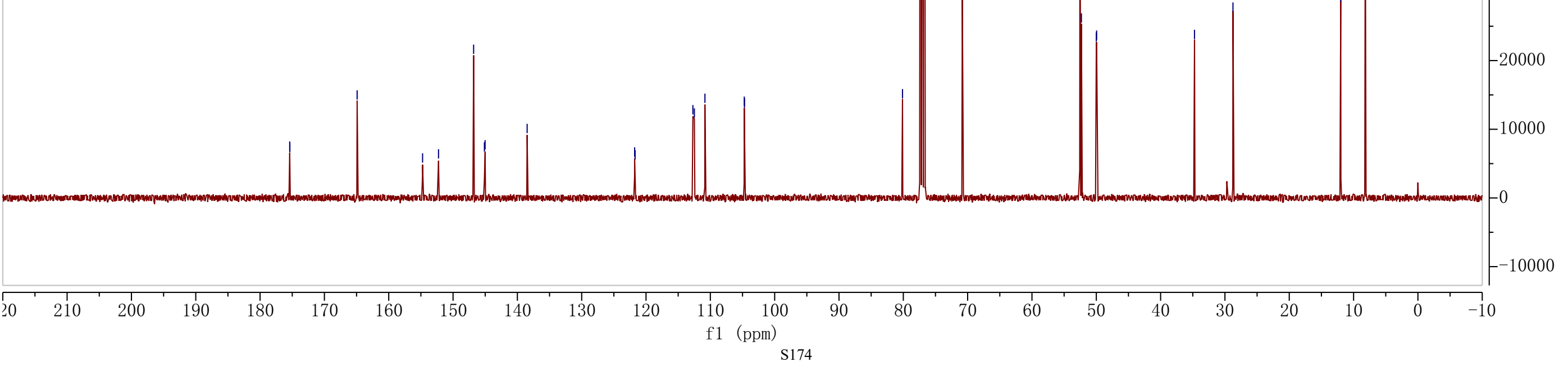




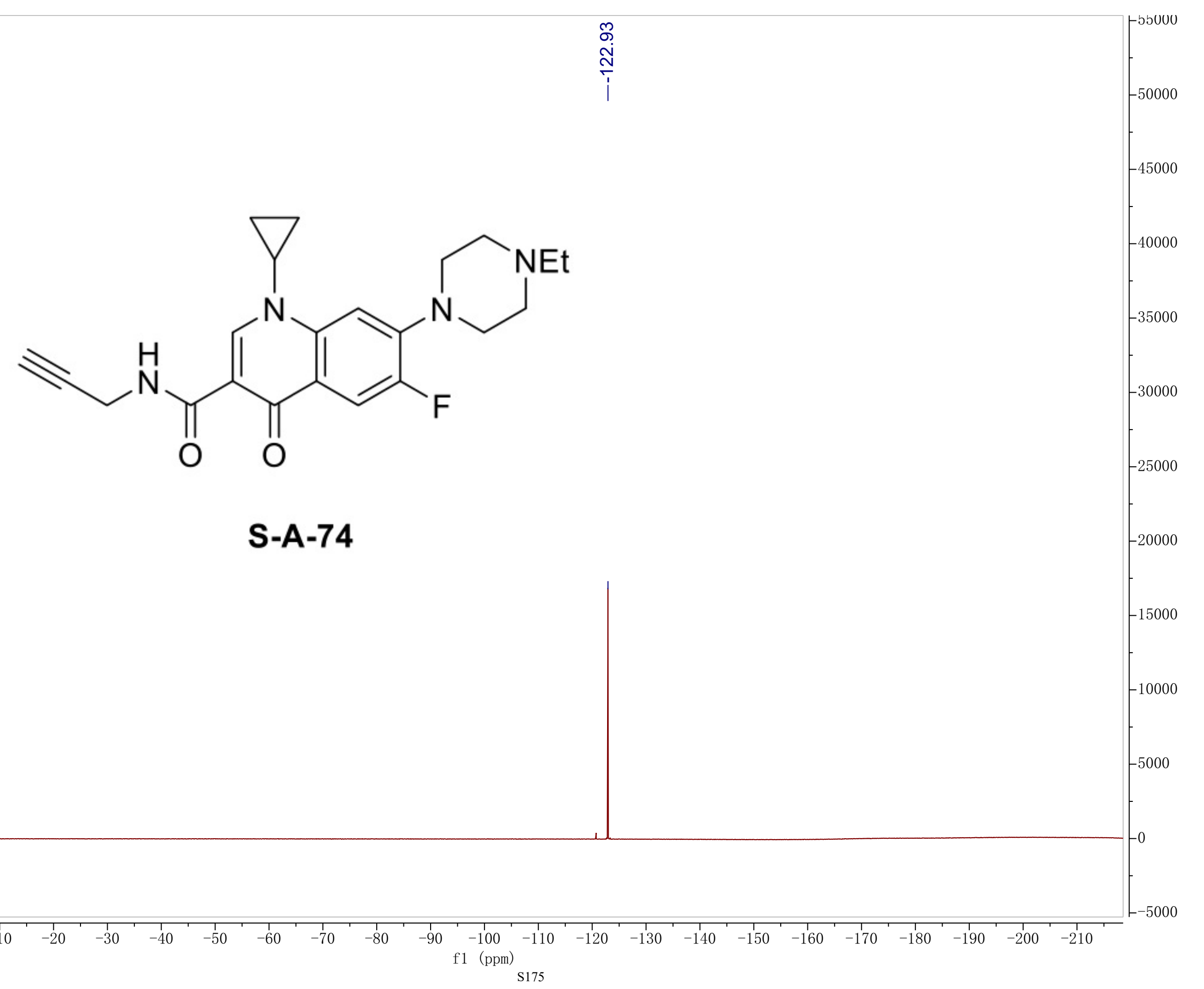



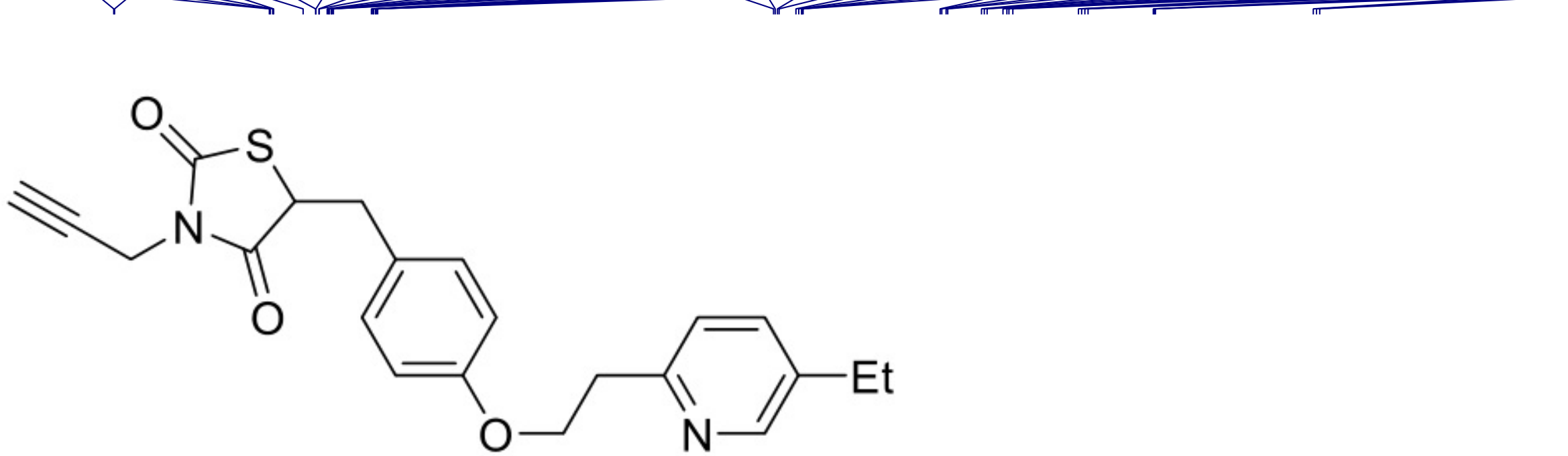

S-A-75

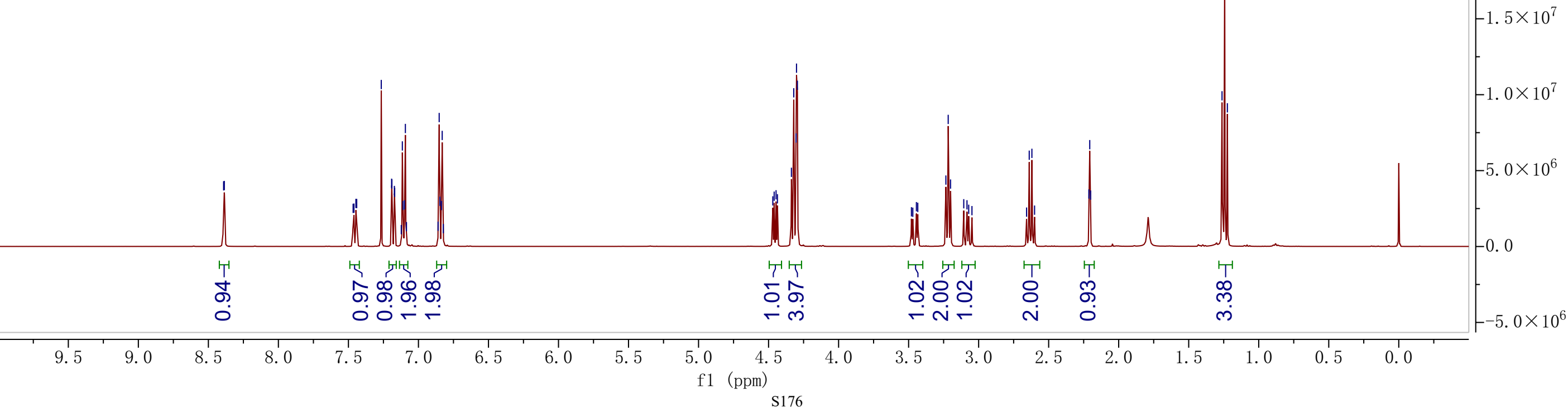




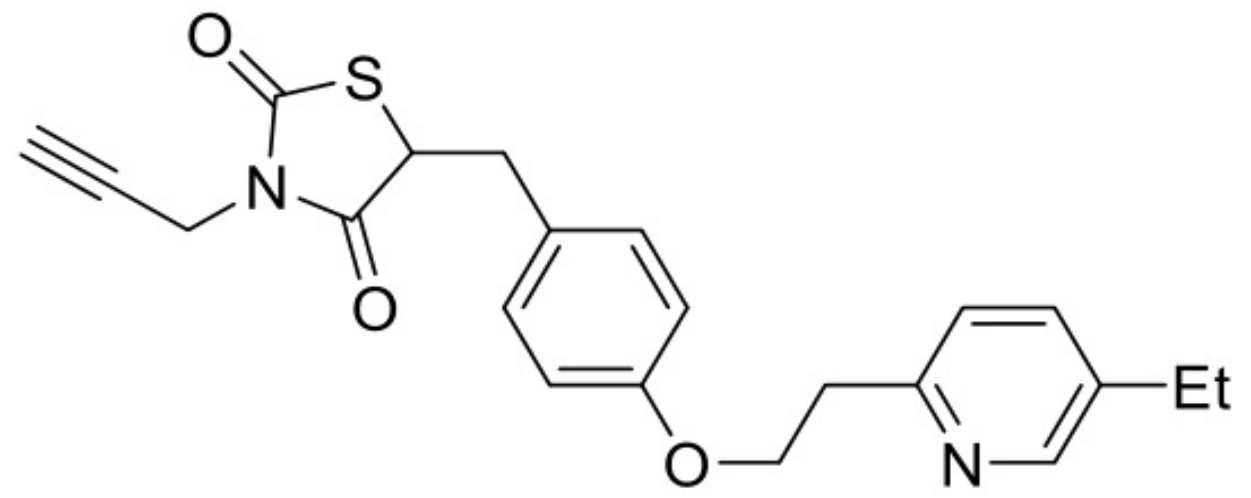

S-A-75

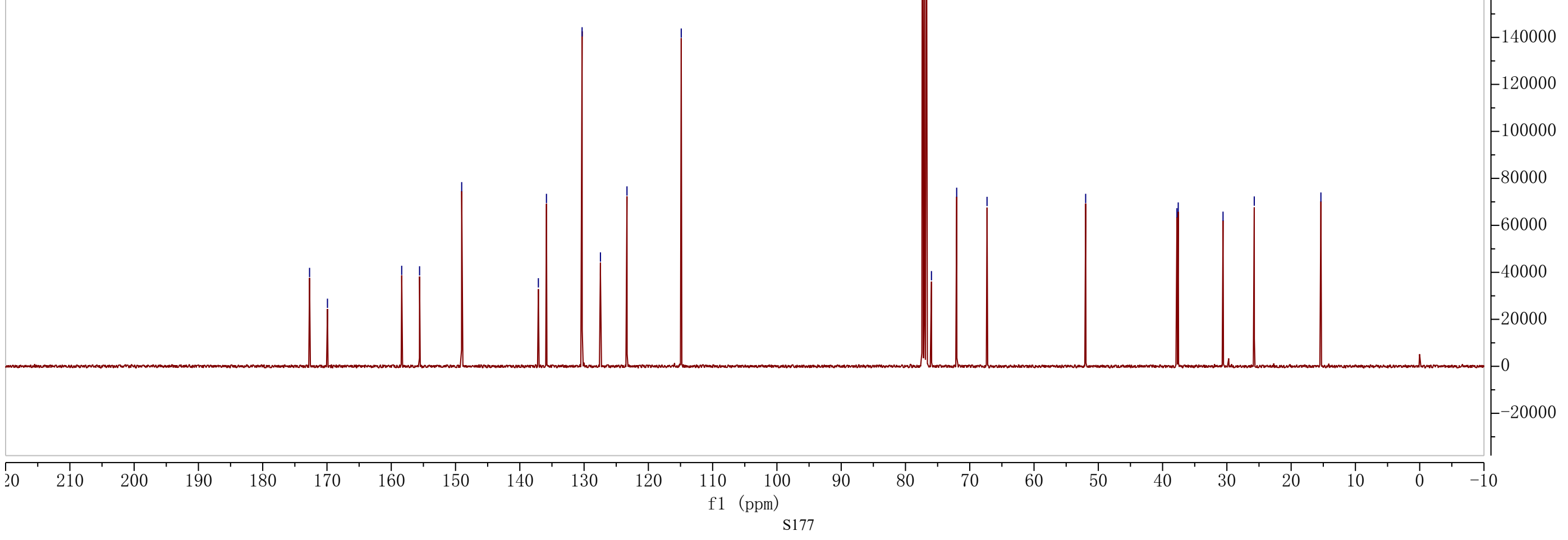




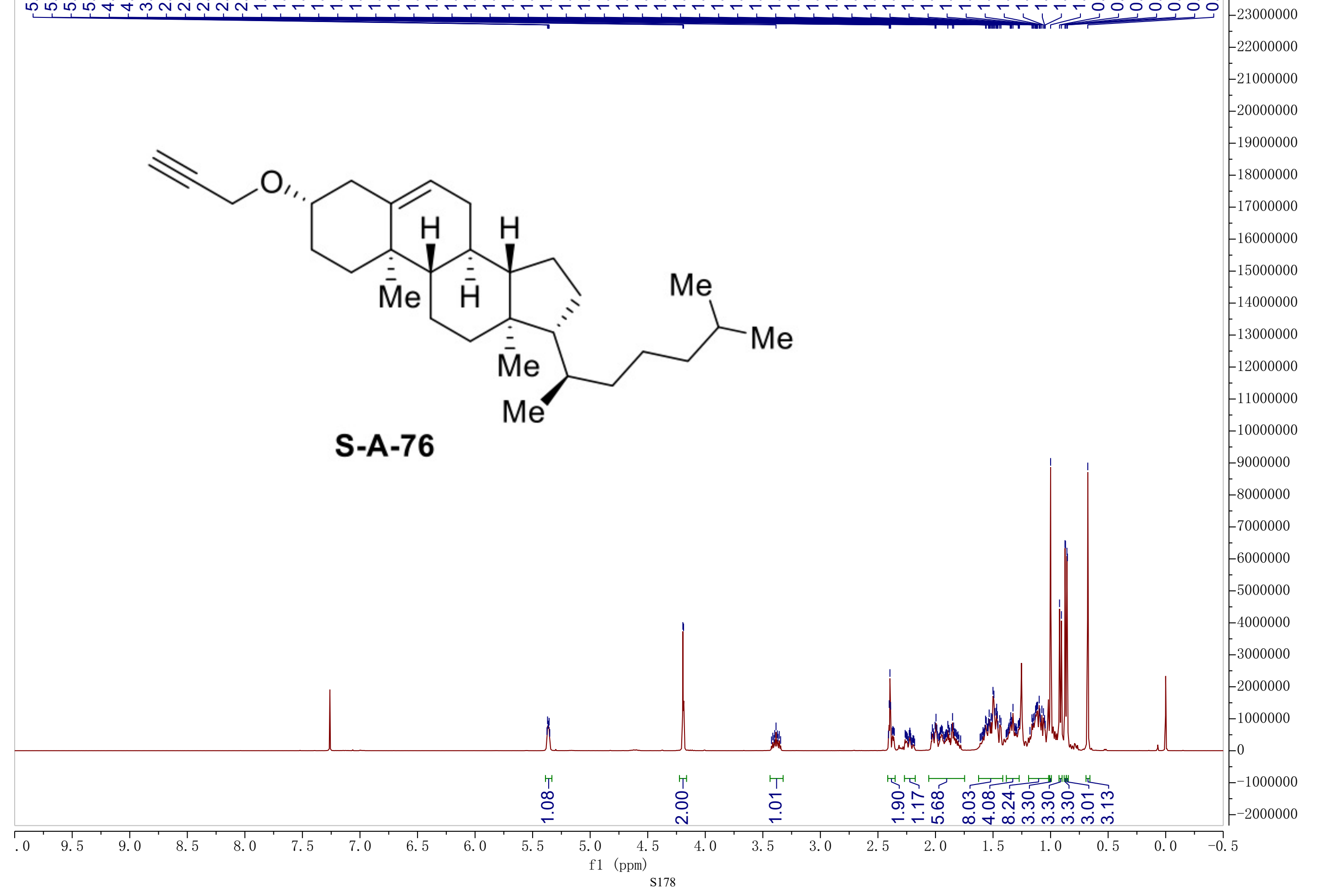



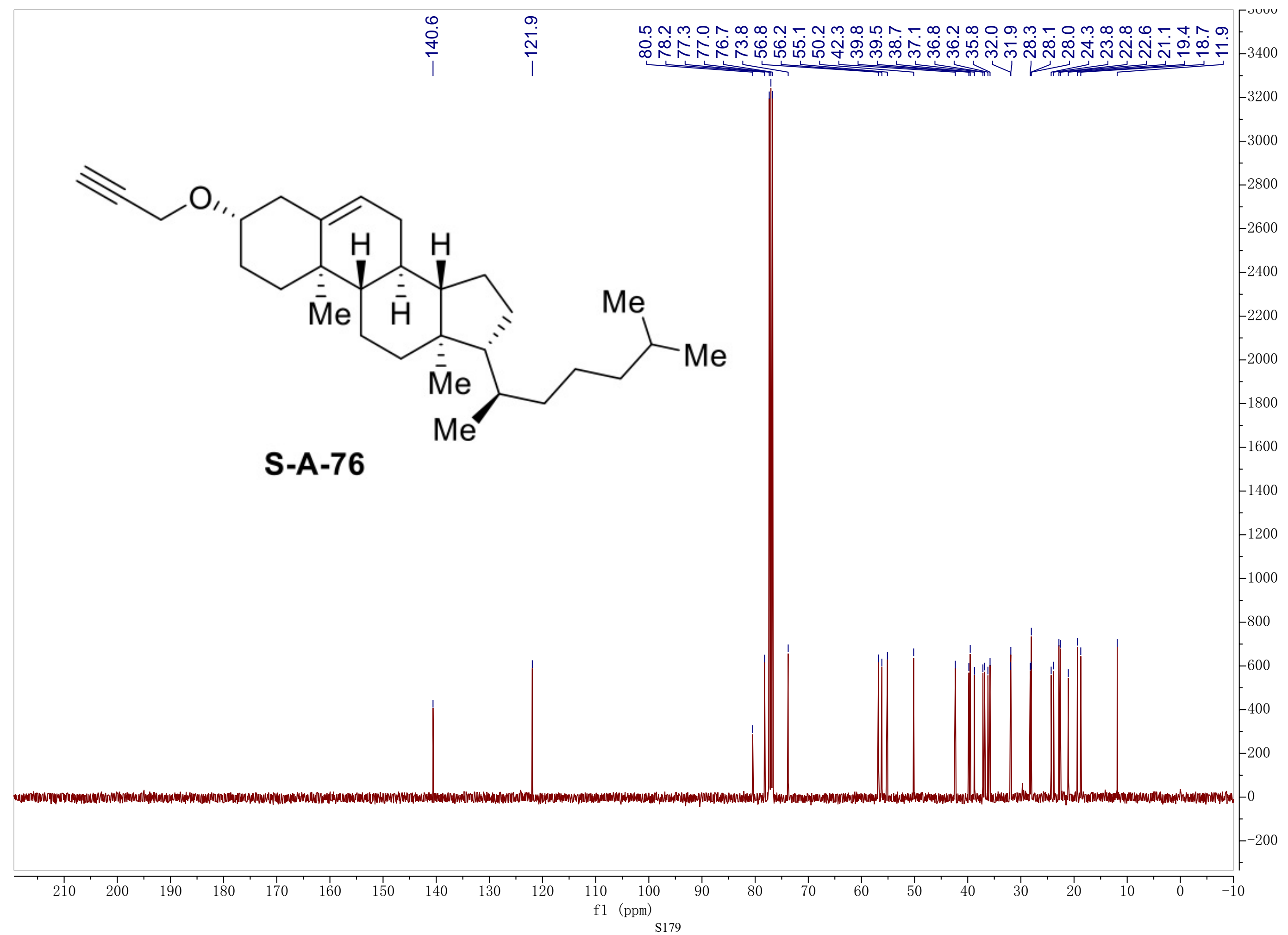


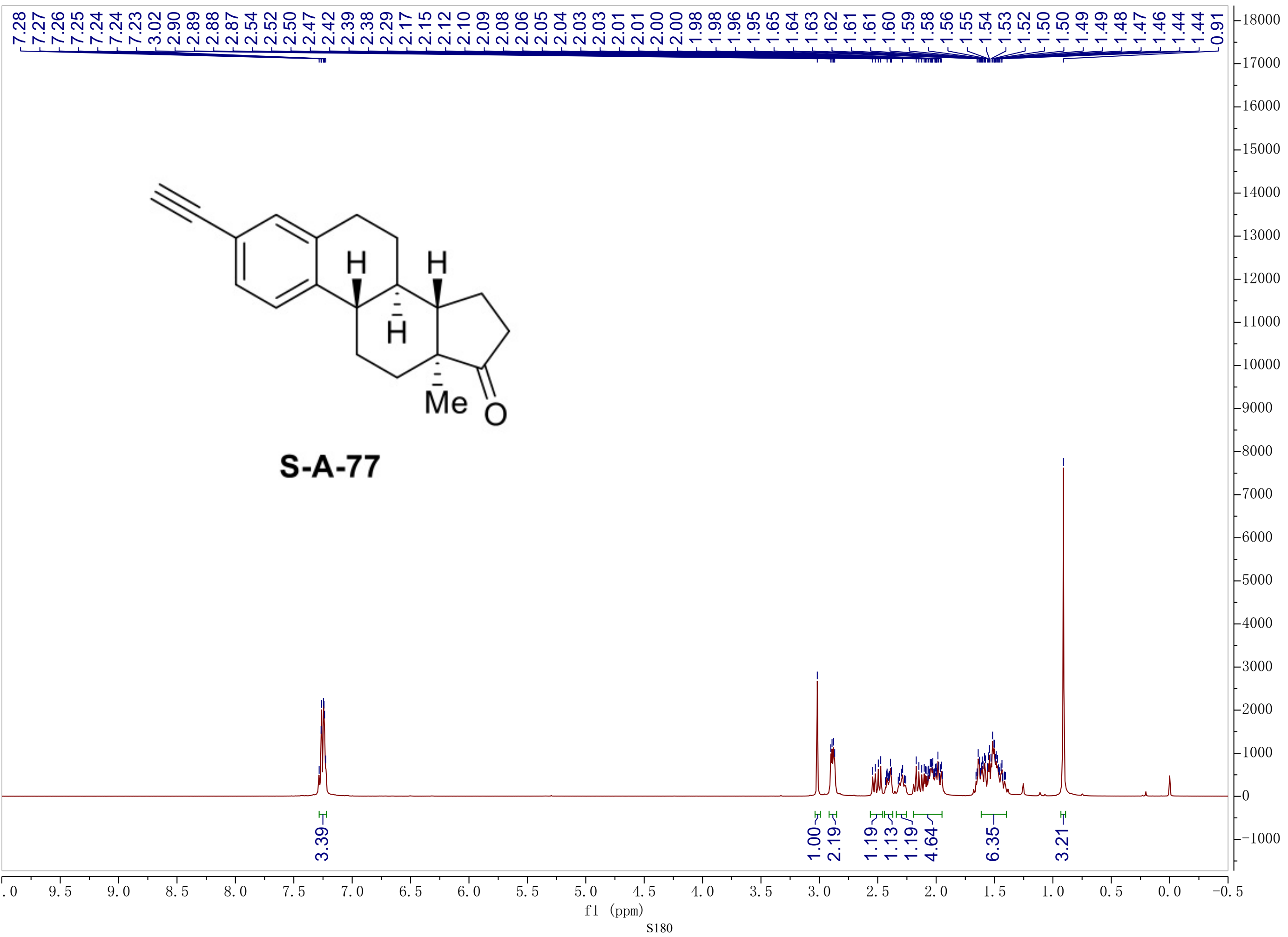


Ts

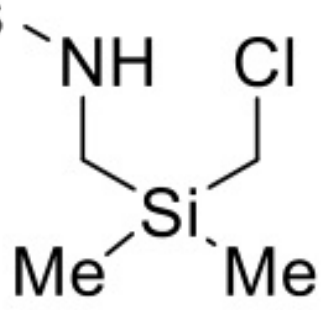

2a

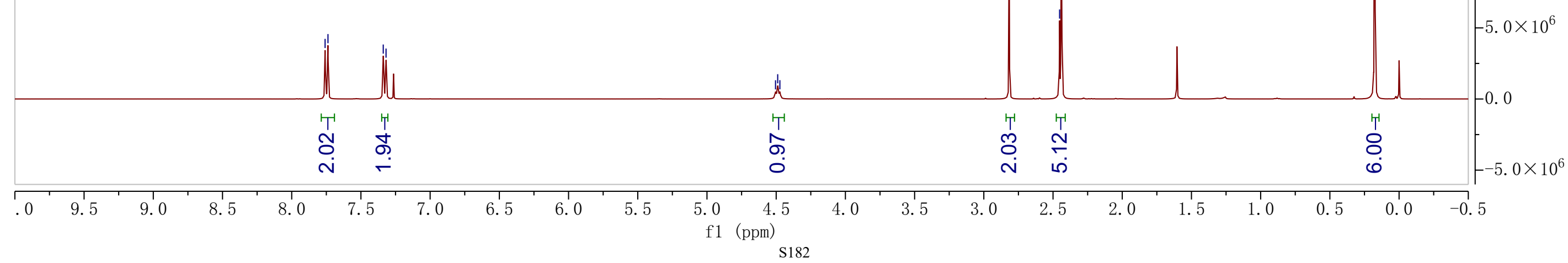




\section{Ts}<smiles>CNC[Si](C)(C)CCl</smiles>

2a

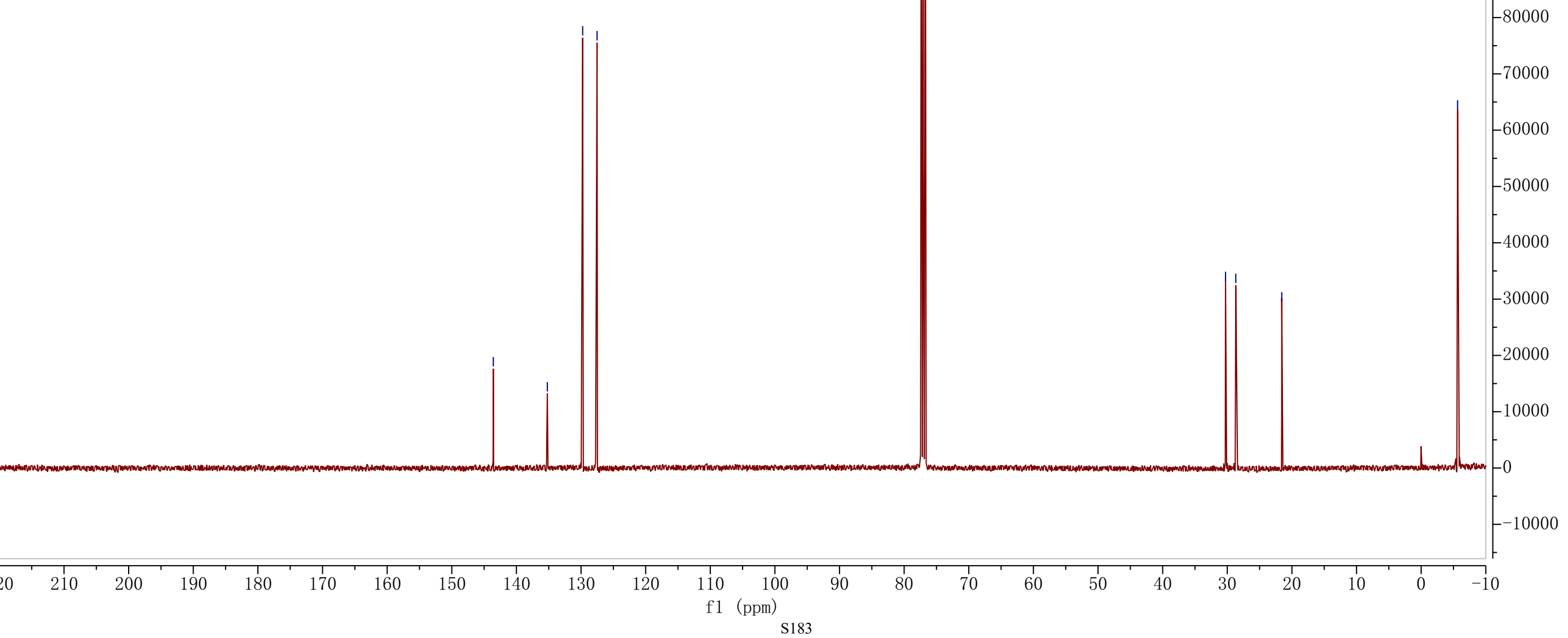




\section{$t-\mathrm{BuSO}_{2} \mathrm{HN} \quad \mathrm{Cl}$}<smiles>C[Si](C)(C[Hg])C[Hg]</smiles>

\section{2b}

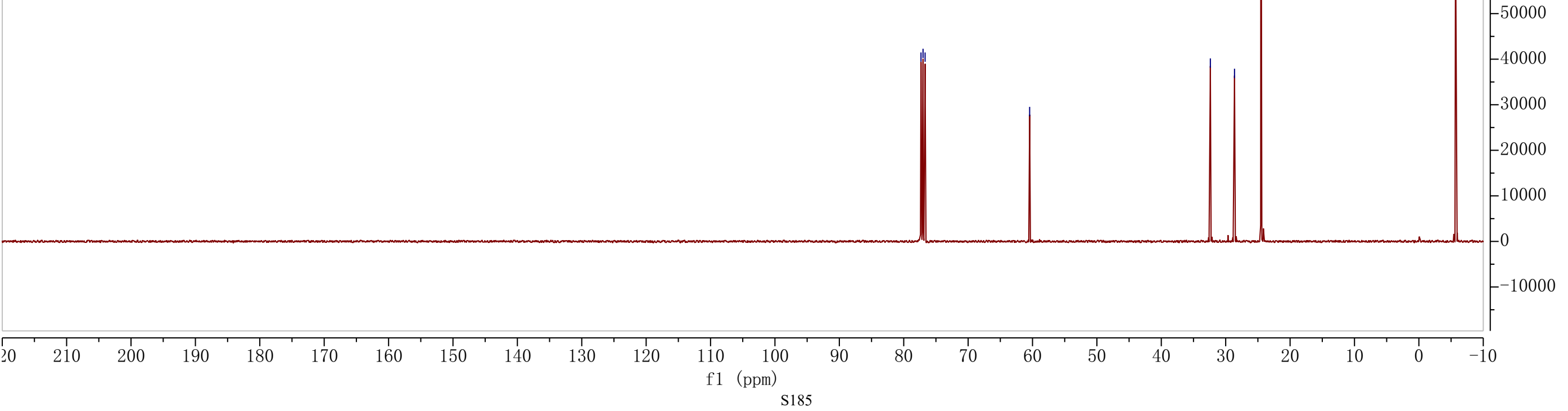


Ms<smiles>CNC[Si](C)(C)CCl</smiles>

\section{2c}

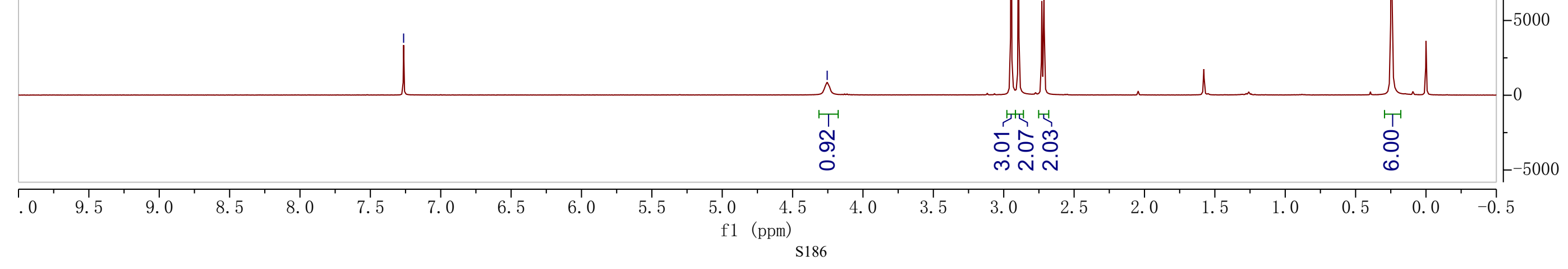


${ }_{\mathrm{Me}^{\prime} \mathrm{Si}}^{\mathrm{SH}} \mathrm{Me}$

2c

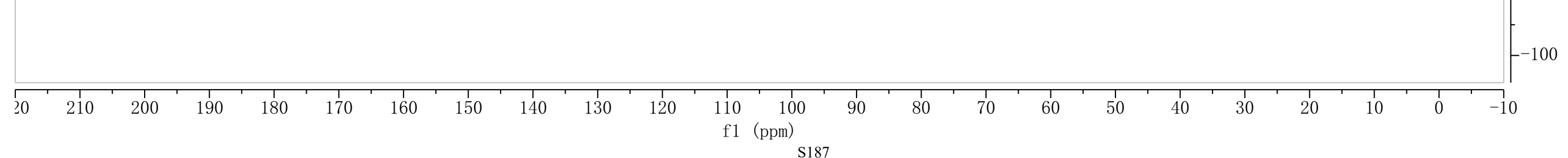


$p-\mathrm{CF}_{3} \mathrm{C}_{6} \mathrm{H}_{4} \mathrm{COHN} \quad \mathrm{Cl}$<smiles>CC[Si](C)(C)CC</smiles>

2d

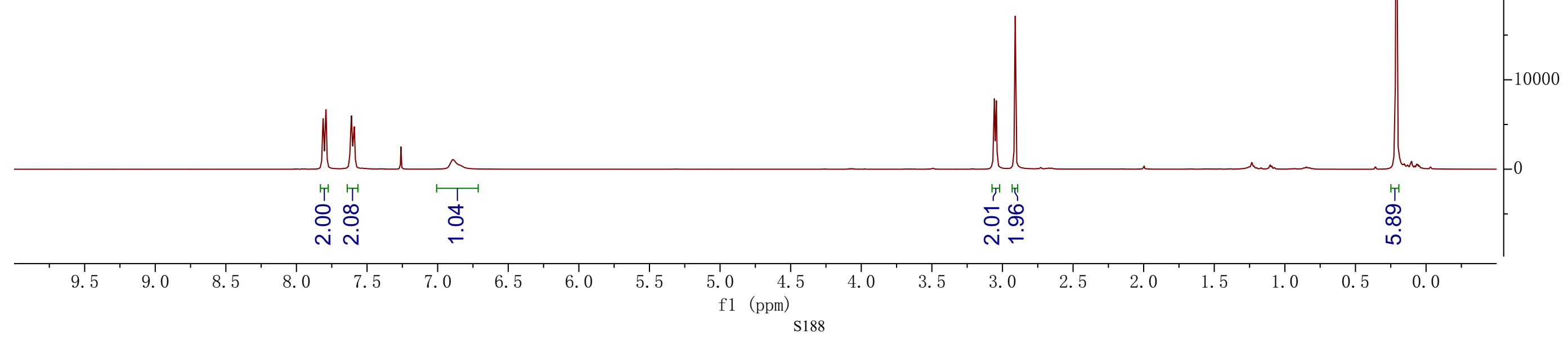


$p-\mathrm{CF}_{3} \mathrm{C}_{6} \mathrm{H}_{4} \mathrm{COHN} \quad \mathrm{Cl}$<smiles>CC[Si](C)(C)CC</smiles>

$2 d$ 
${ }^{19}$ F NMR

$p-\mathrm{CF}_{3} \mathrm{C}_{6} \mathrm{H}_{4} \mathrm{COHN} \quad \mathrm{Cl}$<smiles>CC[Si](C)(C)C</smiles>

2d 
Ts

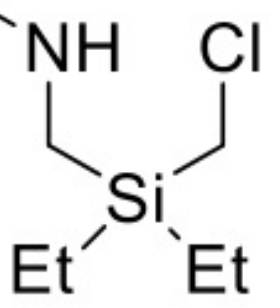

$2 e$

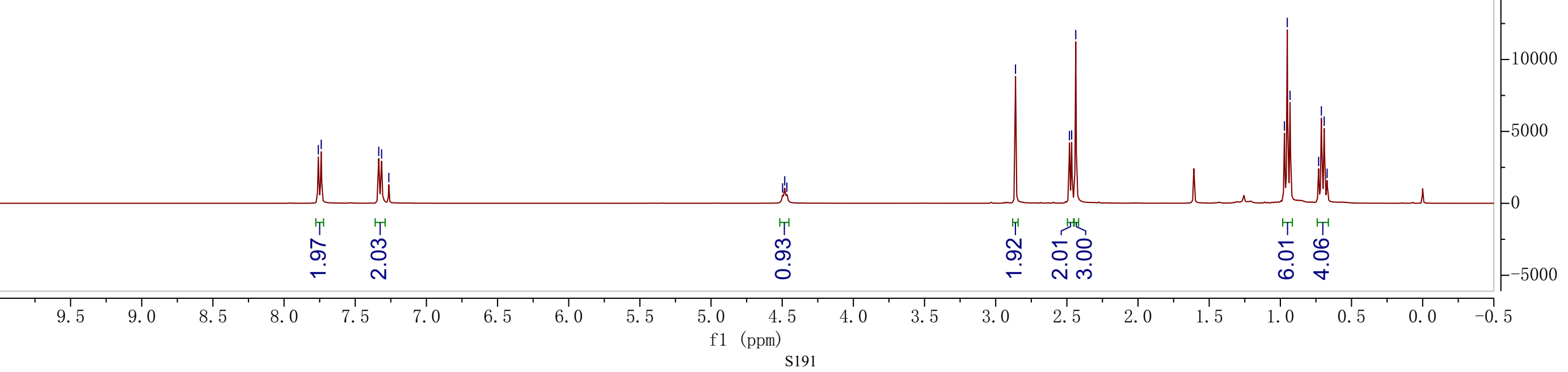


Ts<smiles>CC[Si](CC)(CCl)CNC</smiles>

$2 e$

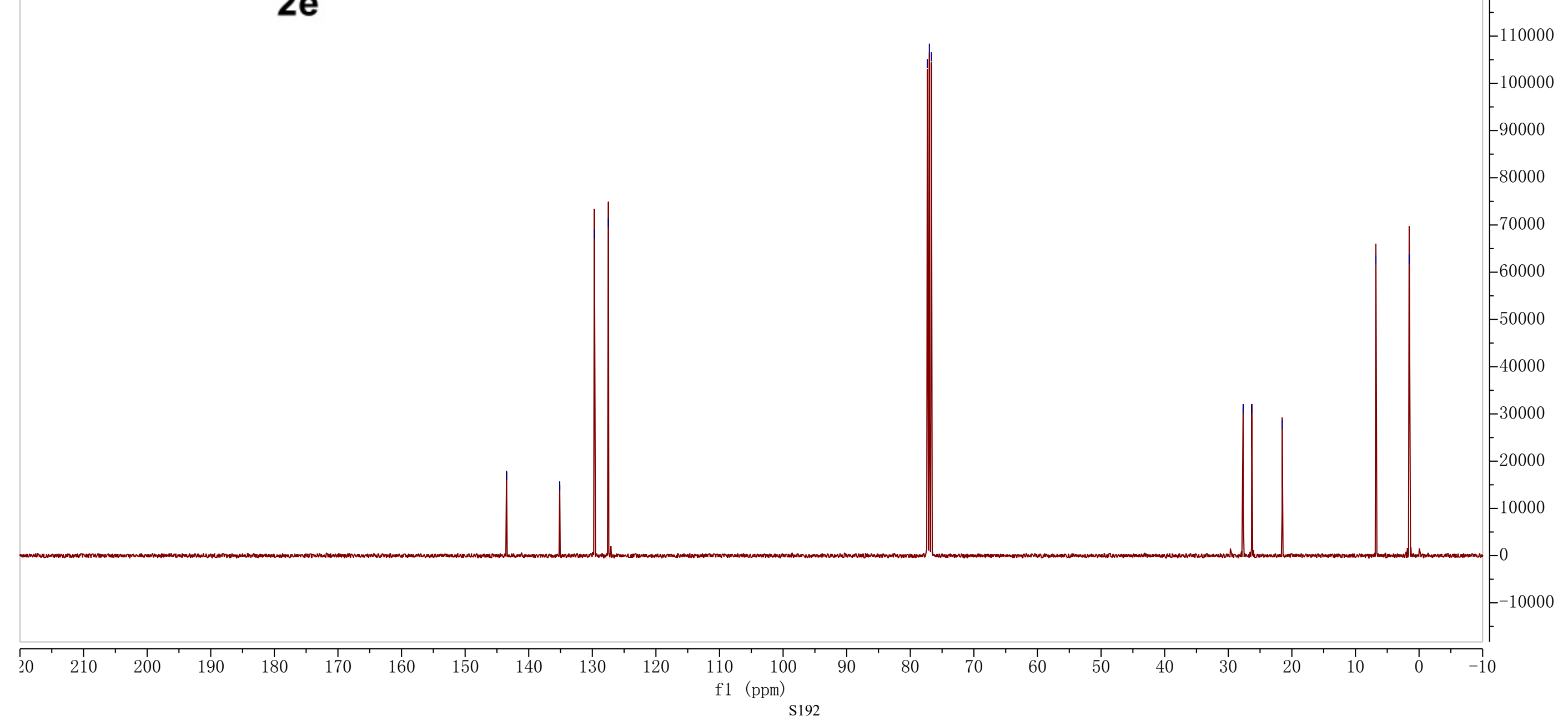


Ts<smiles>CCCC[Si](CCl)(CBr)CNS</smiles>

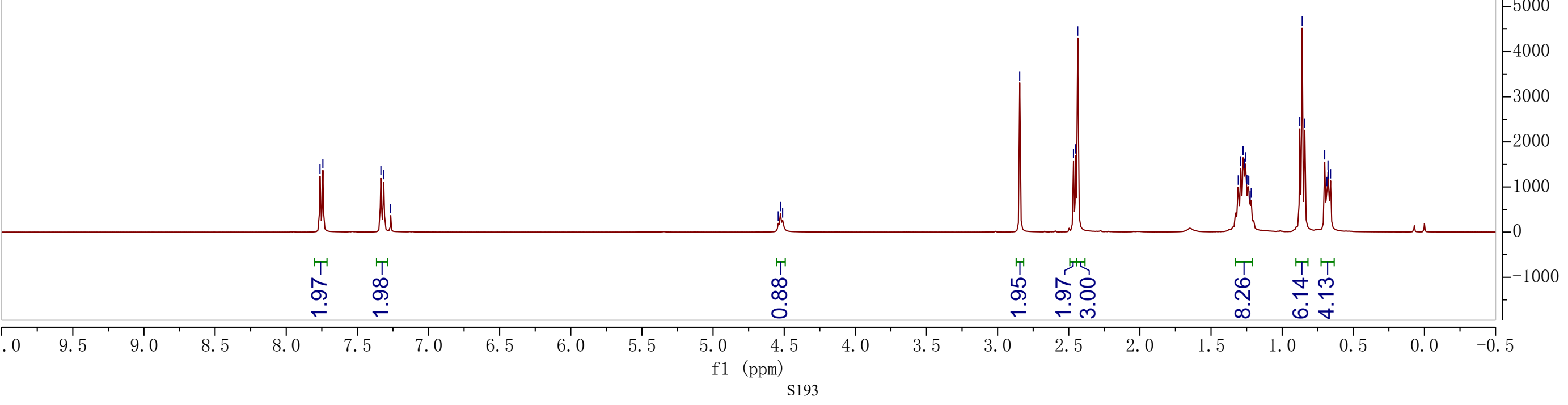


Ts $-\mathrm{NH} \mathrm{Cl}$

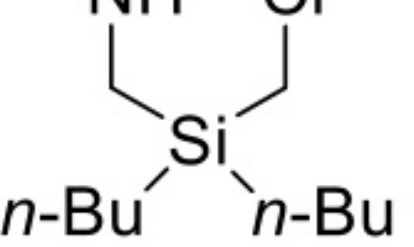

$2 f$

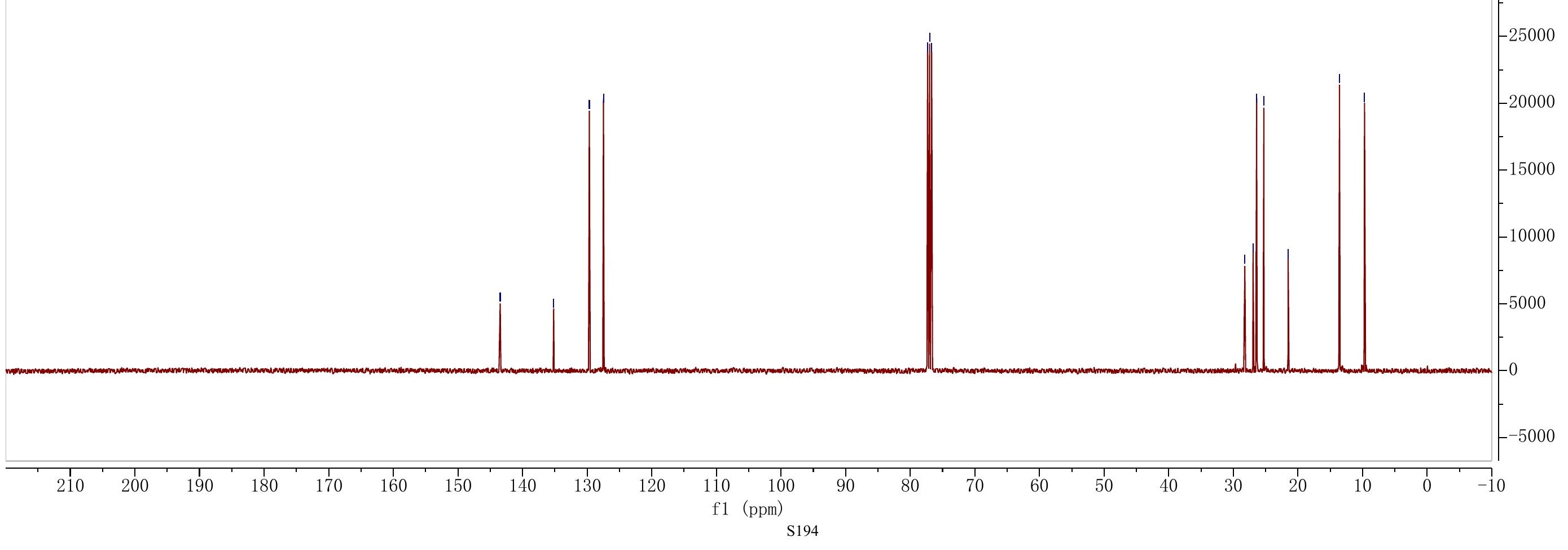


Ts
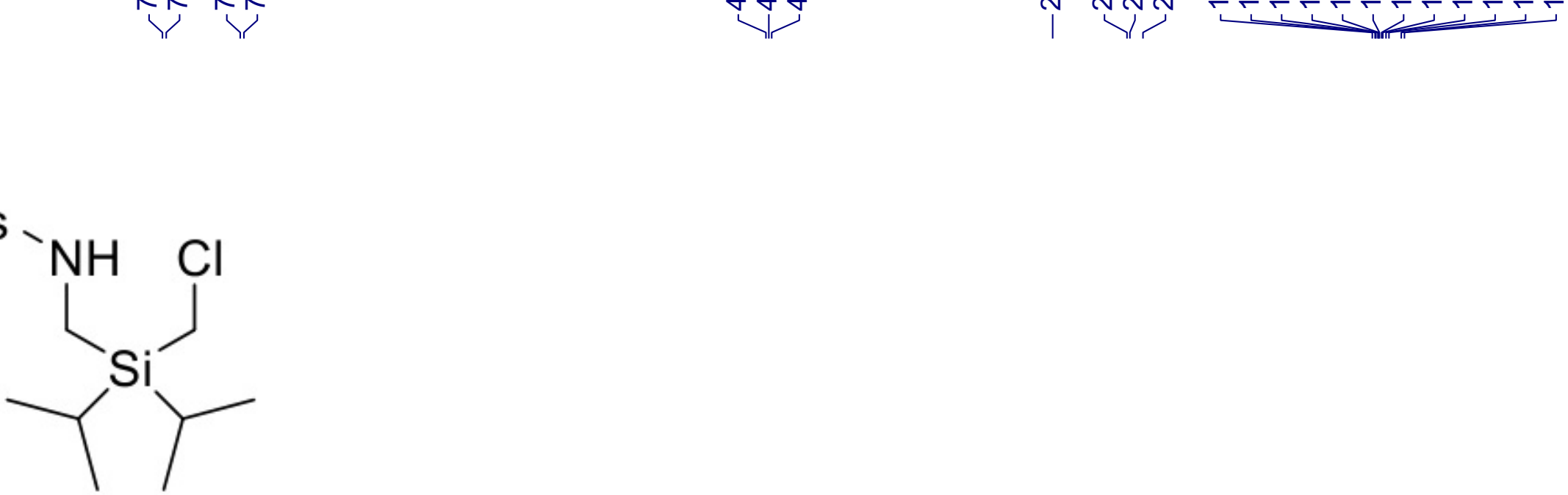

2g

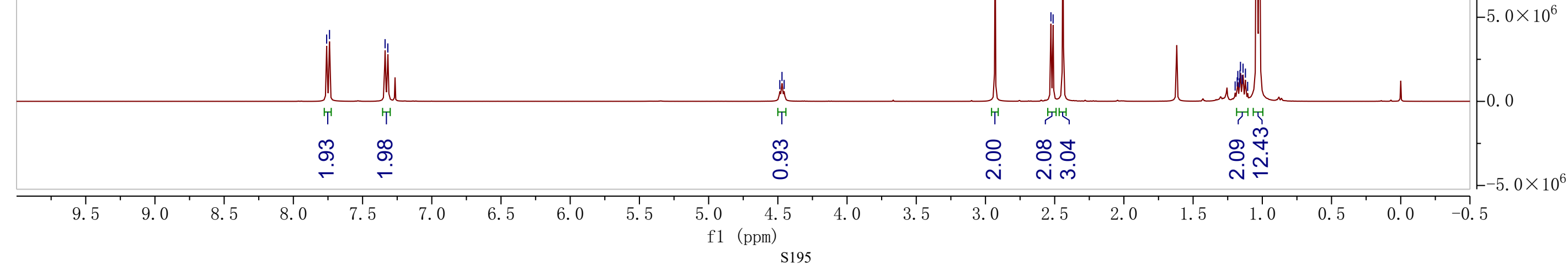




\section{Ts}

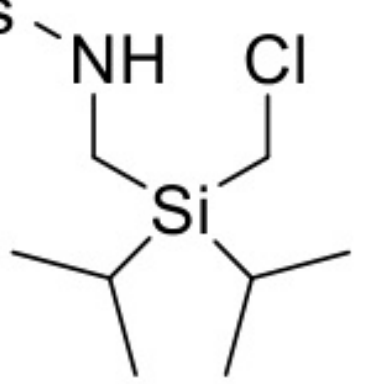

2g 


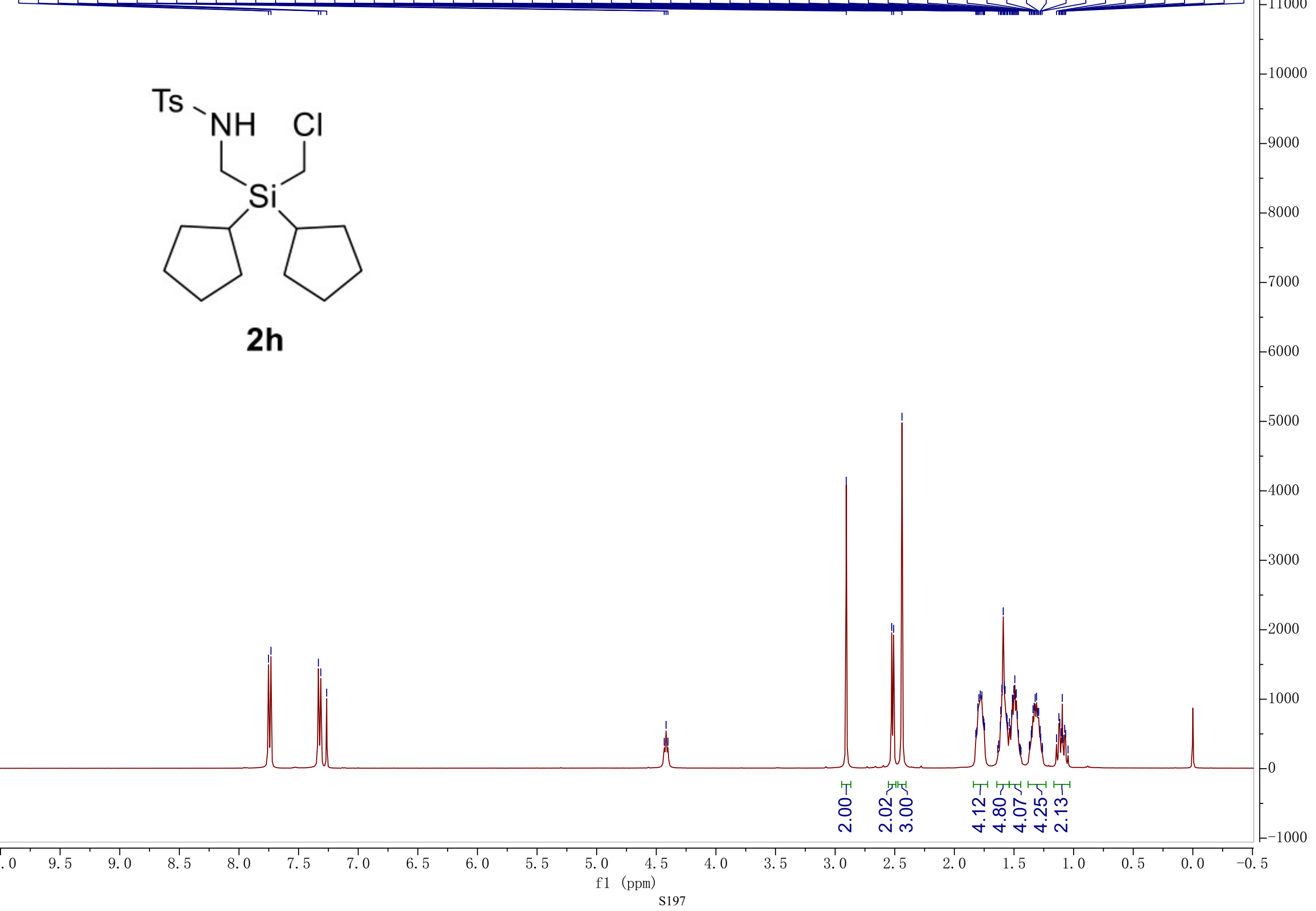


Ts<smiles>SNC[Si](CCl)(C1CCCC1)C1CCCC1</smiles>

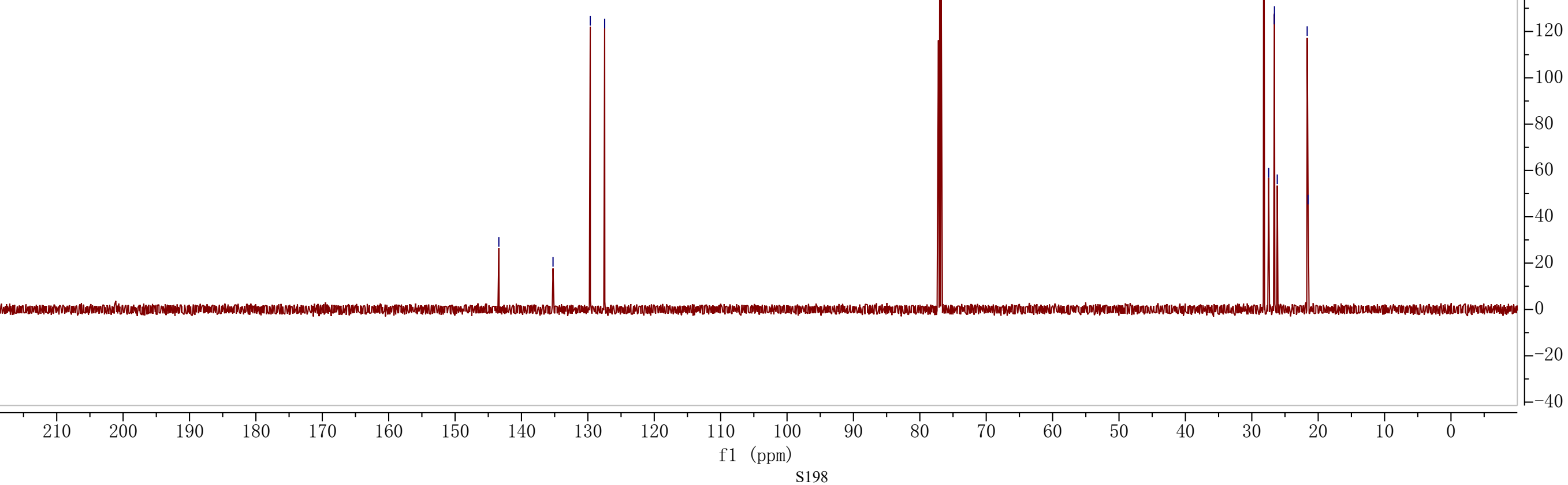


Ts<smiles>C=C[Si](C=C)(CCl)CNC</smiles>

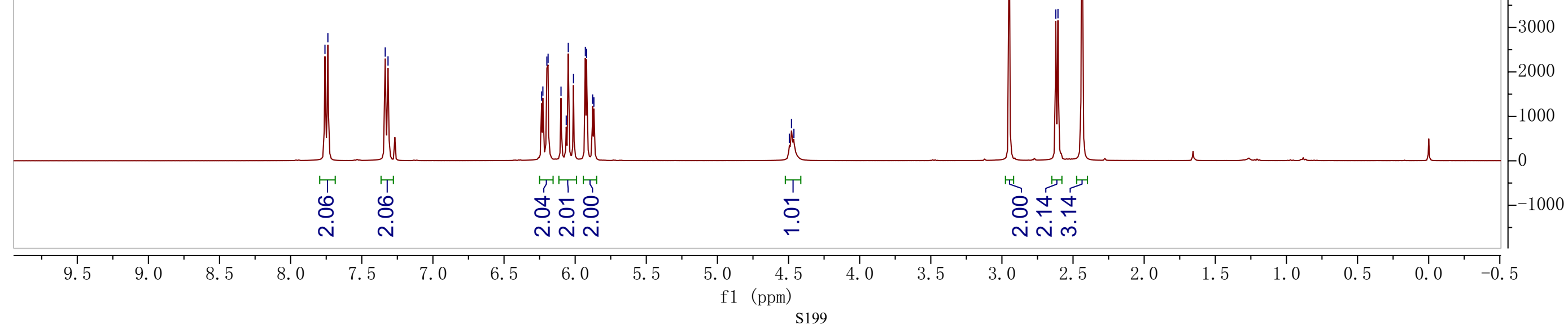


Ts<smiles>C=C[Si](C=C)(CCl)CNC</smiles>

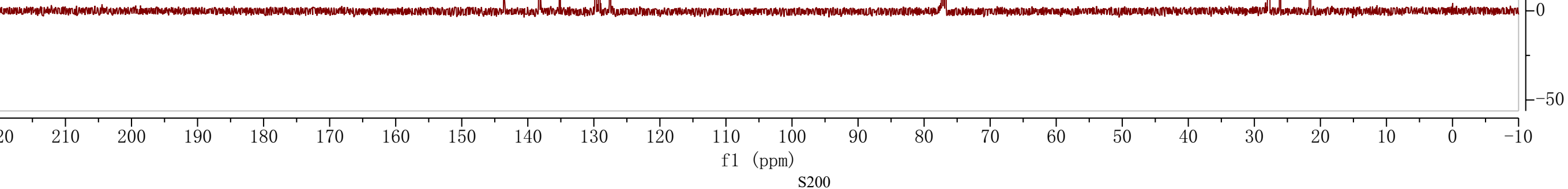



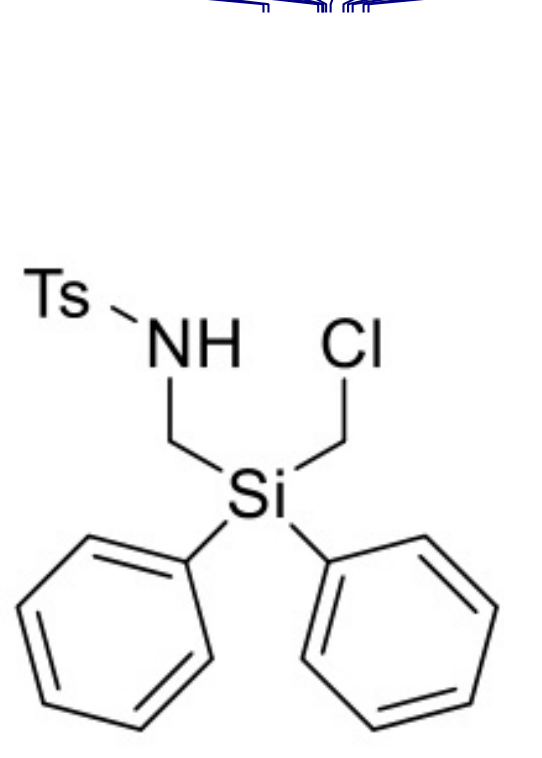

2j

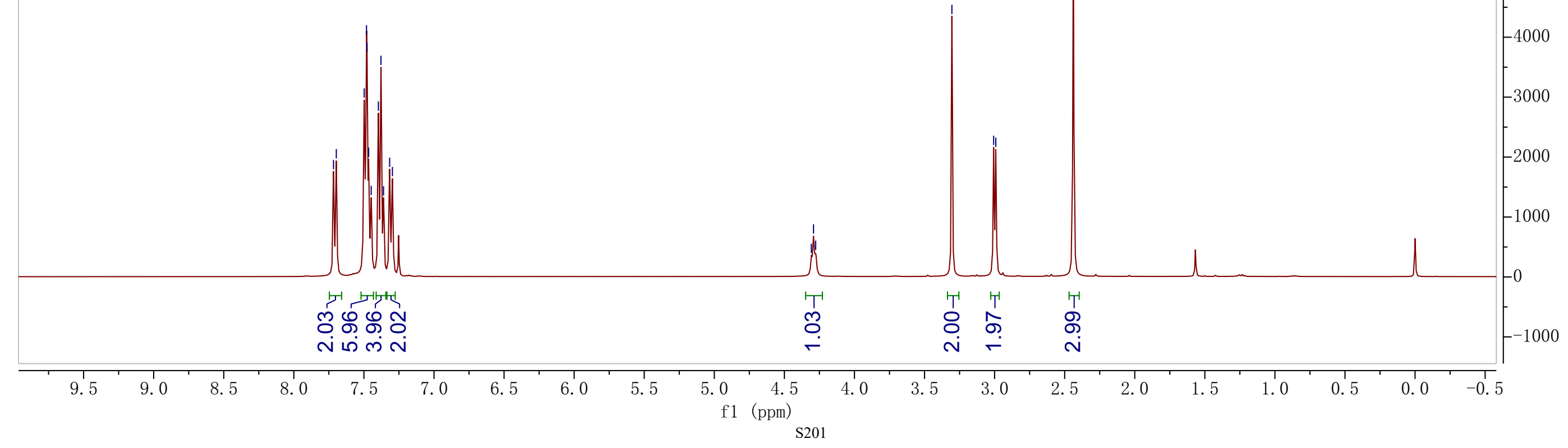


$\mathrm{NH} \mathrm{Cl}$<smiles>CC[Si](CN)(c1ccccc1)c1ccccc1</smiles>

2j 


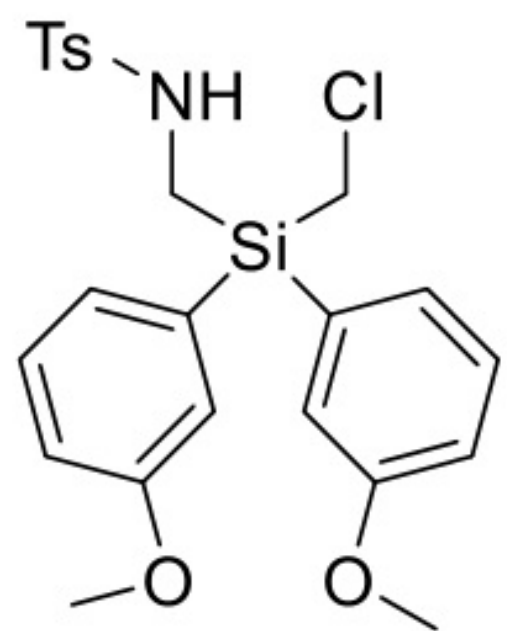

2k

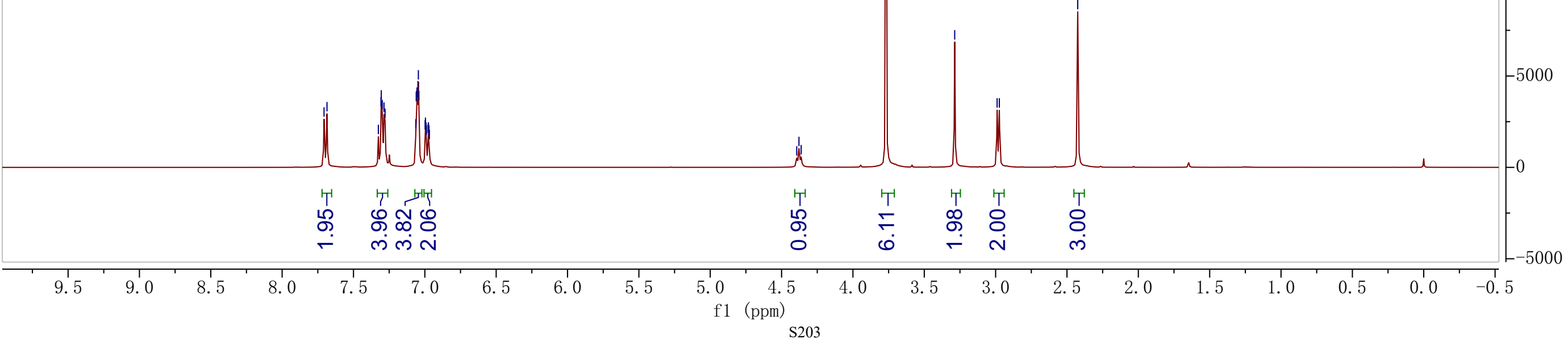


Ts $-\mathrm{NH} \quad \mathrm{Cl}$<smiles>COc1cccc([Si](C[14CH3])(C[14CH3])c2cccc(OC)c2)c1</smiles>

2k 


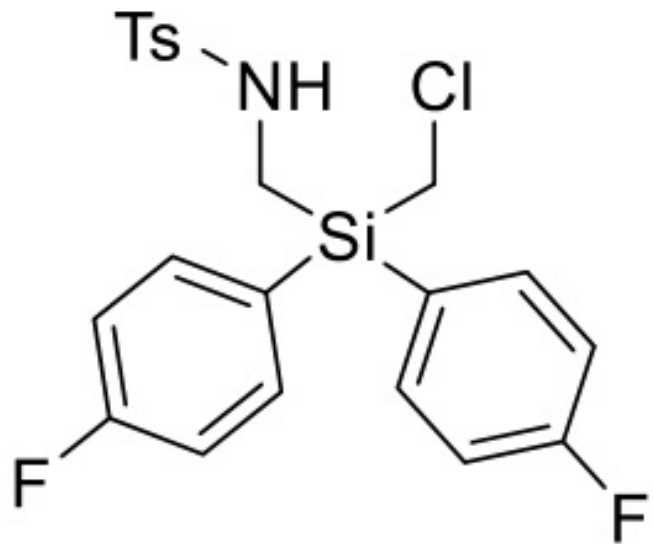

\section{I}

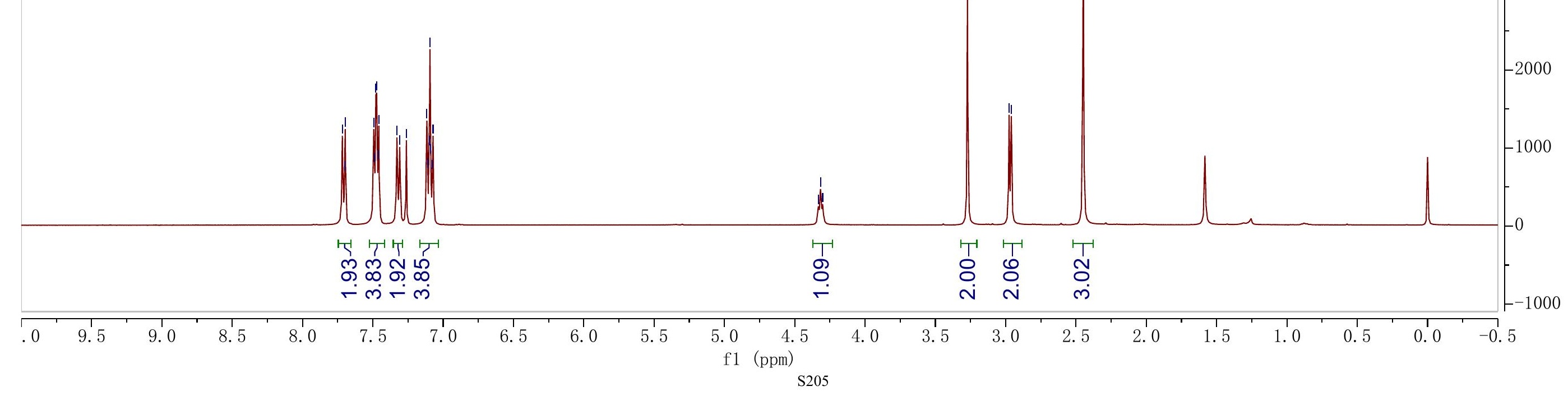




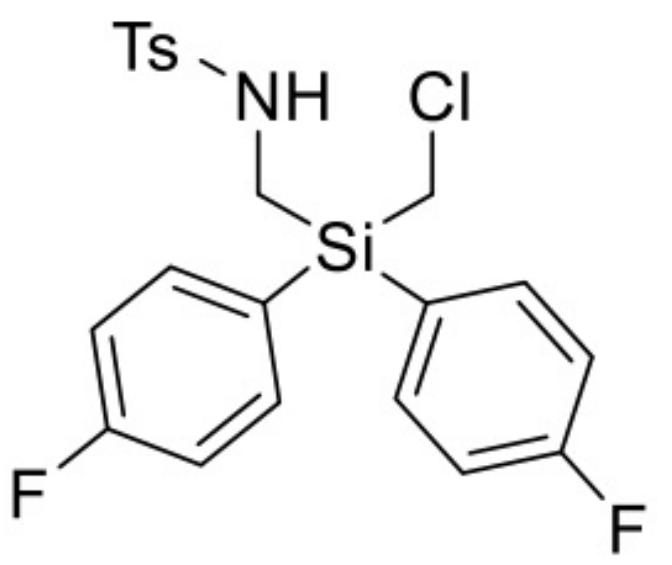

2I

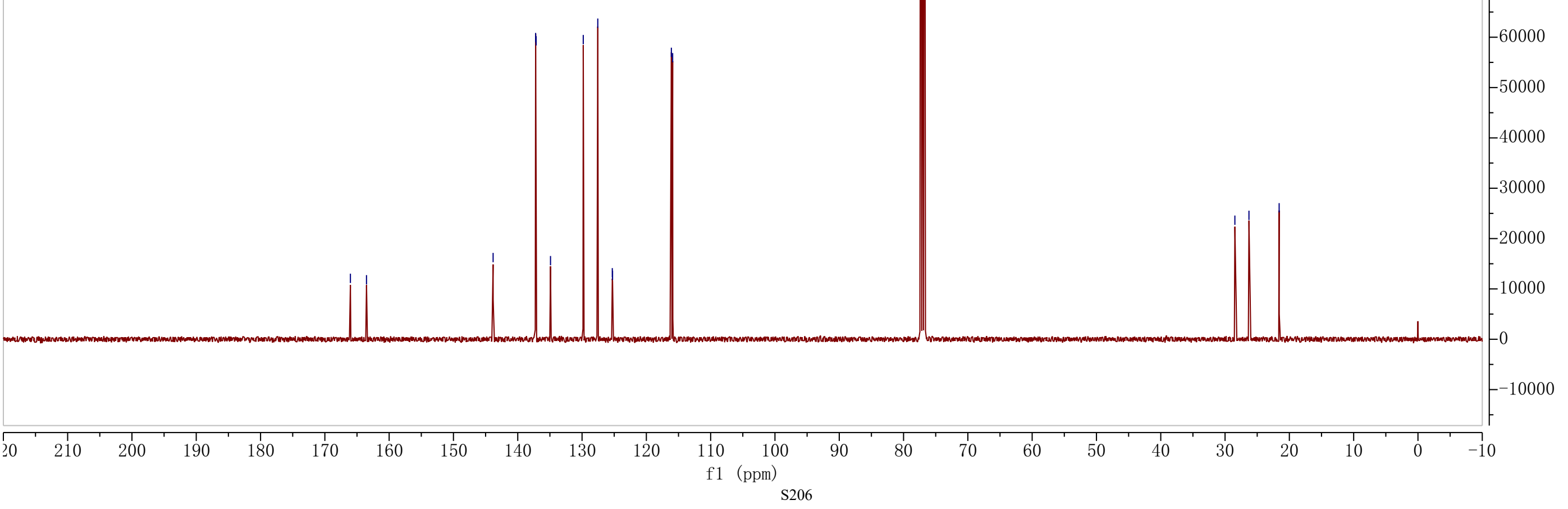


${ }^{19}$ F NMR

Ts<smiles>Fc1ccc([Si](CCl)(CNS)c2ccc(F)cc2)cc1</smiles>

2I 
Ts

$\mathrm{NH} \quad \mathrm{Cl}$<smiles>CC[Si](C)(C)C</smiles><smiles>Cc1ccccc1</smiles>

$2 m$

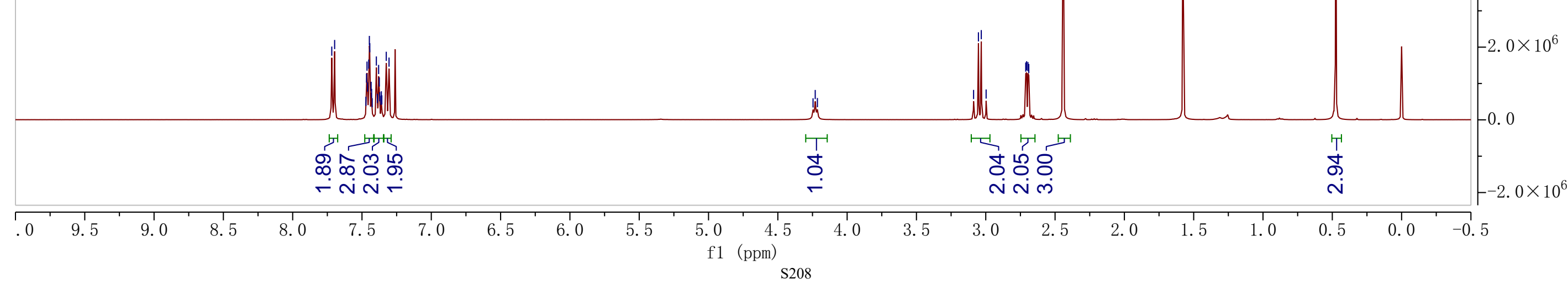




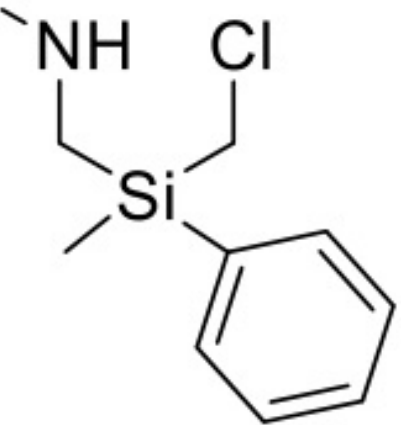

$2 m$ 
Ts<smiles>CNC[Si]1(CCl)CCCC1</smiles>

2n

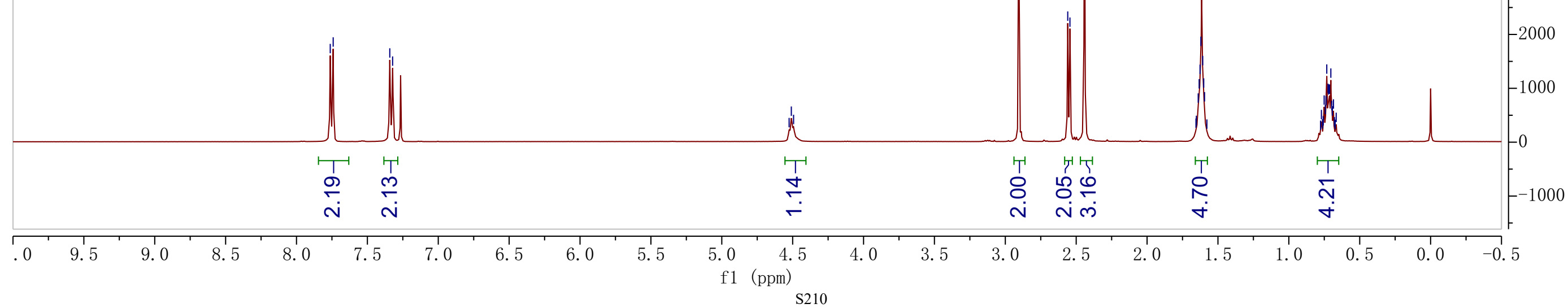


Ts<smiles>CNC[Si]1(CCl)CCCC1</smiles>

2n

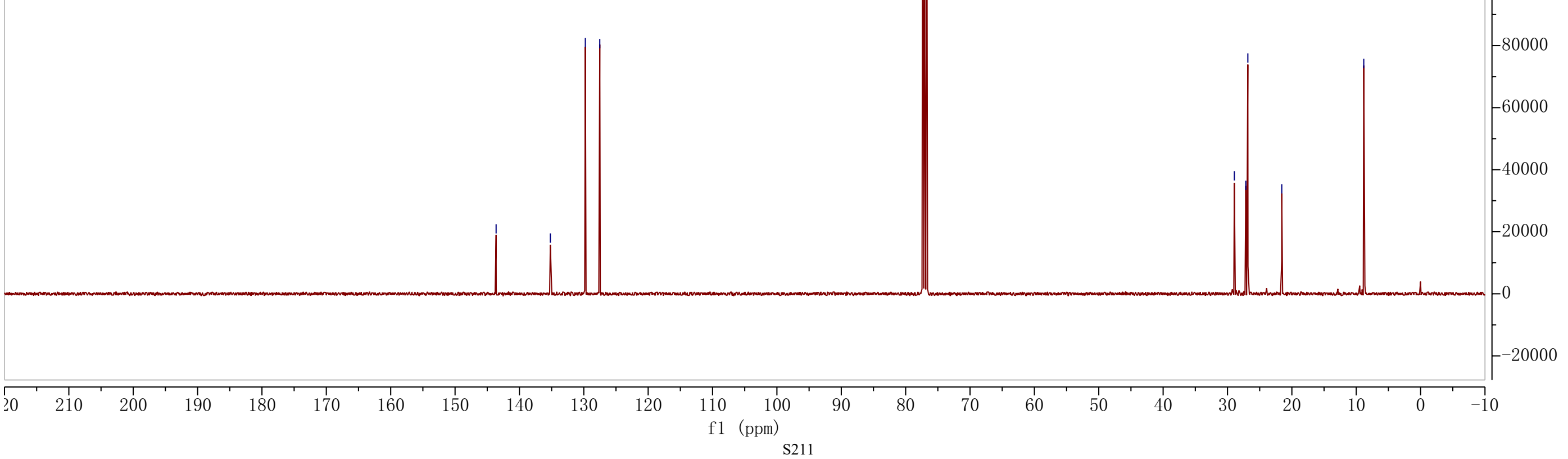




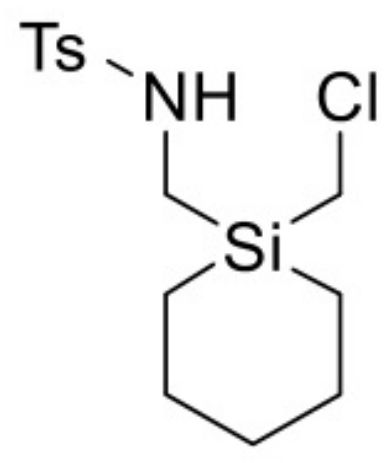

20

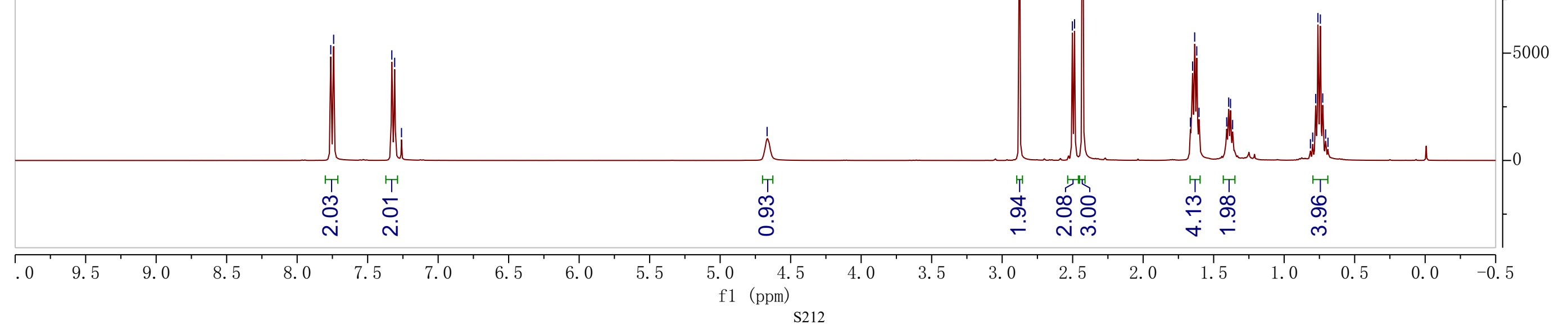


Ts<smiles>CNC[Si]1(CCl)CCCCC1</smiles>

$\mathrm{Cl}$

20

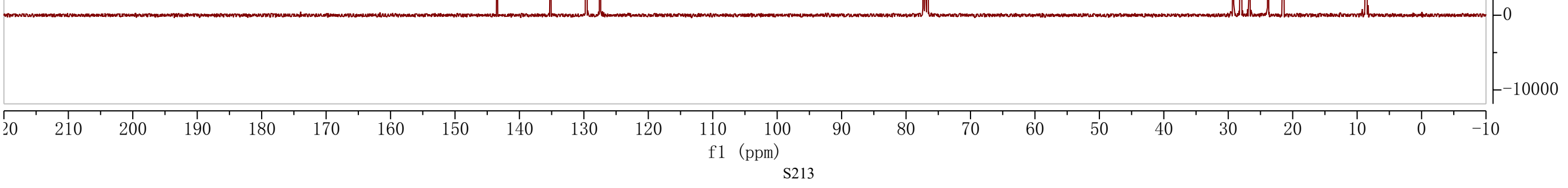


Ts<smiles>NC[Si]1(CCl)CCc2ccccc2C1</smiles>

\section{$2 p$}

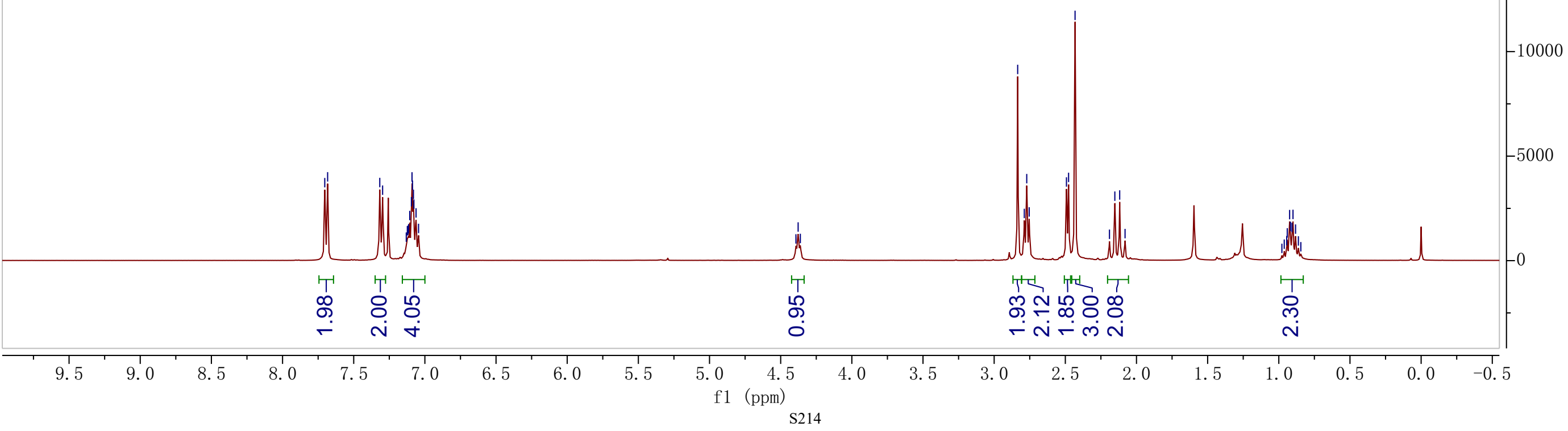


Ts<smiles>CNC[Si]1(CCl)CCc2ccccc2C1</smiles> 


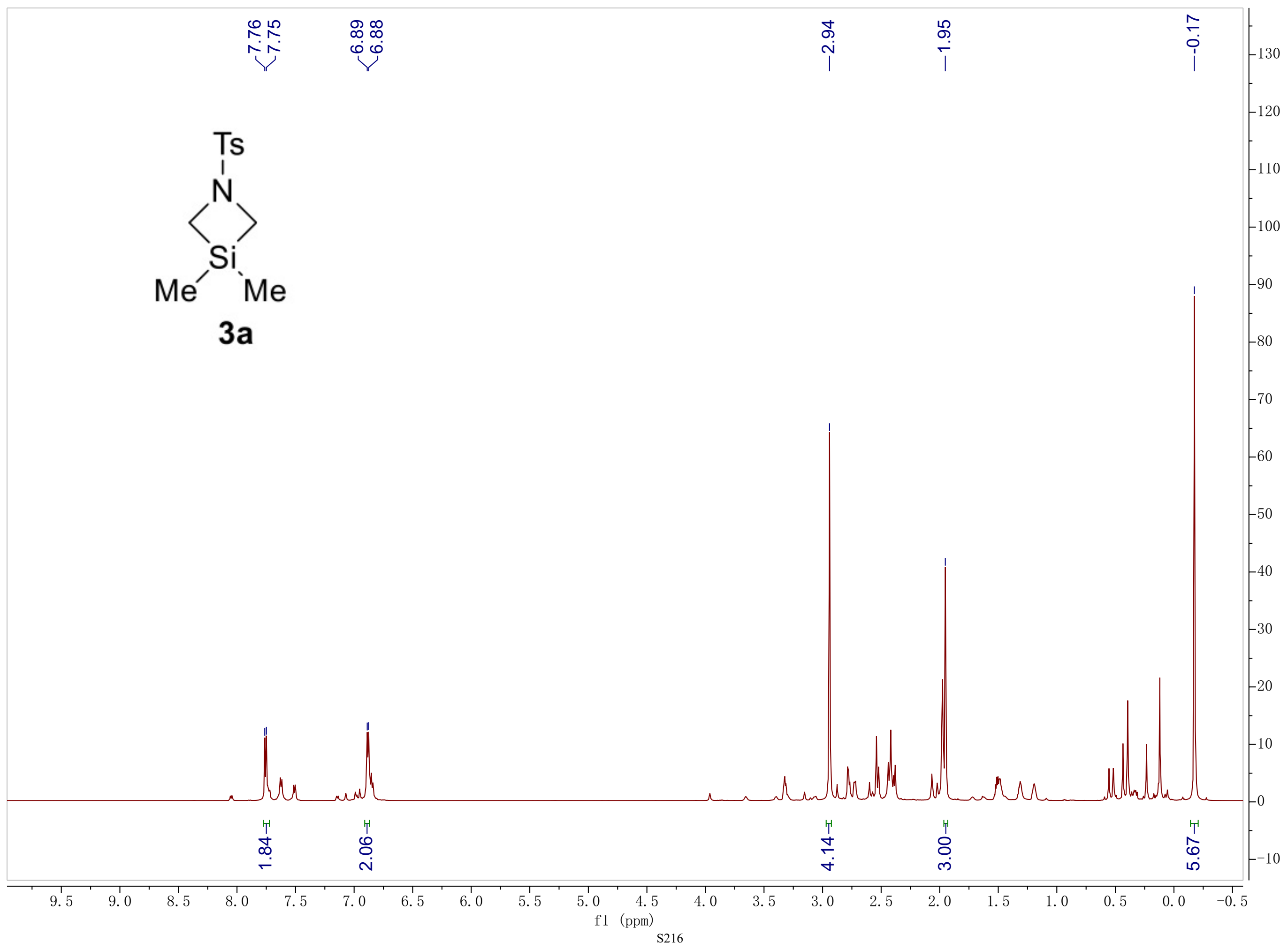


${ }^{29}$ Si NMR

Si NIR

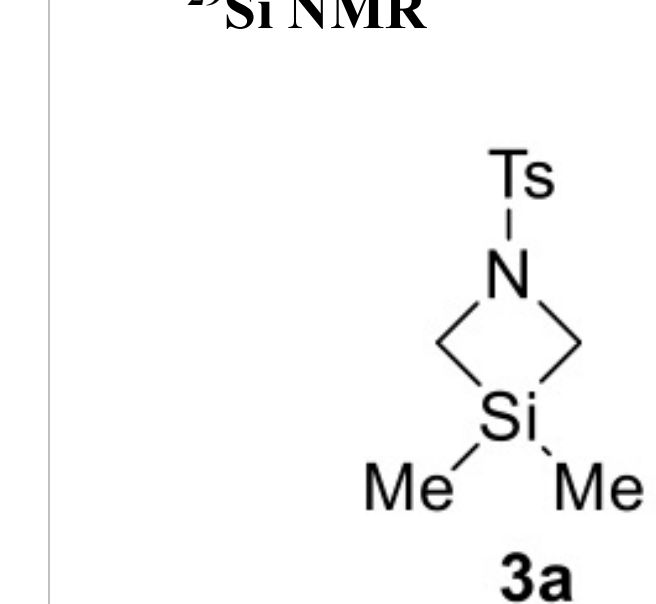



(1)

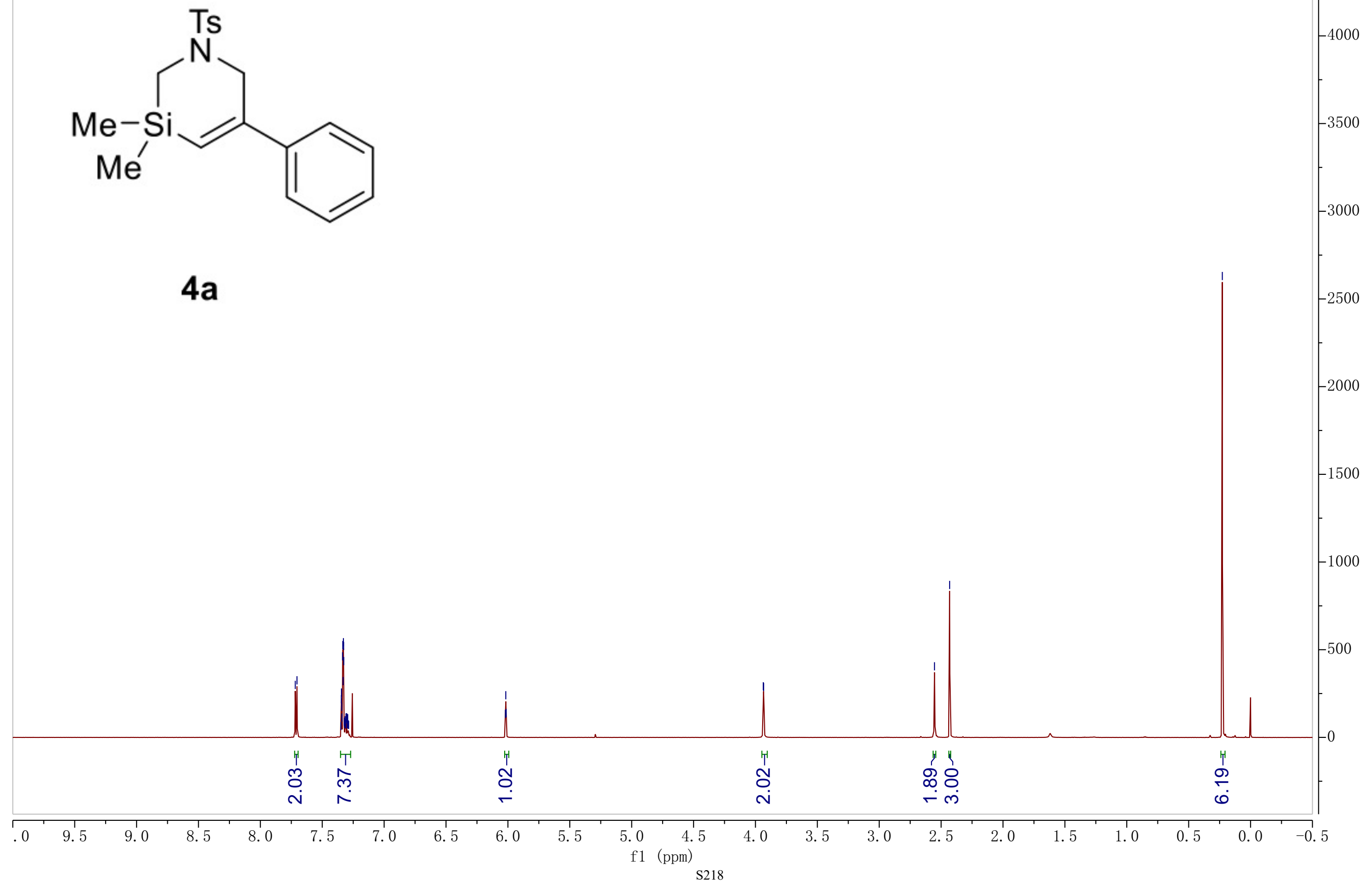




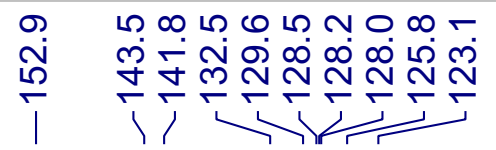

ペ゚

|

n?

กั

$-150000$<smiles>C[Si]1(C)C=C(c2ccccc2)CN([As])C1</smiles>

$4 a$
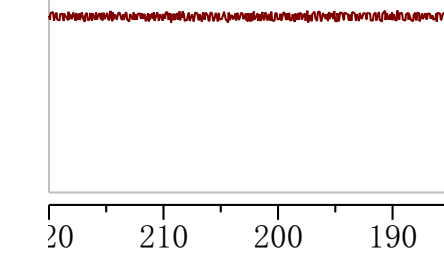

180

170

160

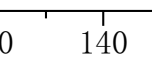

130 
${ }^{29}$ Si NMR<smiles>C[Si]1(C)C=C(c2ccccc2)CN([As])C1</smiles>

$4 a$ 


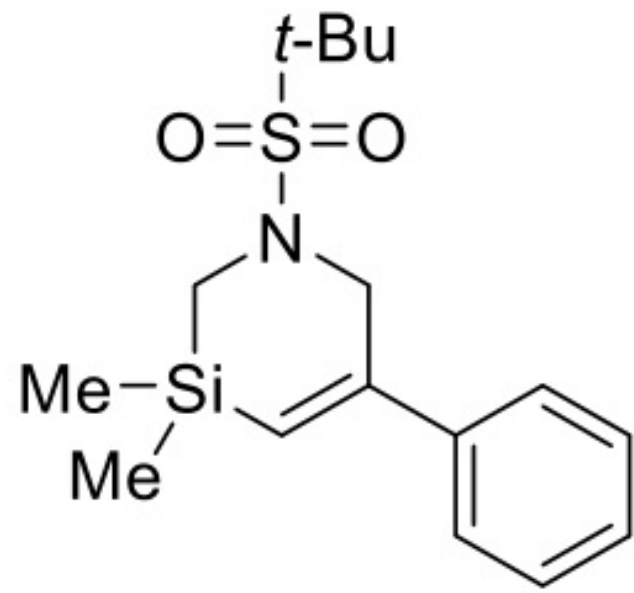

4b

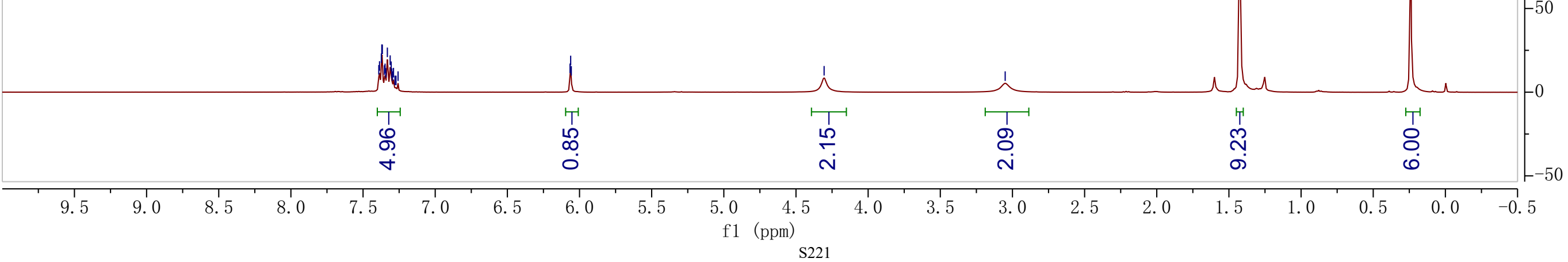




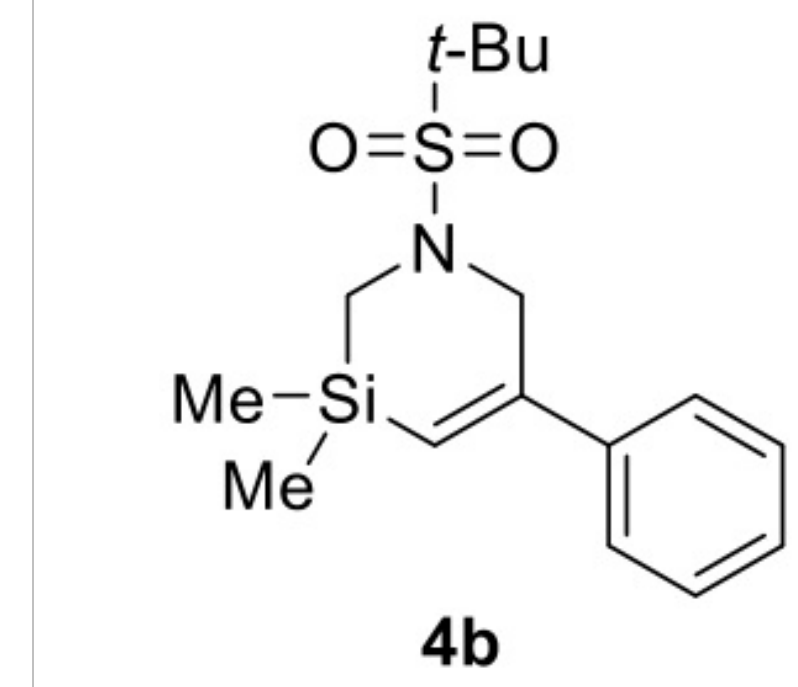


$\mathrm{Me}$<smiles>C[Si]1(C)C=C(c2ccccc2)CN(S(C)(=O)=O)C1</smiles>

4c

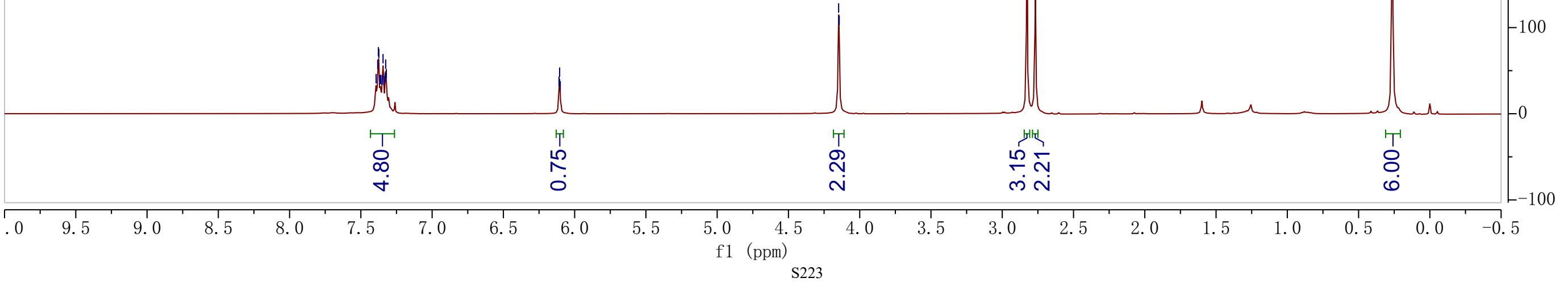


<smiles>C[Si]1(C)C=C(c2ccccc2)CN(S(C)(=O)=O)C1</smiles>

\section{4c}

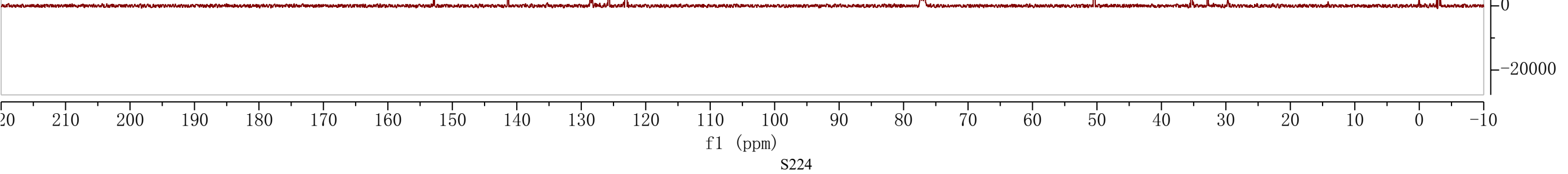


<smiles>Cc1ccccc1C1=C[Si](C)(C)CN([AsH3])C1</smiles>

$4 e$

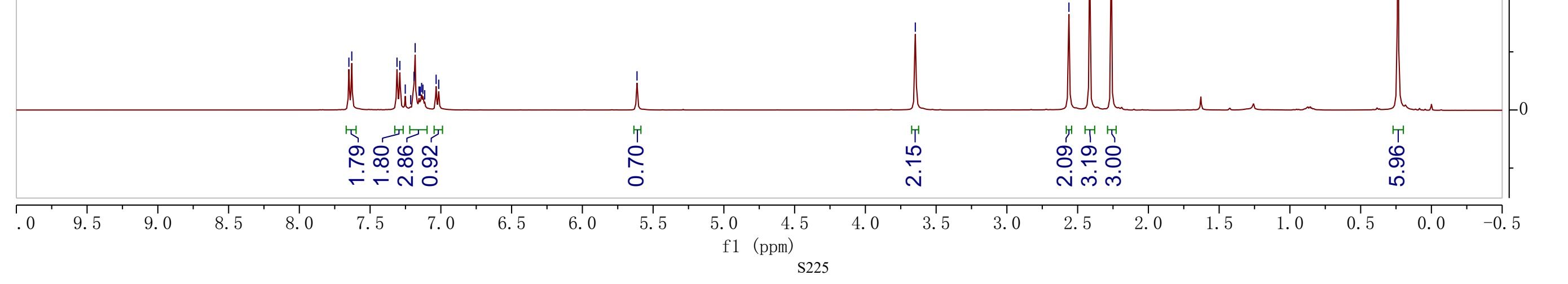


<smiles>Cc1ccccc1C1=C[Si](C)(C)CN([As])C1</smiles>

$4 e$

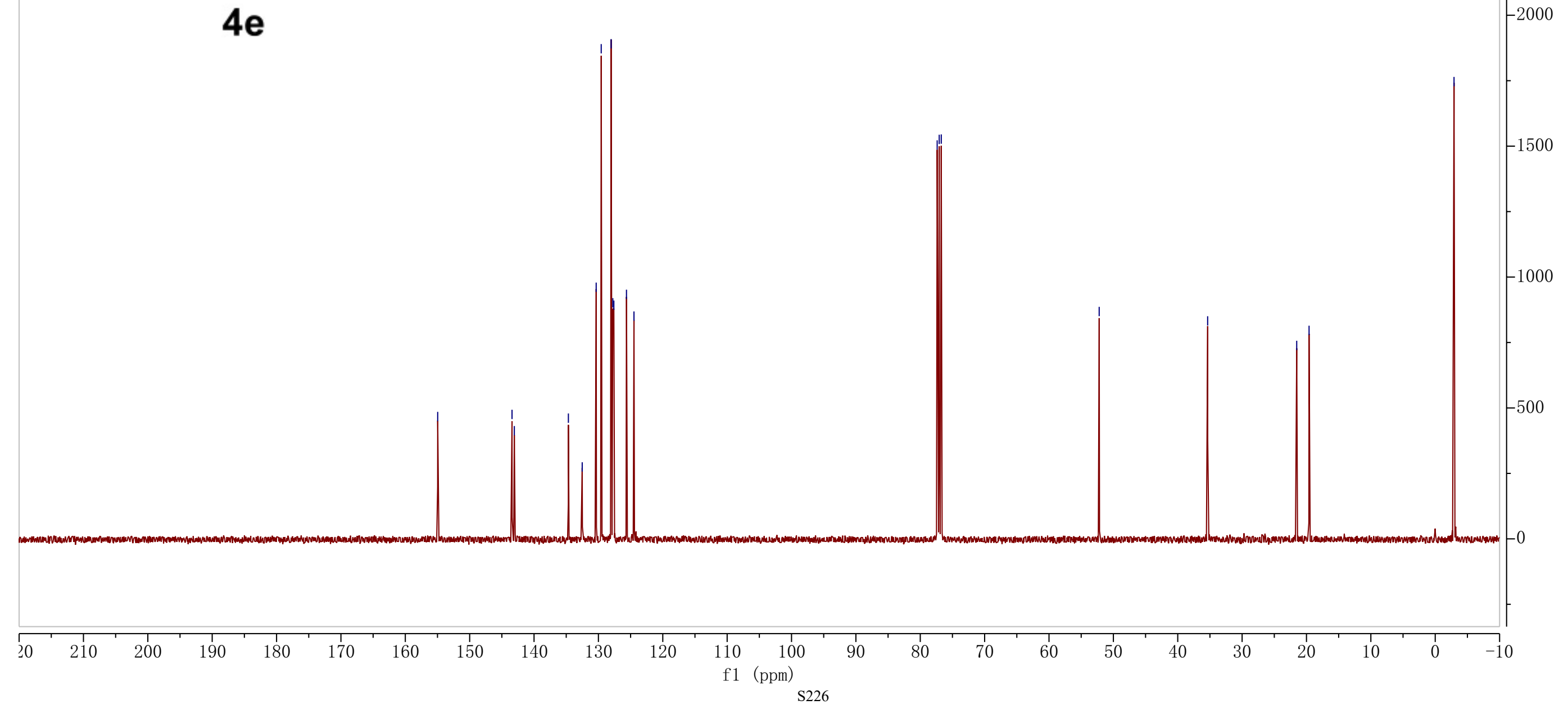


<smiles>Cc1cccc(C2=C[Si](C)(C)CN([As])C2)c1</smiles>

\section{4f}

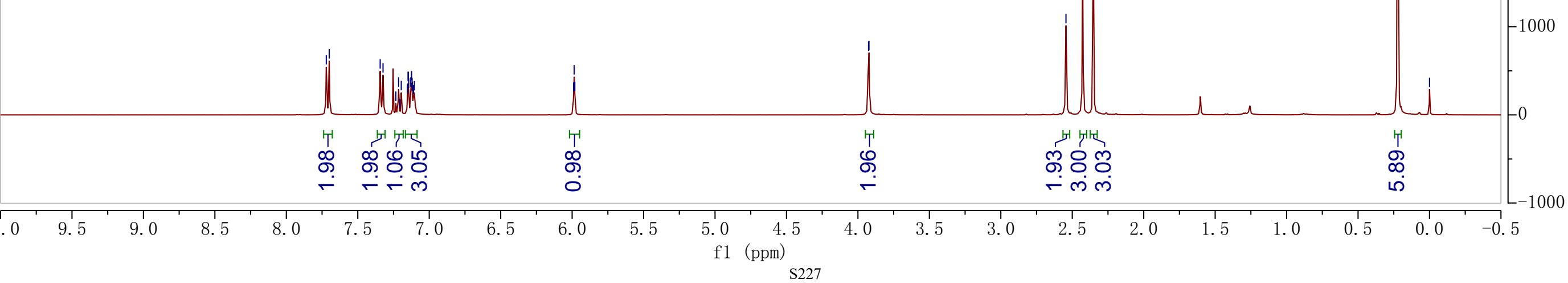


<smiles>Cc1cccc(C2=C[Si](C)(C)CN([As])C2)c1</smiles>

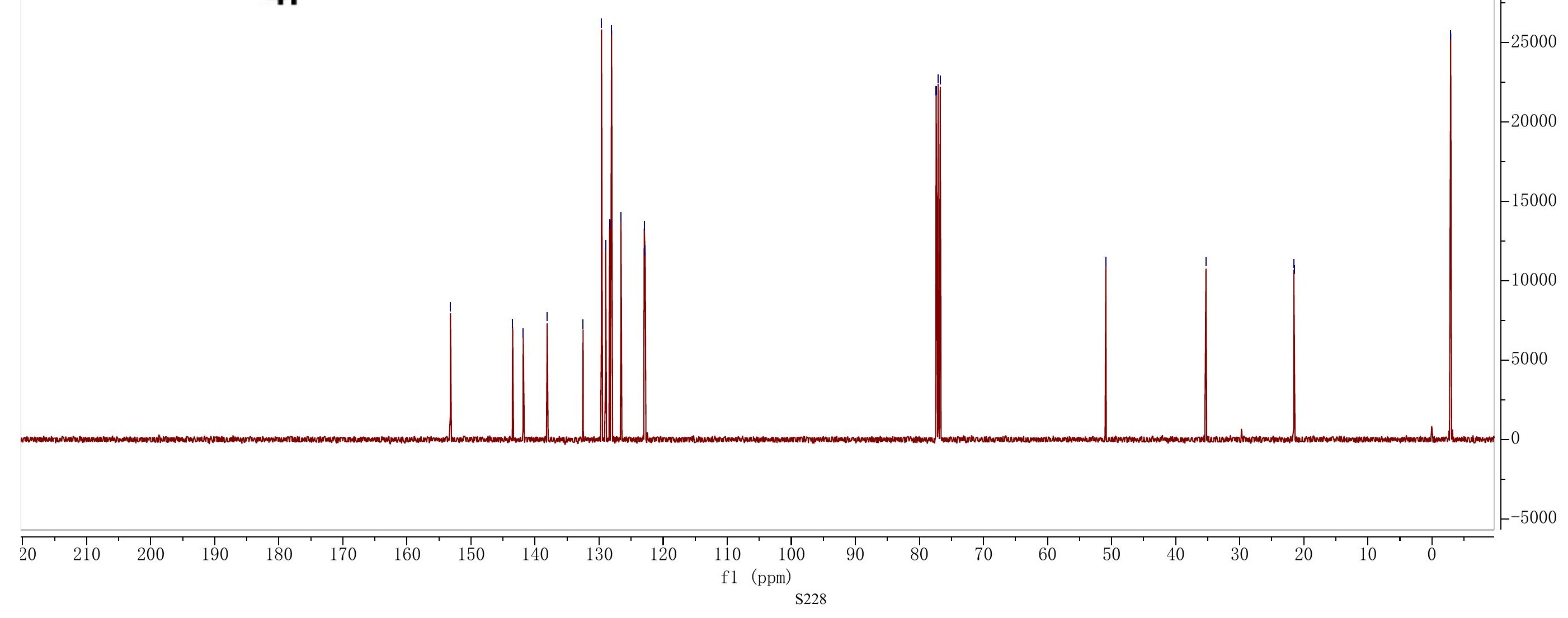


<smiles>Cc1ccc(C2=C[Si](C)(C)CN([As])C2)cc1</smiles>

\section{$4 g$}

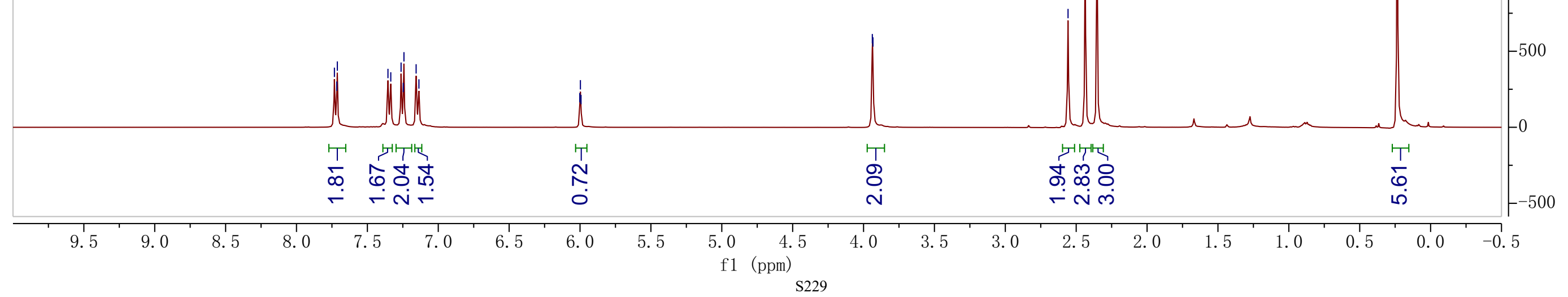


Ts

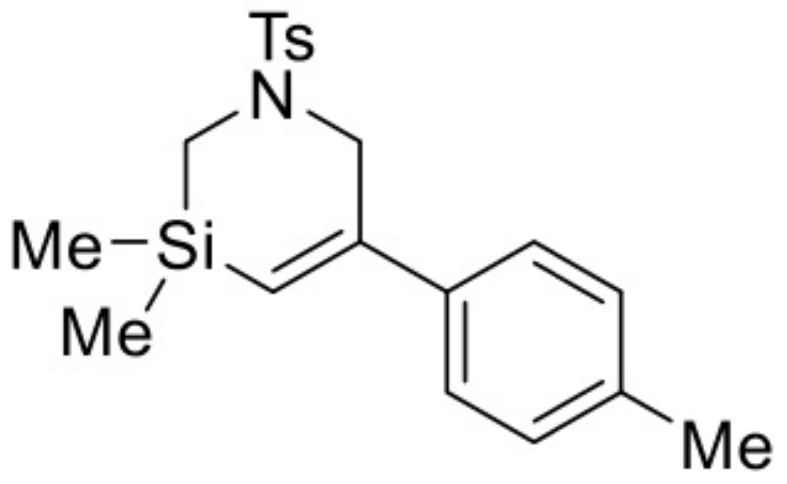

4g

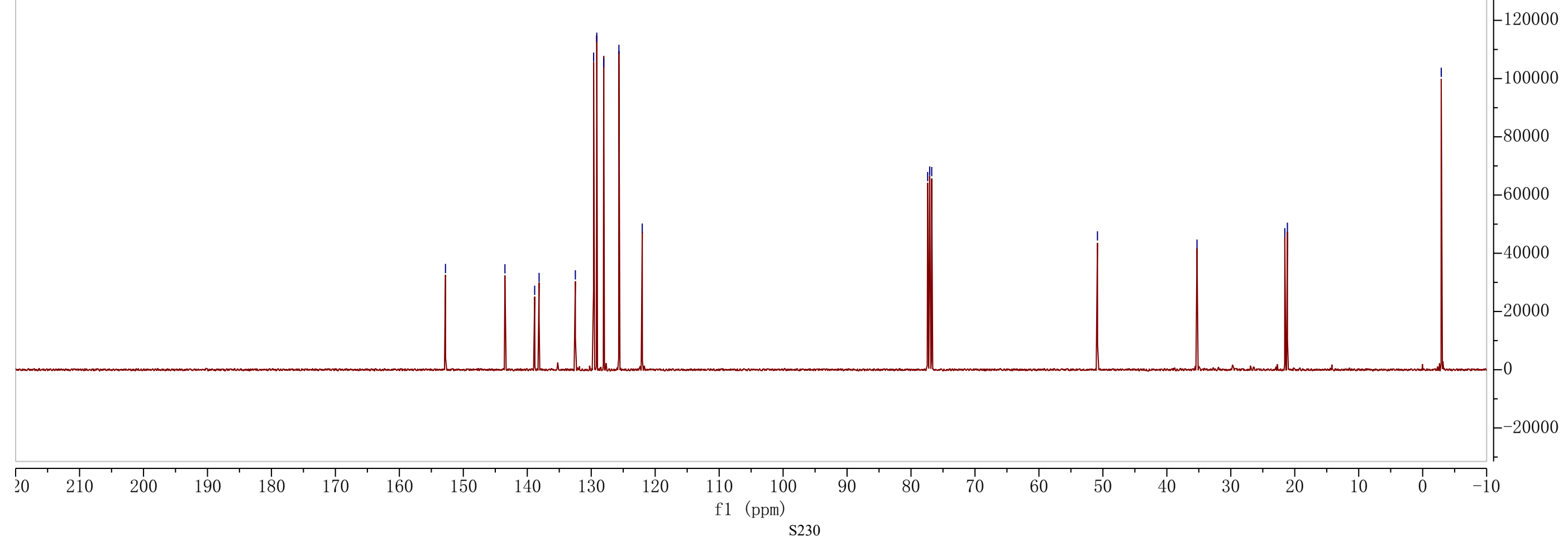




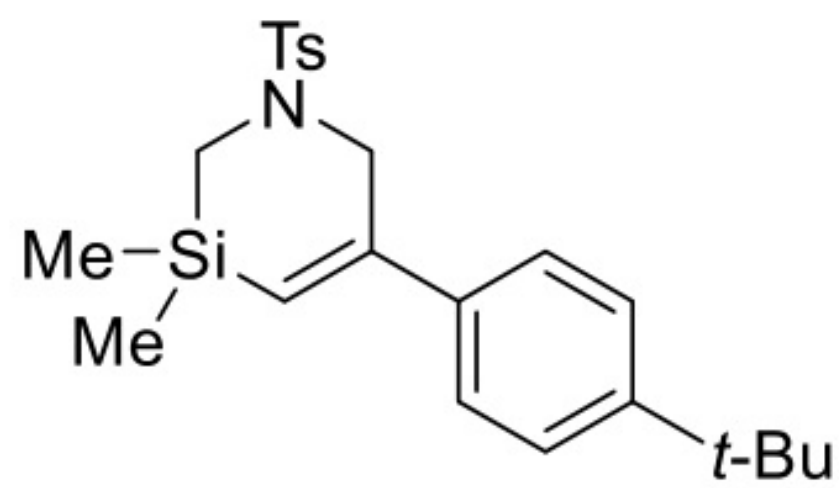

4h

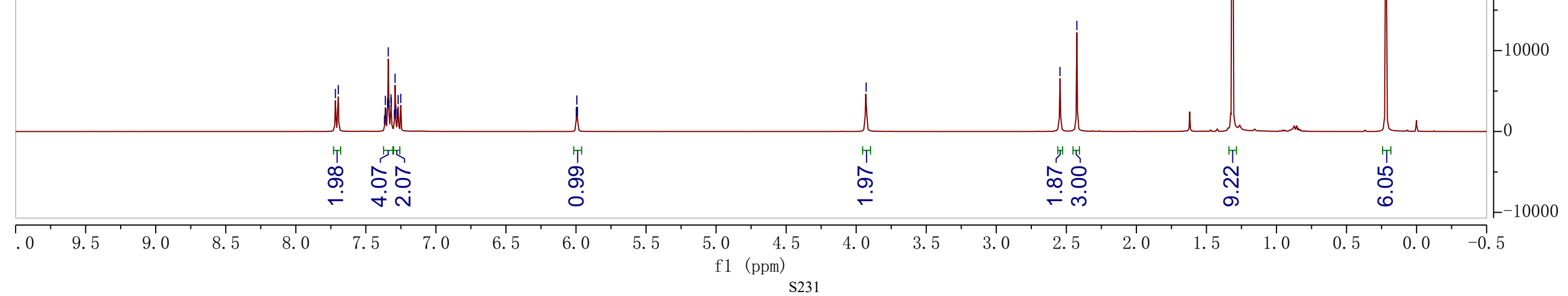




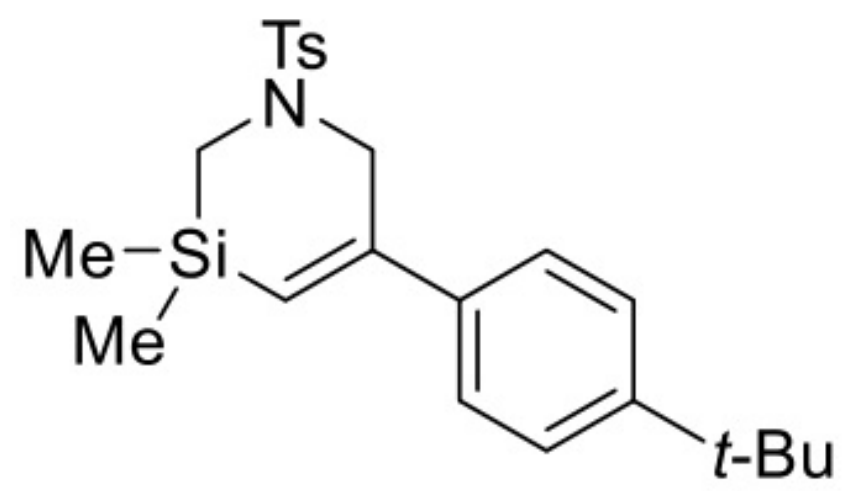

\section{4h}

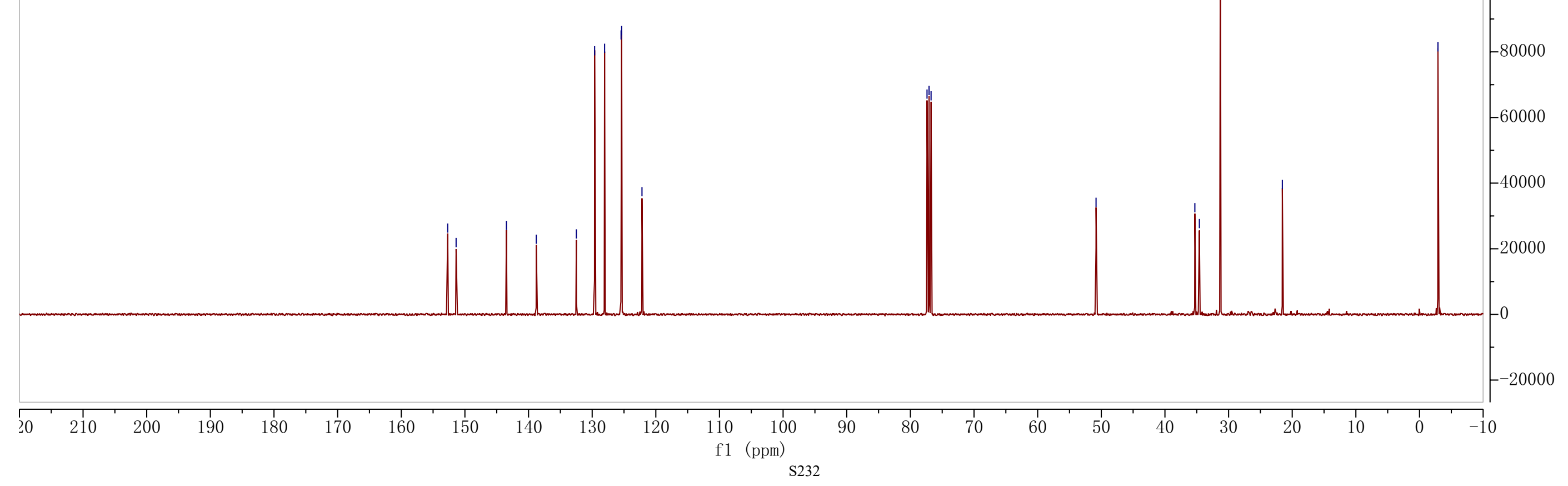




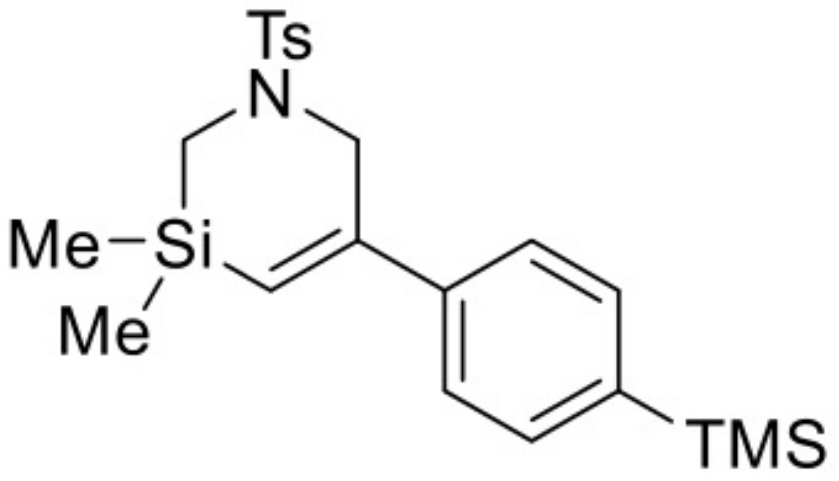

\section{$4 i$}

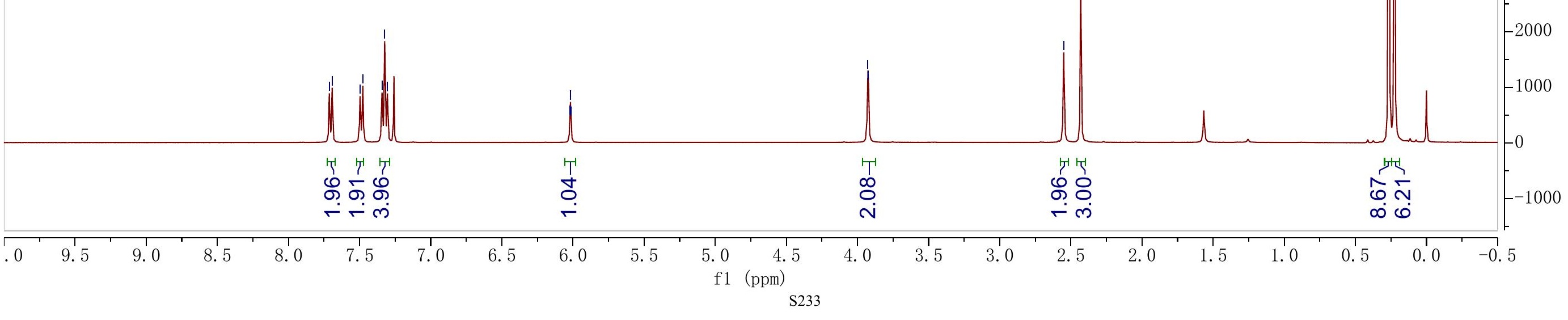


<smiles>[CH2-][NH+]1CC(c2ccc(S(C)(=O)=O)cc2)=C[Si](C)(C)C1</smiles>

$4 \mathbf{i}$

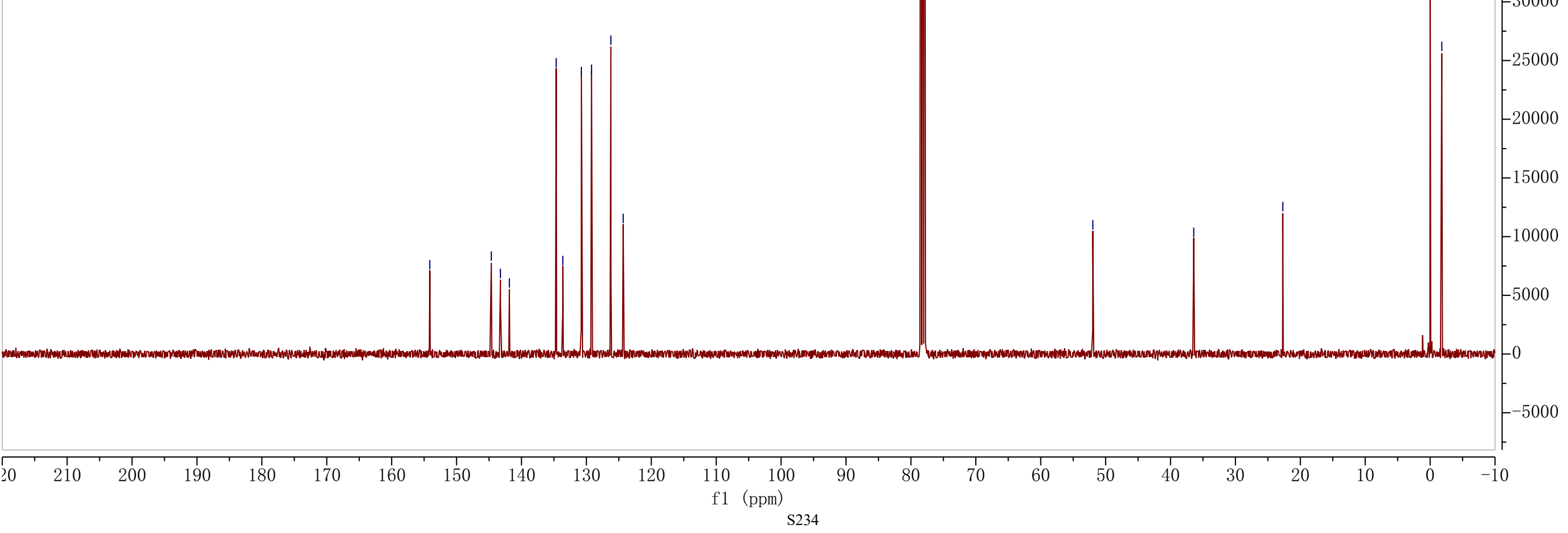


<smiles>C[Si]1(C)C=C(c2ccc(-c3ccccc3)cc2)CN([As])C1</smiles>

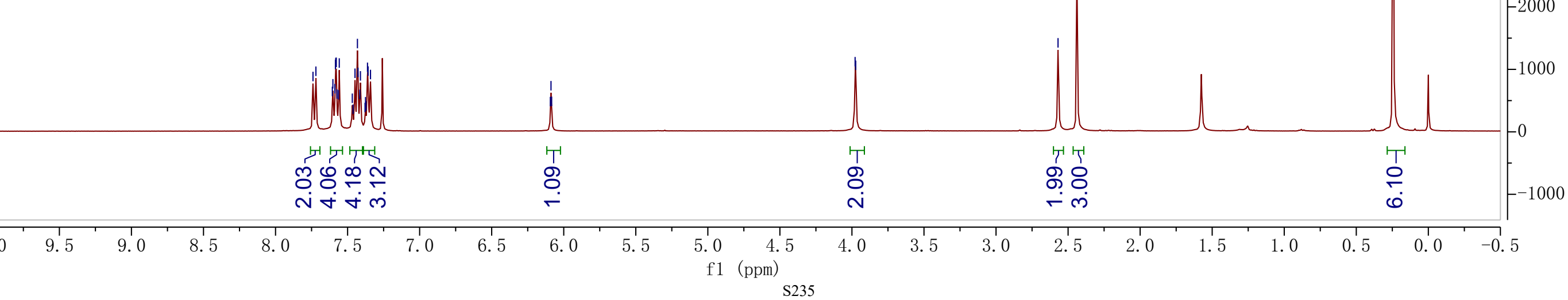


垈<smiles>C[Si]1(C)C=C(c2ccc(-c3ccccc3)cc2)CN([As])C1</smiles>

4j

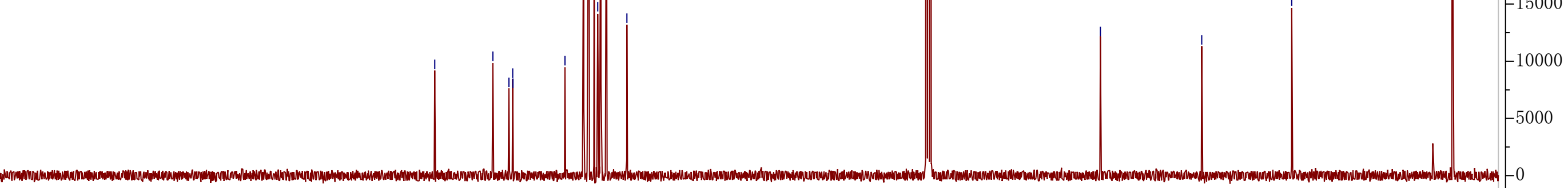


<smiles>COc1ccc(C2=C[Si](C)(C)CN([As])C2)cc1</smiles>

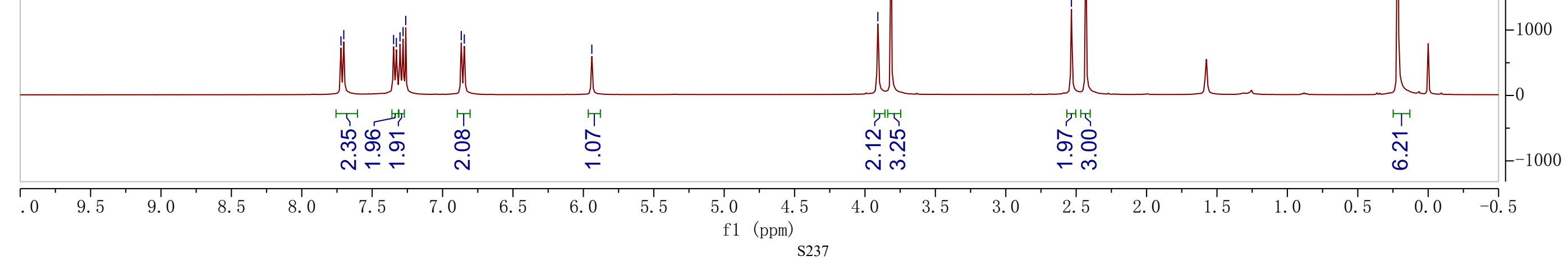


官

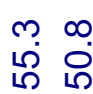

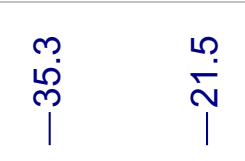
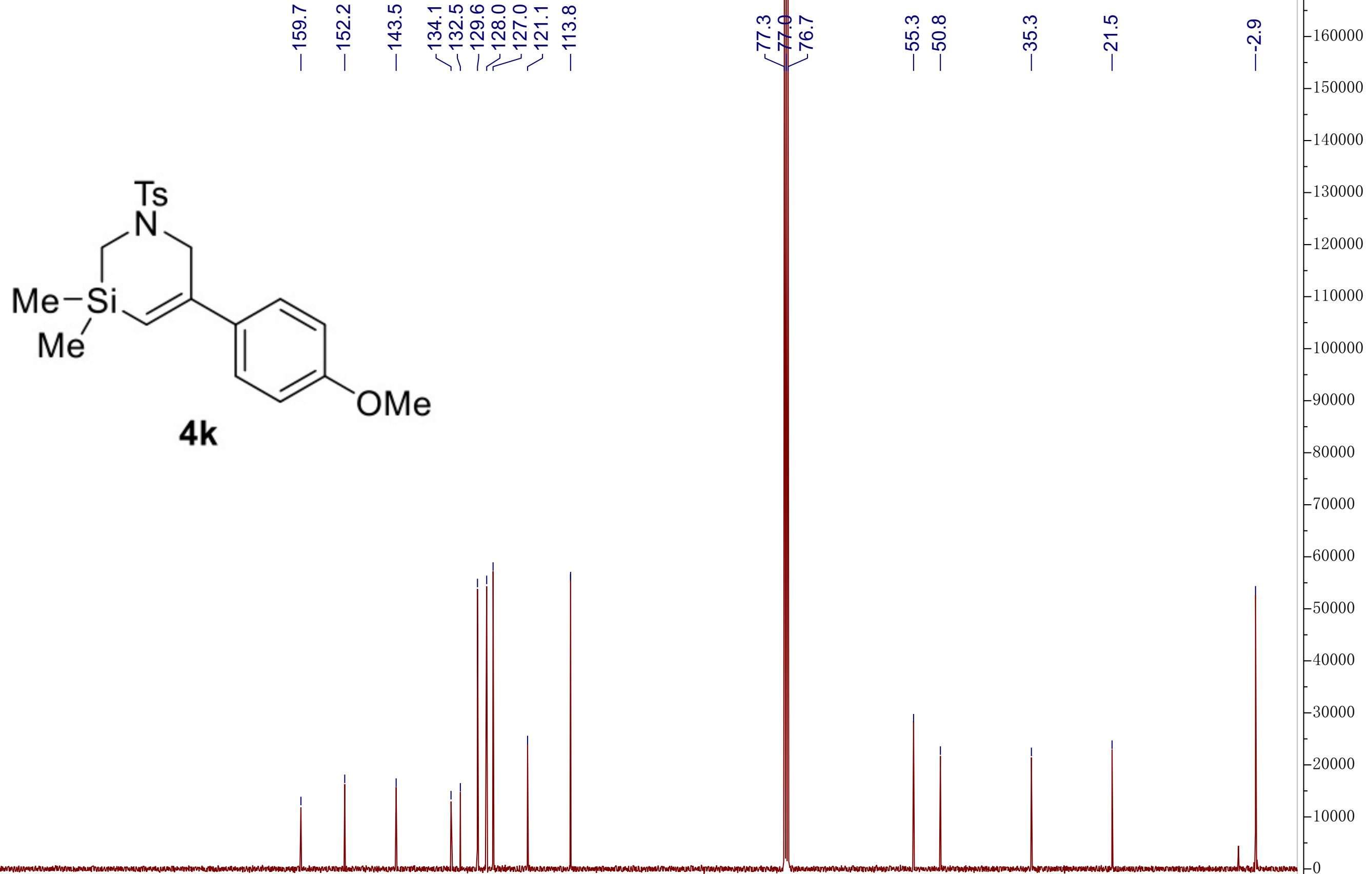


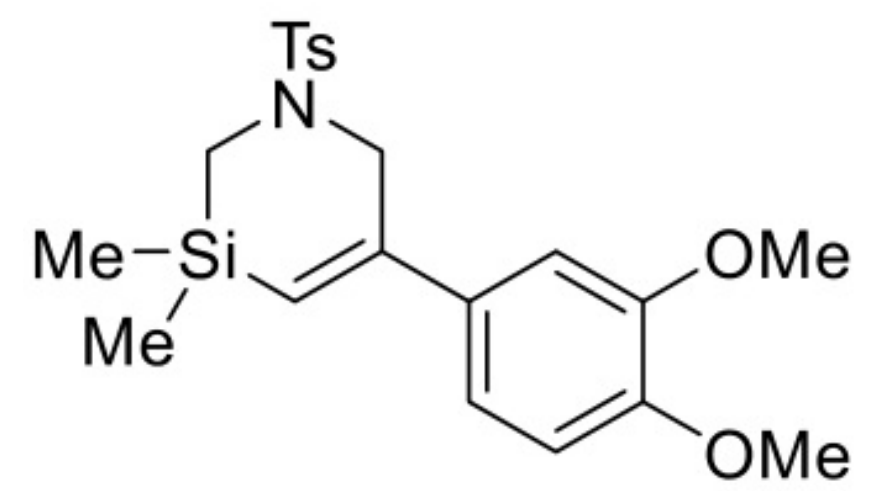

$\mathrm{Me}$

\section{I}

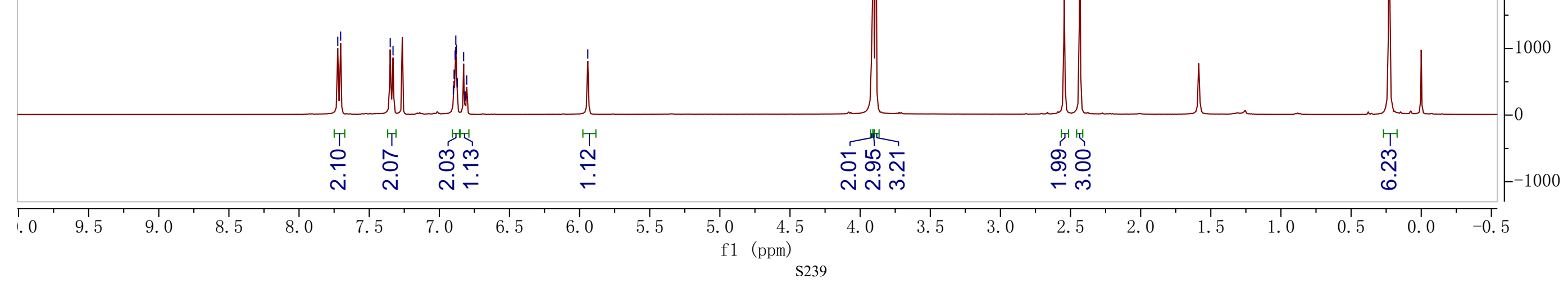




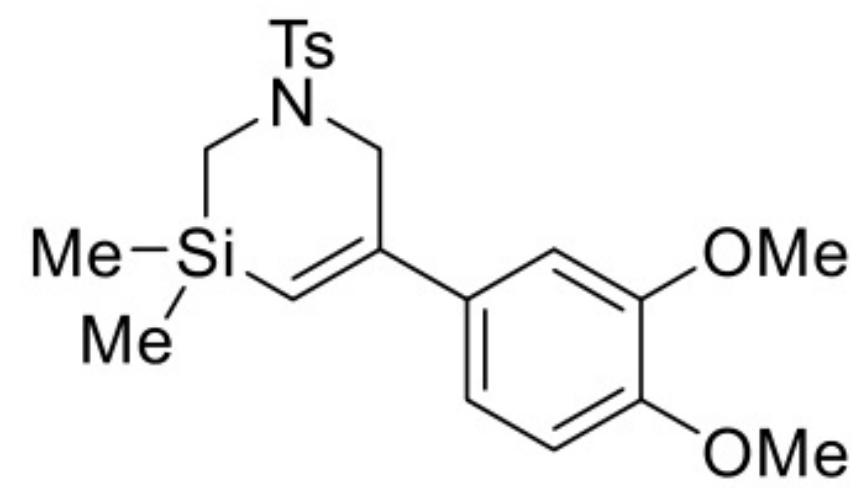

4I

41 
<smiles>C[Si]1(C)C=C(c2ccc3c(c2)OCO3)CN([As])C1</smiles>

\section{$4 m$}

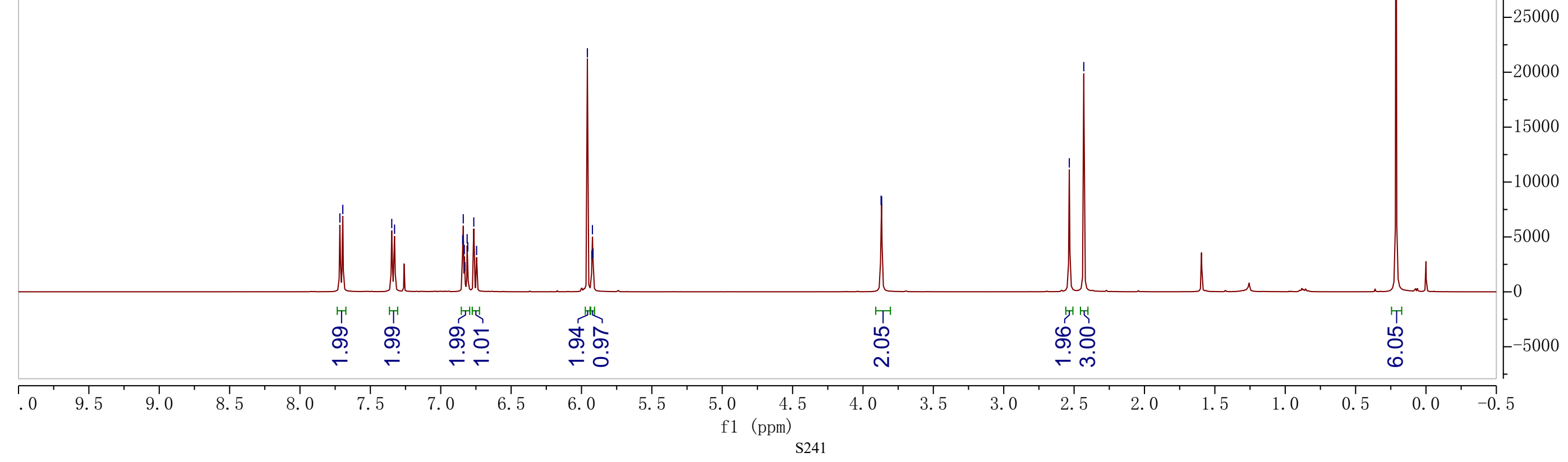


<smiles>C[Si]1(C)C=C(c2ccc3c(c2)OCO3)CN([AsH3])C1</smiles>

$4 m$ 


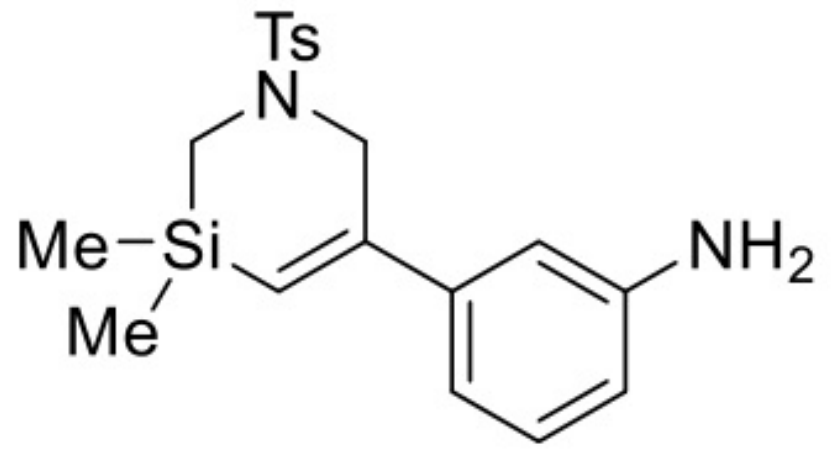

$4 n$

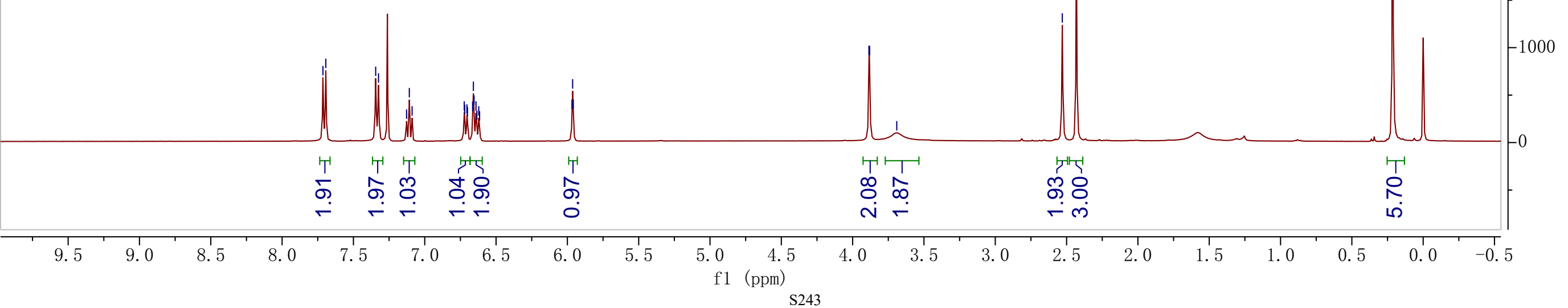


mオL下 L

กี่

政<smiles>C[Si]1(C)C=C(c2cccc(N)c2)CN([As])C1</smiles>

$4 n$ 


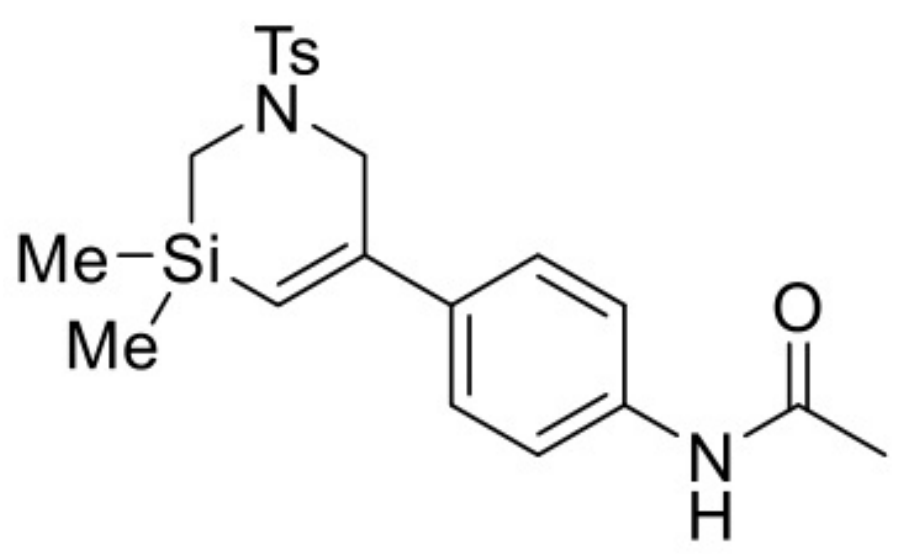

40

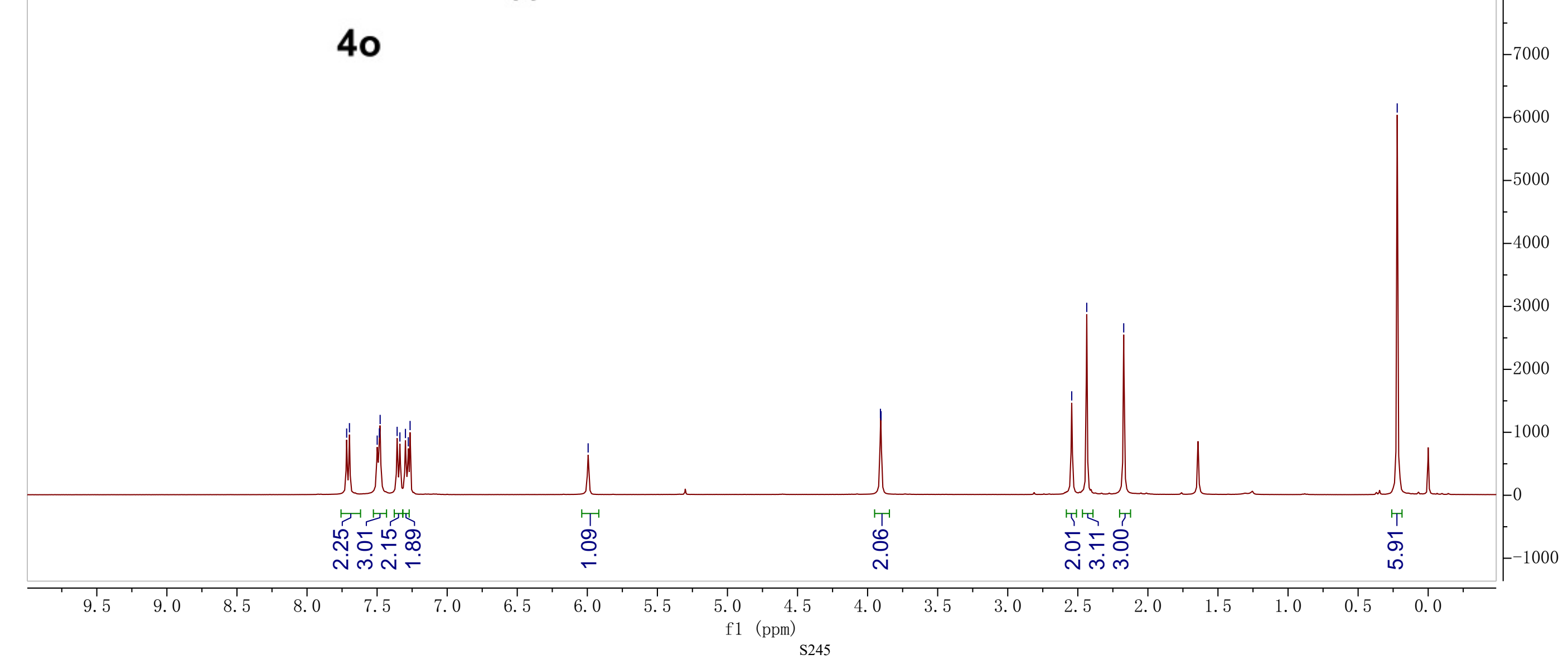


<smiles>[Y9]N1CC(c2ccc(NC(C)=O)cc2)=C[Si]([13CH3])([13CH3])C1</smiles>

40

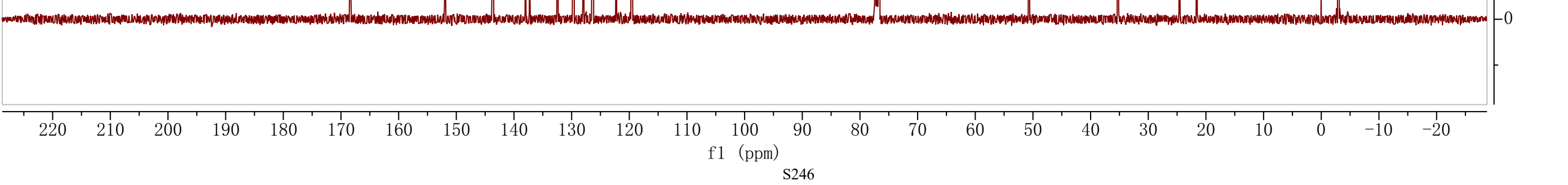




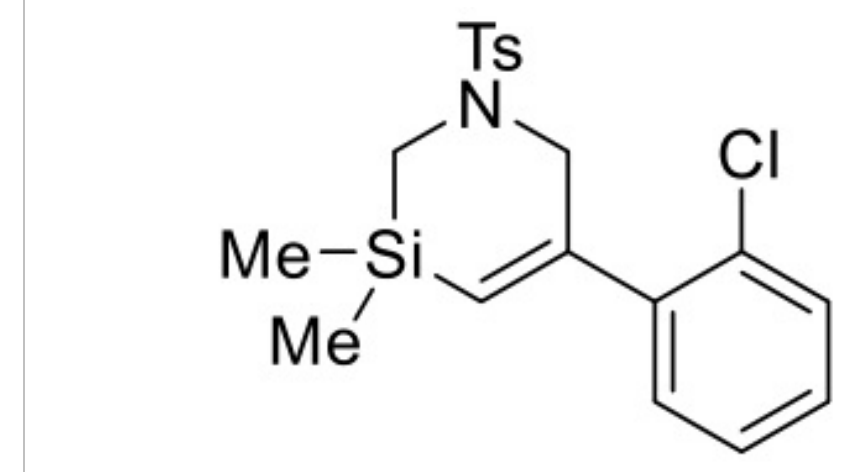

$4 p$

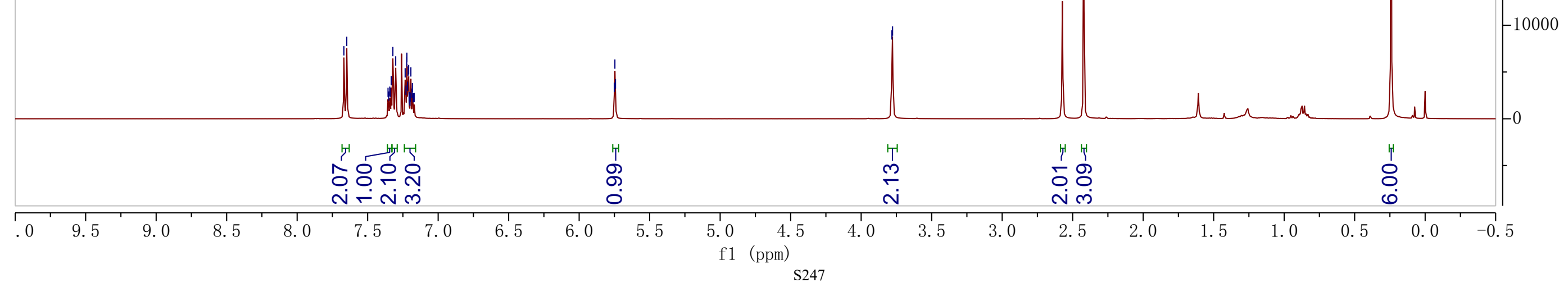


Ts<smiles>C[Si]1(C)C=C(c2ccccc2Cl)CN([As])C1</smiles>

\section{$4 p$}

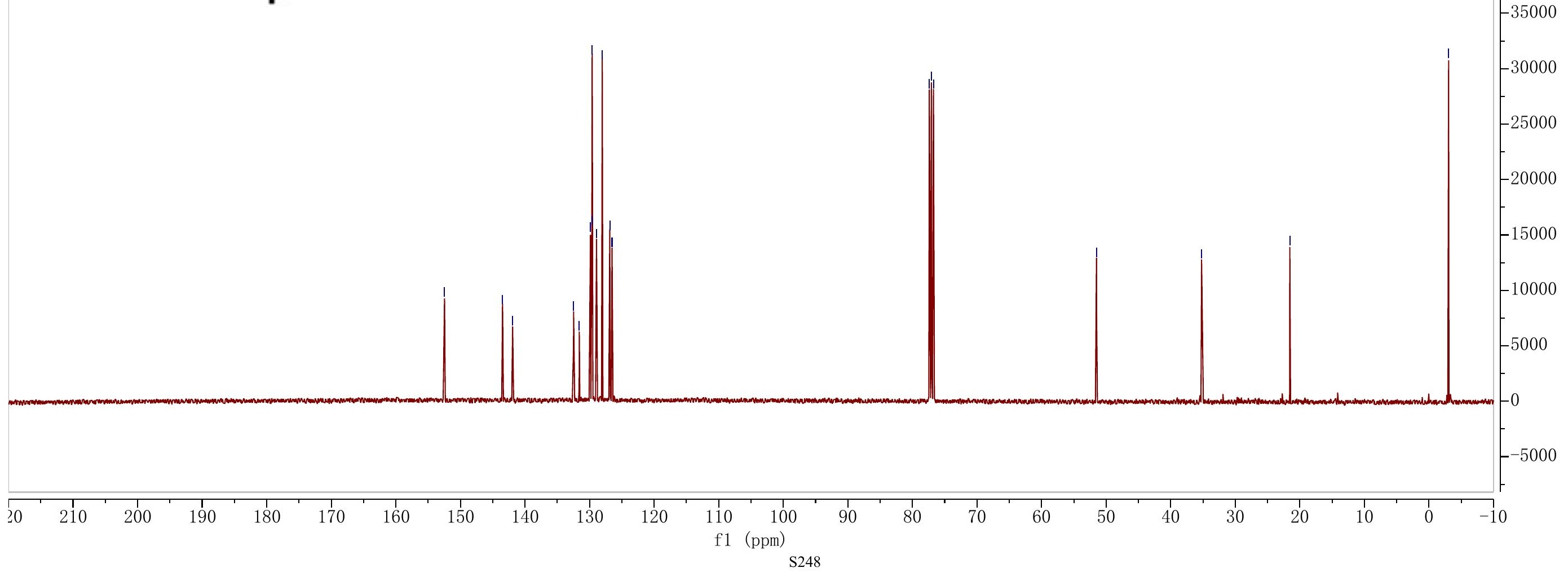


<smiles>C[Si]1(C)C=C(c2cccc(Cl)c2)CN([As])C1</smiles>

$4 q$

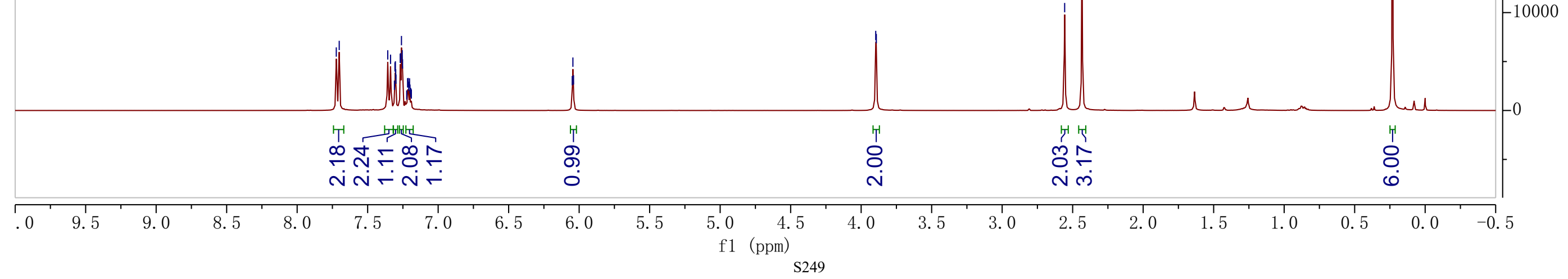


<smiles>C[Si]1(C)C=C(c2cccc(Cl)c2)CN([As])C1</smiles>

$4 q$

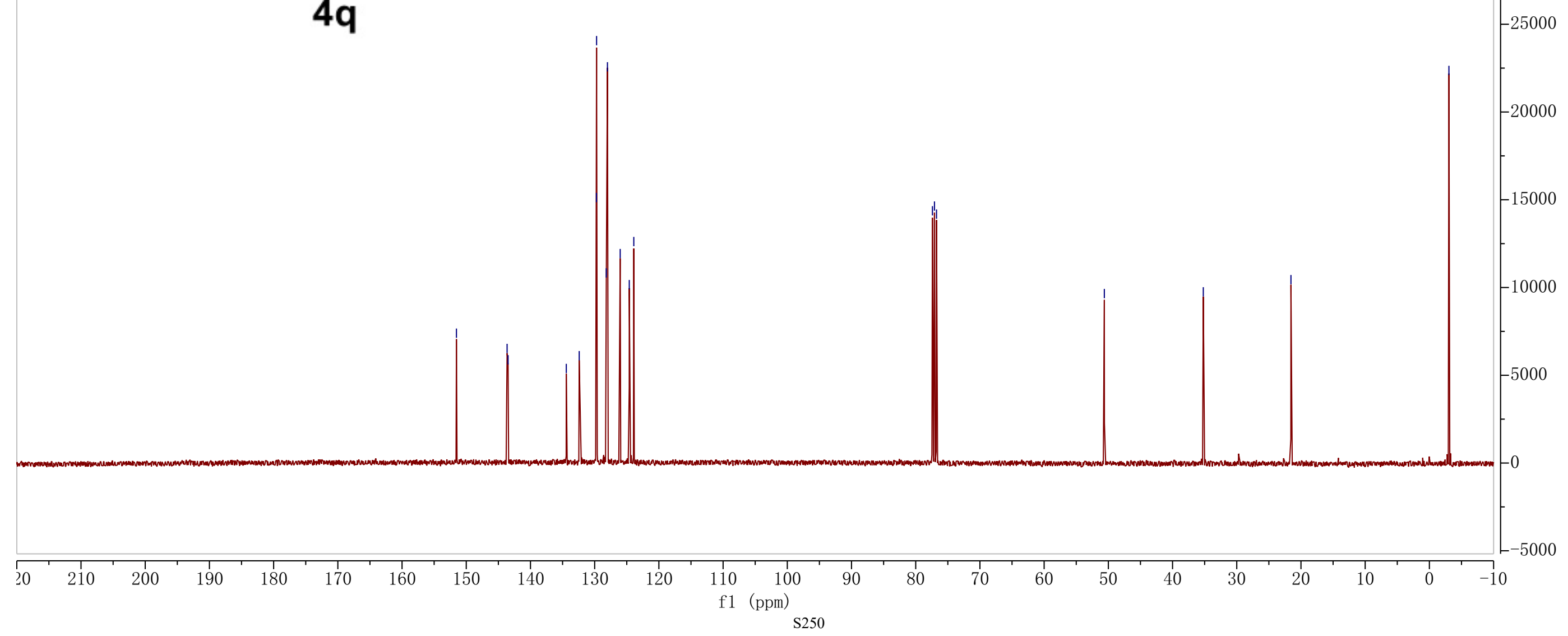


<smiles>C[Si]1(C)C=C(c2ccc(Cl)cc2)CN([As])C1</smiles>

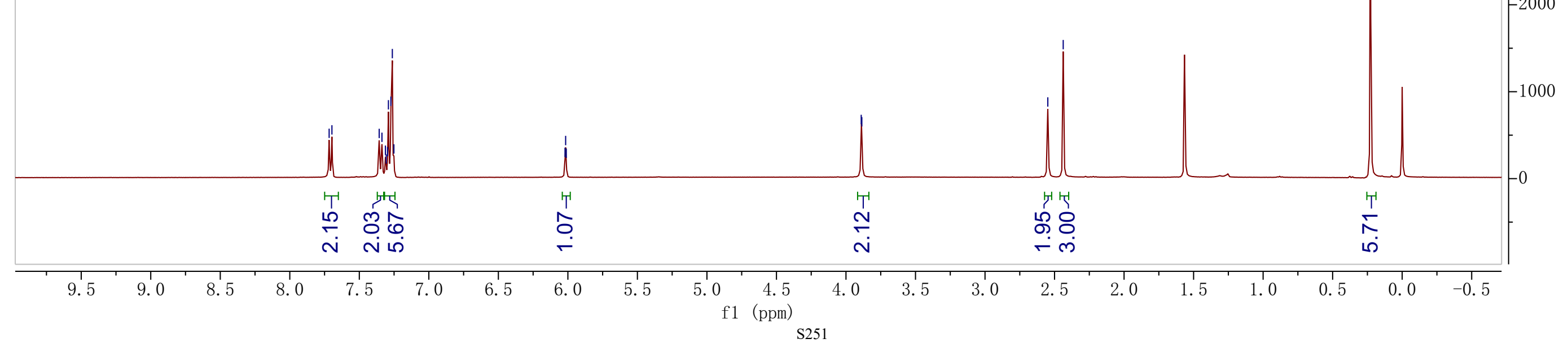


<smiles>C[Si]1(C)C=C(c2ccc(Cl)cc2)CN([As])C1</smiles>

\section{$4 r$}


<smiles>C[Si]1(C)C=C(c2cccc(F)c2)CN([As])C1</smiles>

$4 \mathrm{~s}$

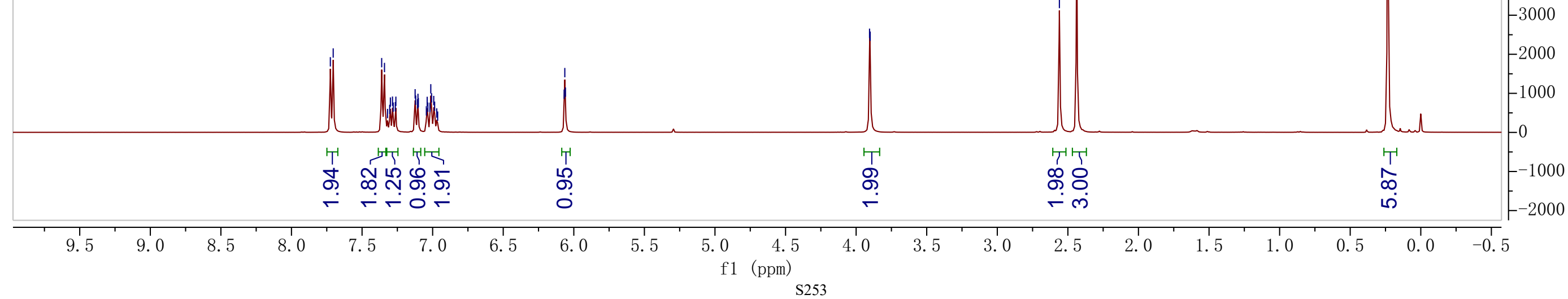


<smiles>C[Si]1(C)C=C(c2cccc(F)c2)CN([As])C1</smiles>

4s

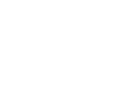




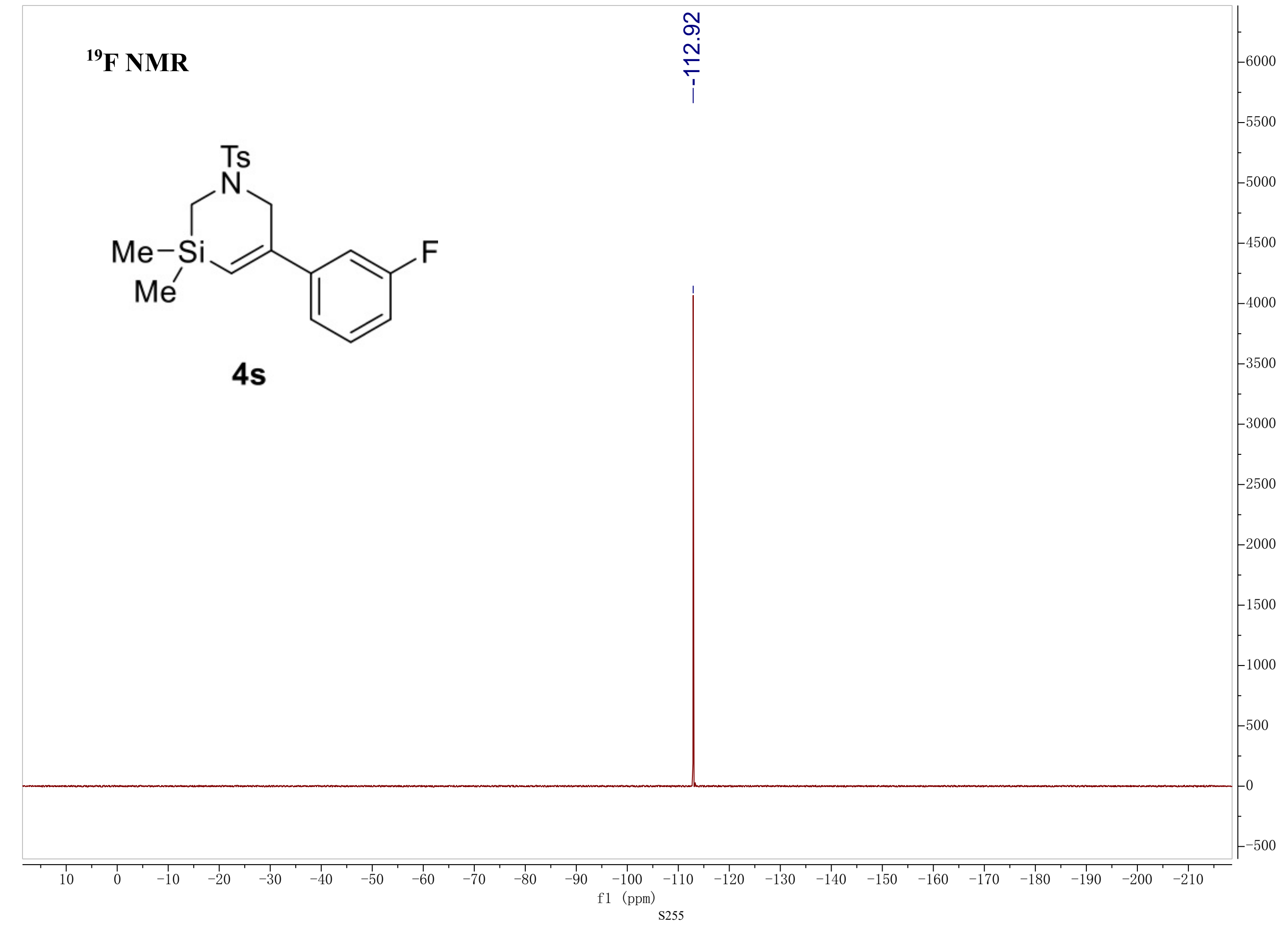




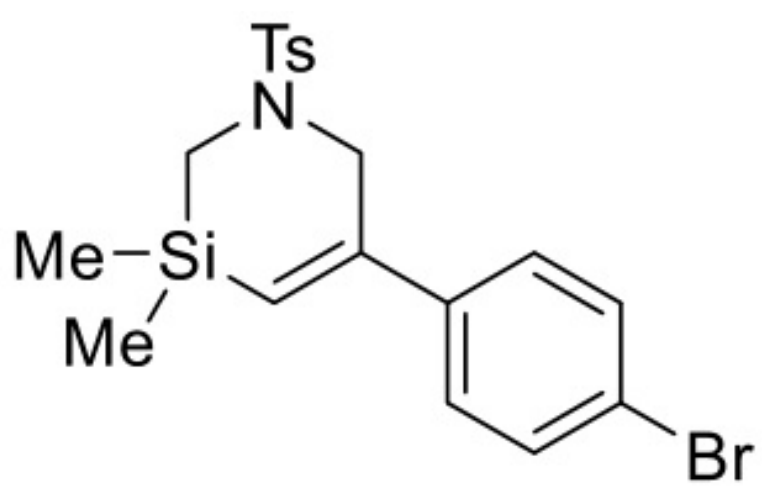

4t

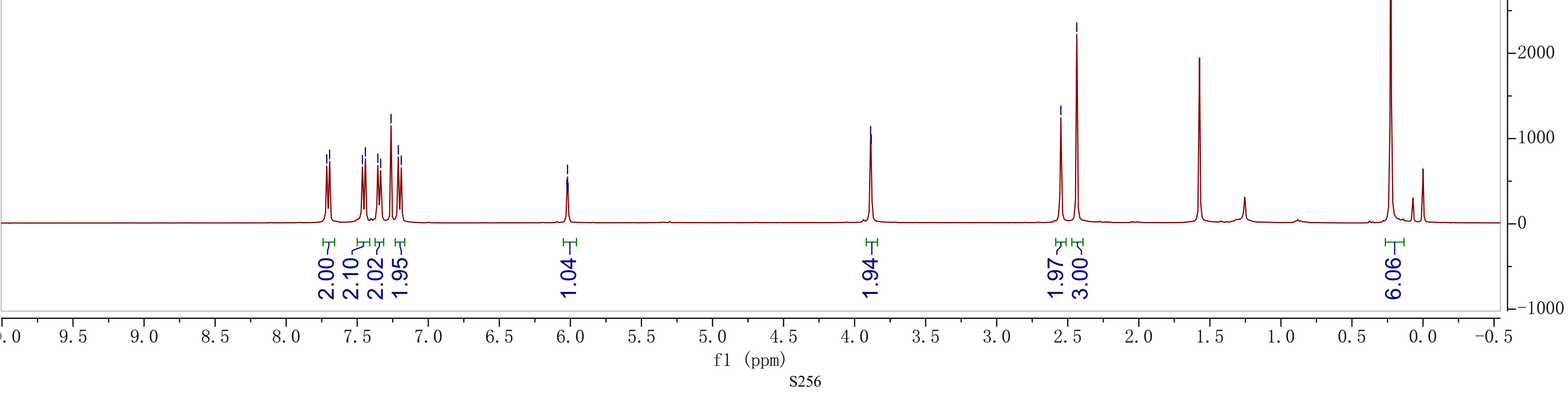


<smiles>C[Si]1(C)C=C(c2ccc(Br)cc2)CN([As])C1</smiles>

$4 t$ 
<smiles>C[Si]1(C)C=C(c2ccc(C(F)(F)F)cc2)CN([As])C1</smiles>

\section{$4 u$}

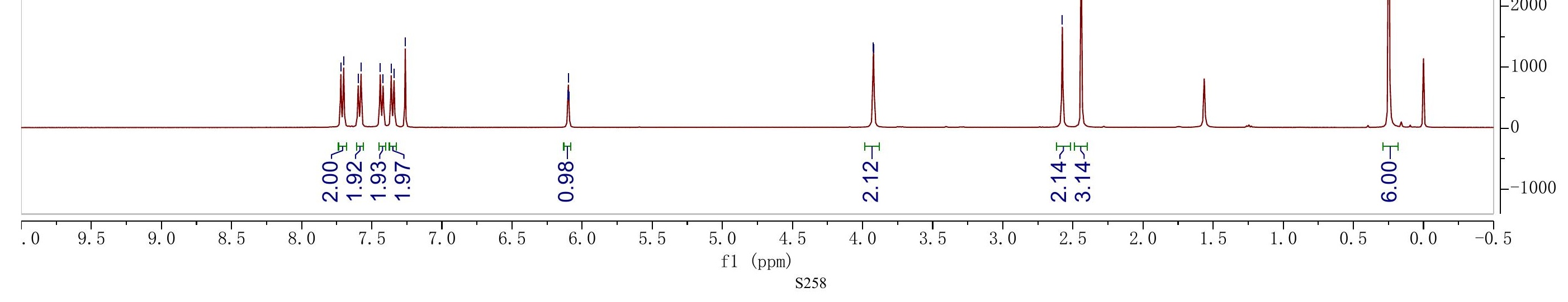




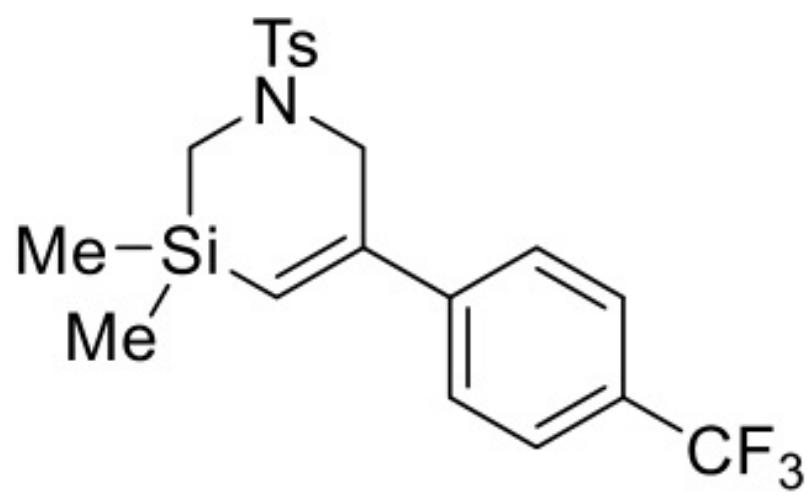

\section{$4 u$}
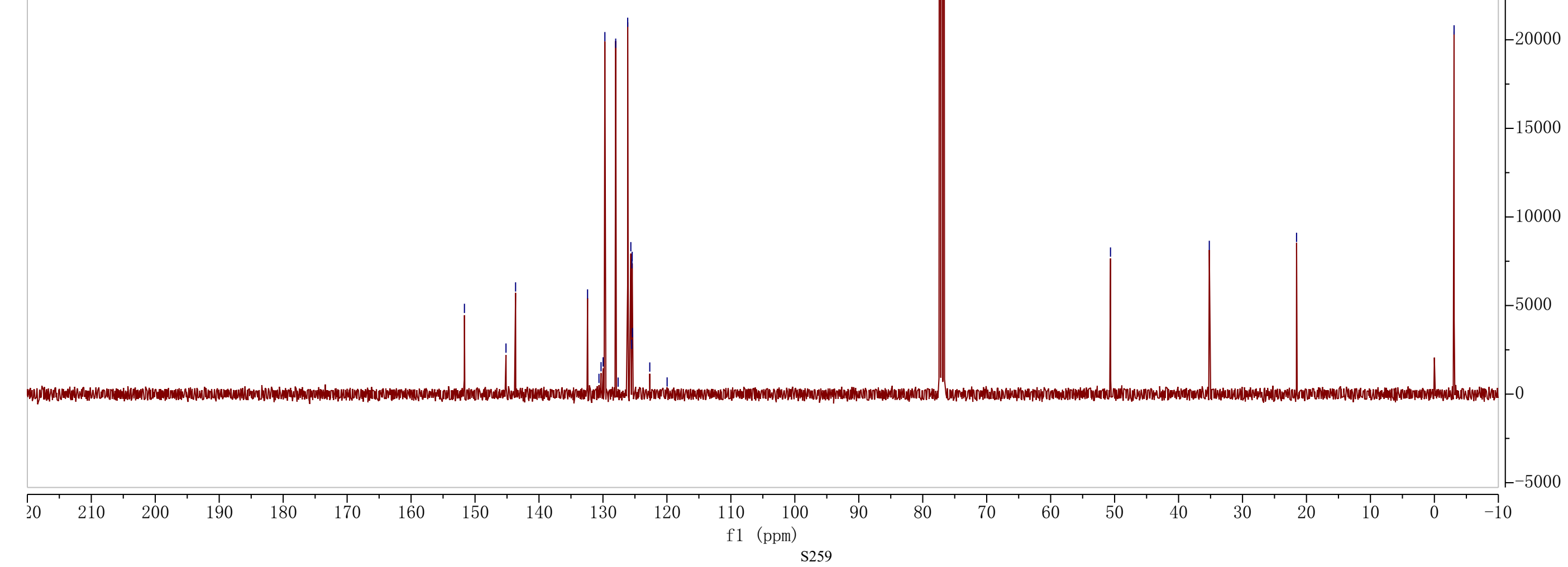
${ }^{19}$ F NMR

Ts<smiles>C[Si]1(C)C=C(c2ccc(C(F)(F)F)cc2)CN([As])C1</smiles>

$4 u$

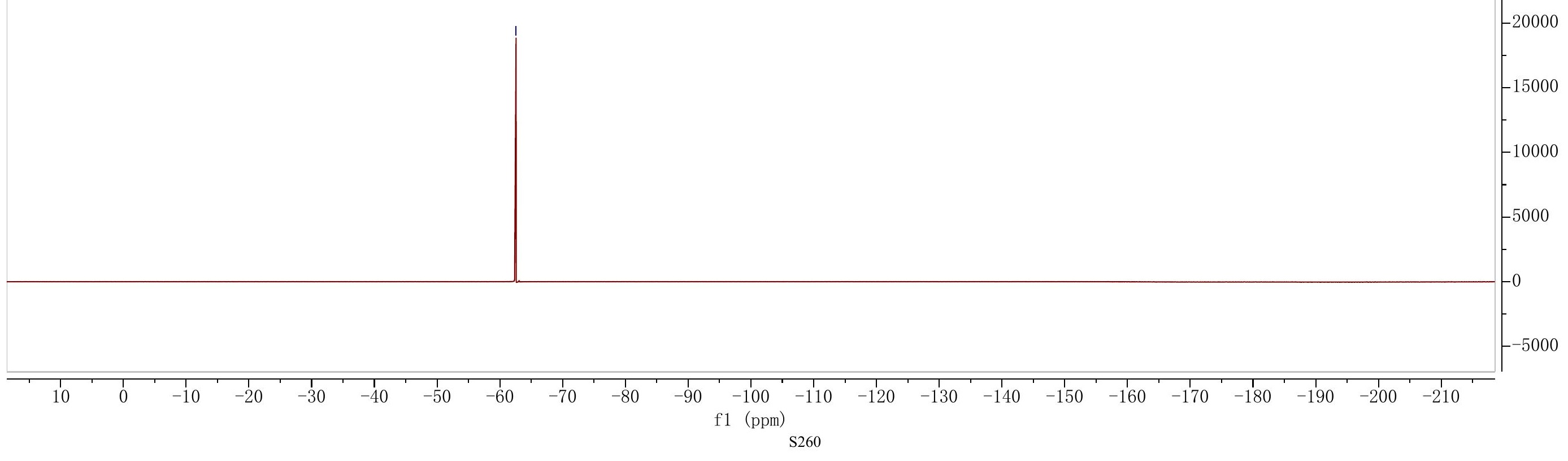




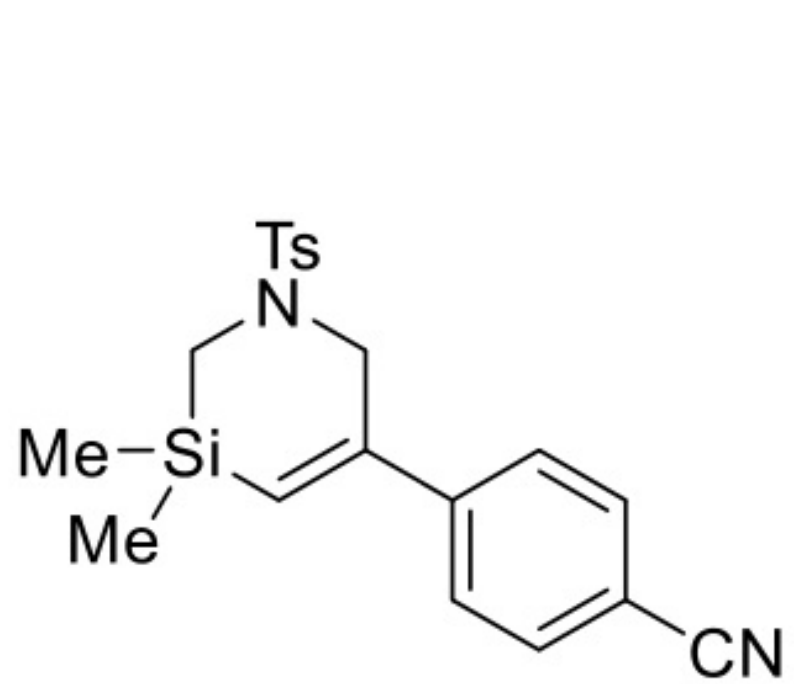

\section{4v}

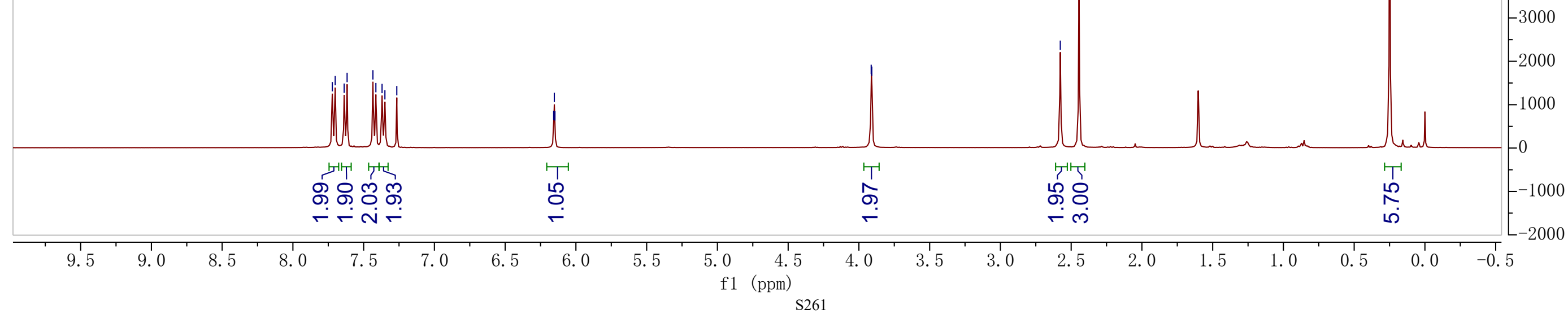


<smiles>C[Si]1(C)C=C(c2ccc(C#N)cc2)CN([As])C1</smiles>

\section{4v}

\section{$\mathrm{CN}$}

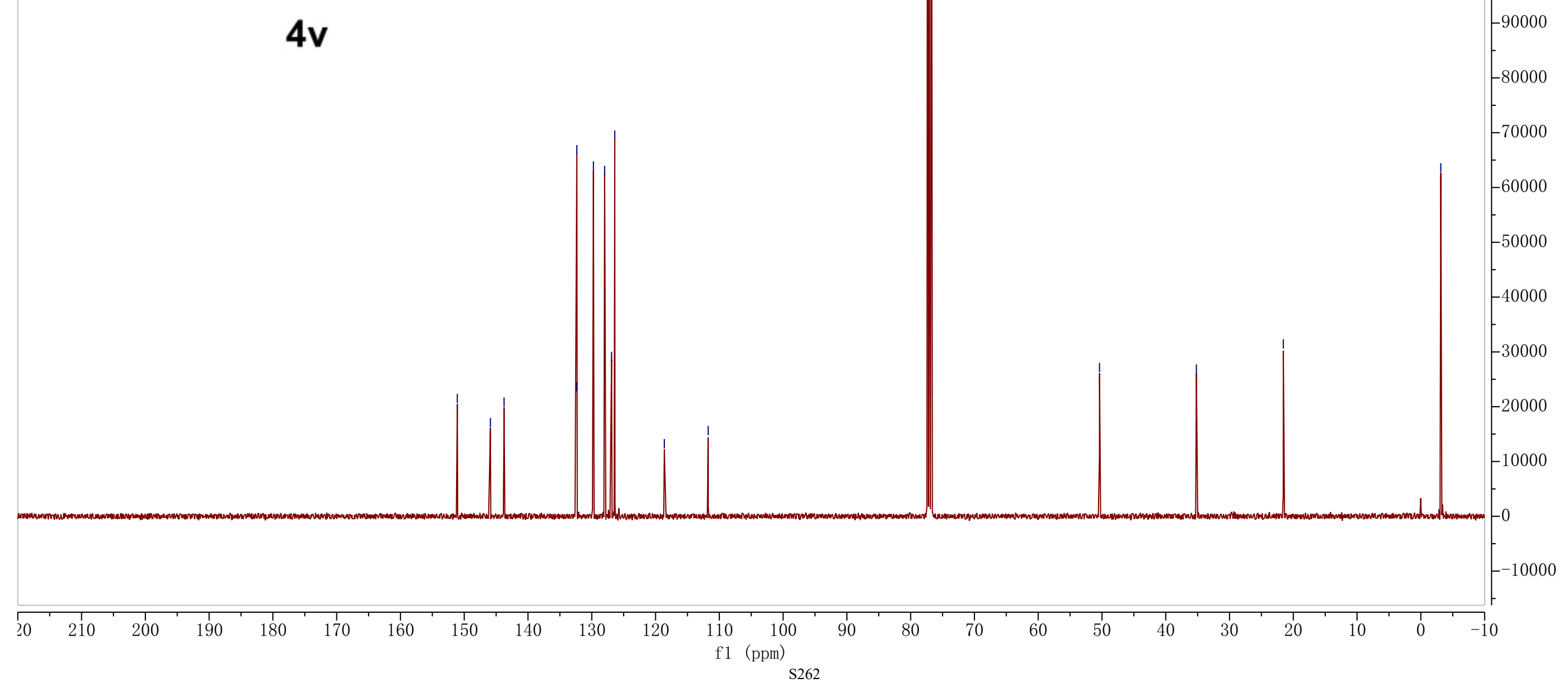


<smiles>C[Si]1(C)C=C(c2ccc(C(=O)c3ccccc3)cc2)CN([As])C1</smiles>

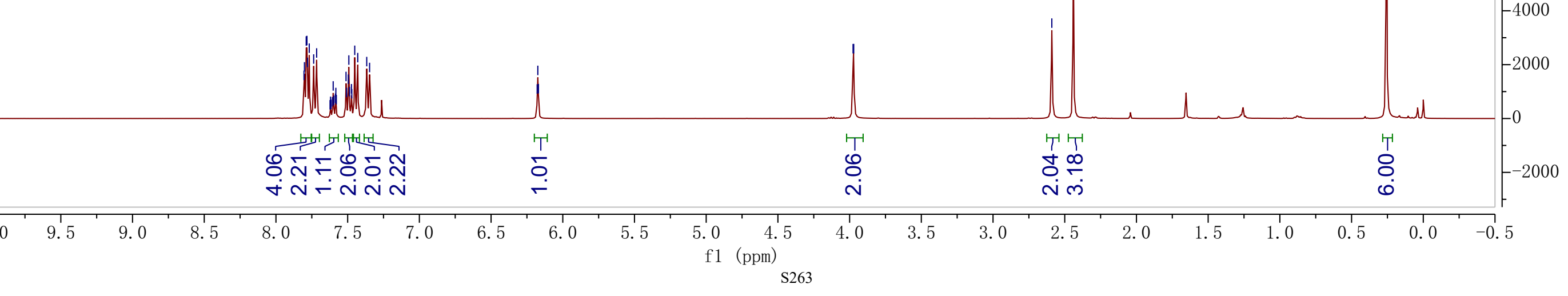


<smiles>C[Si]1(C)C=C(c2ccc(C(=O)c3ccccc3)cc2)CN([As])C1</smiles>

O
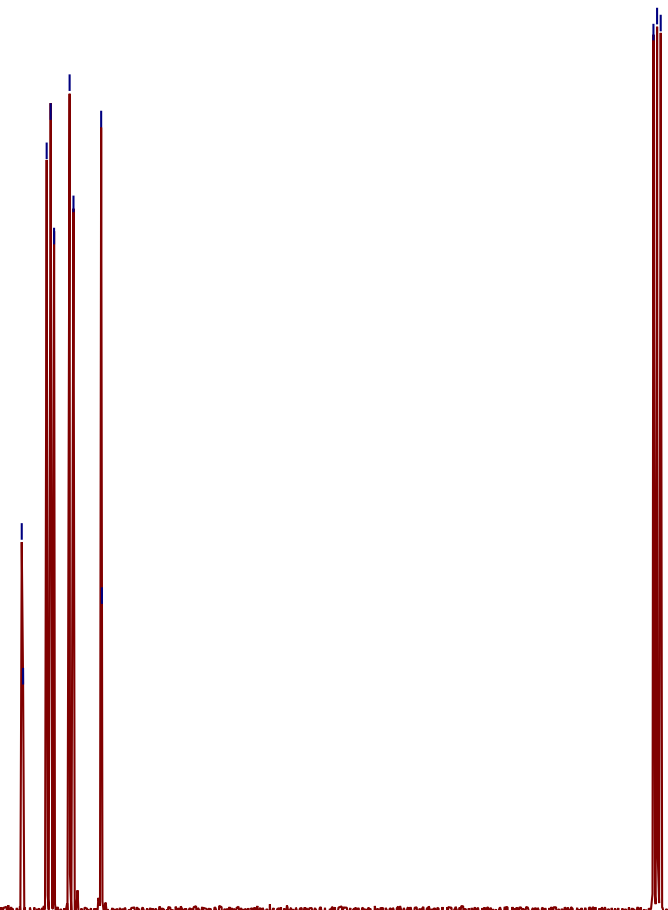


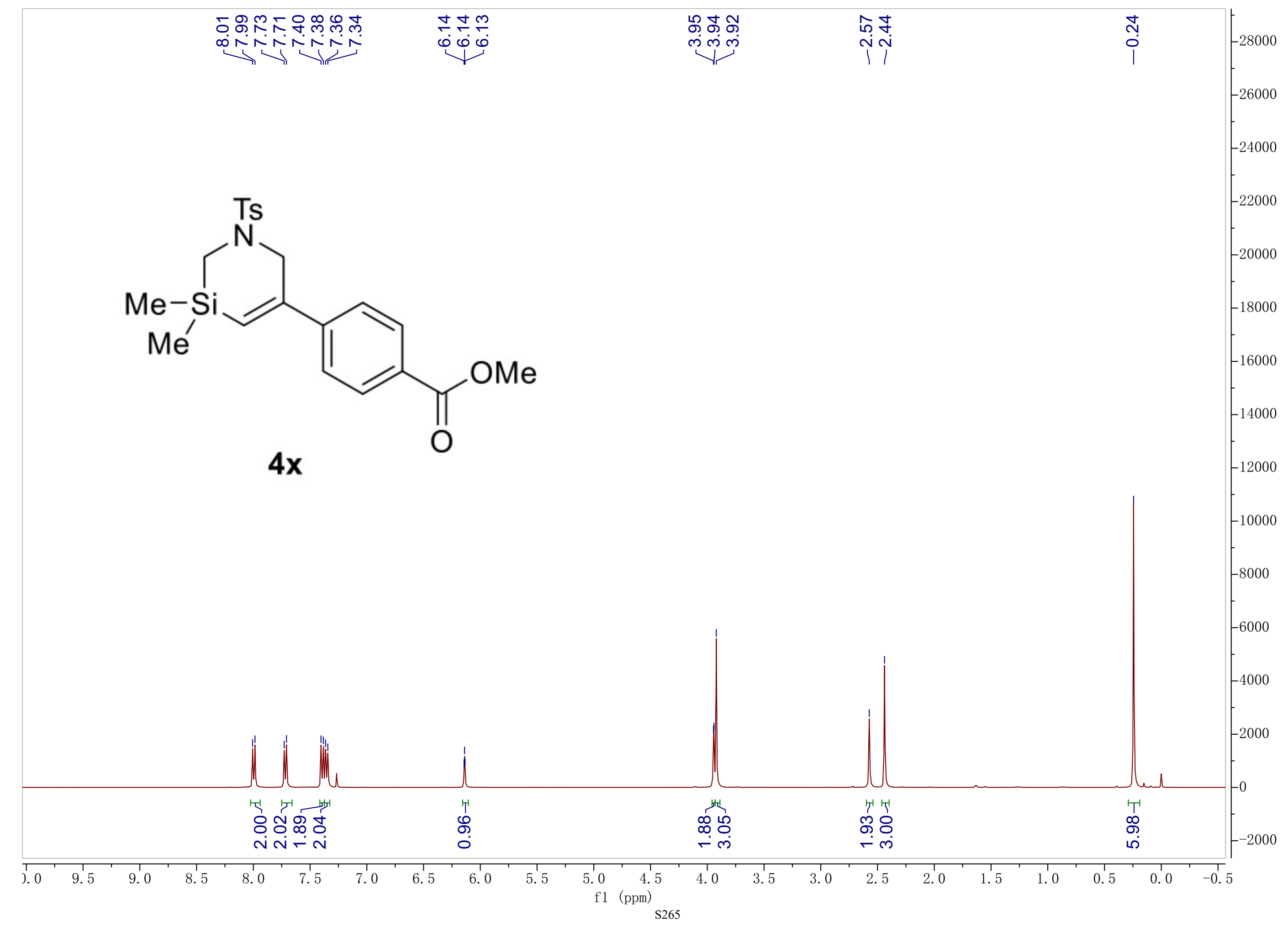




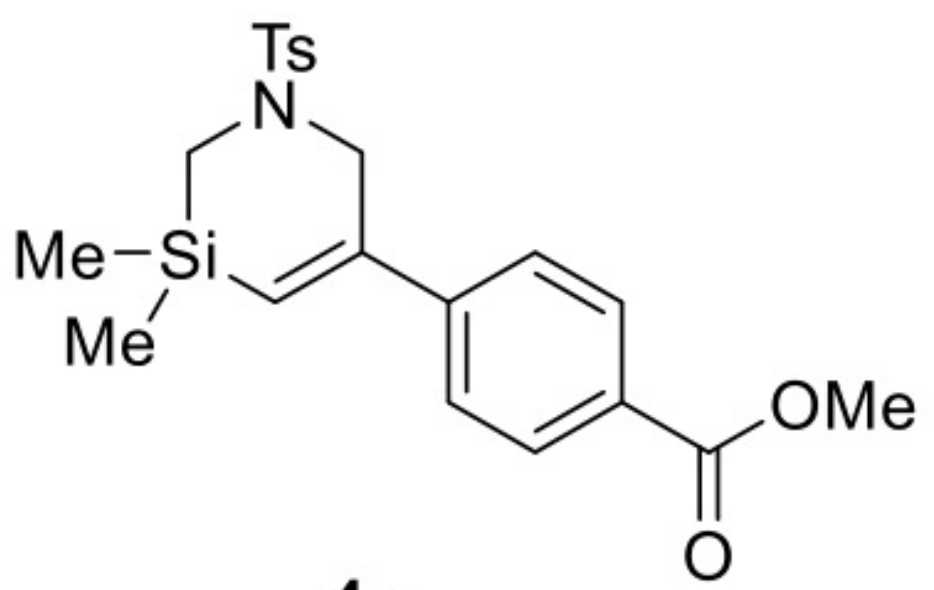

4x
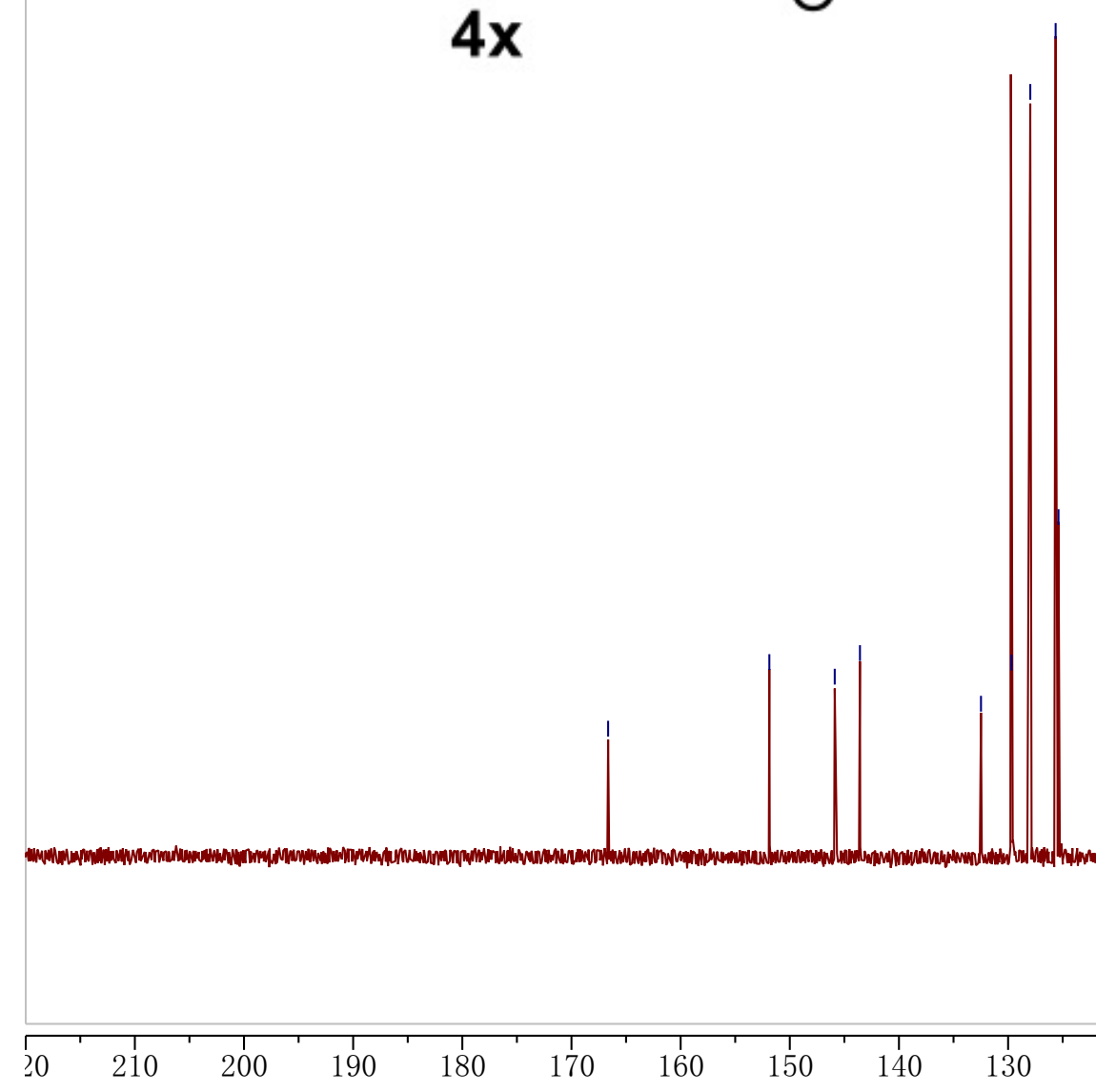

180

170

160

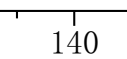

130 


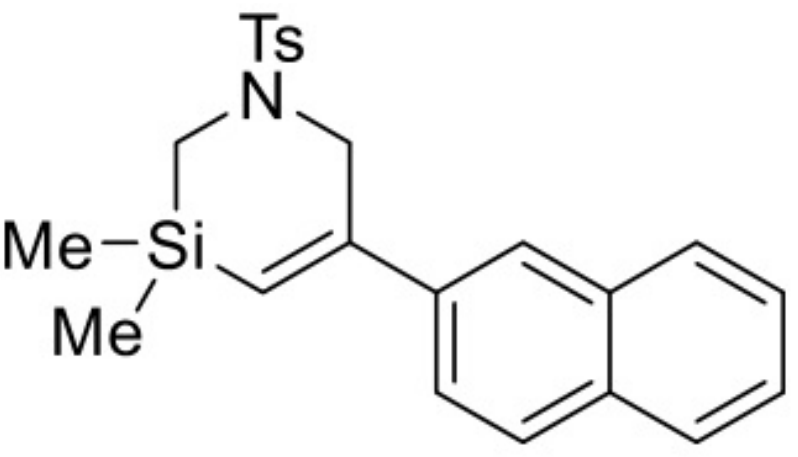

4y

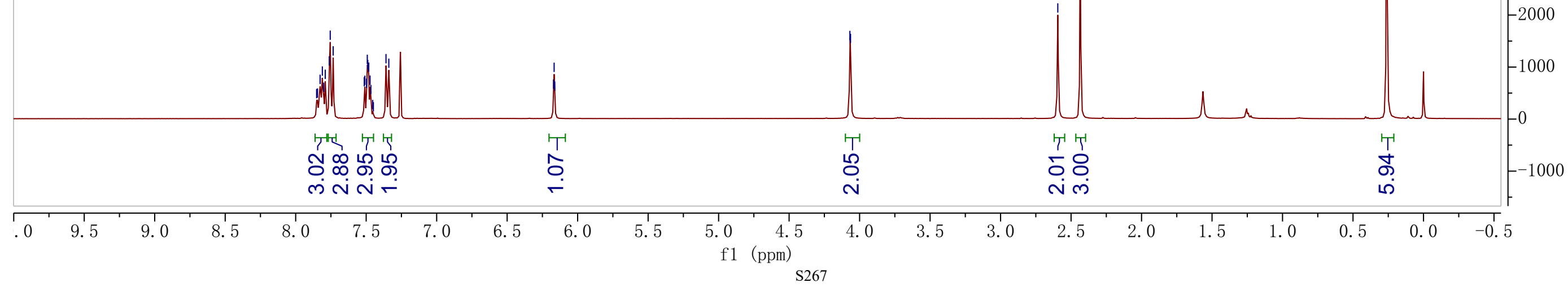


<smiles>C[Si]1(C)C=C(c2ccc3ccccc3c2)CN([As])C1</smiles>

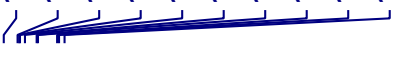

\section{4y}




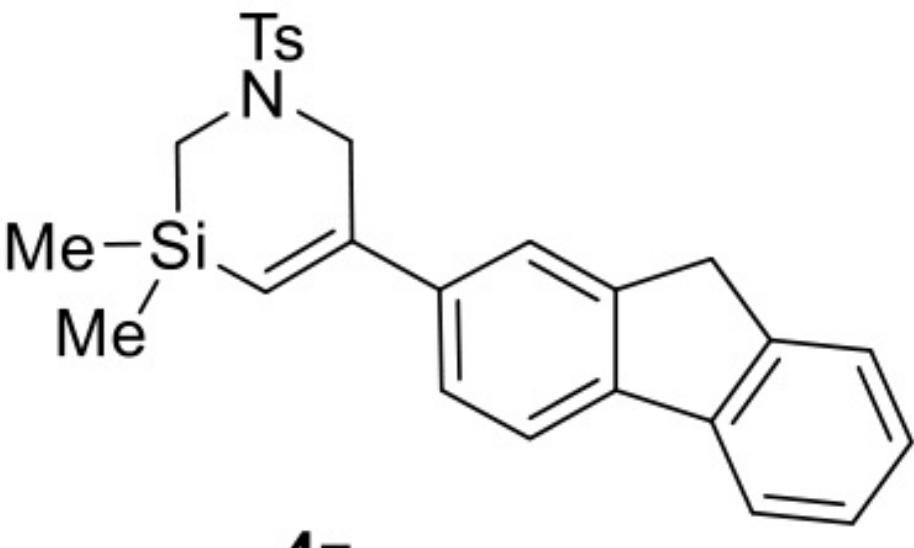

$4 z$

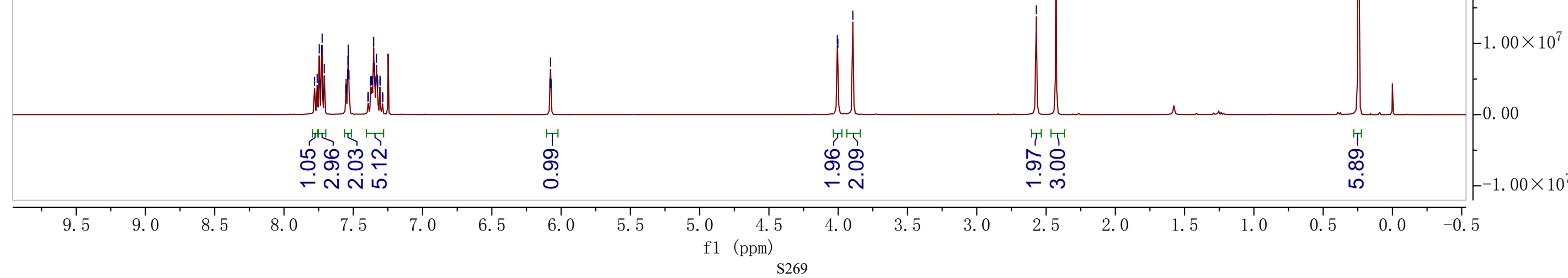




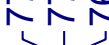

Ts

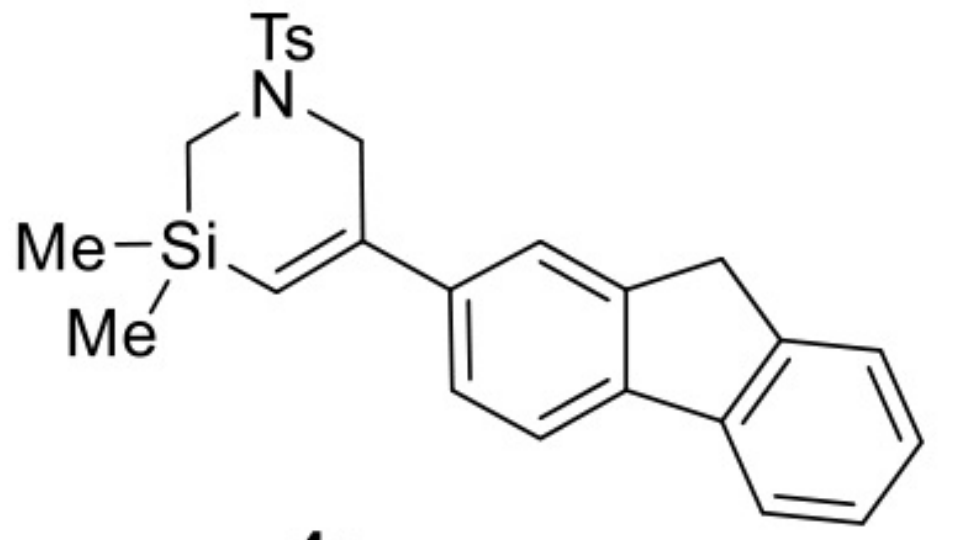

$4 z$
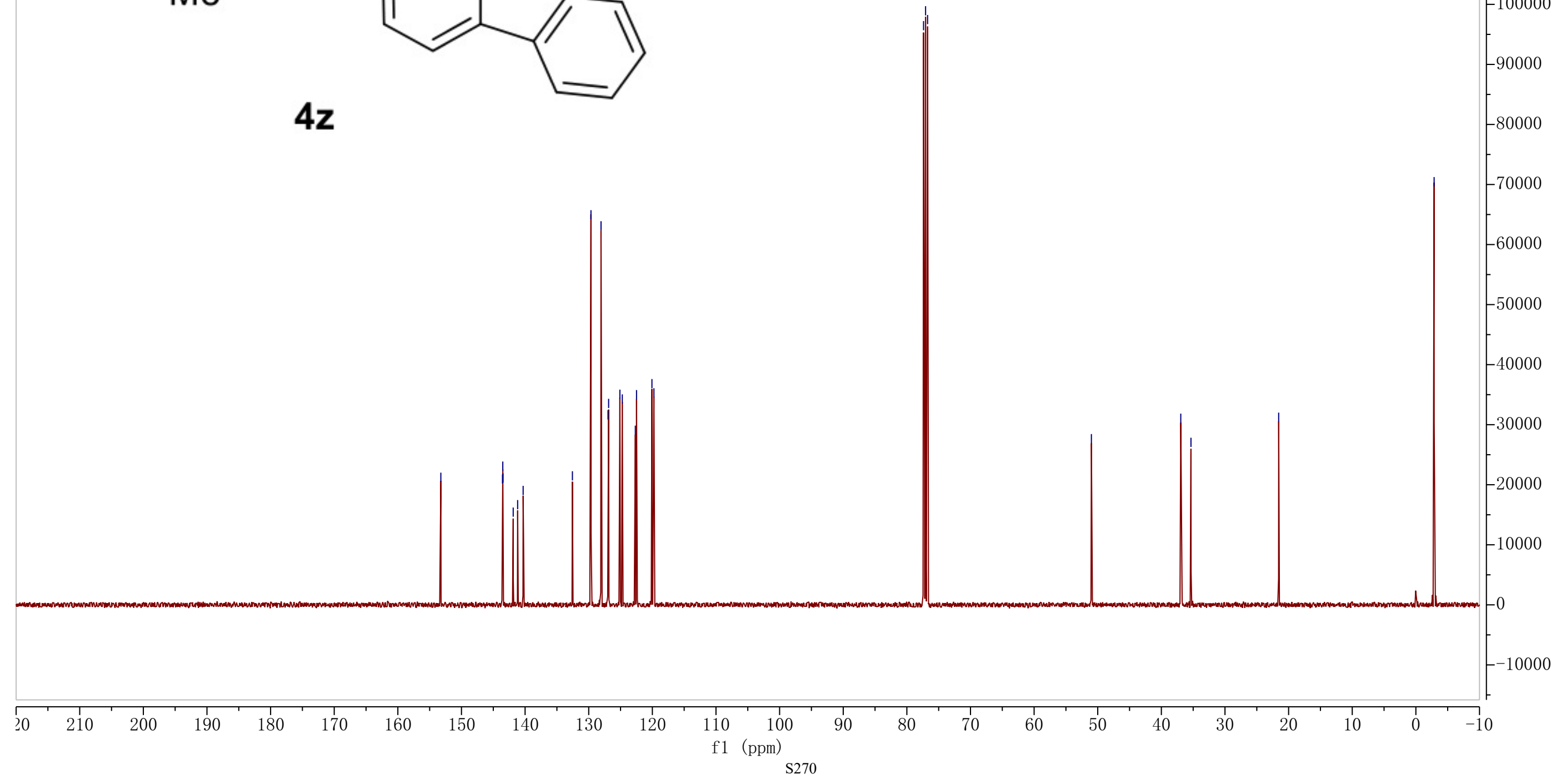


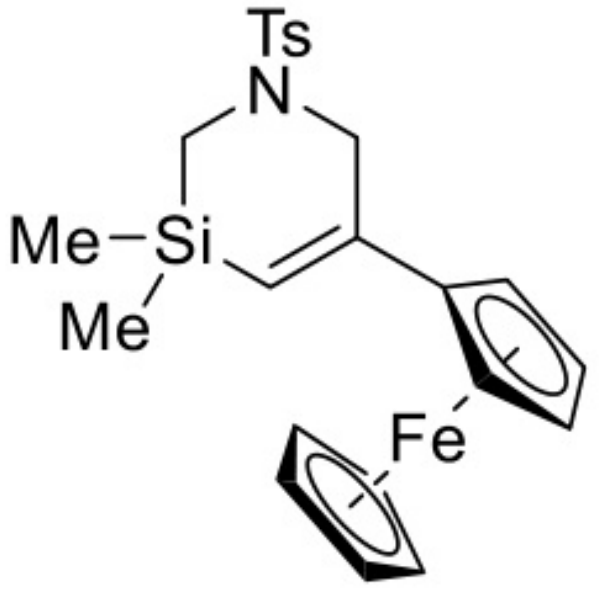

4aa

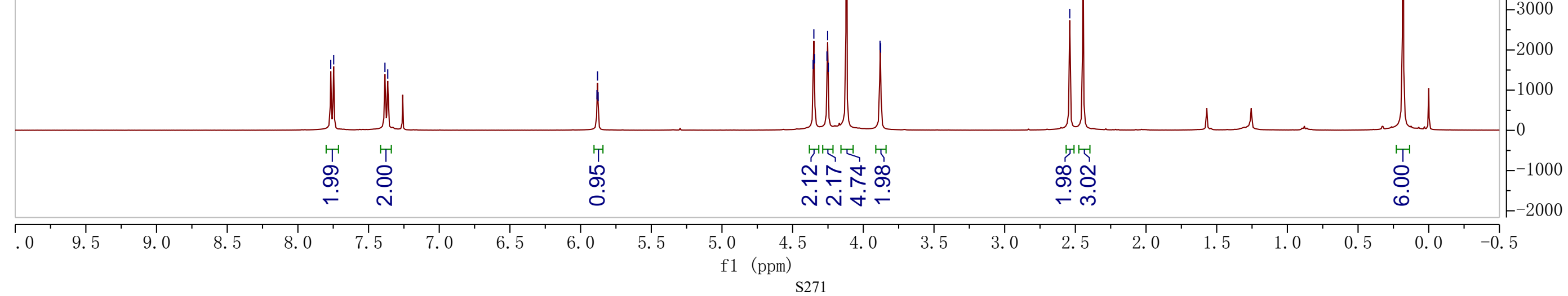


<smiles>C[Si]1(C)C=C(c2cccnc2)CN([As])C1</smiles>

$4 a b$

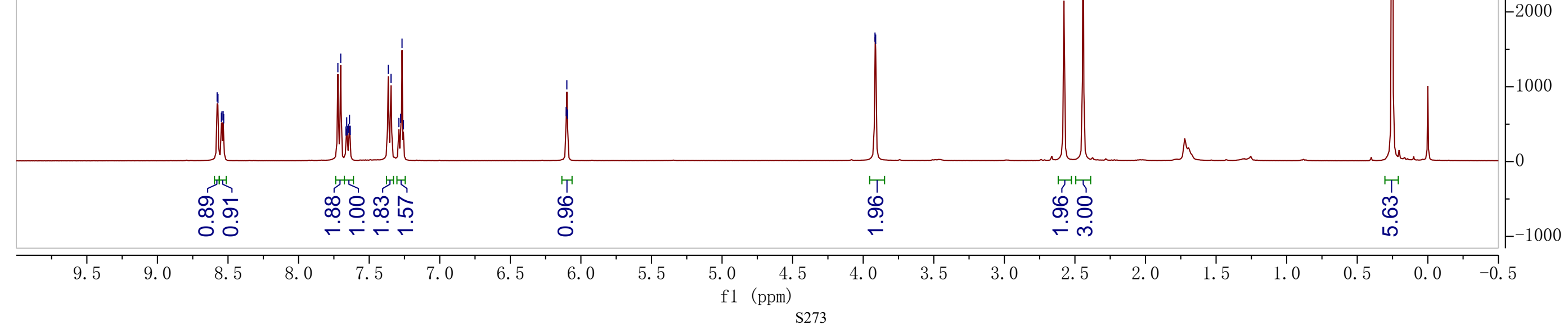


<smiles>C[Si]1(C)C=C(c2cccnc2)CN([As])C1</smiles>

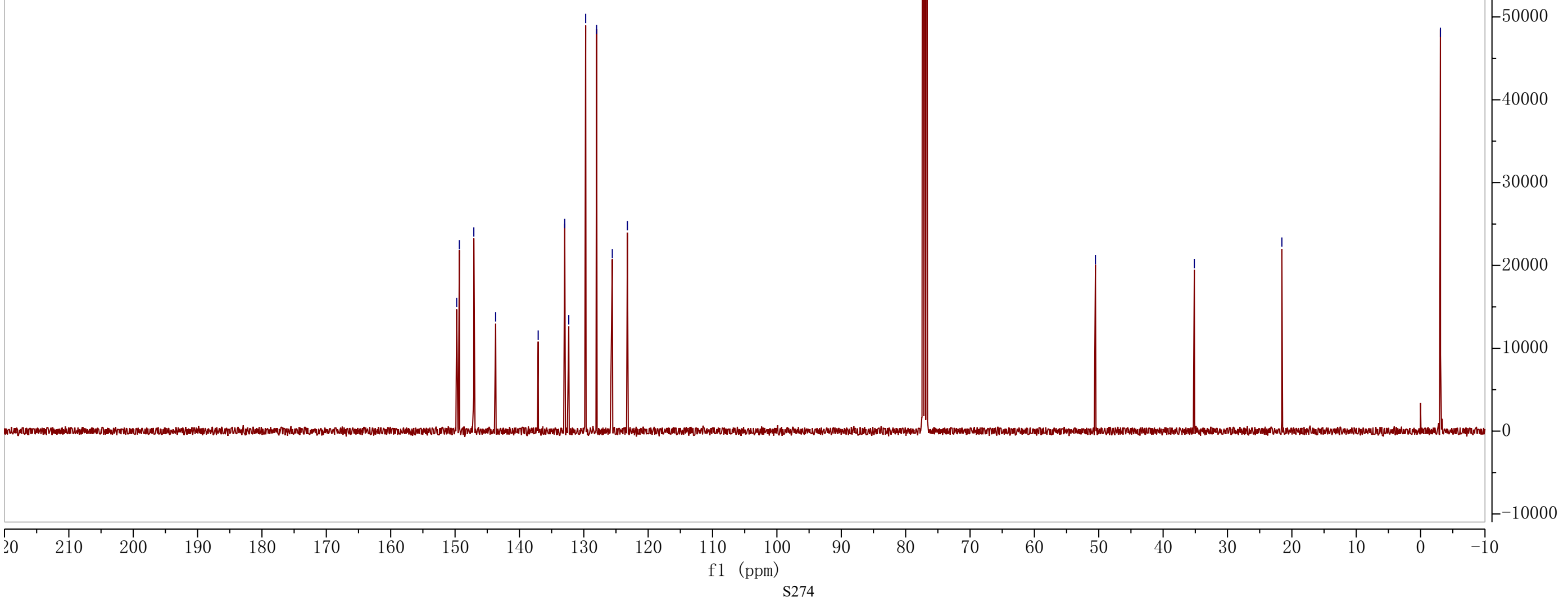




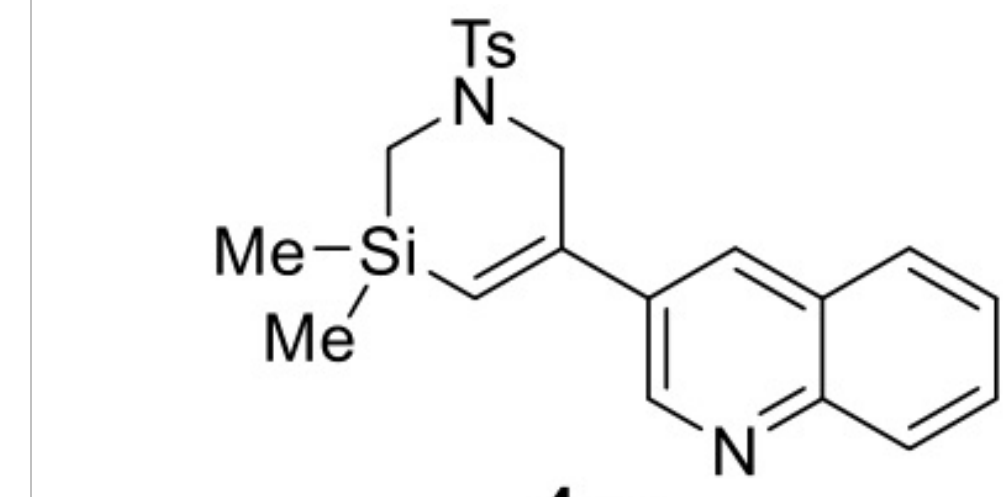

4ac

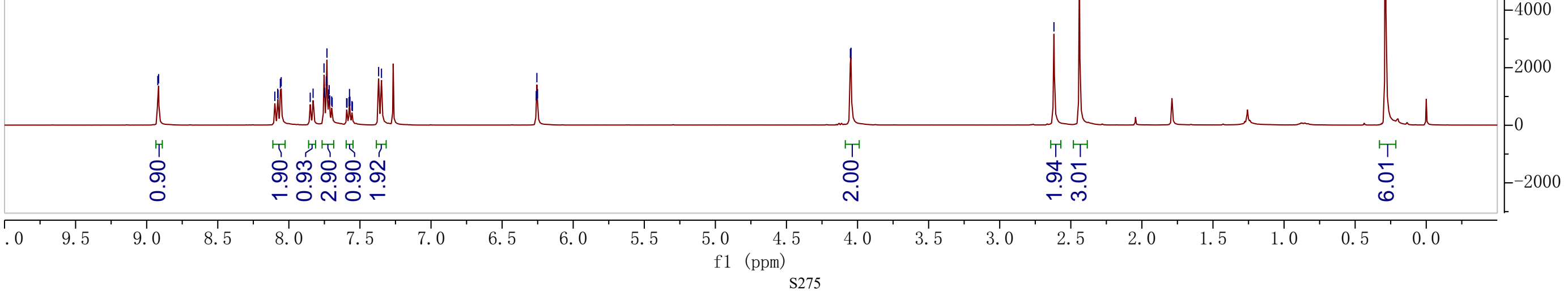


<smiles>CN1CC(c2cnc3ccccc3c2)=C[Si](C)(C)C1</smiles>

4ac 
<smiles>C[Si]1(C)C=C(c2c[nH]c3ccccc23)CN([As])C1</smiles>

\section{4ad}

$\mathrm{Me}$

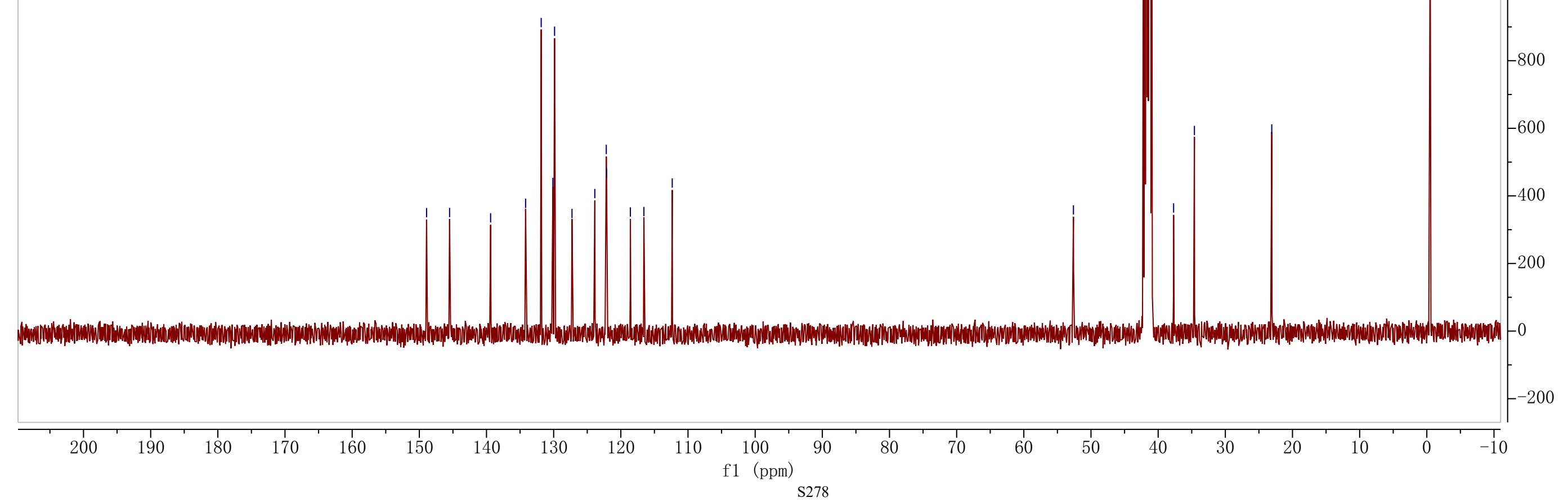




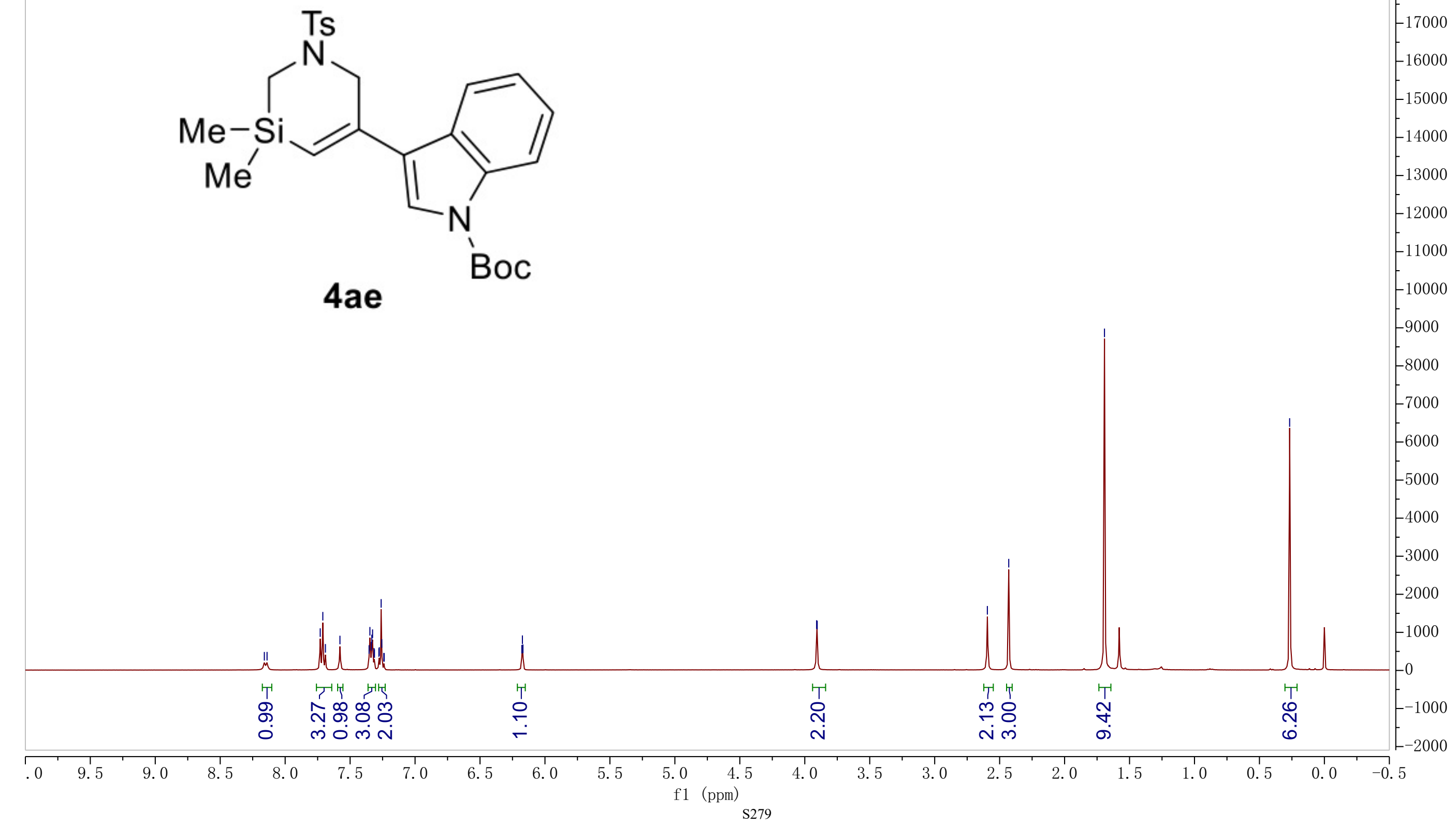


Ts<smiles>C[Si]1(C)C=C(c2cn(C(=O)OCc3ccccc3)c3ccccc23)CN([As])C1</smiles>

$4 a e$

(1) 
ర요욜

你弐

กิ

i

伿寺

ชิ

छั

$\begin{aligned} & \bar{N} \\ & i \\ & i\end{aligned}-28000$<smiles>C[Si]1(C)C=C(c2c[nH]cn2)CN([As])C1</smiles>

$4 a f$

Boc
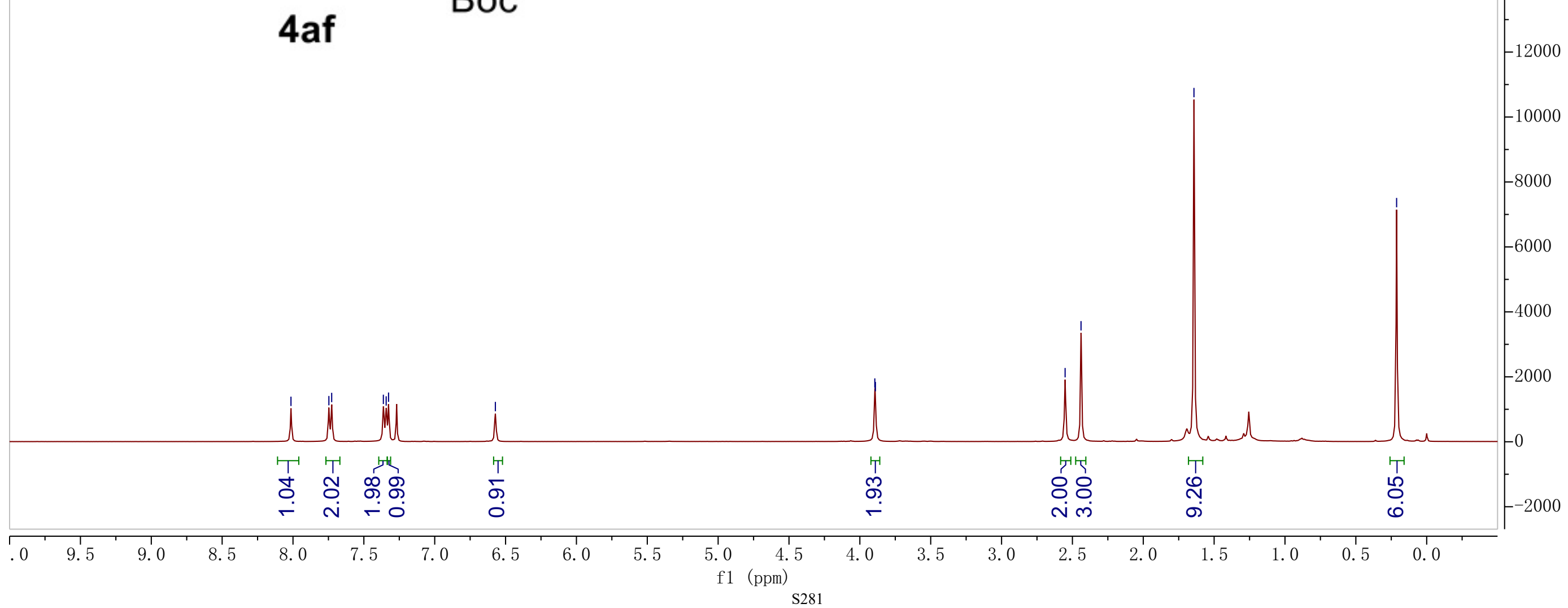


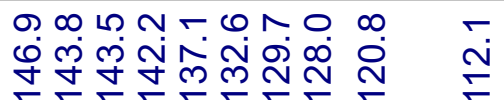

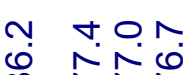

i店

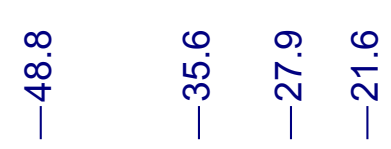

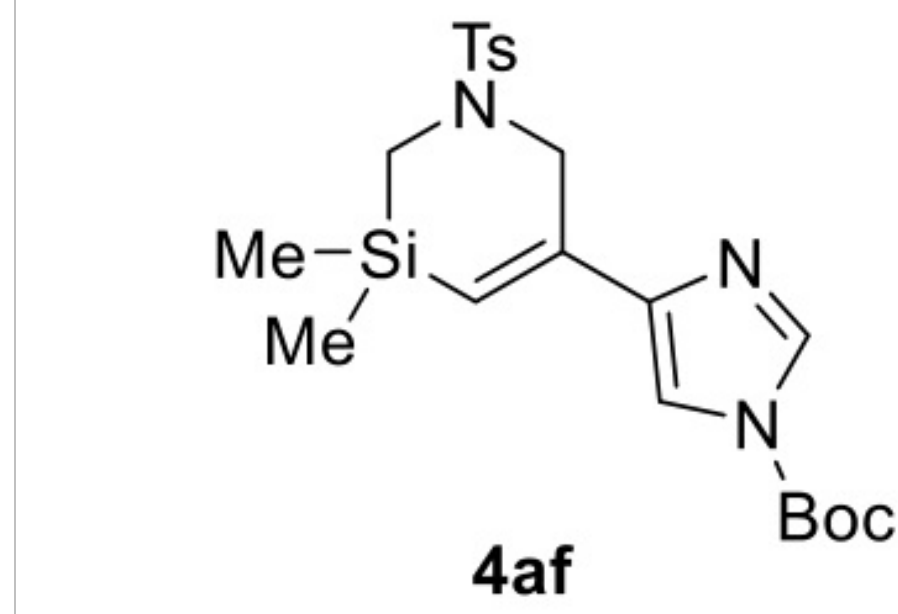

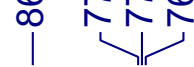




lo

бॅ

i்

ชำ

กั

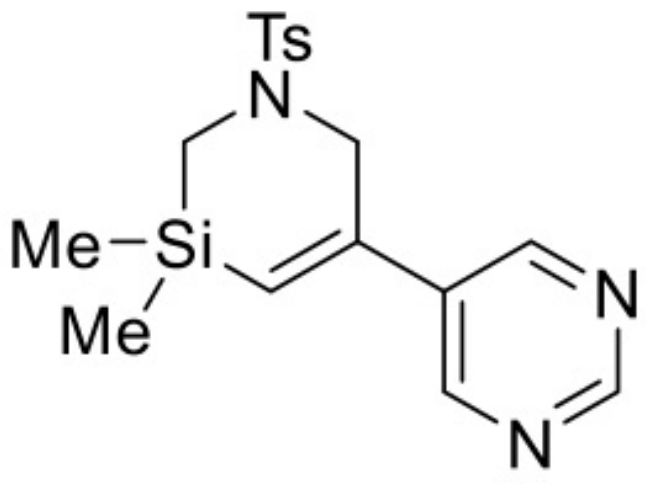

4ag

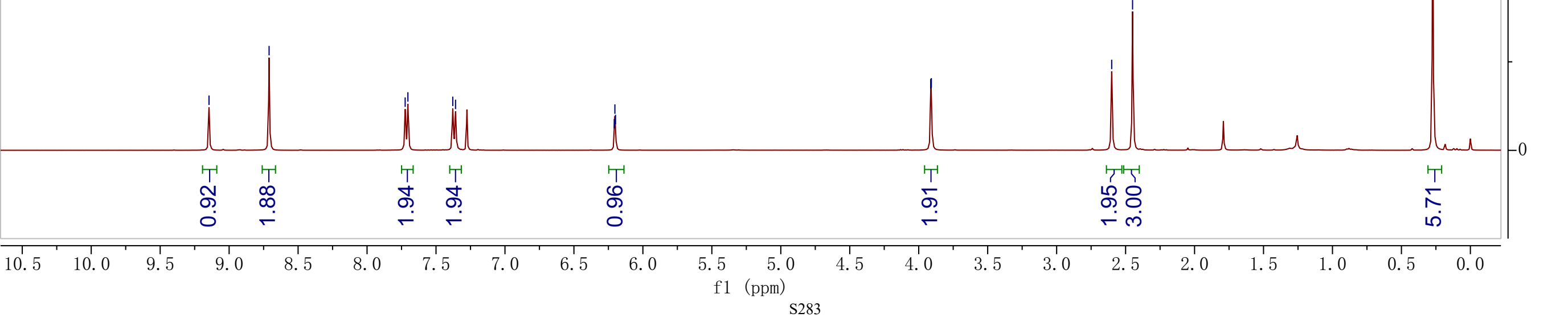


<smiles>CN1CC(c2cncnc2)=C[Si](C)(C)C1</smiles>

\section{4ag}

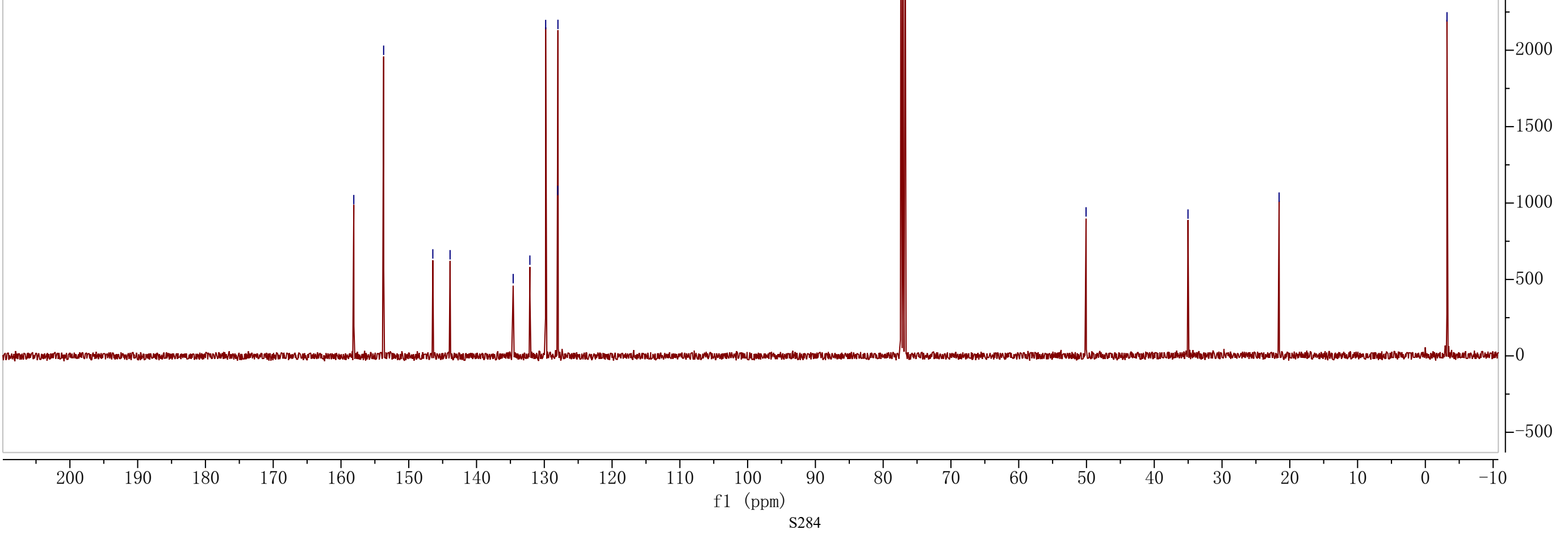


<smiles>C[Si]1(C)C=C(c2cnc3cccnn23)CN([As])C1</smiles>

\section{4ah}

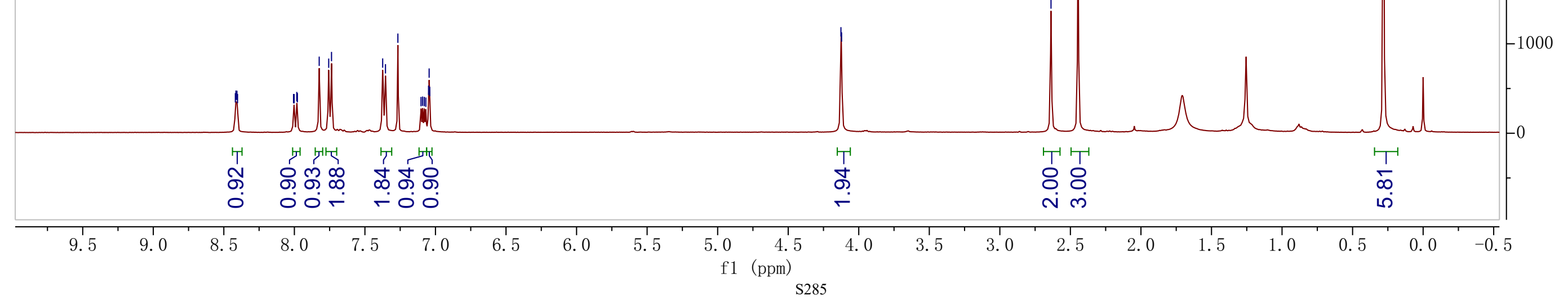


<smiles>C[Si]1(C)C=C(c2cnc3cccnn23)CN([As])C1</smiles>

\section{4ah}

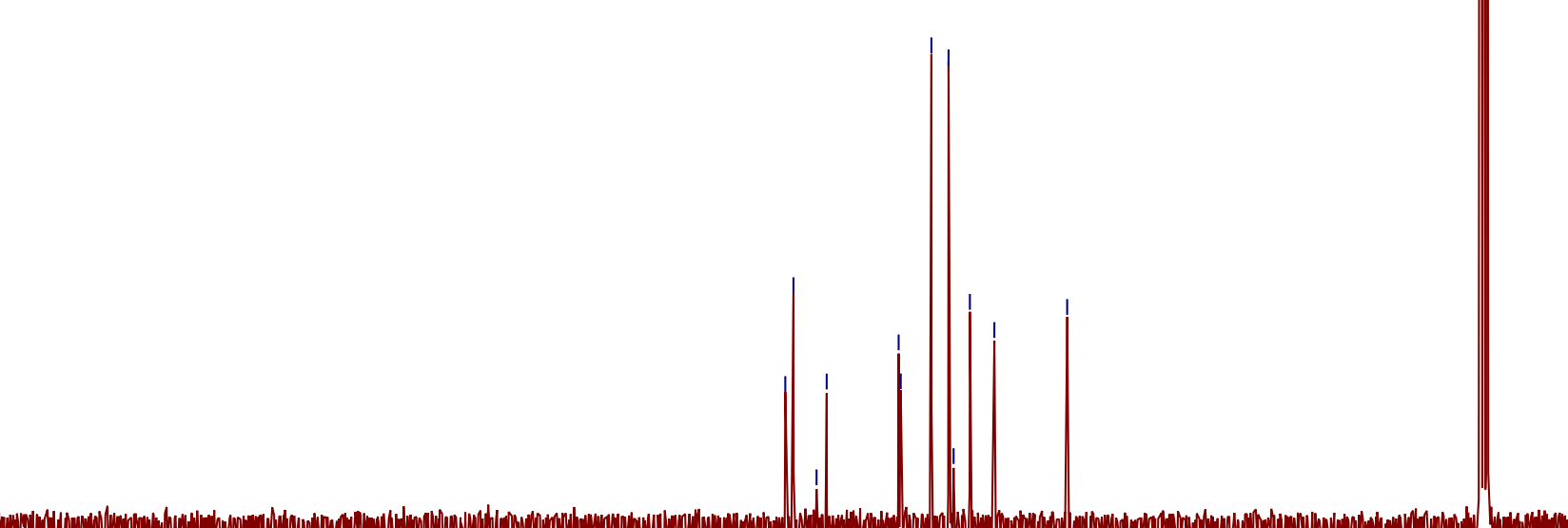




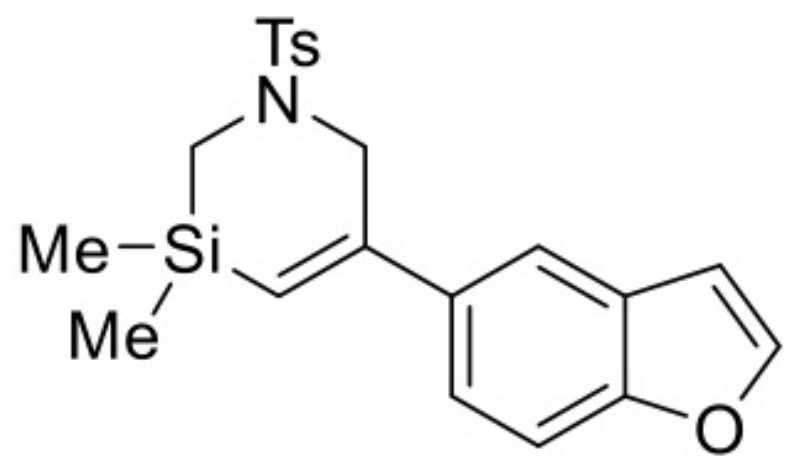

\section{4ai}

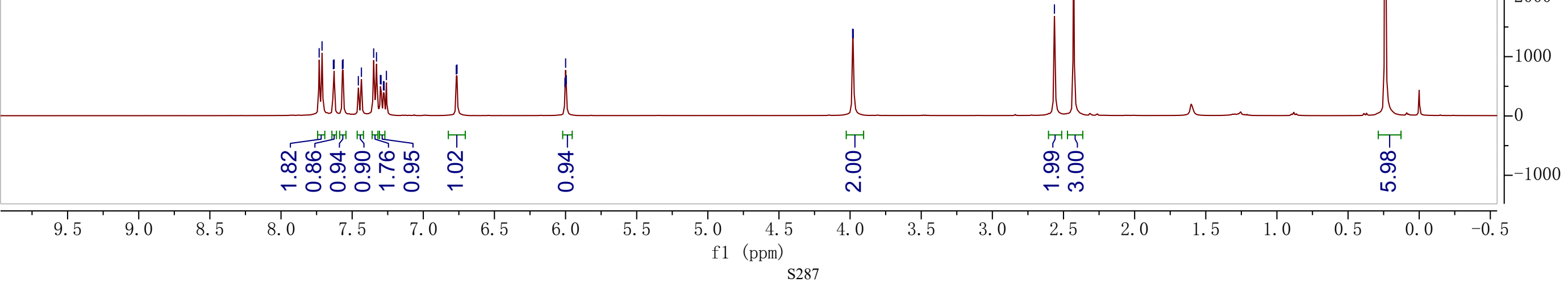




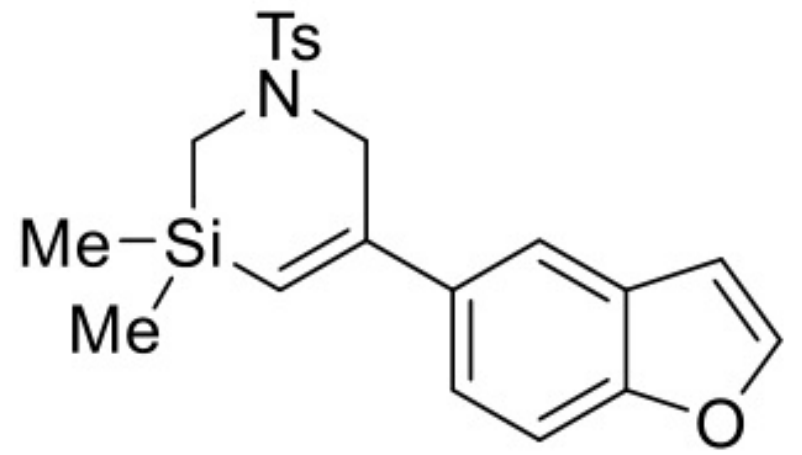

4ai 
<smiles>C[Si]1(C)C=C(c2cccc3c2oc2ccccc23)CN([As])C1</smiles>

4aj

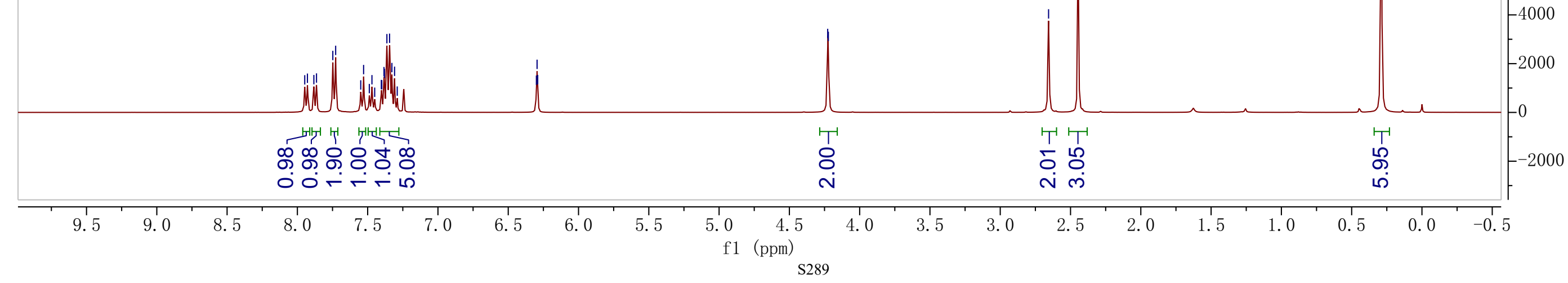


<smiles>C[Si]1(C)C=C(c2cccc3c2oc2ccccc23)CN([AsH2])C1</smiles>

4aj 
<smiles>C[Si]1(C)C=C(c2cccs2)CN([As])C1</smiles>

4ak

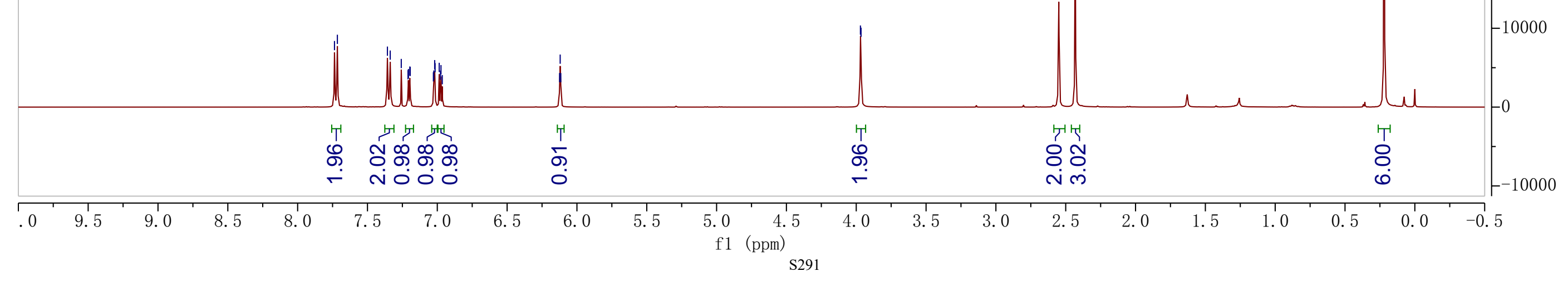




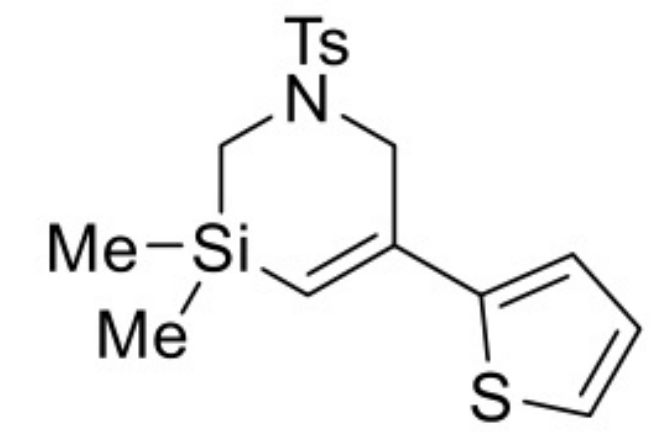

4ak

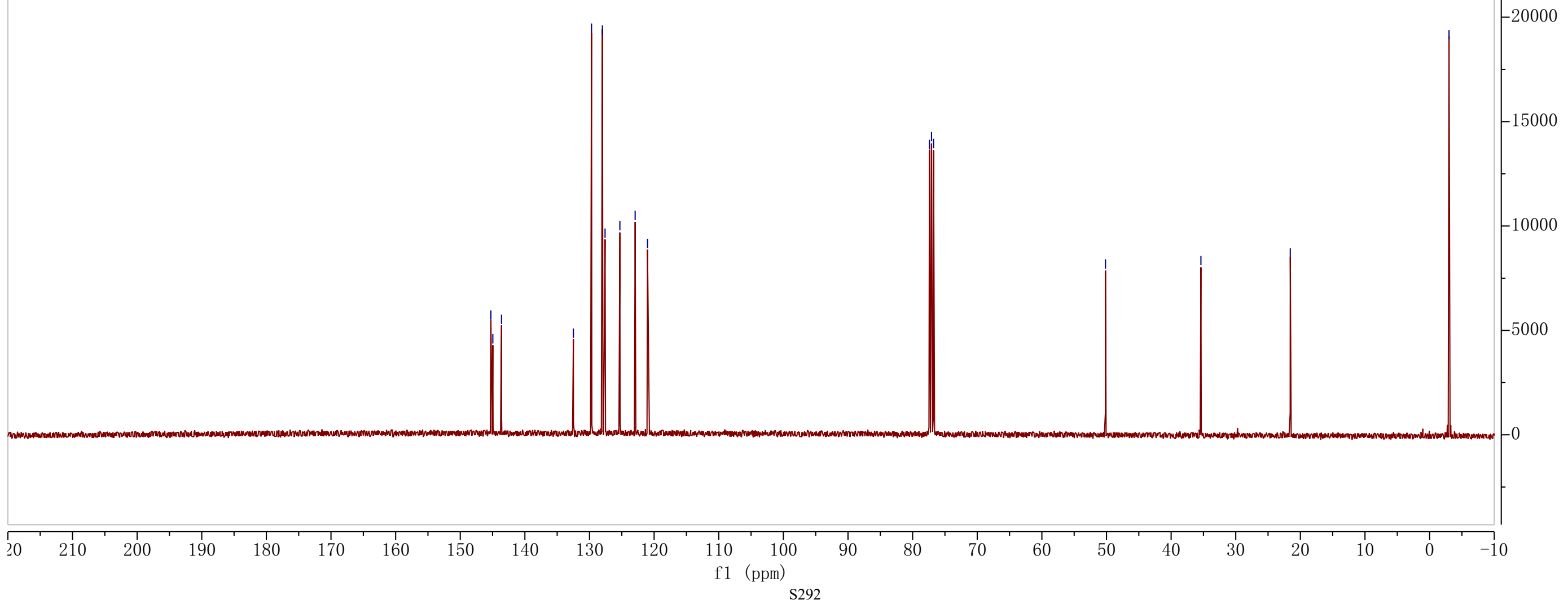


<smiles>CC1(C)CCSc2ccc(C3=C[Si](C)(C)CN([As])C3)cc21</smiles>

4al

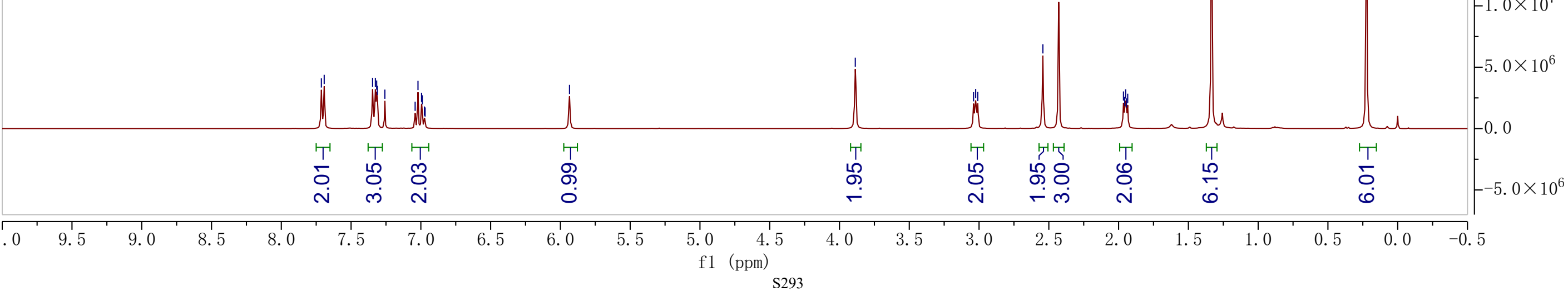


Ts<smiles>CC1(C)CCSc2ccc(C3=C[Si](C)(C)CN([As])C3)cc21</smiles>

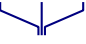

11/1!

4al 


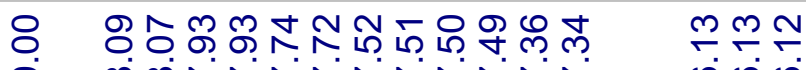

क

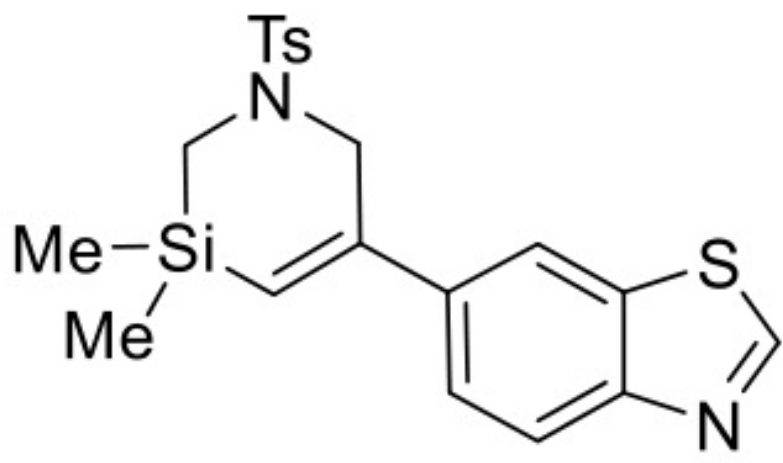

\section{4am}

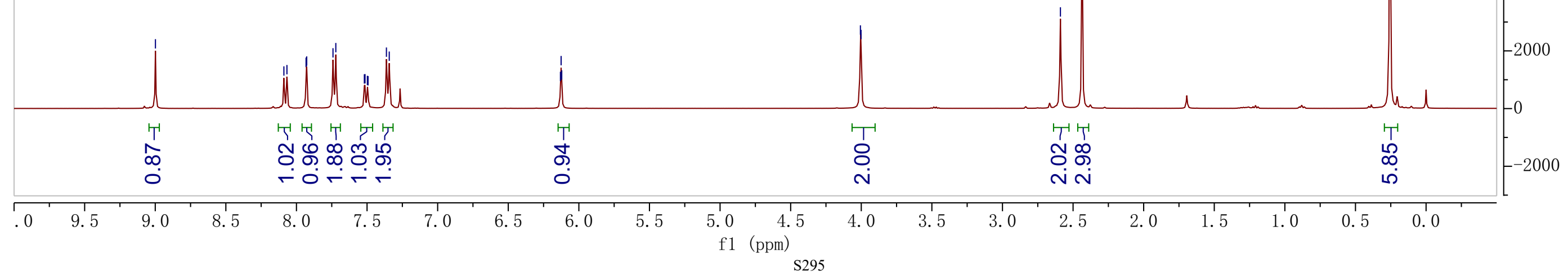


<smiles>C[Si]1(C)C=C(c2ccc3ncsc3c2)CN([AsH3])C1</smiles>

\section{4am}




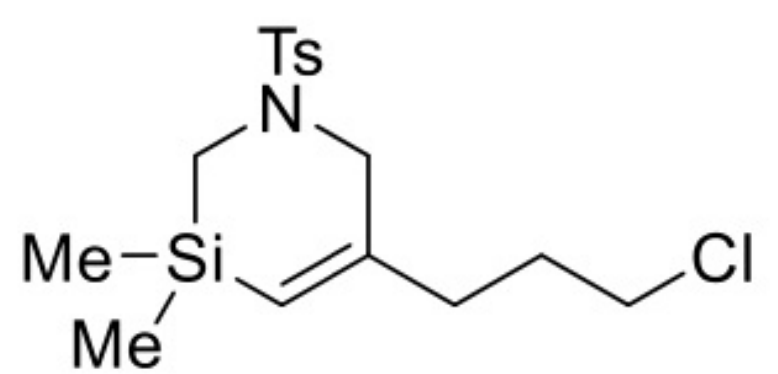

\section{4an}

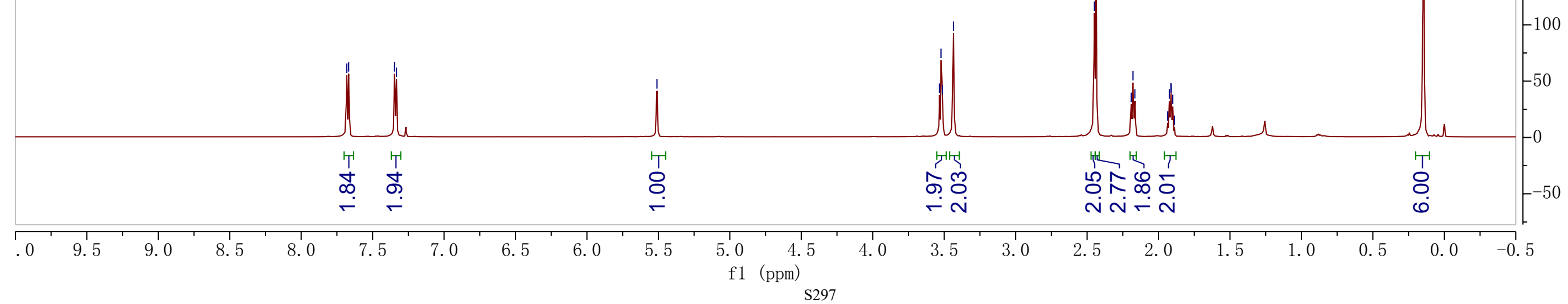


<smiles>C[Si]1(C)C=C(CCCCl)CN([As])C1</smiles>

$4 a n$

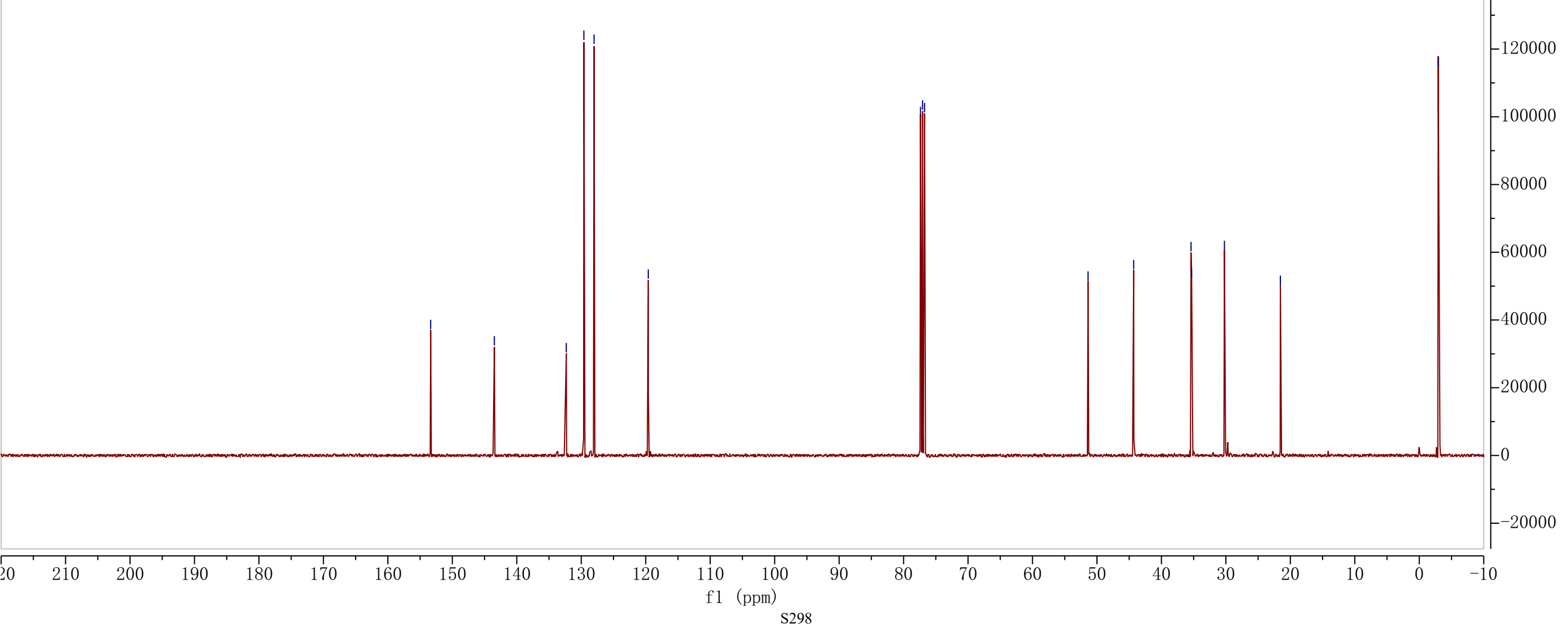


Ts<smiles>C[Si]1(C)C=C(C2CCCCC2)CN([As])C1</smiles>

4 ao

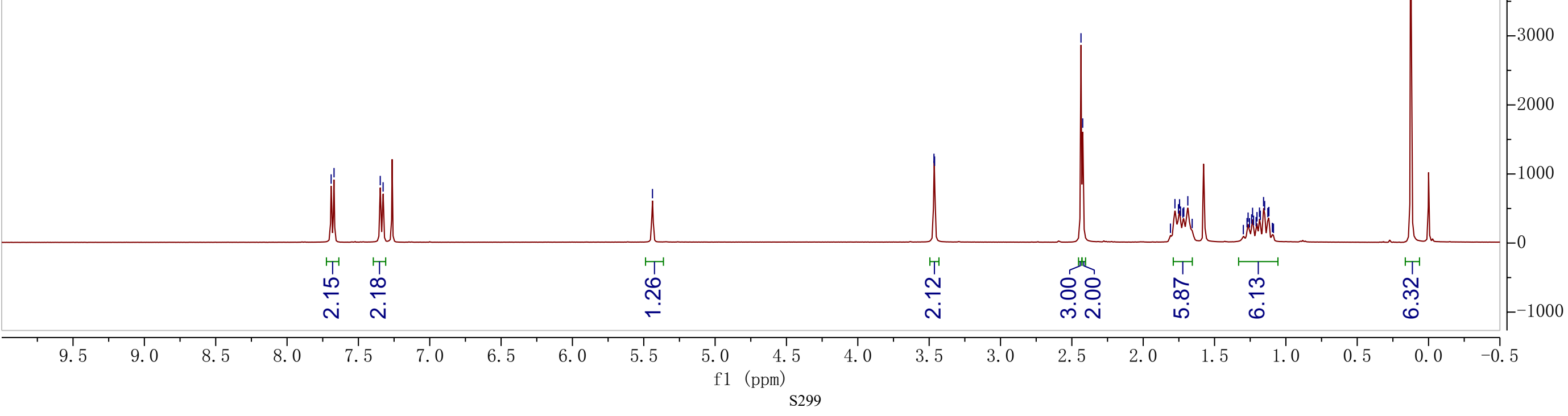


<smiles>C[Si]1(C)C=C(C2CCCCC2)CN([AsH2])C1</smiles>

$4 a o$ 


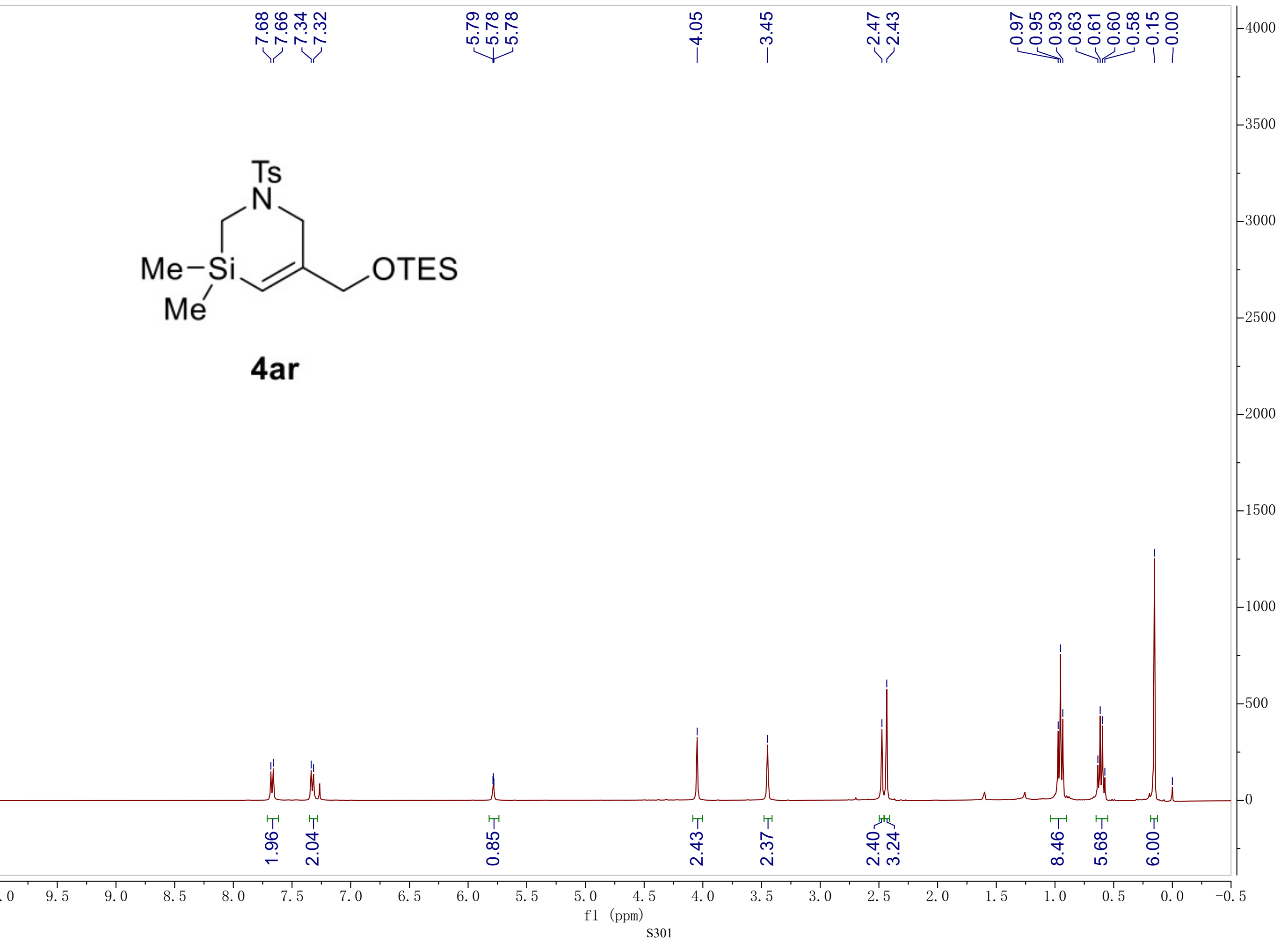


<smiles>C[Si]1(C)C=C(C[O+]C#[SH])CN([As])C1</smiles>

4ar 


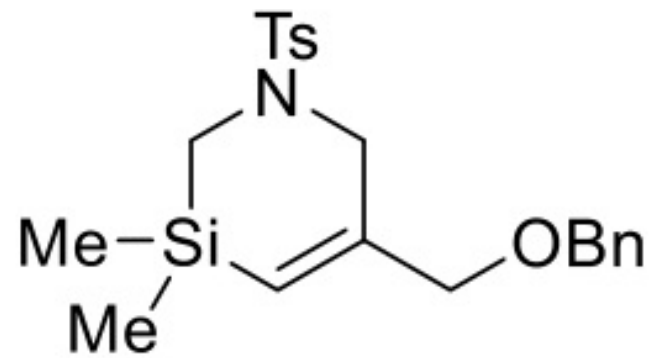

4as

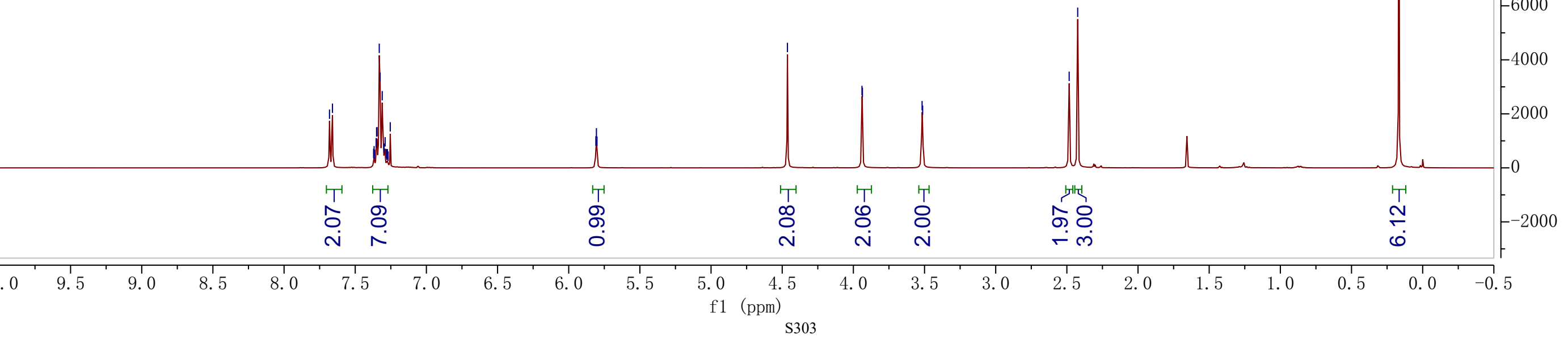




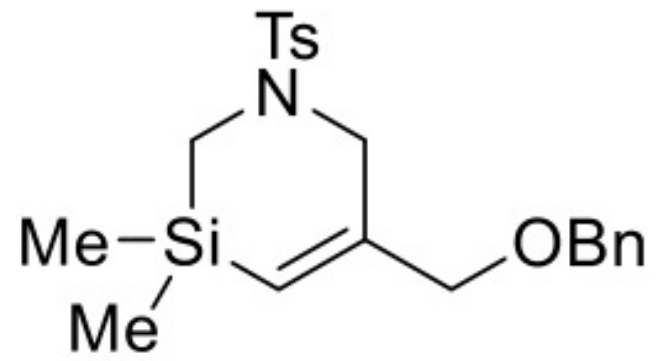

4as

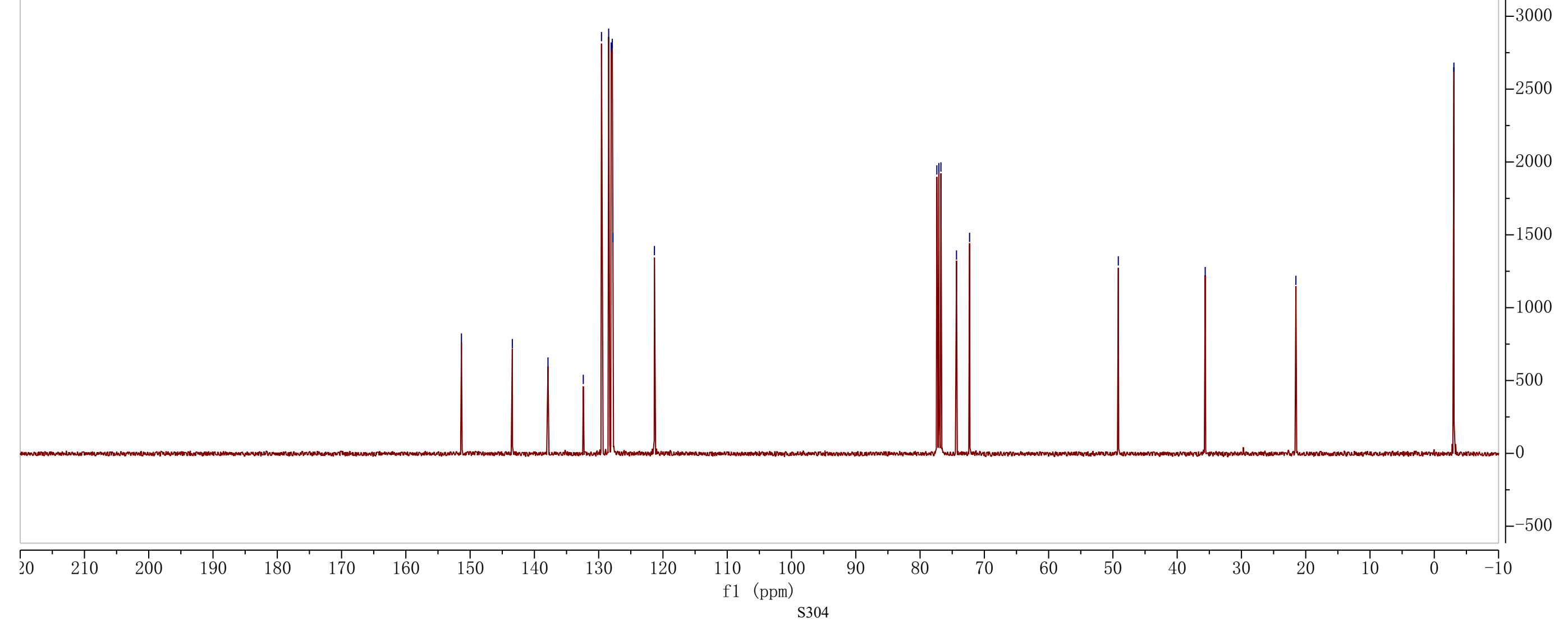




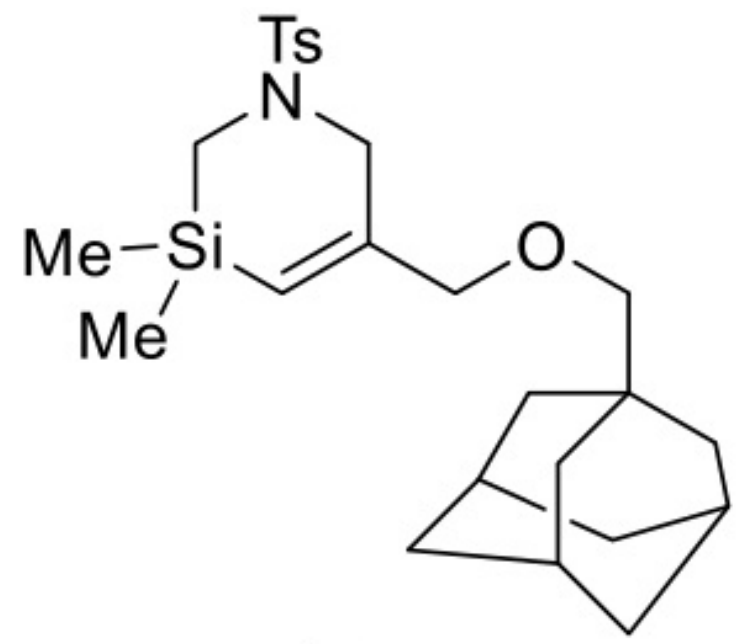

4at

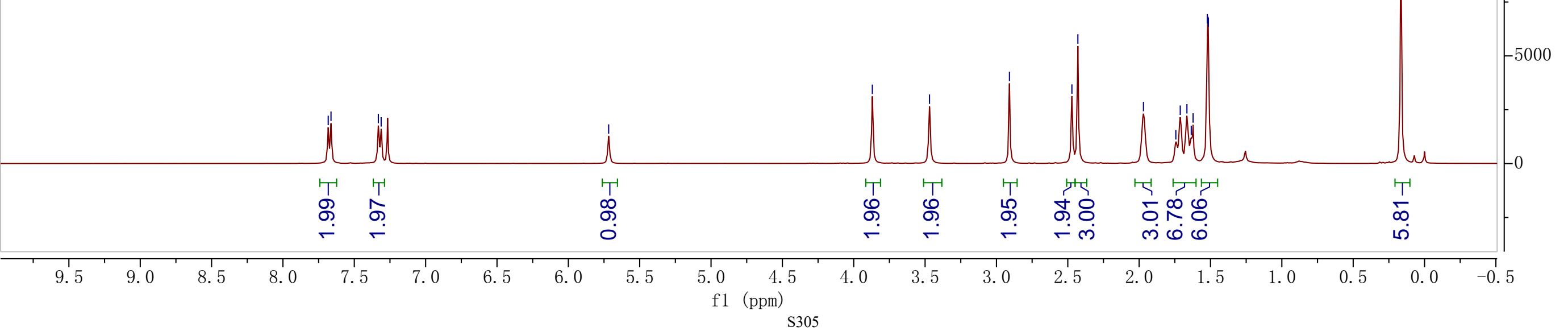




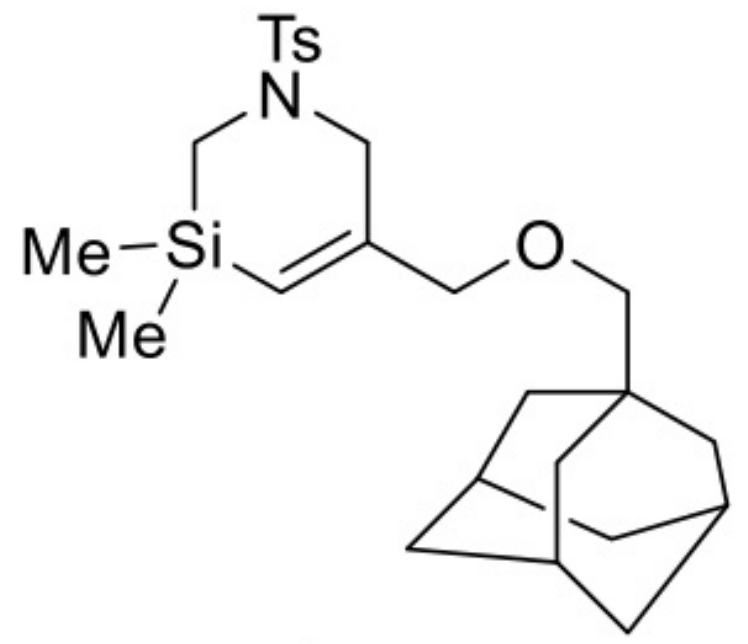

4at

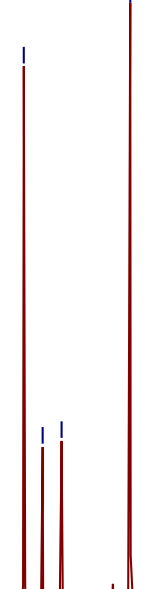




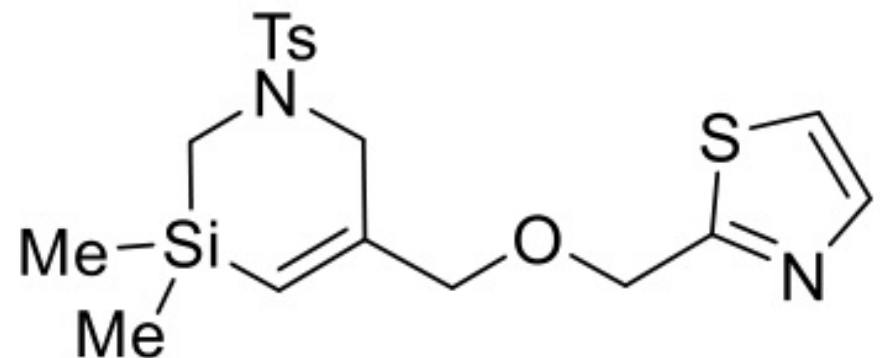

\section{4au}

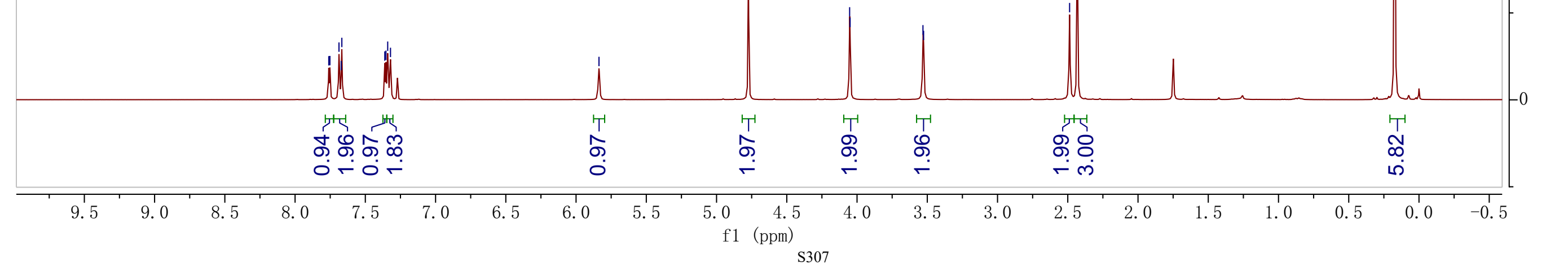




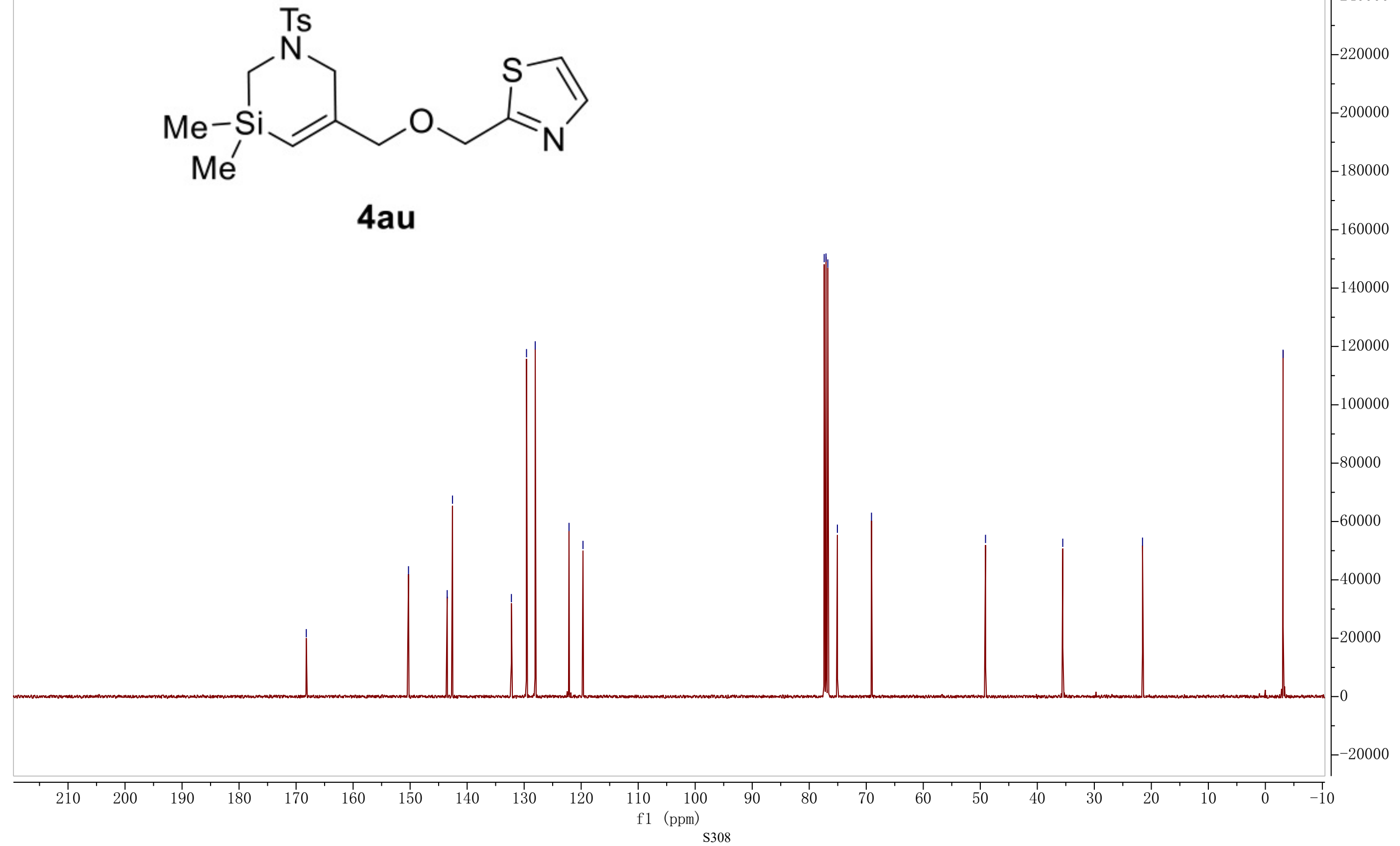




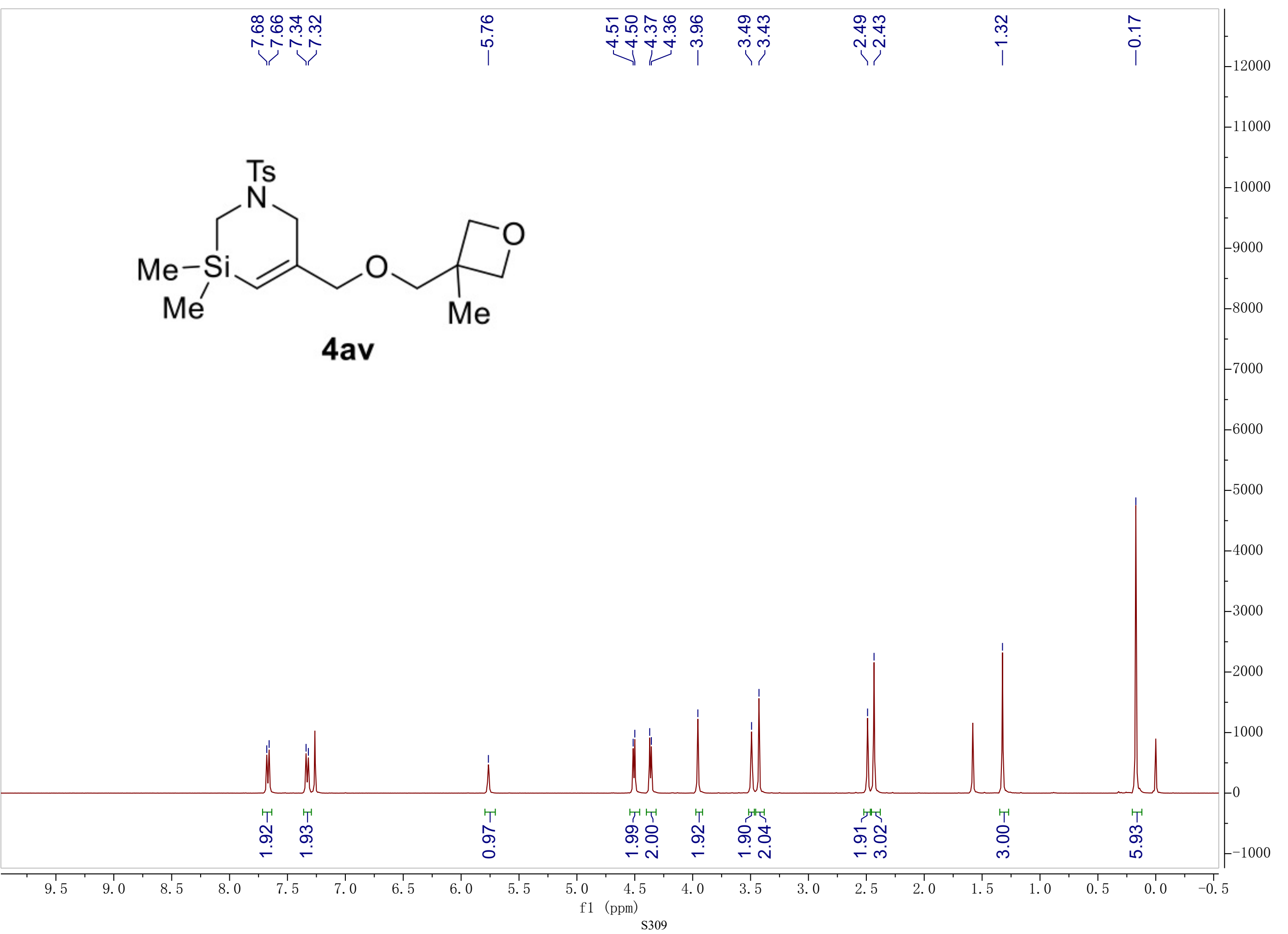


lim

广

$\mathrm{Me}^{-\mathrm{Si}}$

4av

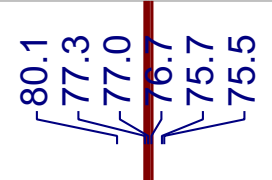

$\stackrel{\circ}{i}$

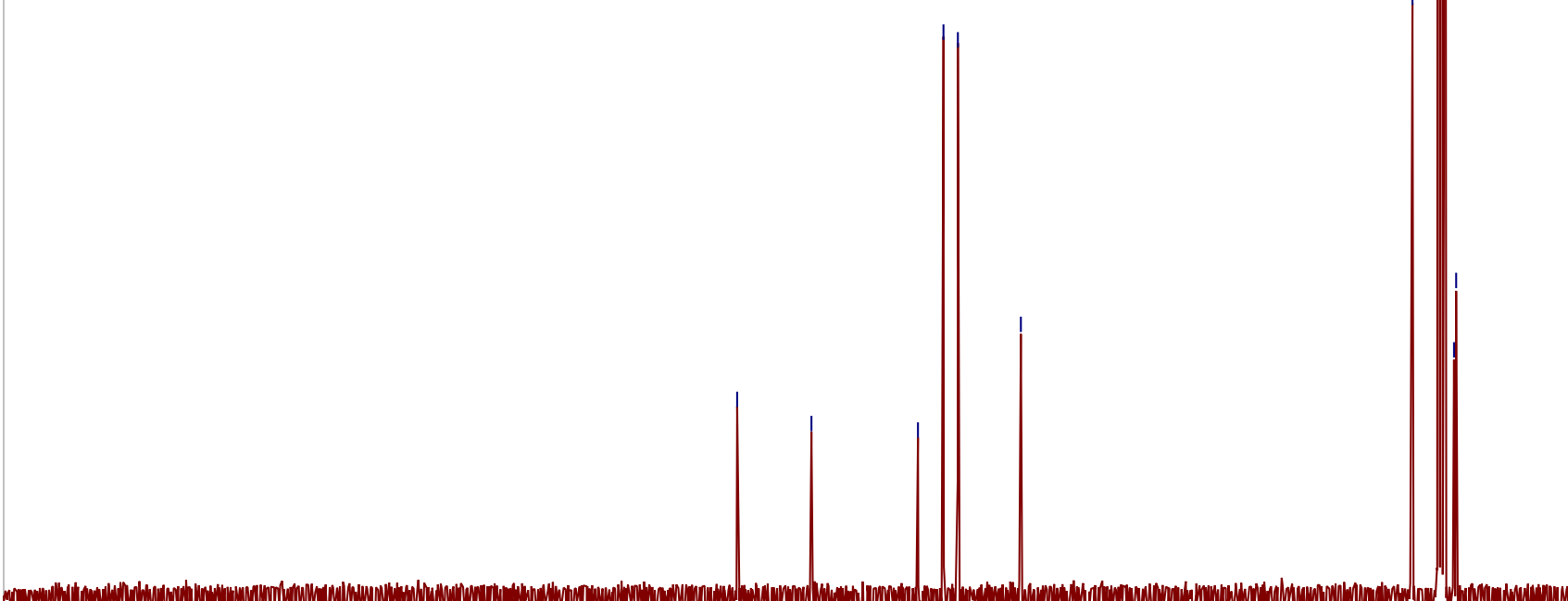


S

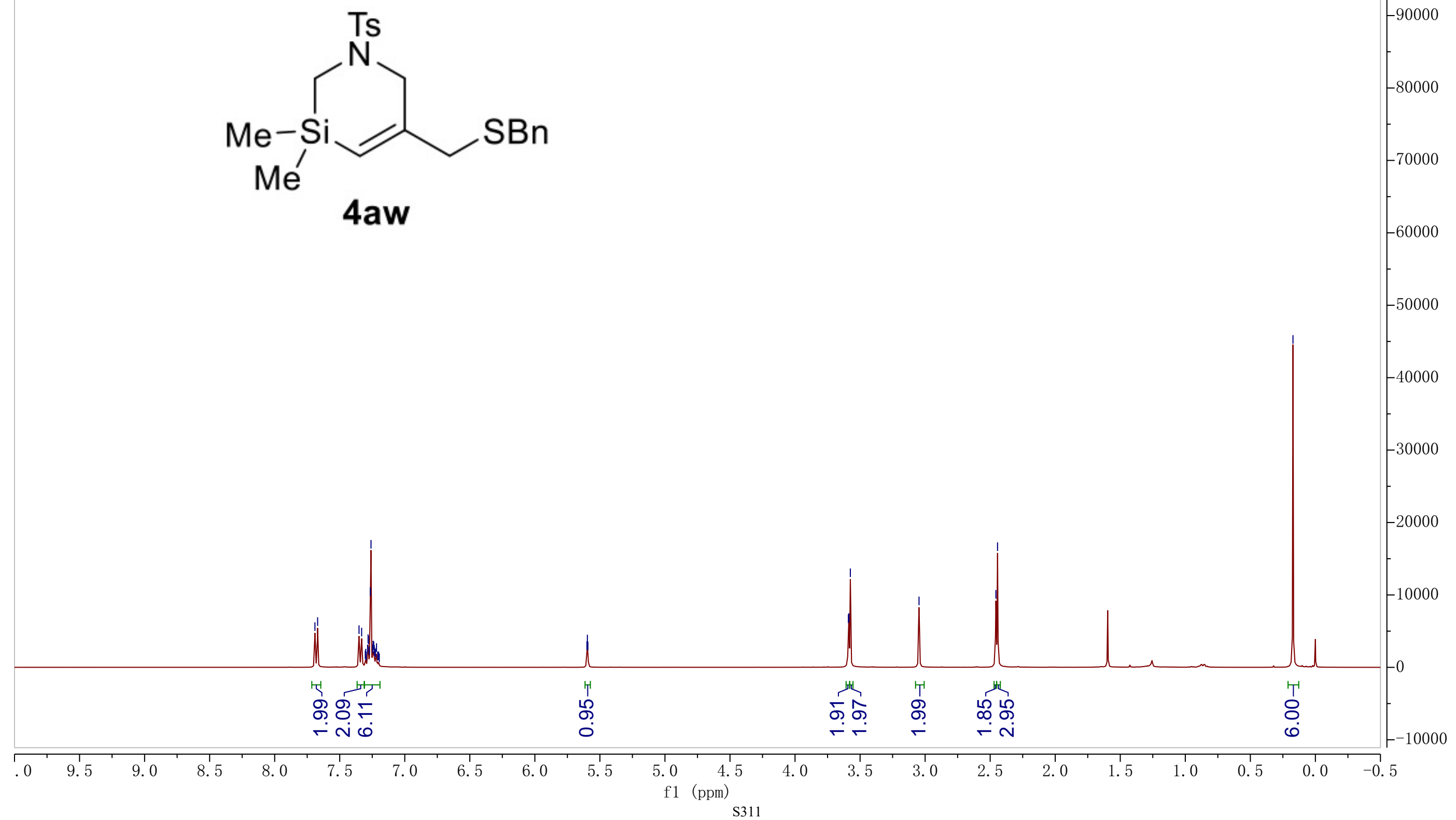


Ts<smiles>C[Si]1(C)C=C(CSc2ccccc2)CN([AsH2+])C1</smiles> 
<smiles>C[Si]1(C)C=C(CNc2ccccc2)CN([AsH2])C1</smiles>

4ax กํ. 
Ts<smiles>C[Si]1(C)C=C(CNc2ccccc2)CN([AsH2-])C1</smiles>

4ax

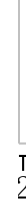









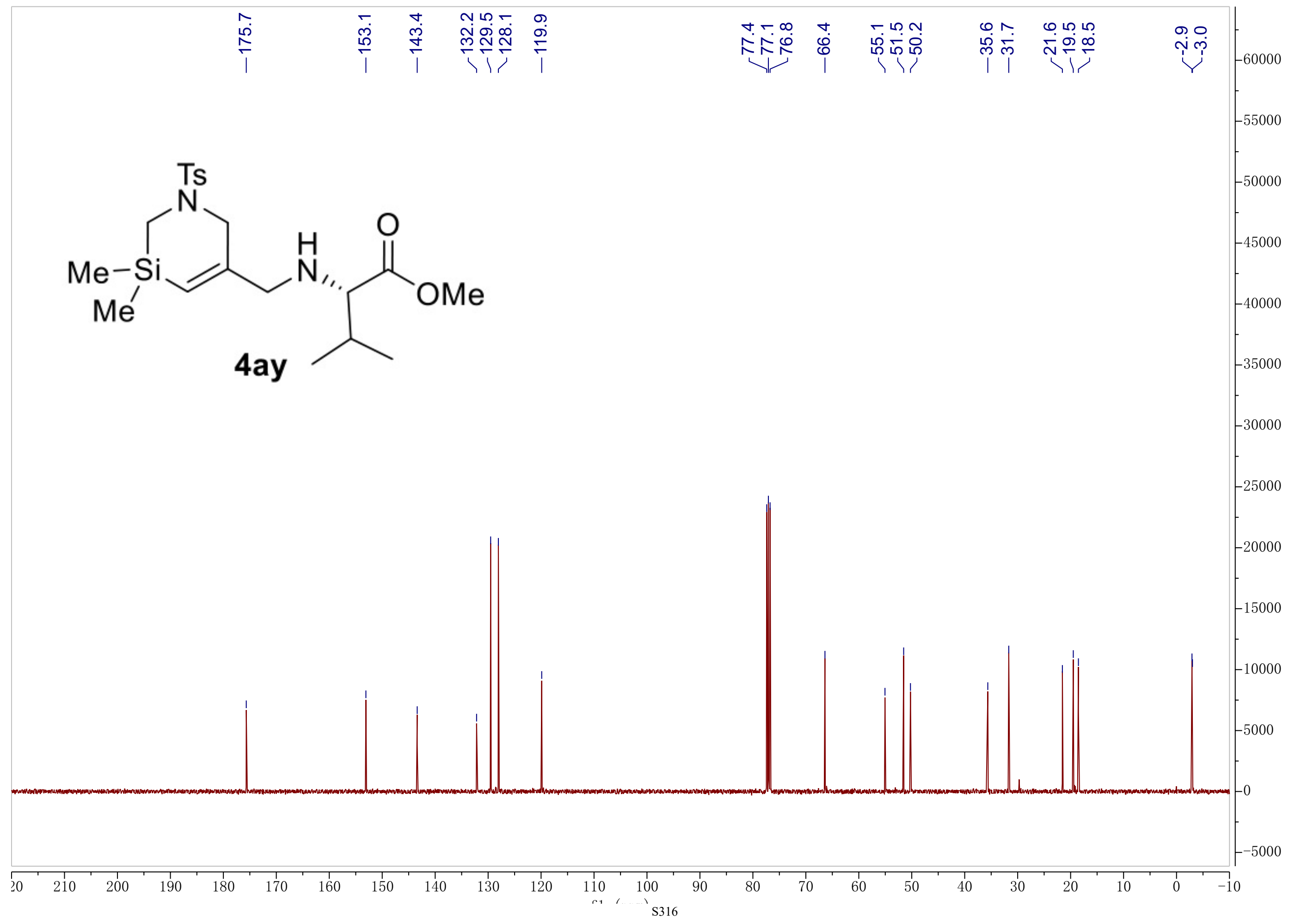




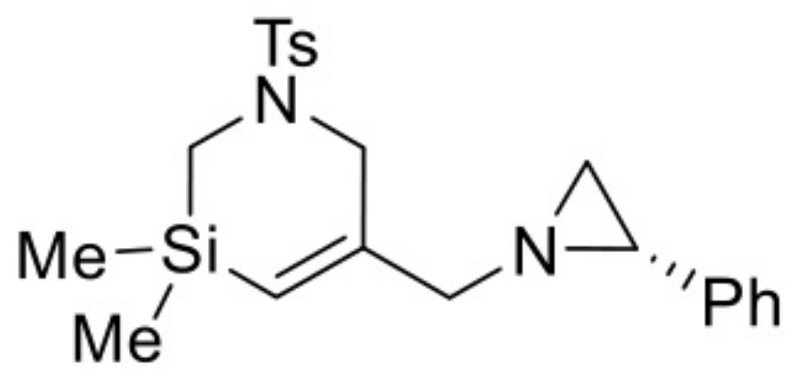

$4 a z$

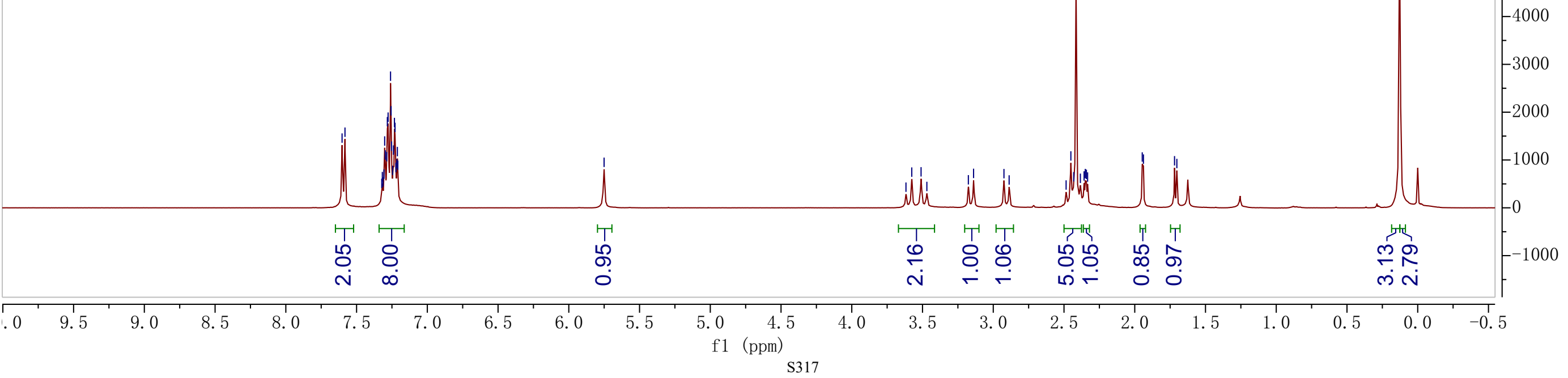


<smiles>C[Si]1(C)C=C(CN2C[C@H]2c2ccccc2)CN([AsH2])C1</smiles>

\section{$4 a z$}



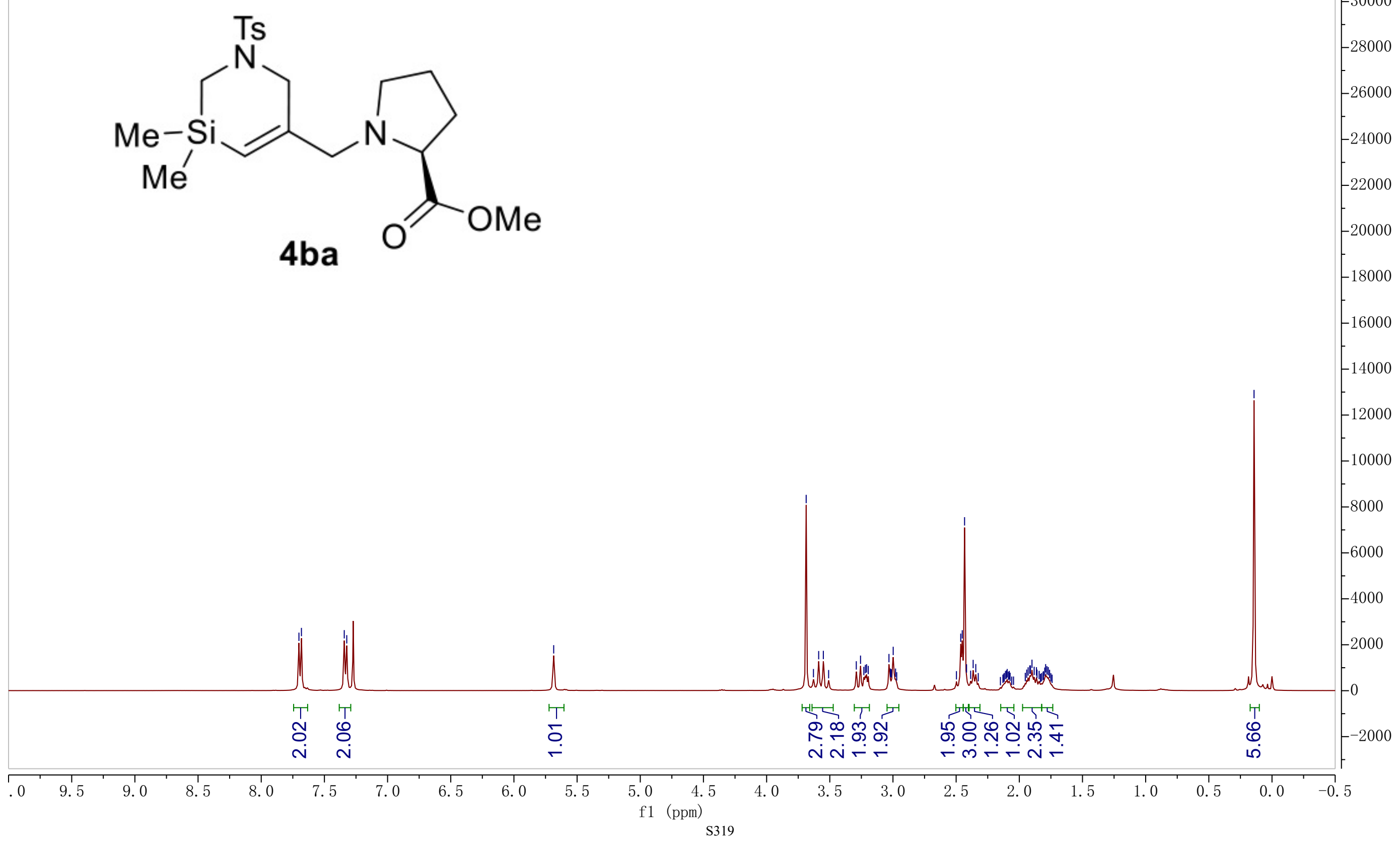


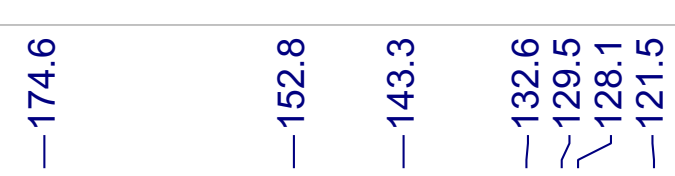

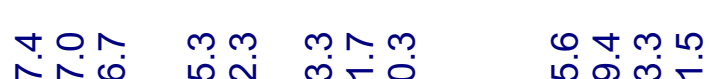

상

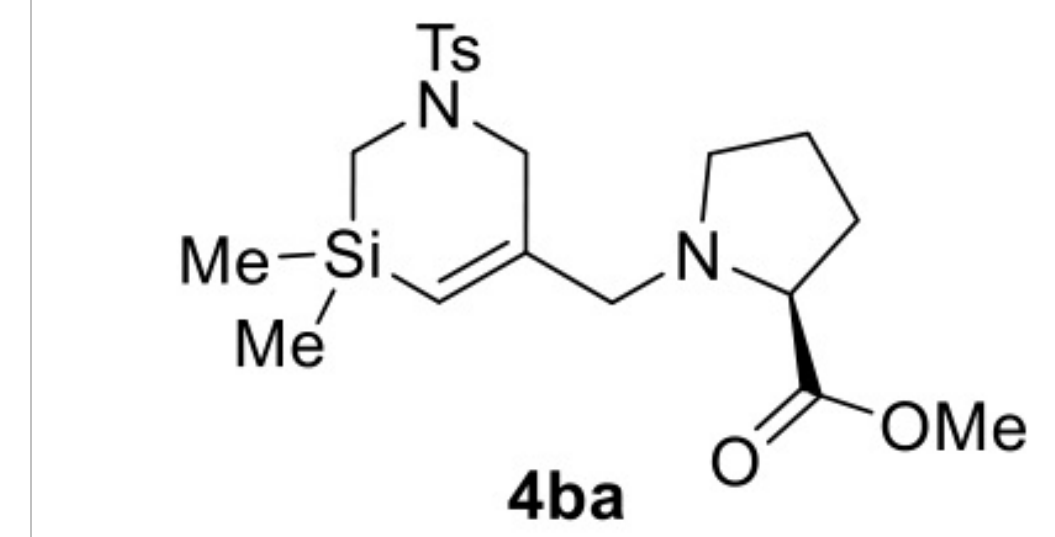




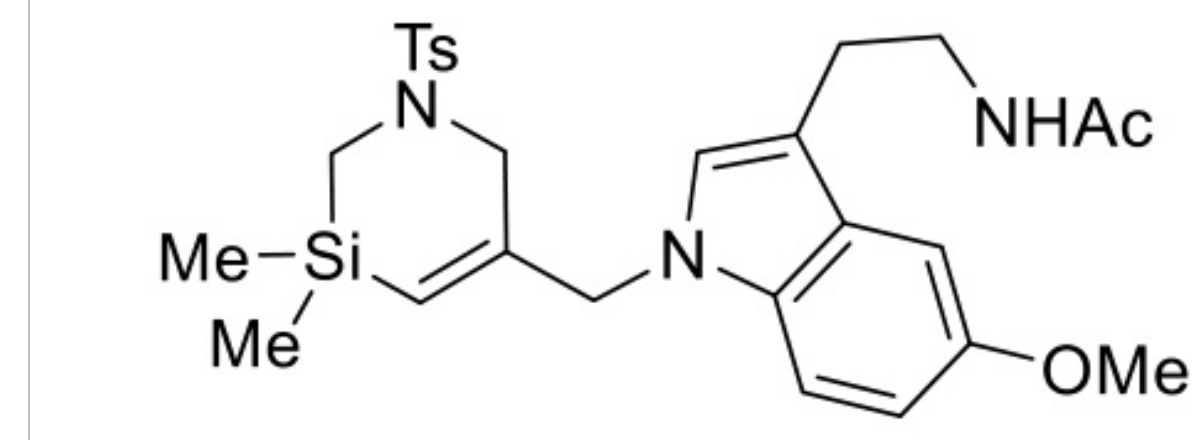

4bb

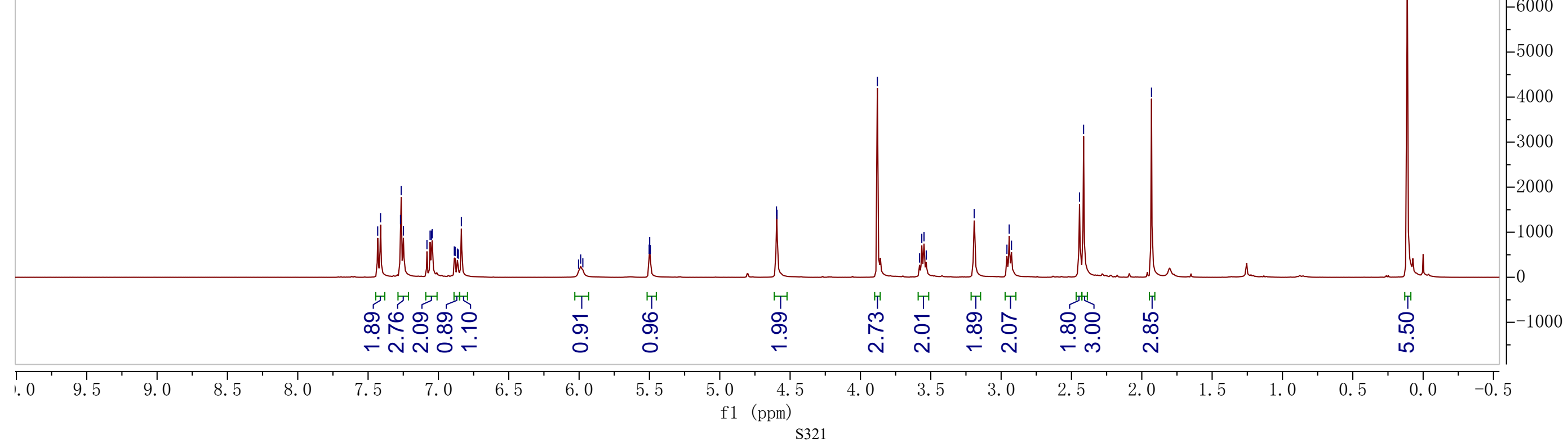




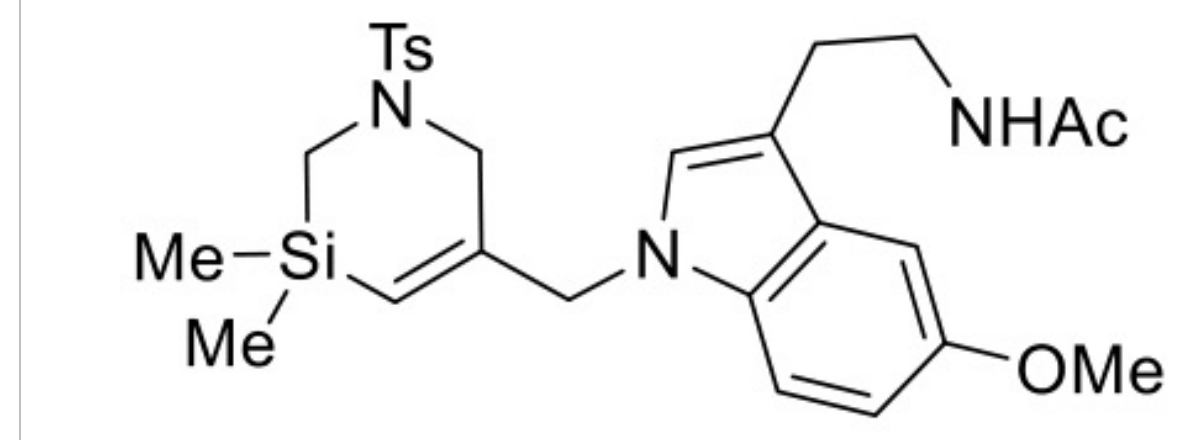

4bb

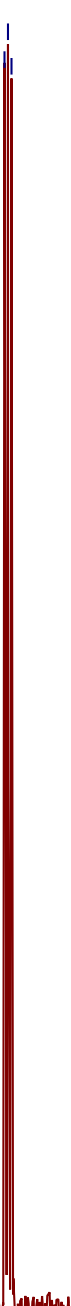




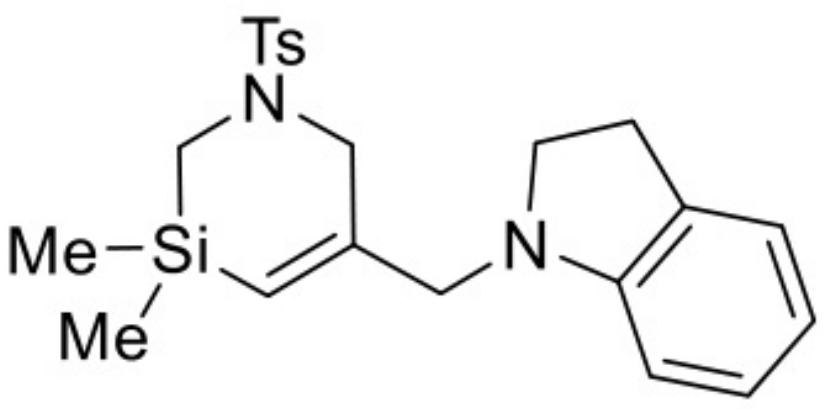

4bc

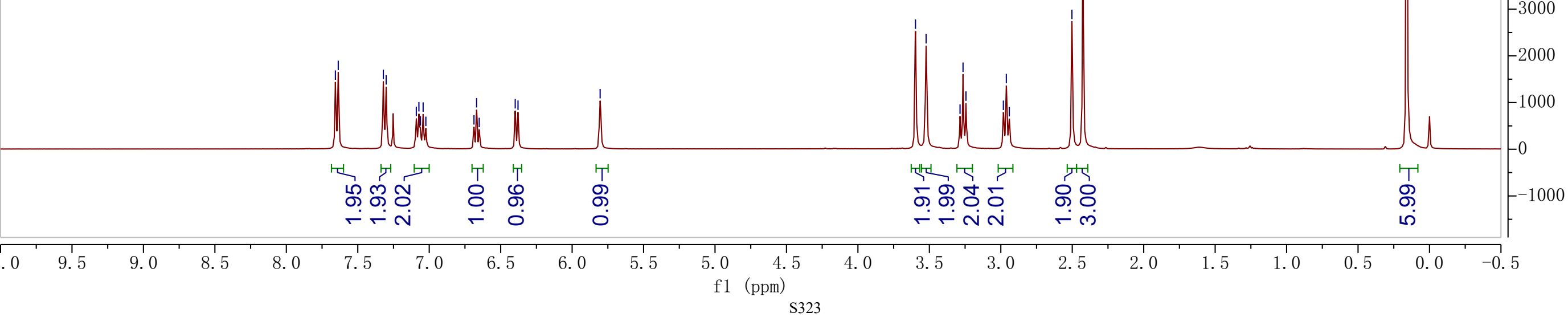


<smiles>C[Si]1(C)C=C(CN2CCc3ccccc32)CN([As])C1</smiles> 
<smiles>C[Si]1(C)C=C(CN2CCc3sccc3C2)CN([AsH])C1</smiles>

4bd

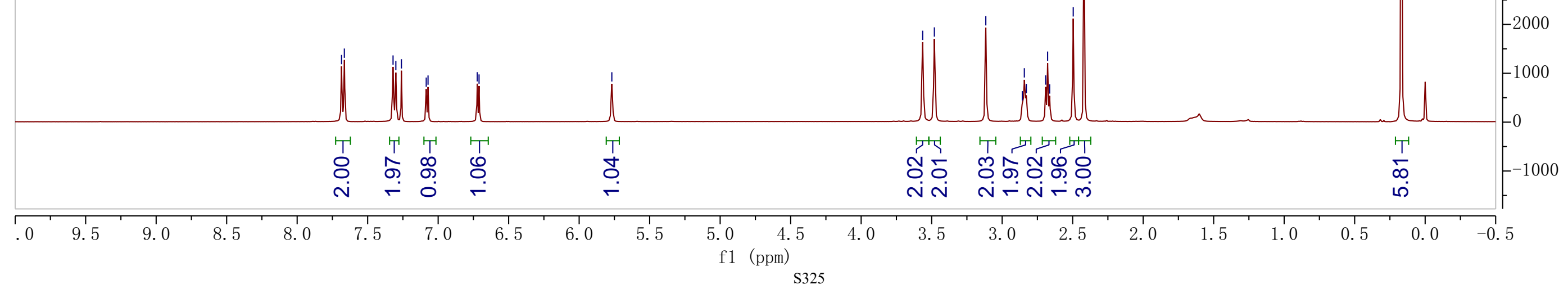




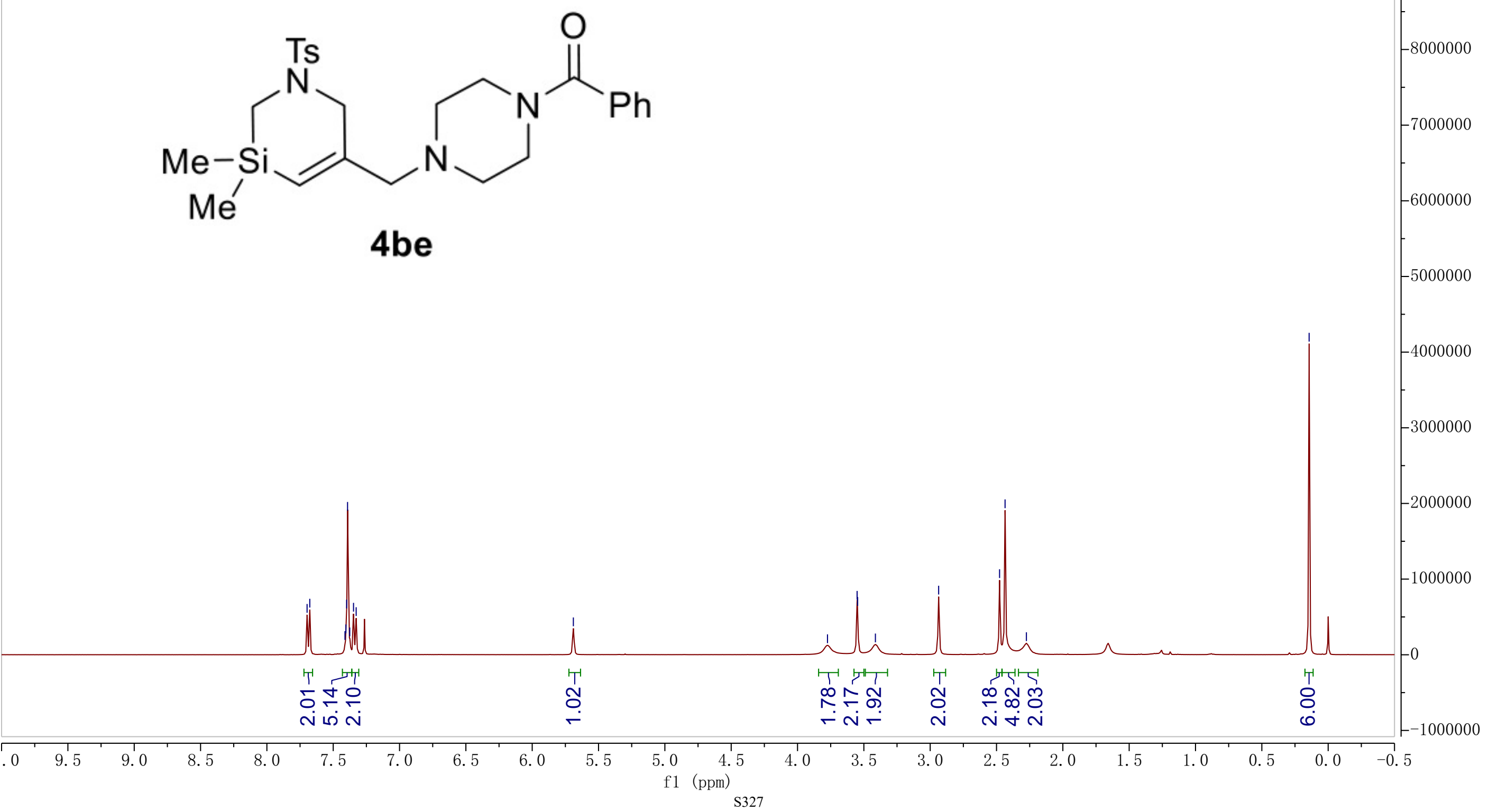


m. 它守舟

$\frac{1}{1}$

|

๑๐

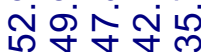

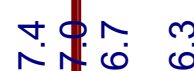

今Ne \&

1)

$\stackrel{0}{i}$<smiles>C[Si]1(C)C=C(CN2CCN(C(=O)c3ccccc3)CC2)CN([AsH2])C1</smiles>

\section{4be}

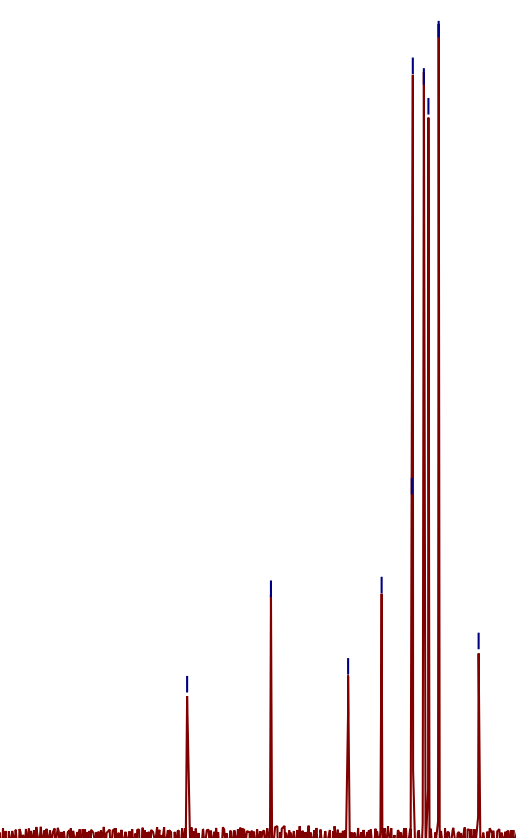



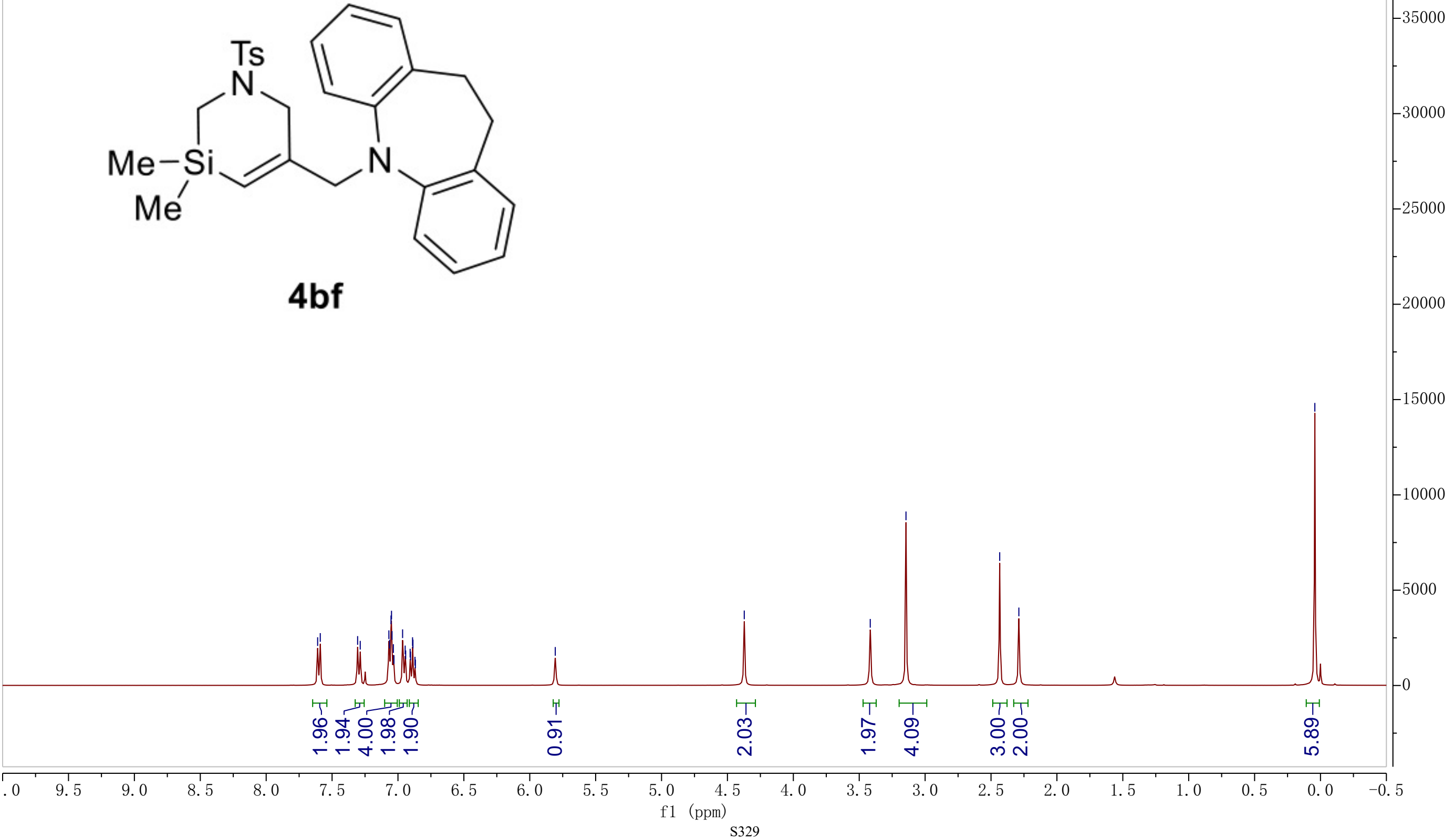


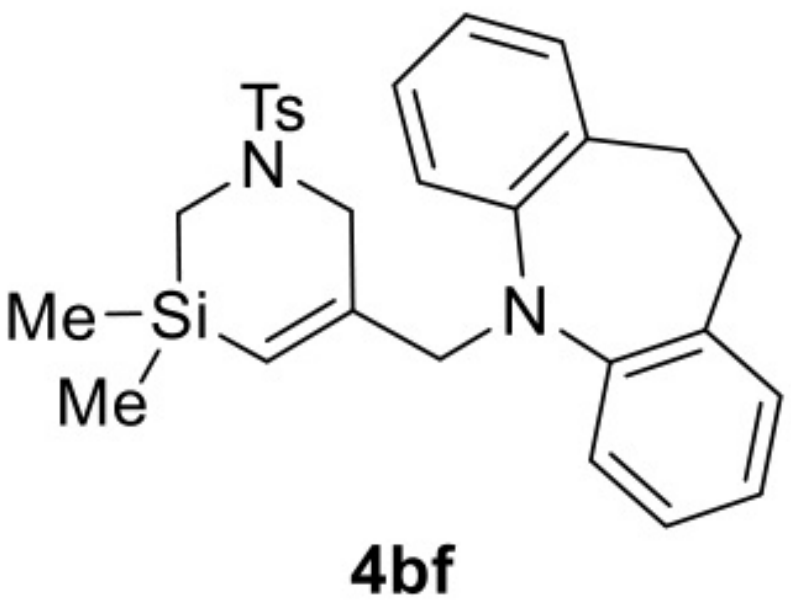


<smiles>[R6]N(C)CC1=C[Si](C)(C)CN([AsH-])C1</smiles>

4bg

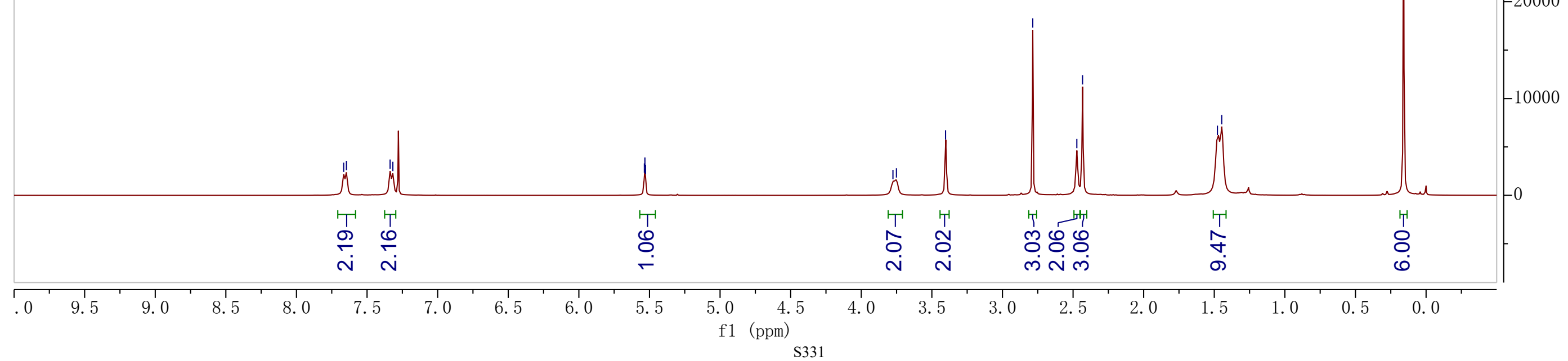




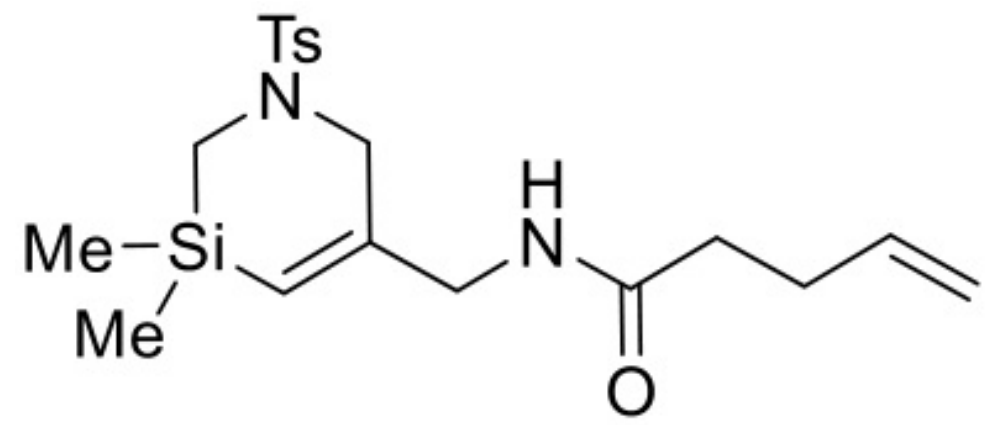

4bh

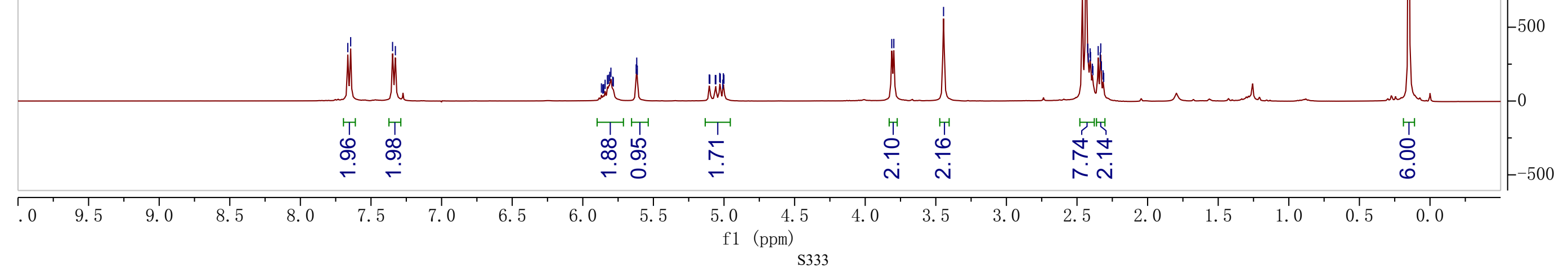




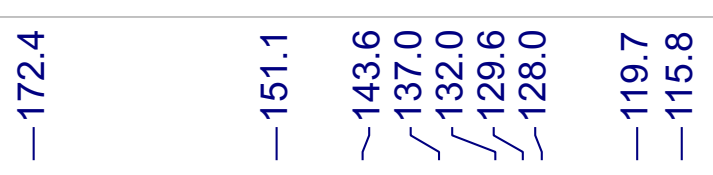

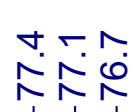

守

riouvuv

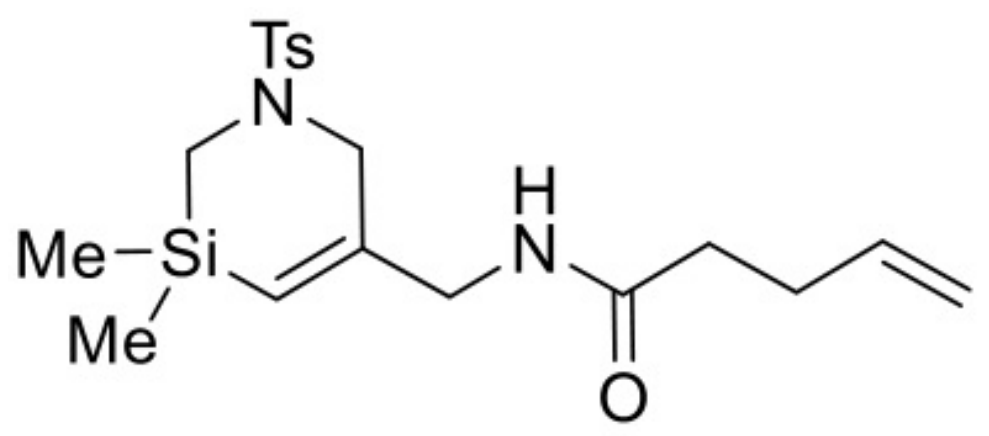

4bh

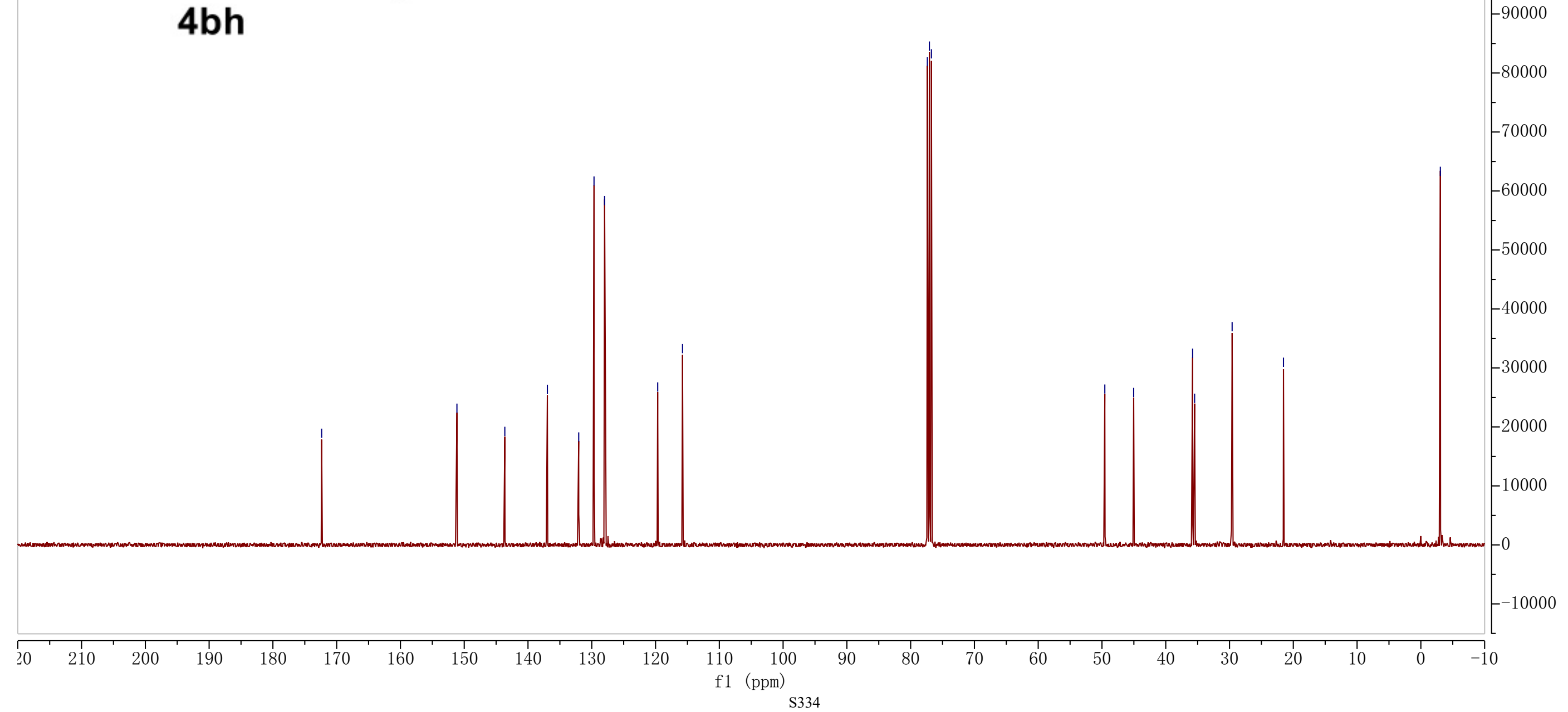


<smiles>C[Si]1(C)C=C(CNC(=O)C2CC2)CN([As])C1</smiles>

4bi

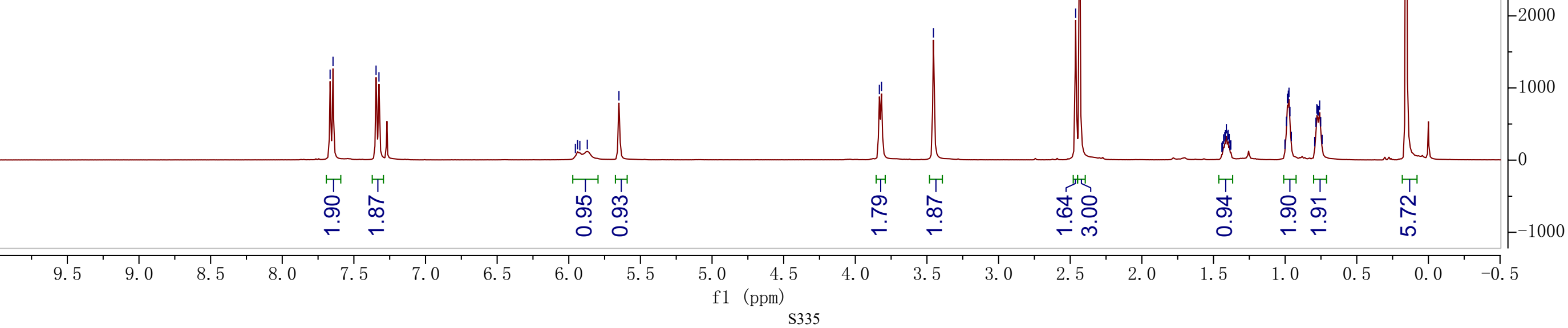


Ts<smiles>C[Si]1(C)C=C(CNC(=O)C2CC2)CN([As])C1</smiles>

4bi 


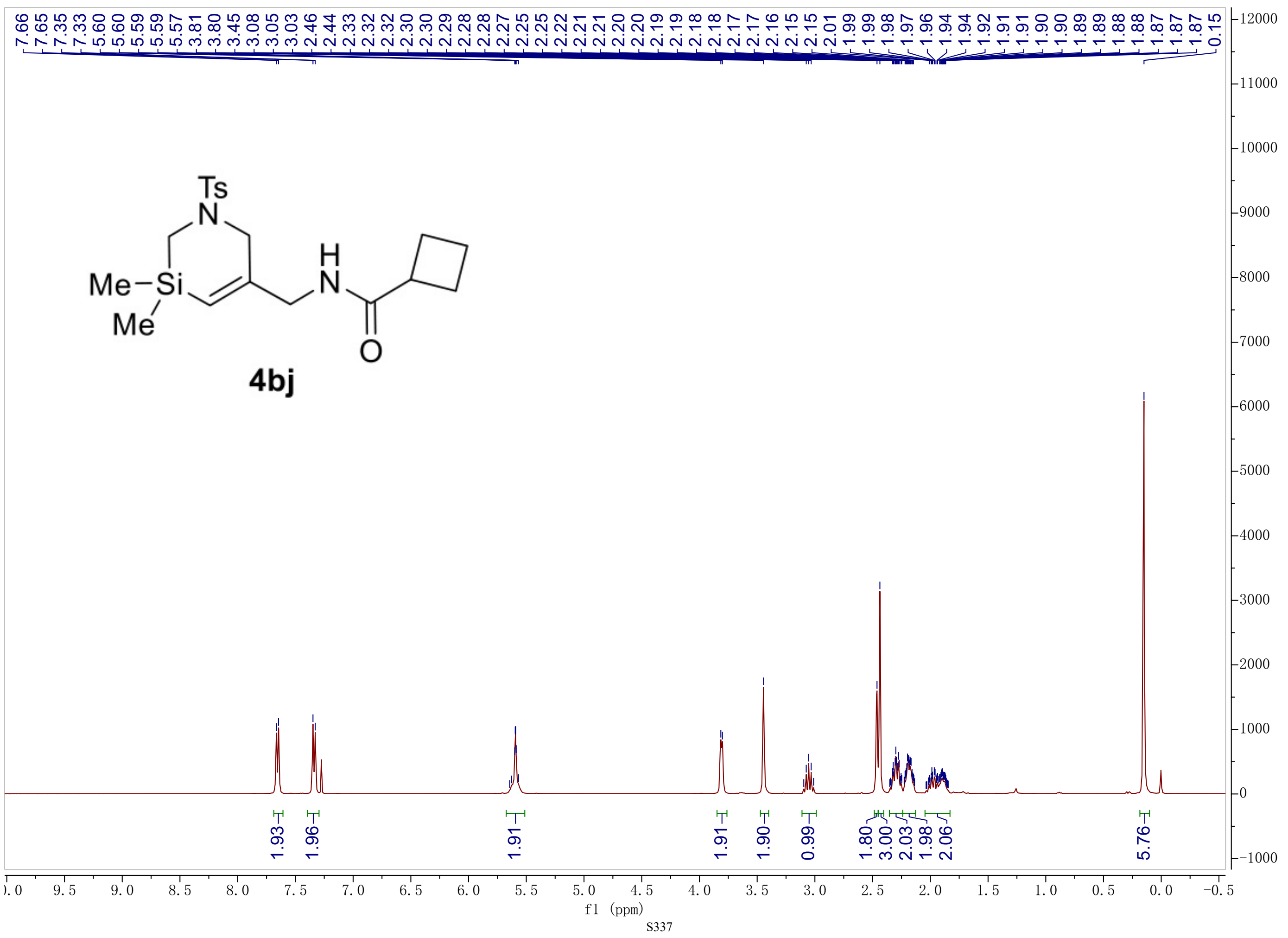


Ts<smiles>C[Si]1(C)C=C(CNC(=O)C2CCC2)CN([AsH3])C1</smiles>

4bj 


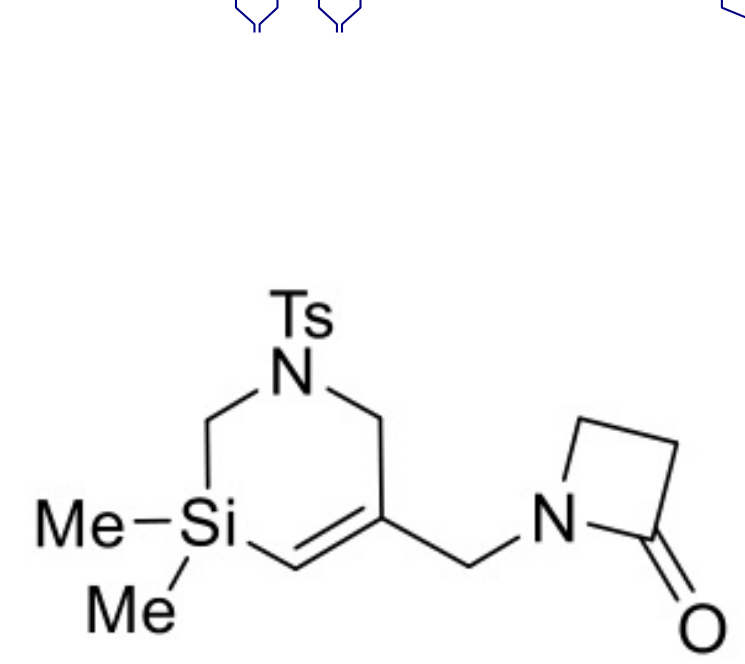

\section{4bk}

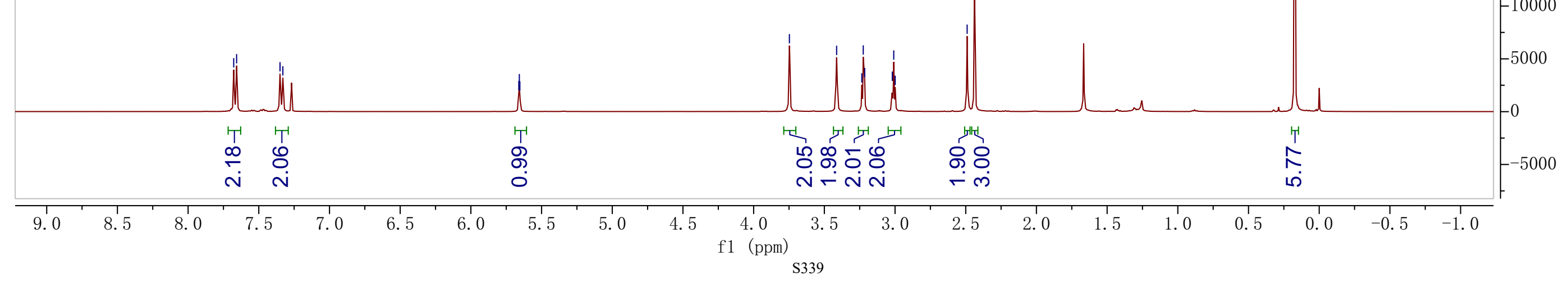




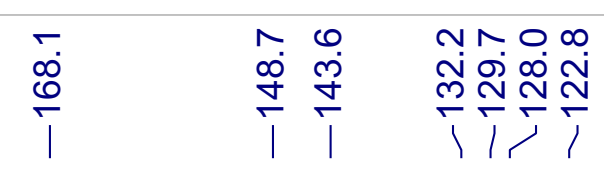

$\mathrm{Me}^{\mathrm{Me}}$

\section{4bk}

ํำ

삭

mo mọ

ஓீ ஓंர்

$\stackrel{1}{\frac{1}{4}}$ 


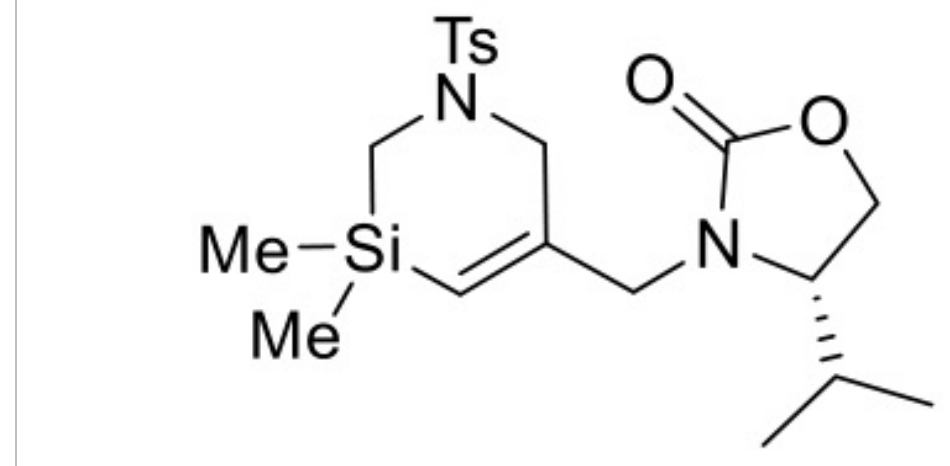

4bl

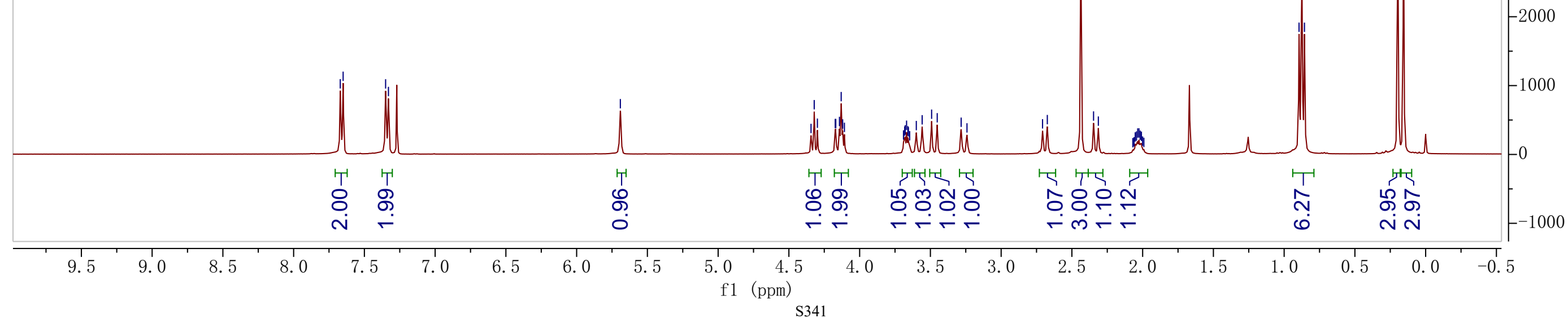


<smiles>CC(C)[C@H]1COC(=O)N1CC1=C[Si](C)(C)CN([As])C1</smiles>

4bl

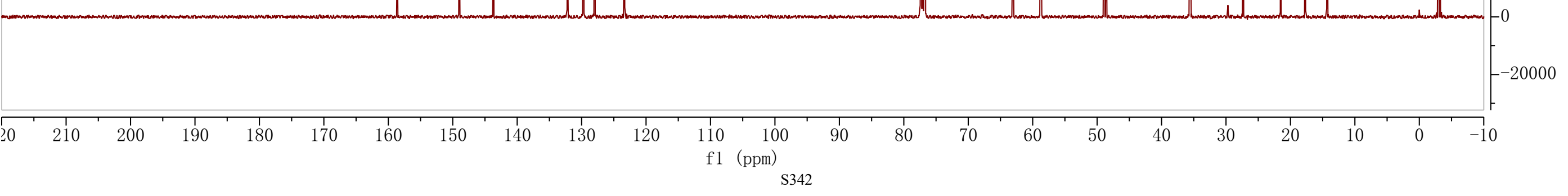




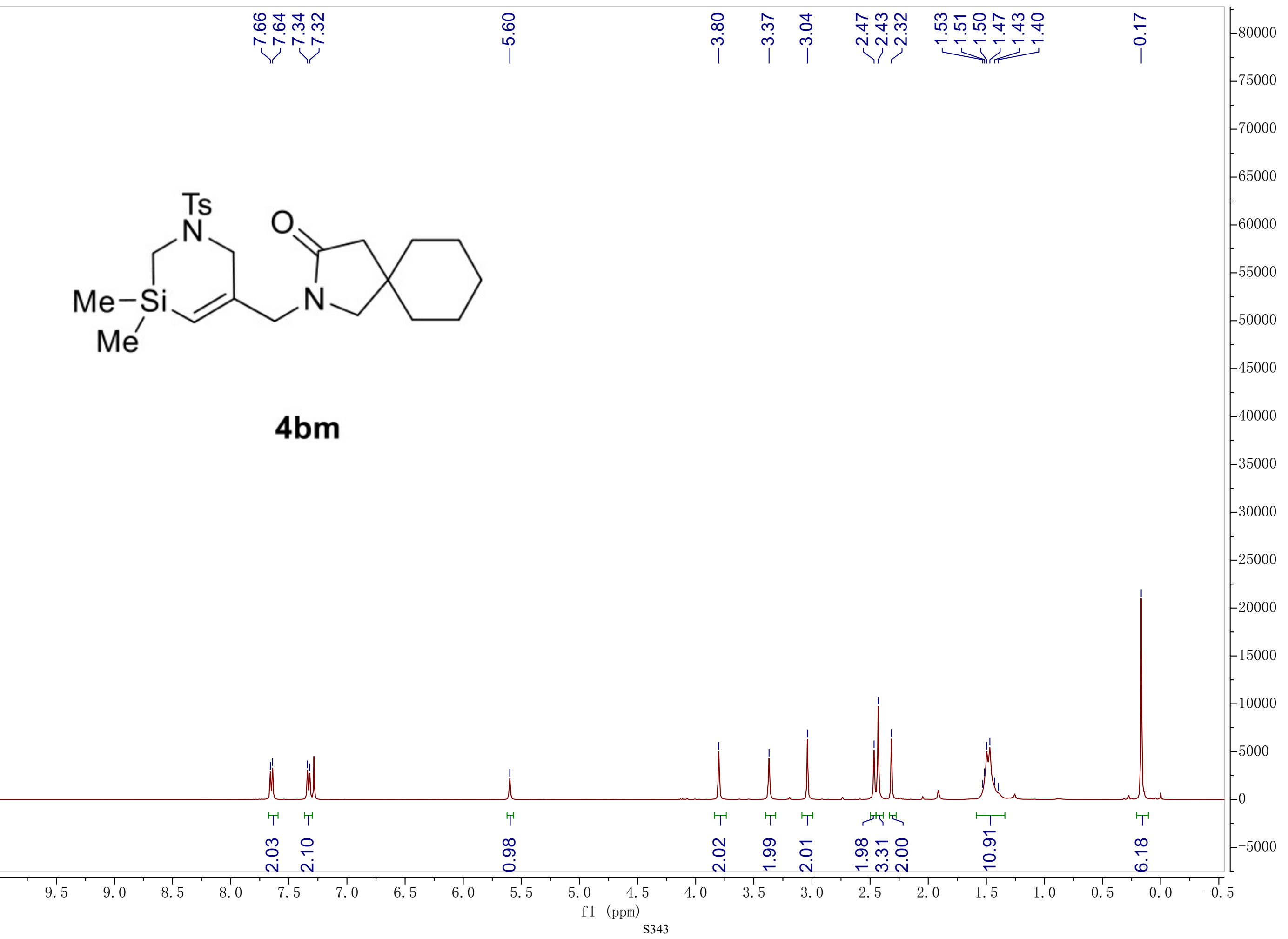




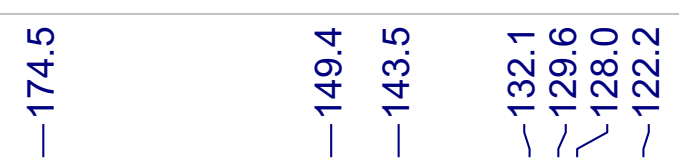

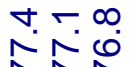

송

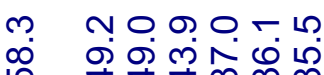

ம்iñ

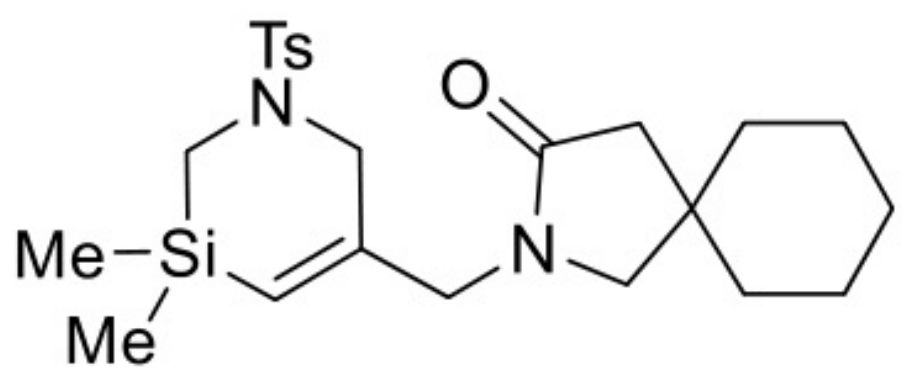

4bm 
<smiles>C[Si]1(C)C=C(CN2CCCCC2=O)CN([As])C1</smiles>

4bn

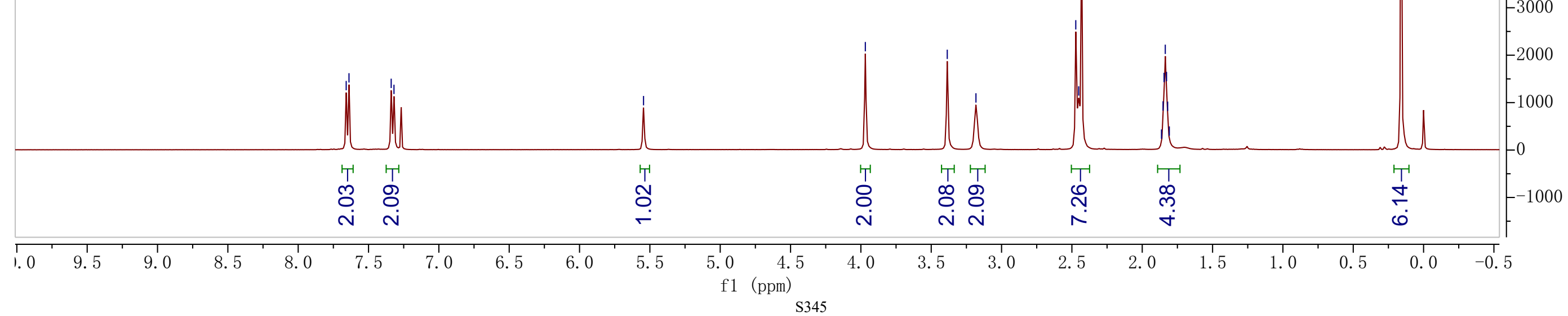


<smiles>C[Si]1(C)C=C(CN2CCCCC2=O)CN([AsH2])C1</smiles>

4bn 


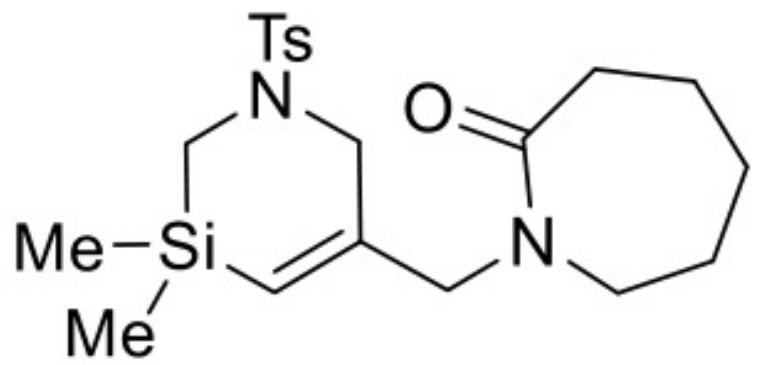

4 bo

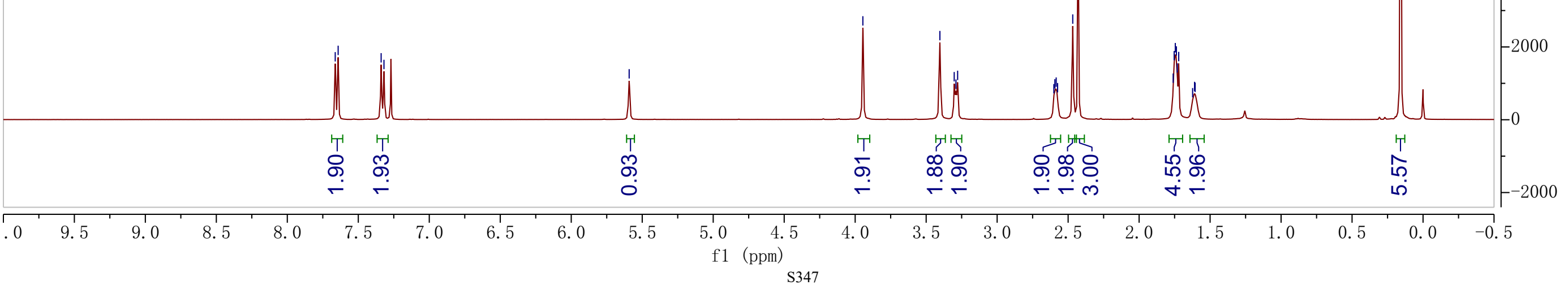




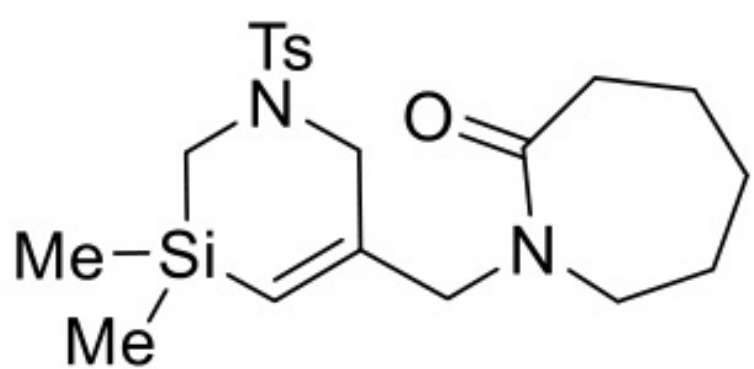

4bo

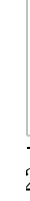


<smiles>C[Si]1(C)C=C(CN2CCCCCCC2=O)CN([As])C1</smiles>

$4 b p$

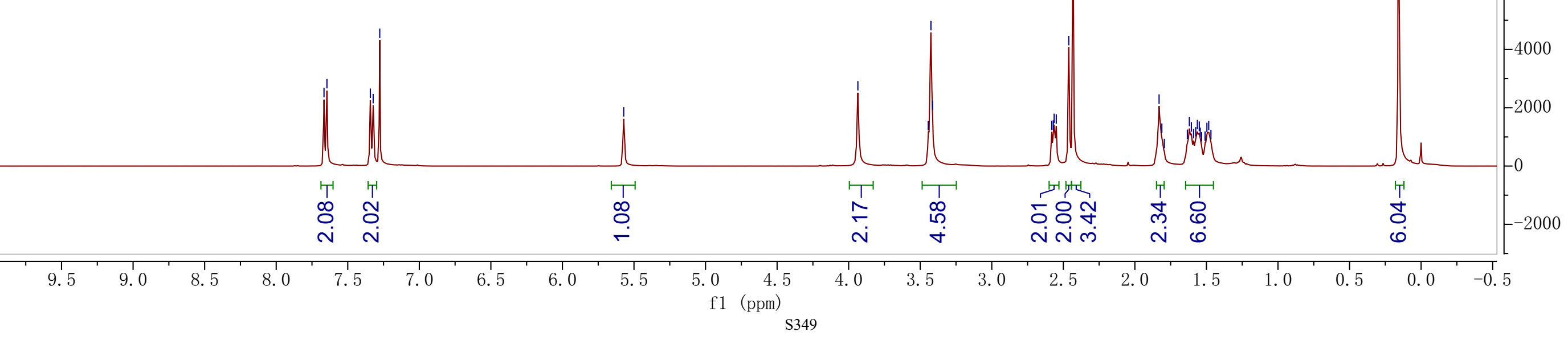


<smiles>C[Si]1(C)C=C(CN2CCCCCCC2=O)CN([AsH3])C1</smiles>

4bp

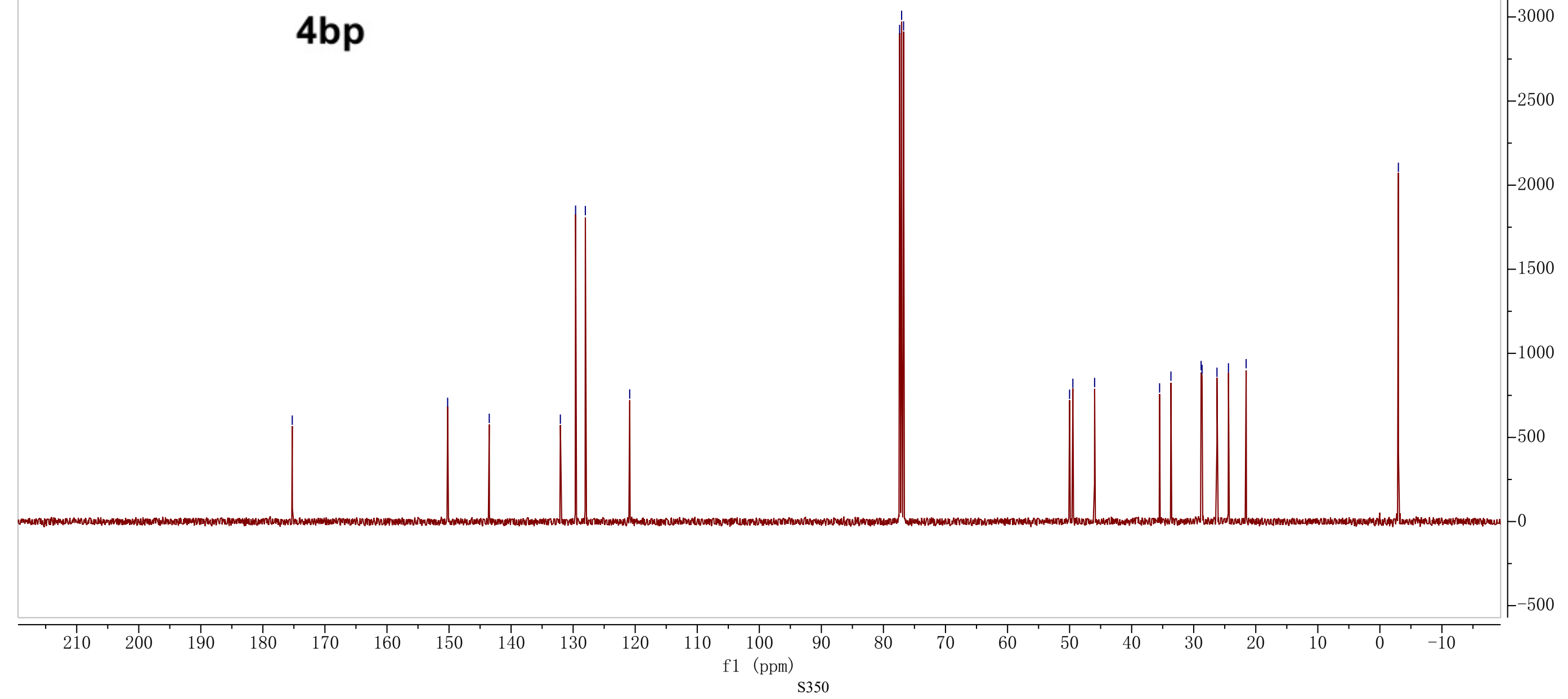


<smiles>C[Si]1(C)C=C(/C=C/c2ccccc2)CN([As])C1</smiles>

$4 \mathrm{bq}$

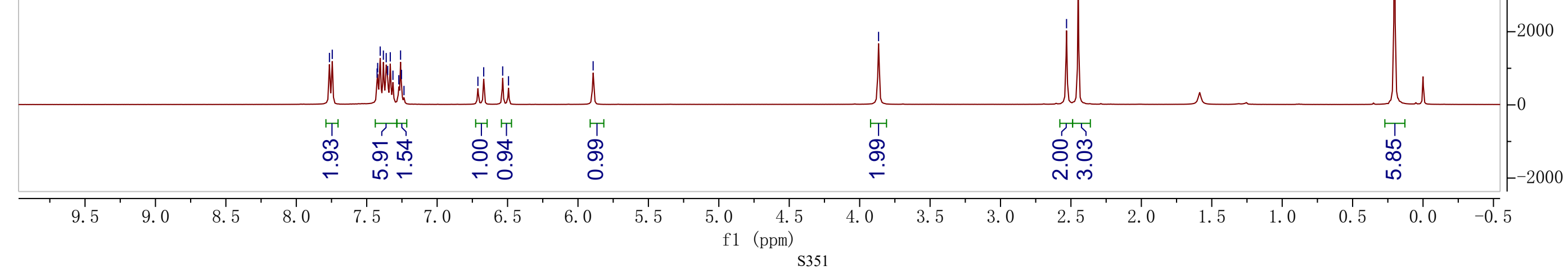


Ts<smiles>C[Si]1(C)C=C(/C=C/c2ccccc2)CN([As])C1</smiles>

$4 \mathrm{bq}$

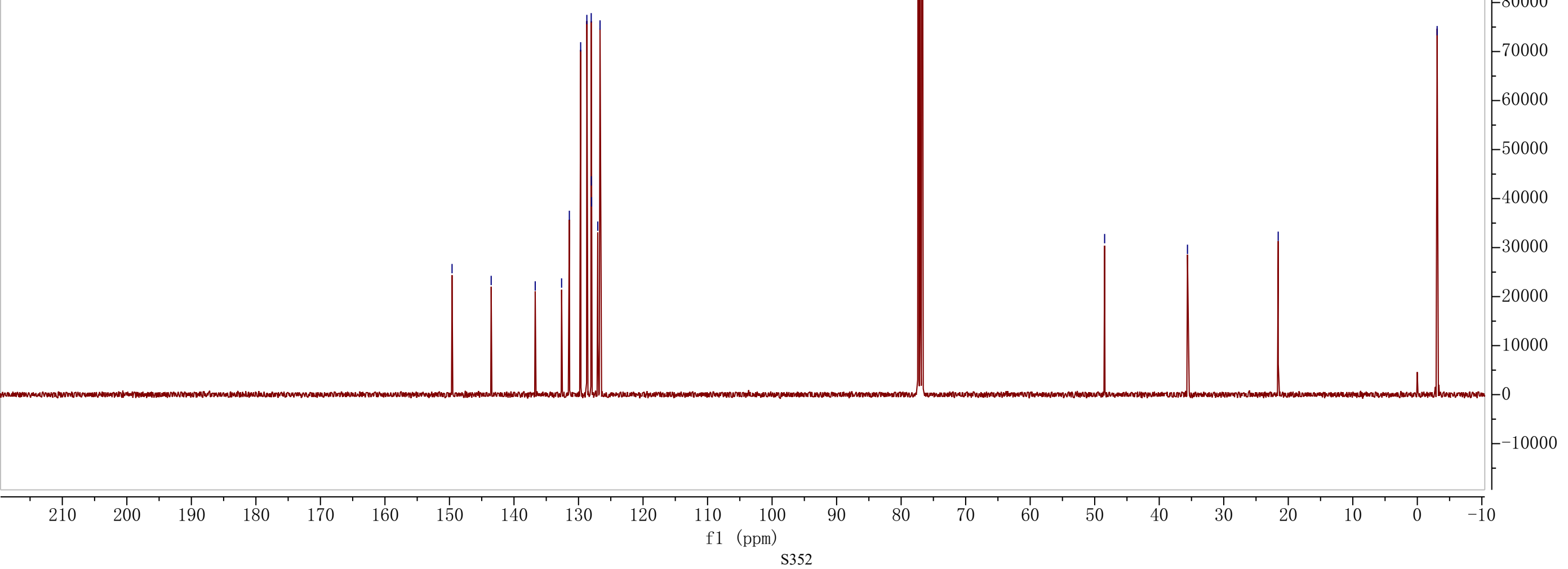



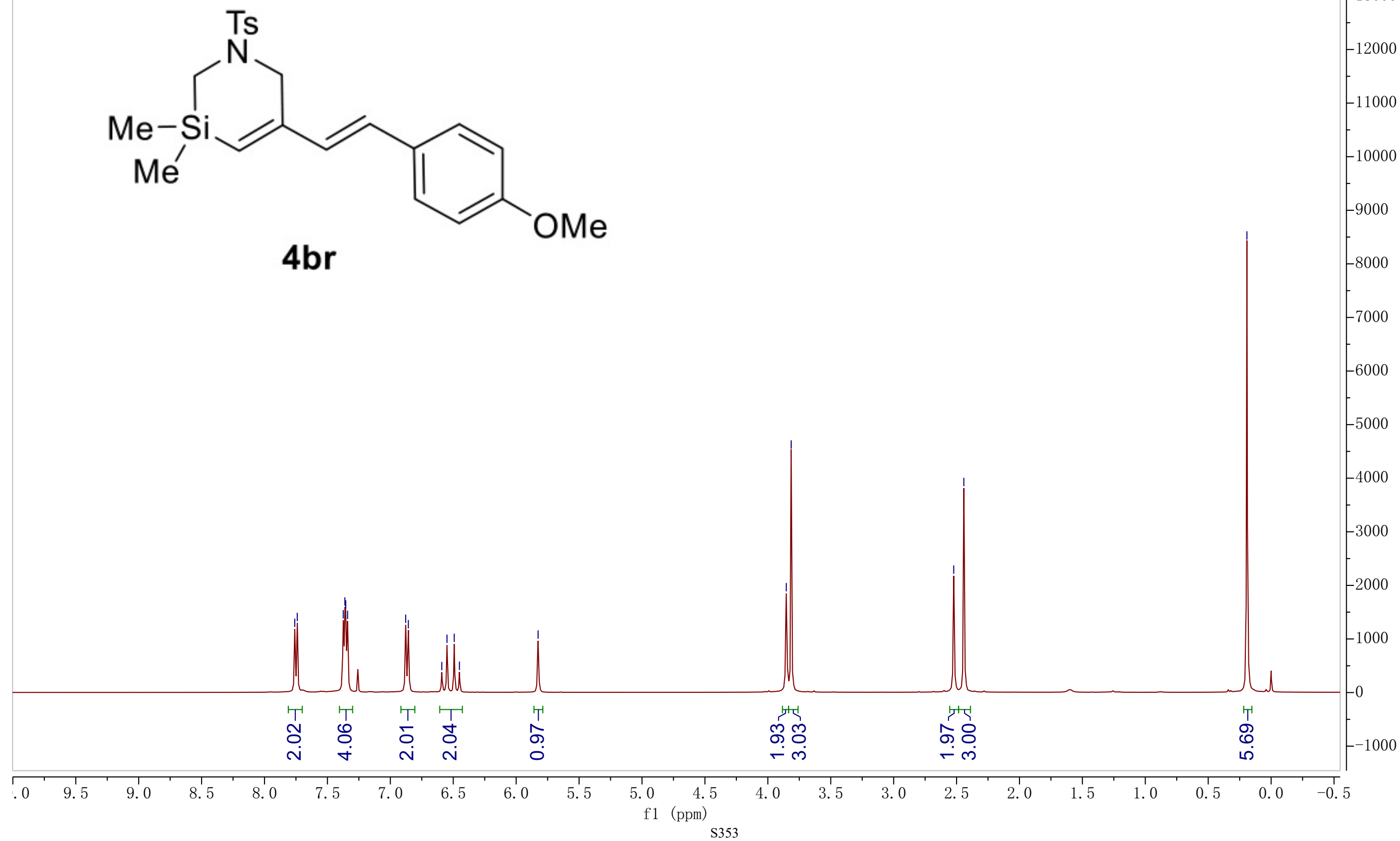


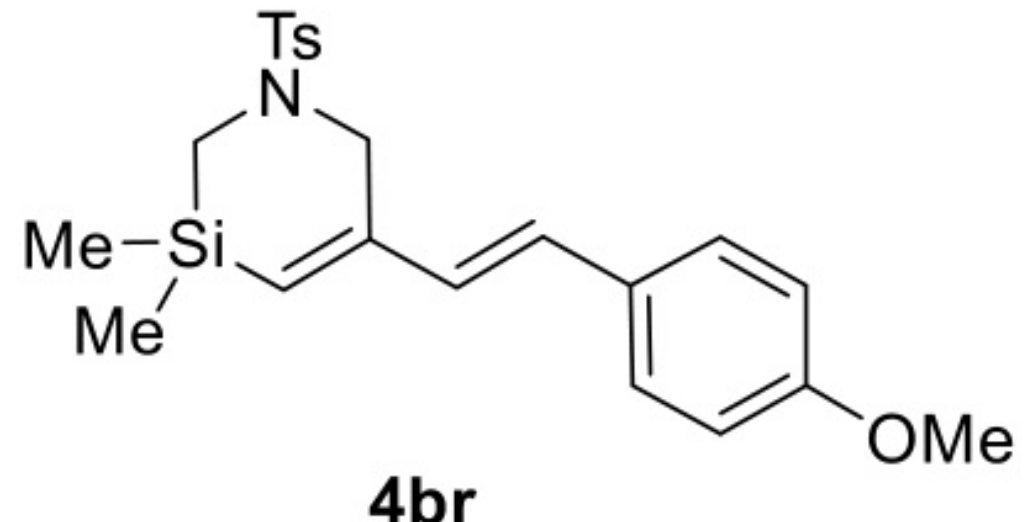


Ts<smiles>C[Si]1(C)C=C(/C=C/c2ccc(F)cc2)CN([As])C1</smiles>

\section{4bs}

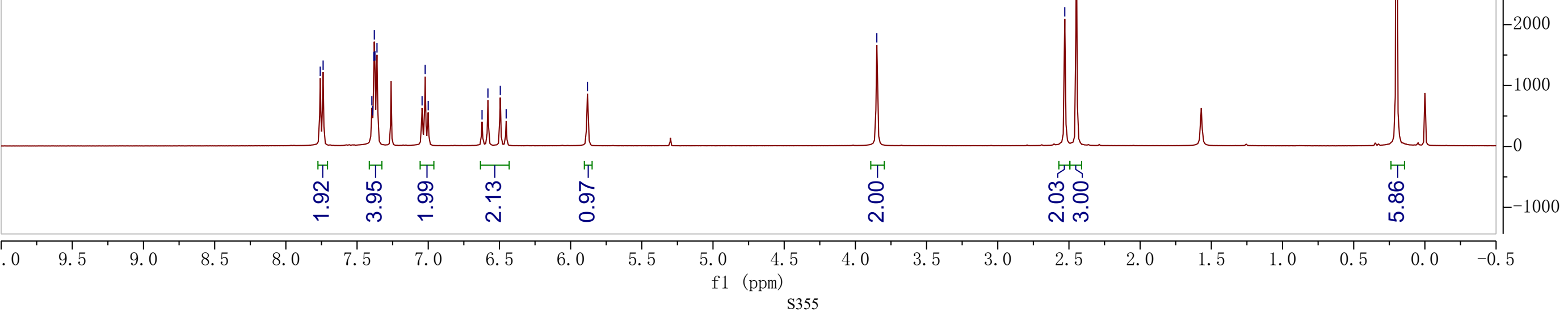


<smiles>C[Si]1(C)C=C(/C=C/c2ccc(F)cc2)CN([As])C1</smiles>

4bs

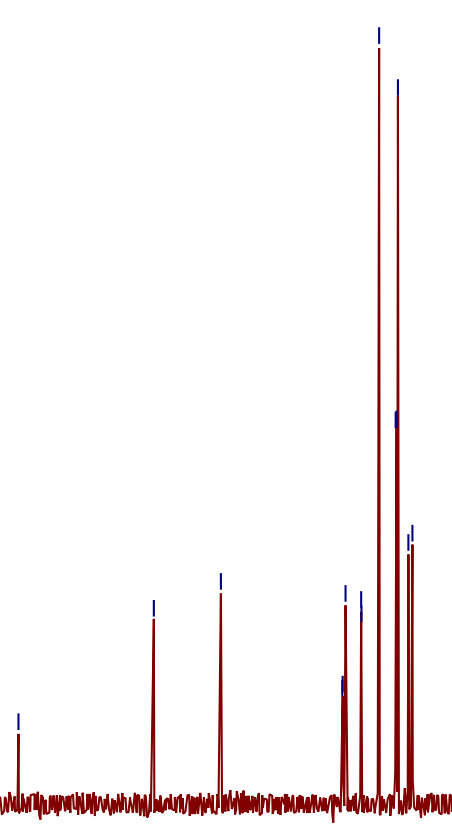


${ }^{19}$ F NMR

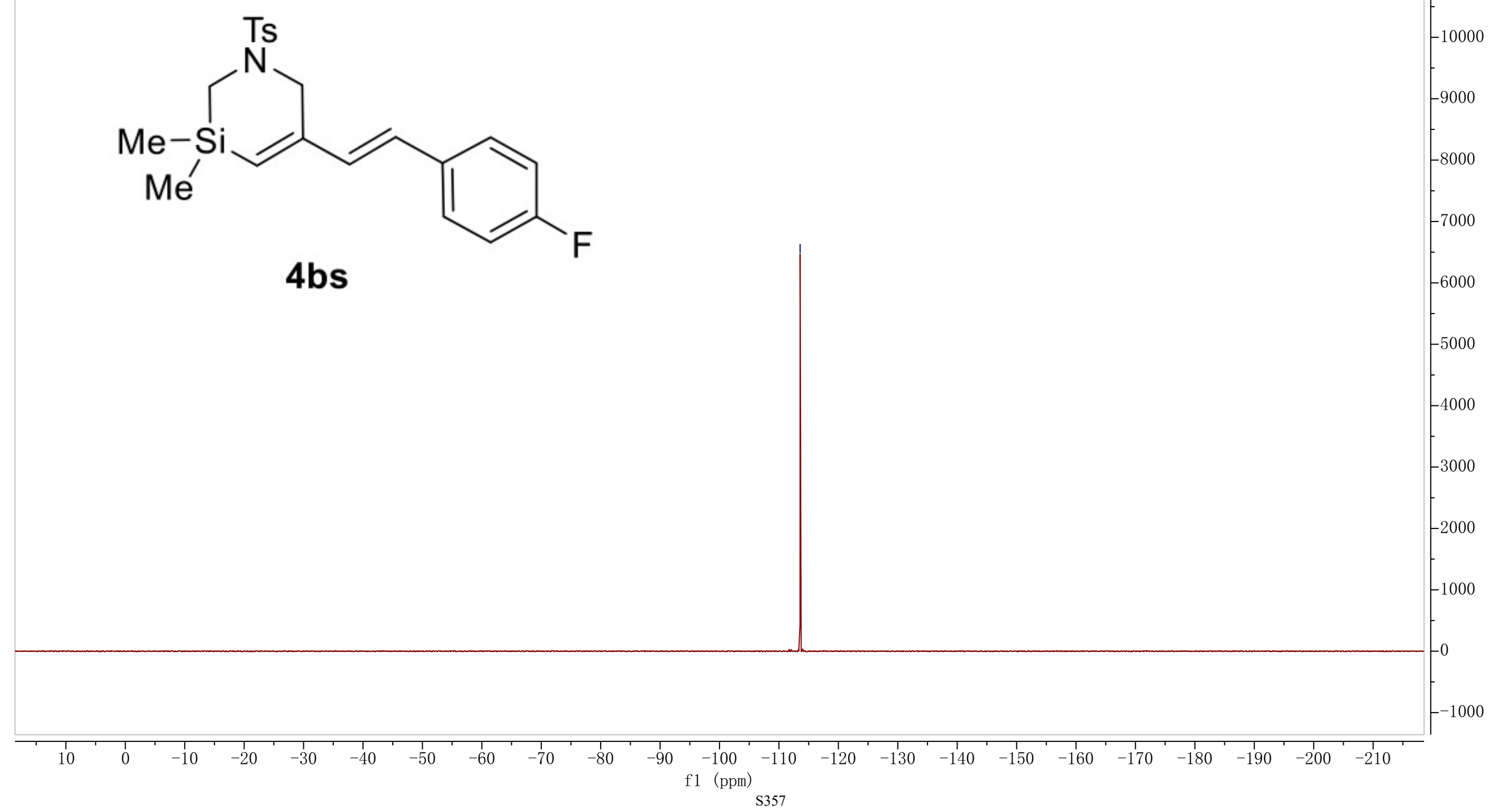




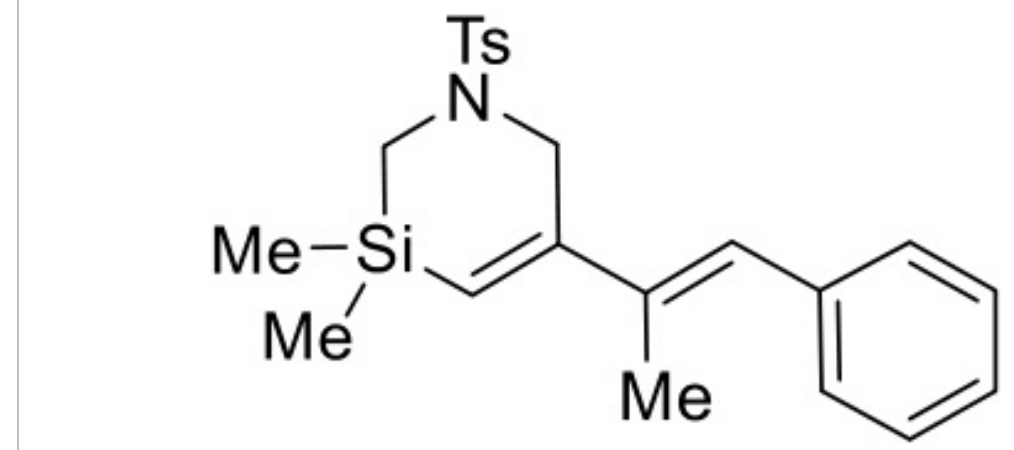

\section{4bt}

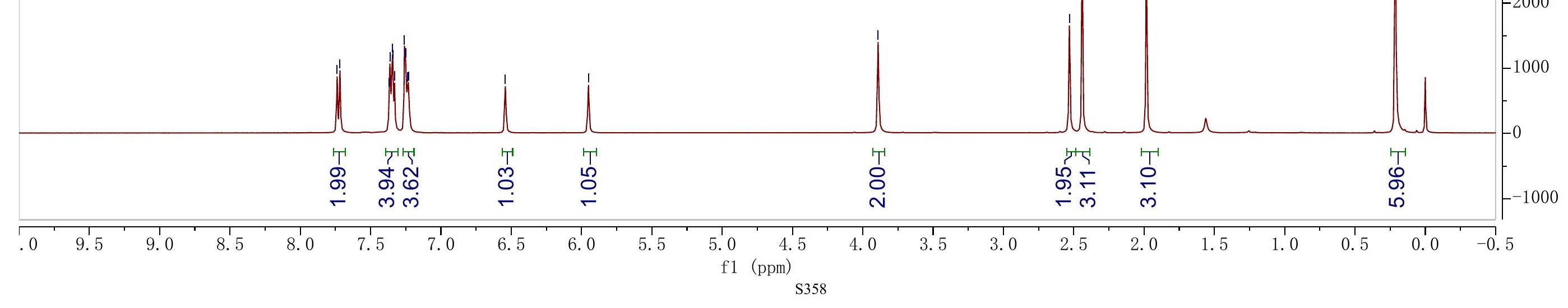


4bt

$\mathrm{Me}$<smiles>CC(=Cc1ccccc1)C1=C[Si](C)(C)CN([AsH3])C1</smiles>

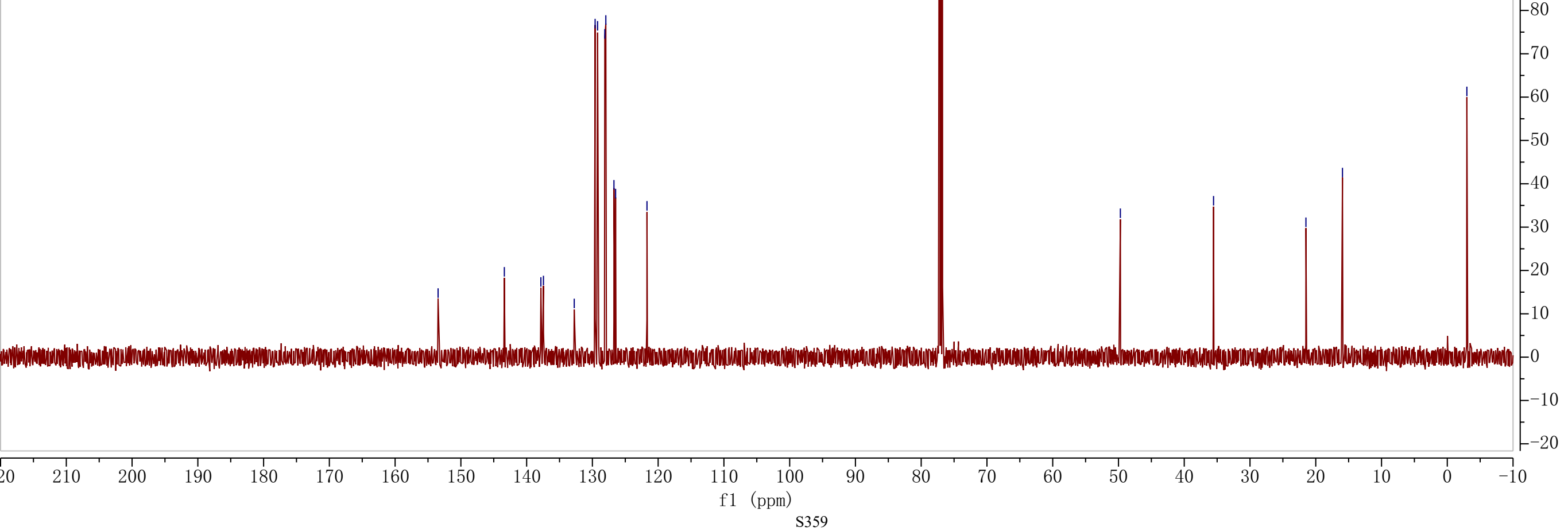




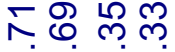

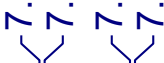

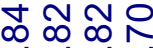

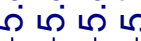

4

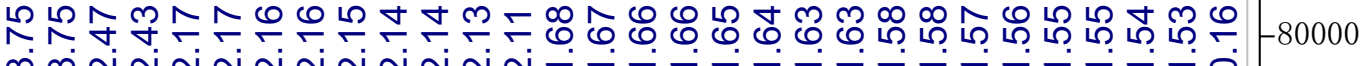

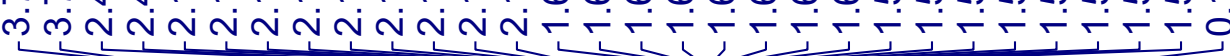<smiles>C[Si]1(C)C=C(C2=CCCCC2)CN([As])C1</smiles>

4bu

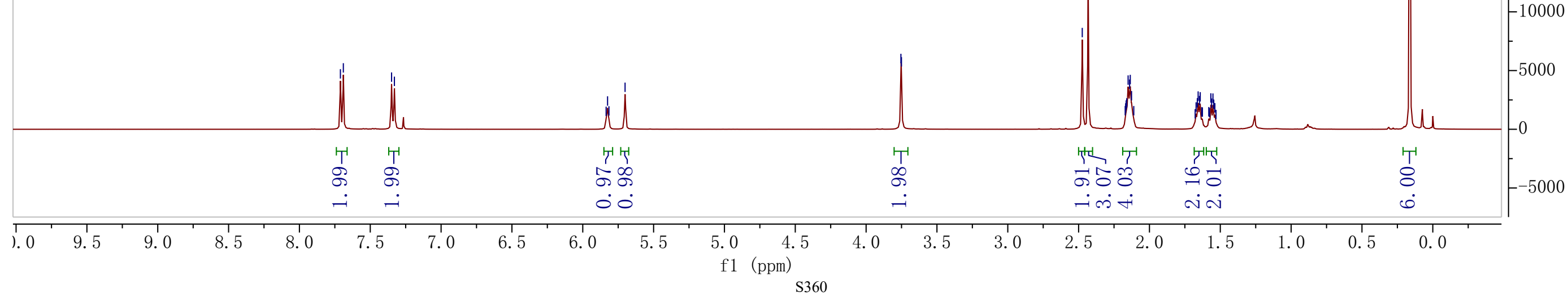




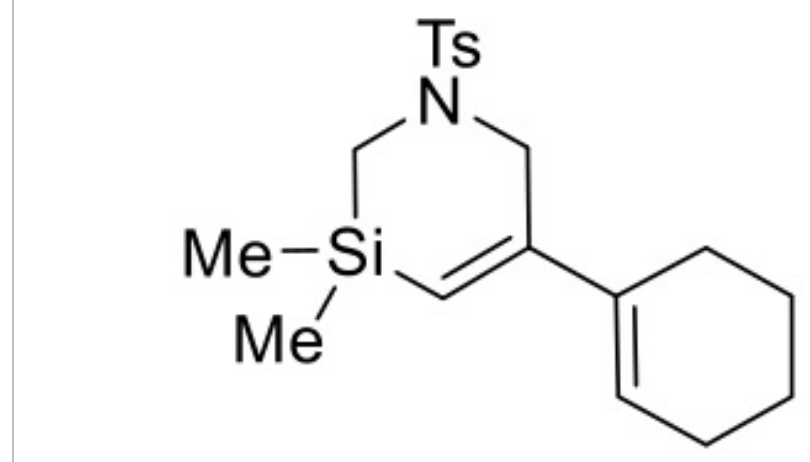

4bu

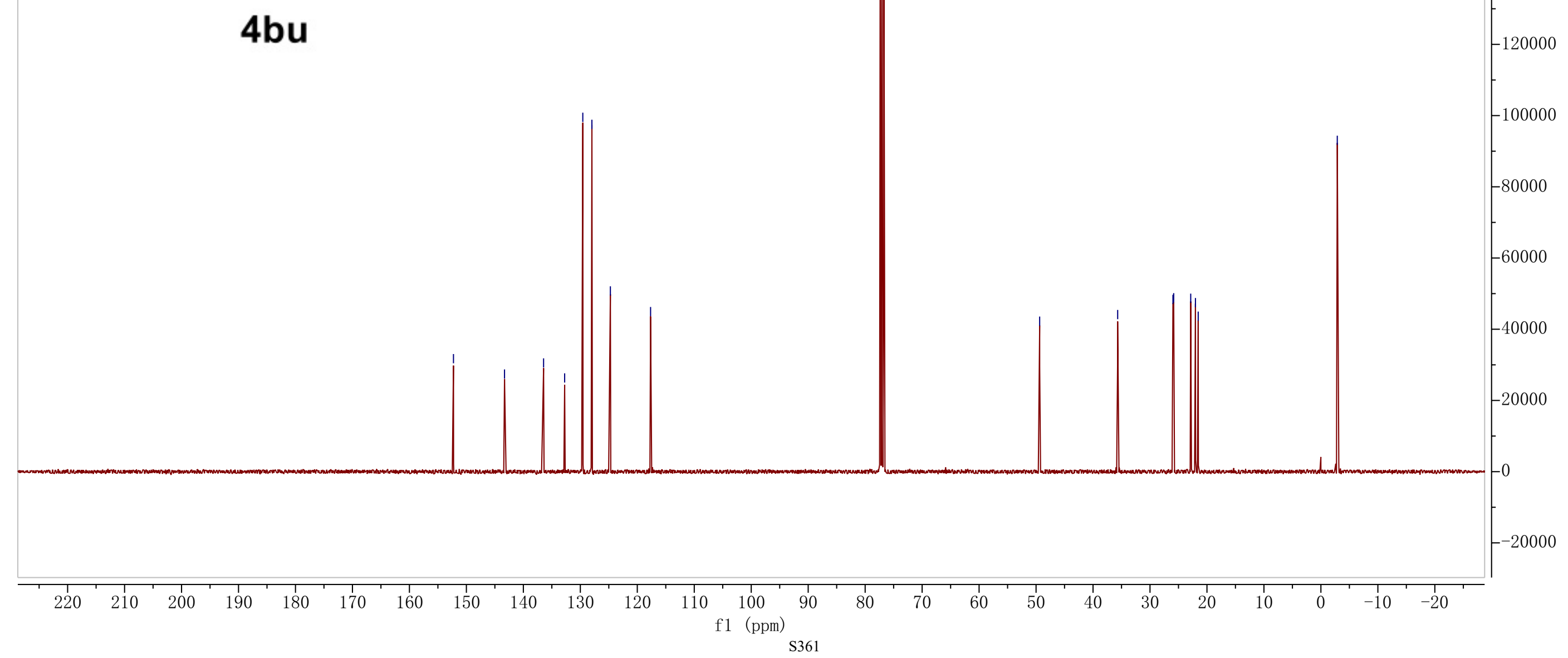


Ts<smiles>COC(=O)C1=C[Si](C)(C)CN([As])C1</smiles>

4bw

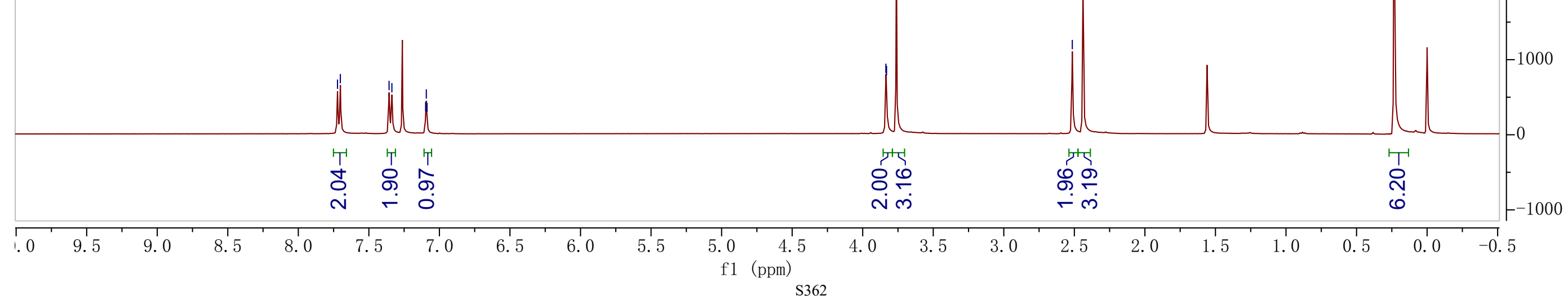




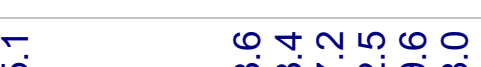

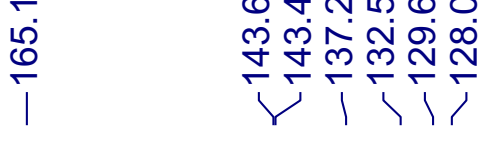

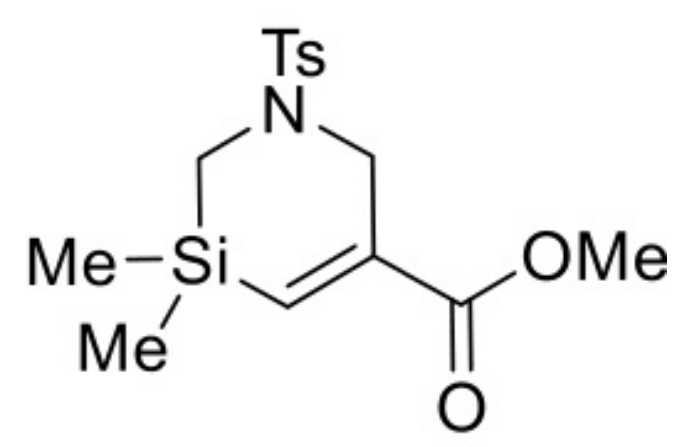

4bw

4bw

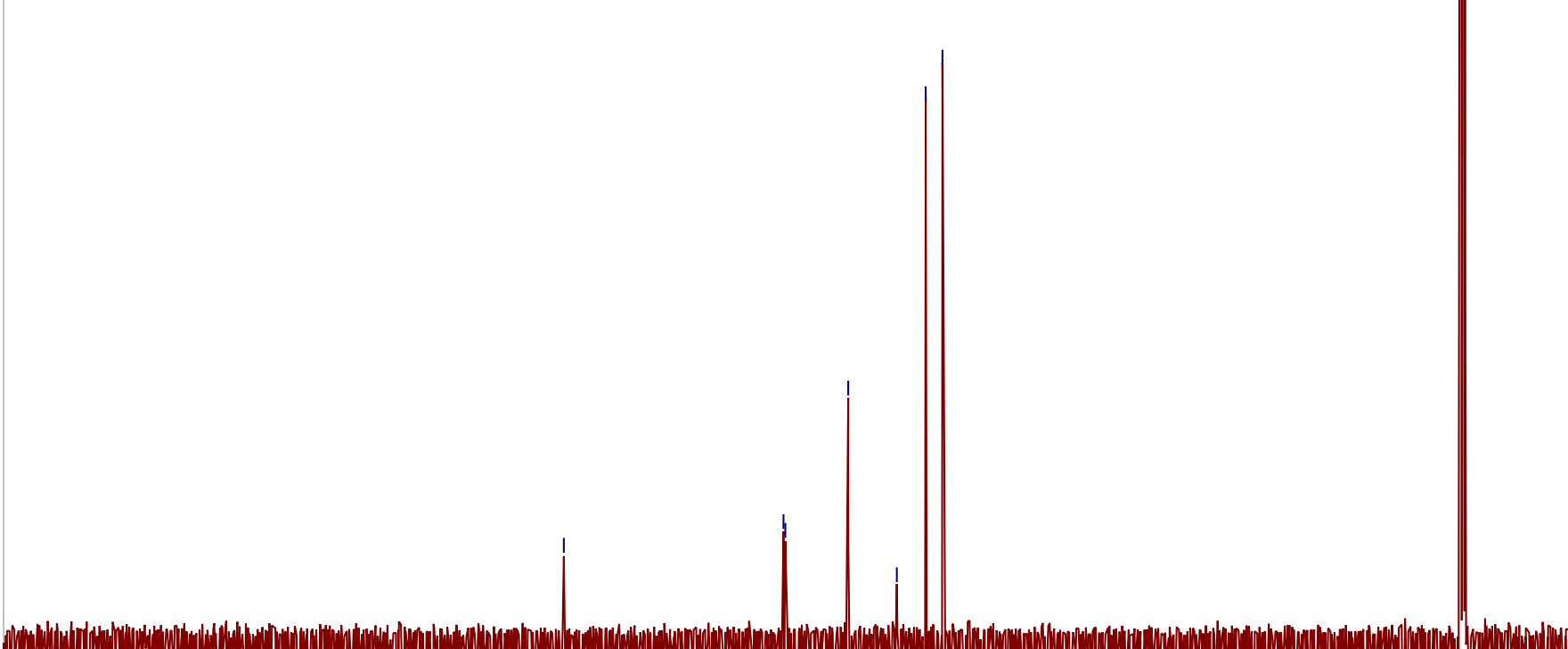

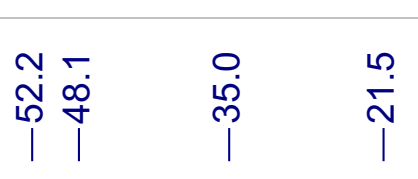

260 
<smiles>CN(CC1=C[Si](C)(C)CN([AsH])C1)Cc1ccccc1</smiles>

$4 b x$

Me

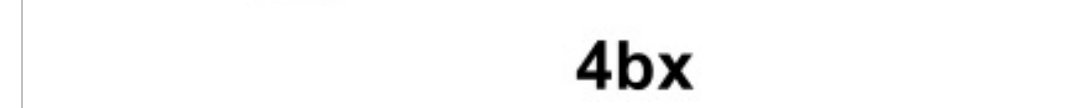




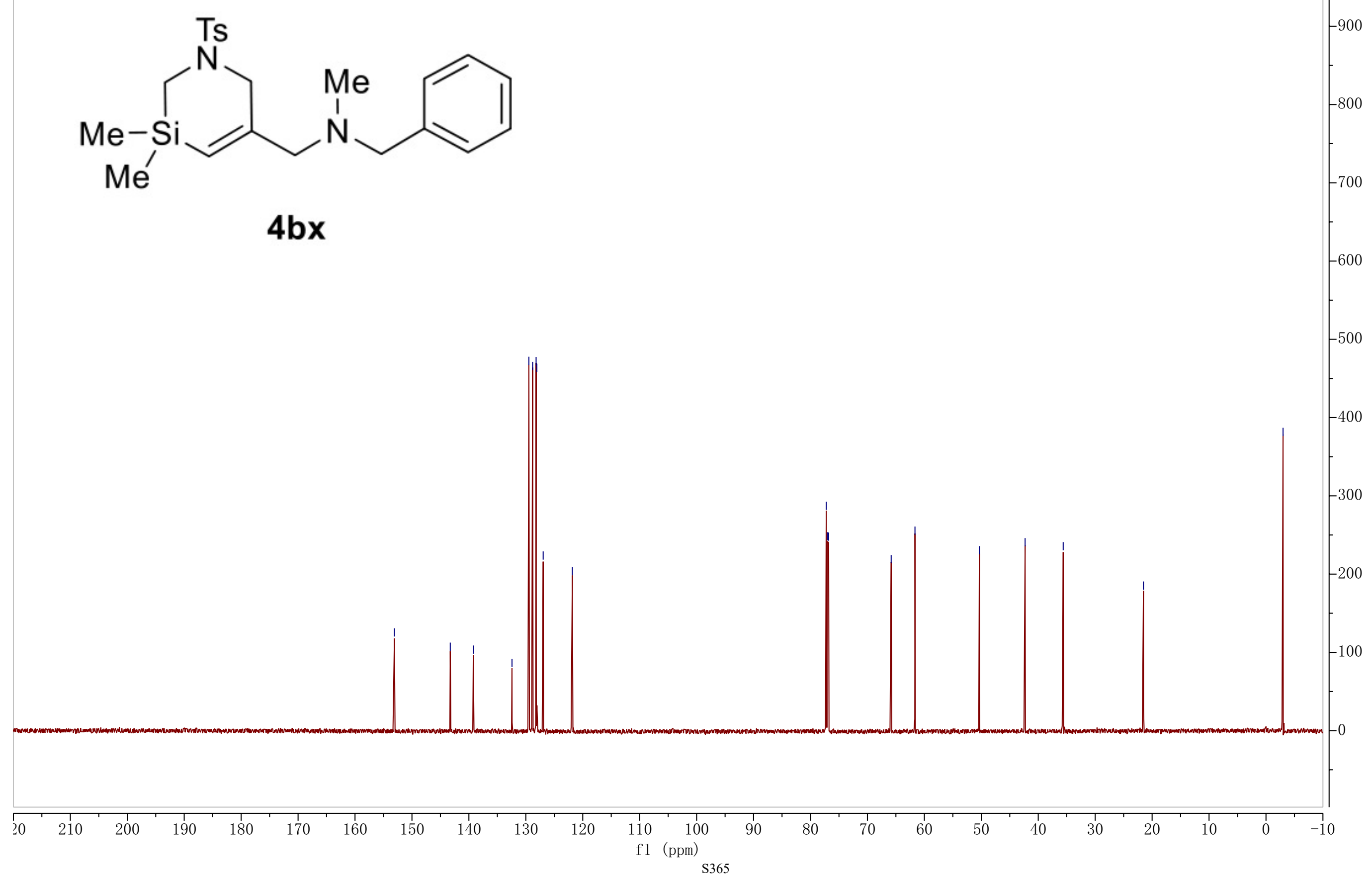


<smiles>CC(C)(NC(=O)c1cc(Cl)cc(Cl)c1)C1=C[Si](C)(C)CN([As])C1</smiles>

\section{4by}

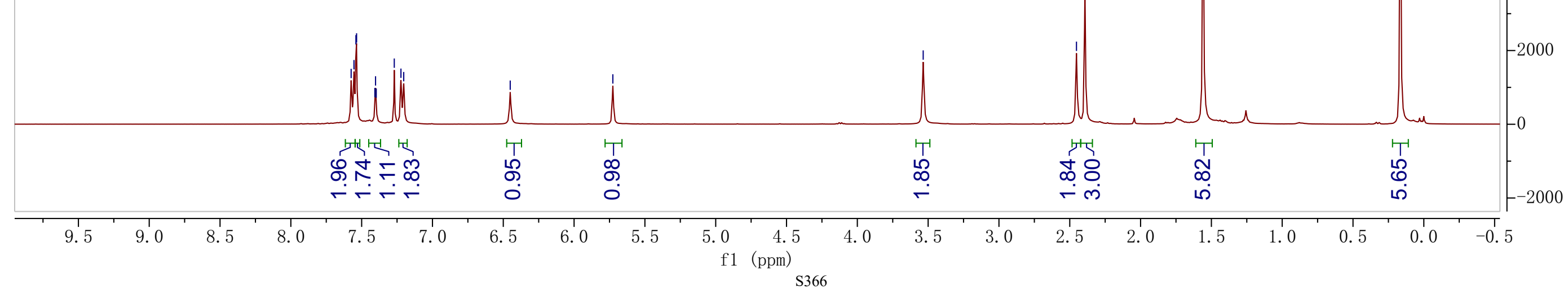


<smiles>[Y5]C1C=C(C(C)([18OH])NC(=O)c2cc(Cl)cc(Cl)c2)C[Si](C)(C)C1</smiles>

\section{4by}


<smiles>CCOc1cc2ncnc(Nc3cccc(C4=C[Si](C)(C)CN([As])C4)c3)c2cc1OCC</smiles>

\section{$4 b z$}

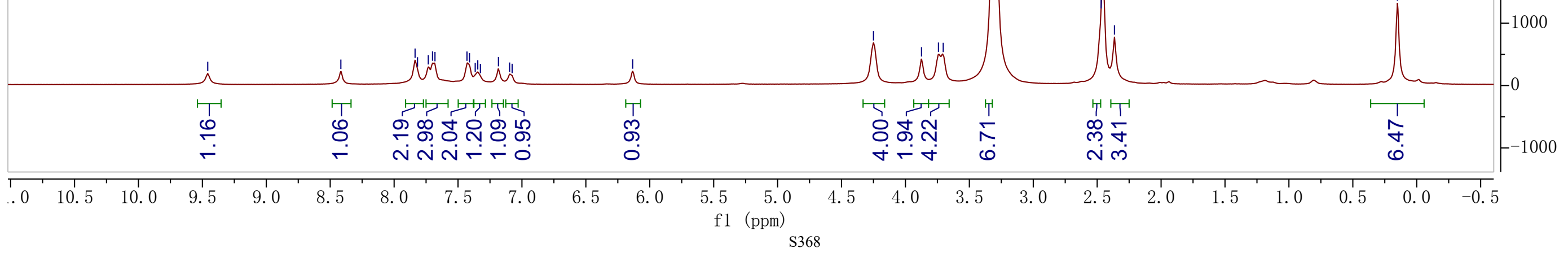




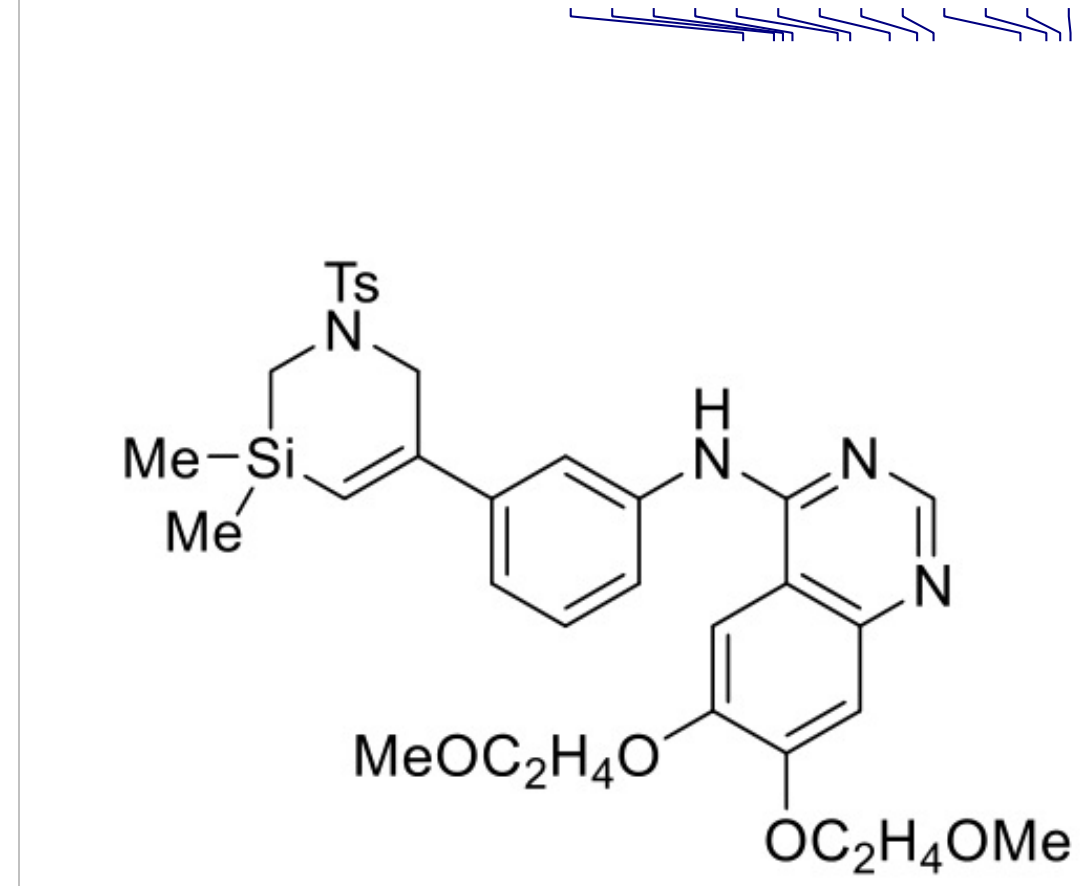

$4 \mathrm{bz}$

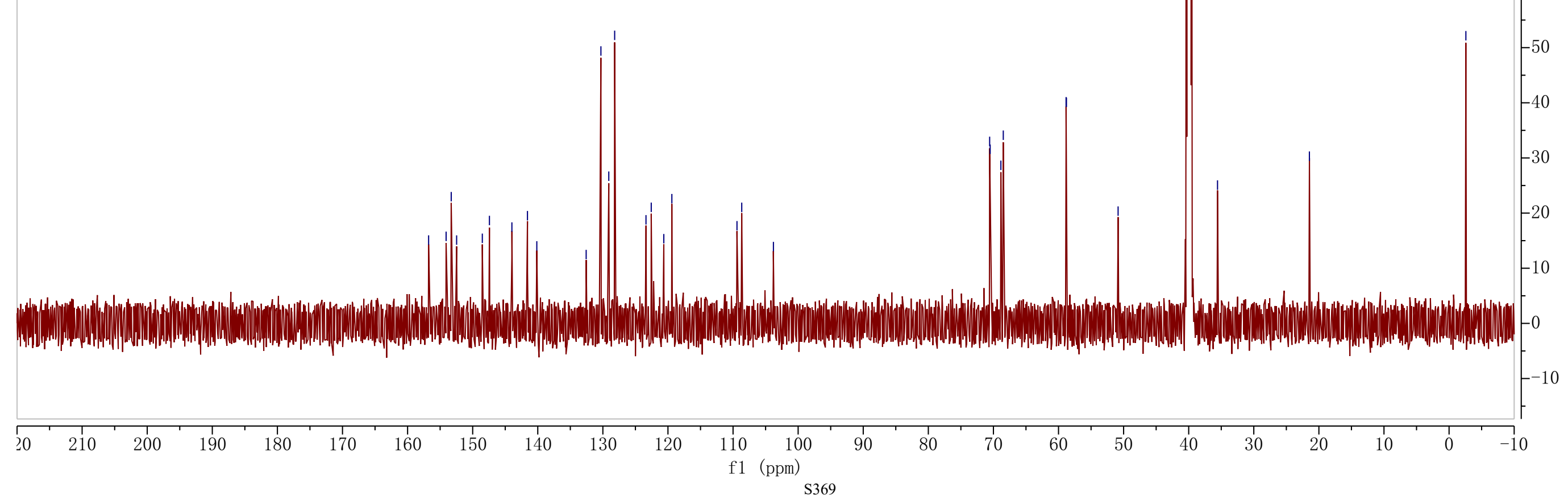


<smiles>Cc1sc(-c2ccc(OCC(C)C)c(C#N)c2)nc1C(=O)NCC1=C[Si](C)(C)CN([AsH])C1</smiles>

$4 c a$

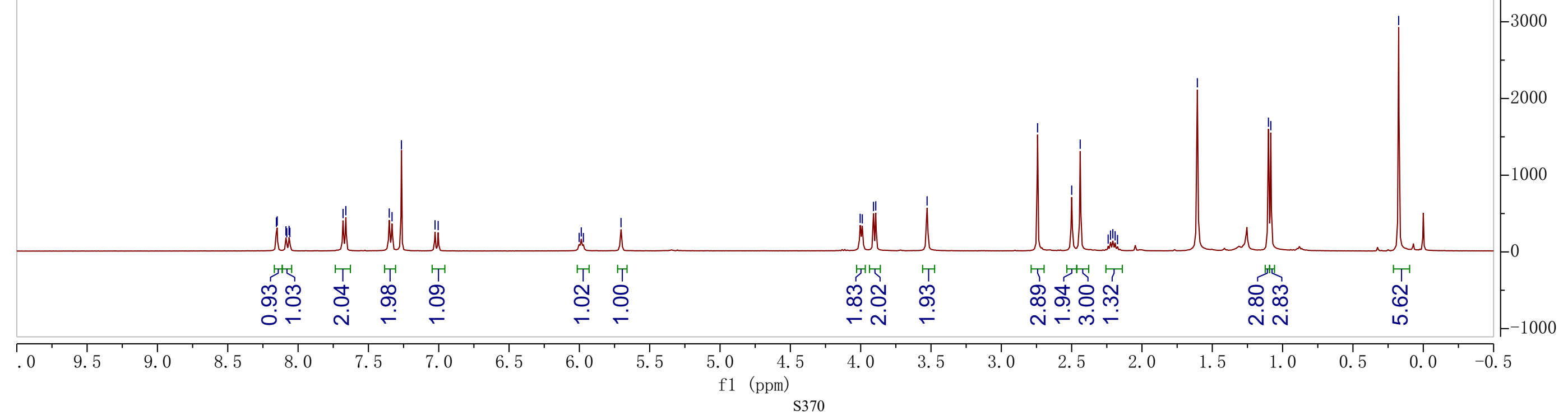




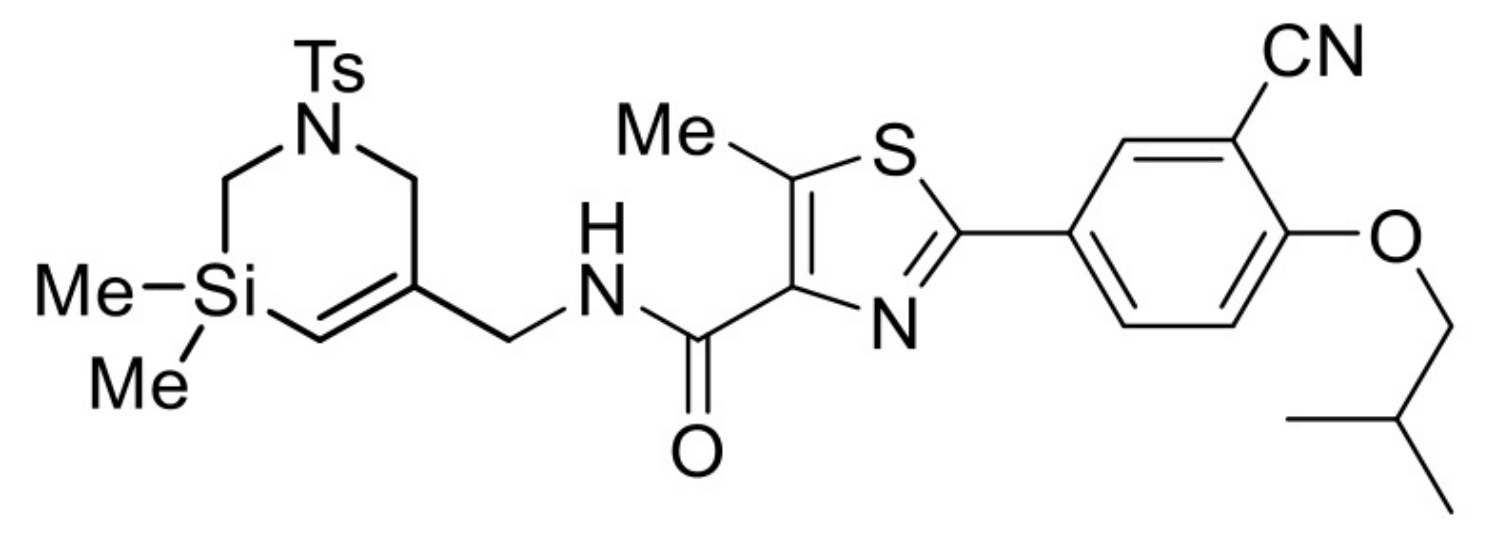

$4 c a$

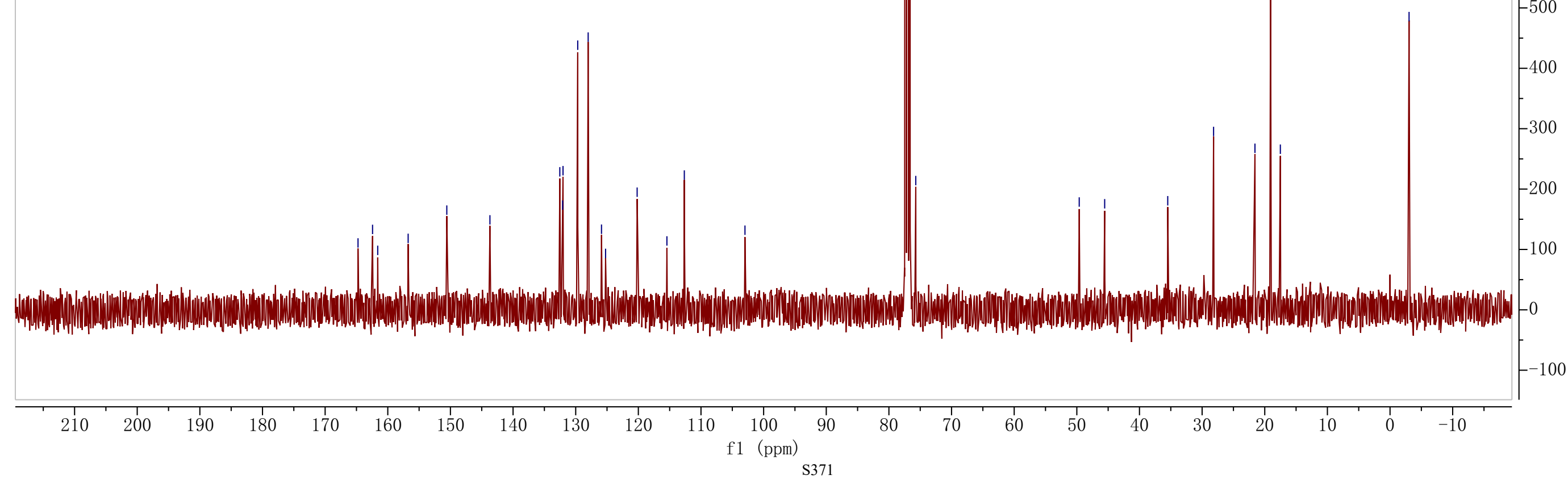




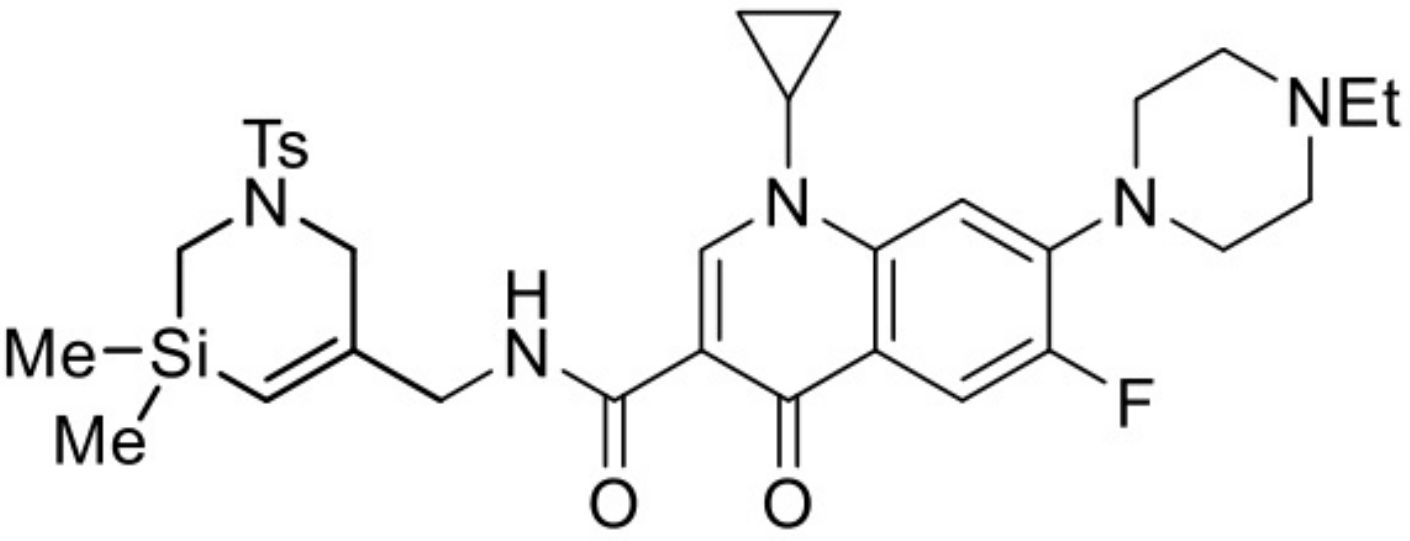

$4 c b$

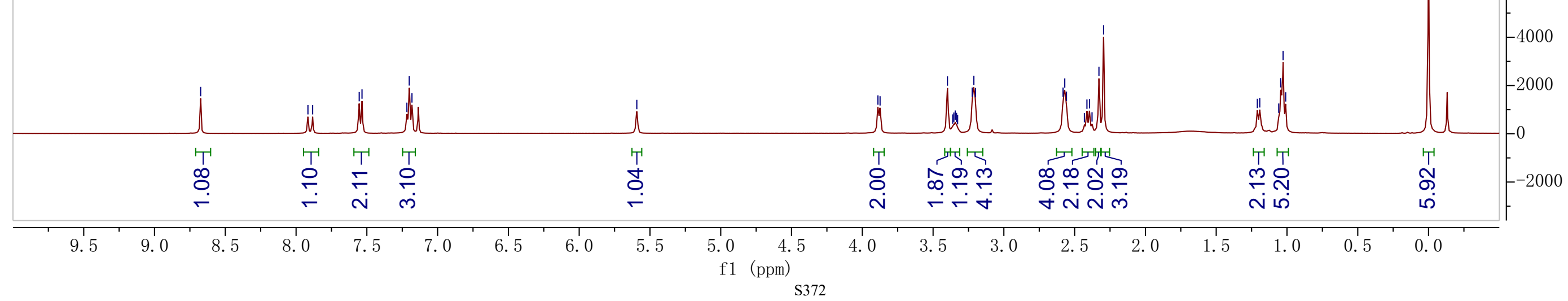




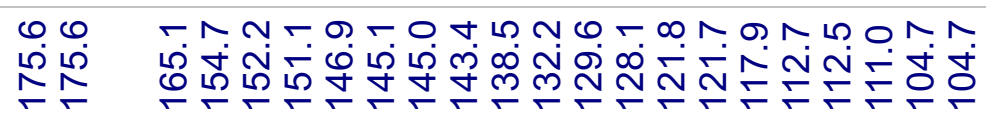

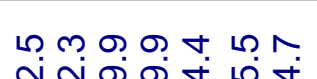

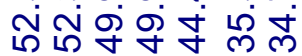

त)

in

$-150000$<smiles>[1H][Si]1([1H])C=C(CNC(=O)c2cn(C3CC3)c3cc(N4CC[NH+](CC)CC4)c(F)cc3c2=O)CN([As])C1</smiles>

4cb

t

पर 
${ }^{19}$ F NMR

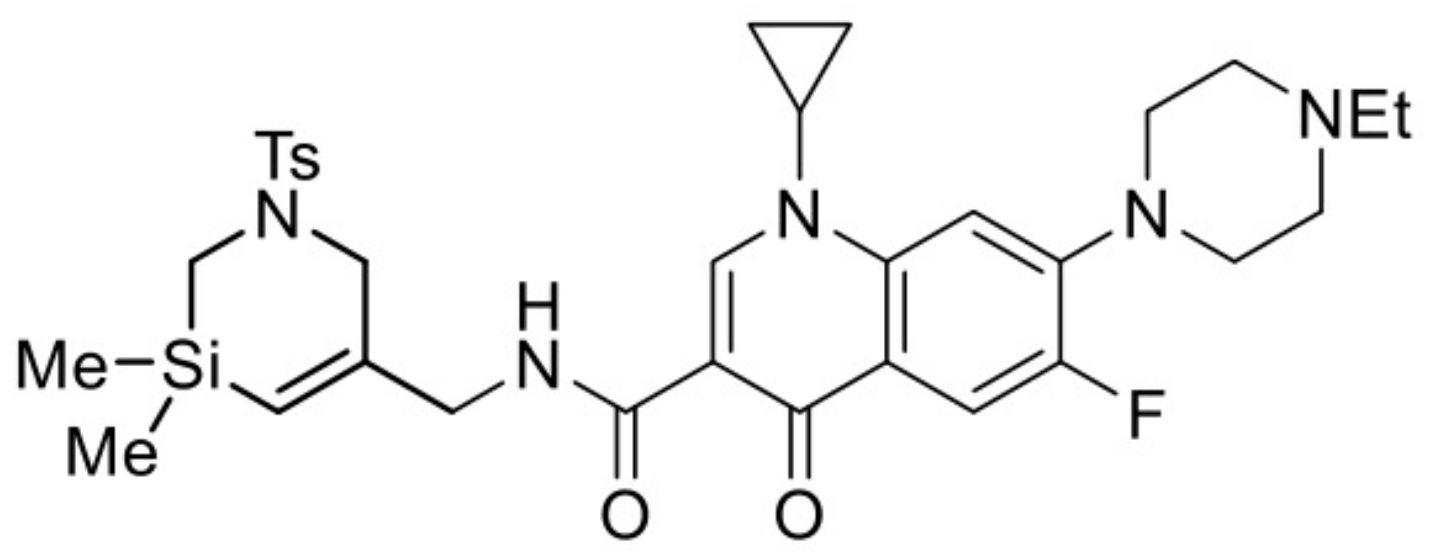

4cb

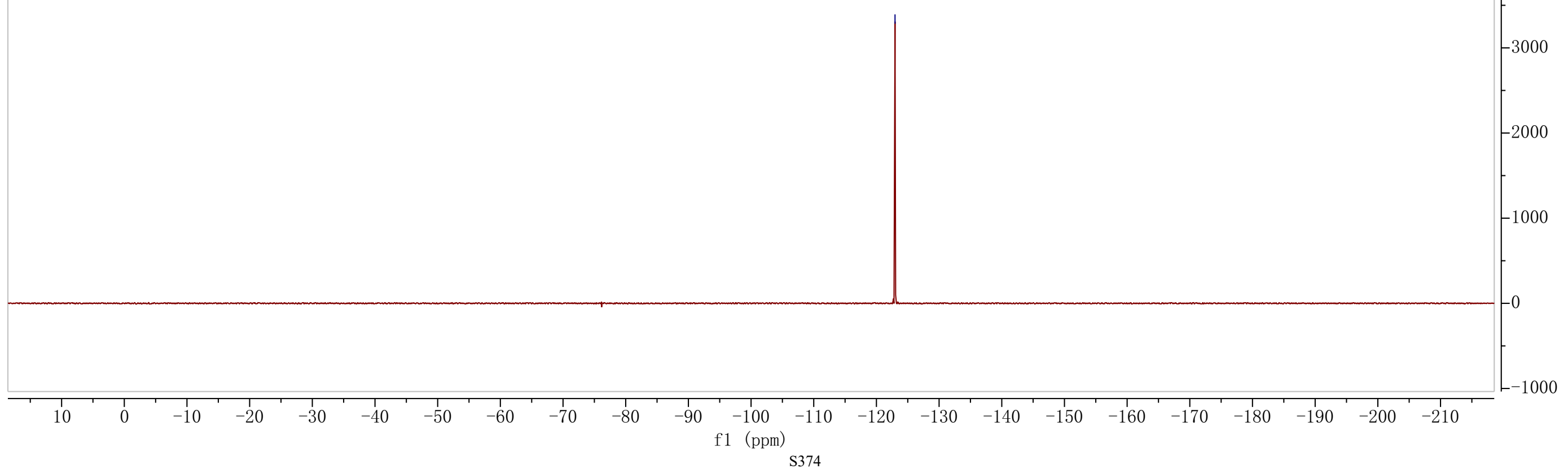




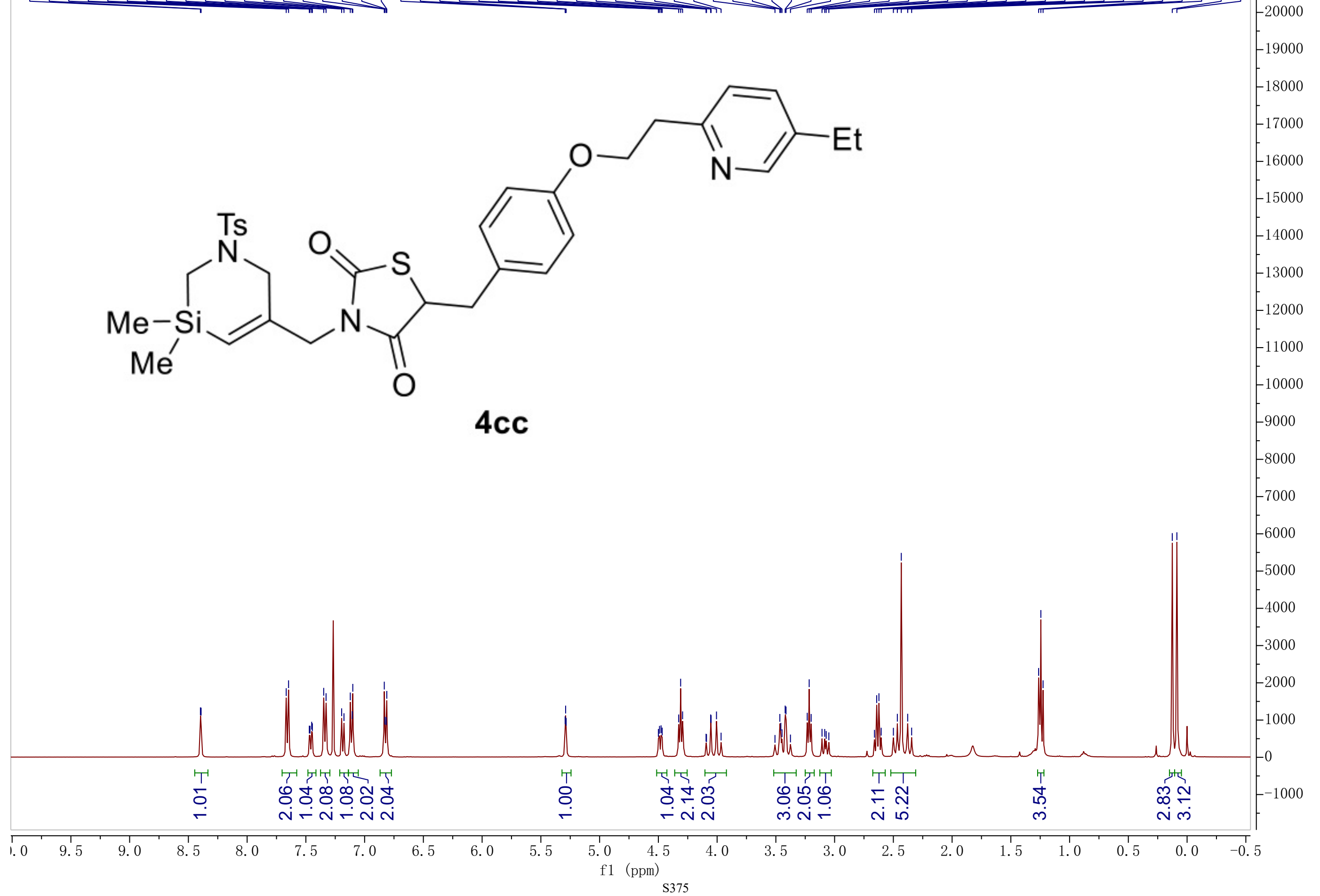



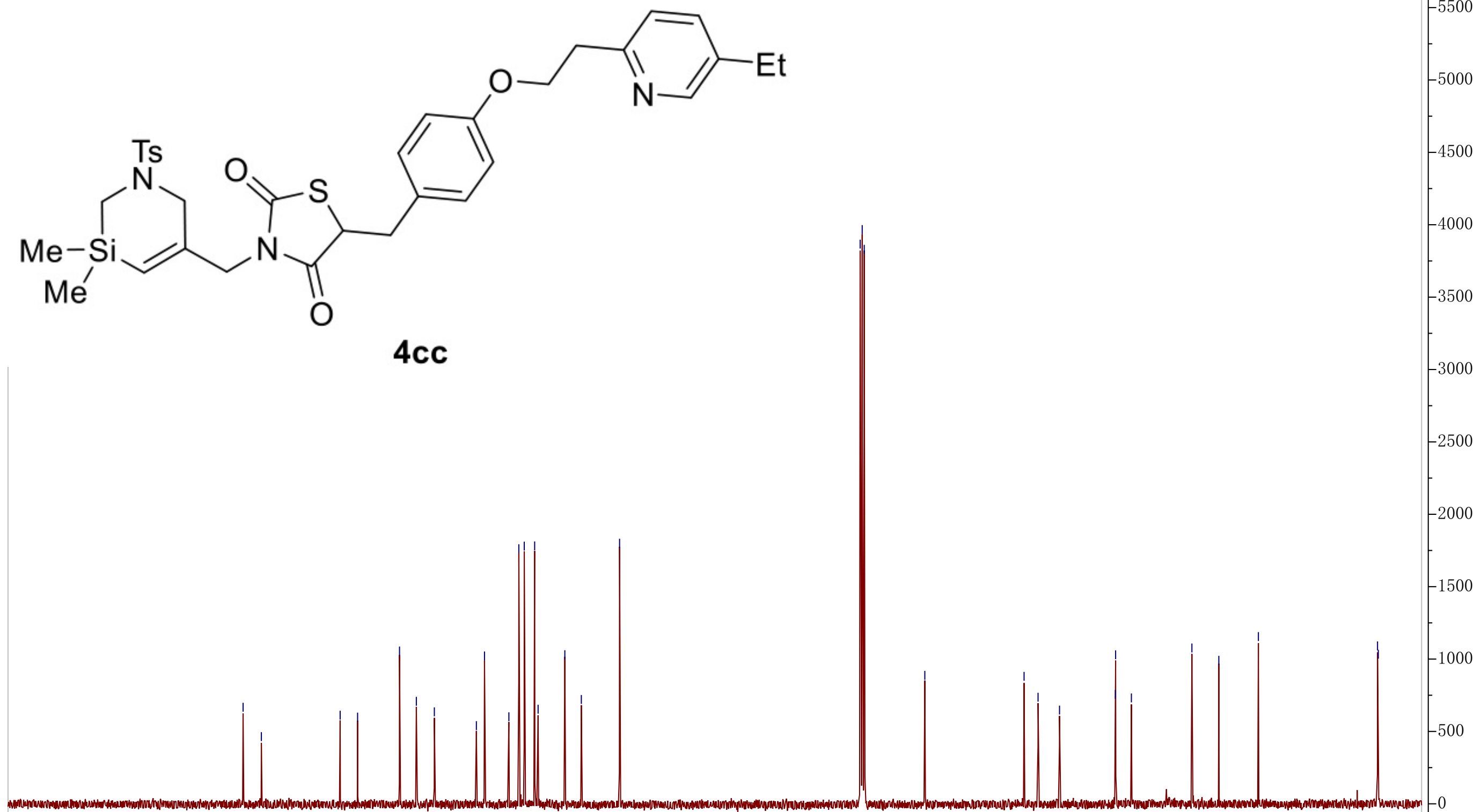

$\cos$ 


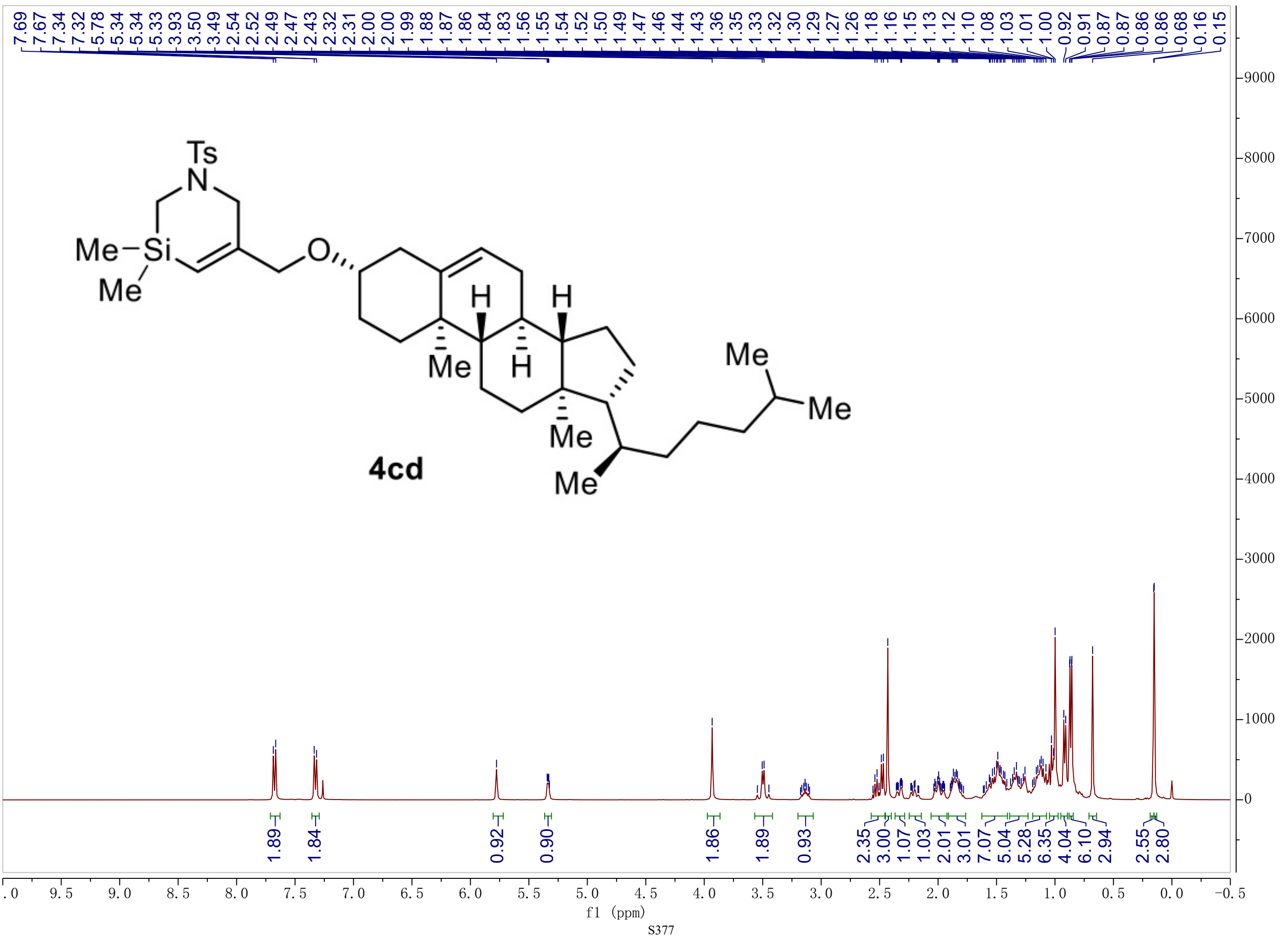




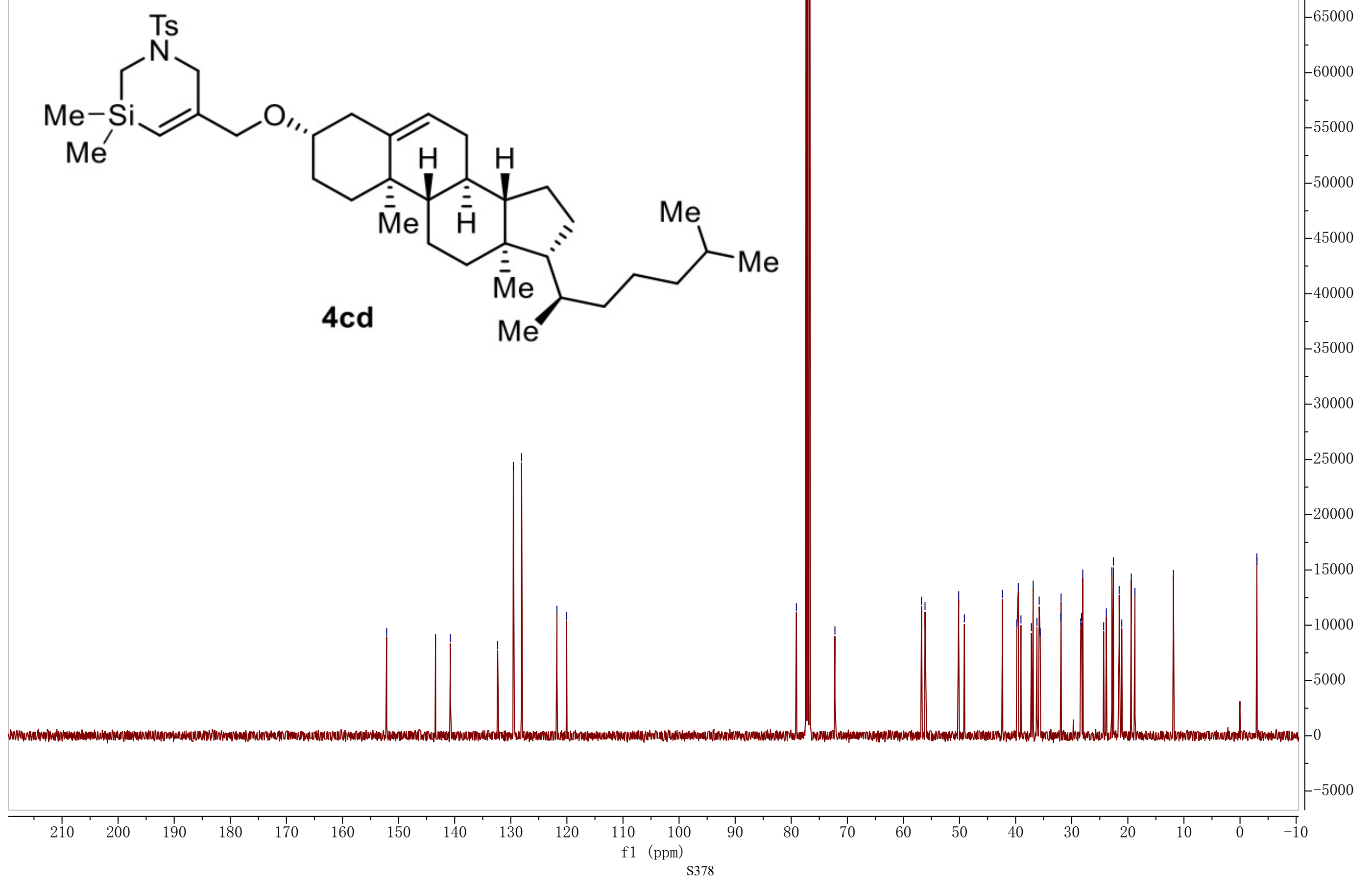




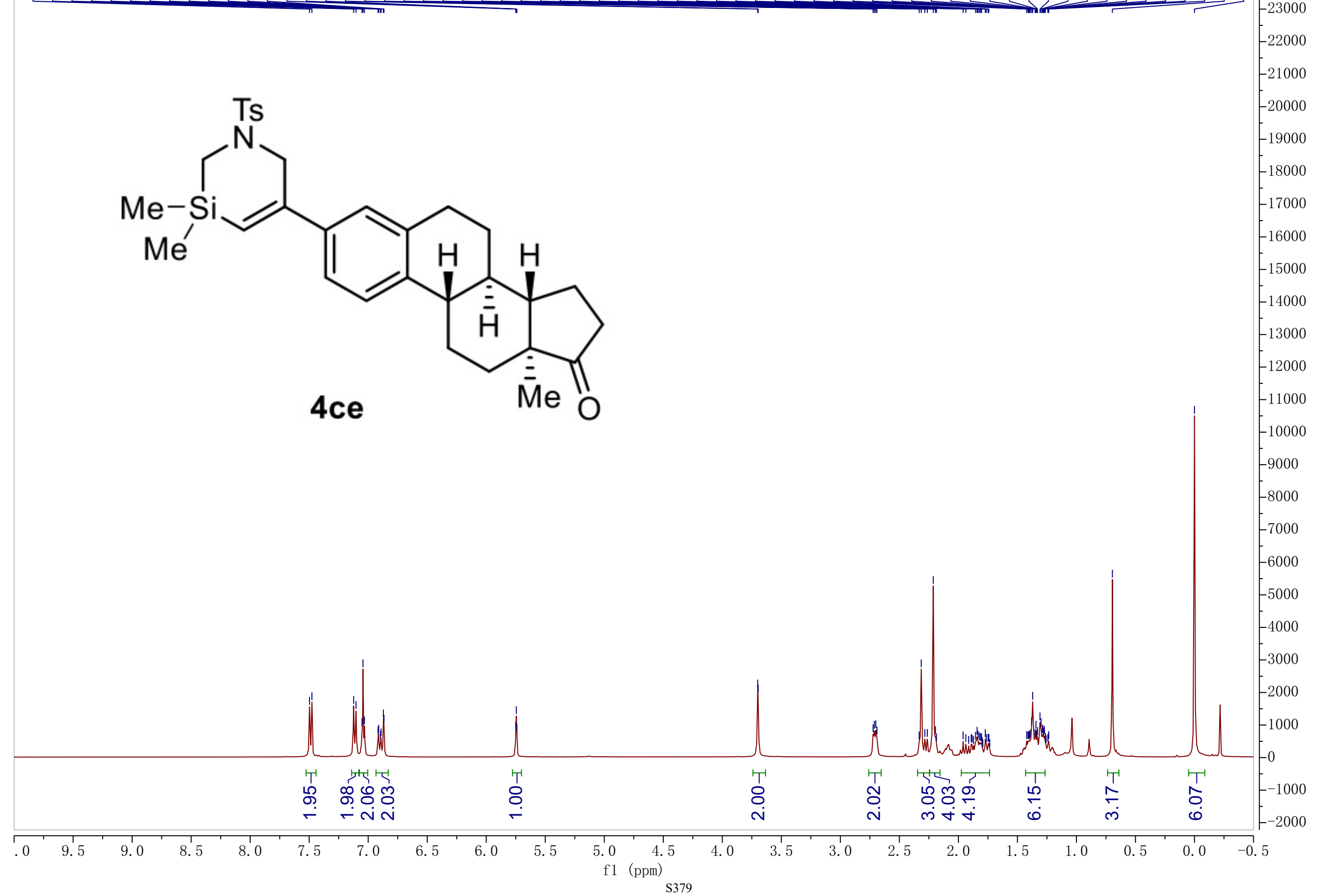


<smiles>C[C@@]12CC[C@@H]3c4ccc(C5=C[Si](C)(C)CN([AsH2])C5)cc4CC[C@@H]3[C@@H]1CCC2=O</smiles>

Me 


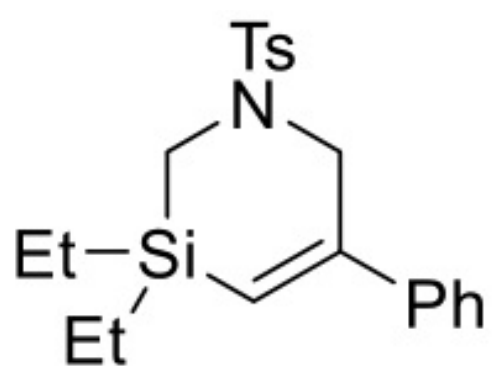

4cf

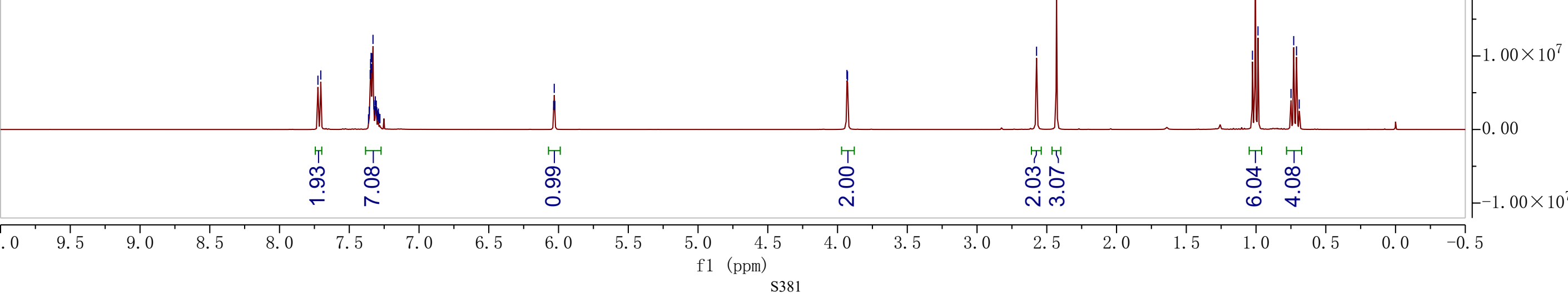




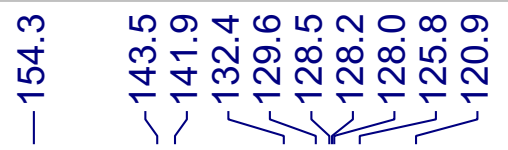

A. $-\infty$

ヘN゚

อุ

min

$-160000$

1

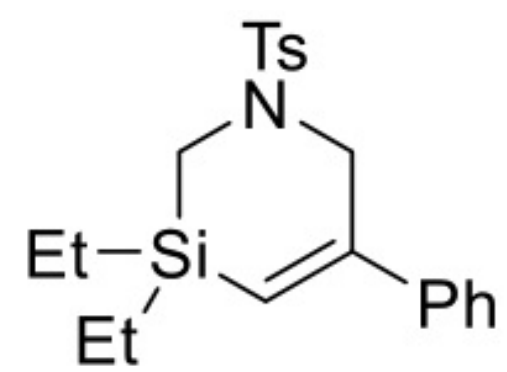

4cf

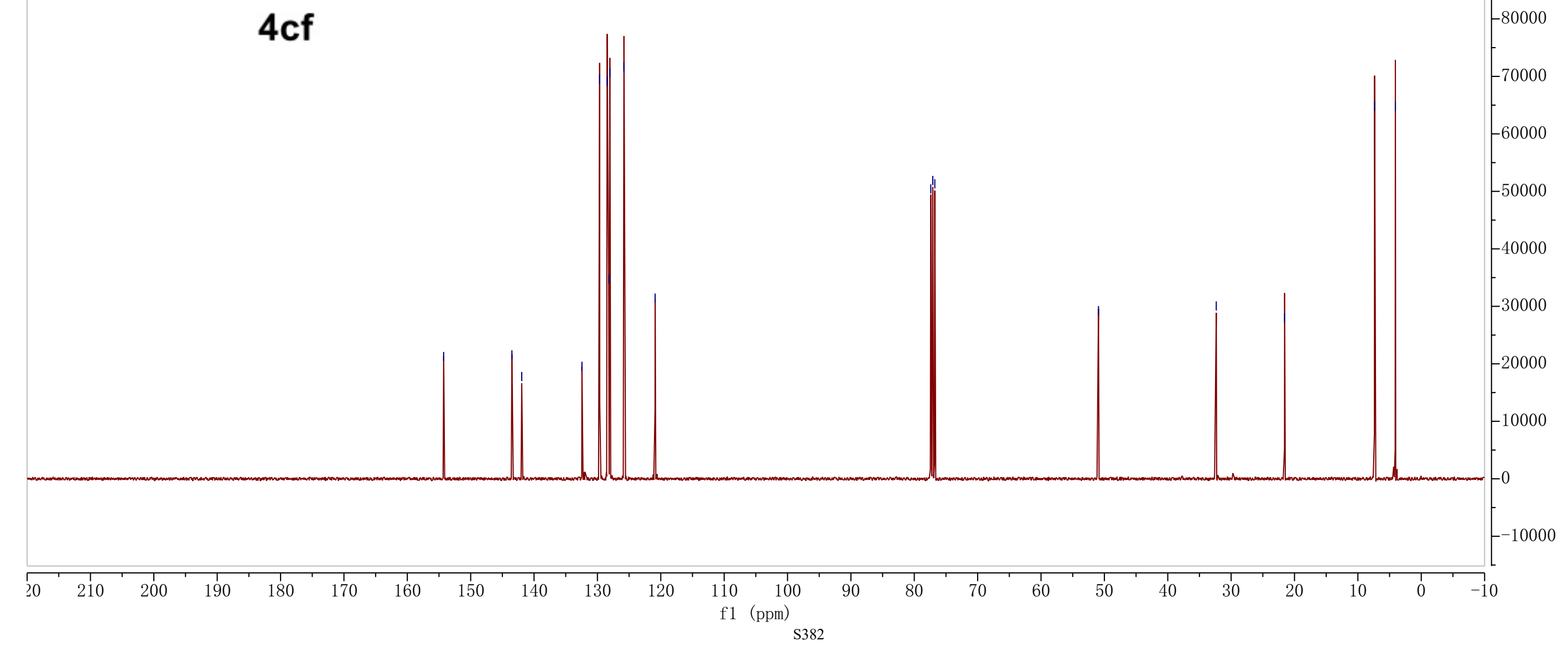




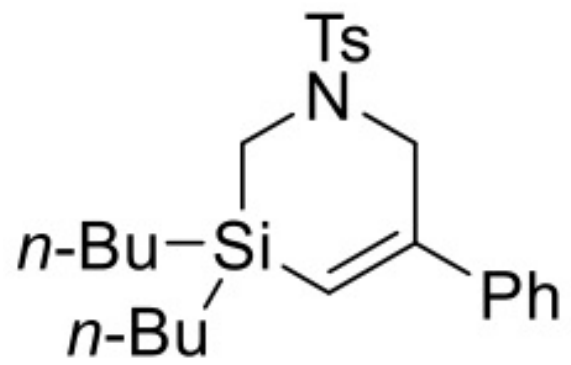

\section{$4 \mathrm{cg}$}

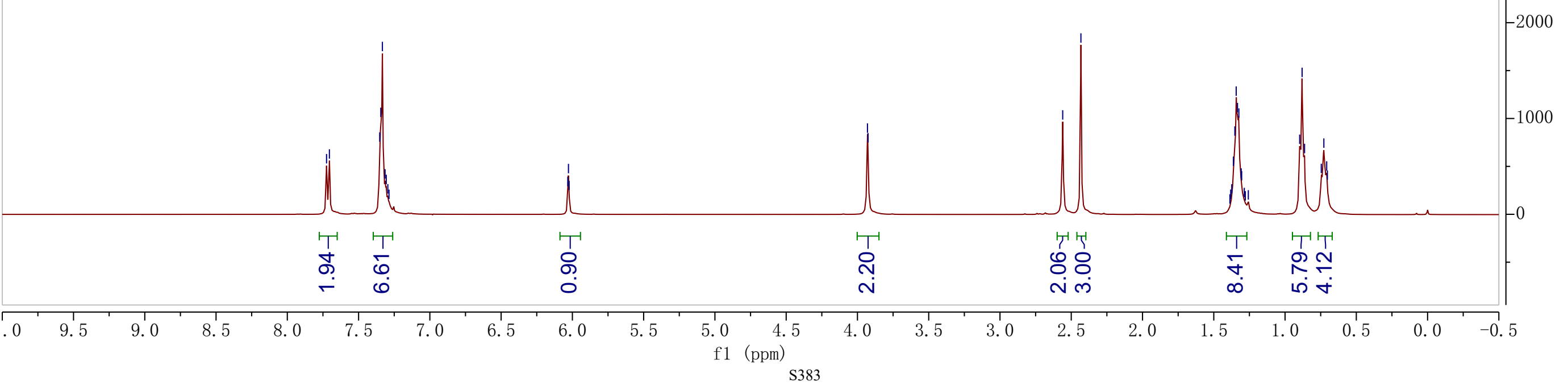




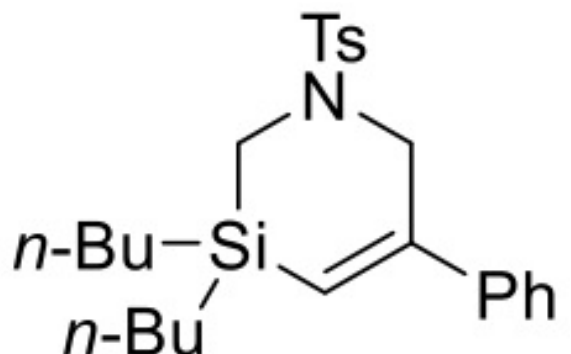

$4 c g$ 


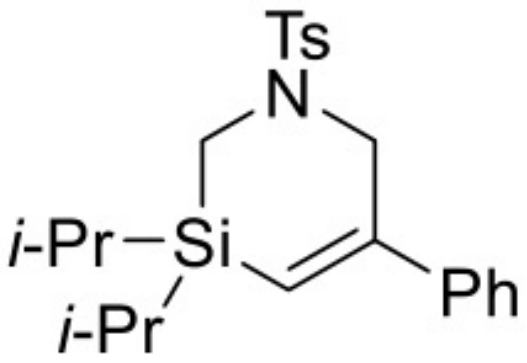

\section{$4 \mathrm{ch}$}

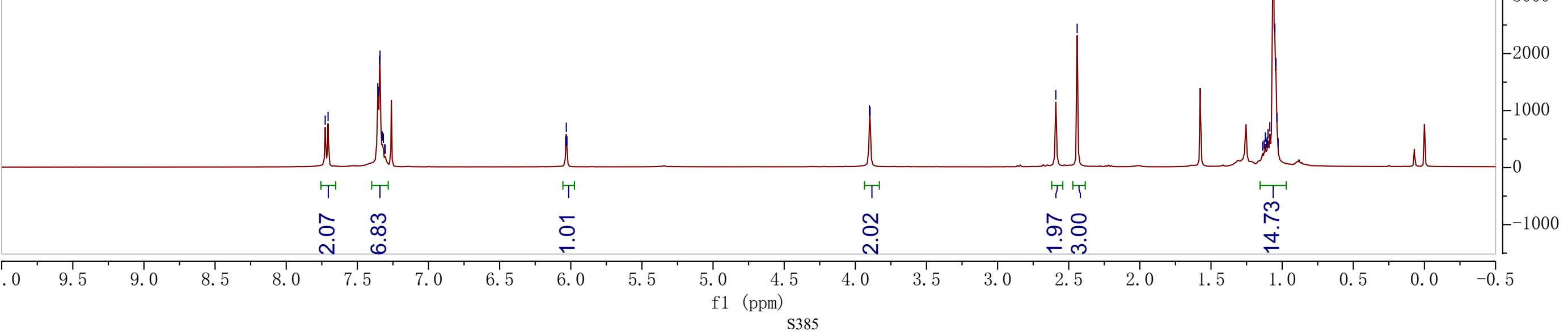




$$
\text { 业 }
$$<smiles>[3H]N1CC(c2ccccc2)=C[Si](P)(P)C1</smiles>

\section{4ch}

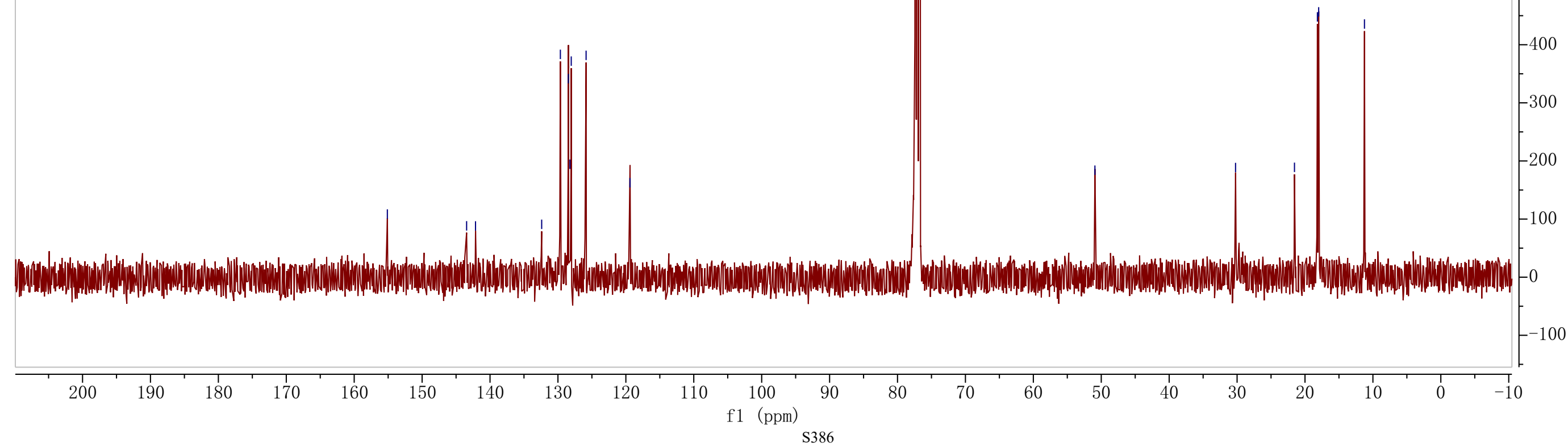




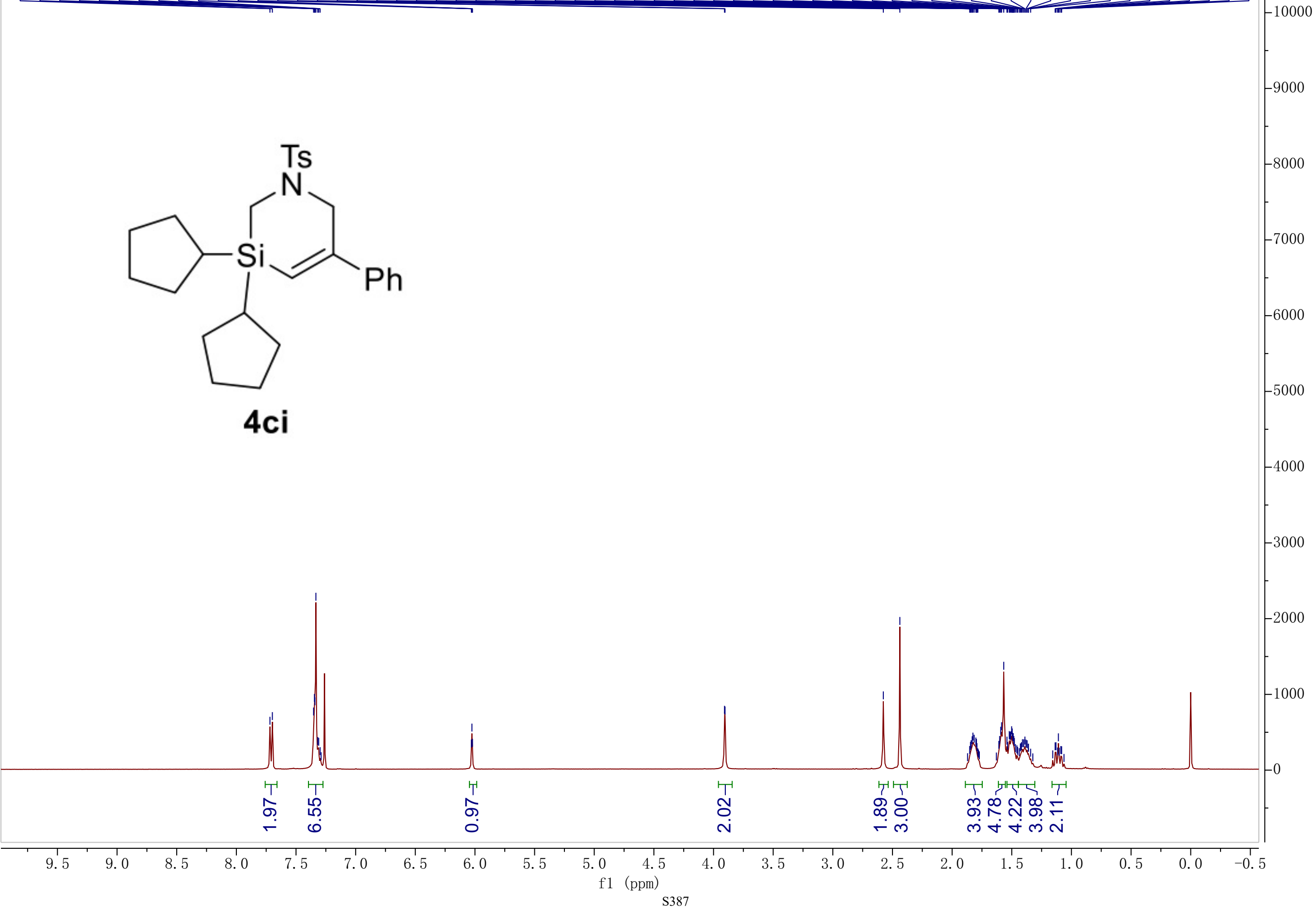




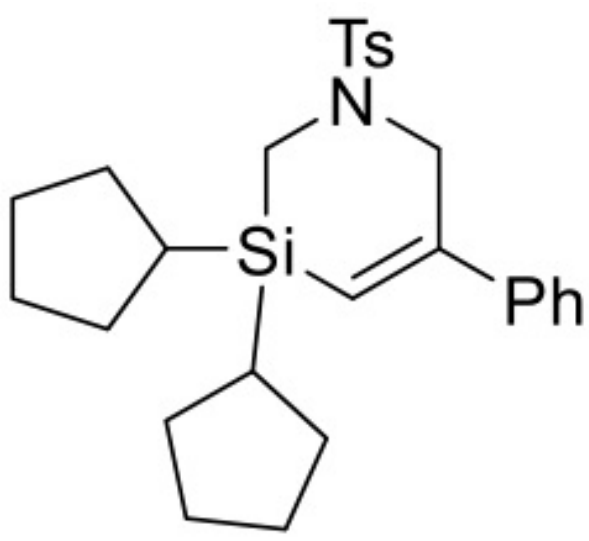

$4 c i$

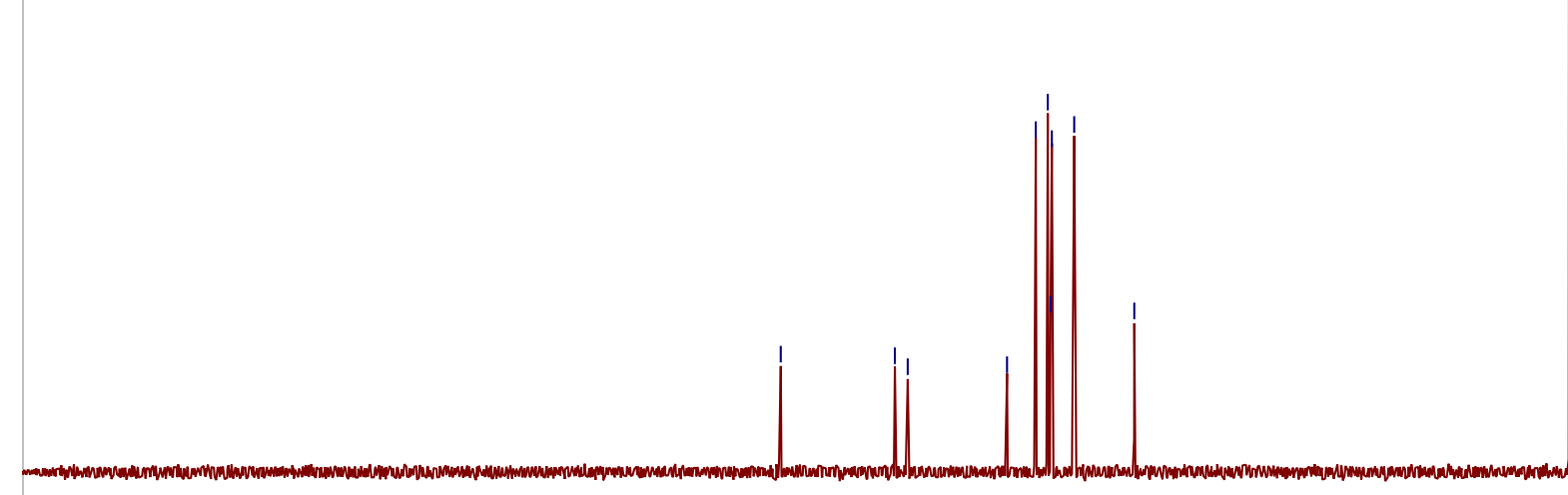




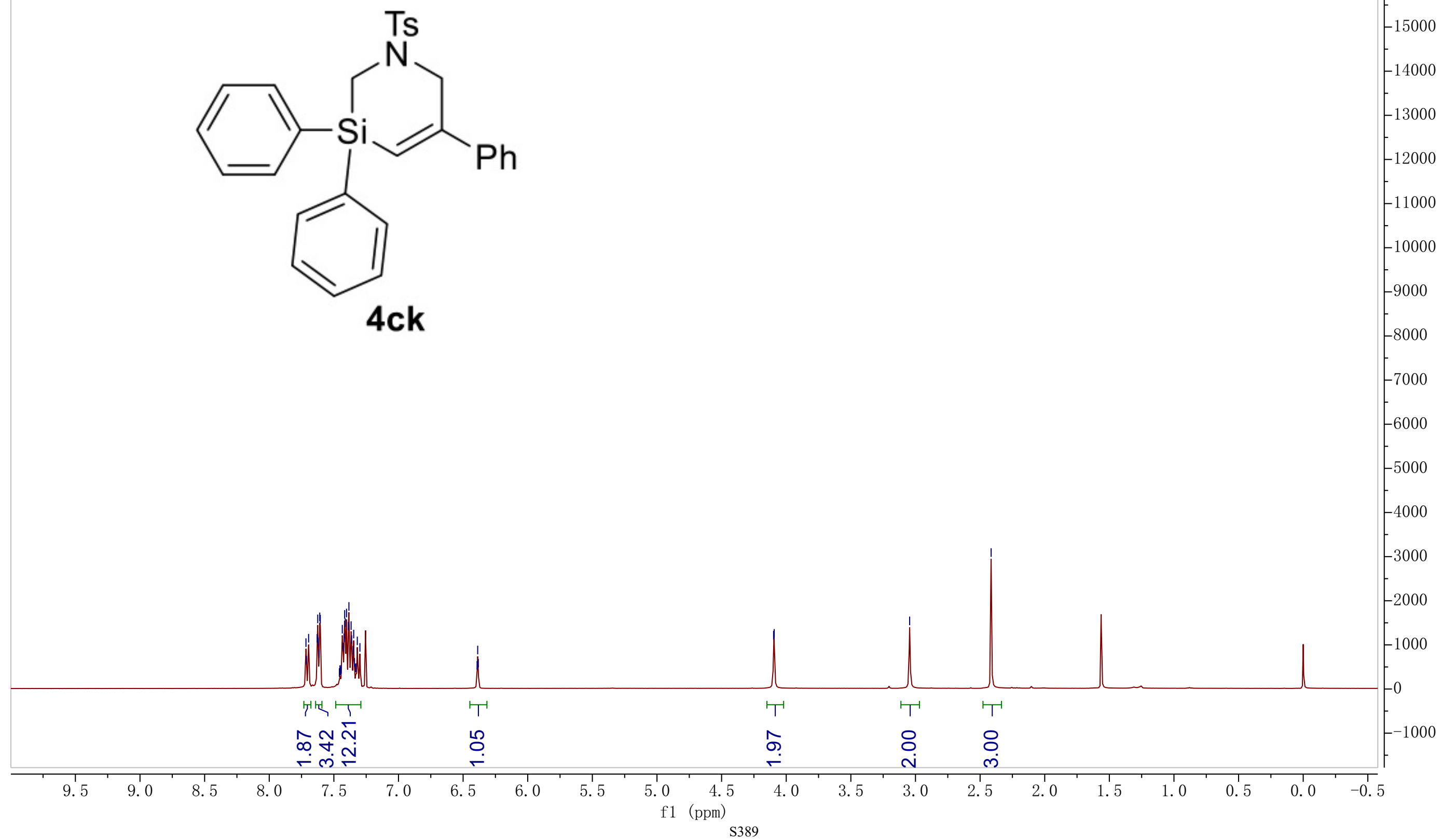




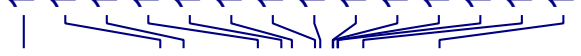

숭ำ

숫

?

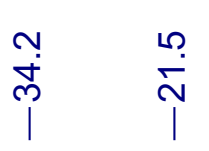

Ts
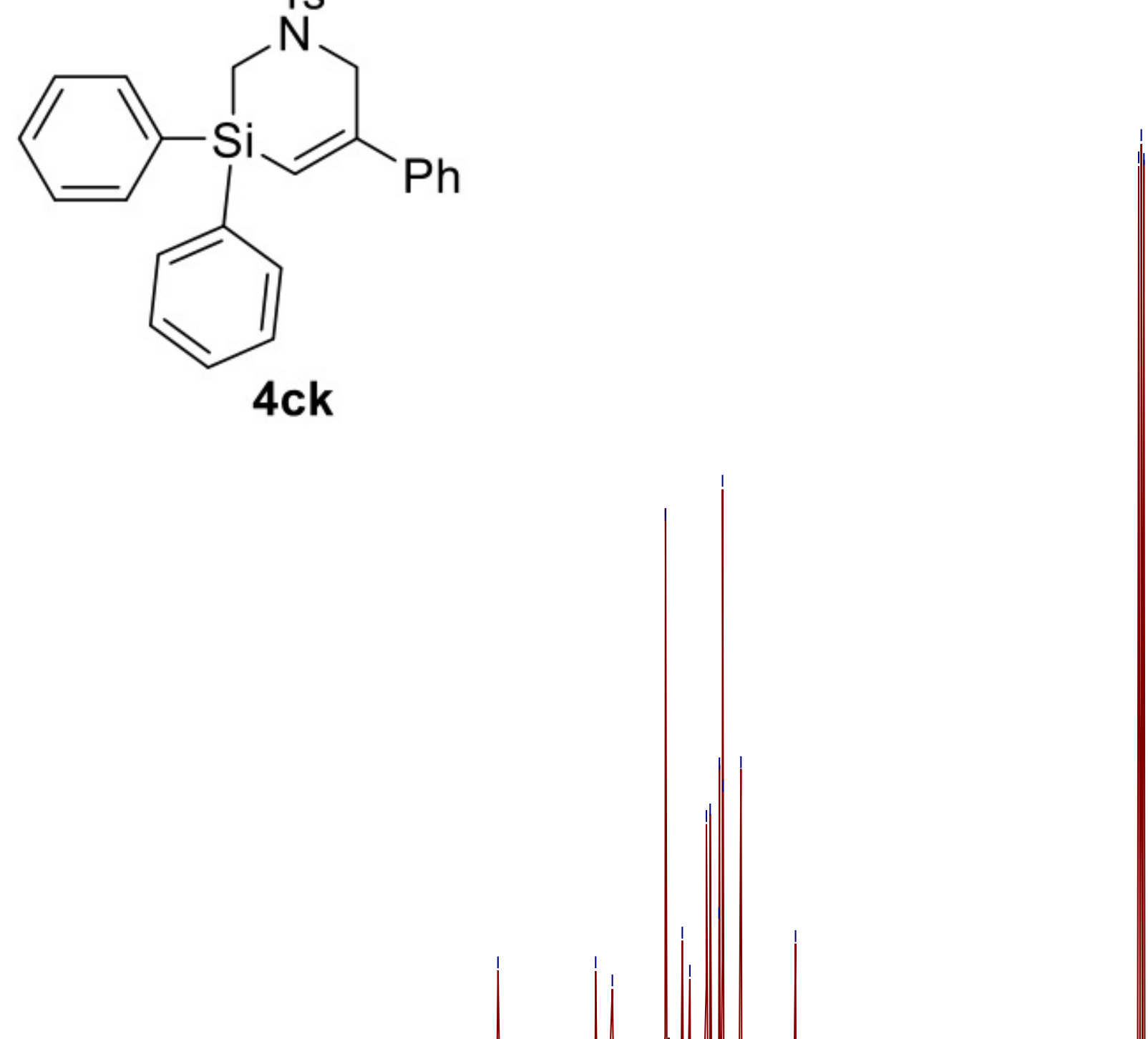

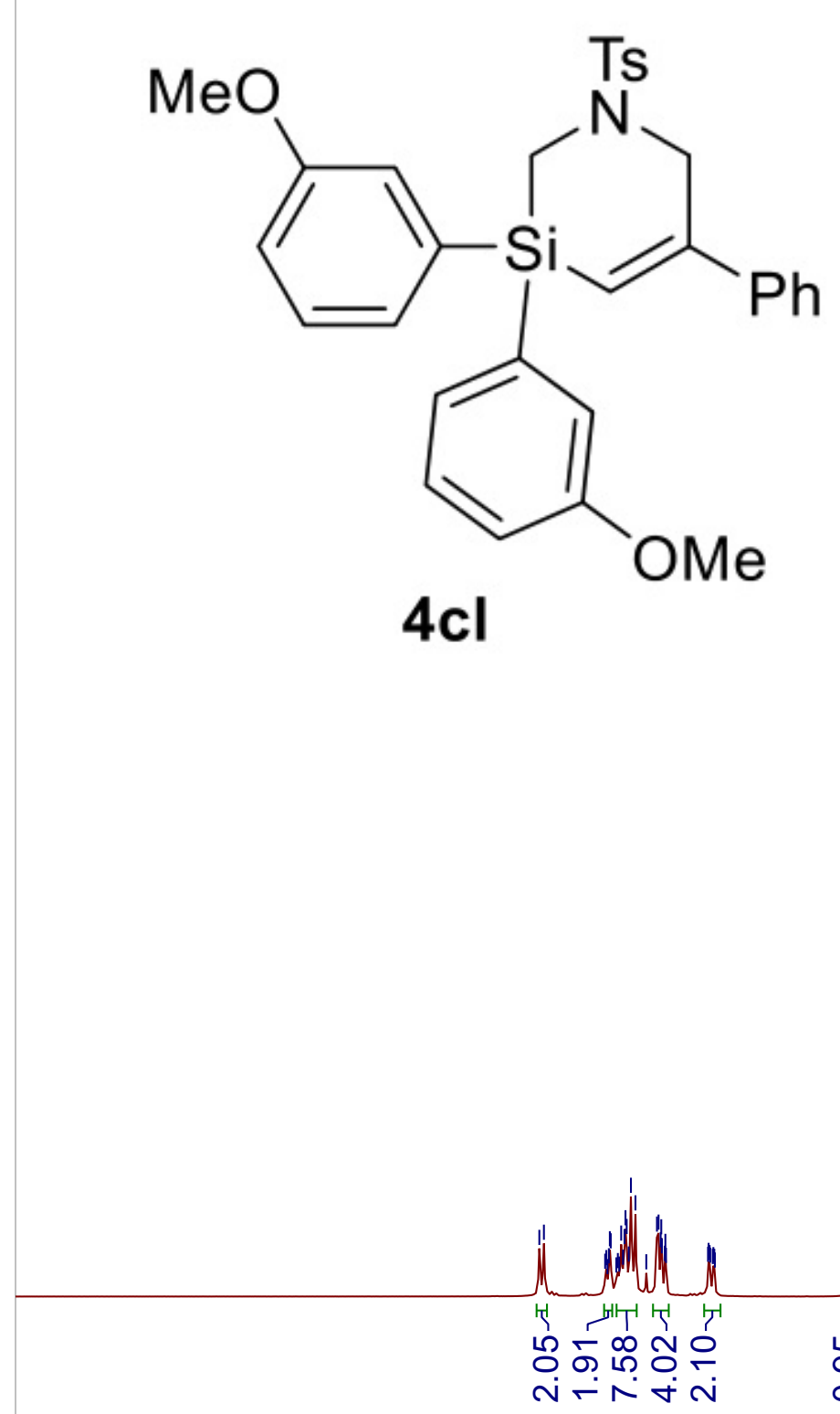
l $1 \underbrace{2}$

A. $-\infty$

Nล

n?

$\stackrel{m}{\tilde{m}}$

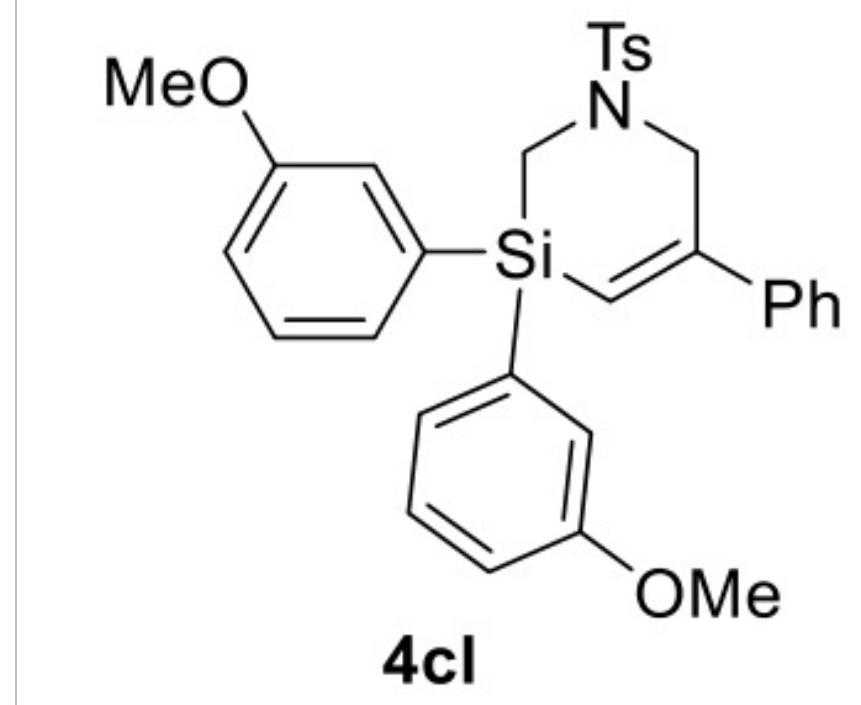



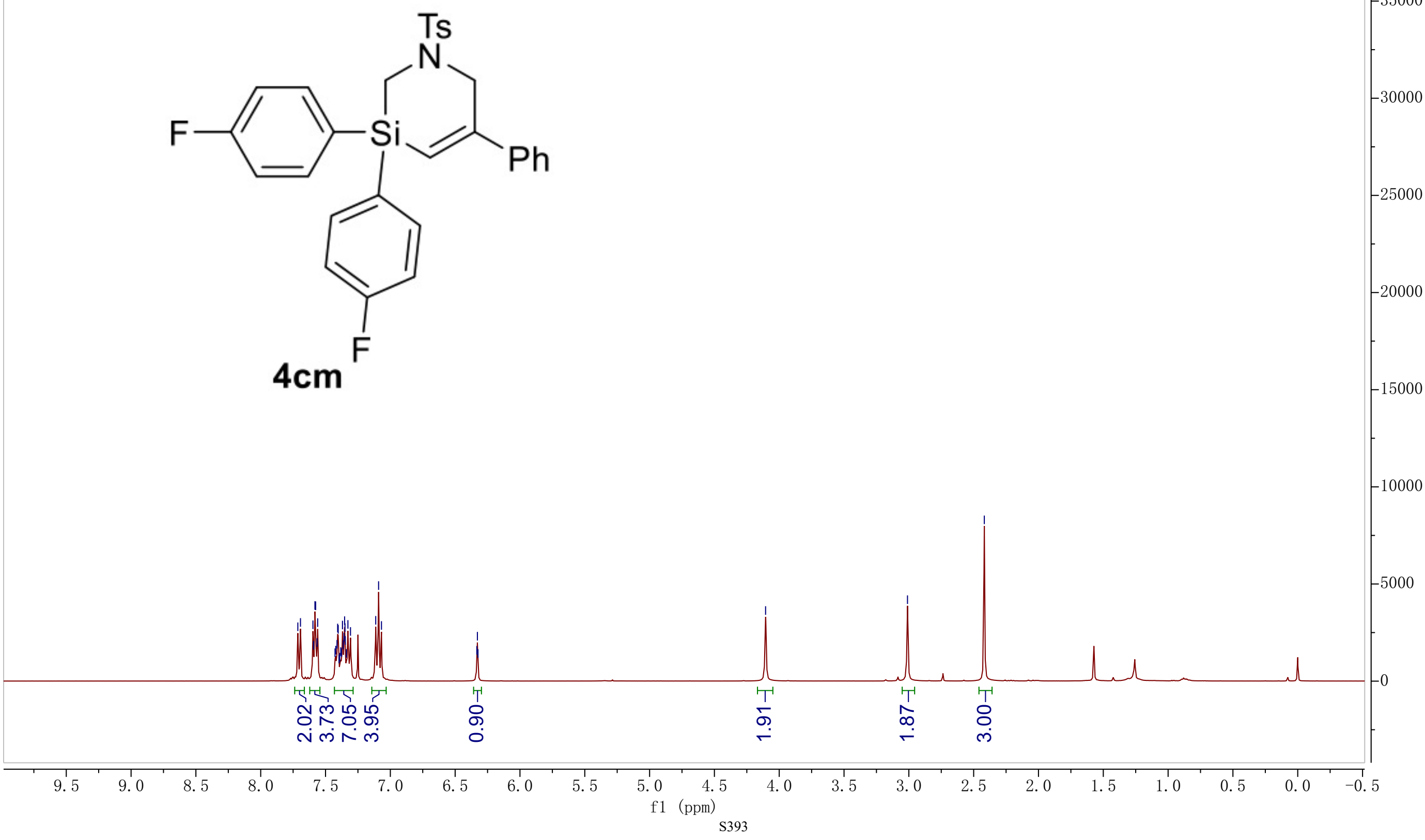


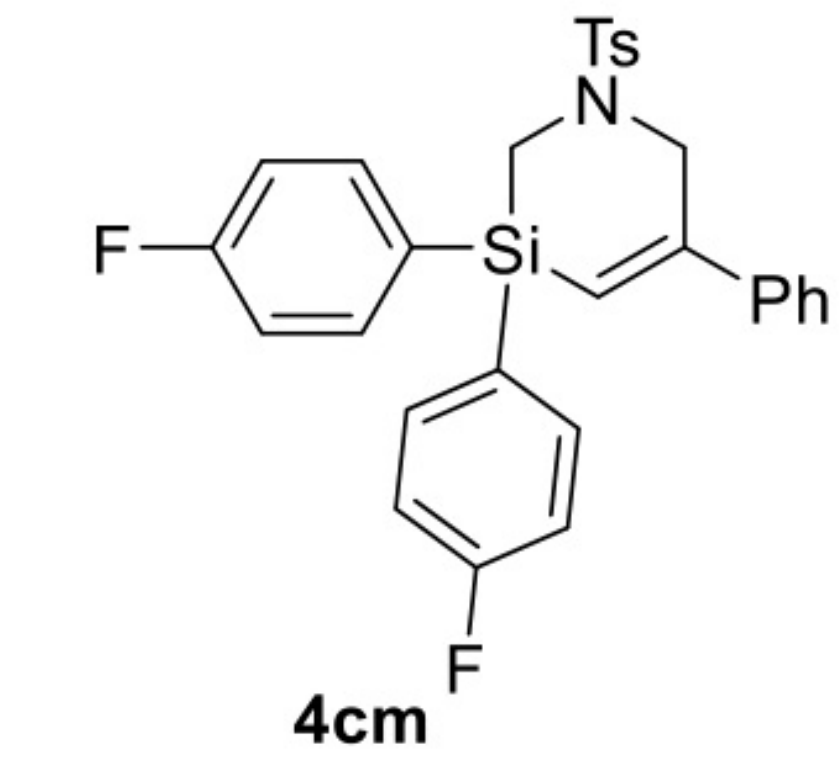


${ }^{19}$ F NMR

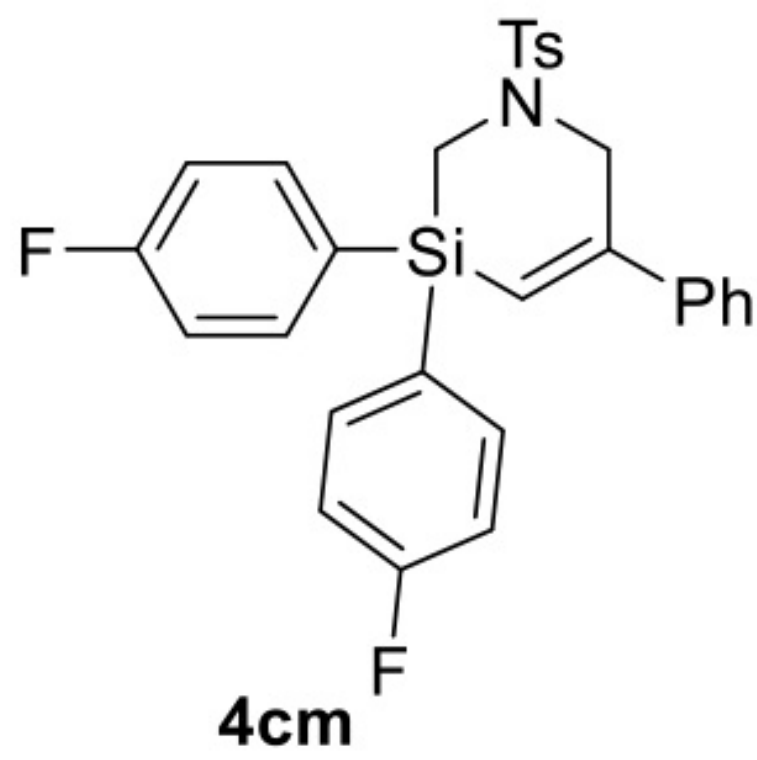


<smiles>C[Si]1(c2ccccc2)C=C(c2ccccc2)CN([As])C1</smiles>

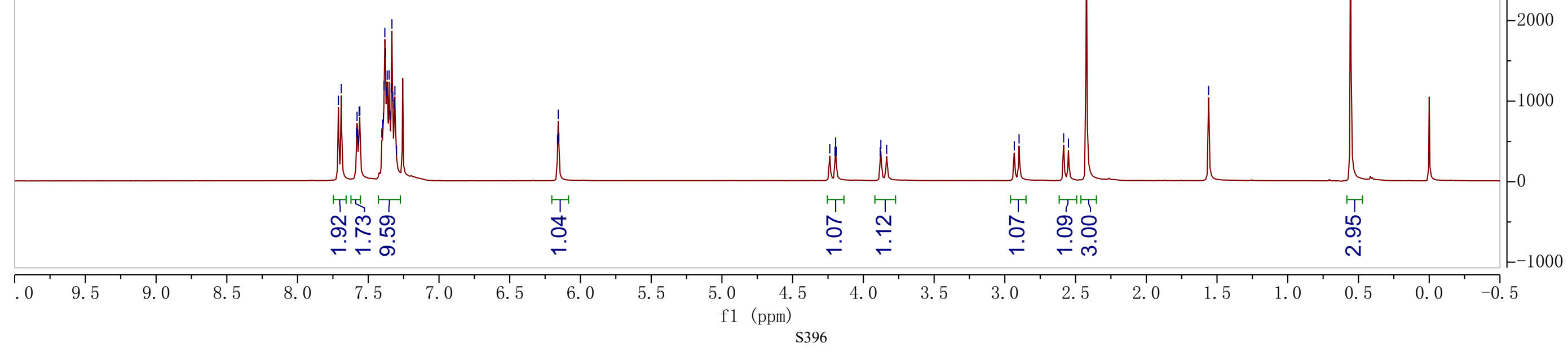


Ts<smiles>C[Si]1(c2ccccc2)C=C(c2ccccc2)CN([As])C1</smiles> 
Nㅜ윴 mల్లెల్

NiNiNin

ธุดำ

نं

$8 \%$

iं

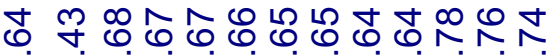

iN

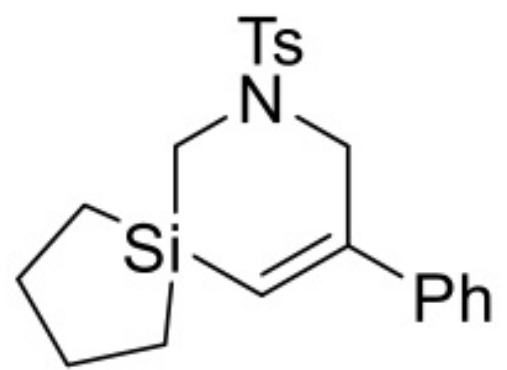

$4 c o$

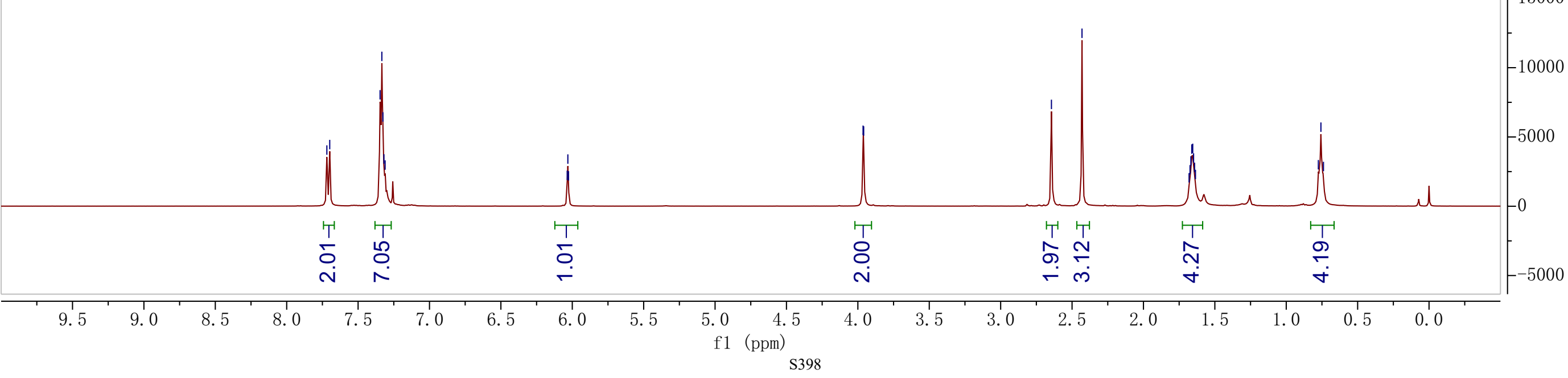




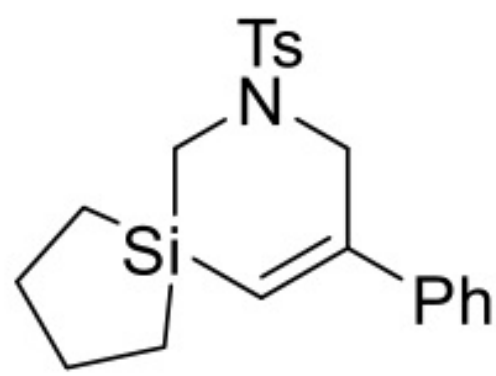

$4 c o$

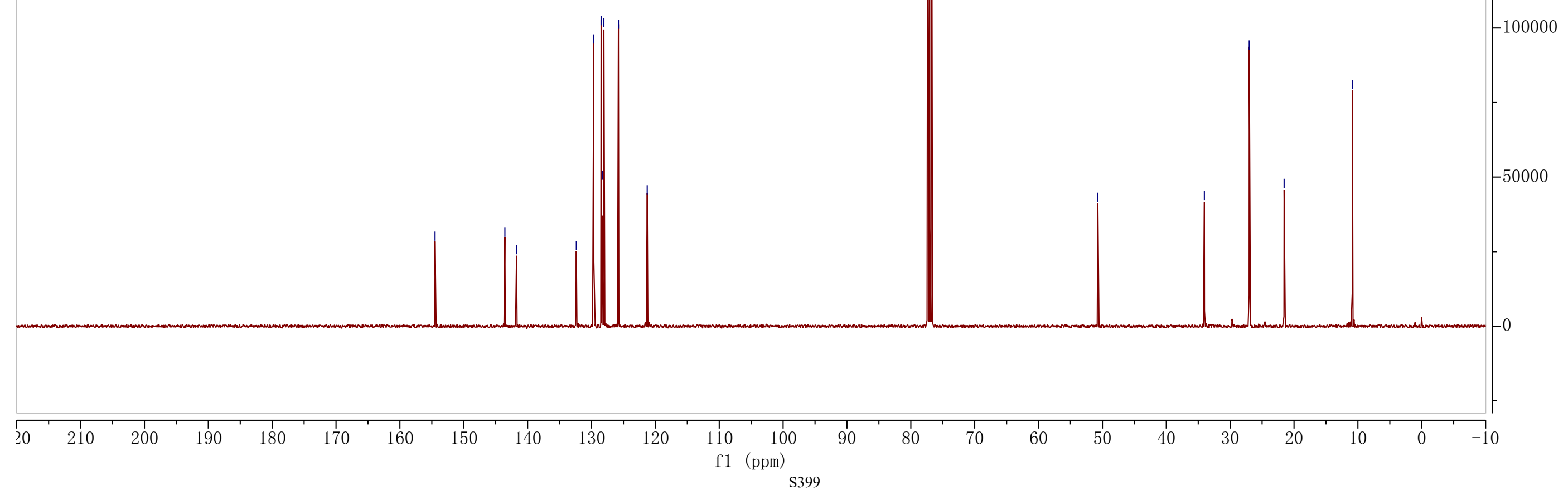




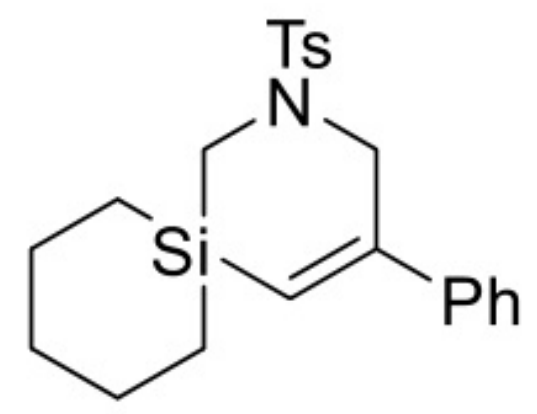

4cp
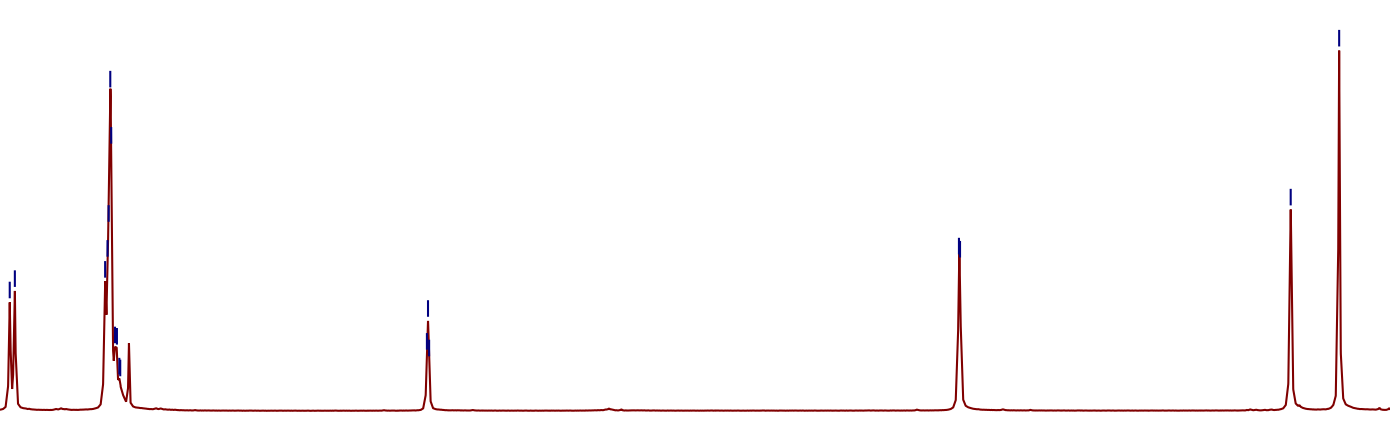

ctin

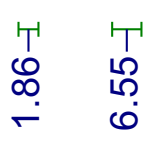

गे

गै ?

ติ

당ำ

ले

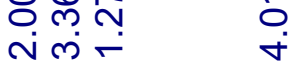

6. $5.5 \quad 5.0$

4.0

1. 5 


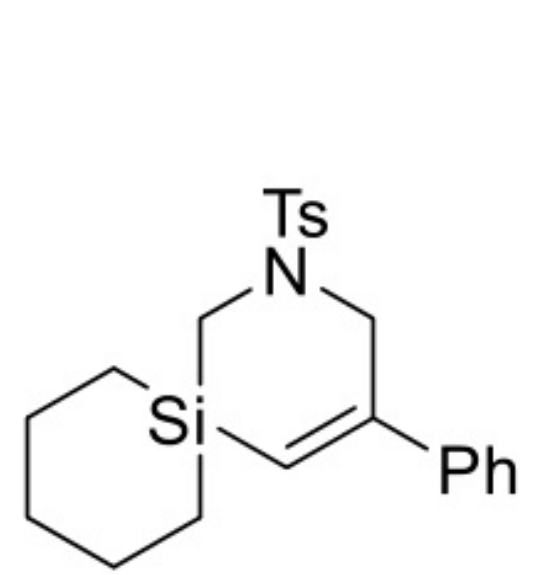

\section{4cp}

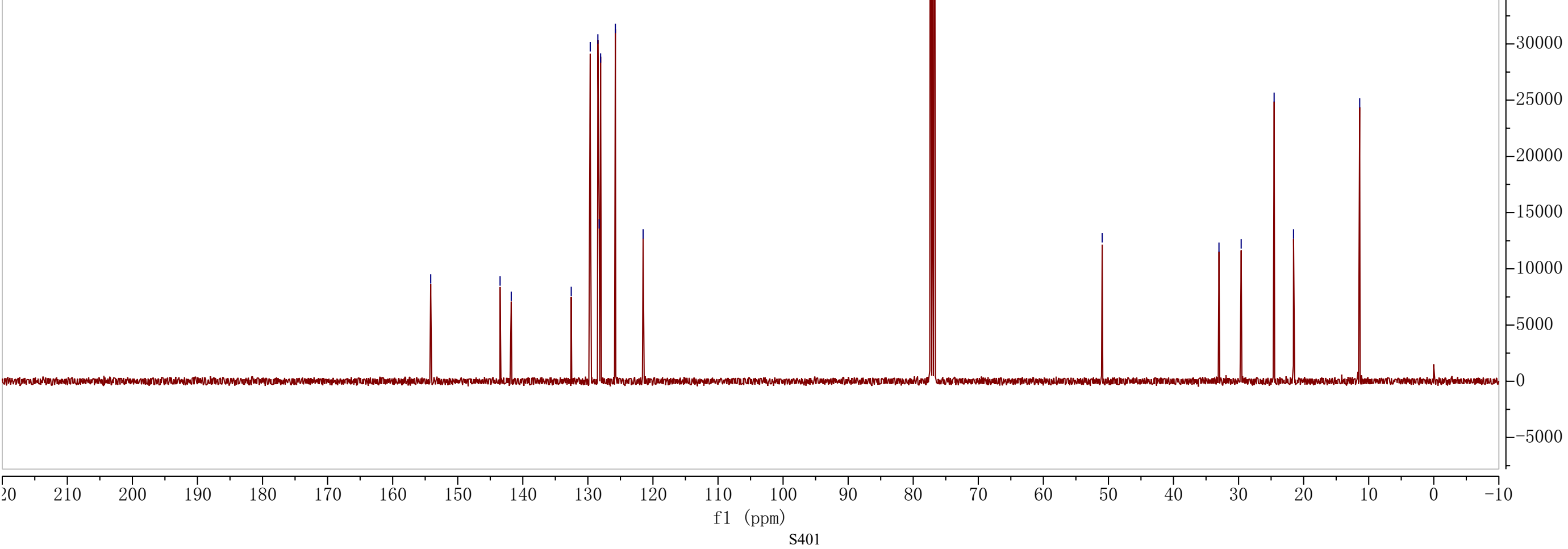




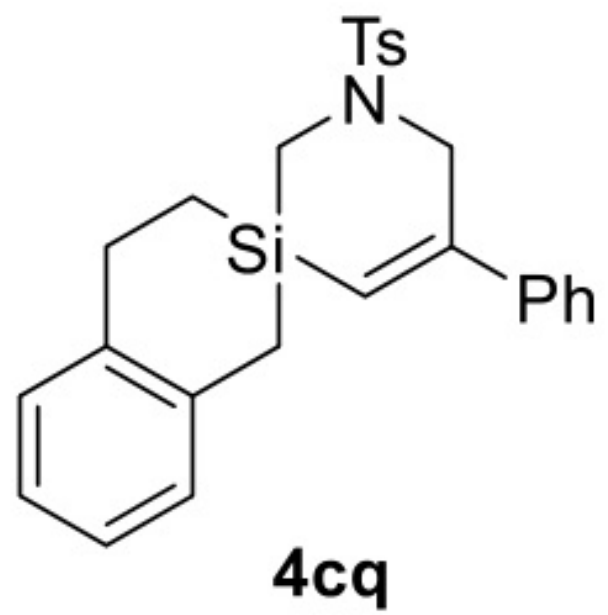

$4 c q$

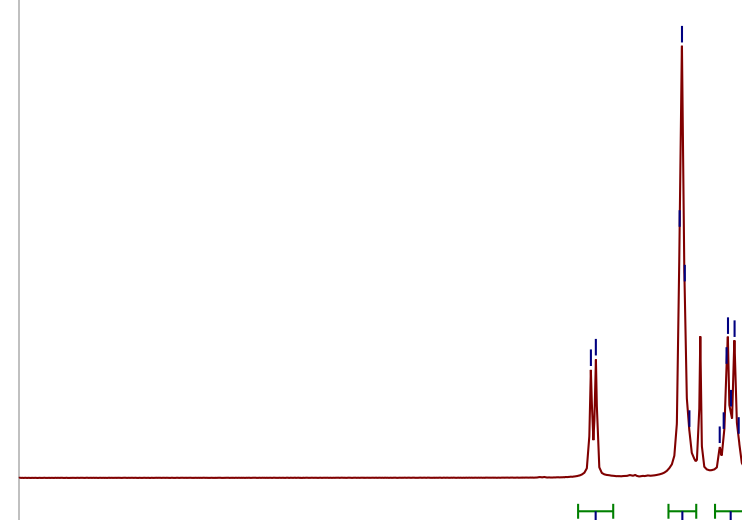

m

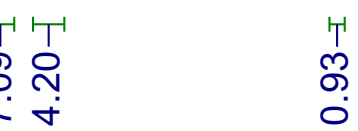

萝


<smiles>ClC(Cl)(Cl)Cl</smiles>

$4 \mathrm{cq}$ 
${ }_{T s N}-M e{ }^{-M T s}$

$\mathrm{Me}^{-\mathrm{Si}^{-}{ }^{-}{ }_{\mathrm{Me}} \mathrm{Si}_{\mathrm{Me}} \mathrm{Me}}$

$5 a$

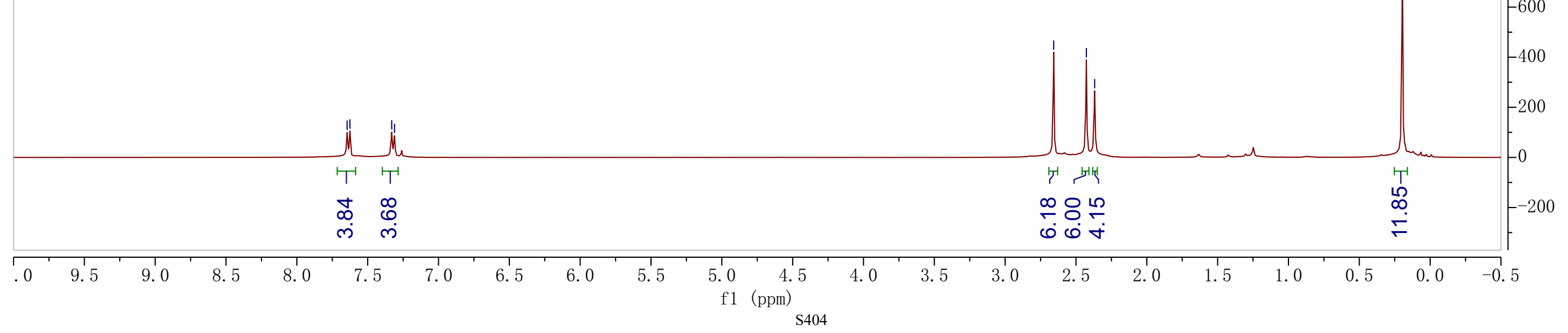




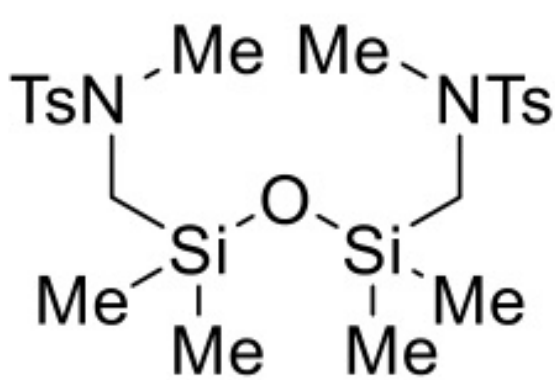

$5 a$

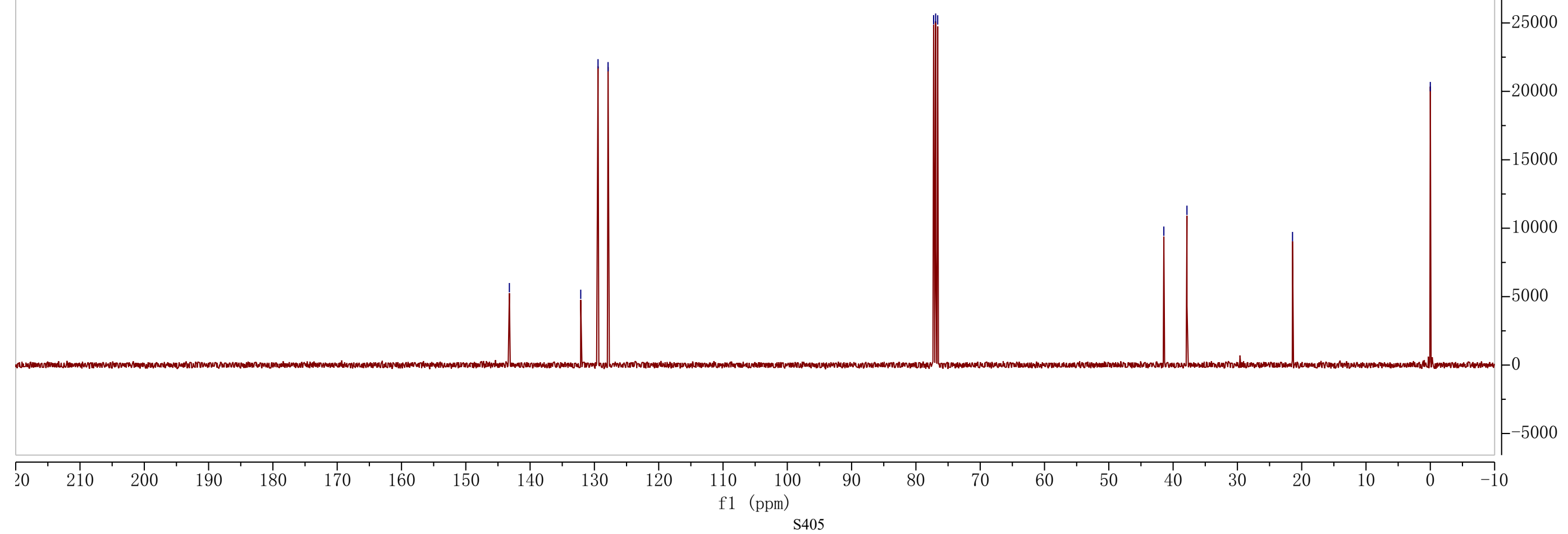


TsHN

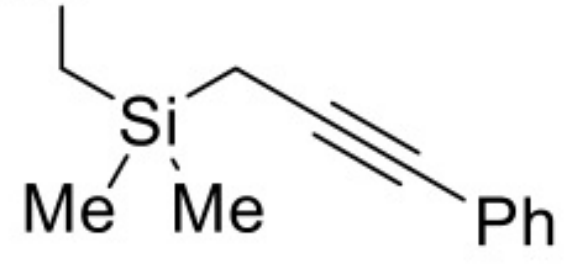

$6 a$

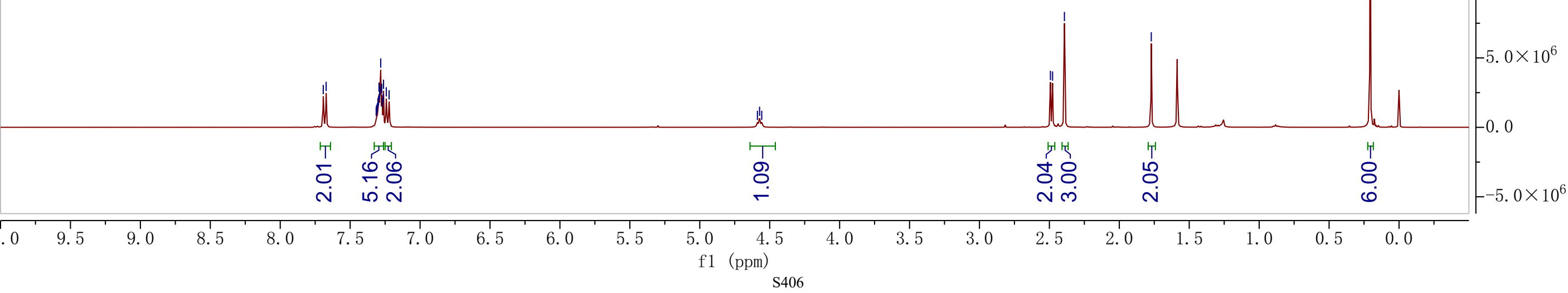


TsHN

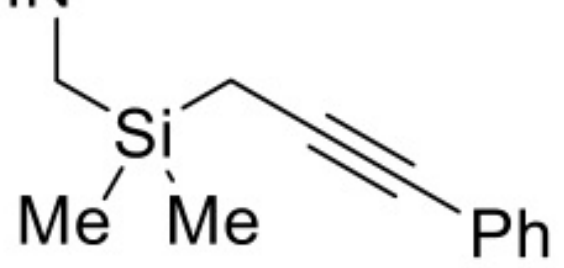

$6 a$

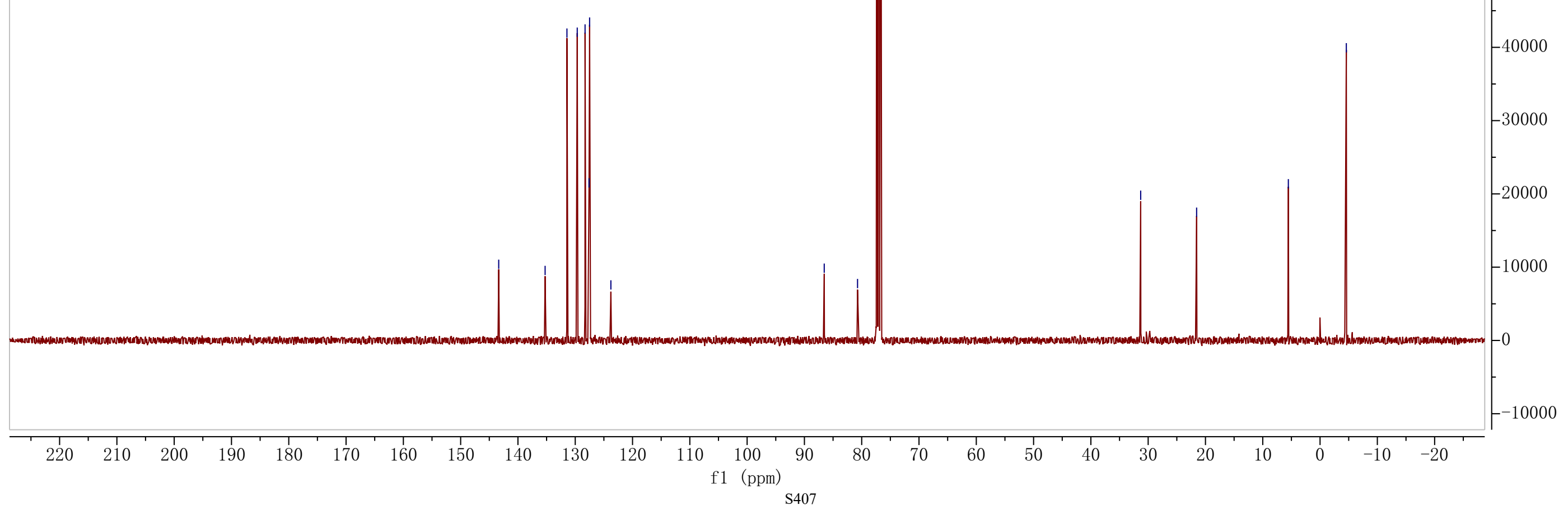




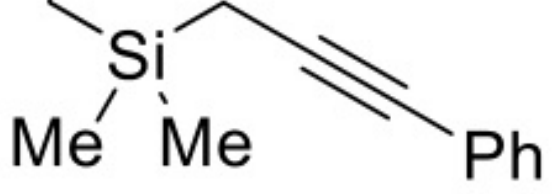

6d

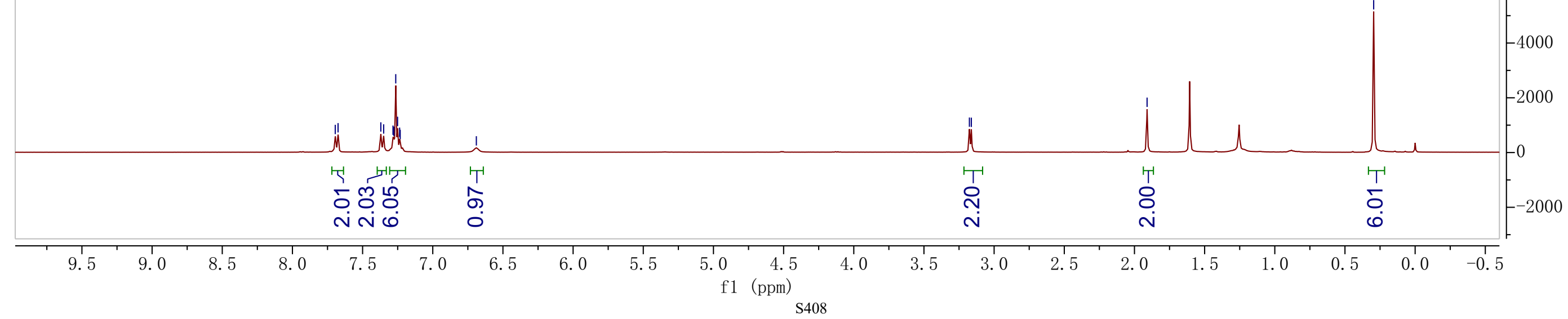




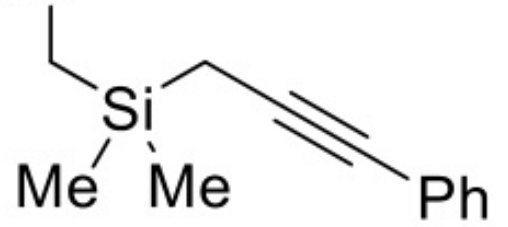

6d 
${ }^{19}$ F NMR

$p-\mathrm{CF}_{3} \mathrm{C}_{6} \mathrm{H}_{4} \mathrm{COHN}$

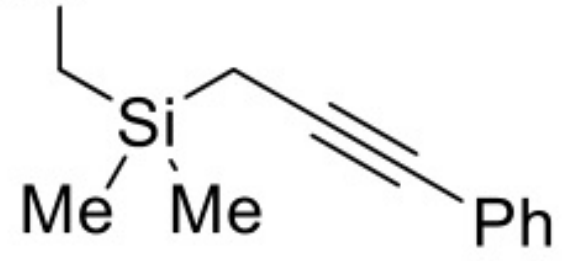

6d 


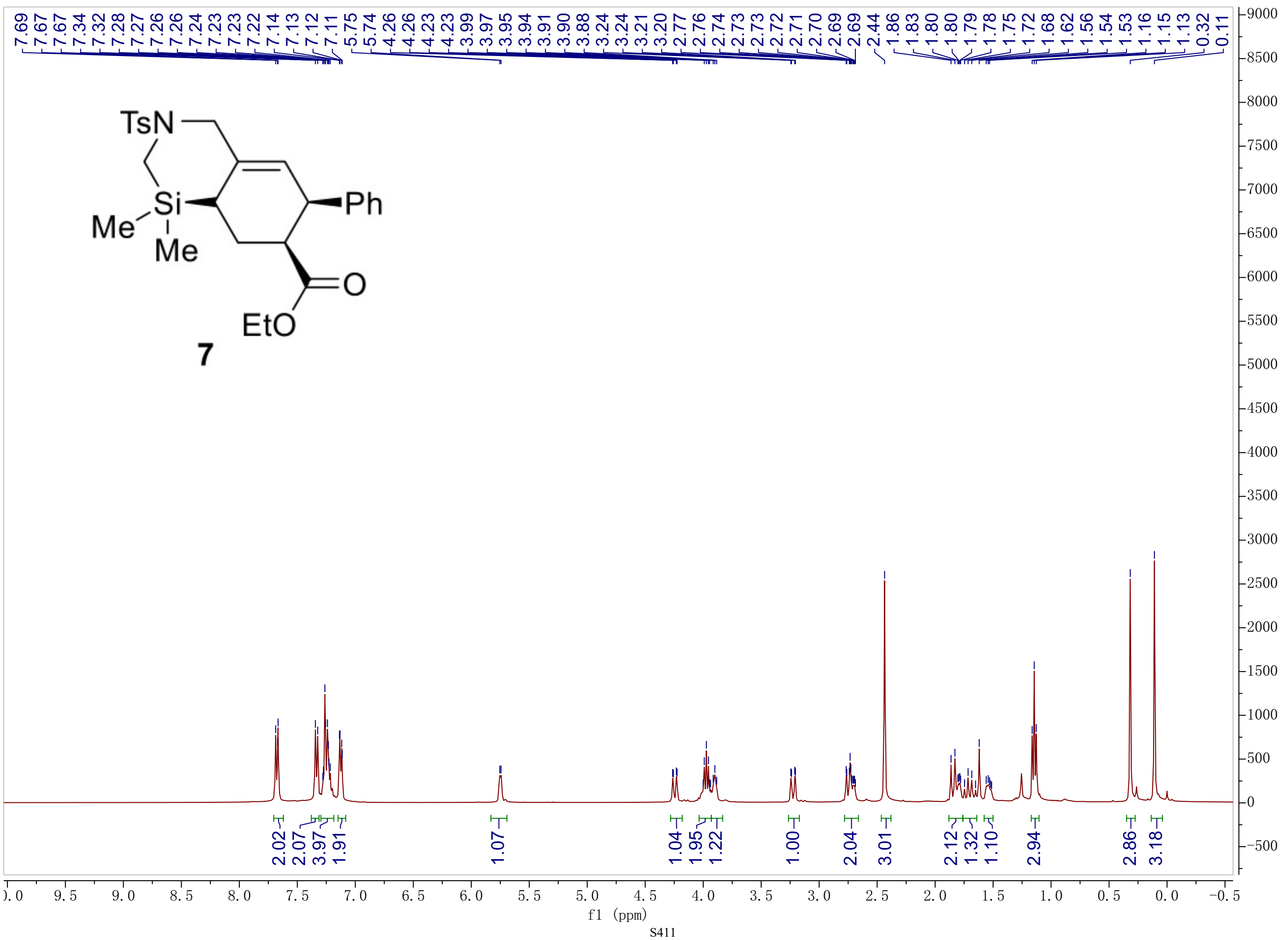




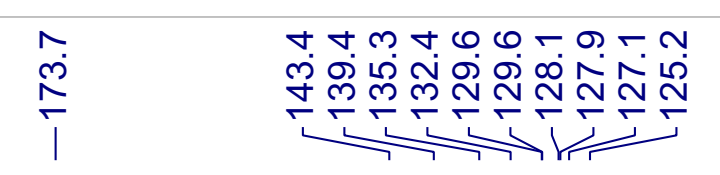

年先

กิ

อ

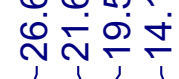

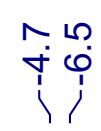<smiles>CCOC(=O)[C@H]1CC2C(=C[C@H](c3ccccc3)[C@@H]2C)CN([As])C[Si]1(C)C</smiles> 


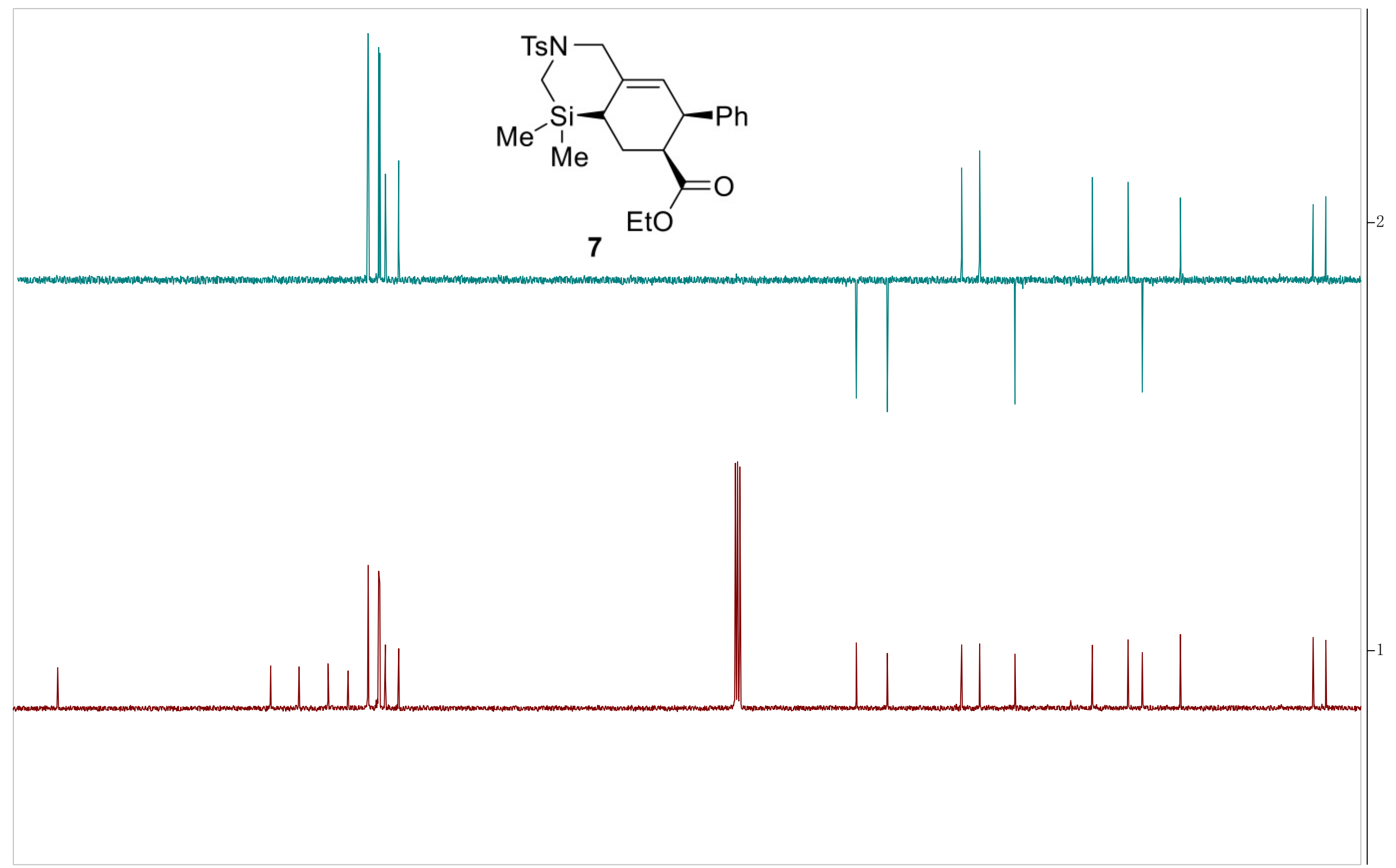

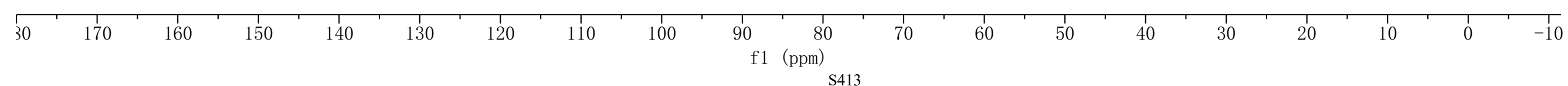




\section{H- H COSY}

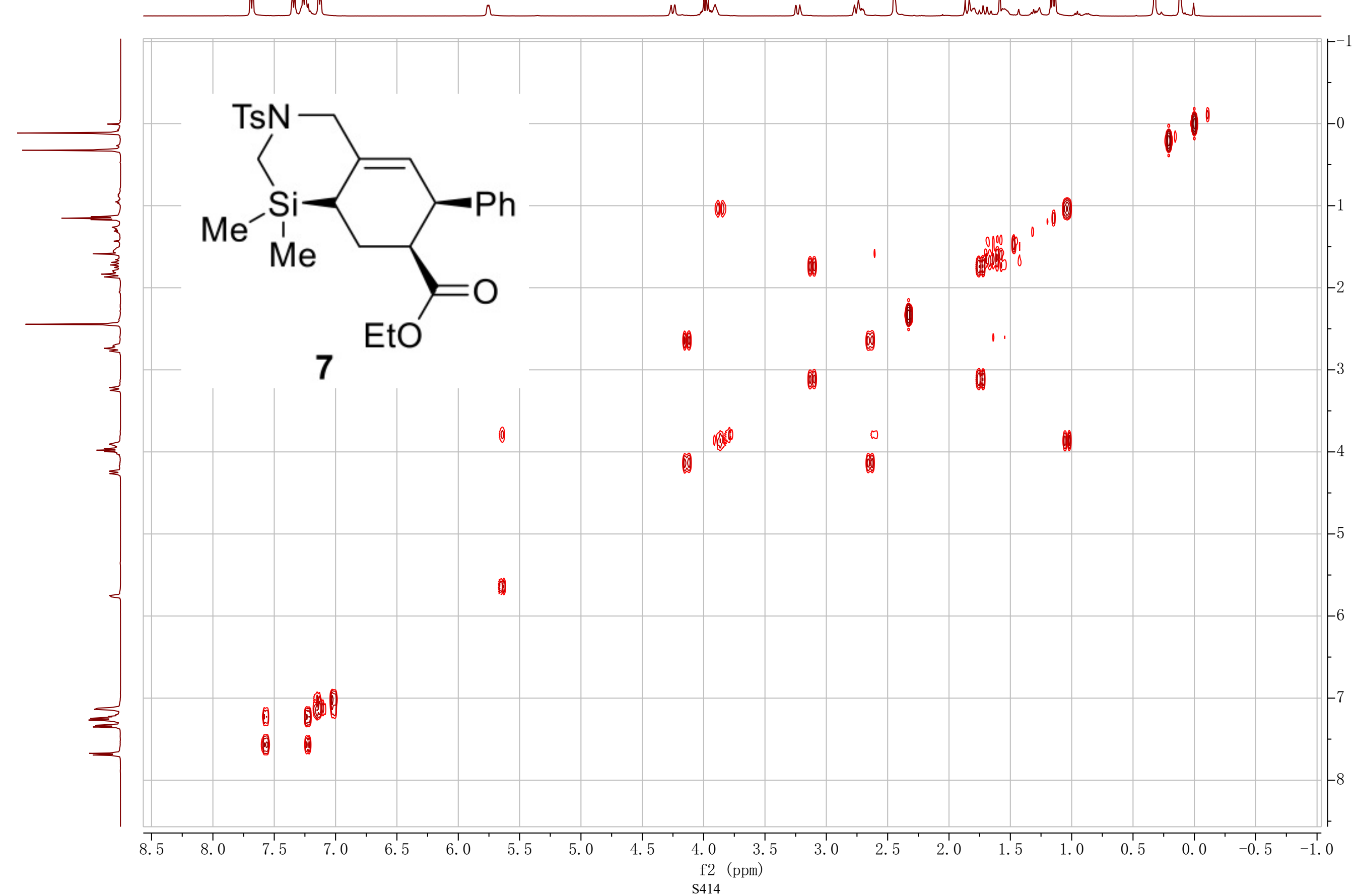


HMQC

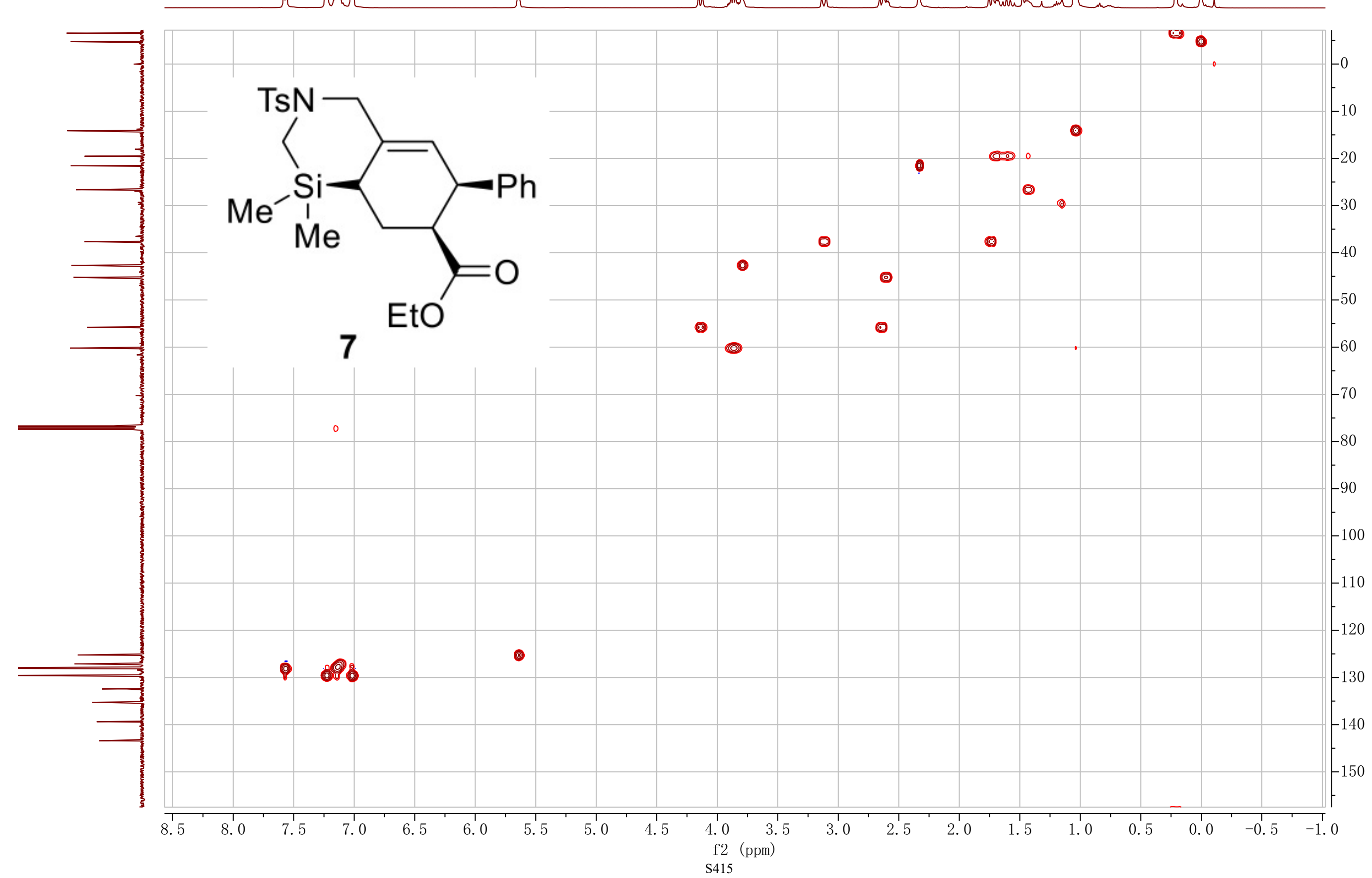


HMBC

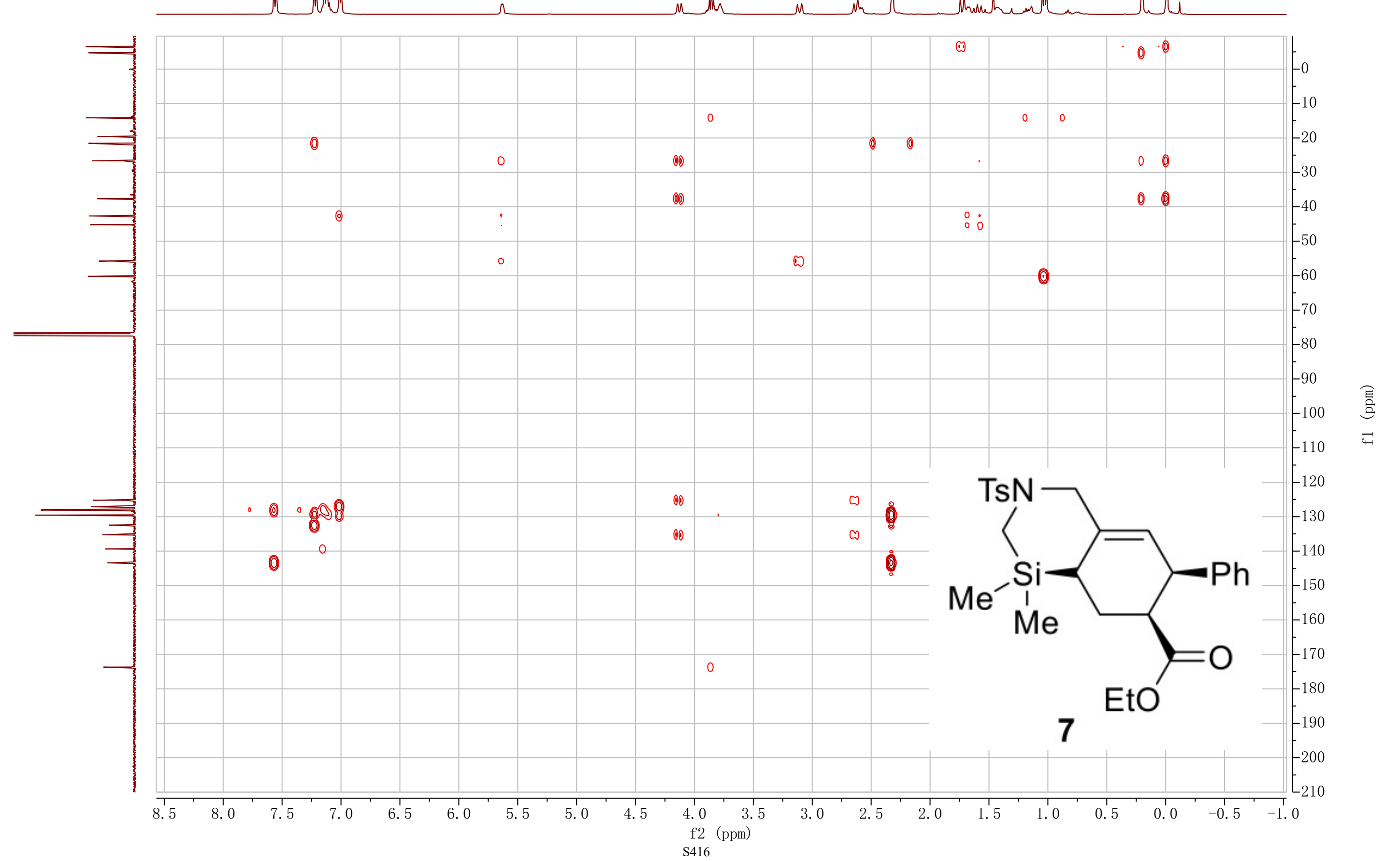




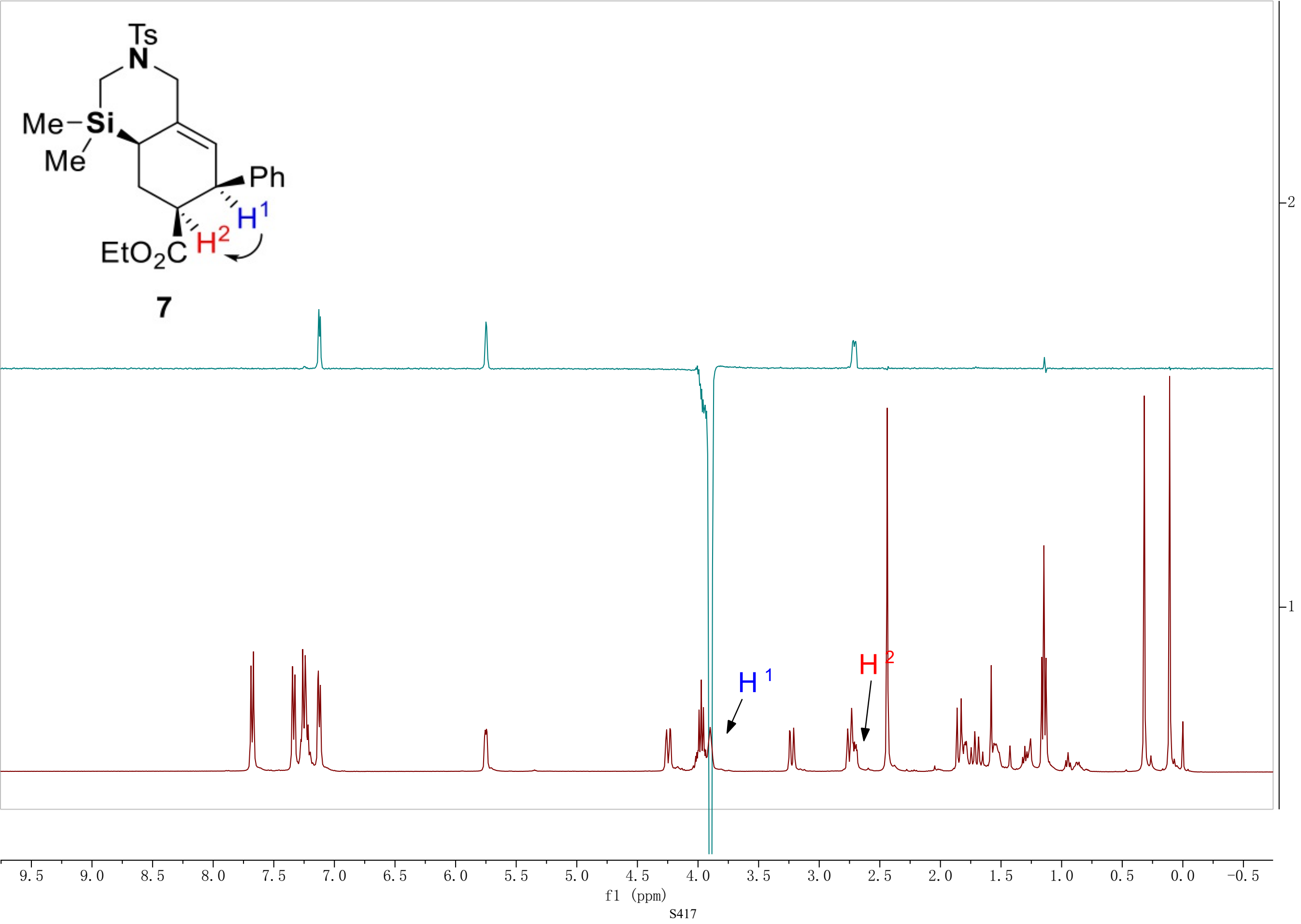




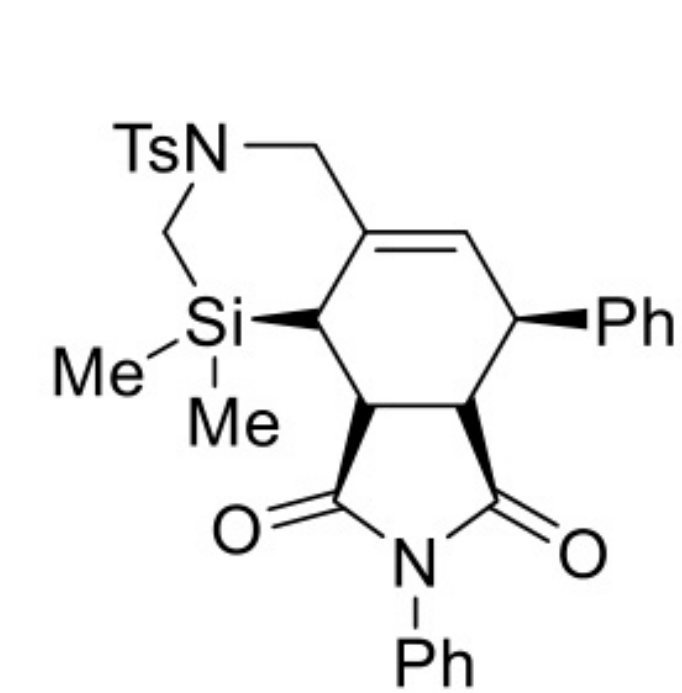

8

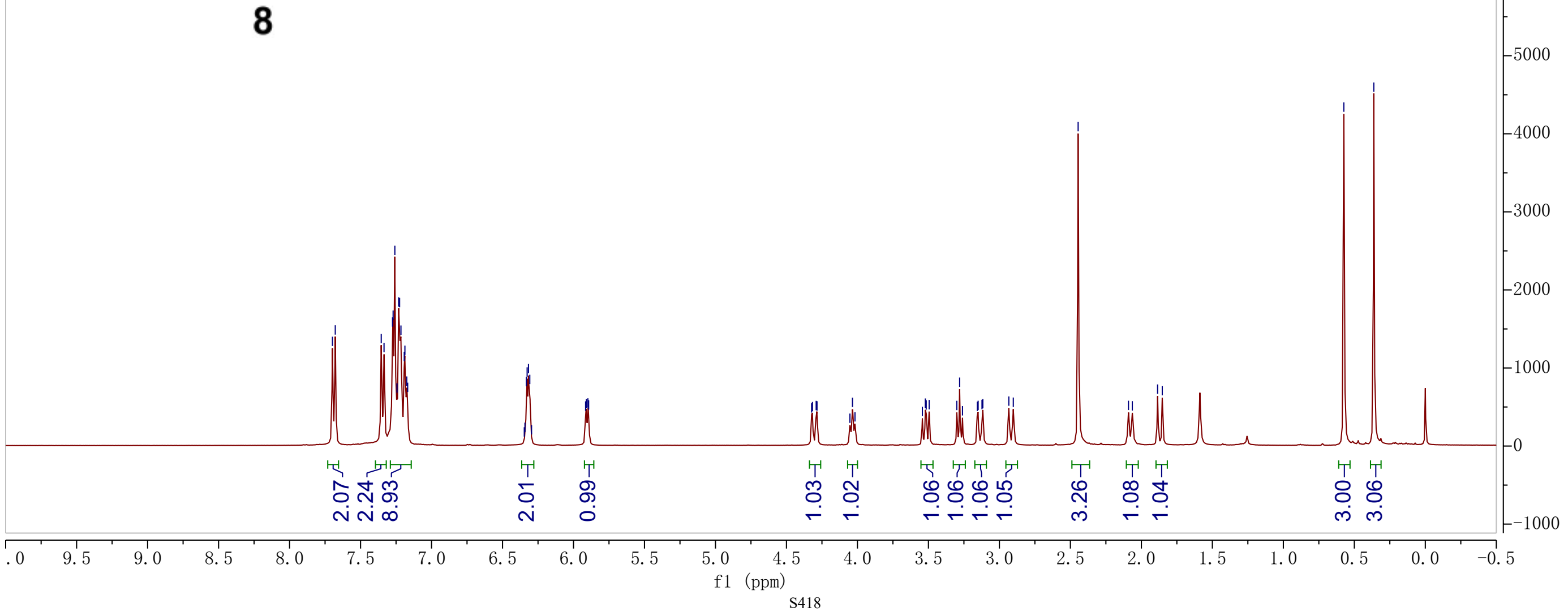




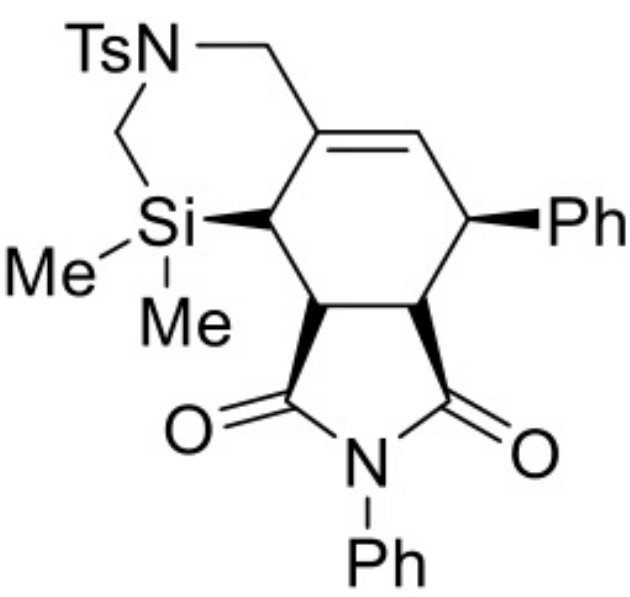

8 

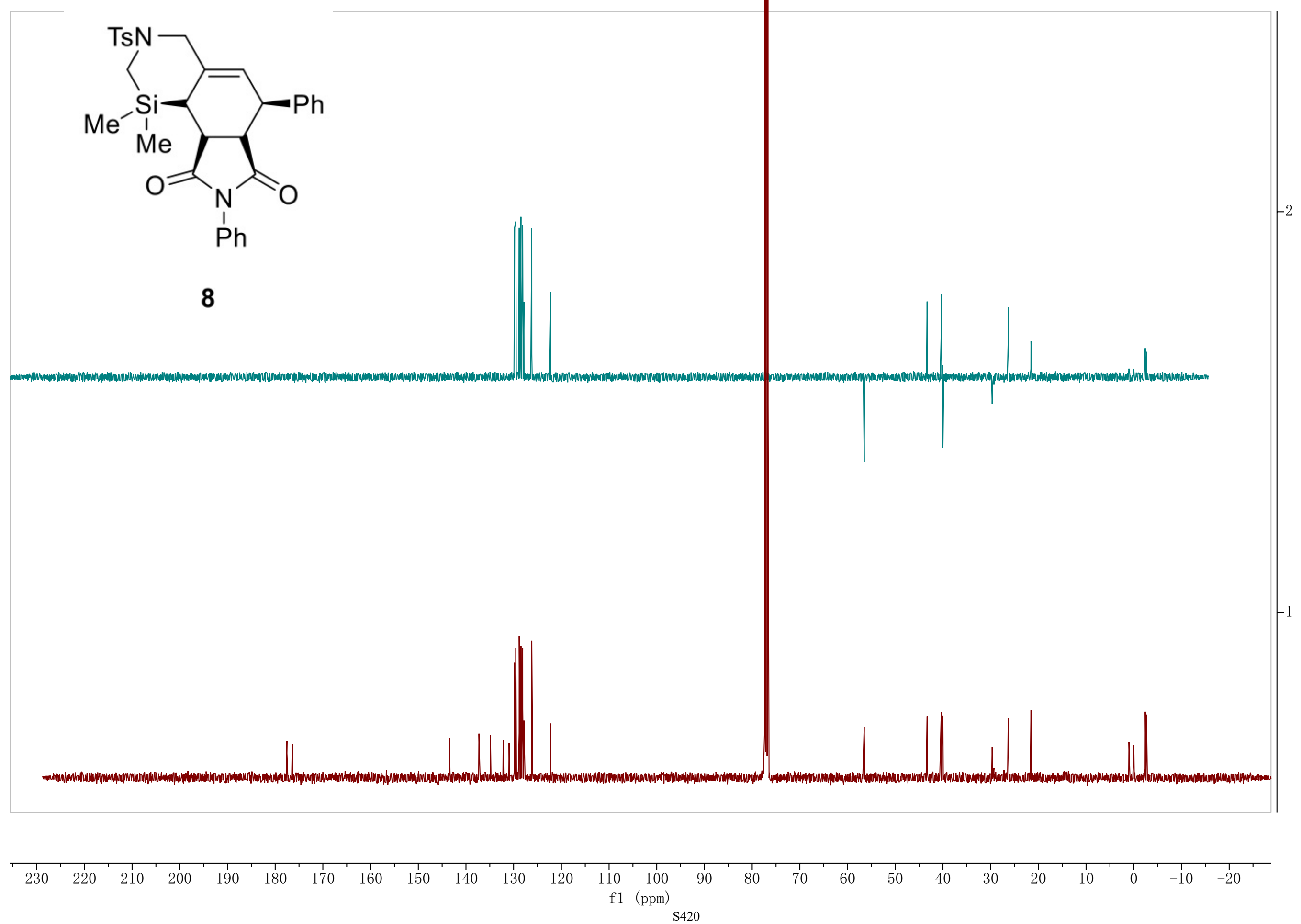
H- H COSY
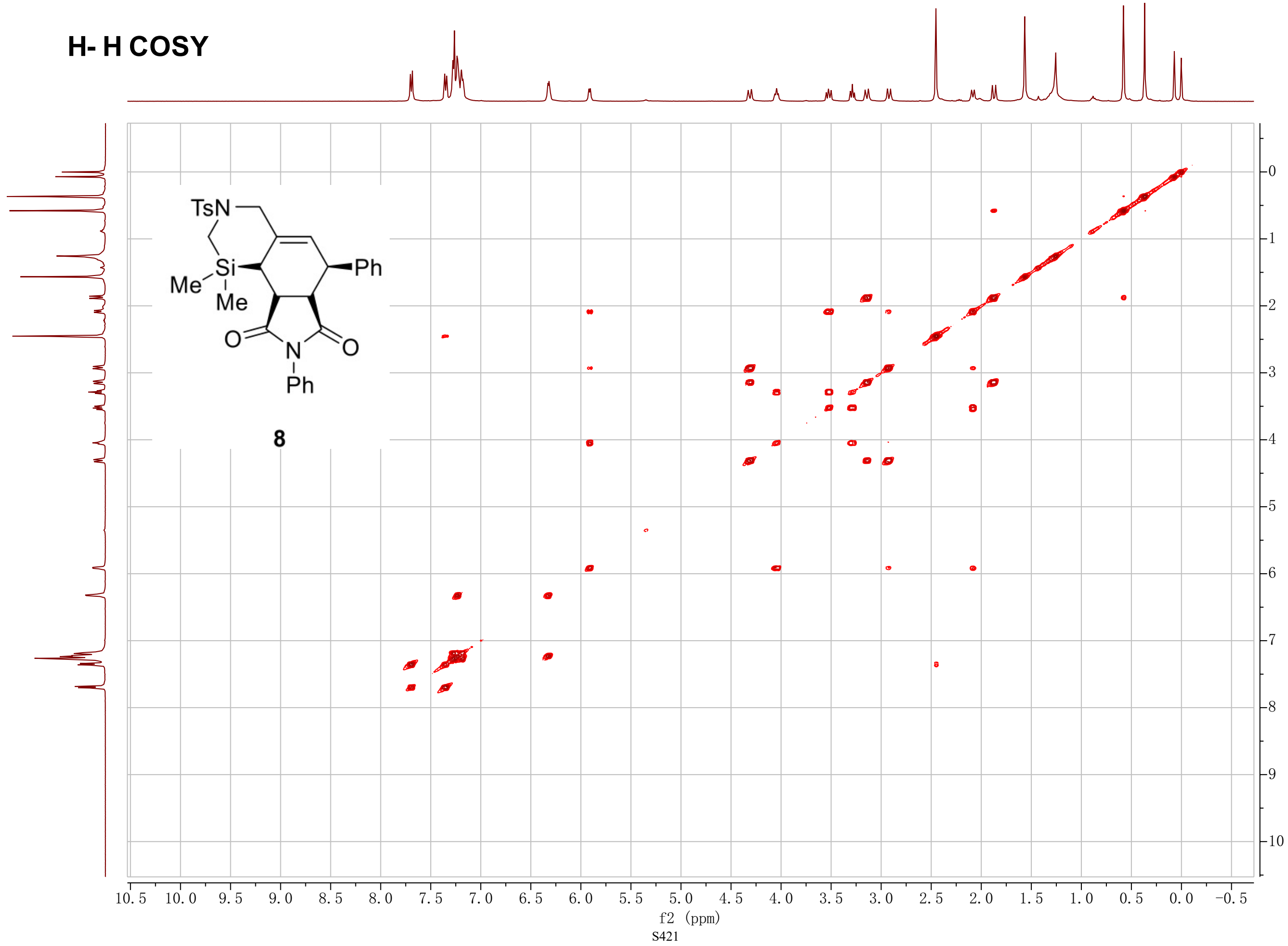


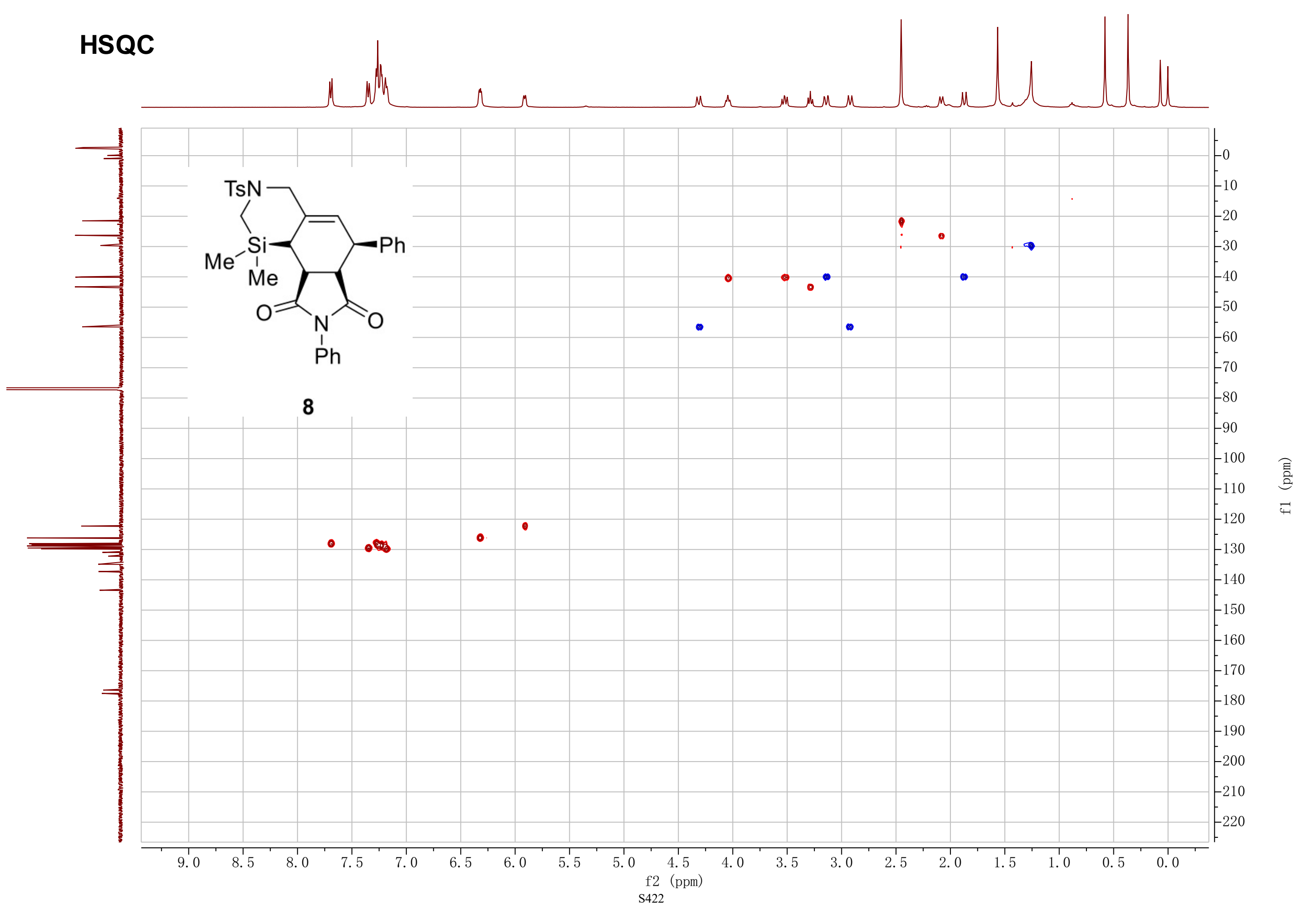


HMBC

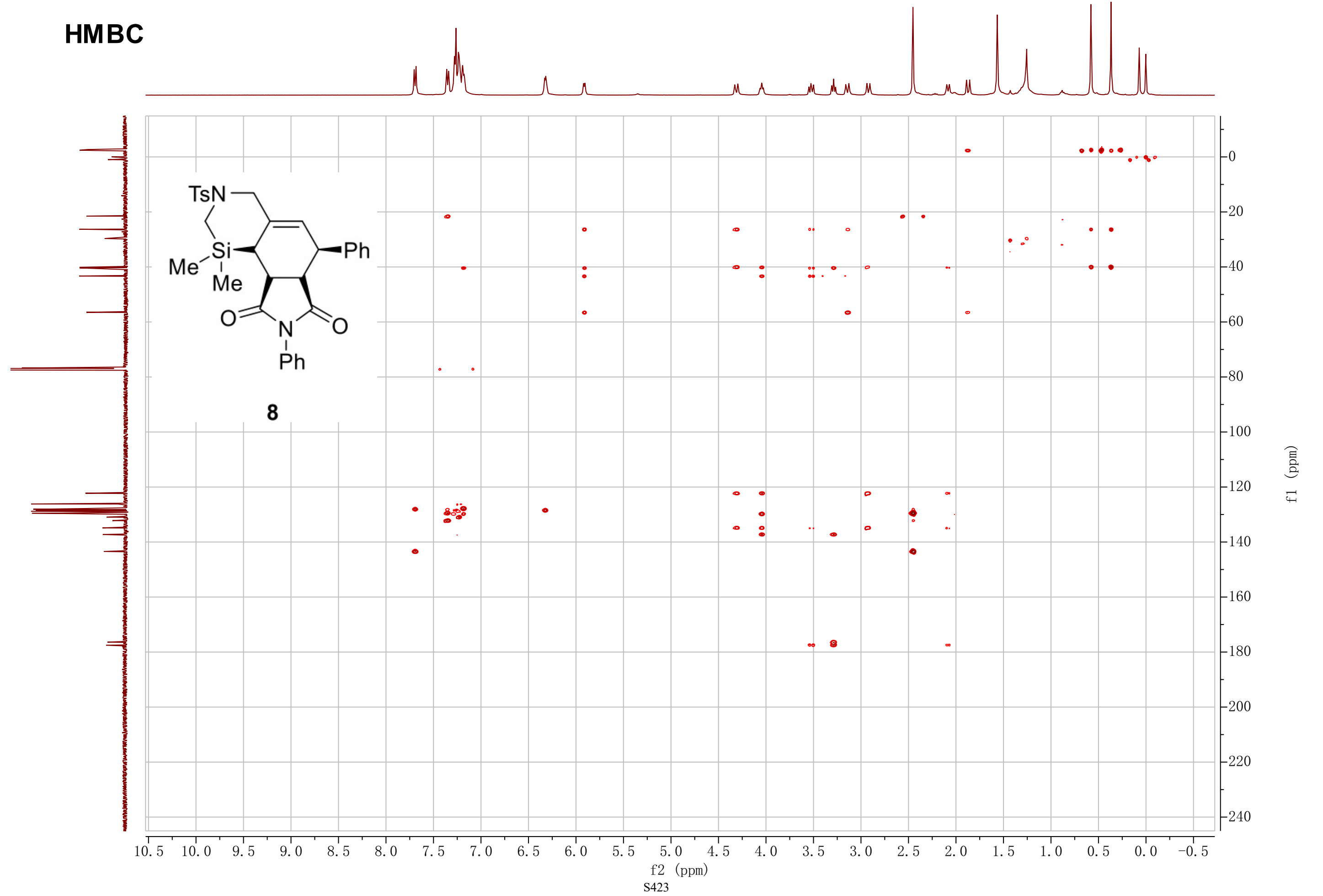




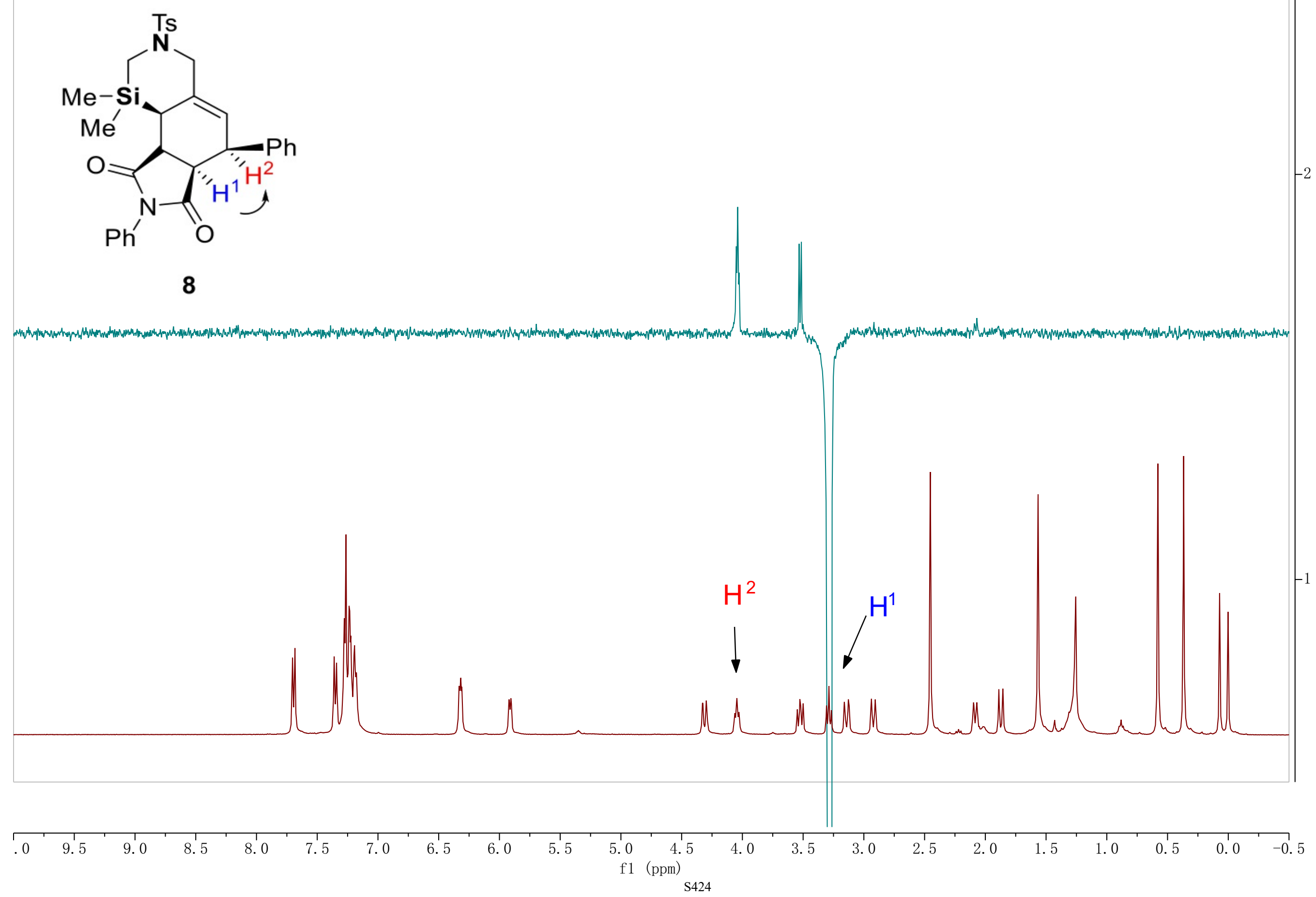




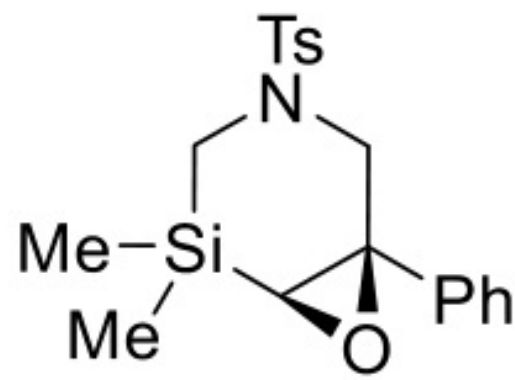

9

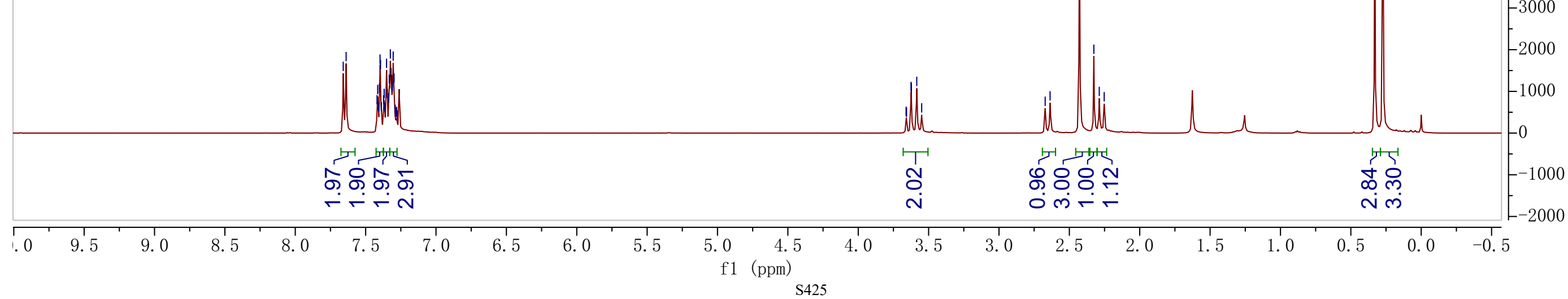




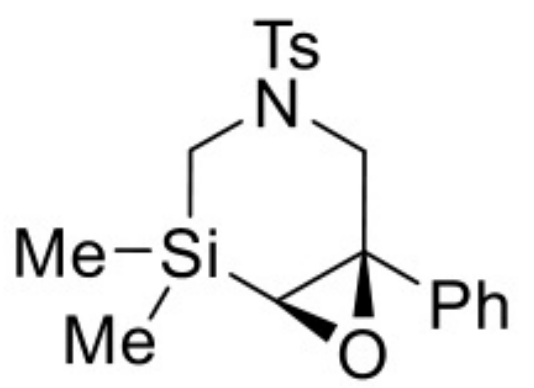

9

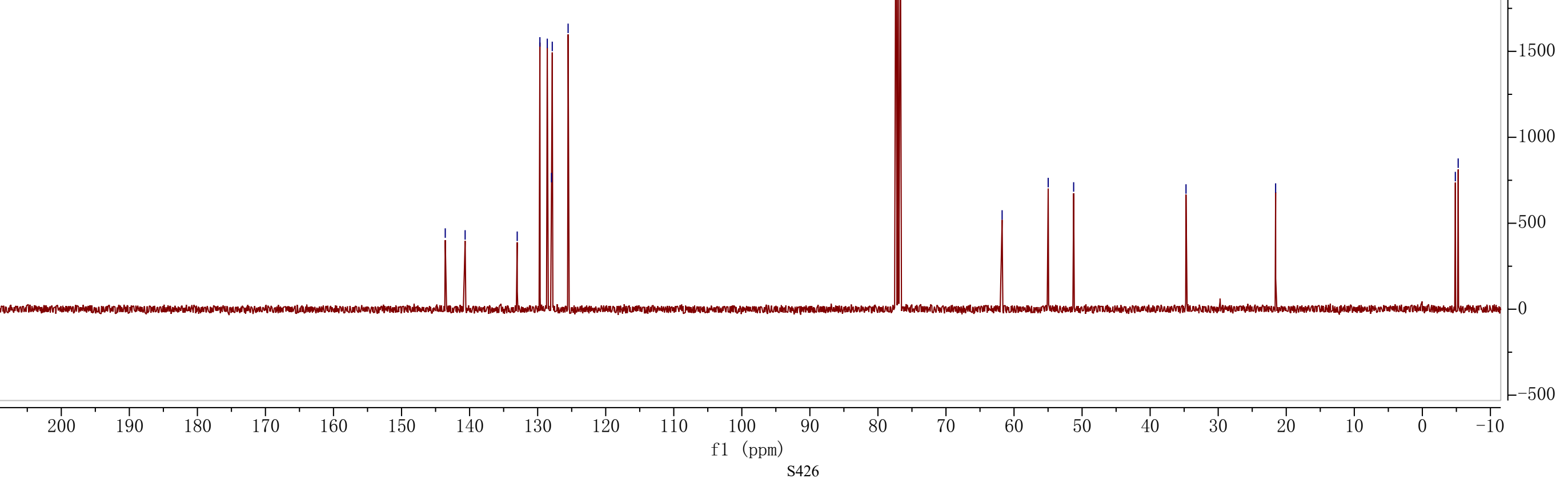


<smiles>C[Si]1(C)CN([As])C[C@@](O)(c2ccccc2)[C@H]1N</smiles>

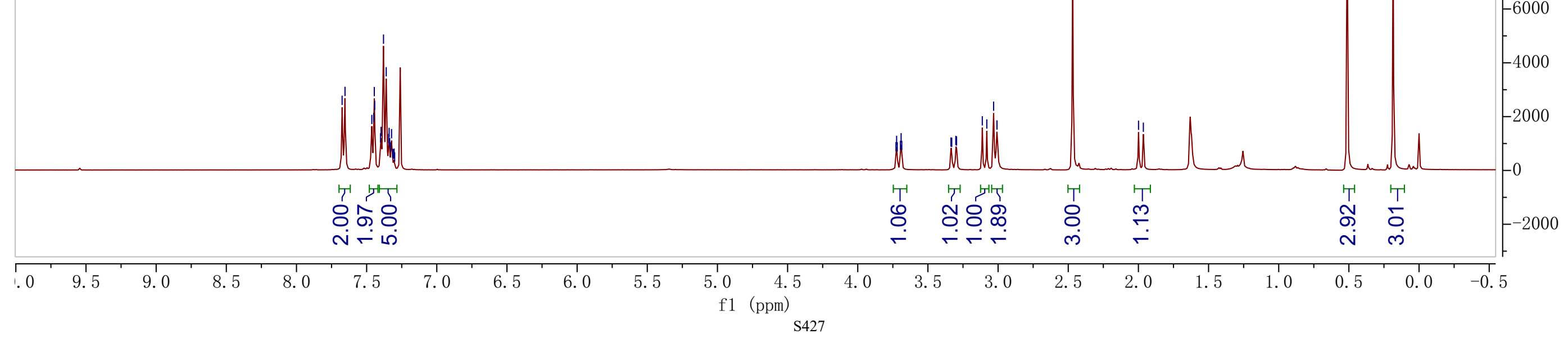


<smiles>C[Si]1(C)CN([As])CC(O)(c2ccccc2)[C@H]1N</smiles>
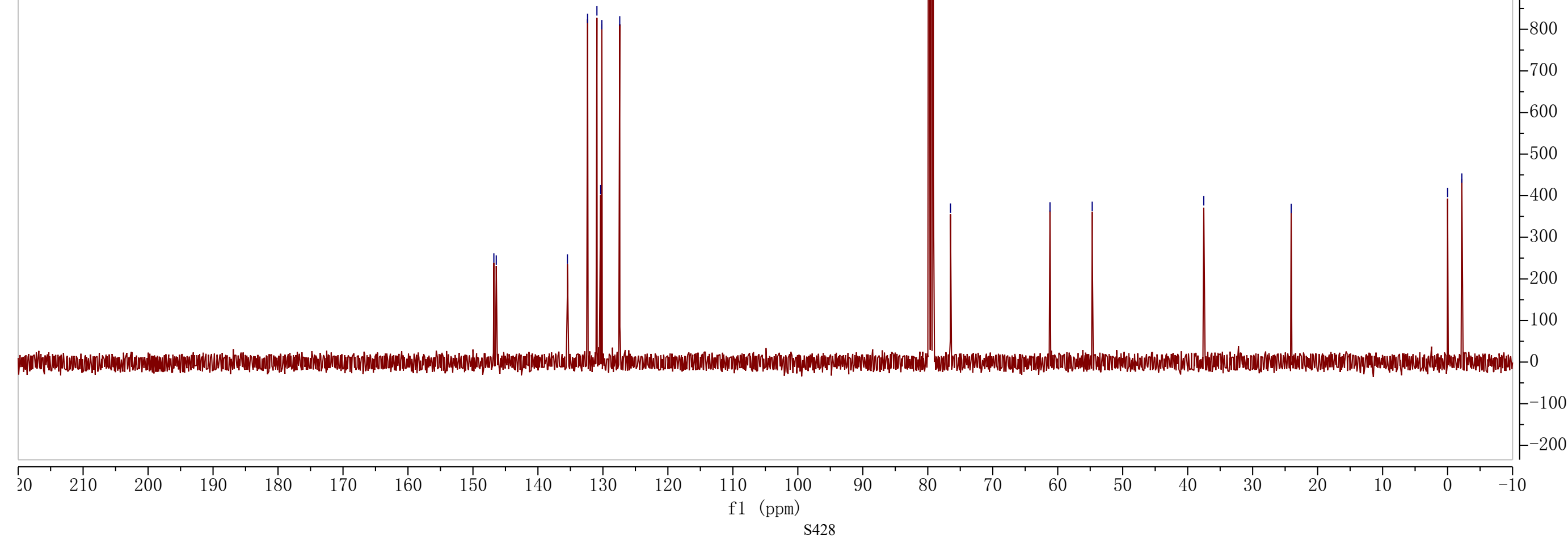
<smiles>C[Si]1(P(c2ccccc2)c2ccccc2)CC(c2ccccc2)CN([AsH3])C1</smiles>

11

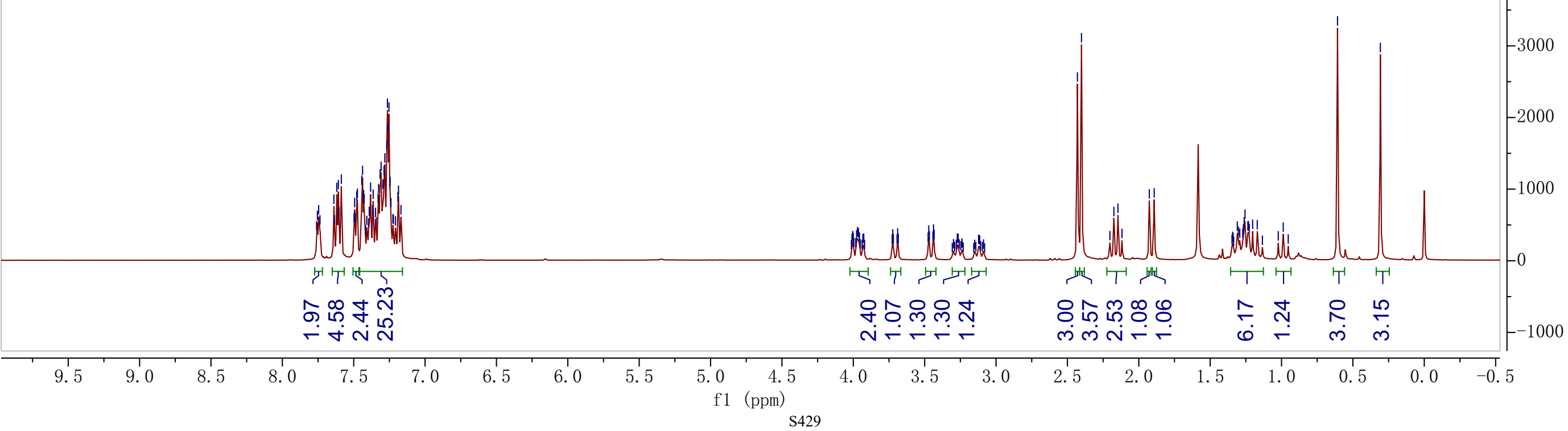


<smiles>C[Si]1(P)CC(c2ccccc2)CN([AsH3])C1</smiles> 


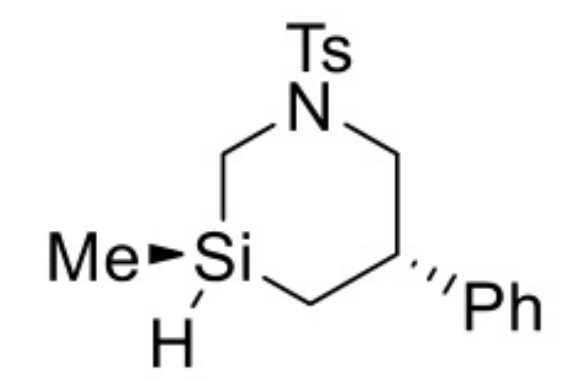

$12 a$

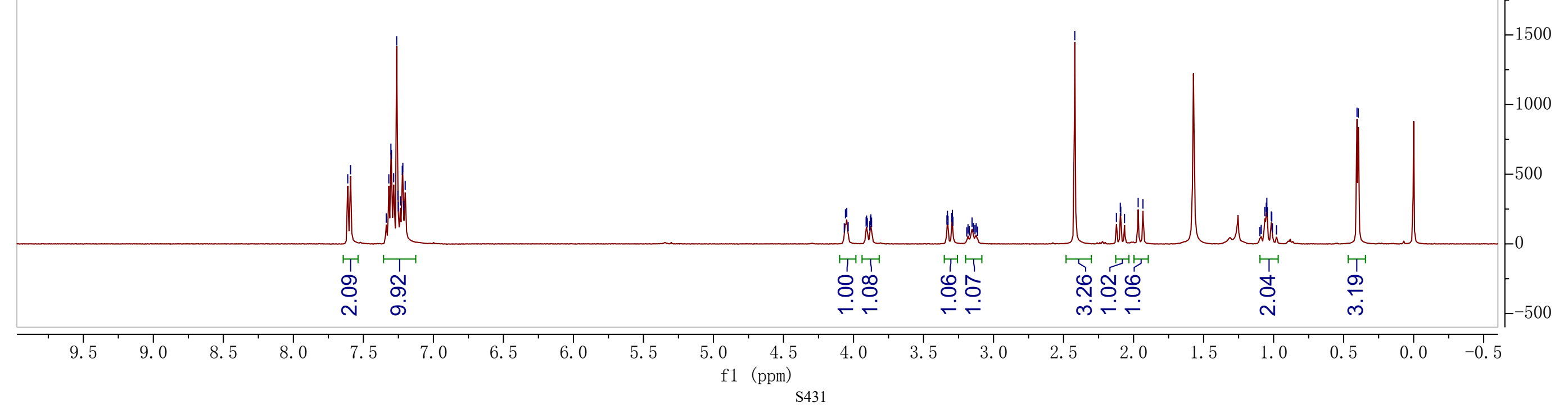


Ts<smiles>C[Si@@H]1CC([PbH])CN([AsH3])C1</smiles>

$12 a$$$
\text { . }
$$ 


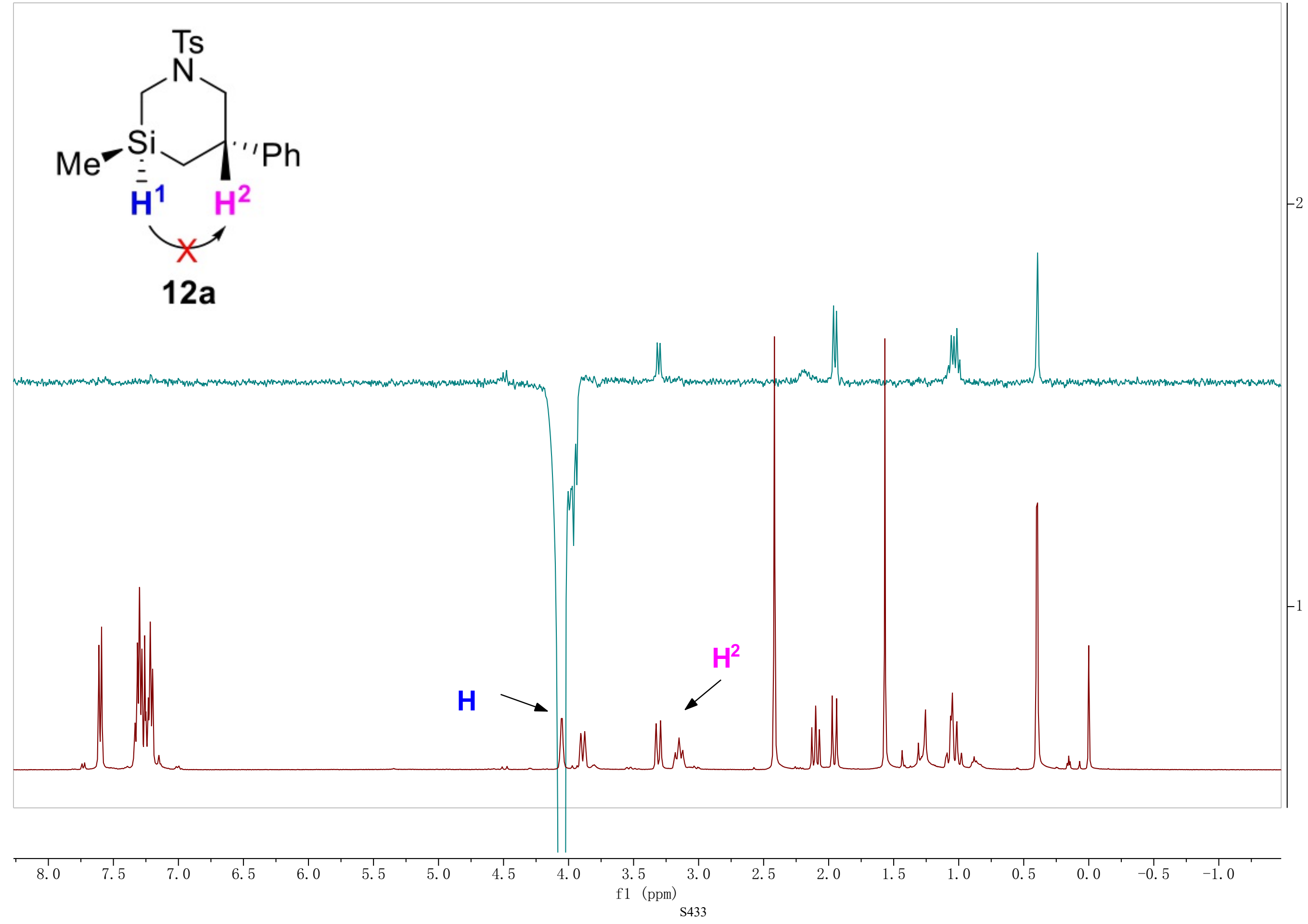




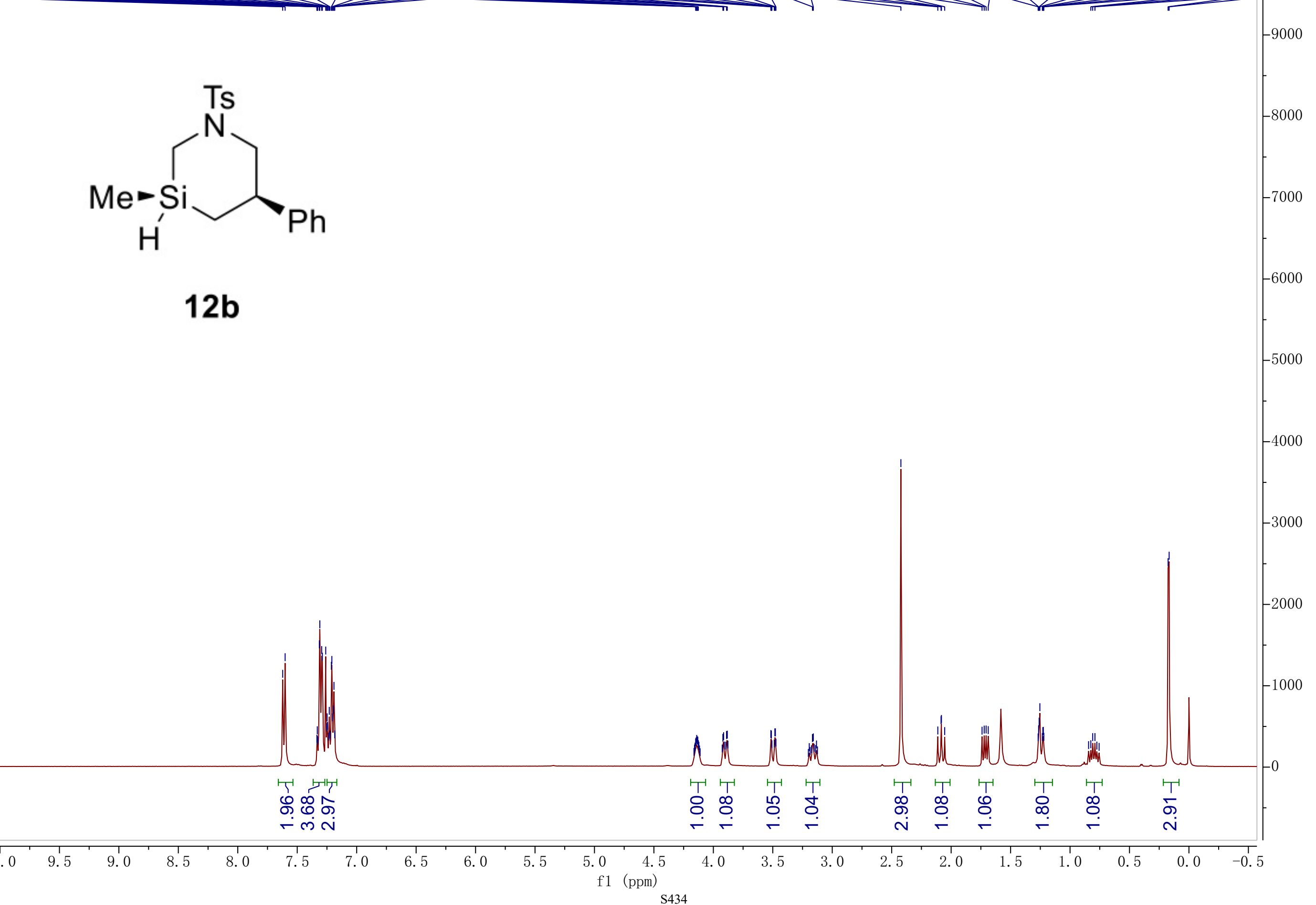


Ts<smiles>C[Si]1([SiH3])C[C@@H](c2ccccc2)CN([AsH3])C1</smiles>

$12 b$

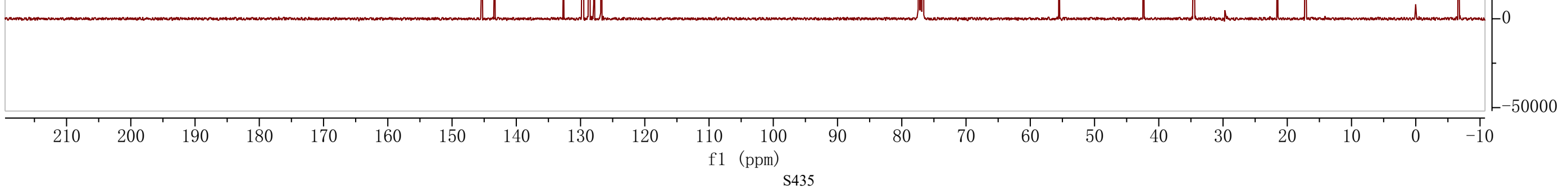




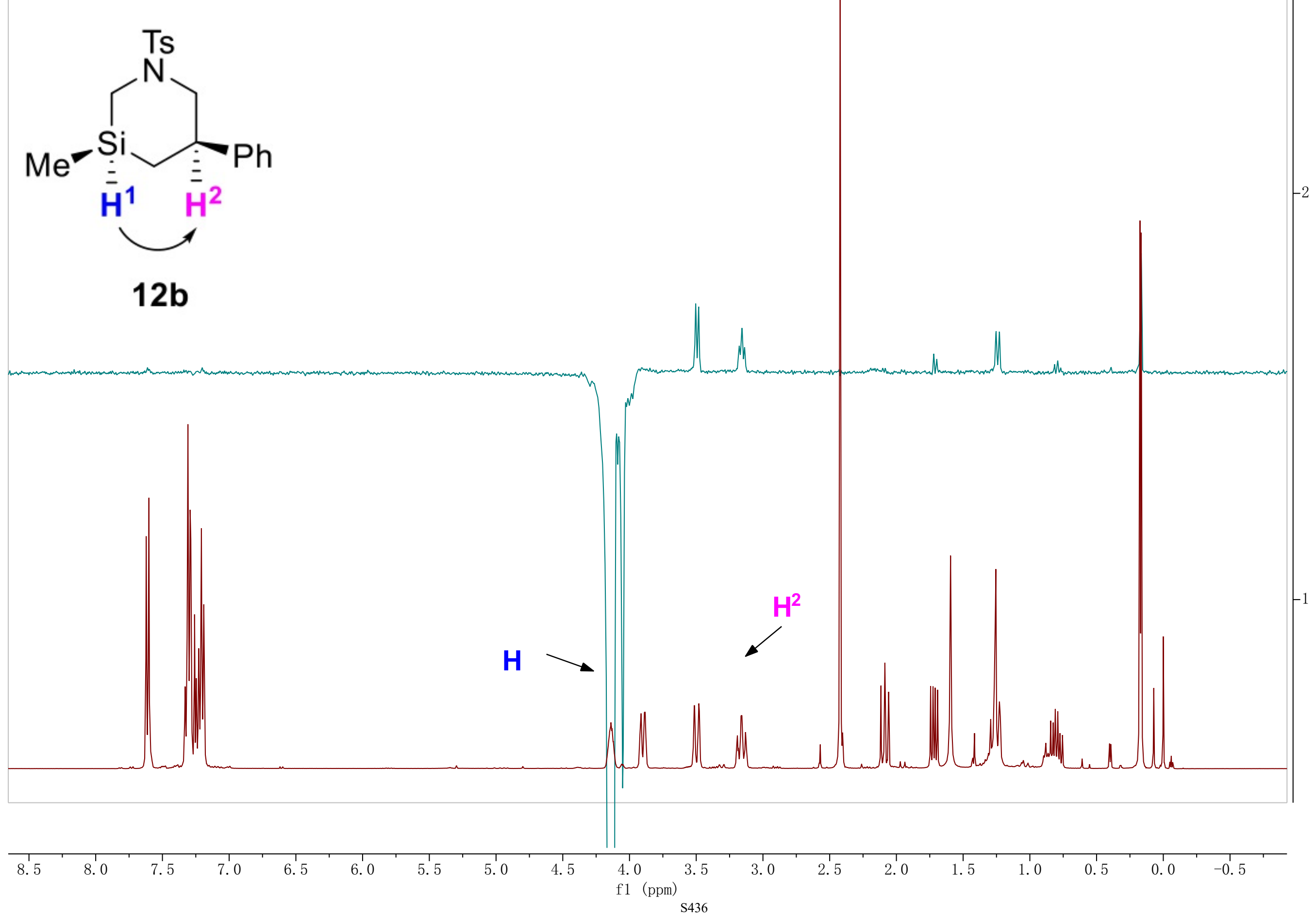


<smiles>C[Si]1(C)CC(c2ccccc2)C[NH+](Cl)C1</smiles>

13

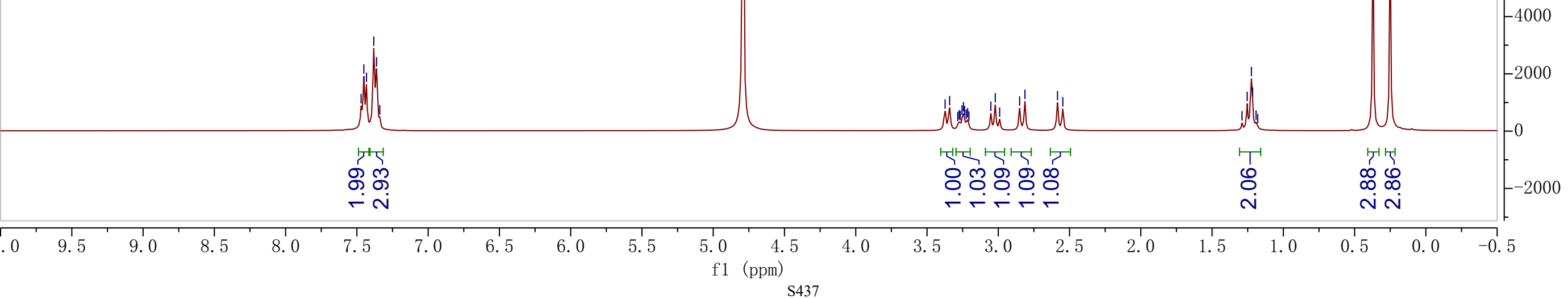




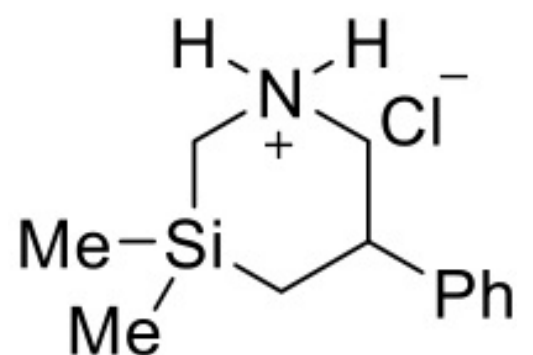

13

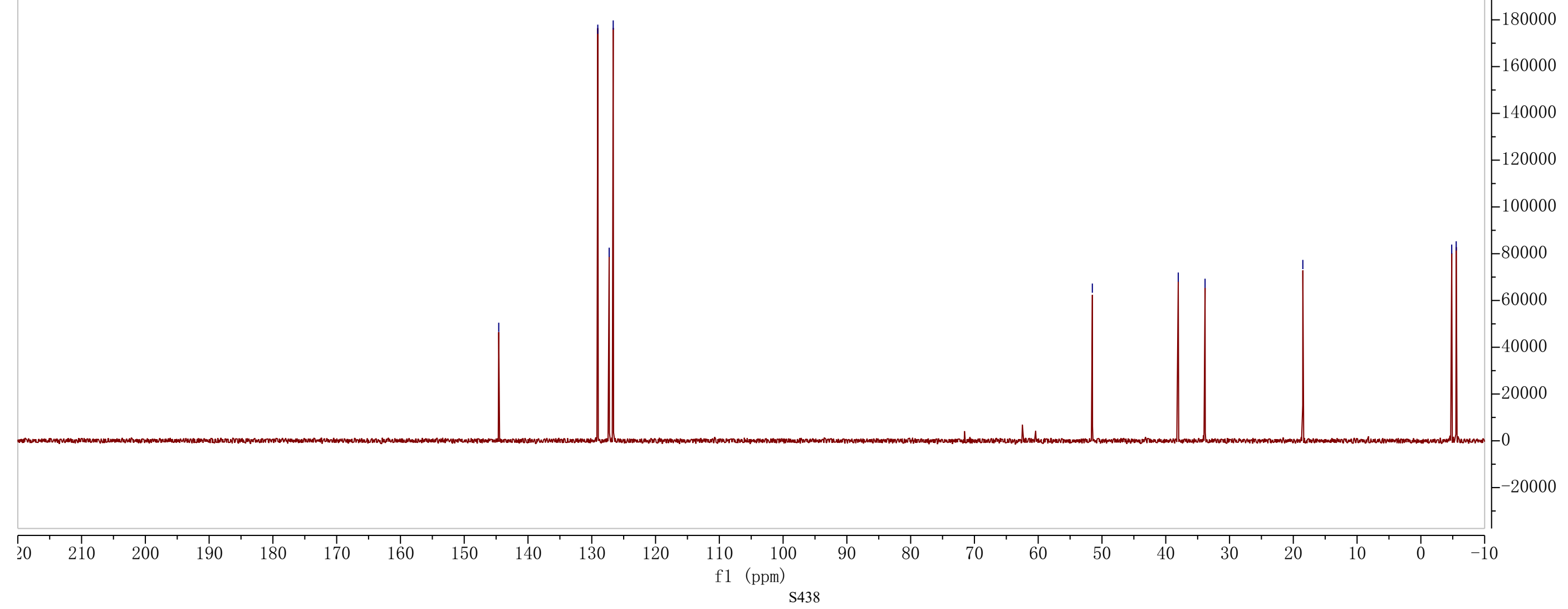



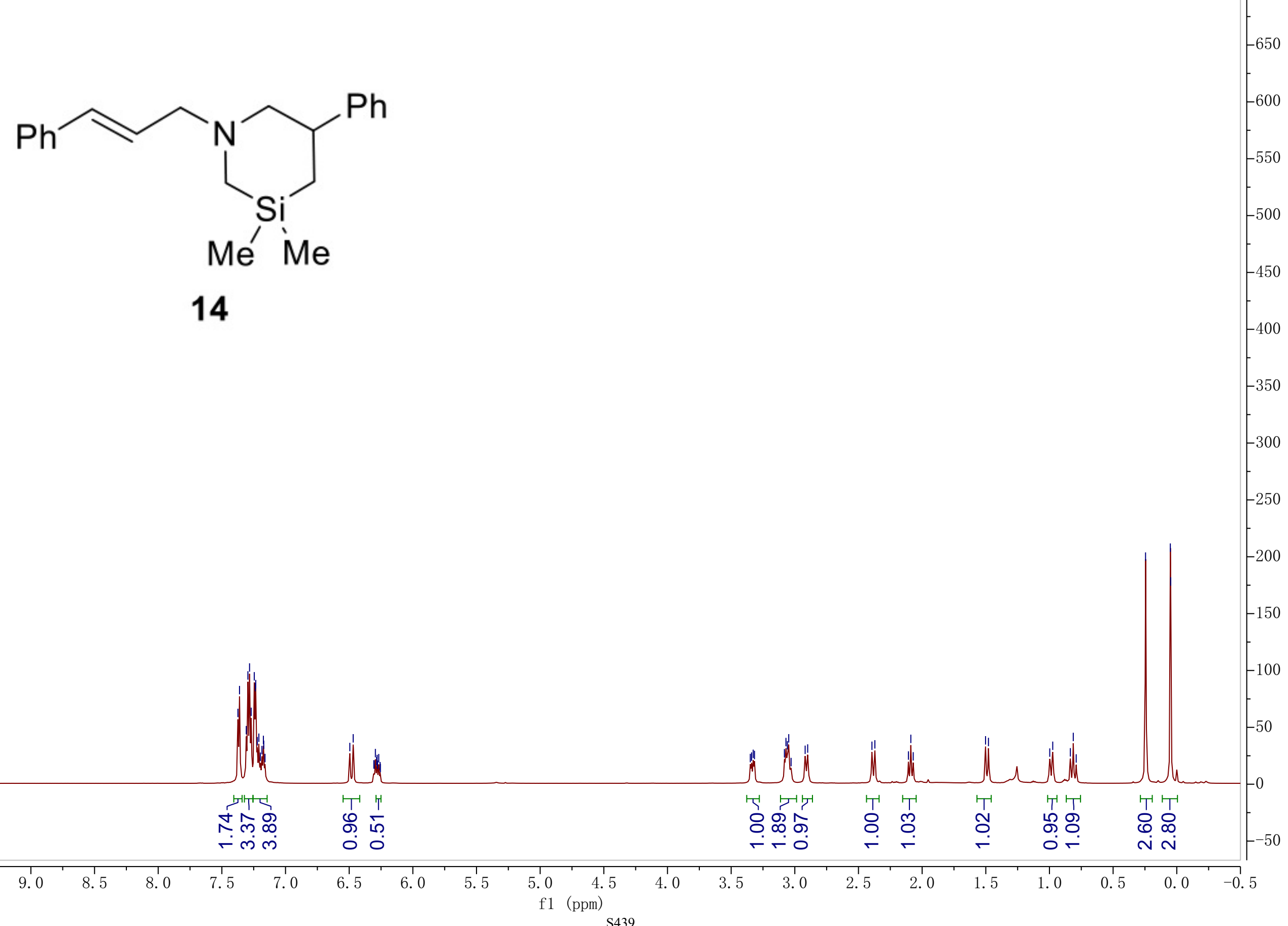
<smiles>CC(CN(C)C/C=C/c1ccccc1)c1ccccc1</smiles>

$\mathrm{Ph}$<smiles>CN1C[SiH2]CC(c2ccccc2)C1</smiles>

Me $\mathrm{Me}$

14

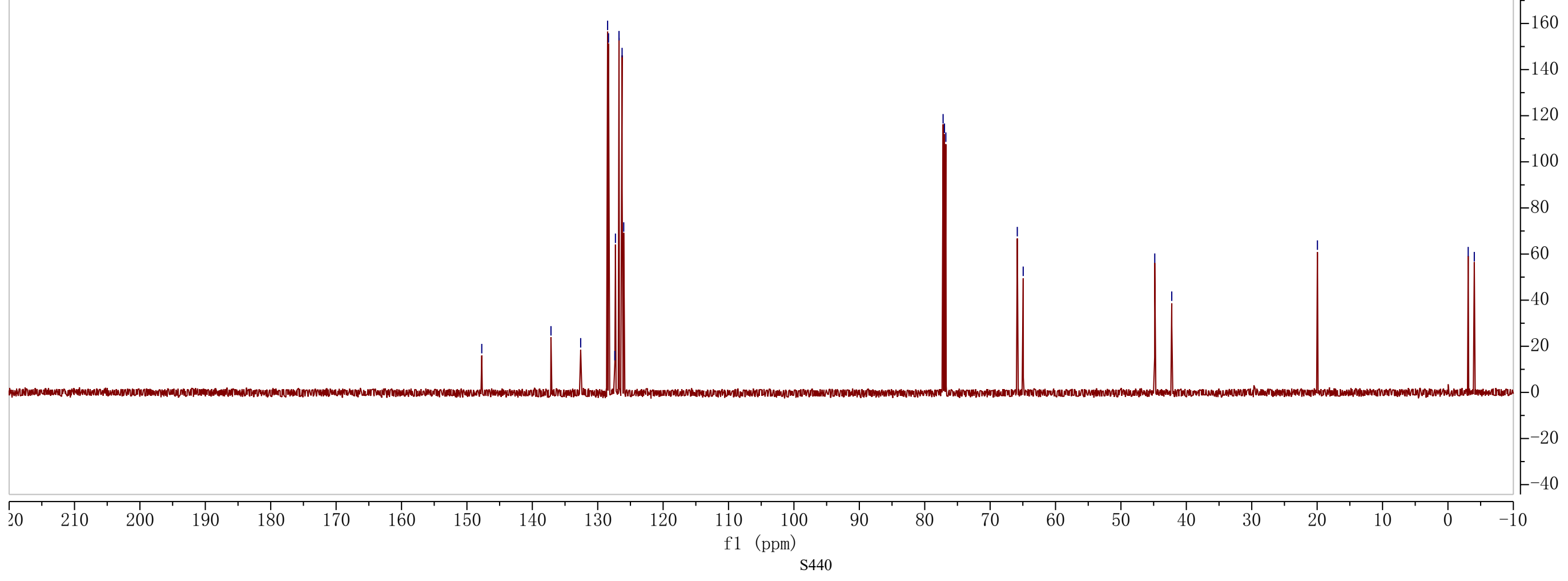




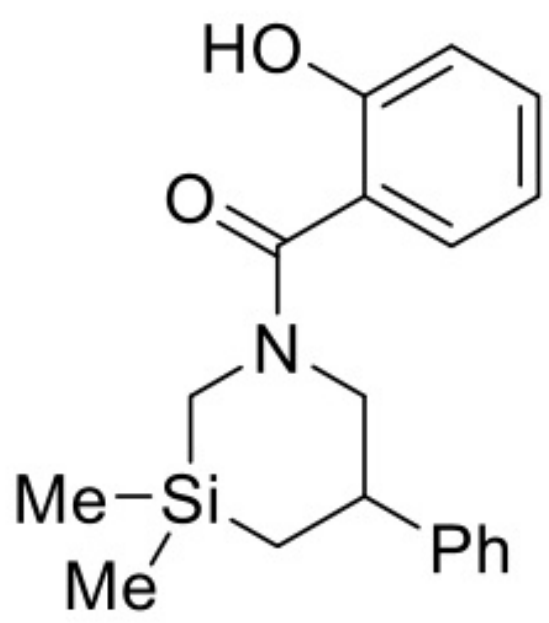

15

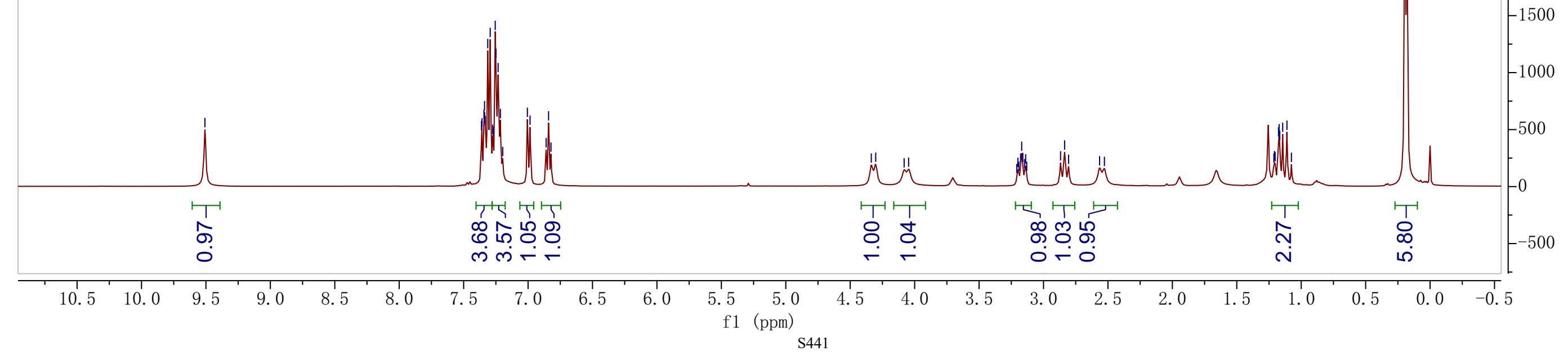




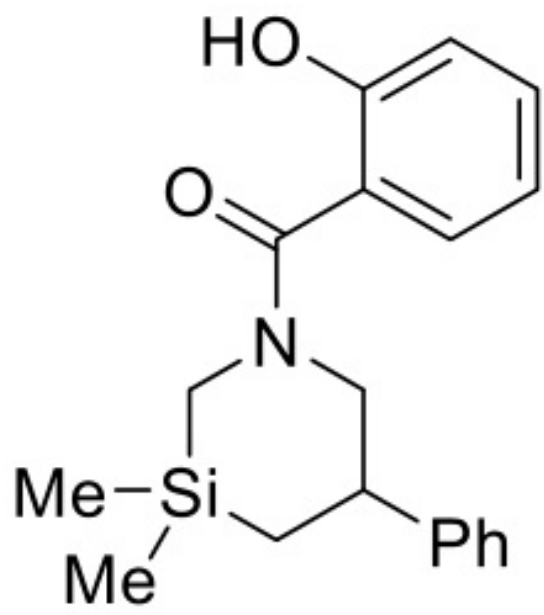

15 